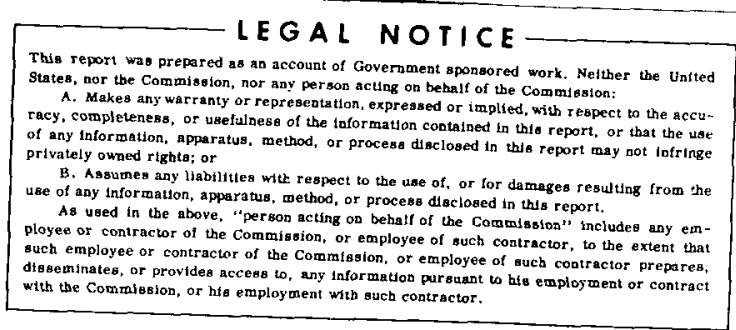

SUPPLEMENTARY REPORT ON EVALUATION OF MASS SPECTROMETRIC AND RADIOCHEMICAL_ANALYSES OF YANKEE CORE I SFENT FUEL, INCLUDING ISOTOPES OF ELEMENTS THORIUM THROUGH CURIUM

R. J . Nodvik

Nuclear Operations Analysis

With contributions by:

J. Alsina

N. Azziz

J. A. Corbett

H. E. Krug

J. E. O1hoeff

S. Semanderes

\title{
August 1969
}

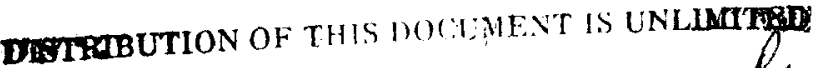

Prepared for the New York Operations Office

U. S. Atomic Energy Commission

Under AEC Contract Number AT (30-1)-3017

T̃echnical Approval:

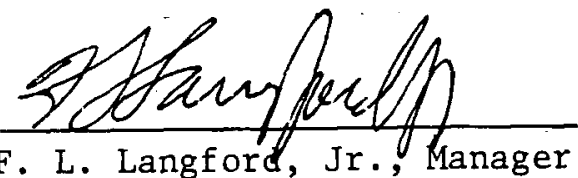

Nuclear Operations

PWR Systems Engineering
Project Manager Approval

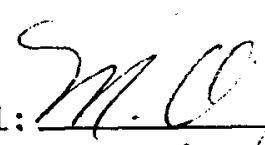

M. J. O'Boýle, Monager Yankee Cofe

Evaluation Program 


\section{DISCLAIMER}

This report was prepared as an account of work sponsored by an agency of the United States Government. Neither the United States Government nor any agency Thereof, nor any of their employees, makes any warranty, express or implied, or assumes any legal liability or responsibility for the accuracy, completeness, or usefulness of any information, apparatus, product, or process disclosed, or represents that its use would not infringe privately owned rights. Reference herein to any specific commercial product, process, or service by trade name, trademark, manufacturer, or otherwise does not necessarily constitute or imply its endorsement, recommendation, or favoring by the United States Government or any agency thereof. The views and opinions of authors expressed herein do not necessarily state or reflect those of the United States Government or any agency thereof. 


\section{DISCLAIMER}

Portions of this document may be illegible in electronic image products. Images are produced from the best available original document. 


\begin{tabular}{|c|c|c|}
\hline J. Alsina & A.S. Kitzes & M. Raymund \\
\hline H.N. Andrews & F. Kramer & E. Roberts \\
\hline B.H. Axelson & H.E. Krug (5) & J.B. Roll (5) \\
\hline N. Azziz & E.L. Kuno & B.M. Rothleder \\
\hline G.H. Bantley (15) & P.G. Lacey & E.S. Schwartz \\
\hline R.F. Barry (5) & J.C. Lee & S. Semanderes \\
\hline D.C. Beatty & F.L. Langford, Jr. (12) & W.R. Smalley \\
\hline E.S. Beckjord & W.D. Leggett (8) & T. Stern \\
\hline W.A. Bezella & W. Lester & A.P. Suda (6) \\
\hline L. Chajson & A.F. McFarlane (10) & G.R. Taylor \\
\hline J.A. Corbett (5) & R.G. McGrath & J.J. Taylor \\
\hline R.A. Dannels (5) & J. Melehan & D.E. Thorn \\
\hline W.F. Davis & D.L. Miller (10) & L.S. Tong \\
\hline R.A. Dean & R.S. Miller & H.J. von Hollen \\
\hline W.J. Dollard & G.H. Minton & H.E. Wa1ch1i \\
\hline W.J. Eich & J.S. Moore & J.H. Wright \\
\hline G.H. Farbman & B.N. Naft & G.D. Workman \\
\hline H.M. Ferrari & R.J. Nodvik (40) & J.M. Yadon \\
\hline R.J. French & M.J. O'Boyle & T.I.C. (3) \\
\hline J.M. Gallagher & J.E. Olhoeff & \\
\hline D.T. Galm & E. Paxson & \\
\hline P.B. Haga & W.L. Orr (5) & \\
\hline J. Haley & L.P. Picone & \\
\hline W.S. Hazelton & C.G. Poncelet & \\
\hline A.J. Impink & D.J. Povejsil & \\
\hline K.R. Jordan & E.U. Powel1 & \\
\hline S. Kellman & E.P. Rahe & \\
\hline
\end{tabular}


EXTERNAL DISTRIBUTION

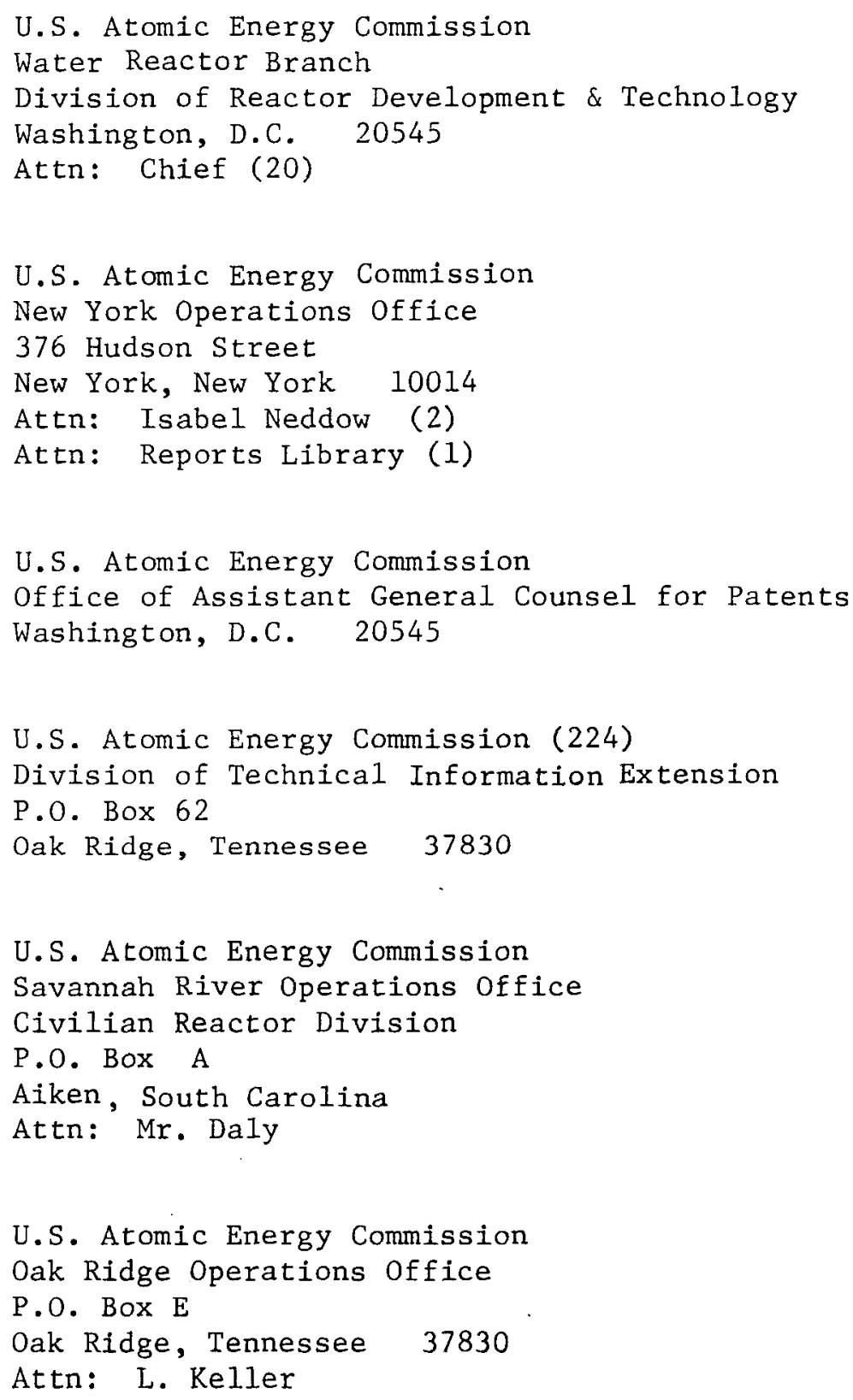




\section{EXTERNAL DISTRIBUTION (Cont)}

Yankee Atomic Electric Company 441 Stuart Street

Boston, Massachusetts

Attn: Roger J. Coe (6)

Oak Ridge National Laboratory

Chemical Technology Division

P.O. Box X

Oak Ridge, Tennessee 37839

Attn: E.D. Arnold

Carolina Virginia Nuclear Power Associates, Inc.

Parr Site

Parr, South Carolina

Attn: Mr. H.T. Babb, Manager 


\section{ABSTRACT}

This report presents the results of mass and alpha spectrometric, x-ray spectrographic, and radiochemical analyses of spent fuel samples irradiated in YankeeRowe Cores I, II, and IV. In addition to the analyses for the main chain uranium and plutonium isotopes, analyses for certain other isotopes of the actinide series were performed, including Th-232, Pa-231, U-232, and U-233 in unirradiated fuel samples, and U-232, U-233, Np-237, $\mathrm{Pu}-236, \mathrm{Pu}-238$, $\mathrm{Am}-241, \mathrm{Am}-243, \mathrm{Cm}-242$, and $\mathrm{Cm}-244$ in irradiated fuel samples.

A significant aspect of this study is the comparison of the measured isotopic data with results obtained with a matrix exponential calculational method incorporated in the LASER-HIC Computer program. This method uses the ENDF/B cross section data for those isotopes for which such data are available, and calculated cross sections for those isotopes for which such data have not been established. These comparisons indicate the validity of the theoretical model and the relative accuracy of the basic nuclear data documented within this report.

Several methods for the determination of fuel burnup are applied to measured data and evaluated. As an example, the value of burnup inferred from $\mathrm{Nd}-148$ concentration is compared with corresponding values of burnup inferred from Cs-137 and Sr-90 activity, and the conservation of heavy element nuclei. 


\section{ACKNOWLEDGMENTS}

The author gratefully acknowledges the efforts of the many individuals of the Westinghouse Nuclear Energy Systems who contributed to the successful completion of this program. Special thanks are extended to the contributors listed at the front of this report, and to W. J. Eich, J. Jedruch, and C. G. Poncelet for their many helpful suggestions, and to D.B. Vogtman who assisted in the LASER-HIC program modifications. Appreciation is extended to R. Forbes, D. L. Mckown, J. M. Moran, and R. L. Hofer for their aid in the reduction of the voluminous data collected in this program. 
TABLE OF CONTENTS

Section

Title

Page

ABSTRACT

$\mathrm{v}$

1

INTRODUCTION . . . . . . . . . . . . . . . . 1-1

2

DESCRIPTION OF THE YANKEE CORE . . . . . . . . . . . 2-1

3

SAMPLING PLAN . . . . . . . . . . . . . . . 3-1

3.1 Selection of Phase 3 Fuel Rods . . . . . . . 3-2

3.2 Selection of Fuel Samples Within a Fuel Rod . . . 3-5

3.3 Selection of Fuel Samples for EYC Program

Extension . . . . . . . . . . . . . 3-9

4

ANALYSES PERFORMED ON SPENT FUEL SAMPLES . . . . . . . 4-1

4.1 Description of Analyses Performed . . . . . . . . 4-2

4.2 Results of Analyses Performed . . . . . . . . 4-3

5

TREATMENT OF DATA . . . . . . . . . . . . . 5-1

5.1 End-of-Life Isotopic Inventory Equations . . . . 5-1

5.2 Isotopic Decay Corrections . . . . . . . . . 5-5

6 EVALUATION OF SEVERAL METHODS FOR DETERMINING FUEL BURNUP . . . . . . . . . . . . . . . 6-1

6.1 Determination of Fuel Burnup . . . . . . . . 6-2

6.2 Comparison of $\mathrm{Cs}-137$ and $\mathrm{Sr}-90$ Burnup Indicators

With Heavy Element Burnup Indicator . . . . . 6-11

6.3 Comparison of $\mathrm{C} s-137$ and Heavy Element Burnup

Indicators With $\mathrm{Nd}-148$ Burnup Indicator . . . . 6-13

7

GAMMA ACTIVITY MEASUREMENTS. . . . . . . . . . . 7-1

7.1 Single-Channel Gross Gamma Scans . . . . . . 7-2

7.2 Multi-Channel Gamma Scans . . . . . . . . 7-19 
TABLE OF CONTENTS (Cont)

Section

Title

Page

8

THEORY FOR CALCULATIONS OF ISOTOPES OF ELEMENTS

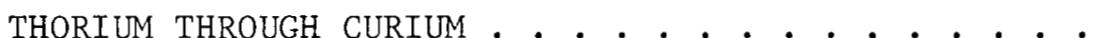

8.1 Calculational Model . . . . . . . . . . 8-1

8.1.1 Mathematical Description . . . . . . 8-2

8.1.2 Numerical Solution . . . . . . . . 8-5

8.1.3 Computer Calculation . . . . . . . . 8-7

8.1.4 Separation of the A Matrix . . . . . . 8-8

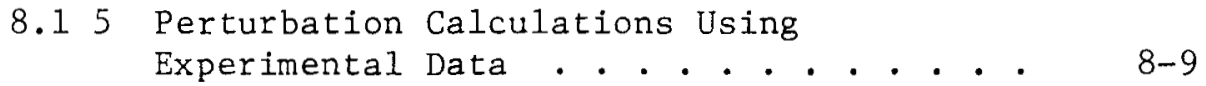

8.2 Nuclide Transmutation Chain Description . . . . 8-10

BASIC NUCLEAR DATA USED IN CALCULATIONS . . . . . . 9-1

9.1 Cross Section Values for EYC Program

Extension .............. . . 9-1

9.1.1 Cross Sections for $(n, \gamma)$ Reaction . . . . 9-11

9.1.2 Cross Sections for $(n, f)$ Reaction . . . . 9-12

9.1.3 Cross Sections for $(n, 2 n)$ Reaction. . . 9-12

9.1.4 Cross Sections for Isomers of $\mathrm{Am}-242$. . . . . . . . . . . . . 9 9-13

9.1.5 Definition of Group Structure and Group Cross Section . . . . . . . . 9-13

9.2 Decay Constant Values for EYC Program

Extension ............. . 9-15

10 DESCRIPTION OF UNIT CELL CALCULATIONS PERFORMED IN THE EYC PROGRAM . . . . . . . . . . . . . . . . 10-1

10.1 Representation of Irradiation History in Unit Ce11 Calculations .. . . . . . . . 10-3

10.2 Yankee Core IV Environmental Effect on Neutron Spectrum ............. 1... 10-4

10.3 Initial Isotopic Composition of Yankee Core I

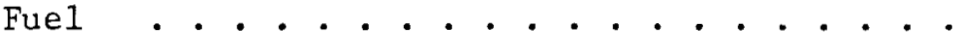

10.4 Cross Sections Used in LASER-HIC Calculations ............ . . 10-18

10.5 Estimate of Reactivity Effect Due to Buildup of Special Isotopes ........... . 10-18 
TABLE OF CONTENTS (Cont)

Section

Title

Page

10.6 Comparison of HIC and LASER Calculations of Main Chain Uranium and Plutonium

Isotopic Concentrations . . . . . . . . 10-30

11 COMPARISON BETWEEN CALCULATED AND MEASURED ISOTOPIC BUILDUP AND DEPLETION IN YANKEE CORE I FUEL • . . . . . . . . . . . . . . . . . . 11-1

11.1 Main Chain $\mathrm{U}$ and $\mathrm{Pu}$ Isotopic Abundances and $\mathrm{Pu} / \mathrm{U}$ Mass Ratio . . . . . . . . . . . . 11-3

11.2 Main Chain Uranium and Plutonium Isotopic Concentration . . . . . . . . . . . . 11-7

11.3 Concentration of Special Isotopes . . . . . . . 11-22

12 SUMMARY AND CONCLUSIONS • • • • • • • • • • • . • • 12-1

13

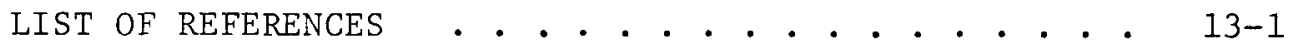

Appendix A Summary of Results from Mass Spectrometric and Radiochemical Analyses . . . . . . . . . . . . A-1

Appendix B Summary of Burnup, Atom Densities, and Fuel Characteristics Determined From Mass Spectrometric, Alpha Spectrographic, and Radiochemical Analyses . . . B-1

Appendix C Cross Section Library For LASER-HIC . . . . . . . . . . C-I

Appendix D Summary of Measurements for Special Isotopes . . . . . D-I 


\section{LIST OF ILLUSTRATIONS}

Figure

Title

Page

2-1 Core Location of Fuel Assemblies and Control Rod Groups in the Yankee Reactor

$2-3$

$2-2$

Fuel Cycling in First Four Yankee Cores

$2-5$

$2-3$

Power History of Yankee Core, January 1961 to

December 1962

$2-7$

$2-4$

Power History of Yankee Core, January 1963 to

December 1964

$2-8$

$2-5$

Power History of Yankee Core, January 1965 to

August 1965

$2-9$

3-1 Plan View of EYC Phase 3 Fuel Assembly Showing

Locations of Fuel Rods Removed for Physics

Analysis

$3-3$

$3-2$

Typical Yankee Flux Wire Activity Trace

$3-6$

$3-3$

Axial Location of Spent Fuel Samples Within a Fuel Rod

$3-7$

$3-4$

Core Location of EYC Fuel Rods Selected for PostIrradiation Analyses

$3-10$

$5-1$

Parameters Used in Reduction of Isotopic Data

$5-4$

$6-1$

Calculated Energy Release Per Fission in U-235, $\mathrm{U}-238, \mathrm{Pu}-239$, and $\mathrm{Pu}-241$

$6-5$

Calculated Correction Factor for Nd-148 Burnout

versus Fuel Burnup

$6-10$

Comparison of Total Fissions Inferred from Cs -137

and Sr-90 with Total Fissions Inferred from Heavy

Elements

$6-12$

$6-4$

Comparison of Total Fissions Inferred from Heavy Elements and Cs-137 with Total Fissions Inferred from $\mathrm{Nd}-148$

6-5 U-235 Fractional Depletion versus Total Accumulated Fissions

Fuel Burnup versus Total Accumulated Fissions 


\section{LIST OF ILLUSTRATIONS (Cont)}

\begin{tabular}{|c|c|c|}
\hline Figure & Title & Page \\
\hline $7-1$ & $\begin{array}{l}\text { Axial Distribution of Gross Gamma Activity in } \\
\text { EYC Phase } 3 \text { Fuel Rod E6-NW-al }\end{array}$ & $7-4$ \\
\hline $7-2$ & $\begin{array}{l}\text { Axial Distribution of Gross Gamma Activity in } \\
\text { EYC Phase } 3 \text { Fuel Rod E6-C-a1 }\end{array}$ & $7-6$ \\
\hline $7-3$ & $\begin{array}{l}\text { Normalized Axial Gross Gamma Activity and } \\
\text { Burnup Distributions in Fuel Rod No. } 369 \text {, Core } \\
\text { Location E6-C-al }\end{array}$ & $7-7$ \\
\hline $7-4$ & $\begin{array}{l}\text { Normalized Axial Gross Gamma Activity and Burnup } \\
\text { Distributions in Fuel Rod No. 5352, Core Location } \\
\text { E6-C-a6 }\end{array}$ & $7-8$ \\
\hline $7-5$ & $\begin{array}{l}\text { Normalized Axial Gross Gamma Activity and Burnup } \\
\text { Distributions in Fuel Rod No. } 536 \text {, Core Location } \\
\text { E6-C-fl }\end{array}$ & $7-9$ \\
\hline $7-6$ & $\begin{array}{l}\text { Normalized Axial Gross Gamma Activity and Burnup } \\
\text { Distributions in Fuel Rod No. } 354 \text {, Core Location } \\
\text { E6-C-f6 }\end{array}$ & $7-10$ \\
\hline $7-7$ & $\begin{array}{l}\text { Normalized Axial Gross Gamma Activity and Burnup } \\
\text { Distributions in Fuel Rod No. } 3198 \text {, Core Location } \\
\text { E6-SE-c2 }\end{array}$ & $7-11$ \\
\hline $7-8$ & $\begin{array}{l}\text { Normalized Axial Gross Gamma Activity and Burnup } \\
\text { Distributions in Fuel Rod No. } 3187 \text {, Core Location } \\
\text { E6-SE-e } 4\end{array}$ & $7-12$ \\
\hline $7-9$ & $\begin{array}{l}\text { Normalized Axial Gross Gamma Activity and Burnup } \\
\text { Distributions in Fuel Rod No. } 3113 \text {, Core Location } \\
\text { E6-SE-f5 }\end{array}$ & $7-13$ \\
\hline $7-10$ & $\begin{array}{l}\text { Normalized Axial Gross Gamma Activity and Burnup } \\
\text { Distributions in Fuel Rod No. } 44 \text {, Core Location } \\
\text { E6-NW-al }\end{array}$ & $7-14$ \\
\hline $7-11$ & $\begin{array}{l}\text { Axial Distribution of } 2 r-95-- \text { Nb- } 95 \text { Activity in } \\
\text { EYC Phase } 3 \text { Fuel Rods Determined from Multi-Channel } \\
\text { Gamma Scans }\end{array}$ & $7-22$ \\
\hline $7-12$ & $\begin{array}{l}\text { Axial Distribution of } 2 r-95 \text { Activity in EYC Phase } 3 \\
\text { Fuel Rods Determined from Radiochemical Analysis }\end{array}$ & $7-23$ \\
\hline $7-13$ & $\begin{array}{l}\text { Axial Distribution of } \mathrm{Zr}-95 \text { in Several EYC Phase } 3 \\
\text { Fuel Rods Determined by Radiochemical and Gamma } \\
\text { Spectrometric Analyses }\end{array}$ & $7-24$ \\
\hline $8-1$ & Transmutation Processes Affecting Isotope $N_{i}$ & $8-3$ \\
\hline
\end{tabular}


LIST OF ILLUSTRATIONS (Cont)

\begin{tabular}{|c|c|c|}
\hline Figure & Title & Page \\
\hline $8-2$ & HIC Depletion Chains & $8-11$ \\
\hline $8-3$ & Transmutation Chain Description for Am-242 & $8-13$ \\
\hline $10-1$ & $\begin{array}{l}\text { Calculated Fast-to-Thermal Flux Ratio versus U-235 } \\
\text { Depletion in the Yankee Recycled Fuel Assembly }\end{array}$ & $10-9$ \\
\hline $10-2$ & $\begin{array}{l}\text { Calculated Pu-239 Atom Density versus U-235 Depletion } \\
\text { in Yankee Cores I, II, and IV }\end{array}$ & $10-10$ \\
\hline $10-3$ & $\begin{array}{l}\text { Calculated Concentrations of the Isotopes of the } \\
\text { Elements Thorium Through Curium Versus Accumulated } \\
\text { Fissions in Yankee Core I Fuel }\end{array}$ & $10-19$ \\
\hline $11-1$ & Uranium Isotopic Abundances versus Total Fissions & $11-4$ \\
\hline $11-2$ & Plutonium Isotopic Abundance versus Total Fissions & $11-5$ \\
\hline $11-3$ & $\begin{array}{l}\text { Calculated and Measured Pu/U Mass Ratio versus } \\
\text { Total Fissions }\end{array}$ & $11-6$ \\
\hline $11-4$ & U-234 Concentrations versus Accumulated Fissions & $11-8$ \\
\hline $11-5$ & U-235 Concentrations versus Accumulated Fissions & $11-9$ \\
\hline $11-6$ & U-236 Concentrations versus Accumulated Fissions & $11-10$ \\
\hline $11-7$ & U-238 Concentrations versus Accumulated Fissions & $11-11$ \\
\hline $11-8$ & $\begin{array}{l}\text { Effective Capture-to-Fission Ratio in U-235 versus } \\
\text { Total Accumulated Fissions }\end{array}$ & $11-14$ \\
\hline $11-9$ & Pu-239 Concentrations versus Accumulated Fissions & $11-15$ \\
\hline $11-10$ & Pu-240 Concentrations versus Accumulated Fissions & $11-16$ \\
\hline $11-11$ & Pu-241 Concentrations versus Accumulated Fissions & $11-17$ \\
\hline $11-12$ & Pu-242 Concentrations versus Accumulated Fissions & $11-18$ \\
\hline $11-13$ & $\begin{array}{l}\text { Fissile Atoms Discharged/Fissile Atoms Loaded versus } \\
\text { Total Accumulated Fissions }\end{array}$ & $11-20$ \\
\hline $11-14$ & NGD/MWD versus Total Accumulated Fissions & $11-21$ \\
\hline $11-15$ & $\begin{array}{l}\text { Calculated and Measured } \mathrm{Np}-237 \text { as a Function of } \\
\text { Accumulated Fissions }\end{array}$ & $11-23$ \\
\hline $11-16$ & $\begin{array}{l}\text { Calculated and Measured Pu-238 Concentration as a } \\
\text { Function of Accumulated Fissions }\end{array}$ & $11-25$ \\
\hline $11-17$ & $\begin{array}{l}\text { Calculated and Measured Pu-236 Concentration as a } \\
\text { Function of Accumulated Fissions }\end{array}$ & $11-27$ \\
\hline $11-18$ & $\begin{array}{l}\text { Calculated and Measured U-232 Concentrations versus } \\
\text { Total Fissions }\end{array}$ & $11-28$ \\
\hline
\end{tabular}




\section{LIST OF ILLUSTRATIONS (Cont)}

Figure

Title

Page

11-19 Calculated and Measured U-233 Concentrations versus Burnup in Yankee Core I Fuel

$11-32$

11-20 Calculated and Measured Am-241 Concentrations as a Function of Accumulated Fissions

11-21 Calculated and Measured Am-243 Concentrations as a Function of Accumulated Fissions

$11-36$

11-22 Calculated and Measured $\mathrm{Cm}-242$ Concentration as a Function of Accumulated Fissions

$11-38$

11-23 Calculated and Measured $\mathrm{Cm}-244$ Concentration as a Function of Accumulated Fissions

$11-39$ 
2-2 Operating Summary for Yankee Cores I, II, III, and IV

Fuel Rods Selected for Physics Analysis in Phase 3 of the EYC Program

Axial Location of Spent Fuel Samples Within a Fuel Rod

Summary of Phase 3 and Program Extension Sample Locations and Analyses

6-1 Parameters Used to Determine Fuel Burnup from Isotopic and Fission Product Measurements

Nd-148 Percent Yield from Fission

Summary of Gross Gamma Activity of EYC Phase 3 Spent Fuel Rods

7-2 Comparison of Gross Gamma Activity with Fuel Sample Burnup in EYC Phase 3 Fuel Rods

7-3 Summary of Rod Average Gross Gamma Activity and Burnup

$$
7-20
$$

Summary of Gamma Activity Peaking Factors in the EYC Phase 3 Fuel Assembly

Summary of Sources of Cross Section Data

9-5 Values of Decay Constants Used in EYC Program Extension

10-1 Summary of LEOPARD Unit Cell Calculations for EYC Axial Zones 1, 3, 5, and 6 
LIST OF TABLES (Cont)

Table

Title

Page

$\mathrm{C}-1$

HIC Isotopic Identification Numbers

$\mathrm{C}-2$

LASER Thermal Energy Mesh

$\mathrm{C}-2$

$\mathrm{C}-3$

Library of Cross Sections for LASER - HIC Calculations

$\mathrm{C}-3$

D-1

Summary of Measurements for U-232

$\mathrm{C}-4$

D -3

D-2

D-3

D-4

D-5

Summary of Measurements for Np-237

D-4

Summary of Measurements for Am-241

D-5

Summary of Measurements for Am-243

D-7

Summary of Measurements for Cm-242 and $\mathrm{Cm}-244$

$\mathrm{D}-8$ 


\section{SECTION 1}

\section{INTRODUCTION}

This report presents the mass spectrometric, the alpha spectrometric, the $X$-ray spectrographic, and the radiochemical data from a number of spent Yankee fuel samples which were analyzed under Phase 3 of the Yankee Core Evaluation (EYC) Program, ${ }^{[1]}$ plus additional samples from Phases 1, 2, and 3 which were analyzed under an extension ${ }^{[2]}$ to the EYC Program.

The EYC Program Extension represents a broadening of program scope to include, in addition to the analysis of the main chain uranium and plutonium isotopes (U-234, U-235, U-236, U-238, Pu-239, Pu-240, Pu-241, and Pu-242), the analysis of certain other isotopes of the actinide series of elements including Th-232, $\mathrm{Pa}-231, \mathrm{U}-232$, and $\mathrm{U}-233$ in unirradiated fuel samples, and U-232, Pu-236, $\mathrm{Np}-237, \mathrm{Pu}-238, \mathrm{Am}-241, \mathrm{Am}-243, \mathrm{Cm}-242$, and $\mathrm{Cm}-244$ in depleted fuel samples. Also, the Program Extension includes the analysis and evaluation of the stable non-volatile fission product $\mathrm{Nd}-148$ as an accurate fuel burnup indicator. A significant aspect of the EYC Program Extension is the comparison of the measured isotopic data with results obtained with a matrix exponential calculational method* used in conjunction with the LASER ${ }^{[4]}$ computer program, and employing ENDF/B cross section data for those isotopes for which such data are available.

In addition to the analyses described above, non-destructive gamma activity measurements were carried out and the results are compared with measured burnup distributions. The information in this report supplements the Phase 1 and Phase 2 data which are reported and evaluated in WCAP-6068. [5]

* The matrix exponential method of analysis was introduced at Westinghouse by Alsina[1] and is similar to that described previously by Duane.[3] 
Under the EYC Program, 56 fuel rods were removed from fourteen Core I fuel assemblies (Phase 1), 7 fuel rods were removed from one of two Core I assemblies recycled in Core II (Phase 2), and 8 fuel rods were removed from the Core I assembly which was recycled in Cores II and IV for extended burnup (Phase 3). These fuel rods were subjected to extensive post-irradiation examinations, destructive and non-destructive. Non-destructive examinations consisted of visual inspections, dimensional checks, ${ }^{[6]}$ and gamma-activity measurements. [7] Destructive examinations included: chemical defilming of cladding surfaces, [8] fission gas collection and analysis; hardness, burst and tensile testing of cladding specimens; ${ }^{[6]}$ and $X$-ray spectrographic, mass spectrometric, and radiochemical analysis of spent fuel, and, in some instances, cladding. [5] The mass spectrometric data reported in Reference 5 include only the main chain uranium and plutonium isotopes.

Phase 1 represents the analysis of pellet-size samples from 191 preselected locations within Yankee Core I, spanning a range of burnups from 1,300 to 18,000 megawatt days per metric ton of initial uranium loading (MWD/MTU). The data obtained in Phase 1 of the 5-year EYC Program have been utilized to infer the assembly and core-total uranium and plutonium isotopic inventory and burnup of Core I. A complete description of the assembly and core-total results can be found in topical report WCAP-6071, [9] and a comparison of these results with the fuel input accountability determined during reprocessing of the Core I fuel* is given in WCAP-3896-1. [10]

Phase 2 represents the analyses of pellet-size samples from 28 locations in the Core I assembly which was recycled in Core II, and spans a burnup range from 10,000 to 31,000 MWD/MTU. As a test of analytical methods, the Phase 1 and Phase 2 data have been compared with depletion calculation results in topical report WCAP-6077. [11]

Phases 1 and 2 also include the analysis of microsamples along the radii of the cross section of a few pellets. The microsample results are reported and evaluated in topical report WCAP-6069.

* Fifty metric tons of fuel discharged from the first four Yankee cores were reprocessed by Nuclear Fuel Services of West Valley, New York during the period from June through September, 1967. 
Phase 3 represents the analyses of pellet-size samples from 32 locations in the Core I assembly which was recycled in Core II and again in Core IV for extended burnups, ranging from 16,000 to $46,000 \mathrm{MWD} / \mathrm{MTU}$. In addition to the pellet-size samples, microsamples were obtained along the radii of two pellet cross sections.

Upon the completion of Phase 3, the EYC Program was extended to include analysis of 26 additional Phase 1, 2, and 3 samples for the special fuel isotopes listed above, as well as for the main chain uranium and plutonium isotopes. Small concentrations of $\mathrm{U}-232$ and $\mathrm{U}-233$ are formed as byproducts in irradiated fuel, and accumulate through the fuel enrichment - irradiation - reprocessing cycle. The strong and persistent gamma activity of U-232, U-233, and their daughter products is of concern in processing of spent fuel. Since the gamma activity interferes with operation of the enrichment process, limits on the allowable concentrations in diffusion plant level have been established by the Atomic Energy Commission; thus, it is important to determine theoretically and/or experimentally the degree to which these isotopes are present in spent fuel. In the case of the transuranium isotopes, interest centers on the alpha-emitters Pu-238, $\mathrm{Cm}-242$, and $\mathrm{Cm}-244$ and their usefulness as power-generating isotopes (heat sources) and their immediate precursors which could be separated and irradiated as targets to produce the desired isotopes. Increasing requirements for power-generating isotopes have directed attention to spent power-reactor fuel as a potential source of supply, developing a need for reliable quantitative data over a wide range of burnups. It is pointed out that the consideration of the negative reactivity contribution of some of these special isotopes, particularly Np-237 and Am-241, becomes increasingly important to the nuclear designer, especially as present generation reactor designs are directed toward higher average discharge burnup and plutonium recycle.

This report is organized so that a brief description of Yankee Cores I, II, and IV is given in the next section. The Phase 3 sampling plan is presented in Section 3, and the origin and treatment of the spent fuel data are given in Sections 4 and 5. The fuel pellet burnup and a comparison of several methods used to obtain the burnup are given in Section 6 , with particular 
emphasis placed on the evaluation of the stable fission product $\mathrm{Nd}-148$ as an accurate burnup indicator. In Section 7, the fuel rod gross gamma scan data are compared with corresponding burnup data. The analytic model and cross section data used in the unit cell calculations are discussed in sections 8 and 9; a description of the calculations performed is given in section 10. Calculated isotopic data are compared with results of measurements for pellet-sized samples in section 11. A summary and the conclusions reached in this study are stated in section 12 . 


\section{SECTION 2}

DESCRIPTION OF THE YANKEE CORE

Core I of the Yankee power reactor ${ }^{[12,13]}$ consisted of 76 fuel assemblies, each loaded with either 304 or 305 fuel rods arranged in a square lattice. In each assembly one fuel rod, located at approximately the center of the assembly, was omitted from the lattice to provide a space for the insertion of a flux wire thimble. (Only 22 assemblies subsequently were equipped with thimbles.) The total number of fuel rods in the core was 23,142. General data of the first core are listed in Table 2-1.

The basic component of the core was a stainless steel tube filled with approximately 150 slightly enriched $\mathrm{UO}_{2}$ pellets, each with a nominal length of 0.6 inch. The average height of the $\mathrm{UO}_{2}$ columns was 90.57 inches with a cross sectional area of 0.0590 square inch. In addition to the $\mathrm{UO}_{2}$ pellets, each fuel rod contained stainless steel spacer discs of total height of 0.61 inch and axial expansion voids of a total height of 0.71 inch, adding up to an active core height of 91.89 inches from the bottom of the lowest $\mathrm{UO}_{2}$ pellet to the top of the highest pellet.

The arrangement of the fuel assemblies in the core is shown in Figure 2-1. Also shown on the figure are the active control rods (solid black) which at some time during core operation presented their Ag-In-Cd absorbing sections to the neutron flux. The cross checked outer ring of control rod positions was filled with Zircaloy during power operation. (Half of these positions contain movable Group 6 control rods, while the other half contain cruciform shaped Zircaloy shim pieces.) On the periphery of the core are shown the stainless steel fillers, displacing water from the recesses in the fuel assemblies. The alpha-numeric matrix notation used to describe the core location of a particular fuel assembly is also shown on the figure. 
TABLE 2-1

YANKEE CORE I GENERAL DATA

Uranium fuel loading ( $\mathrm{kg}$ )

Fuel enrichment (w/o U-235)

Average core diameter (inches)

Active core height (inches)

Number of Ag-In-Cd control rods

Number of $\mathrm{Zr}$ shims

Nominal system pressure (psi)

Nominal main coolant temperature $\left({ }^{\circ} \mathrm{F}\right)$

Number of fuel assemblies

Number of fuel rods

Fuel rod pitch (inches)

Fuel rod outside diameter (inches)

Fuel rod stainless steel cladding

thickness (inches)

Fuel pellet diameter (inches)

Unit $\operatorname{cell} \mathrm{W} / \mathrm{U}$ $20,908^{(a)}$

3.4

75.1

91.9

24

8

2000

514

76

23,142

0.422 and 0.456

0.340

0.021

0.294

2.67

a. Determined from gravimetric measurements of pre-irradiated fuel. 


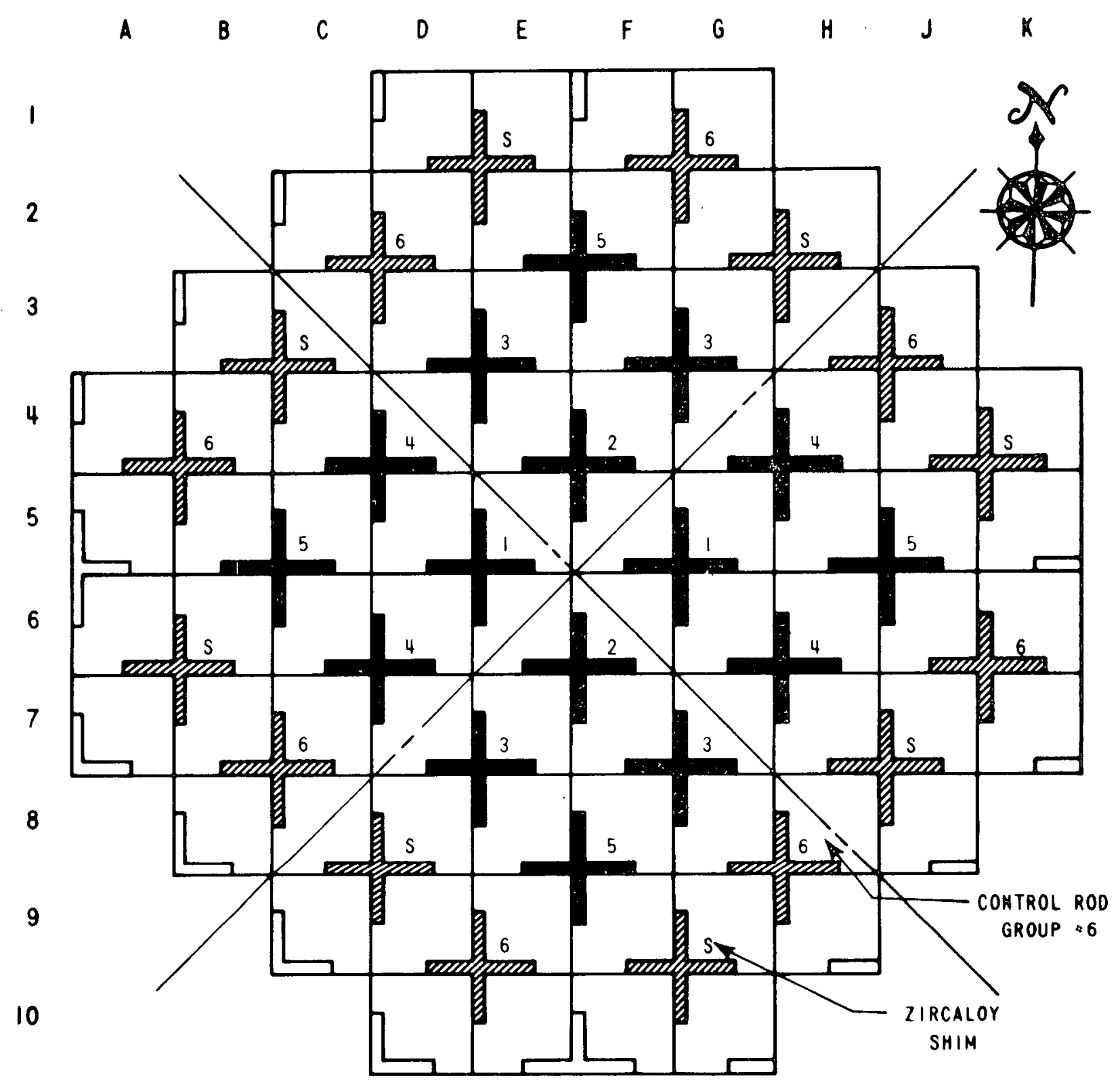

Figure 2-1. Core Location of Fuel Assemblies and Control Rod Groups in the Yankee Reactor 
The second Yankee core was nominally identical to Core I except for the presence of two spent Core I fuel assemblies located in the central region of the core and a modified control rod design.

A modified fuel cycling program was initiated in the third Yankee core and continued in the fourth core. The fuel cycling scheme and the enrichment of the feed assemblies are shown on Figure 2-2. Note that the Phase 3 fuel assembly occupied core position E6 and was irradiated in Cores I, II, and IV. During Core III operation, the Phase 3 assembly was stored in the Yankee fuel storage pit.

The operating summaries for the first four Yankee cores are given in Table 2-2. An accurate record exists of the total energy release in the first four Yankee cores based on the calorimetric measurements obtained during reactor operation. A plot of the reactor power versus time for the periods during which Cores $I$, II, III, and IV were operated at power is shown in Figures 2-3 through 2-5. These periods extend from January 1961 until May 1962 for Core I, from September 1962 until September 1963 for Core II, from November 1963 unti1 August 1964 for Core III, and from September 1964 until August 1965 for Core IV. In addition, some low power physics and startup tests [14] were made on Core I between November 1960 and January 1961. The relatively small amount of energy released during those tests is included in the totals given for Core I in Table 2-2. 


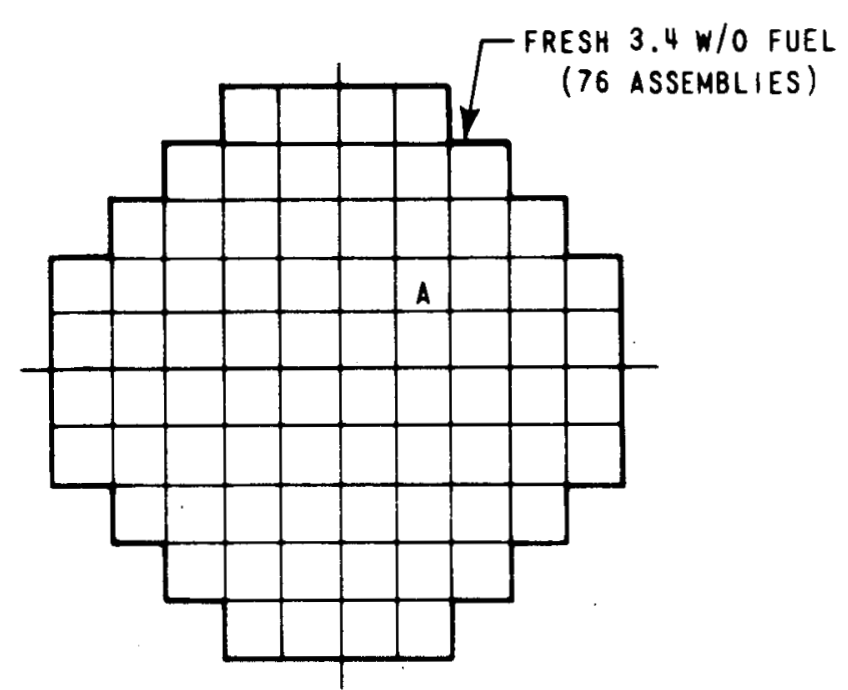

CORE I (8470 MWD/MTU)

N

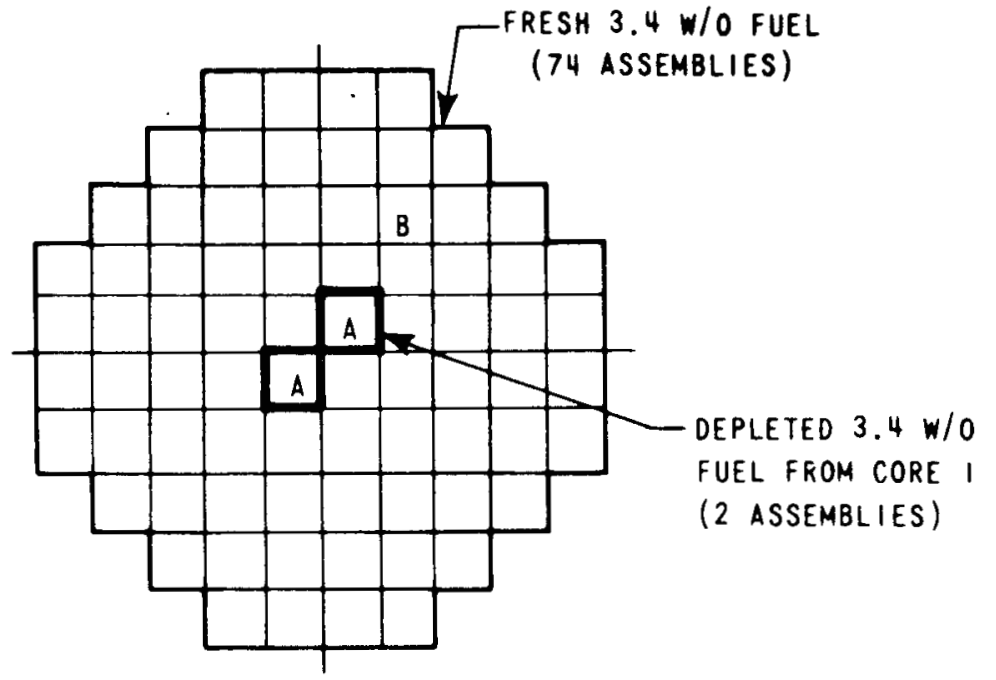

CORE II (7870 MWO/MTU)

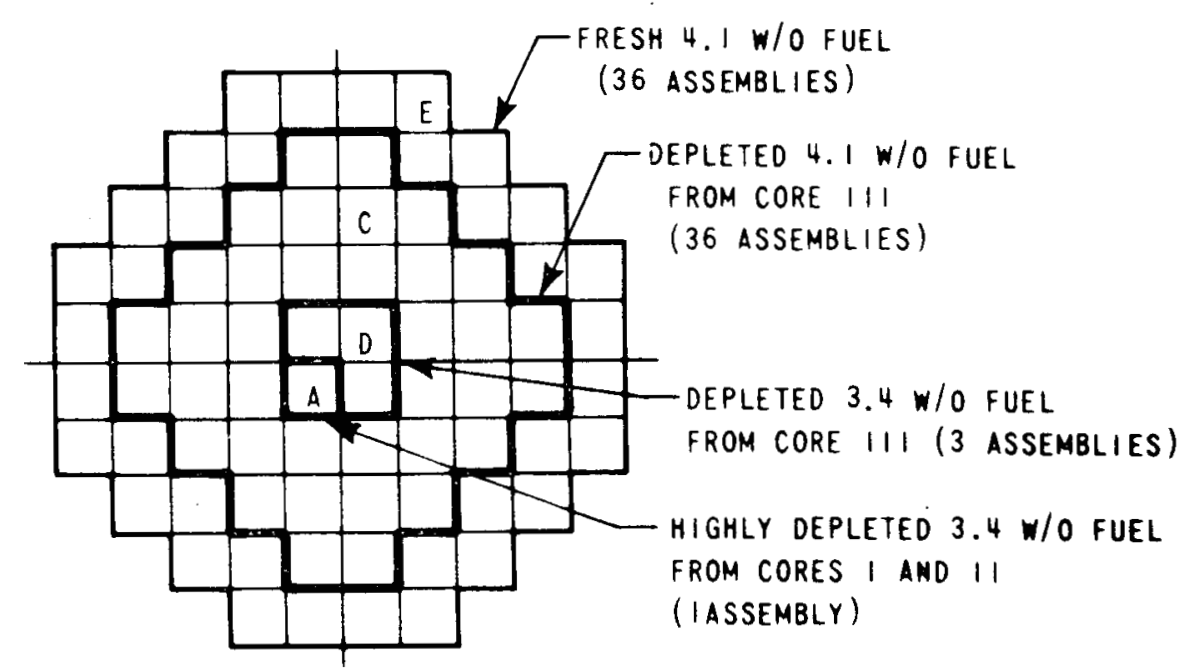

CORE IV ( 8720 MHD/MTU)

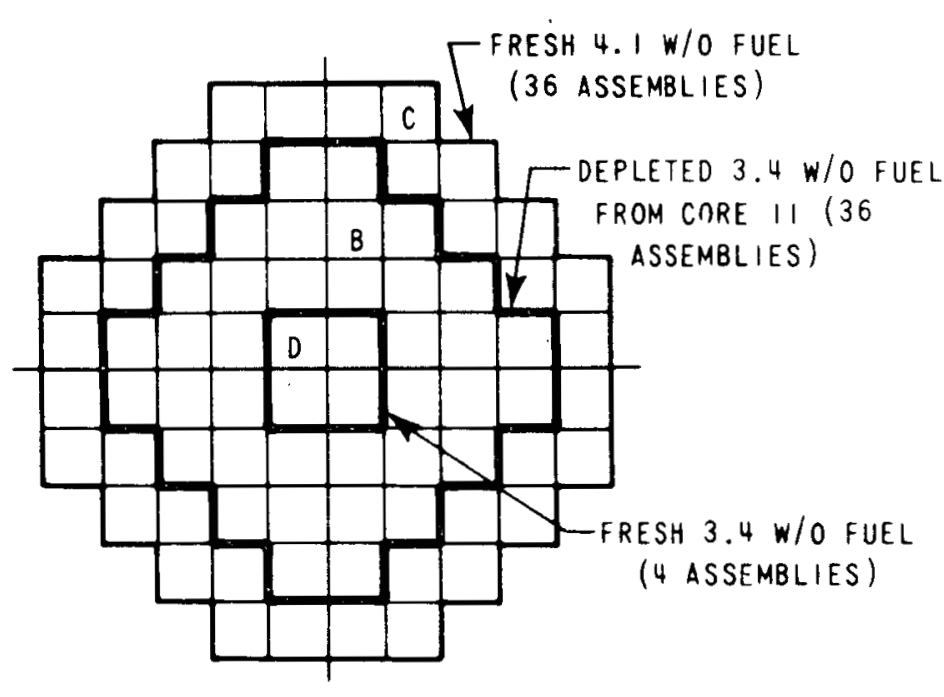

CORE 111 (6330 MNO/MTU)

Figure 2-2. Fuel Cycling in First Four Yankee Cores 
TABLE 2-2

OPERATING SUMMARY FOR YANKEE CORES I, II, III, AND IV

\begin{tabular}{|c|c|c|c|c|}
\hline . & Core I & Core II & Core III & Core IV \\
\hline Initial criticality & $8-19-60$ & $9-12-62$ & $10-23-63$ & $8-31-64$ \\
\hline At power & $1-16-61$ & $9-24-62$ & $11-14-63$ & $9-6-64$ \\
\hline Maximum power (MWe) & 150 & 170 & 185 & 185 \\
\hline Maximum power (MWt) & 485 & 540 & 600 & 600 \\
\hline Average power during generation (MWe) & $1.19 *$ & $150 *$ & $154 *$ & $164 *$ \\
\hline Gross energy (MWH elèctrical) & $1,330,521$ & $1,205,597$ & 944,772 & $1,309,059$ \\
\hline Net energy (MWH electrical) & $1,222,960$ & $1,125,863$ & 882,072 & $1,224,892$ \\
\hline Gross energy (MWH thermal) & $4,249,646$ & $3,926,634$ & $3,174,626$ & $4,349,046$ \\
\hline Times critical & 262 & 44 & 19 & 32 \\
\hline Hours critical & $13,247.43$ & $8,305.68$ & 6257.38 & 8160.27 \\
\hline Times scrammed & 30 & 8 & 6 & 5 \\
\hline Core average burnup (MWD/MTU) & 8,470 & 7,866 & 6,329 & 8,724 \\
\hline Final shutdown & $5-18-62$ & $9-2-63$ & $8-2-64$ & $8-9-65$ \\
\hline Conditions at End of Core Life & & & & \\
\hline Power (MWe) & 78.4 & 83.3 & 92.0 & 96 \\
\hline Power (MWt) & 260 & 308 & 340 & 320 \\
\hline Condenser back pressure ("Hg) & 0.9 & 2.0 & 1.95 & 2.10 \\
\hline Avg. moderator temperature $\left({ }^{\circ} \mathrm{F}\right)$ & 429.5 & 441.0 & 452.6 & 479 \\
\hline Control rod configuration & al1 rods out & all rods out & all rods out & all rods out \\
\hline
\end{tabular}

* Includes core extension period (coast-down) at reduced power and coolant temperature. 

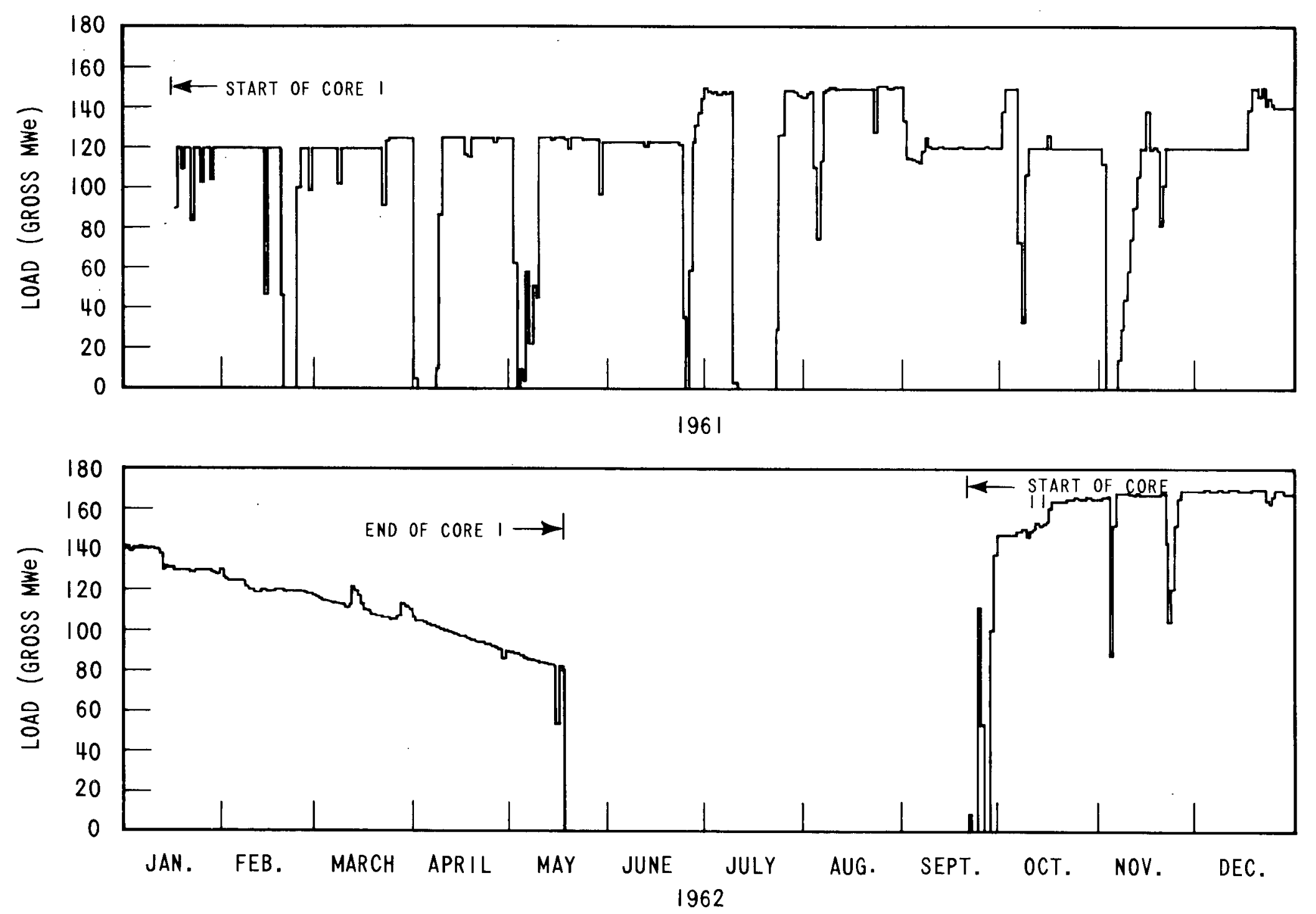

Figure 2-3. Power History of Yankee Core, January 1961 to December 1962 

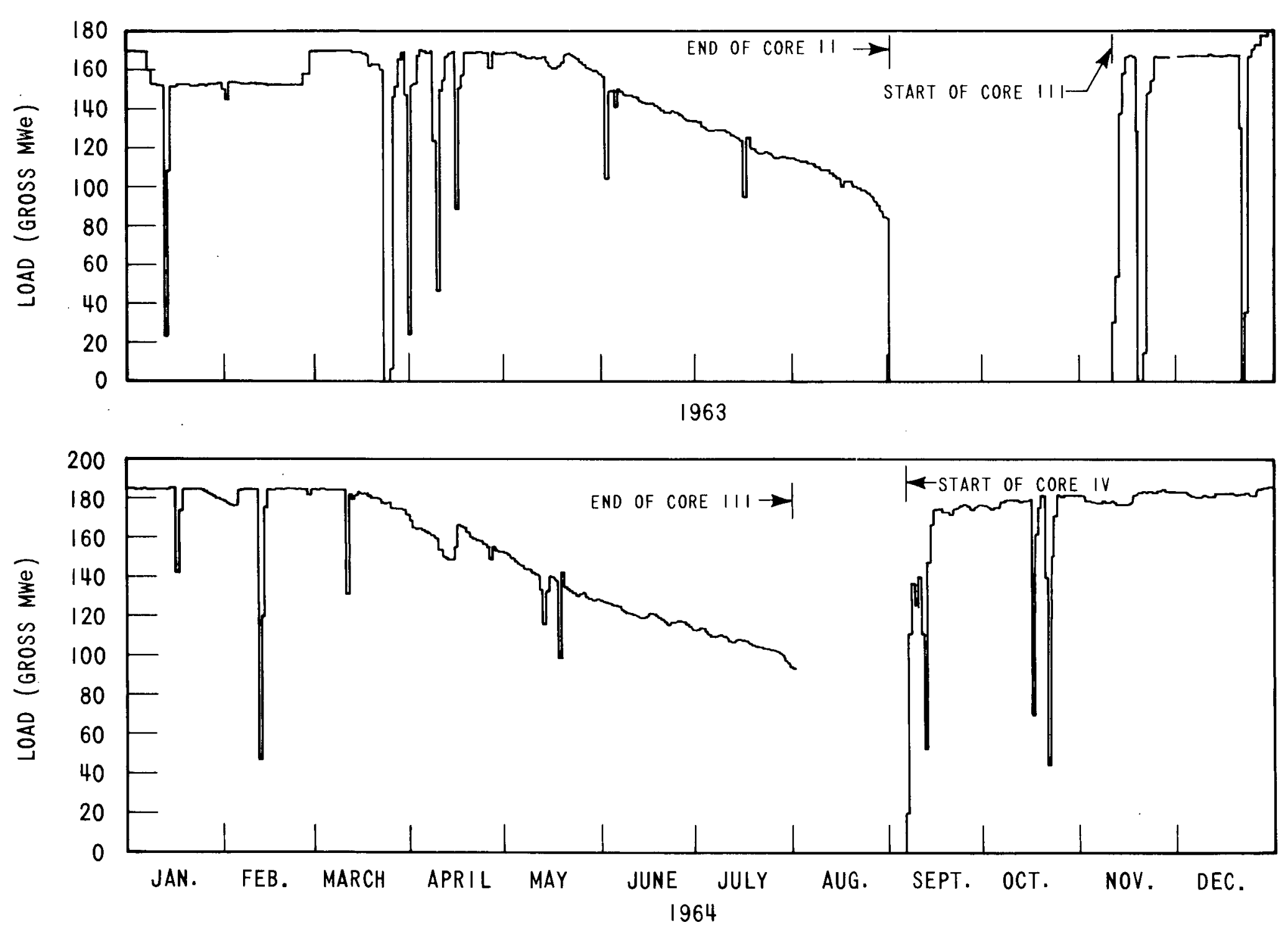

Figure 2-4. Power History of Yankee Core. January 1963 to December 1964 


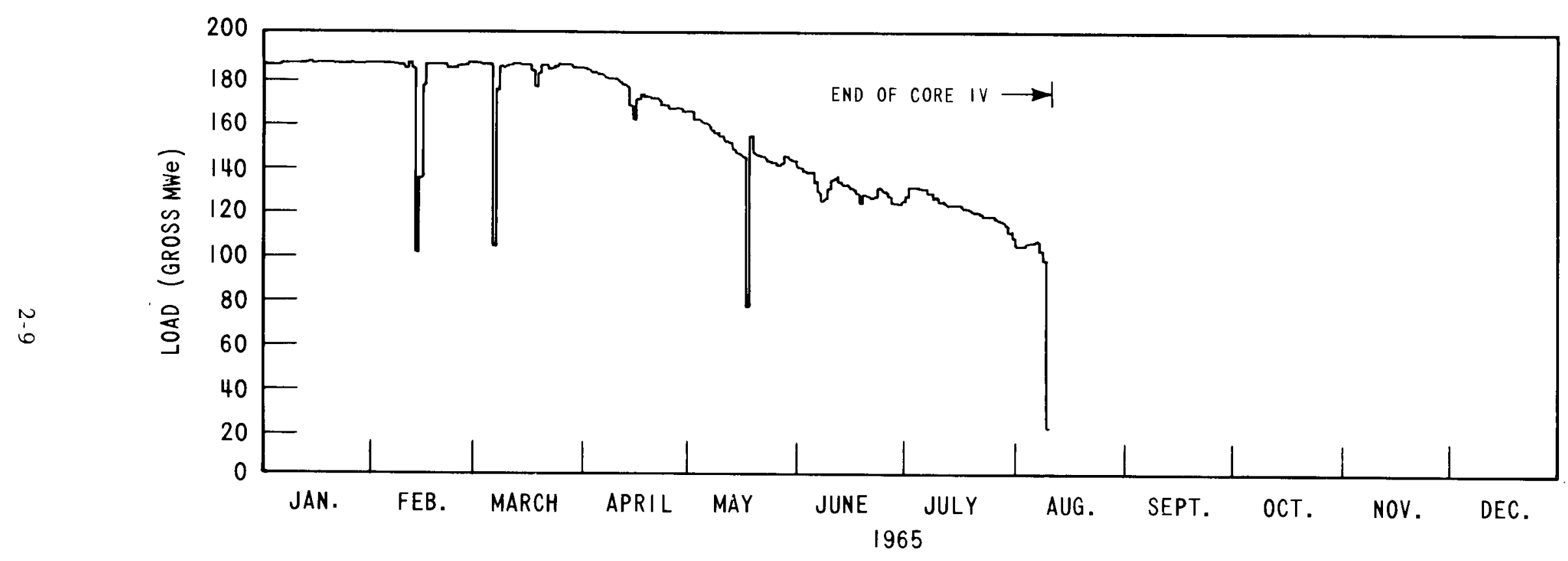

Figure 2-5. Power History of Yankee Core. January 1965 to August 1965 
SECTION 3

SAMPLING PLAN

The objectives in the selection of the spent fuel samples for the physics portion of Phase 3 of the EYC Program were to determine:

1. The main chain uranium and plutonium isotopic composition of the fuel as a function of burnup in the asymptotic and perturbed neutron spectra,

2. The spatial distribution of burnup and fuel composition in the pellets, in the rods, and in the Phase 3 assembly,

3. The total uranium and plutonium isotopic inventory of the assembly

4. The fuel characteristics, including conversion ratio and the captureto-fission ratio in $\mathrm{U}-235$.

The objectives of the Program Extension were to investigate the production of the special isotopes U-232, Np-237, Pu-236, Pu-238, Am-241, Am-243, Cm-242, and $\mathrm{Cm}-244$ and to evaluate the use of the fission product $\mathrm{Nd}-148$ as an accurate fuel burnup indicator, over a wide range of burnup in the asymptotic and perturbed neutron spectra.

As used above, the perturbed neutron spectra refer to those fuel regions in the vicinity of the water slots which surround the cruciform control rod positions. When the absorber sections of control rod groups 1 and 2 (see Figure 2-1) are in the core, the thermal neutron flux in the vicinity of the control rods is depressed. When the control rods are withdrawn from the core and replaced by the Zircaloy control rod follower sections, the excess moderation due to the water slots causes a peaking in the thermal flux. These perturbations affect only those fuel rods which are within 2 or 3 lattice pitches of the water slots. 
The perturbed neutron spectra also refer to those fuel pellets which are adjacent to the stainless steel spacer discs and axial expansion voids which separate stacks of pellets within a fuel rod, and also to those fuel pellets which are in the neighborhood of the stainless steel ferrules used to braze together the individual fuel rods to form a fuel subassembly. (The fuel assembly consists of 9 subassemblies, in a 3 x 3 array.) The spacer discs and axial voids cause an increase in the water-to-uranium ratio, thus producing a peak in the local neutron flux. The ferrules, which are in the coolant channels, displace water, thereby decreasing the water-to-uranium ratio and causing a depression in the local flux.

The so-called asymptotic neutron spectrum refers to those regions of the core which are well away from and unaffected by the perturbations described above (i.e., where the fast-to-thermal flux ratio is constant).

\subsection{SELECTION OF PHASE 3 FUEL RODS}

A plan view of the Phase 3 fuel assembly which occupied Yankee core position E6 is shown on Figure 3-1. With the assembly oriented as shown, the nine subassemblies are identified with a compass-point notation as indicated on the sketch in the lower right corner of the figure. In each subassembly individual fuel rods are identified with an alpha-numeric notation whereby the columns of fuel rods, reading left to right, are headed with the letters a through $e$ in the case of subassembly NW, and a through $f$ for the eight other subassemblies. Reading top to bottom in each subassembly, the rows of fuel rods are numbered 1 through 5 in the case of subassembly SE, and 1 through 6 for the eight other subassemblies.

Each fuel rod is identified with a three-part notation which indicates the assembly core position, the subassembly, and the fuel rod lattice position within the subassembly. The eight fuel rods which were selected for analysis are indicated as solid symbols in Figure 3-1, and are 1isted in Table 3-1. 


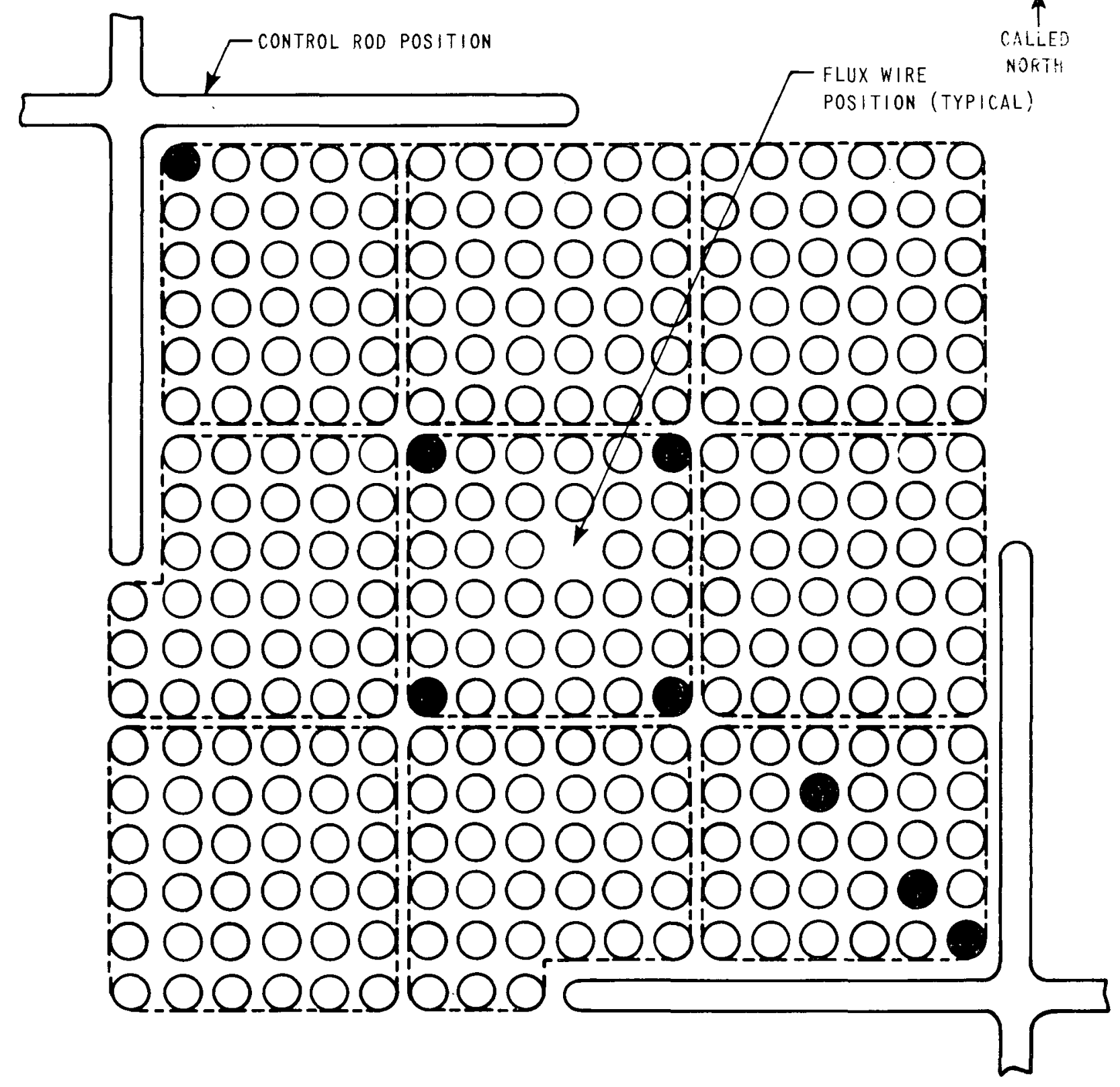

fuel rod in place

FUEL ROD REMOVED

\begin{tabular}{|c|c|c|}
\hline NW & N & NE \\
\hline W & C & E \\
\hline SW & $S$ & SE \\
\hline
\end{tabular}

Figure 3-1. Plan View of EYC Phase 3 Fuel Assembly Showing Locations of Fuel Rods Removed for Physics Analys is 
TABLE 3-1

FUEL RODS SELECTED FOR PHYSICS ANALYSIS IN PHASE 3 OF THE EYC PROGRAM*

Fuel Rod

Location

E6-C-al

E6-C-a6

E6-C-f I

E6-C-f6

E6-SE-C2

E6-SE-e4

E6-NW-a1

E6-SE-f 5
Fuel Rod

Number

369

5352

536

354

3198

3187

44

3113
Neutron

Spectrum

Asymptotic

Asymptotic

Asymptotic

Asymptotic

Intermediate

Intermediate

Perturbed

Perturbed

* A ninth fuel rod, E6-W-a4, was removed and subjected to engineering evaluations. 


\subsection{SELECTION OF FUEL SAMPLES WITHIN A FUEL ROD}

Analysis of flux wire manganese activation data obtained during core operation revealed that the axial flux distribution in the core was perturbed by the presence of ferrules, axial voids, and spacer discs, as discussed earlier. Since the number of fuel pellets affected by these perturbations is small compared to the total number of pellets in the assembly, it was felt that samples should not be taken from these regions. Samples more representative of the asymptotic condition could be obtained from positions along the fuel rod stack, midway between the local perturbations described above.

The Phase 3 assembly occupied core position $\mathrm{E} 6$ which did not contain flux wire instrumentation; consequently, no flux wire data are available for this assembly. However, the flux-wire measurements made in any Core I assembly can be considered representative for the local flux perturbations caused by the presence of spacer discs and ferrules.

A typical axial flux wire trace obtained during Core $I$ operation is shown in Figure 3-2. The peaks and depressions along the activation distribution are characteristic of all the flux wire traces in all assemblies. The spacer discs and voids are located at nominal 16-inch intervals along the fuel rod pellet stack, while the ferrules occur at nominal 8-inch intervals along the rod. Consequently, 6 relatively unaffected axial regions or zones, bracketed by consecutive pairs of ferrules, are available for sampling. The 6 axial sample zones, and the location of the positions which were sampled, are shown in the sketch on Figure 3-3. To obtain all the samples in each axial sample zone from the same $x-y$ plane, the measurements shown on Figure 3-3 are from a reference point on that end of the fuel rod which was in the bottom of the core during reactor operation.

The axial location information is summarized in Table 3-3. The normalized weighting factors listed in the table have been used to obtain fuel rod average data by volume weighting the pellet data. It is emphasized that the dimensional data and the weighting factors are nominal, and represent the average conditions in the core for the following reasons:

1. Not all fuel rods contained exactly the same number of pellets, due to manufacturing tolerances on the fuel pellets. 


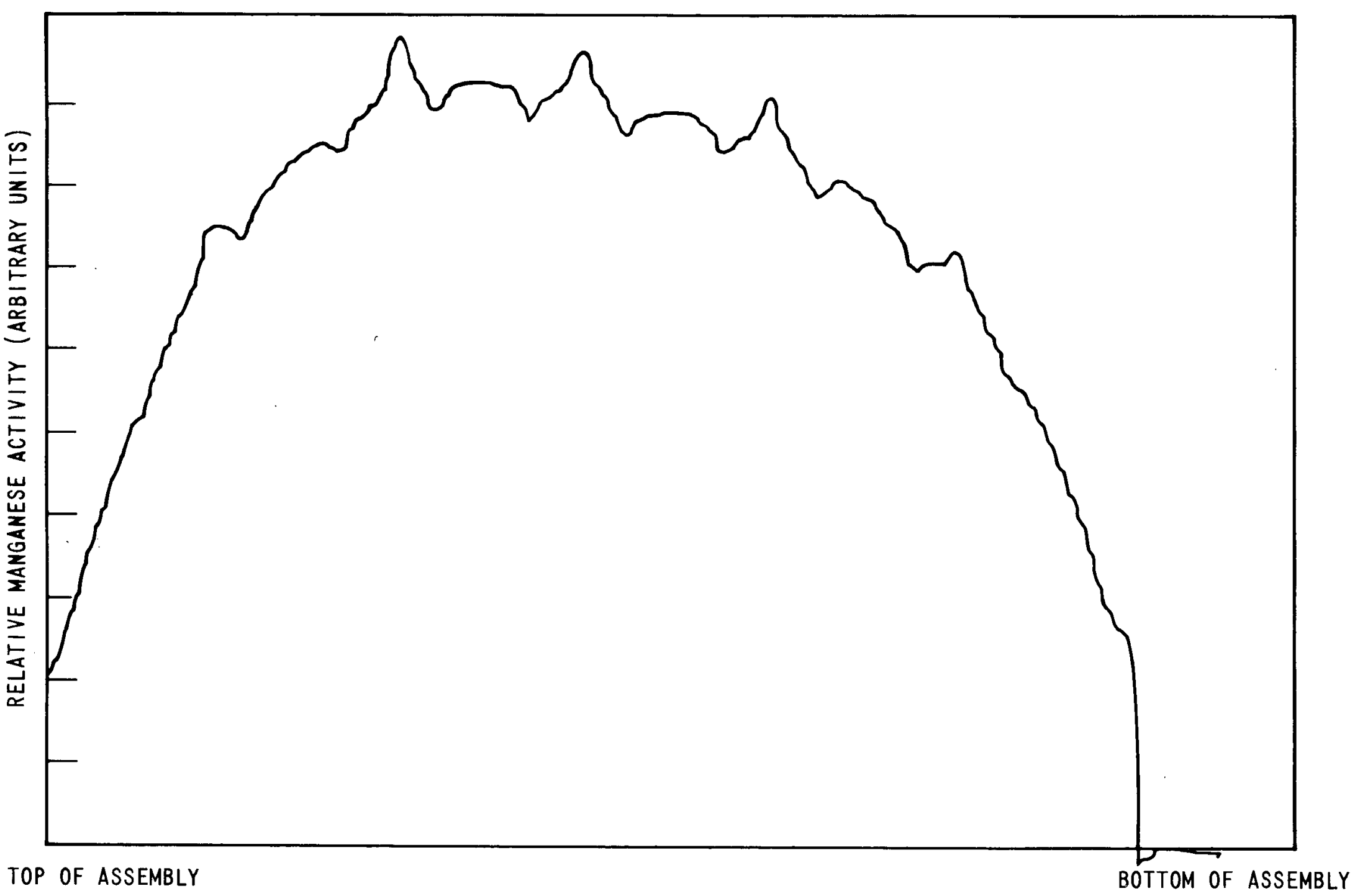

Figure 3-2. Typical Yankee Flux Wire Activity Trace 


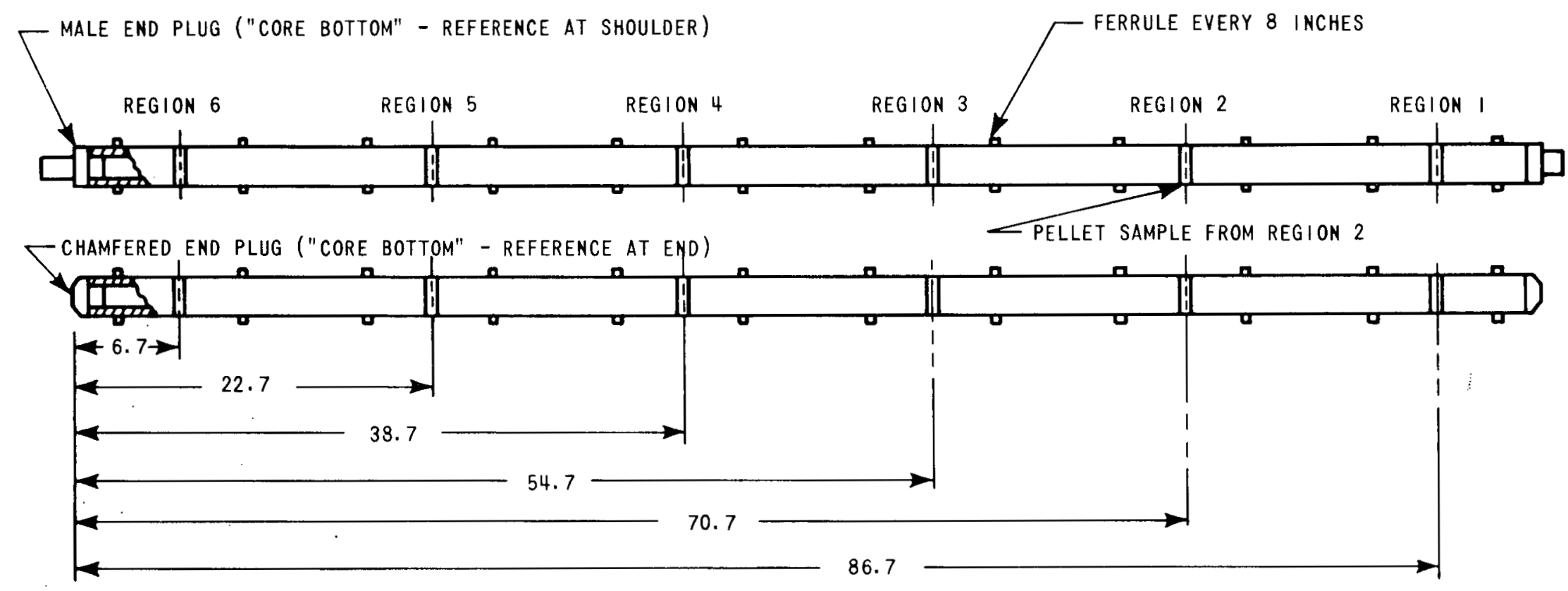

ALL DIMENSION IN INCHES

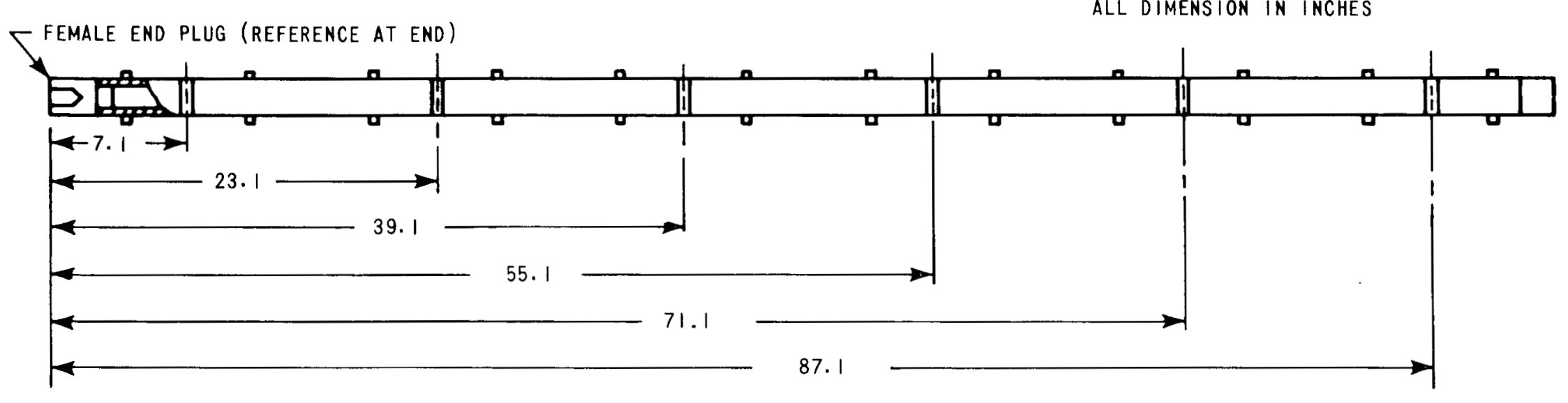

Figure 3-3. Axial Location of Spent Fuel Samples within a Fuel Rod 
TABLE $3-2$

AXIAL LOCATION OF SPENT FUEL SAMPLES WITHIN A FUEL ROD

\begin{tabular}{|c|c|c|c|c|}
\hline $\begin{array}{l}\text { Type of } \\
\text { End Plug }\end{array}$ & $\begin{array}{l}\text { Axial } \\
\text { Sample } \\
\text { Zone }\end{array}$ & $\begin{array}{c}\text { Distance From } \\
\text { Reference Point } \\
\text { to Sample } \\
\text { (inches) }\end{array}$ & $\begin{array}{c}\text { Length of } \\
\text { Pellet Stack } \\
\text { Per Sample Zone } \\
\text { (inches) }\end{array}$ & $\begin{array}{l}\text { Volume } \\
\text { Weighting } \\
\text { Factor }\end{array}$ \\
\hline \multirow{6}{*}{$\begin{array}{c}\text { Male } \\
\text { or } \\
\text { Chamfered }\end{array}$} & 1 & 86.7 & 13.30 & 0.1470 \\
\hline & 2 & 70.7 & 15.77 & 0.1741 \\
\hline & 3 & 54.7 & 15.77 & 0.1741 \\
\hline & 4 & 38.7 & 15.77 & 0.1741 \\
\hline & 5 & 22.7 & 15.77 & 0.1741 \\
\hline & 6 & 6.7 & 14.18 & 0.1566 \\
\hline \multirow[t]{4}{*}{ Total } & & & 90.56 & 1.0000 \\
\hline & 1 & 87.1 & 13.30 & 0.1470 \\
\hline & 2 & 71.1 & 15.77 & 0.1741 \\
\hline & 3 & 55.1 & 15.77 & 0.1741 \\
\hline \multirow{3}{*}{ Female } & 4 & 39.1 & 15.77 & 0.1741 \\
\hline & 5 & 23.1 & 15.77 & 0.1741 \\
\hline & 6 & 7.1 & 14.18 & 0.1566 \\
\hline Total & & & 90.56 & 1.0000 \\
\hline
\end{tabular}


2. During fabrication of the fuel assemblies, fewer handling fixtures were required if some types of subassemblies were inserted in the assembly in an inverted position.

These fabrication procedures resulted in slight non-uniformity in the axial locations of the spacer discs and ferrules; that is, with respect to the bottom of the fuel assembly, the locations of the spacer discs (and axial voids) and ferrules are staggered, and are not all in the same $x-y$ plane in each axial sample zone. These conditions are obvious in the photographs of some spent fuel assemblies shown, for example, on page 340.3 and 340.4 of Reference 15.

\subsection{SELECTION OF FUEL SAMPLES FOR EYC PROGRAM EXTENSION}

The selection of fuel samples for the Program Extension was Iimited to those fuel rods which had been sectioned during the execution of EYC Phase 1, 2, and 3. To provide sample continuity over a wide range of fuel burnup, an attempt was made to select the samples of Phase 1 fuel from the assembly which occupied core Location E5*; however, due to a limitation on the availability of Phase 1 fuel (at this stage of the program, much of the Phase 1 fuel had been shipped to Nuclear Fuel Services for reprocessing), some samples were selected from the assembly which occupied core location F4. Figure 3-4 shows the core locations of all the fuel rods removed for physics evaluations in Phases 1, 2, and 3; the solid symbols in Figure 3-4 indicate the fuel rods which had provided samples for the Program Extension.

Since the objective of the EYC Program Extension is to investigate the production of U-232, $\mathrm{Pu}-236, \mathrm{~Np}-237, \mathrm{Pu}-238, \mathrm{Am}-241, \mathrm{Am}-243, \mathrm{Cm}-242$, and $\mathrm{Cm}-244$ in the Yankee fuel, and since these isotopes are present in the spent fuel in relatively small concentrations (which requires time-consuming and relatively

* With respect to core geometry and the programmed control rod withdrawal pattern, the Phase 1 assembly in position E5 is quarter-core symmetric with the Phase 2 and 3 assemblies in core positions F5 and E6. 
NOTE: THE 8 PHASE III RODS ARE IN ASSEMBLY E6.

THE 7 PHASE II RODS ARE IN ASSEMBLY F5.

THE 56 PHASE I RODS ARE IN THE REMAINING 14 ASSEMBLIES

AS SHOWN.

3

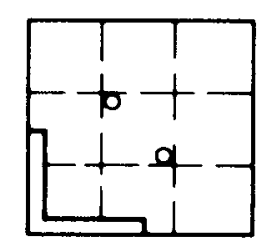

C

5
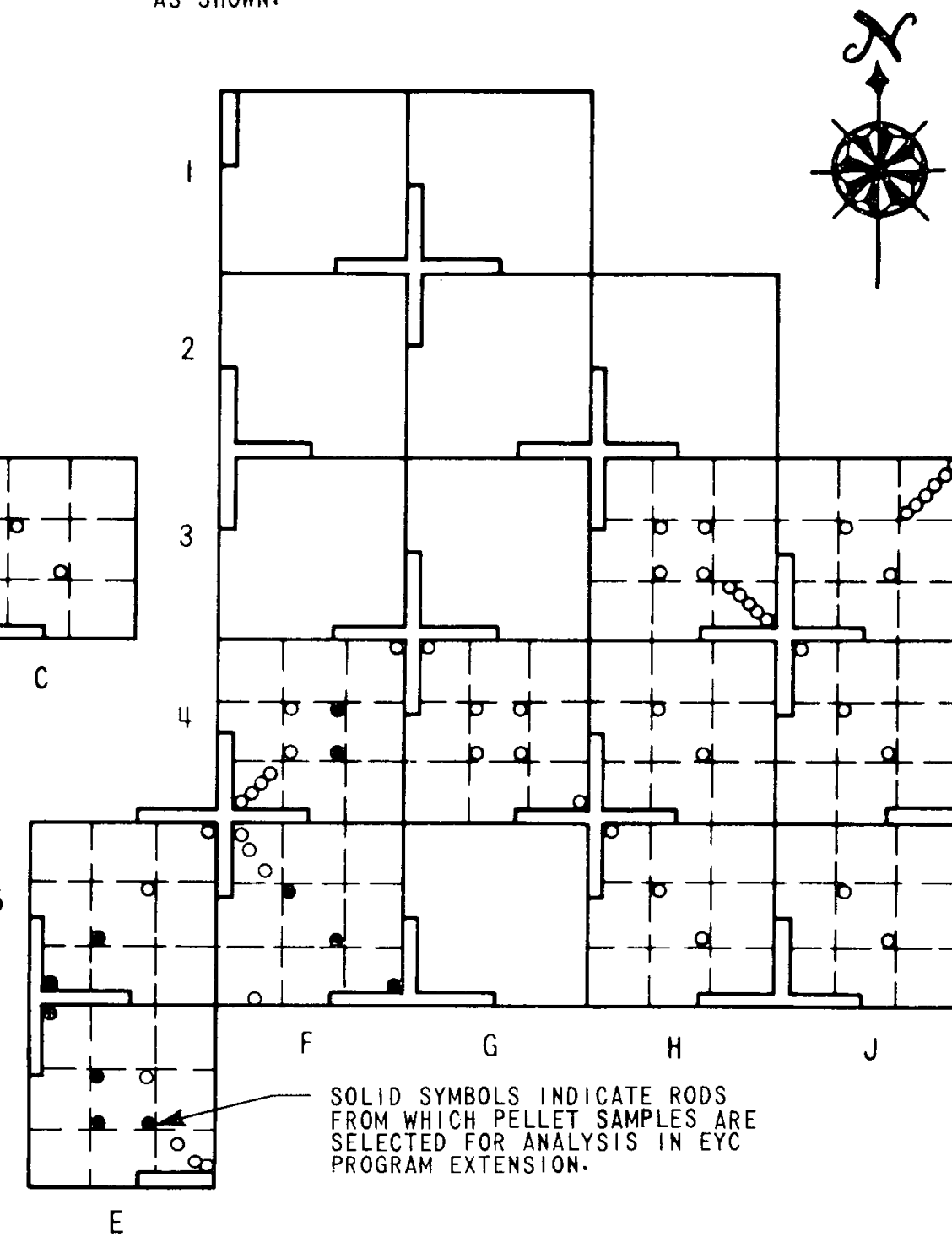

0
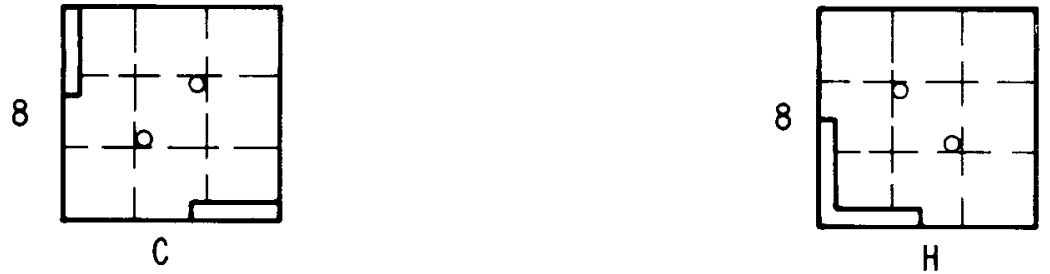

Figure 3-4. Core Location of EYC Fuel Rods Selected for Post-Irradiation Analyses 
expensive analysis), it is important that the samples selected for analyses be typical of the fuel which they represent. The EYC Program Extension samples had been labeled and stored for as long as five years. Due to the elapsed time between removal from the core and analysis, and the handing and storing operations, the possibility existed that the fuel samples had deteriorated or become contaminated.

To ensure against this possibility, the samples selected for the Program Extension were subjected first to a relatively inexpensive radiochemical analysis for Cs-137 activity. If the results were consistent with measurements made previously on Phase 1, 2, and 3 samples,* the Program Extension samples were then submitted for routine alpha-spectroscopy and mass spectrometric isotopic dilution analyses for the main chain uranium and plutonium isotopes, as well as for analyses for the special isotopes listed above. Three checks were employed to evaluate the main chain uranium and plutonium isotopic data obtained in the Program Extension:

1. Program Extension data were compared with results obtained in Phases 1, 2, and 3 of the existing EYC Program

2. A monitor sample solution was included in each batch of samples submitted for analysis

3. In some cases, solutions of the same sample were submitted to two laboratories for cross check purposes.

The number and types of sample analyses performed in Phase 3 and in the Program Extension are summarized in Table 3-3.

* In most cases, the Program Extension sample was removed from a position within 2 or 3 pellets of the nominal axial zone position which had been sampled previously in Phases 1,2 , and 3 . 
TABLE 3-3

SUMMARY OF PHASE 3 AND PROCRAM EXTENSTON SAMPLE LOCATIONS AND ANALYYSES

\section{c}

I

\begin{tabular}{|c|c|c|c|c|c|c|c|c|c|c|c|c|c|c|c|c|c|c|c|}
\hline & & & & & & & ASE & $\mathrm{AM}$ & TENSTOS & SANPLE & vs $t$ & ss & & & & & & & \\
\hline $\begin{array}{c}\text { Fuel } \\
\text { Roor } \\
\text { Core } \\
\text { Location }\end{array}$ & 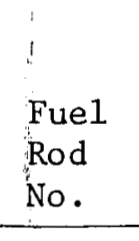 & $\begin{array}{c}\text { Neutron }(\mathrm{a} \\
\text { Spectrum }\end{array}$ & $\begin{array}{l}\text { EYC (b) } \\
\text { Phase }\end{array}$ & $\begin{array}{c}\text { Alixal } \\
\text { Sanple } \\
\text { zone } \\
\end{array}$ & 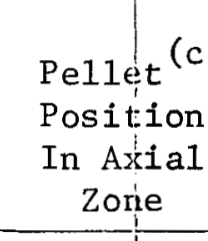 & 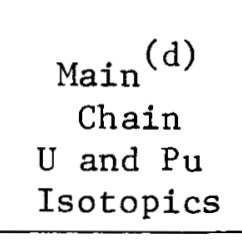 & $\begin{array}{l}x-\text { ray } \\
\text { Pu/v }\end{array}$ & Cs-137 & $s_{r-90}$ & $2 \mathrm{r}-95$ & $\mathrm{Nd}-148$ & $\mathrm{v}-232$ & ${ }_{\mathrm{Pu}-236}$ & $\mathrm{P}_{\mathrm{u}-238}$ & $\mathrm{~Np}_{\mathrm{p}-237}$ & $\mathrm{Am}-241$ & $\mathrm{Am}-243$ & $\mathrm{Cm}-242$ & $\mathrm{~cm}-244$ \\
\hline E5-C-a6 & 7992 & Asymp & 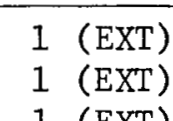 & $\begin{array}{l}3 \\
5\end{array}$ & $\begin{array}{l}+1 \\
-3 \\
-3\end{array}$ & $\frac{1}{1}$ & $\begin{array}{l}1 \\
1\end{array}$ & $\frac{1}{1}$ & & & 1 & $\frac{1}{1}$ & $\frac{1}{1}$ & $\begin{array}{l}1 \\
1\end{array}$ & $\mid \begin{array}{l}1 \\
2\end{array}$ & $\frac{1}{1}$ & & $\frac{1}{1}$ & $\frac{1}{1}$ \\
\hline ES-SBH-aS & 15025 & Pert & $\begin{array}{l}1(\mathrm{EXT}) \\
1\end{array}$ & 3 & +1 & 1 & 1 & 1 & & & 1 & & 1 & 2 & 1 & 2 & & 2 & ${ }_{1(\mathbb{B})}$ \\
\hline $\mathrm{F}_{4}-\mathrm{C}-\mathrm{f} 1$ & 8977 & Asymp & 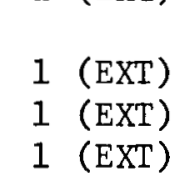 & $\begin{array}{l}1 \\
4 \\
5\end{array}$ & $\begin{array}{c}+1 \\
+1 \\
+1 \\
++1\end{array}$ & $\begin{array}{l}1 \\
1\end{array}$ & $\begin{array}{l}1 \\
1 \\
1\end{array}$ & $\begin{array}{l}1 \\
1 \\
1\end{array}$ & & 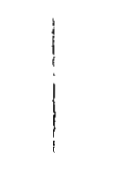 & $\begin{array}{l}1 \\
1\end{array}$ & $\frac{1}{1}$ & $\begin{array}{l}1 \\
1 \\
1\end{array}$ & $\begin{array}{l}1 \\
1\end{array}$ & $\begin{array}{l}2 \\
1\end{array} \mid$ & 2 & & 2 & $\begin{array}{l}18) \\
7(8)\end{array}$ \\
\hline $\mathrm{F} 4-\mathrm{C}-\mathrm{f} 6$ & 19579 & Asymp & $\begin{array}{l}1 \\
1(E X I T) \\
1(E X T)\end{array}$ & $\begin{array}{l}3 \\
4\end{array}$ & $\begin{array}{l}-1 \\
-2 \\
-2\end{array}$ & ${ }_{1}^{1}$ & $\frac{1}{1}$ & $\begin{array}{l}1 \\
1\end{array}$ & & & $\begin{array}{l}1 \\
1\end{array}$ & & $\frac{1}{1}$ & $\frac{1}{1}$ & & & & & \\
\hline G4-C-a & 0994 & Asymp & 1 & $3^{(e)}$ & $\mathrm{w}$ & 6 & & 6 & & & & 2 & 4 & 5 & & 1 & & 1 & 1 \\
\hline F5-C-Aal & 553 & Asymp & 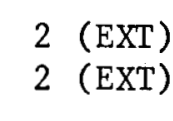 & $\frac{1}{3}$ & $\begin{array}{l}+1 \\
-1\end{array}$ & $\frac{1}{2}$ & $\frac{1}{1}$ & $\frac{1}{1}$ & & & $\frac{1}{1}$ & $\frac{1}{1}$ & ${ }_{1}^{1}$ & $\frac{1}{2}$ & 1 & $\frac{1}{2}$ & & $\frac{1}{2}$ & $\frac{1}{2}$ \\
\hline F5-C--f6 & 541 & Asymp & (F) & $\begin{array}{l}1 \\
2 \\
4 \\
5\end{array}$ & 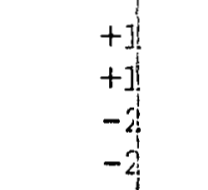 & $\begin{array}{l}1 \\
1\end{array}$ & $\begin{array}{l}1 \\
1 \\
1 \\
1\end{array}$ & $\frac{1}{1}$ & & & $\frac{1}{1}$ & $\begin{array}{l}1 \\
1 \\
1\end{array}$ & $\begin{array}{l}1 \\
1 \\
1 \\
1\end{array}$ & $\begin{array}{l}1 \\
\frac{1}{2} \\
1\end{array}$ & $\begin{array}{l}2 \\
{ }^{2}\end{array}$ & $\begin{array}{l}1 \\
2 \\
2\end{array}$ & & $\begin{array}{l}1 \\
\frac{1}{2} \\
2\end{array}$ & $\begin{array}{l}1 \\
1(8) \\
1(B)\end{array}$ \\
\hline F5-SE-f5 & 8717 & Pert & 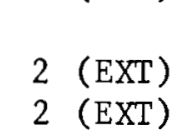 & $\begin{array}{l}3 \\
6\end{array}$ & $\begin{array}{c}-3 \\
+1 \\
+1\end{array}$ & 1 & $\frac{1}{1}$ & $\begin{array}{l}1 \\
1\end{array}$ & & & $\frac{1}{1}$ & & $\begin{array}{l}1 \\
1\end{array}$ & $\frac{1}{1}$ & & 2 & & 2 & $1(\mathrm{~B})$ \\
\hline $\mathrm{E} 6-\mathrm{c}-\mathrm{a}$ & 369 & Asymp & $\begin{array}{l}3 \\
3 \\
3 \\
3 \\
3 \\
3(\mathrm{EXII}) \\
3 \\
3 \\
3 \\
3 \\
3 \\
3\end{array}$ & $\begin{array}{l}1 \\
2 \\
2 \\
3 \\
3 \\
3 \\
4 \\
5 \\
6\end{array}$ & \begin{tabular}{c|}
$\mathrm{N}$ \\
$\mathrm{N}$ \\
-1 \\
$\mathrm{~N}$ \\
$\mathrm{~N}$ \\
$\mathrm{~N}$ \\
$\mathrm{~N}$ \\
$\mathrm{~N}$ \\
-2 \\
$\mathrm{~N}$
\end{tabular} & $\frac{1}{1}$ & $\begin{array}{l}1 \\
1 \\
1\end{array}$ & 1 & $\begin{array}{l}1 \\
1 \\
1 \\
1 \\
1 \\
1\end{array}$ & $\begin{array}{l}1 \\
1 \\
1 \\
1 \\
1 \\
1\end{array}$ & $\begin{array}{l}1 \\
2\end{array}$ & $\begin{array}{l}1 \\
1\end{array}$ & $\frac{1}{1}$ & $\begin{array}{l}1 \\
1 \\
1 \\
1\end{array}$ & $\begin{array}{l}1^{1} \\
2 \\
1 \\
1\end{array}$ & $\begin{array}{l}1 \\
2 \\
1\end{array}$ & & $\begin{array}{l}1 \\
2 \\
1\end{array}$ & $\begin{array}{l}1 \\
2\end{array}$ \\
\hline
\end{tabular}




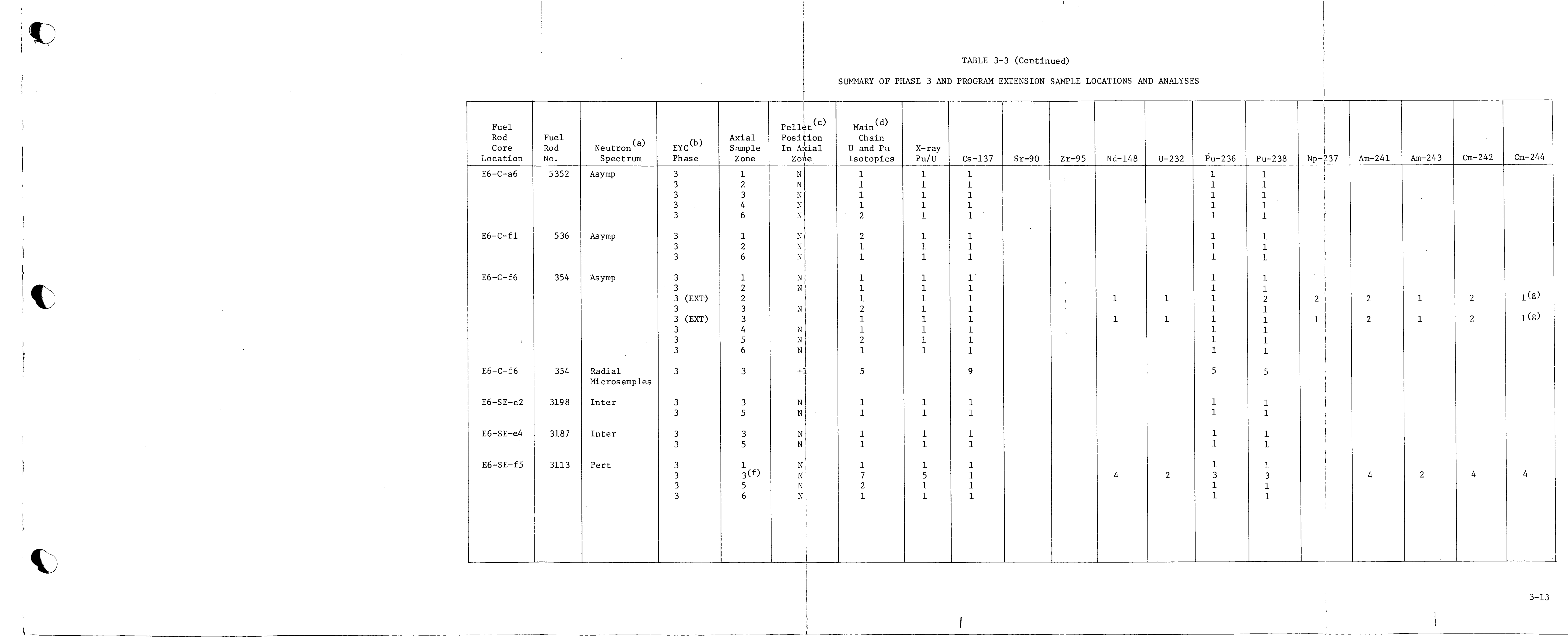


TABLE 3-3 (Continued)

SUMMARY OF PHASE 3 AND PROGRAM EXTENSTON SAMPLE LOCATIONS AND ANALYSES

\begin{tabular}{|c|c|c|c|c|c|c|c|c|c|c|c|c|c|c|c|c|c|c|c|}
\hline $\begin{array}{l}\text { Fue1 } \\
\text { Rod } \\
\text { Core } \\
\text { Location } \\
\end{array}$ & $\begin{array}{l}\text { Fue1 } \\
\text { Rod } \\
\text { No. }\end{array}$ & $\begin{array}{c}\text { Neutron (a) } \\
\text { Spectrum } \\
\end{array}$ & $\begin{array}{l}\text { EYc (b) } \\
\text { Phase }\end{array}$ & $\begin{array}{l}\text { Axial } \\
\text { Sample } \\
\text { Zone }\end{array}$ & $\begin{array}{l}\text { Pellet (c) } \\
\text { Position } \\
\text { In Axial } \\
\text { Ione } \\
\text { Zone }\end{array}$ & $\begin{array}{l}\operatorname{Main}^{(\mathrm{d})} \\
\text { Chain } \\
\text { Uand Pu } \\
\text { Isotopics } \\
\text { Issotopics }\end{array}$ & $\begin{array}{l}\mathrm{X}-\mathrm{ray} \\
\mathrm{Pu} / \mathrm{U}\end{array}$ & $\mathrm{Cs}-137$ & $\mathrm{Sr}-90$ & $2 \mathrm{z}-95$ & Nd-148 & $\mathrm{U}-232$ & $\mathrm{Pu}-236$ & $\mathrm{Pu}-238$ & $\mathrm{~Np}_{\mathrm{p}-2,37}$ & $\mathrm{Am}-241$ & $\mathrm{Am}-243$ & $\mathrm{Cm}-242$ & $\mathrm{Cm}-2$ \\
\hline E6-NW-a1 & 44 & Pert & $\begin{array}{l}3 \\
3 \\
3 \\
3 \text { (EXI) } \\
3 \\
3 \text { (EXI) } \\
3 \\
3\end{array}$ & $\begin{array}{l}1 \\
2 \\
3 \\
3 \\
4 \\
4 \\
5 \\
6 \\
6\end{array}$ & $\begin{array}{l}\mathrm{N} \\
\mathrm{N} \\
\mathrm{N} \\
\mathrm{N} \\
\mathrm{N} \\
\mathrm{N}\end{array}$ & $\begin{array}{l}1 \\
1 \\
2 \\
1 \\
2 \\
1 \\
1 \\
1 \\
\end{array}$ & $\begin{array}{l}1 \\
1 \\
1 \\
1 \\
1 \\
1 \\
1 \\
\end{array}$ & $\begin{array}{l}1 \\
1 \\
1 \\
1 \\
1 \\
1 \\
1 \\
\end{array}$ & $\begin{array}{l}1 \\
1 \\
1 \\
1 \\
1 \\
1 \\
\end{array}$ & $\begin{array}{l}1 \\
1 \\
1 \\
1 \\
1 \\
1 \\
1 \\
\end{array}$ & $\begin{array}{l}1 \\
1\end{array}$ & $\begin{array}{l}1 \\
1\end{array}$ & $\begin{array}{l}1 \\
1 \\
1 \\
1 \\
1 \\
1 \\
1 \\
\end{array}$ & $\begin{array}{l}1 \\
1 \\
1 \\
1 \\
1 \\
1 \\
1 \\
\end{array}$ & $\begin{array}{l}1 \\
2\end{array}$ & $\begin{array}{l}2 \\
2\end{array}$ & 1 & $\begin{array}{l}2 \\
2\end{array}$ & $\begin{array}{l}1^{(g)} \\
1^{(g)}\end{array}$ \\
\hline \multirow{2}{*}{\multicolumn{6}{|c|}{ Total, Asymptotic Spectrum }} & 55 & 40 & 46 & 6 & 6 & 21 & 17 & 44 & 50 & 21 & 26 & 2 & 26 & 19 \\
\hline \multirow{2}{*}{\multicolumn{6}{|c|}{$\begin{array}{l}\text { Tota1, Interniediate Spectra } \\
\text { Total, Perturbed Spectra }\end{array}$}} & 4 & 4 & 4 & 0 & $\dot{\varphi}$ & 0 & 0 & 4 & 4 & 0 & 0 & 0 & 0 & 0 \\
\hline & & & & & & 25 & 20 & 16 & 6 & 6 & 10 & 4 & 18 & 19 & 3 & 12 & 3 & 12 & 8 \\
\hline \multirow{2}{*}{\multicolumn{6}{|c|}{$\begin{array}{l}\text { Total, Radial| Microsamples } \\
\text { Grand Totals }\end{array}$}} & 5 & 0 & 9 & 0 & 0 & 0 & 0 & 5 & 5 & 0 & 0 & 0 & 0 & 0 \\
\hline & & & & & & 89 & 64 & 75 & 12 & 12 & 31 & 21 & 71 & 78 & 24 & 38 & 5 & 38 & 27 \\
\hline
\end{tabular}

NOTES (a) The asymptotic neutron spectrum refers to fuel samples from the center subassembly of the fuel assembly, well away from any perturbing
influences; the perturbed neutron spectrum refers to fuel samples from corner fuel rods, adjacent to the water slot which surrounds the

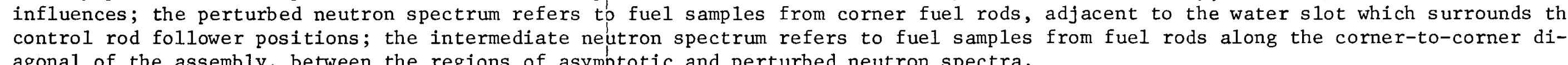

(b) Phase 1 samples were irradiated in Yankee Core I; phase 2 samples were irradiated in Cores I and II; Phase 3 samples were irradiated in
Cores I, II, and IV: EXT indicates samples obtained in EXC Program Extension.

(c) The letter $N$ indicates nominal sample position at mid-point of axial sample zone; plus or minus numerals indicate pellet position in
axial sample zone, relative to nominal position.

(d) Mass spectrometric analysis for $\mathrm{U}-234, \mathrm{U}-235, \mathrm{U}-236, \mathrm{U}-238, \mathrm{Pu}-239, \mathrm{Pu}-240, \mathrm{Pu}-241$, and $\mathrm{Pu}-242$.

(e) EYC Phase 1 monitor sample; includes only analyses in EYC Phase 3 and Program Extension.

(f) EYC Phase 3 monitor sample.

(g) Analyzed for $\mathrm{dpm} / \mathrm{ml}$ by both WARD and TRACERLAB. 
SECTION 4

ANALYSES PERFORMED ON SPENT FUEL SAMPLES

After a cooling period of approximately ten months following the shutdown of Yankee Core I on May 18, 1962, the fourteen depleted fuel assemblies selected for sampling in Phase 1 of the EYC Program were shipped ${ }^{[16]}$ from the Yankee site at Rowe, Massachusetts to the Transfer Building at the Westinghouse Post Irradiation Facility (WPIF) at Waltz Mill, Pennsylvania. The Phase 2 assembly, which had been irradiated in Yankee Cores I and II in core position F5, was shipped to WPIF after a cooling time of about five months (September 1963 to January 1964). The Phase 3 assembly, which had been irradiated in Cores I, II, and IV in core position E6,* was shipped to WPIF after a cooling time of about two months (August 1965 to October 1965).

In each phase of the program, pre-selected fuel rods were removed intact from the assemblies with the fuel rod hole-saw, described in Reference 15, and transferred in a shielded cask, via a canal, to the WPIF Hot Cell Laboratory. After performing dimensional, gamma scanning, and fission gas measurements, the fuel rods were cut with an abrasive wheel at the locations given in Table 3-2, and up to six pellet-size sections of cladding and fuel were removed from each fuel rod.

The fuel was separated from the cladding by dissolution in nitric acid, and sample solutions were prepared by pipetting aliquots of the fuel solution into the water. The final acid concentration of the sample solution was approximately $2 \mathrm{M} \mathrm{HNO}_{3}$. The following paragraphs briefly describe the various types of analyses performed on the spent fuel samples.

* During the operation of Core III, the Phase 3 assembly was stored in the Yankee fuel storage pit. 
4.1 DESCRIPTION OF ANALYSES PERFORMED

Utilizing the technique of isotopic dilution, mass spectrometric analyses for the main chain uranium and plutonium isotopes were performed by two subcontractors. These were:

a TRACERLAB, Reactor Monitoring Center, a Division of Laboratory for Electronics, Inc., 2030 Wright Avenue, Richmond, California, under Messrs. Buddy L. Brock, and Leon Leventhal.

b. Vallecitos Atomic Laboratory, General Electric Atomic Products Division, P.0. Box 846, Pleasanton, California, under Messrs. W. O. Wade, J. F. Hanus, and W. W. Sabol.

The Phase 3 samples were analyzed by TRACERLAB (TL), and the Program Extension samples were analyzed by General Electric - Vallecitos (GE), with a few cross check analyses by TRACERLAB. Also, several cross check analyses were performed by the USAEC New Brunswick Laboratories (NB) at New Brunswick, New Jersey. In addition, the GE analyses of the Program Extension samples included the determination of the fission product $\mathrm{Nd}-148$ by mass spectrometric measurements on separated neodymium fractions both with and without a spike of Nd-150. The method used by TRACERLAB is outlined in Reference 5, while the method used by GE is given in detail in Reference 17. Measurements for the concentrations of the special isotopes in the Program Extension were performed by Westinghouse Nuclear Energy Systems.* Uranium232 was determined by alpha spectrometry on highly purified uranium fractions; each sample required 30 to 60 hours of counting time. Plutonium-236 and plutonium-238 were determined by alpha pulse height analysis of freshly separated plutonium using a surface barrier alpha particle detector to prepare a high resolution alpha spectrum. The americium and curium isotopes were measured by alpha counting and spectrometry on fractions separated and purified by ion exchange. Neptunium-237 was measured by an extraction procedure

* Sample preparation and measurements performed under Mr. J. A. Corbett, Manager of Analytical Laboratories of the Westinghouse Advanced Reactor Division, Waltz Mill, Pennsylvania. 
involving thenoyltrifluoracetone (TTA) and triiso-octylamine (TIOA) extractions with neptunium-239 used as a tracer to measure recovery. Some analyses for special isotopes were performed by TRACERLAB. During Phase 3, the TRACERLAB analyses included $\mathrm{Pu}-236$ and $\mathrm{Pu}-238$ along with the main chain uranium and plutonium isotopes; in the Program Extension, TRACERLAB analyzed some samples for Am-241, Cm-242, and $\mathrm{Cm}-244$.

Radiochemical analyses for $\mathrm{Cs}-137$ and $\mathrm{Sr}-90$, and X-ray spectrographic analysis for $\mathrm{Pu} / \mathrm{U}$ mass ratio were performed by WNES using the methods outlined in Reference 5 and Reference 18.

\subsection{RESULTS OF ANALYSES PERFORMED}

The results obtained from the mass spectrometric analyses are given in Appendix A. The data consist of the main chain uranium and plutonium isotopic composition of the fuel, the atomic ratio of $\mathrm{Pu}-239$ to U-238, and the atomic ratio of $\mathrm{Nd}-148$ to $\mathrm{U}-238$. Also included are the results obtained from alpha pulse height analyses for $\mathrm{Pu}-236$ and $\mathrm{Pu}-238$, radiochemical analyses for $\mathrm{Cs}-137$ and $\mathrm{Sr}-90$, and $\mathrm{X}$-ray fluorescence analysis for $\mathrm{Pu} / \mathrm{U}$ mass ratio.

The data obtained from the measurements for the special isotopes U-232, $\mathrm{Np}-237, \mathrm{Am}-241, \mathrm{Am}-243, \mathrm{Cm}-242$ and $\mathrm{Cm}-244$ are given in Appendix D; these measured isotopic data are compared with the results of unit cell calculations in Section 11. 


\section{SECTION 5}

TREATMENT OF DATA

A material balance, performed with pre-irradiated and post-irradiated fuel composition data, can be used to infer the various fuel characteristics and nuclear parameters of interest (e.g., fuel burnup, specific production of uranium and plutonium isotopes, and U-235 capture-to-fission ratio). However, the required computations are somewhat long and laborious, and involve several correction factors which are dependent upon the parameters to be calculated. For these reasons, and due to the great number of spent fuel analyses performed in the EYC Program, the REBUF ${ }^{[5]}$ computer code was written to facilitate the reduction of the mass spectrometric data. Provisions have been included in the code to process Cs-137, Sr-90, Nd-148, and X-ray fluorescence Pu/U data. The data reduction methods used to infer fuel burnup are similar to those outlined in Reference 19.

The treatment accorded the mass spectrometric and radiochemical data in this study is given in detail in References 5 and 20; a general outline of the methods is given below. Discussed is the main chain uranium and plutonium isotopes, and the out-of-core decay corrections made to the measurements for the special isotopes included in the Program Extension.

\subsection{END-OF-LIFE ISOTOPIC INVENTORY EQUATIONS}

When considering the composition of spent reactor fuel, the relationship between the abundances of the various isotopes of uranium and plutonium and their fission products can be expressed through a number of comparatively simple equations. Since the preponderant mode of fission is binary, i.e., since a fission-product pair results from one fission, the fission-product nuclei from one fission can be counted as one hypothetical nucleus, and the total number of nuclei can be conserved. 
The symbols and equations defined below are equally valid when applied to any $1 \mathrm{~cm}^{3}$ of fuel to represent atom densities, or when applied to the total core volume to represent total core inventories of the uranium and plutonium isotopes or fission-product pairs.

Some material balance equations are:

$$
\begin{aligned}
& \mathrm{N}_{0}^{24}+\mathrm{N}_{0}^{25}+\mathrm{N}_{0}^{26}+\mathrm{N}_{0}^{28}=\mathrm{N}^{24}+\mathrm{N}^{25}+\mathrm{N}^{26}+\mathrm{N}^{28} \\
& +N^{49}+N^{40}+N^{41}+N^{42} \\
& +F^{25}+F^{28}+F^{49}+F^{41} \\
& +A^{26}+A^{42}+D^{41} \\
& \mathrm{~F}^{25}=\mathrm{N}_{0}^{24}+\mathrm{N}_{0}^{25}+\mathrm{N}_{0}^{26}-\mathrm{N}^{24}-\mathrm{N}^{25}-\mathrm{N}^{26}-\mathrm{A}^{26} \\
& F^{49}=\left(N^{40}+N^{41}+N^{42}+F^{41}+A^{42}+D^{41}\right) / a^{49} \\
& F^{41}=\left(N^{42}+A^{42}\right) /\left(\alpha^{41}+1\right) \\
& F^{28}=\delta^{28}\left(F^{25}+F^{49}+F^{41}\right) \\
& A^{26}=\left(N^{26}-N_{0}^{26}\right)(\operatorname{COR} 26-1) \\
& A^{42}=N^{42}(\operatorname{COR} 42-1) \\
& D^{41}=N^{41}[1-\exp (-\lambda T)] / \lambda T
\end{aligned}
$$


The symbols $\mathrm{N}^{i}$ and $\mathrm{F}^{i}$ denote, respectively, the density of isotope $i$ (atoms $/ \mathrm{cm}^{3}$ ) and of pairs of products from the fission of isotope $i$ (atom pairs $/ \mathrm{cm}^{3}$ ); the symbols $\mathrm{A}^{i}$ and $\mathrm{D}^{i}$ account for losses due to absorbtion and decay in isotope $i$. The in-core decay of $\mathrm{Pu}-241$ is approximated by equation (8), where $\lambda$ is the decay constant and $T$ is the irradiation time for the sample under consideration. The standard indexing system is used; for example, the two-digit index consists of the last digits of the respective atomic and mass numbers of the nuclide in question. Subscript 0 signifies the beginningof-life value of parameter.

To relate the final (i.e., end-of-life) isotopic concentrations to the initial U-238 concentration, Equations (1) through (8) are divided by $\mathrm{N}_{0}^{28}$.

From unit cell calculations of the Yankee fuel, the following parameters are obtained as functions of U-235 fractional depletion and input to the REBUF code:

$$
\begin{aligned}
\alpha^{49}= & f(D 25)=\text { ratio of total captures to total fissions in Pu-239 } \\
\alpha^{41}= & f(D 25)=\text { ratio of total captures to total fissions in Pu-24I } \\
\delta^{28}= & f(D 25)=\text { total fissions in } U-238 \text { relative to sum of total fissions } \\
& \text { in U-235, Pu-239, and Pu-241 }
\end{aligned}
$$

$$
\begin{aligned}
& \text { COR26 }=\mathrm{f}(\mathrm{D} 25)=\text { ratio of gross to net } \mathrm{U}-236 \text { production } \\
& \text { COR42 }=\mathrm{f}(\mathrm{D} 25)=\text { ratio of gross to net } \mathrm{Pu}-242 \text { production }
\end{aligned}
$$

The fractional depletion of $\mathrm{U}-235$ is obtained with:

$$
\mathrm{D} 25=1-\frac{\mathrm{N}^{25}}{\mathrm{~N}^{28}} \times \frac{\mathrm{N}^{28}}{\mathrm{~N}_{0}^{28}} \times \frac{\mathrm{N}_{0}^{28}}{\mathrm{~N}_{0}^{25}}
$$

The above system of equations are solved by iteration in the REBUF code. The values of $\alpha^{49}, \alpha^{41}, \delta^{28}, \operatorname{COR} 26$, and COR42 have been obtained from a LASER ${ }^{[21]}$ calculation and are shown on Figure 5-1. 


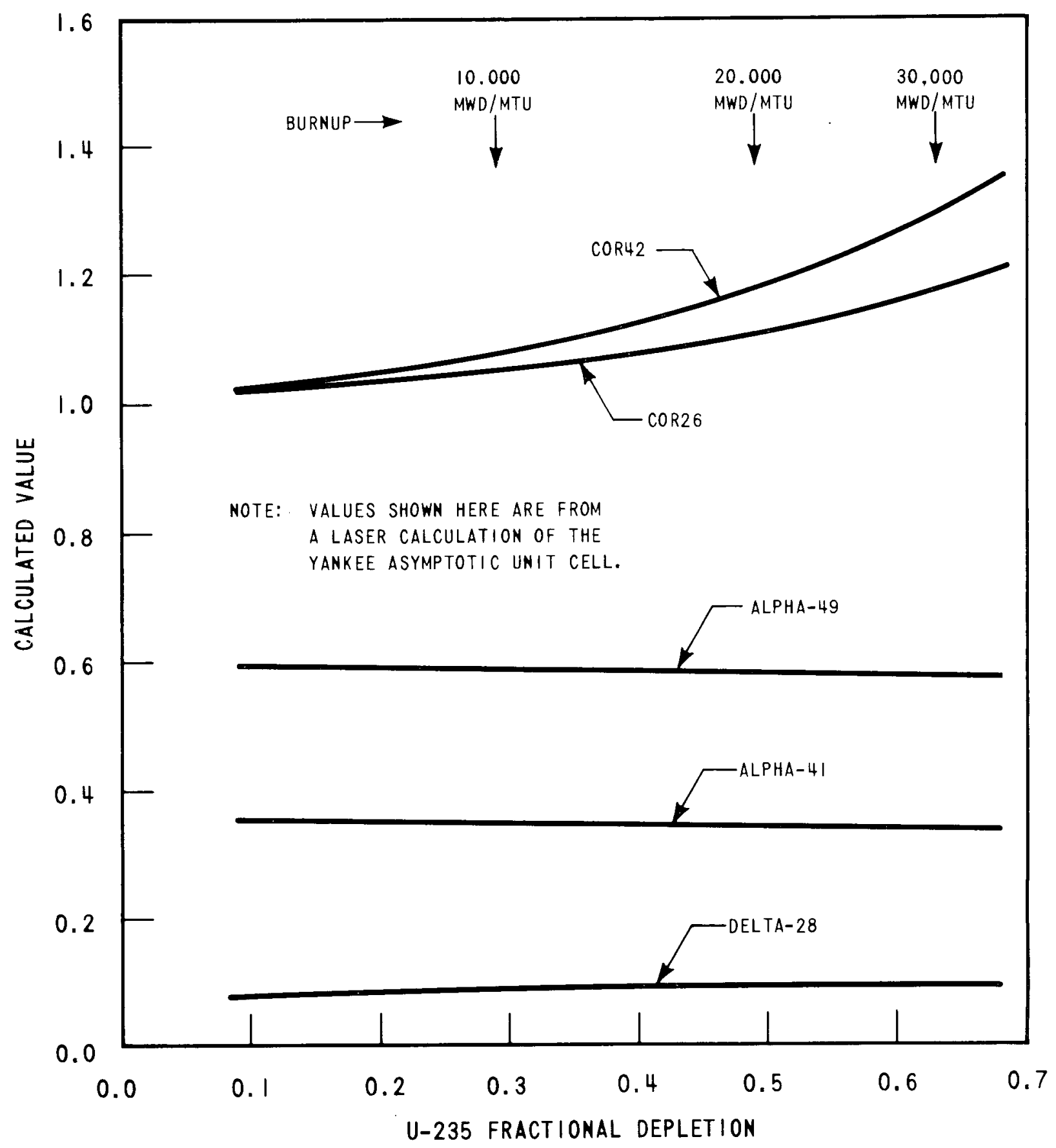

Figure 5-1. Parameters Used in Reduction of Isotopic Data 
Some nuclear fuel characteristics determined from the isotopic measurements are given in Appendix $B$.

\subsection{ISOTOPIC DECAY CORRECTIONS}

The measurements on spent fuel samples are made after various intervals of cooling time after core shutdown. To place the results obtained for those isotopes of relatively short half-life on the same basis with the results obtained for those isotopes of relatively long half-life, and to make meaningful comparisons with the results of calculations, it is necessary to decay-correct the measured data to some given reference time. In this study, the reference time is taken to be the core end-of-life.

The equations used to account for radioactive decay from the core end-of-life (EOL) to the sample time-of-analysis (TOA) are:

$$
\begin{aligned}
& \mathrm{A}_{0}=\mathrm{A} \mathrm{e}^{\lambda \mathrm{a}^{\mathrm{T}} \mathrm{a}} \\
& \mathrm{B}_{0}=\mathrm{B} \mathrm{e}^{\lambda_{\mathrm{b}} \mathrm{T} b}-\frac{\lambda_{\mathrm{a}}}{\lambda_{\mathrm{b}}-\lambda \mathrm{a}} \mathrm{A} e^{\lambda_{\mathrm{a}} \mathrm{T} a}\left[\mathrm{e}^{\left(\lambda_{\mathrm{b}}-\lambda_{\mathrm{a}}\right) \mathrm{T} b}-1\right]
\end{aligned}
$$

where isotope A decays to isotope B, and isotope B itself undergoes decay, and

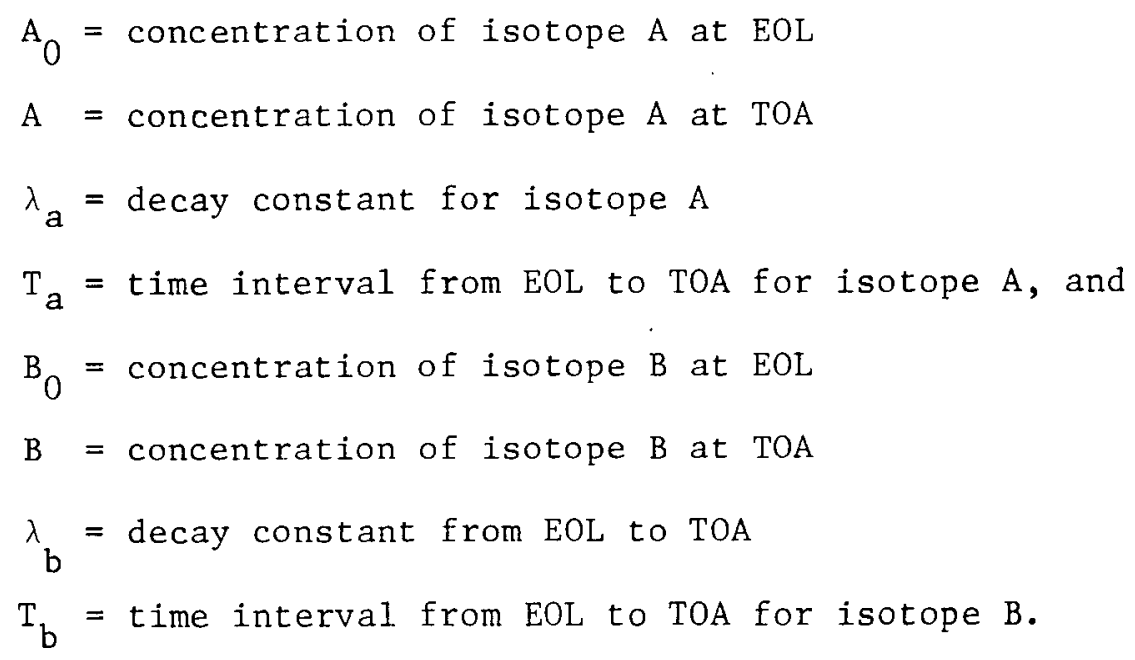


In the actual application of the above equations, $A$ and $B$ are atomic ratios, relative to some constant, rather than absolute values of atoms $/ \mathrm{cm}^{3}$. For example, in the case of $\mathrm{Pu}-236$ and U-232, A could be $\mathrm{N}^{46} / \mathrm{N}_{0}^{28}$ and $\mathrm{B}$ would be $\mathrm{N}^{22} / \mathrm{N}_{0}^{28}$.

Phase 1 data, for samples irradiated in Core I, are referenced to Core I EOL on May 18, 1962; Phase 2 data, for samples irradiated in Cores I and II, are referenced to Core II EOL on September 2, 1963; Phase 3 data, for samples irradiated in Cores I, II, and IV, are referenced to Core IV EOL on August 9, 1965. 
SECTION 6

EVALUATION OF SEVERAL METHODS FOR

DETERMINING FUEL BURNUP

The main objectives for obtaining the burnup from destructive analysis of depleted fuel samples are:

1. To determine the total energy release of the fuel of a reactor core, or of regions of fuel irradiated during one or more core cycles; the results can then be used to evaluate the total energy release inferred fron non-destructive calorimetric measurements, used in conjunction with power distribution measurements obtained with in-core instrumentation (e.g., flux-wires, fission detectors, thermocouples) during reactor operation.

2. To provide an independent parameter with which changes in isotopic inventory can be correlated; the resulting correlations can then be used to evaluate the adequacy of calculational models and the accuracy of the basic nuclear data used in the calculations.

3. To determine various nuclear fuel characteristics such as conversion ratios and capture-to-fission ratios.

4. To provide a check on the self-consistency of isotopic data obtained from destructive analysis when more than one burnup indicator is used per sample.

In the case of Yankee fuel, the results of these objectives are discussed and evaluated to some degree in References $5,9,10,11,20,27,28,29,41$, and 62 .

In obtaining the specific energy release for each sample, the total accumulated fissions is determined first, and then converted to burnup with appropriate values of $\mathrm{MeV} / \mathrm{fission}$. In this study the parameter $\mathrm{F}_{\mathrm{TOT}} / \mathrm{N}_{28}^{0}$, the total accumulated fissions relative to the initial U-238 atoms, is used as the independent variable to evaluate uranium depletion and plutonium 
buildup. As will be shown in later sections of this report, the comparisons between experiment and theory are down to about the two to three percent level for the main chain uranium and plutonium isotopes. For these comparisions to be valid, the values of burnup, or total fissions, with which the uranium and plutonium isotopic data are correlated, must be accurate and free from bias.

In the following paragraphs, several methods for obtaining fuel burnup are described; the methods are compared using the values of total fissions inferred by applying the several methods to the data measured for each sample.

\subsection{DETERMINATION OF FUEL BURNUP}

Two basic methods can be used to determine the fuel burnup from the destructive analysis of spent fuel. These are based on the reduction of isotopic composition of spent fuel (heavy element or HE method) and the concentration of fission products. The descriptions given below include the fission products cesium-137, strontium-90 and neodymium-148.

The parameters necessary to calculate the burnup with the HE method are the energy-conversion factors (MeV/fission), $\delta_{28}, \alpha_{49}, \alpha_{41}$. In addition, the initial composition of the pre-irradiated fuel must be known. The parameters necessary to determine the burnup using cesium-137, strontium-90 or neodymium-148 are MeV/fission, the decay constant (zero in the case of the stable fission product neodymium-148), and the isotopic fission yields. The values of these parameters used in this study are summarized in Table 6-1. The effective values of energy release per fission are from a LEOPARD [22] calculation performed for a Yankee unit cell; the values of $\delta_{28}, \alpha_{49}$, and $\alpha_{41}$ are from a LASER calculation, and are shown on Figure 5-1 of the previous section. The fission product data shown in Table 6-1 are from References $23,24,25,30$, and 59 .

The accumulated fissions relative to initial $\mathrm{U}-238$ atoms $\left(\mathrm{F}_{i}^{\mathrm{HE}} / \mathrm{N}_{28}^{\mathrm{O}}\right)$ and the burnup $\left(\mathrm{BU}_{i} \mathrm{in}\right.$ GWD/MTU) due to fissions in each of the fissionable isotopes

* Please note that, relative to the indexing method of Section 5 and subsequent sections, the superscripts and subscripts used in this section are reversed, consistent with the method used in Reference 20. 
TABLE 6-1

PARAMETERS USED TO DETERMINE FUEL BURNUP FROM ISOTOPIC AND FISSİON PRODUCT MEASUREMENTS

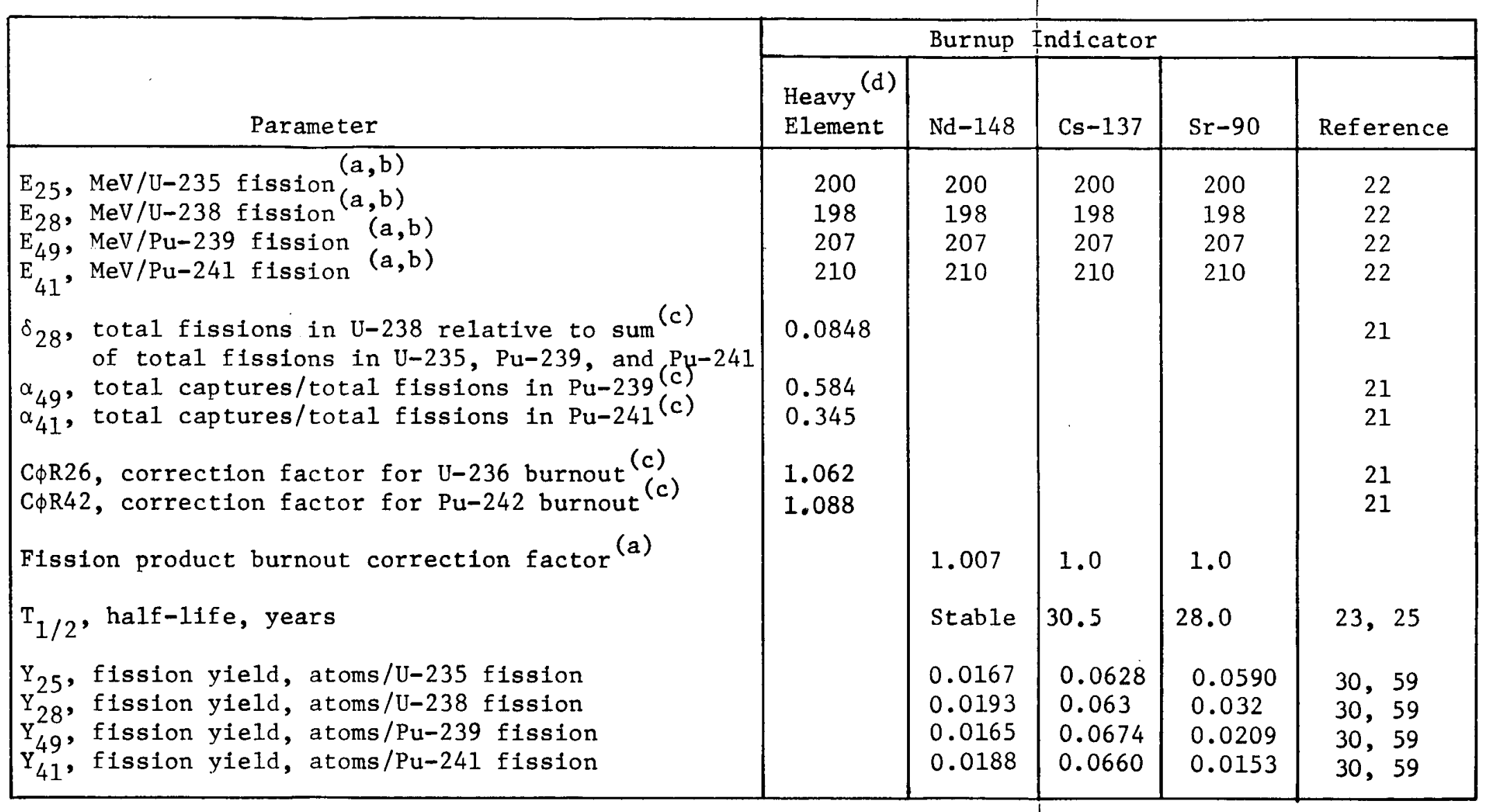

a. Nominal values shown here were obtained from a LEOPARD calculation of a Yankee 3.4 w/o unit cell at 13,500 MWD/MTU burnup. Actual values used in data reduction are burnup-dependent.

b. Includes kinetic energy of fission fragments and neutrons, energy of beta and gamma decay, and energy of radiative capture in fuel, cladding, and moderator; see Reference 9 for breakdown of various energy sources.

c. Nominal values shown here were obtained from a LASER calculation of a Yankee $3.4 \mathrm{w} / \mathrm{o}$ unit cell at 13,500 MWD/MTU burnup. Actual values used in data reduction are burnup-dependent.

d. The Heavy Element method also requires the pre-irradiated isotopic distribution in the fuel. Values of initial fuel composition used in this program are $0.021,3.44,0.020$ and 96.519 atom percent for U-234, U-235, U-236 and $\mathrm{U}-238$, respectively, 
can be calculated from

$$
\begin{gathered}
\mathrm{F}_{i}^{\mathrm{HE}} / \mathrm{N}_{28}^{0}=\frac{\mathrm{F}_{i}}{\mathrm{~N}_{28}^{0}} \\
\mathrm{BU}_{i}^{\mathrm{HE}}=\mathrm{KE}_{i} \frac{\mathrm{N}_{28}^{0}}{\rho} \frac{\mathrm{F}_{i}}{\mathrm{~N}_{28}^{0}}
\end{gathered}
$$

where $E_{i}$ is defined in Table 6-1 and is shown as a function of U-235 fraction depletion in Figure 6-1. The quantity $\rho$ is the density of uranium feed in $\mathrm{MTU} / \mathrm{cm}^{3}$, and $\mathrm{K}=1.854 \times 10^{-27} \mathrm{GWD} / \mathrm{MeV}$, an energy unit conversion factor. The method of calculating the ratios $\mathrm{F}_{i} / \mathrm{N}_{28}^{0}$ was shown previously in section 5 . The quantity $\mathrm{N}_{28}^{0} / \rho$ is evaluated explicitly from the initial fuel composition on a unit mass basis with

$$
\frac{\mathrm{N}_{28}^{0}}{\rho}=\frac{\mathrm{A}_{0}(\mathrm{a} / 0)_{28}^{0} \times 10^{6}}{\mathrm{~W}_{24}(\mathrm{a} / \mathrm{o}){ }_{24}^{0}+\mathrm{W}_{25}(\mathrm{a} / 0)_{25}^{0}+\mathrm{W}_{26}(\mathrm{a} / 0)_{26}^{0}+\mathrm{W}_{28}(\mathrm{a} / 0)_{28}^{0}}
$$

where $(a / 0)_{i}^{0}$ is the isotopic composition of the pre-irradiated fuel in atom percent, $W_{i}$ is the atomic weight of isotope $i$, and $A_{0}$ is the Avagadro number. The total accumulated fissions relative to initial U-238 atoms and the fuel burnup determined from the heavy elements is:

$$
\begin{aligned}
\mathrm{F}_{\mathrm{TOT}}^{\mathrm{HE}} / \mathrm{N}_{28}^{0} & =\sum_{i} \mathrm{~F}_{i}^{\mathrm{HE}} / \mathrm{N}_{28}^{0} \\
\mathrm{BU}^{\mathrm{HE}} & =\sum_{i} \mathrm{BU}_{i}
\end{aligned}
$$

where $i$ includes the fissionable isotopes uranium-235, uranium-238, plutonium-239 and plutonium-241. 


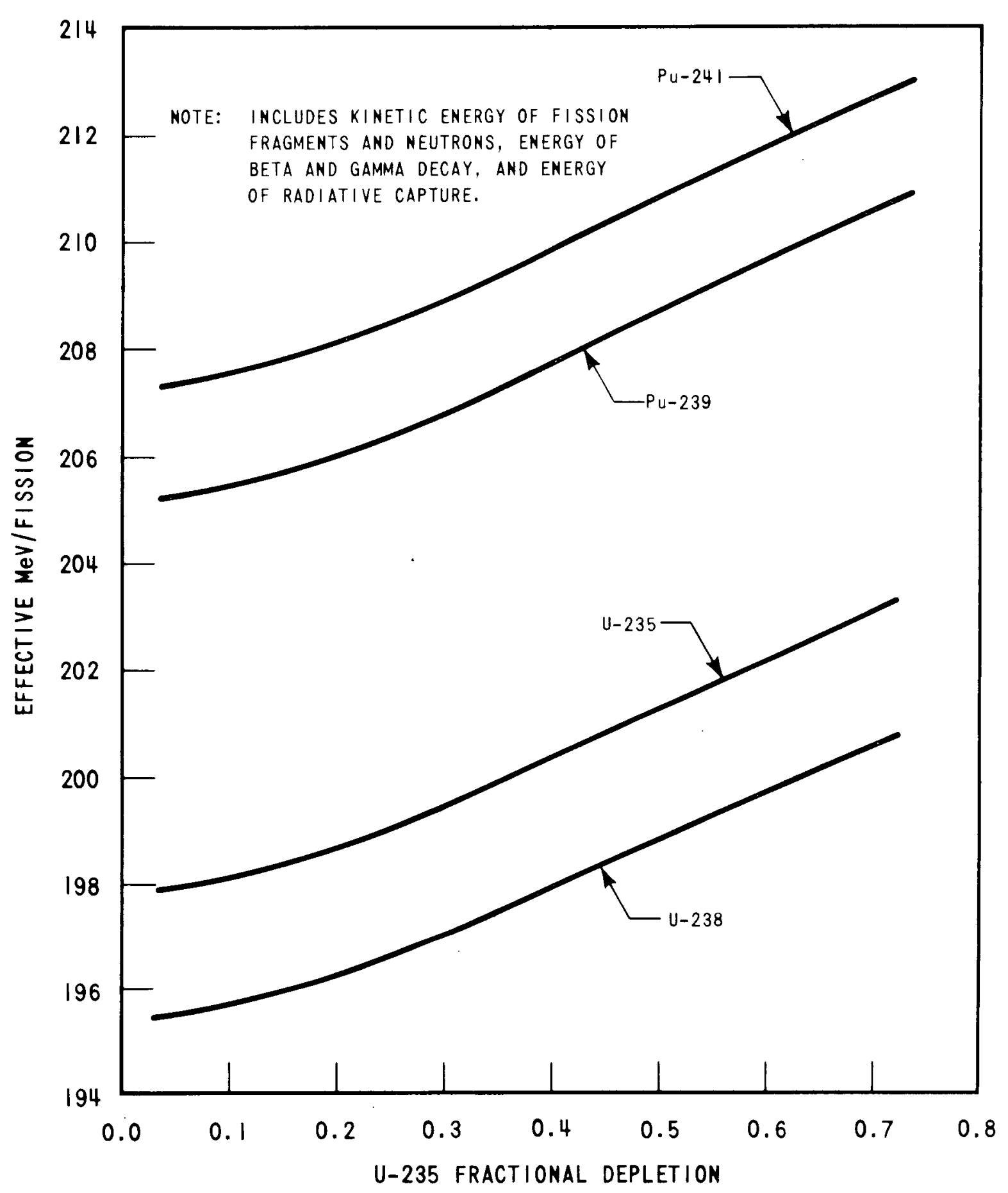

Figure 6-1. Calculated Energy Release Per Fission in U-235. $\mathrm{U}-238$. $\mathrm{Pu}-239$, and $\mathrm{Pu}-241$ 
The burnup of the fuel can also be calculated from the density of any fission product whose yield in fission and half life are reasonably well known and which has a low enough neutron-capture cross section so as to require relatively small or negligible corrections for its subsequent destruction by neutron captures. In the case of a fuel sample which represents but one or several fuel pellets, the fission product must be nonvolatile or its use must be restricted to samples of fuel which has not been subjected to severe temperature gradients during irradiation. In the case of a fuel sample which represents a whole fuel rod or bundle of rods, the migration of the fission product is not a problem. To a greater or lesser degree, cesium-137, strontium-90, and neodymium-148 satisfy these conditions.

The total accumulated fissions relative to initial U-238 atoms and the fuel burnup can be obtained from cesium-137 concentration by application of the following equations:

$$
\begin{aligned}
& \mathrm{F}_{\mathrm{TOT}}^{\mathrm{Cs}} / \mathrm{N}_{28}^{0}=\frac{\mathrm{A}^{\mathrm{Cs}} t_{2} \times 10^{6}}{\mathrm{Y}_{\text {eff }}^{\mathrm{Cs}}} \frac{\exp \left(\lambda_{57} t_{1}\right)}{1-\exp \left(-\lambda_{57} t_{2}\right)} \frac{\mathrm{N}_{28}^{0}}{\rho}
\end{aligned}
$$

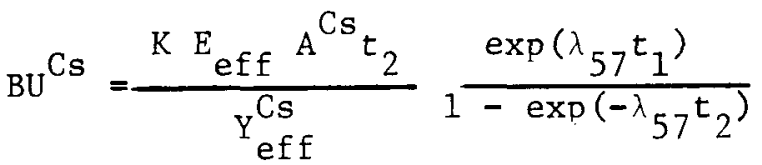

where $t_{1}$ is the time between core shutdown and cesium-137 analysis (min), $t_{2}$ is the in-core irradiation time ( $\left.\mathrm{min}\right), \mathrm{A}^{\mathrm{Cs}}$ is the cesium-137 activity $\mathrm{dis} /$ (min MTU) at the time of analysis, $\lambda_{57}$ is the cesium-137 decay constant $\left(\min ^{-1}\right.$ ), and $\mathrm{Y}_{\text {eff }}^{\mathrm{Cs}}$ is the effective fission yield of cesium-137, weighted by isotope (fission ${ }^{-1}$ ). The symbol $E_{\text {eff }}$ denotes the effective energy per fission, weighted by isotope (MeV/fission). $\mathrm{K}$ and $\rho / \mathrm{N}_{28}^{0}$ were defined earlier. 
Similarly, total fissions and burnup can be obtained from strontium-90 concentration with:

$$
\begin{aligned}
\mathrm{F}_{\mathrm{TOT}}^{\mathrm{Sr}} / \mathrm{N}_{28}^{O} & =\frac{\mathrm{A}_{\mathrm{t}_{2}}^{\mathrm{Sr}} \times 0^{6}}{\mathrm{Y}_{\text {eff }}^{\mathrm{Sr}}} \frac{\exp \left(\lambda_{80} t_{1}\right)}{1-\exp \left(-\lambda_{80} t_{2}\right)} \frac{\mathrm{N}_{28}^{O}}{\rho} \\
\mathrm{BU}^{\mathrm{Sr}} & =\frac{\mathrm{K} \mathrm{E}_{\text {eff }} \mathrm{A}^{\mathrm{Sr} t_{2}}}{\mathrm{Y}_{\text {eff }}^{\mathrm{Sr}}} \frac{\exp \left(\lambda_{80} t_{1}\right)}{1-\exp \left(-\lambda_{80} \mathrm{t}_{2}\right)}
\end{aligned}
$$

To determine the total accumulated fissions and the fuel burnup from the neodymium-148 data, the following expressions can be used:

$$
\begin{gathered}
\mathrm{F}_{\mathrm{TOT}}^{\mathrm{Nd}} / \mathrm{N}_{28}^{\mathrm{O}}=\frac{\mathrm{N}_{08}}{\mathrm{~N}_{28}} \frac{\mathrm{N}_{28}}{\mathrm{~N}_{28}^{0}} \frac{\mathrm{C}}{\mathrm{Y}_{\text {eff }}^{\mathrm{Nd}}} \\
\mathrm{BU}^{\mathrm{Nd}}=\mathrm{K} C \frac{\mathrm{N}_{28}^{0}}{\rho} \frac{\mathrm{N}_{08}}{\mathrm{~N}_{28}} \frac{\mathrm{N}_{28}}{\mathrm{~N}_{28}^{0}} \frac{\mathrm{E}_{\text {eff }}}{\mathrm{Y}_{\text {eff }}^{\mathrm{Nd}}}
\end{gathered}
$$

where $\mathrm{N}_{08} / \mathrm{N}_{28}$ is the measured $\mathrm{Nd}-148 / \mathrm{U}-238$ atom ratio*; $\mathrm{N}_{28} / \mathrm{N}_{28}^{0}$ is the final to initial uranium-238 atom ratio, determined from isotopic data as outlined in the previous section; $\mathrm{N}_{28}^{0} / \rho$ is the initial uranium-238 atoms per metric ton of uranium loading; $E_{\text {eff }}$ is the effective energy release per fission (including kinetic energy of fission fragments and neutrons, energy of beta and gamma decay, and energy of radiative capture) weighted by isotope, $\mathrm{MeV} /$ fission; $\mathrm{Y}_{\mathrm{eff}}^{\mathrm{Nd}}$ is the effective fission yield of neodymium-148, weighted by isotope, atom/fission; $C$ is a burnup-dependent correction factor for neodymium-148 burnout; and $\mathrm{BU}^{\mathrm{Nd}}$ is the fuel burnup inferred from neodymium-148 concentration, GWD/MTU.

* Corrected for natural neodymium contamination. 
The correction for neodymium-148 burnout ranges up to about 2 percent at 40 GWD/MTU burnup. The correction factor $C$, was determined by calculating the gross and net neodymium-148 production as described in the following paragraphs. An equation which describes the variation of neodymium-148 can be written as:

$$
\begin{aligned}
\frac{d N_{08}(t)}{d t} & =Y_{e f f}(t) \Sigma_{f_{1}}(t) \phi_{1}(t)+\Sigma_{f_{2}}(t) \phi_{2}(t)-\sigma_{a_{1}}(t) \phi_{1}(t) \\
& +g \sigma_{a_{2}}(t) \phi_{2}(t) N_{08}(t)
\end{aligned}
$$

where, as a function of irradiation time $t, Y_{\text {eff }}$ is the effective fission yield of neodymium-148, $\Sigma_{\mathrm{f}_{1}}$ and $\Sigma_{\mathrm{f}_{2}}$ are the fast and thermal macroscopic fission cross sections obtained from the LEOPARD unit cell calculations, $\phi_{1}$ and $\phi_{2}$ are the fast and thermal fluxes from the LEOPARD calculation, and $\mathrm{g}$ is the unit cell thermal disadvantage factor. $\mathrm{N}_{08}$ is the neodymium-148 atom density, and $\sigma_{a_{1}}$ and $\sigma_{a_{2}}$ are the neodymium-148 fast and thermal microscopic absorption cross sections.

Neodymium-148 is also formed through the fission yield of praseodymium and neutron capture in neodymium isotopes of mass number lower than 148 . However, these routes are effectively blocked by neodymium-147 which has a half life of 11.1 days. Assuming a neodymium-147 thermal absorption cross section of 350 barns, the absorption reaction rate is of the order of $6 \times 10^{-9} \mathrm{sec}^{-1}$, which is approximately 1 percent of the neodymium-147 decay constant of $7 \times 10^{-7} \sec ^{-1}$.

The values of neodymium-148 microscopic absorption cross sections used in the above expression are

$$
\sigma_{a_{1}}=\frac{R I}{\Delta u}=\frac{18.7}{16.5884}=1.13 \mathrm{~b}
$$




$$
\sigma_{a_{2}}=\left.\sigma_{a} \frac{1}{v}\right|_{W W}=(2.54)(0.52)=1.32 \mathrm{~b}
$$

where the resonance integral RI $(18.7 \pm 0.5)$ and the $2200 \mathrm{~m} / \mathrm{s}$ cross section $(2.54 \pm 0.18)$ are reported by $C . P$. Ruiz, et al. [26] The lethargy increment $\Delta \mathrm{u}$ applies to neutron energy $>0.625 \mathrm{eV}$, and $1 / \mathrm{v}$ (Wigner-Wilkins) factor converts the $2200 \mathrm{~m} / \mathrm{s}$ value to the Yankee thermal spectrum.

Solution of the differential equation for net neodymium-148 production gives:

$$
N_{08}^{n e t}=\frac{A}{B} Y_{\text {eff }}+\left[N_{08}^{0}-\frac{A}{B} Y_{\text {eff }}\right] e^{-B t}
$$

Equating the neodymium-148 cross sections, $\sigma_{a_{1}}$ and $\sigma_{a_{2}}$, to zero gives the gross neodymium-148 production:

$$
\mathrm{N}_{08}^{\text {gross }}=\mathrm{Y}_{\text {eff }} A t+\mathrm{N}_{08}^{0}
$$

where

$$
\begin{aligned}
\mathrm{A}= & \Sigma_{\mathrm{f}_{1}} \phi_{1}+\Sigma_{\mathrm{f}_{2}} \phi_{2} \\
\mathrm{~B}= & \sigma_{\mathrm{a}_{1}} \phi_{1}+\mathrm{g \sigma}_{\mathrm{a}_{2}} \phi_{2} \\
\mathrm{~N}_{08}^{0}= & \text { neodymium-148 atom density at the beginning of a time interval, and } \\
\mathrm{t}= & \text { time interval in seconds, over which the neodymium-148 production } \\
& \text { is evaluated. }
\end{aligned}
$$

The correction factor for neodymium-148 burnout, C, is simply:

$$
\mathrm{C}=\frac{\mathrm{Nd}-148 \text { (gross production) }}{\mathrm{Nd}-148 \text { (net production }}
$$

The calculated variation of $C$ with fuel burnup is shown in Figure 6-2. This correction factor is applied to the measured $\mathrm{N}_{08} / \mathrm{N}_{28}$ ratio through an 


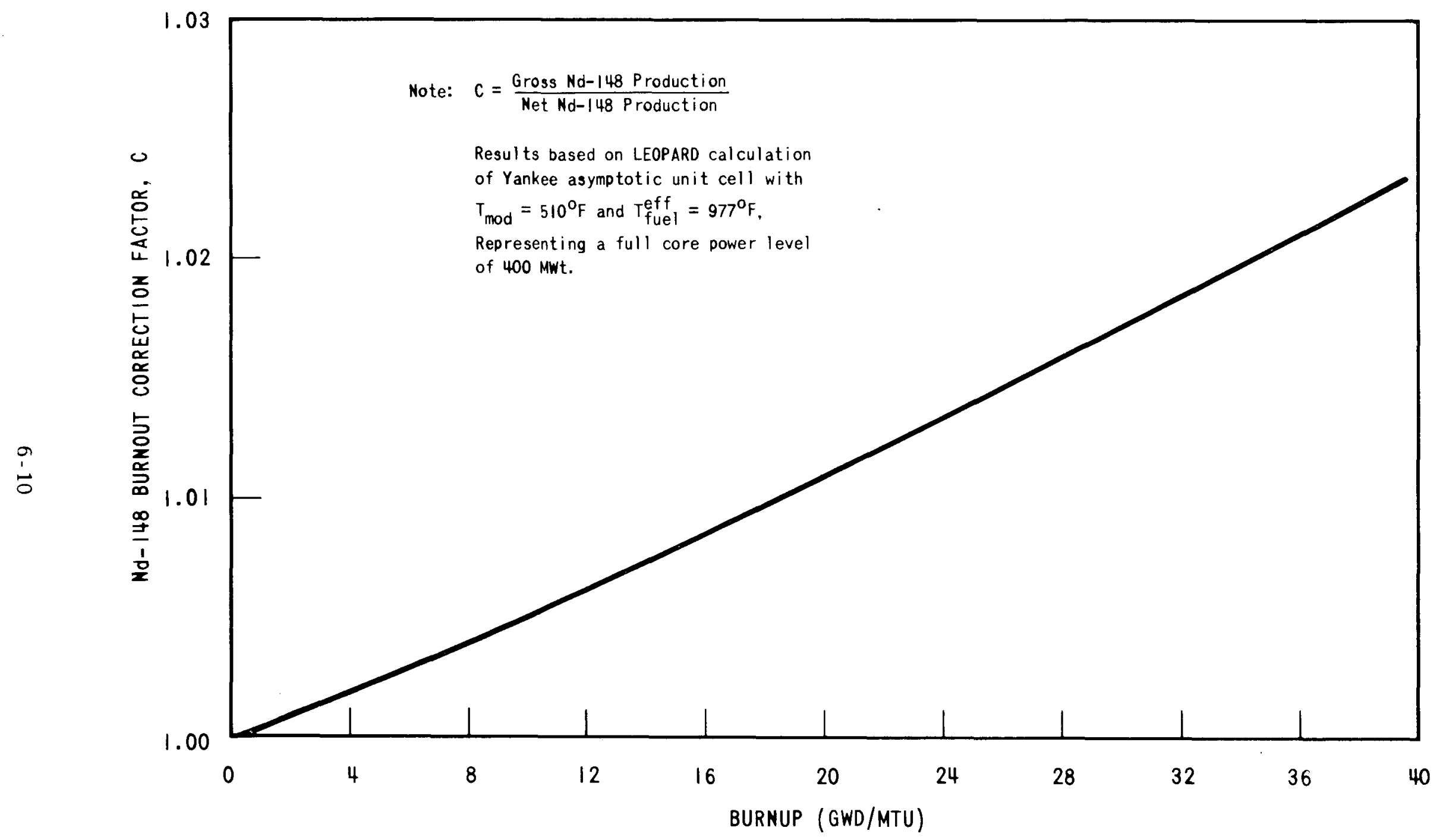

Figure 6-2. Calculated Correction Factor for Nd-148 Burnout versus Fuel Burmup 
iterative procedure in the REBUF code with the following quadratic expression:

$$
\mathrm{C}=1.0+\mathrm{C}_{1}(\mathrm{BU})+\mathrm{C}_{2}(\mathrm{BU})^{2},
$$

where

$$
\begin{aligned}
C_{1}= & 5.019 \times 10^{-4} \\
C_{2}= & 2.275 \times 10^{-6} \text {, and } \\
B U= & \text { fuel burnup inferred from measured } \mathrm{Nd}-148 / \mathrm{U}-238 \text { data, in units } \\
& \text { of GWD/MTU. }
\end{aligned}
$$

Using the methods described above, the values of total accumulated fissions and fuel burnup were determined for each sample. The results obtained for the EYC Phase 3 and the Program Extension samples are given in Appendix B; the Phase 1 and Phase 2 data are reported in Reference 5. The several methods are compared in the following paragraphs.

\subsection{COMPARISON OF Cs-137 AND Sr-90 BURNUP INDICATORS WITH HEAVY ELEMENT BURNUP INDICATOR}

The values of total accumulated fissions determined from the measurements of the concentrations of the $\mathrm{Cs}-137$ and $\mathrm{Sr}-90$ fission products are compared with the total accumulated fissions determined with the heavy element method as shown in Figure 6-3. The upper portion of Figure 6-3 shows the ratio of values of total accumulated fissions determined from Cs -137 relative to the corresponding values determined from the heavy elements, while the lower portion of the figure shows the $\mathrm{Sr}-90$ data relative to the heavy element results; the ratios are plotted versus the fuel burnup inferred with the HE method. The solid line at 1.0 indicates equivalent results from the two methods being compared, while the broken line is a least squares fit of the data.

For each sample, the estimated uncertainties on the Cs -137 and $\mathrm{Sr}-90$ results are on the order of \pm 7 percent while the estimated uncertainty on the HE results is burnup-dependent, ranging from about \pm 8 percent at low burnup to about \pm 3 percent at high burnup. Assuming that the uncertainties on the 


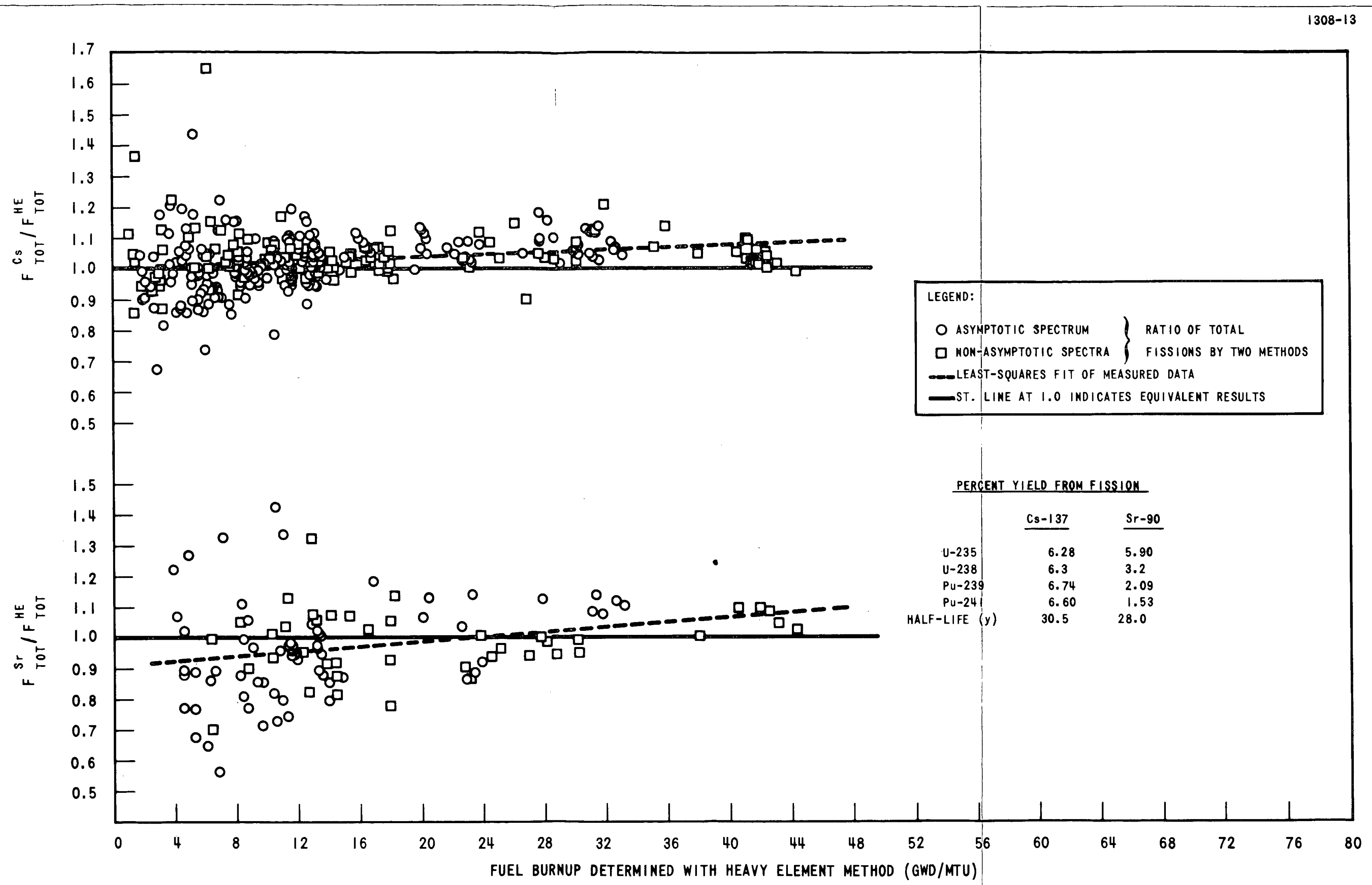

Figure 6-3. Comparison of Total Fissions Inferred from Cs-137 and Sr-90 with from Heavy El ements 
results obtained by the different methods are independent and statistical in nature, the resulting estimated uncertainties on the ratios $\mathrm{F}_{\mathrm{TOT}}^{\mathrm{Cs}} / \mathrm{F}_{\mathrm{TOT}}^{\mathrm{He}}$ and $\mathrm{F}_{\mathrm{TOT}}^{\mathrm{Sr}} / \mathrm{F}_{\mathrm{TOT}}^{\mathrm{HE}}$ range from about \pm 11 percent at low burnup to about \pm 8 percent at high burnup. Referring to Figure 6-3, it is apparent that these estimates are not quite consistent with the measured data, the data scatter being somewhat greater than \pm 11 percent at low burnup, and somewhat less than \pm 8 percent at high burnup.

In spite of the considerable scatter in the data on Figure 6-3, it is concluded that the results from the Cs-137 burnup indicator are biased with respect to the results obtained with the $\mathrm{HE}$ method; the bias is burnupdependent and ranges up to about +7 percent at high burnup. The Sr-90 burnup indicator is also biased with respect to the $\mathrm{HE}$ burnup indicator, and on a least squares basis the bias ranges from about -8 percent at low burnup to about +8 percent at high burnup.

Use of the values of Cs-137 and $\mathrm{Sr}-90$ fission yields reported in Reference 59 would tend to increase the bias in the Cs-137 data, and decrease the bias in the Sr-90 data.

\subsection{COMPARISON OF CS-137 AND HEAVY ELEMENT BURNUP INDICATORS WITH Nd-148 BURNUP INDICATOR}

One of the objectives of the EYC Program Extension is the evaluation of the use of the Nd-148 fission product as an accurate burnup indicator. The evaluation was to have been a comparison of the results obtained with the relatively new method which employs the Nd-148 burnup indicator with the results obtained from the relatively older, and supposedly well established, methods employing the Cs-137 and the HE burnup indicators. However, the scatter in the Cs-137 and HE data, and more particularly, the bias between the Cs-137 and HE methods as shown in the previous paragraphs, preclude the use of these burnup indicators in a meaningful evaluation of the Nd-148 burnup indicator. Instead, the premise is made that the Nd-148 burnup indicator is the most accurate means of determining total fissions and fuel burnup, and the $\mathrm{Nd}-148$ results are used to resolve the bias between the Cs-137 and HE methods. 
At least two sets of fission yields have been reported for $\mathrm{Nd}-148$; these values are listed in Table 6-2. The values reported by lieek and Rider, et al, [59] are designated as Set $A$, while the values for thermal fission reported by Lisman, et al, [30] are designated as set B. In the evaluations made in this study, both sets of fission yield data are used.

The method of comparison is similar to that used in the previous section for Cs-137 and Sr-90; however, in this case, the $\mathrm{Nd}-148$ burnup indicator is used as the reference. Figure $6-4$ shows a plot of the ratios $\mathrm{F}_{\mathrm{TOT}}^{\mathrm{Cs}} / \mathrm{F}_{\mathrm{TOT}}^{\mathrm{Nd}}$ and $\mathrm{F}_{\mathrm{TOT}}^{\mathrm{He}} / \mathrm{F}_{\mathrm{TOT}}^{\mathrm{Nd}}$ versus fuel burnup, where the value of $\mathrm{F}_{\mathrm{TOT}}^{\mathrm{Nd}}$ used in the ratios has been determined using both Set $A$ and Set $B$ fission yields. In each of the four comparisons made in Figure 6-4, the solid line at 1.0 indicates equivalent results from the two methods being compared, while the broken line is a least squares fit of the data.

For each sample, the estimated uncertainty on the $\mathrm{Nd}-148$ data is about \pm 2 percent. Assuming that the uncertainties associated with the different methods are independent and statistical in nature, the resulting estimated uncertainty on the ratio $\mathrm{F}_{\mathrm{TOT}}^{\mathrm{Cs}} / \mathrm{F}_{\mathrm{TOT}}^{\mathrm{Nd}}$ is about \pm 7 percent; the estimated uncertainty on the ratio $\mathrm{F}_{\mathrm{TOT}}^{\mathrm{HE}} / \mathrm{F}_{\mathrm{TOT}}^{\mathrm{Nd}}$ is burnúp-dependent, ranging from about \pm 8 percent at low burnup to about +4 percent at high burnup. Except for a few outlying data points, these estimates are generally consistent with the scatter in the data shown in Figure 6-4.

On a least squares basis, it is concluded that the Cs -137 burnup indicator is biased with respect to the Nd-148 burnup indicator; the bias ranges from +1 percent at low burnup to about +3 percent at high burnup if set A yield data is used for $\mathrm{Nd}-148$; the bias ranges from +2 percent at low burnup to about +5 percent at high burnup if Set B yield data is used for Nd-148. In the case of the $\mathrm{HE}$ versus $\mathrm{Nd}-148$ comparison, the two methods are in statistical agreement regardless of which fission yield set is used for $\mathrm{Nd}-148$. On a least squares basis, the HE method is about one percent high or low, depending upon whether fission yield Set A or Set B is used for $\mathrm{Nd}-148$.

In the correlations of uranium depletion and plutonium buildup versus total accumulated fissions given in later sections of this report, the total accumulated fissions obtained with the HE method is used as the independent 
TABLE $\quad 6-2$

Nd-148 PERCENT YIELD FROM FISSION

\begin{tabular}{llcl} 
& \multicolumn{2}{c}{ Set $A$} & \multicolumn{2}{c}{ Set B } \\
$\frac{\text { Isotope }}{\mathrm{U}-235}$ & $\frac{\text { Thermal }}{1.67 \pm 0.03}$ & $\frac{\text { Thermal }^{(b)}}{1.69 \pm 0.01}$ & $\frac{\text { Fast }}{1.75}$ \\
$\mathrm{U}-238$ & $1.93 \pm 0.1$ & (c) & \\
Pu-239 & $1.65 \pm 0.05$ & $1.70 \pm 0.03$ & $1.73 \pm 0.03$ \\
Pu-241 & $1.88 \pm 0.09$ & $1.89 \pm 0.05$ & $1.92^{\text {(d) }}$
\end{tabular}

a. Reference 24 and 59.

b. Reference 30 .

c. Not reported, assume Set A value here.

d. Not reported. The value shown here is the Pu-241 thermal value, scaled by ratio of fast/thermal Pu-239 values. 


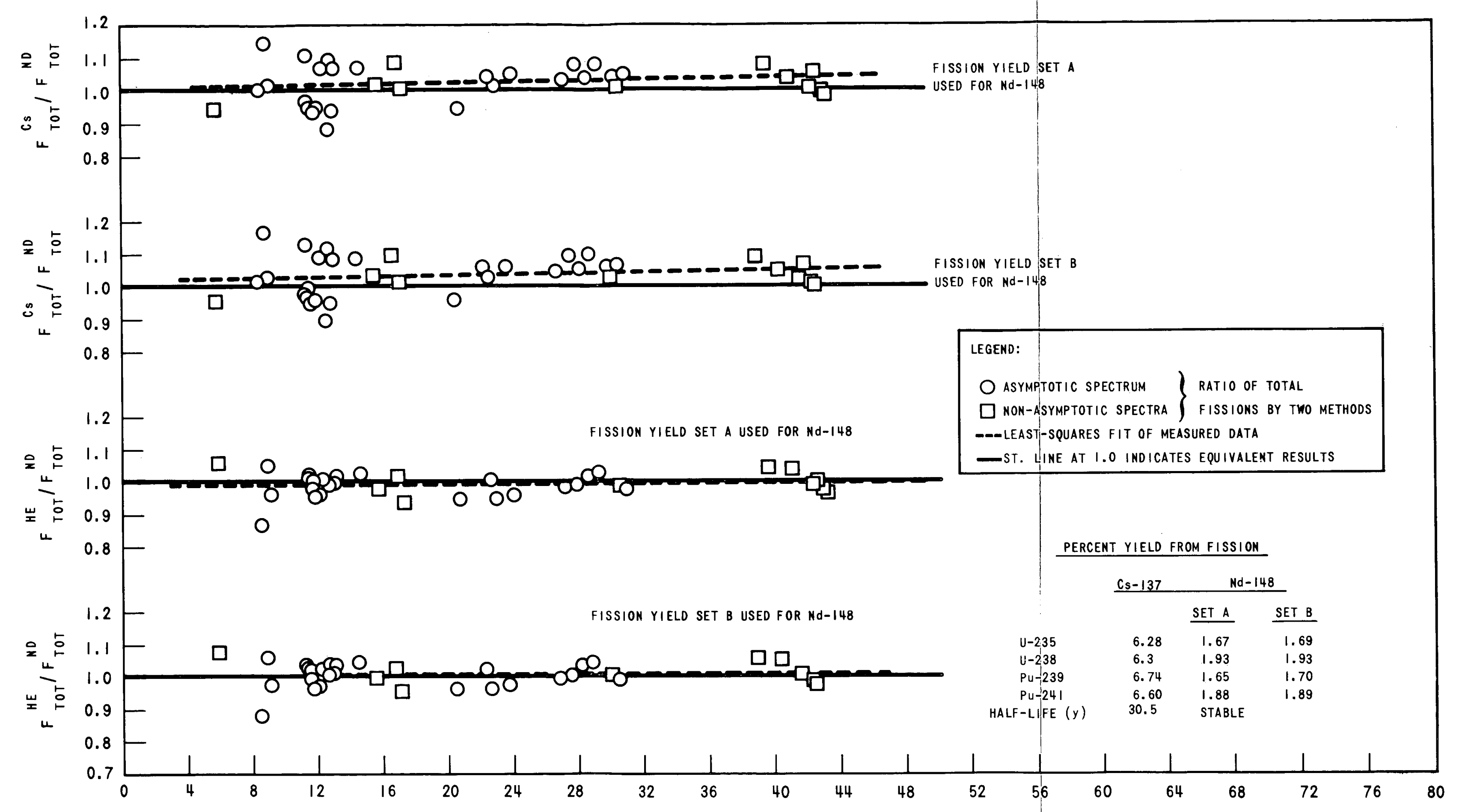

FUEL BURNUP DETERMINED FROM Nd-148 ANALYSIS (GWD/MTU)

Figure 6-4. Comparison of Total Fissions Inferred from Heavy Elements and $\mathrm{Cs}-137$ with Total Fissions Inferred rom Nd-148 
variable. Actually, in correlating the uranium and plutonium isotopic data with $\mathrm{F}_{\mathrm{TOT}}^{\mathrm{HE}} / \mathrm{N}_{28}^{0}$, the parameter $\mathrm{F}_{\mathrm{TOT}}^{\mathrm{HE}} / \mathrm{N}_{28}^{0}$ is not independent since it is inferred from the measured uranium and plutonium isotopic data. However, in view of the excellent agreement observed between the HE and Nd-148 methods, and since the HE burnup was determined for every sample analyzed in the EYC Program while Nd-148 was obtained only for the relatively small number of Program Extension samples, it is felt that the use of the HE method for determination of the independent variable is justified. The use of the Sr-90 and Cs-137 burnup indicators is not acceptable due to the large scatter, and apparent bias, in the results.

For cross reference purposes, Figures $6-5$ and $6-6$ have been prepared;

Figure 6-5 shows the U-235 Eractional depletion versus $\mathrm{F}_{\mathrm{TOT}}^{\mathrm{HE}} / \mathrm{N}_{28}^{0}$ determined for each sample in EYC Phases 1, 2, and 3 and in the Program Extension, while Figure $6-6$ shows the fuel burnup versus $\mathrm{F}_{\mathrm{TOT}}^{\mathrm{HE}} / \mathrm{N}_{28}^{0}$. 


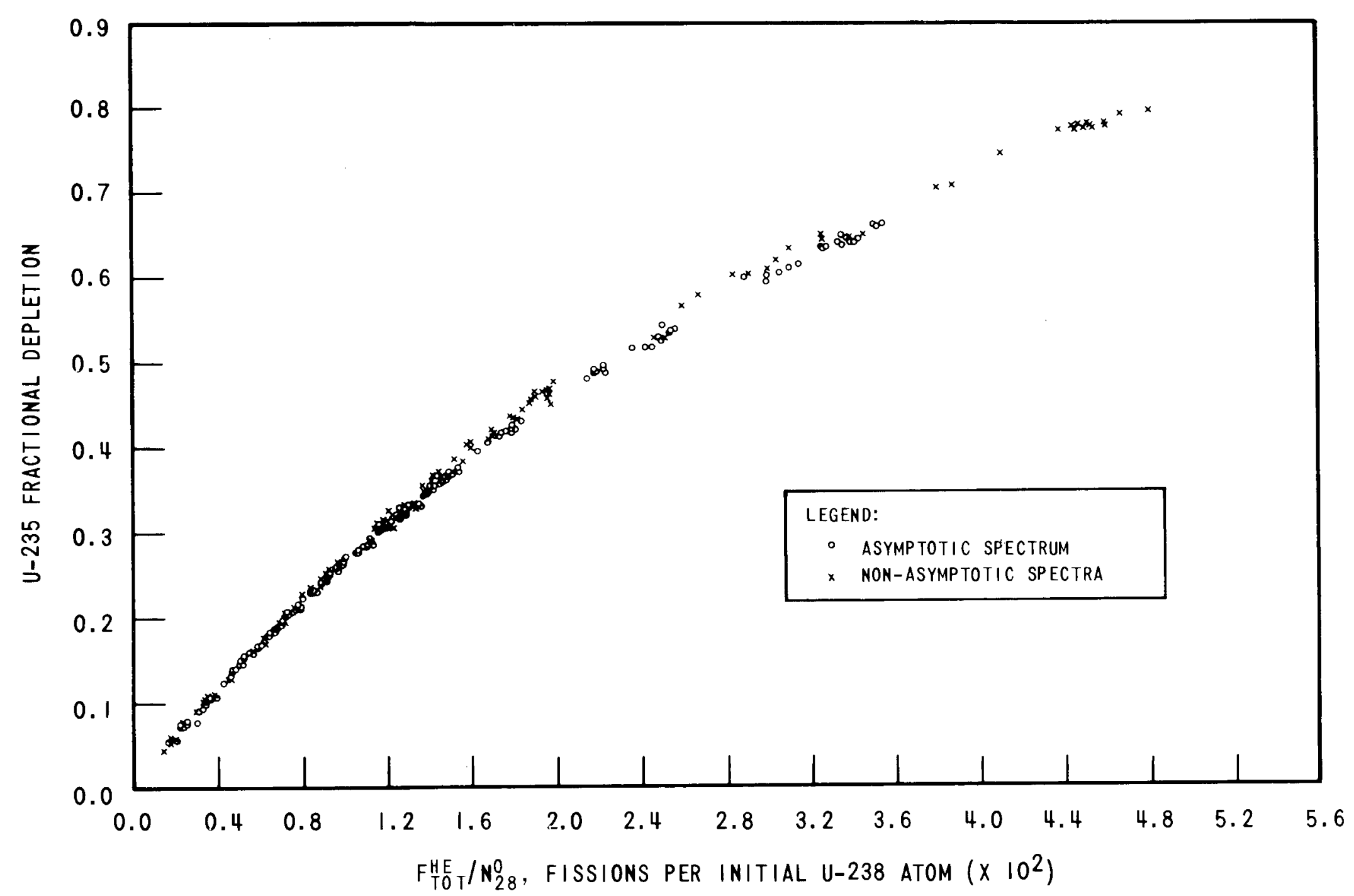

Figure 6-5. U-235 Fractional Depletion versus Total Accumulated Fissions 


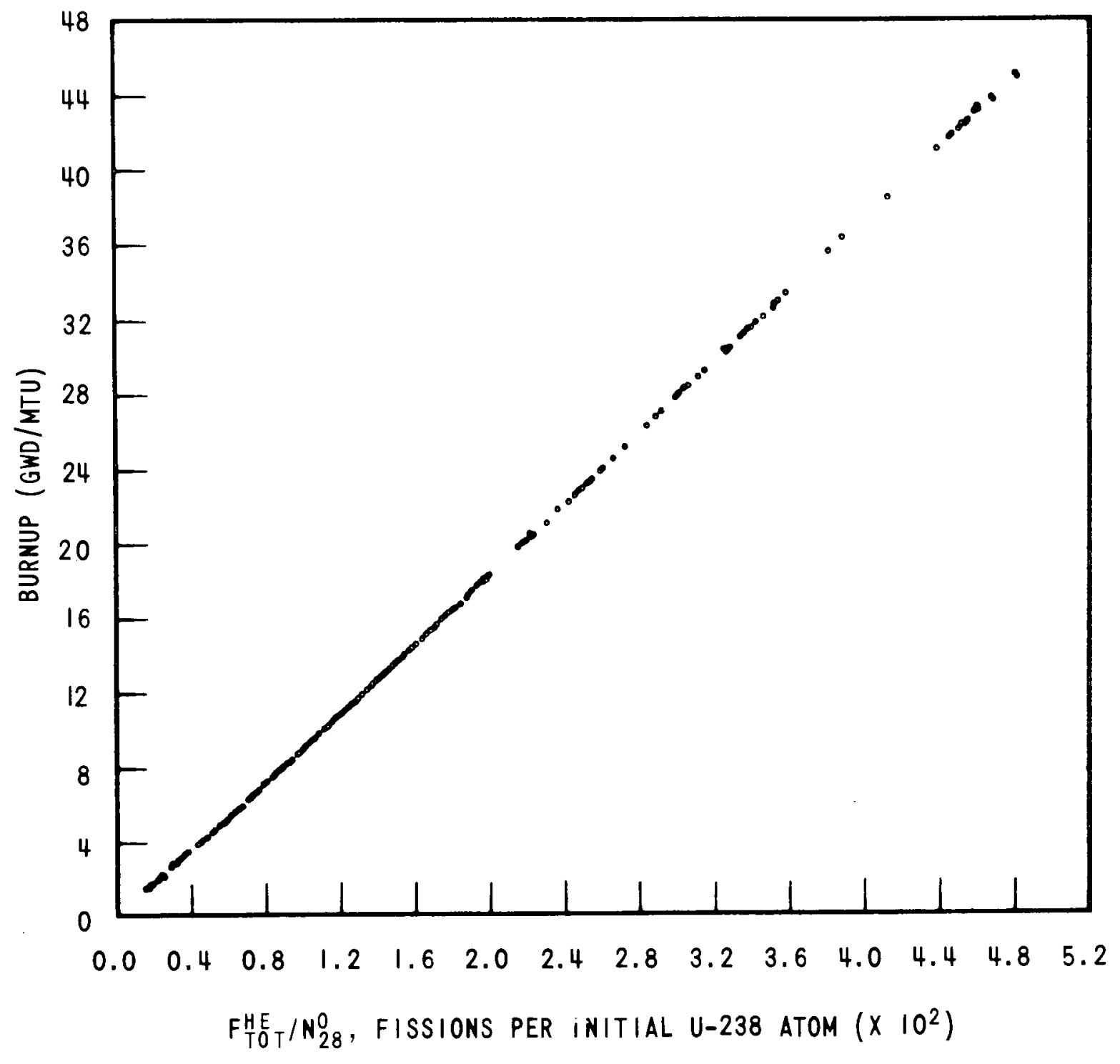

Figure 6-6. Fuel Burnup versus Total Accumulated Fissions 


\section{SECTION 7}

GAMMA ACTIVITY MEASUREMENTS

Both single-channel and multi-channel gamma activity data were obtained for the EYC Phase 3 fuel. The reasons for obtaining the single-channel gross gamma scans are:

1. The distribution of the gross gamma activity along the length of each rod reveals the presence of any gross abnormalities in the fuel pellet stack and aids in the selection of spent fuel samples representative of the fuel.

2. The normalized gamma activity distribution along each rod is compared with the normalized burnup distribution obtained from destructive analysis to determine the degree of accuracy with which the nondestructive gamma activity measurements can be used to predict the axial burnup distribution.

3. The integrated-average gamma activity of each rod is compared with the average burnup of the rod to determine the degree of accuracy with which the gamma activity can predict the assembly corner-to-center burnup ratio.

The reasons for obtaining the multi-channel gamma scans are:

1. The determination of the spectrum of fission product gamma activity at discrete points.

2. The predominate fission product activities can be stripped out of the gamma spectrum; the activity of the long-lived fission products represents the burnup distribution, while the activity of the short-lived fission products is indicative of the end-oflife power distribution. The gamma activity measurements are evaluated in the following paragraphs. 


\subsection{SINGLE-CHANNEL GROSS GAMMA SCANS}

The eight fuel rods removed from the Phase 3 fuel assembly were axially gamma scanned to obtain an estimate of the burnup distributions in the spent fuel. Two different detector systems were utilized to obtain the gross gamma activity of each intact fuel rod. One set of scans was obtained with a sodium-iodide scintillation crystal and a second set of scans was obtained with a miniature ionization chamber. The results of all Phase 3 gross gamma scans are summarized in Table 7-1. The scintillation crystal was used previously to scan the Phase 1 and 2 fuel rods, while the ionization chamber was used to scan the Phase 1 fuel assemblies; the results of these earlier scans, and a general description of the scanning systems, can be found in WCAP-6067. ${ }^{[7]}$

Both sets of gamma activity scans were corrected for background and decay. In addition, due to the high gamma emission, it was necessary to correct the gamma activity data obtained with the scintillation crystal for counter dead time. (No dead time correction was necessary for the ion chamber data.)

The counter dead time was measured by WPIF personnel and was found to be 0.9 $\mu$ seconds. The resulting correction factor is:

$$
f=1.0-9.0 \times 10^{-8} \mathrm{~A}
$$

where $f$ is the factor by which the observed count rate must be divided, and $A$ is the gross gamma activity in units of counts/10 seconds.

The extent of the counter dead time correction is shown on Figure 7-1, where the gamma activity of Fuel Rod 44 (core location E6-NW-al) is shown, before and after the application of the correction factor. The net effect on the axial maximum-to-average ratio is about 2 percent, relative; the net effect on the assembly corner-to-center peaking is about 4 percent relative.

An estimate of the effective decay characteristic was obtained from two scans of Fuel Rod 369 (core location E6-C-a1), made eight days apart. Over this time interval, the decay of the rod average gross gamma activity was found to be approximately 5 percent, which indicates an effective half-life of 107 days, 
TABLE $7-1$

SUMMARY OF GROSS GAMMA ACTIVITY OF EYC PHASE 3 SPENT FUEL RODS

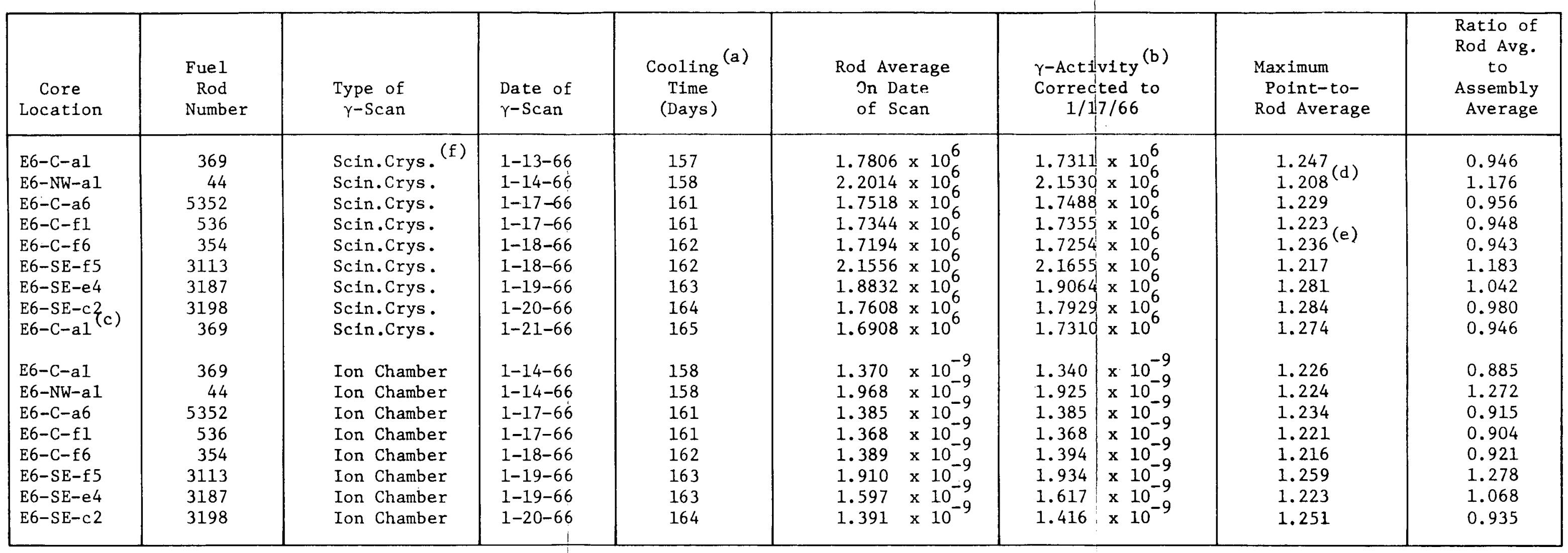

Notes: (a) Elapsed number of days from Core IV shutdown on August 9, 1965 to time of $\gamma$-scan.

(b) Units on values obtained with sodium iodide scintillation crysta1 are counts/10 seconds. Units on values obtained with minature ionization chamber are amps.

(c) This rod was re-scanned to determine the effective decay characteristic.

(d) Spurious activities of 1.308 and 1.368 were recorded.

(e) A spurious activity of 1.319 was recorded.

(f) All gamma scans obtained with the scintillation crystal were gated at $0.05 \mathrm{Mev}$. 


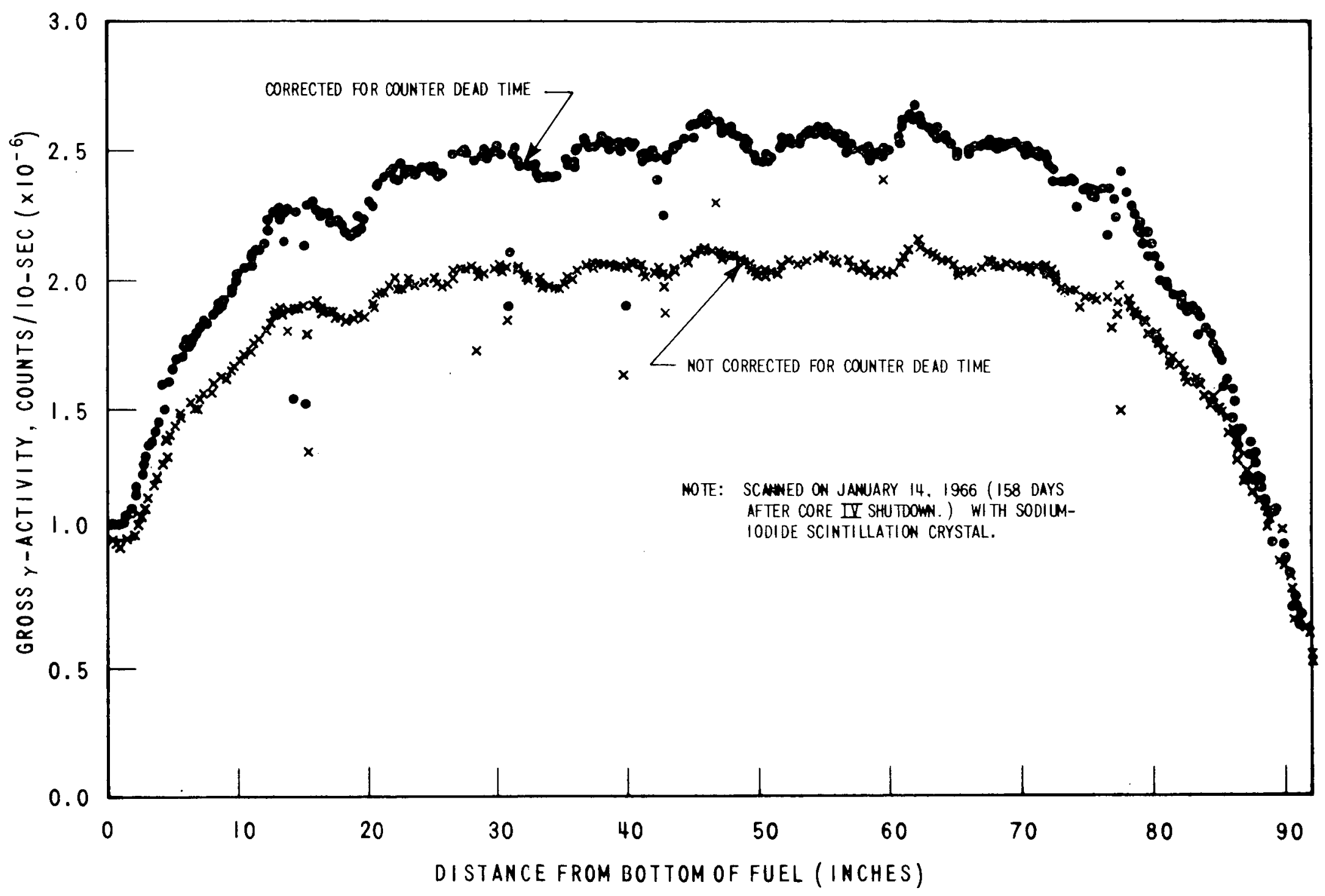

Figure 7-1. Axial Distribution of Gross Gamma Activity in EYC Phase 3 Fue 1 Rod E6-NW-a 1 
after 161 days cooling time. In EYC Phase 1, repeated scans of Core I fuel rod H3-C-f6 indicated an effective half-iife of about 104 days, [7] after 351 days cooling time. The two gross gamma scans of E6-C-al, obtained with the scintillation crystal, and normalized to the integrated rod average gamma activity, are shown in Figure 7-2. Notice that the axial distribution of gamma activity apparently did not remain constant over the 8-day time interval; the bottom half of the rod seems to have undergone 10 percent more decay than the top half of the rod. Since no explanation of this anomaly is readily available, the value of the decay characteristic must be viewed with some uncertainty. Fortunately, the Phase 3 fuel rods were scanned over a time interval of just one week, and the decay corrections, as can be seen in Table 7-1, generally involve a factor of less than 2 percent for most of the rods.

A11 gross gamma scans are characterized by a series of depressions and peaks in the axial distributions. The depressions which occur at nominal 8-inch intervals are due to stainless steel ferrules which displaced water in the coolant channels; the depressions were also evident in the EYC Phase 1 and 2 scans. The large depressions which occur at nominal 16-inch intervals are due to the presence of axial expansion voids and stainless steel spacer disks which separate stacks of pellets within the fuel tube; in the EYC Phase 1 and 2 scans, which were 2 to 3 orders of magnitude less intense than the Phase 3 scans, the spacer disk locations were characterized by peaks in the gamma activity distribution. Finally, in the fuel pellets immediately adjacent to the spacer disk locations, the gamma activity distribution exhibited a 5 to 8 percent peaking relative to the asymptotic distribution; these peaks, caused by the displacement of fuel, are consistent with measured flux-wire activity distributions, and with calculated axial flux distributions.

The axial gamma activity distributions obtained with the sodium-iodide scintillation crystal and the miniature ionization chamber are compared in Figures 7-3 through 7-10. (The solid curve on each figure is a smoothed version of the recorded trace produced by the ionization current.) Except for the approximate 10-inch segments at the top and bottom of the fuel rods, both scanning systems give gamma activity distributions which agree to within 


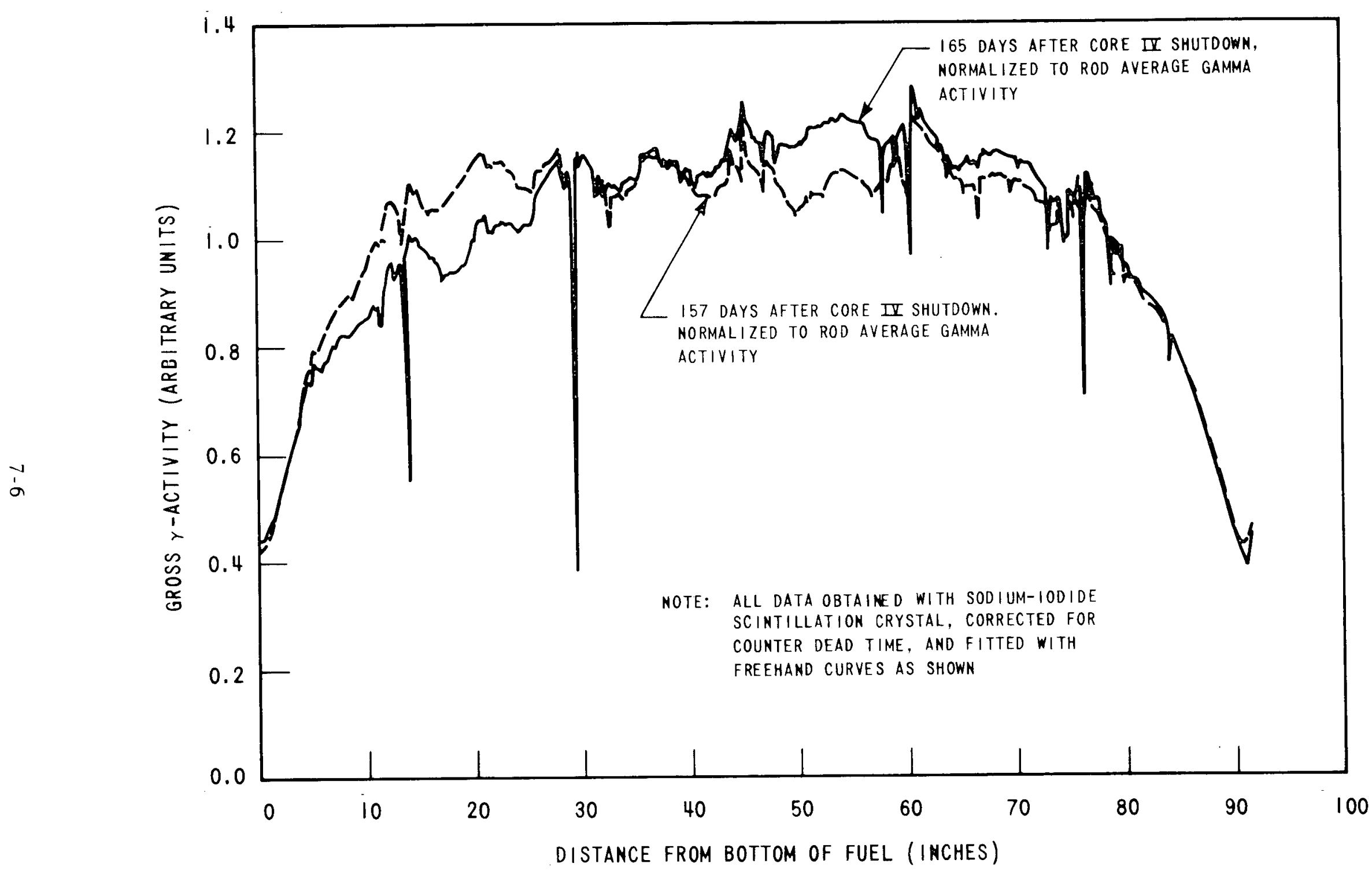

Figure 7-2. Axial Distribution of Gross Gamma Activity in EYC Phase 3 Fuel Rod E6-C-a1 


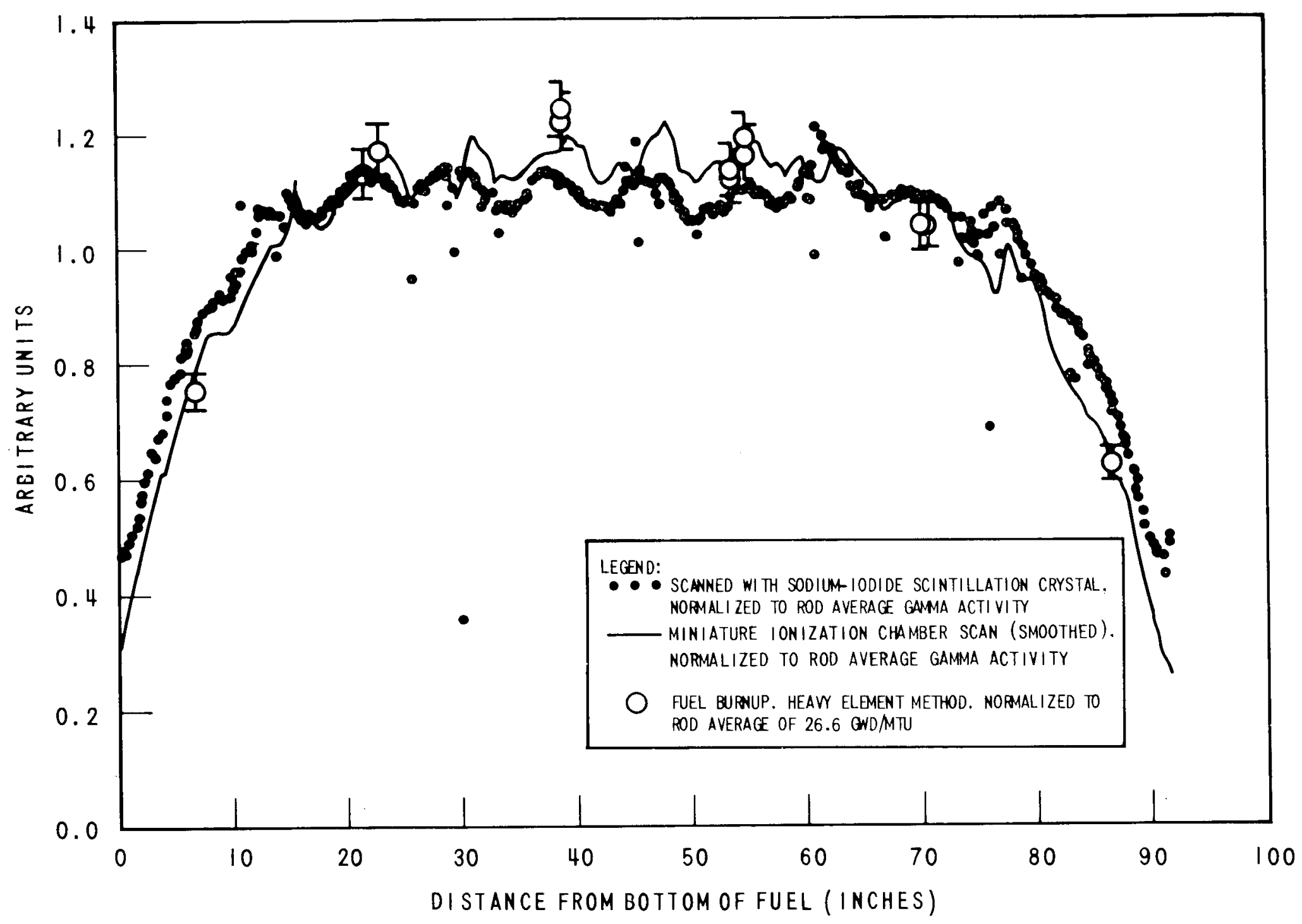

Figure 7 - 3. Normalized Axial Gross Gamma Act ivity and Burnup Dist $r$ ibutions 


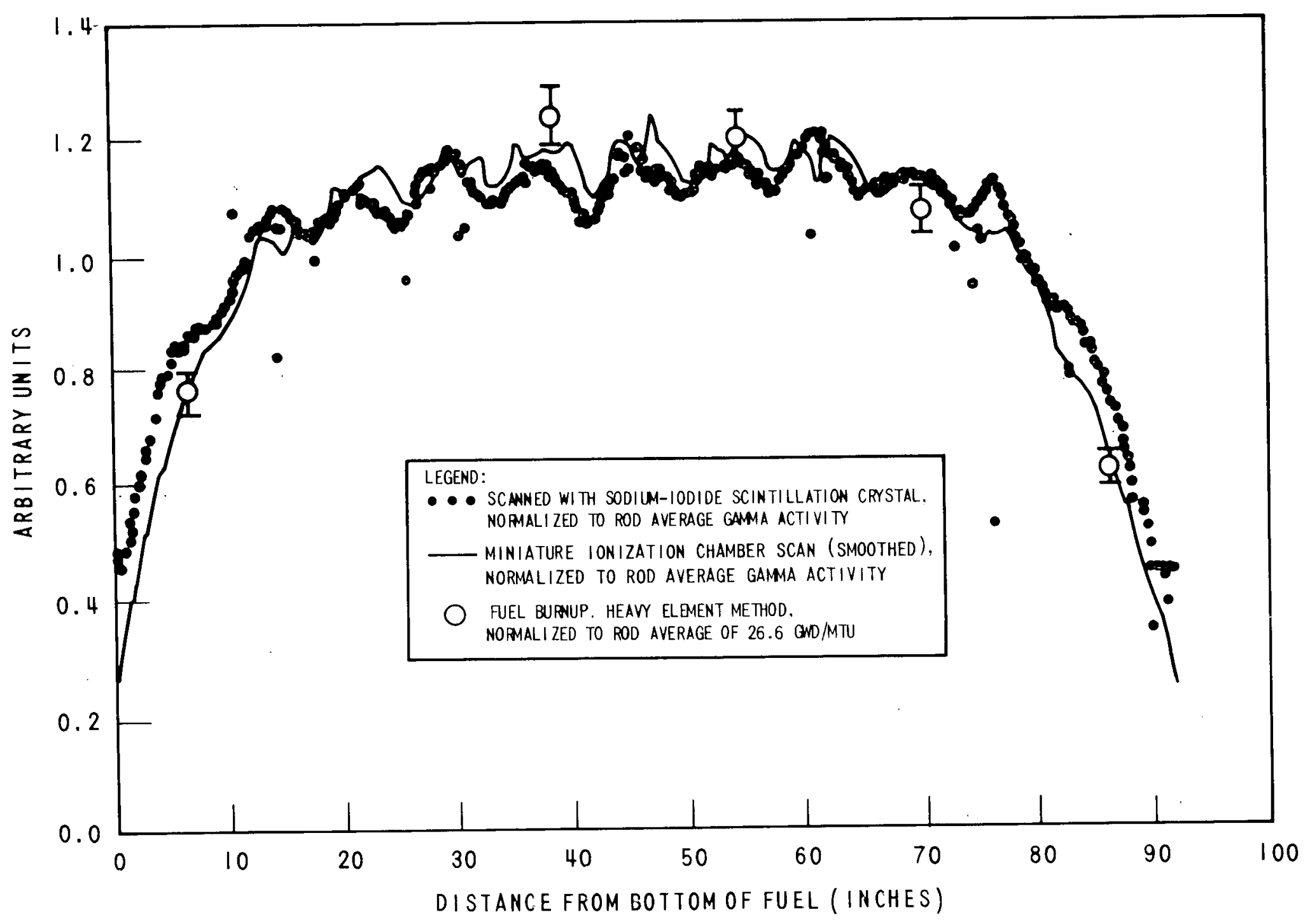

Figure 7=4. Normalized Axial Gross Gamma Activity and Burnup Distributions in in Fuel Kod No. 5352 , Core Locat ion E6-C-a6 


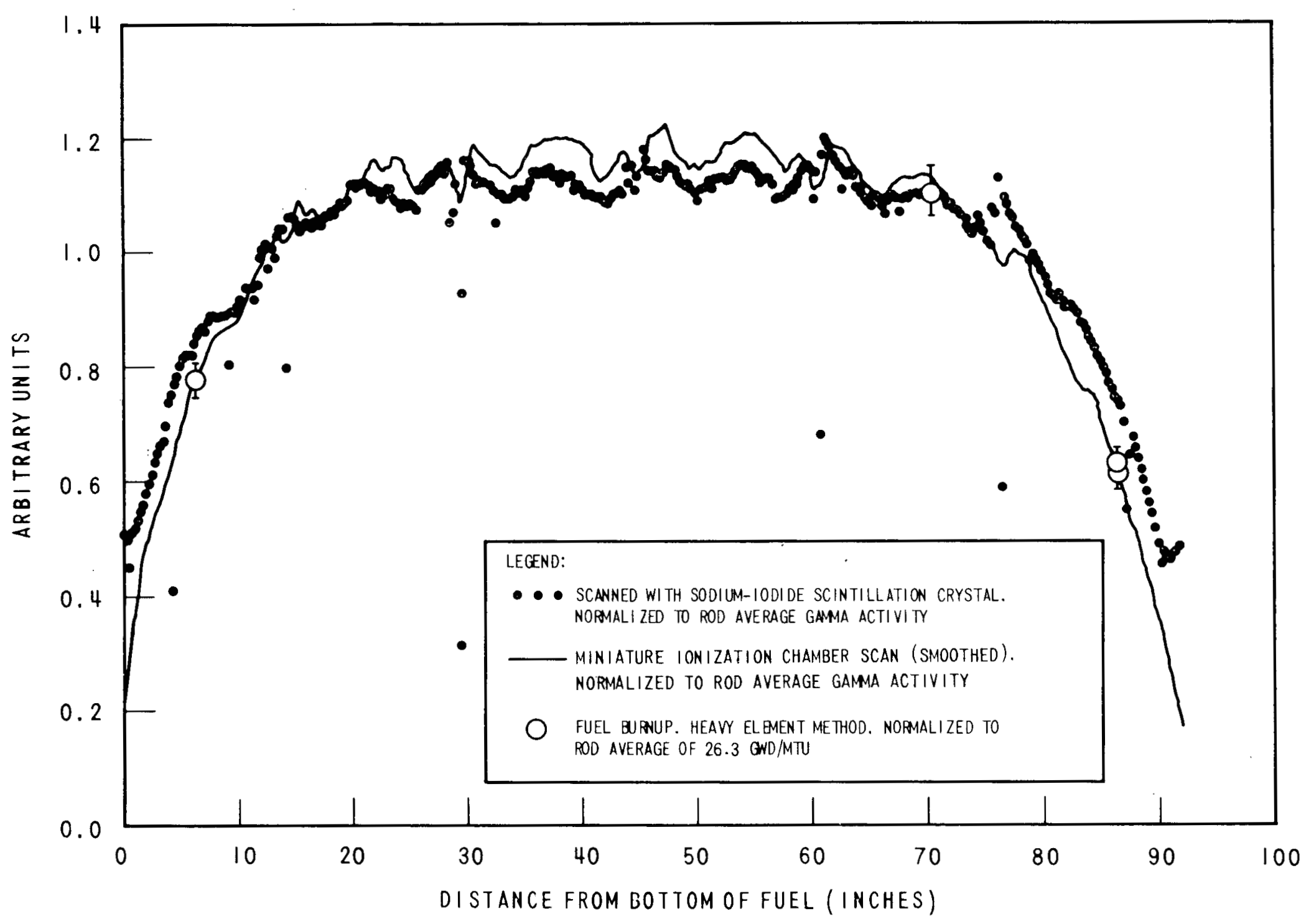

Figure 7 - 5. Normalized Axial Gross Gamma Activity and Burnup Dist ribut ions in Fuel Rod No. 536, Core Locat i on E6-C - f 1 


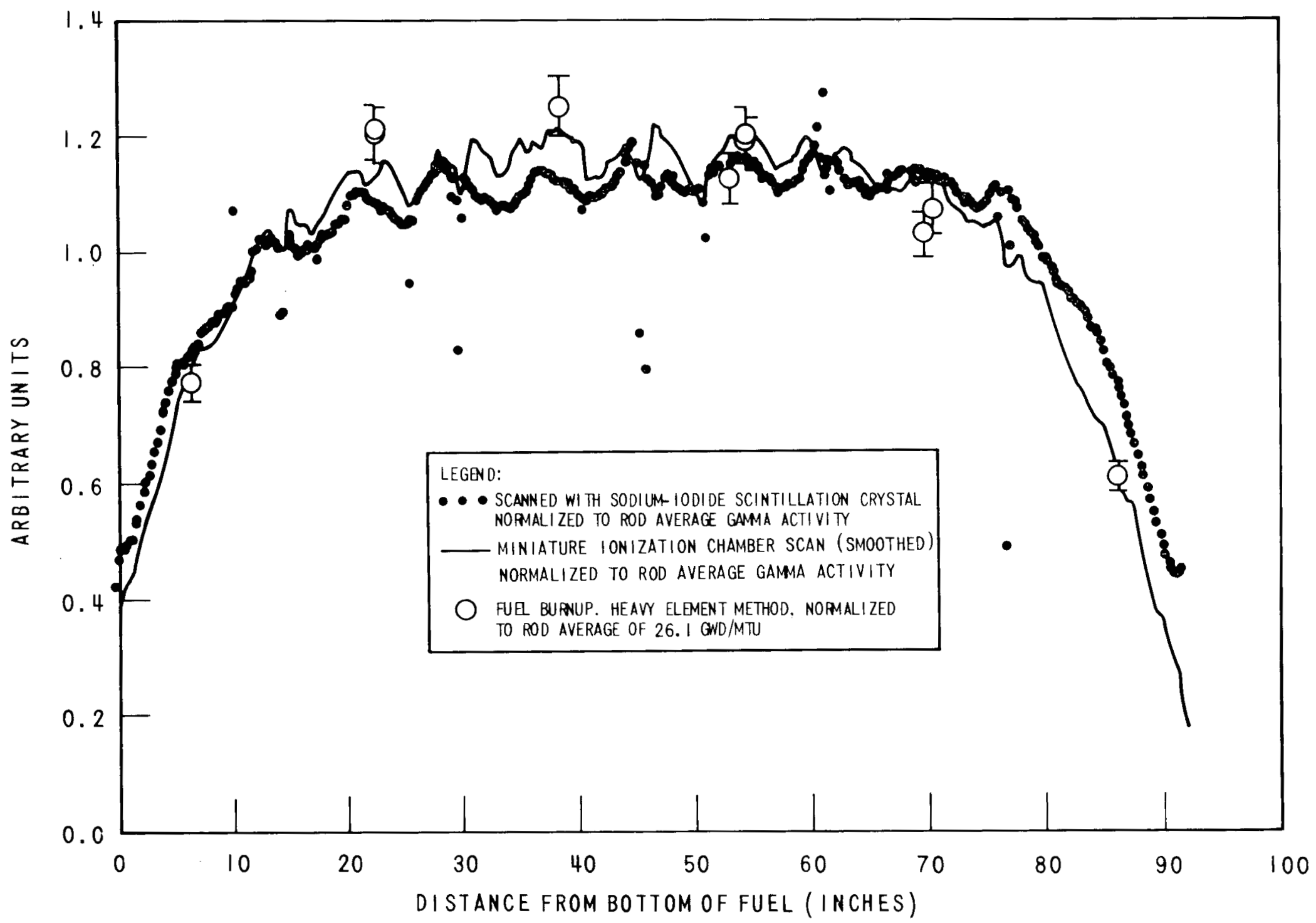

Figure 7-6. Normalized Axial Gross Gamma Activity and Burnup Distributions in Fuel Rod No. 354, Core Location E6-C-f6 


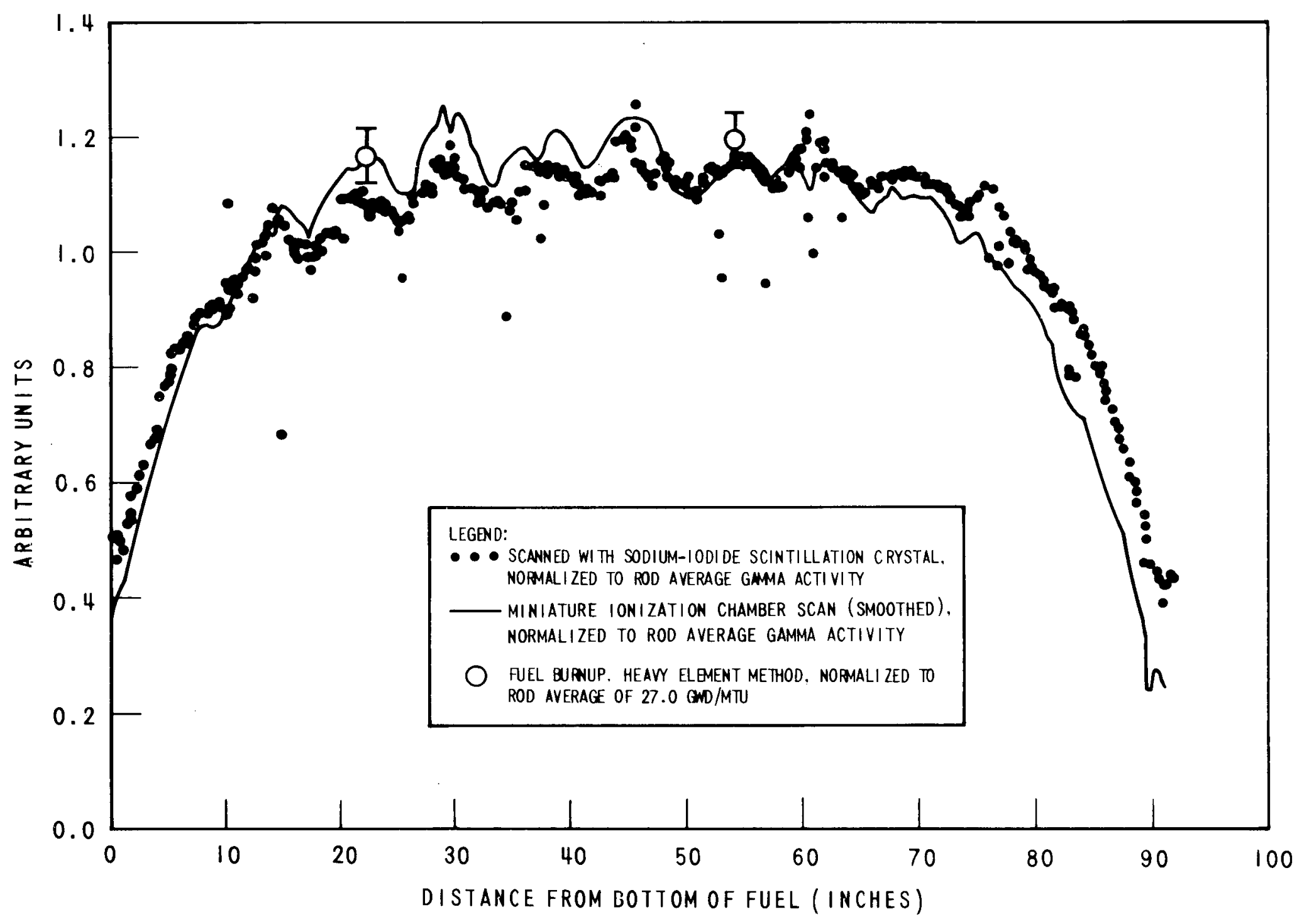

Figure 7-7. Normalized Axial Gross Gamma Activity and Burnup Distributions in Fuel Rod No. 3198, Core Location E6-SE-c2 


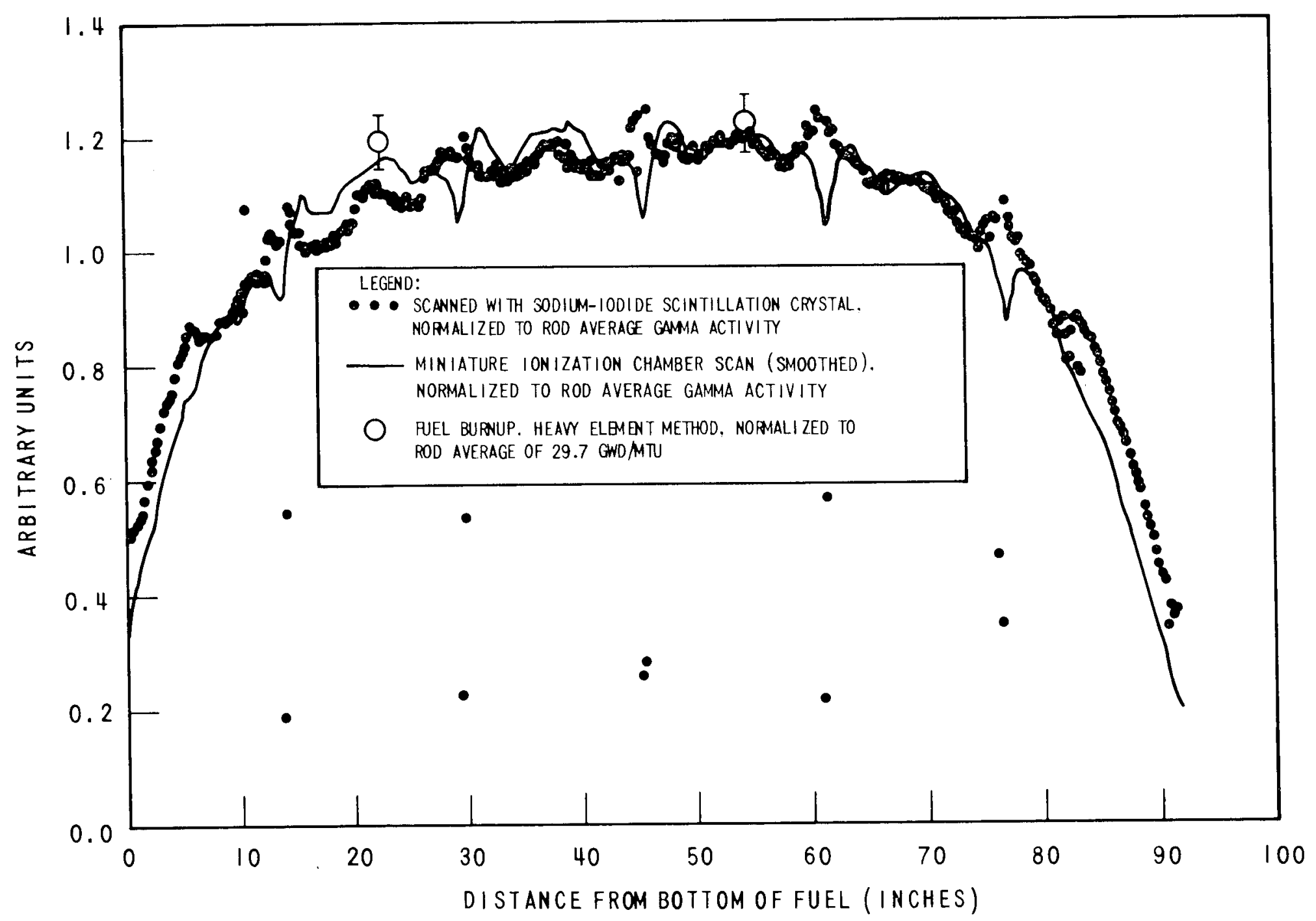

Figure 7-8. Normalized Axial Gross Gamma Activity and Burnup Distributions in Fue 1 Rod No. 3187, Core Locat i on E6-SE- e 4 


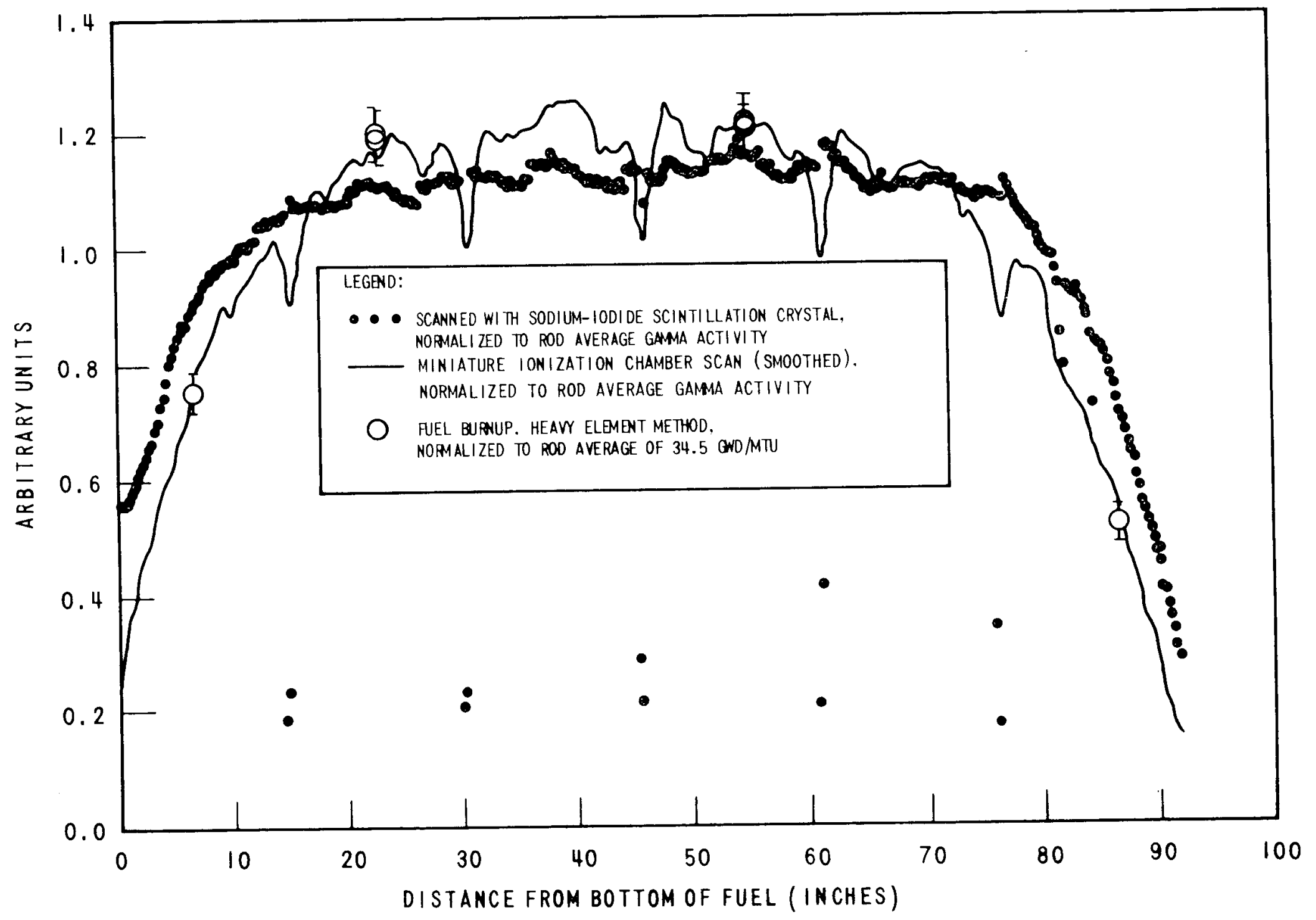

Figure 7 -9. Normalized Axial Gamma Activity and Burnup Distributions in Fue 1 Rod No. 3113, Core Location E6-SE-f 5 


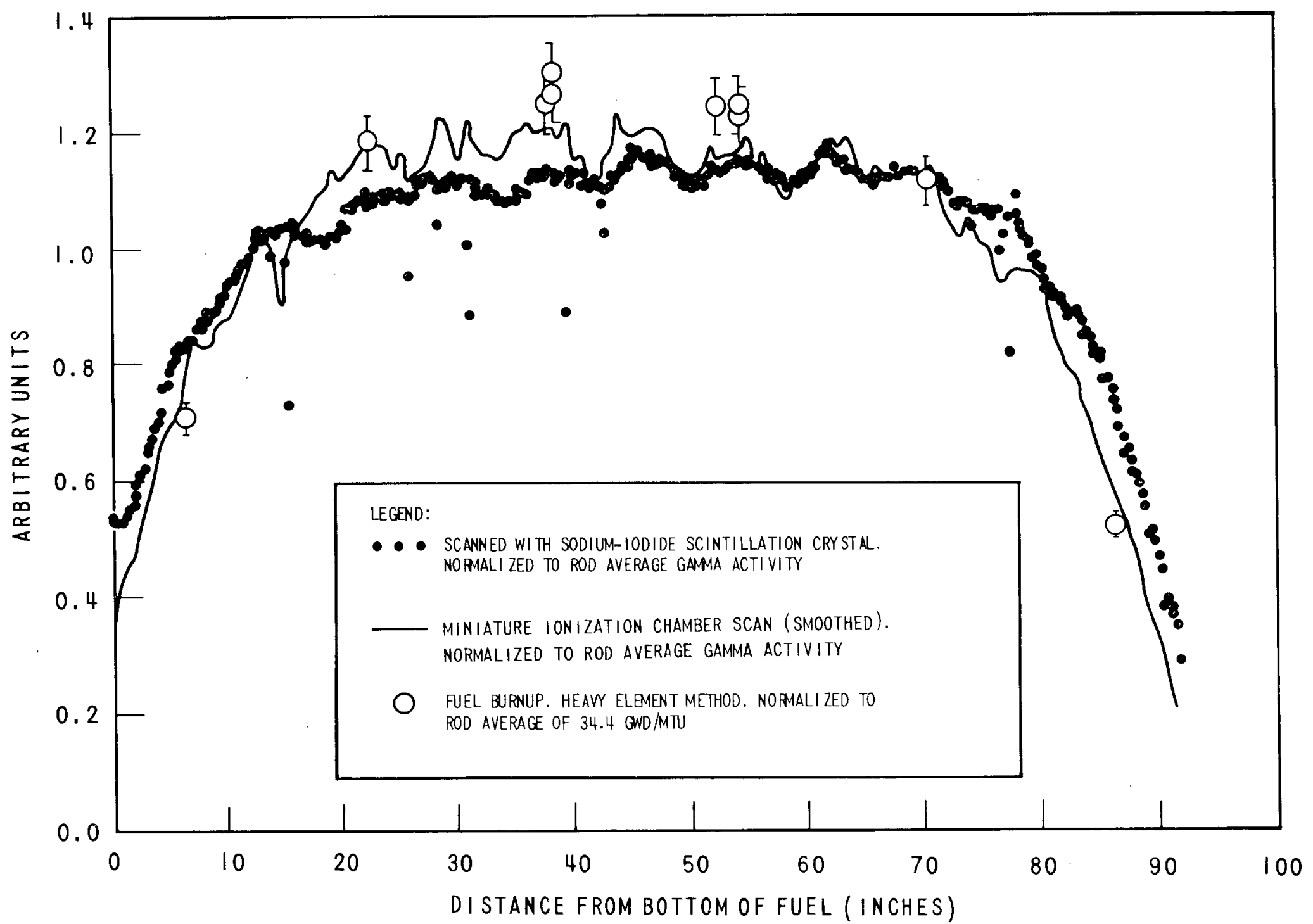

Figure 7 =10. Normalized Axial Gross Gammat Act ivity and Burnup Dist $\mathrm{r}$ ibutions in Fue 1 Rod No. 44, Core Locat ion E6-NW-a1 
7 percent. However, for each fuel rod, and particularly for the two high burnup corner rods (Figures 7-9 and 7-10), the scintillation crystal gives a gross gamma activity distribution which is somewhat flatter than the gross gamma activity distribution measured with the ion chamber. The most probable cause of the apparent discrepancy between the two sets of gross gamma activity distribution is that the two detectors respond differently to the varying spectrum of fission product gamma emission. Another possible, but improbable, cause of the discrepancy may be due to the use of an inaccurate value of counter dead time in the corrections made to the measured count rate data obtained with the scintillation crystal.

Also shown in Figures 7-3 through 7-10 are the normalized burnup distributions obtained from destructive analyses of the fuel. The absolute and normalized values of burnup and gamma activity are summarized on a pointwise basis for each fuel rod in Table 7-2. Comparisons of the gross gamma activity distributions and the burnup (each set of data normalized to the respective average of each fuel rod) lead to the following conclusions:

1. The burnup distribution is skewed somewhat towards the bottom of the core while the gamma activity distributions are nearly symmetrical about the mid-plane, indicating that the axial distributions of gross gamma activity are representative of some combination of burnup and end-of-life power distributions (Cores I and II were controlled with control rods withdrawn from the bottom to the top of the core while Core IV was controlled mainly with boric acid dissolved in the main coolant);

2. The difference between the normalized gross gamma activity distribution obtained with the ion chamber and the normalized burnup distribution obtained from destructive analysis is about 4 percent on the average, ranging from as much as -7 percent in the central portions of the fuel rods to as much as +10 percent at the end portions of the fuel rods;

3. The difference between the normalized gross gamma activity distribution obtained with the scintillation crystal and the normalized 
TABLE 7-2

COMPARISON OF GROSS GAMMA ACTIVITY WITH FUEL SAMPLE BURNUP IN EYC PHASE 3 FUEL RODS

\begin{tabular}{|c|c|c|c|c|c|c|c|c|c|c|c|c|c|c|}
\hline \multirow[b]{3}{*}{$\begin{array}{l}\text { Fuel } \\
\text { Rod } \\
\text { Core } \\
\text { Location }\end{array}$} & \multirow[b]{3}{*}{$\begin{array}{l}\text { Fue1 } \\
\text { Rod } \\
\text { No. }\end{array}$} & \multirow[b]{3}{*}{$\begin{array}{l}\text { Neutron } \\
\text { Spectrum }\end{array}$} & \multirow[b]{3}{*}{$\begin{array}{l}\text { EYC } \\
\text { Phase }\end{array}$} & \multirow[b]{3}{*}{$\begin{array}{c}\text { Axial } \\
\text { Sample } \\
\text { Zone }\end{array}$} & \multirow{3}{*}{$\begin{array}{l}\text { Nominal } \\
\text { Distance } \\
\text { From } \\
\text { Bottom } \\
\text { of Fuel } \\
\text { (inches) }\end{array}$} & \multirow[b]{3}{*}{$\begin{array}{l}\text { Sample } \\
\text { Code No. }\end{array}$} & \multirow{3}{*}{$\begin{array}{l}\text { Heavy } \\
\text { Element } \\
\text { Burnup } \\
\text { (GWD/MTU) }\end{array}$} & \multirow{3}{*}{$\begin{array}{c}\text { Ganma } \\
\text { Activity, } \\
\text { Ion } \\
\text { Chamber } \\
\text { amps (x109) }\end{array}$} & \multirow{3}{*}{$\begin{array}{c}\text { Gamma } \\
\text { Activity, } \\
\text { Scin. } \\
\text { Crystal, } \\
\text { C/10 } \sec \left(\times 10^{-6}\right)\end{array}$} & \multicolumn{3}{|c|}{ Normalized to Rod Average } & \multirow{2}{*}{\multicolumn{2}{|c|}{ Percent Difference }} \\
\hline & & & & & & & & & & B & $\mathrm{A}_{\mathrm{I}}$ & ${ }^{A}{ }_{S c}$ & & \\
\hline & & & & & & & & & & Burnup & $\begin{array}{c}\text { Ion } \\
\text { Chamber } \\
\gamma \text {-Activity }\end{array}$ & $\begin{array}{c}\text { Scin. } \\
\text { Crystal } \\
\gamma \text {-Activity }\end{array}$ & $\frac{\mathrm{A}_{\mathrm{I}}-\mathrm{B}}{\mathrm{B}} \times 100 \%$ & $\frac{\mathrm{A}_{\mathrm{Sc}}{ }^{-\mathrm{B}}}{\mathrm{B}} \times 100 \%$ \\
\hline $\mathrm{E} 6-\mathrm{C}-\mathrm{a} 1$ & 369 & Asymp & \begin{tabular}{|ll} 
& 3 \\
& 3 \\
3 & $(\mathrm{EXT})$ \\
& 3 \\
& 3 \\
3 & $(\mathrm{EXT})$ \\
3 & $(\mathrm{EXT})$ \\
& 3 \\
& 3 \\
& 3 \\
3 & $(\mathrm{EXT})$ \\
& 3
\end{tabular} & $\begin{array}{l}1 \\
2 \\
2 \\
3 \\
3 \\
3 \\
3 \\
4 \\
4 \\
5 \\
5 \\
6\end{array}$ & $\begin{array}{r}86.4 \\
70.4 \\
69.7 \\
54.4 \\
54.4 \\
53.1 \\
53.1 \\
38.4 \\
38.4 \\
22.4 \\
21.1 \\
6.4\end{array}$ & $\begin{array}{l}\mathrm{T}-168 \\
\mathrm{~T}-169 \\
\mathrm{G}-107 \\
\mathrm{~T}-170 \\
\mathrm{~N}-19 \\
\mathrm{G}-108 \\
\mathrm{~T}-212 \\
\mathrm{~T}-171 \\
\mathrm{~N}-20 \\
\mathrm{~T}-172 \\
\mathrm{G}-109 \\
\mathrm{~T}-173\end{array}$ & $\begin{array}{l}16.88 \\
27.86 \\
27.77 \\
31.08 \\
31.70 \\
30.06 \\
30.35 \\
32.62 \\
33.19 \\
31.30 \\
30.22 \\
20.07\end{array}$ & $\begin{array}{l}0.831 \\
1.473 \\
1.447 \\
1.553 \\
1.553 \\
1.548 \\
1.548 \\
1.573 \\
1.573 \\
1.552 \\
1.514 \\
1.059\end{array}$ & $\begin{array}{l}1.269 \\
1.887 \\
1.868 \\
1.916 \\
1.916 \\
1.883 \\
1.883 \\
1.928 \\
1.928 \\
1.944 \\
1.959 \\
1.475\end{array}$ & $\begin{array}{l}0.633 \\
1.045 \\
1.042 \\
1.166 \\
1.189 \\
1.128 \\
1.139 \\
1.224 \\
1.245 \\
1.174 \\
1.134 \\
0.753\end{array}$ & 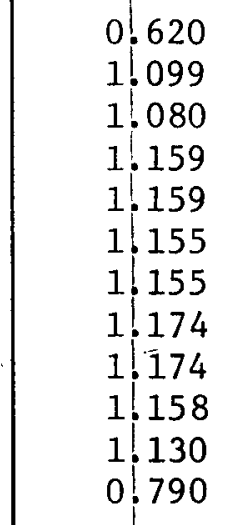 & $\begin{array}{l}0.733 \\
1.090 \\
1.079 \\
1.107 \\
1.107 \\
1.088 \\
1.088 \\
1.114 \\
1.114 \\
1.123 \\
1.132 \\
0.852\end{array}$ & $\begin{array}{l}-2.0 \\
+5.2 \\
+3.6 \\
-0.6 \\
-2.5 \\
+2.4 \\
+1.4 \\
-4.1 \\
-5.7 \\
-1.4 \\
-0.4 \\
+4.9\end{array}$ & $\begin{array}{l}+15.8 \\
+4.3 \\
+3.6 \\
-5.1 \\
-6.9 \\
-3.5 \\
-4.5 \\
-9.0 \\
-10.5 \\
-4.3 \\
-0.2 \\
+13.1\end{array}$ \\
\hline & Rod & Average & & & & & 26.65 & 1.340 & 1.731 & 1.000 & $1: 000$ & 1.000 & & \\
\hline E6-C-a6 & 5352 & Asymp & $\begin{array}{l}3 \\
3 \\
3 \\
3 \\
3 \\
3\end{array}$ & $\left.\begin{array}{l}1 \\
2 \\
3 \\
4 \\
6 \\
6\end{array}\right]$ & $\begin{array}{r}86.4 \\
70.4 \\
54.4 \\
38.4 \\
6.4 \\
6.4\end{array}$ & $\begin{array}{l}\mathrm{T}-153 \\
\mathrm{~T}-154 \\
\mathrm{~T}-155 \\
\mathrm{~T}-156 \\
\mathrm{~T}-157 \\
\mathrm{~N}-18\end{array}$ & $\begin{array}{l}16.42 \\
28.36 \\
31.66 \\
32.77 \\
20.06 \\
20.40\end{array}$ & $\begin{array}{l}0.874 \\
1.534 \\
1.638 \\
1.623 \\
1.048 \\
1.048\end{array}$ & $\begin{array}{l}1.284 \\
1.947 \\
2.015 \\
1.968 \\
1.483 \\
1.483\end{array}$ & $\begin{array}{l}0.617 \\
1.065 \\
1.189 \\
1.231 \\
0.754 \\
0.766\end{array}$ & $\begin{array}{l}0.631 \\
1.108 \\
1 ! 183 \\
1 ! 172 \\
0 ! 757 \\
0 ! 757\end{array}$ & $\begin{array}{l}0.734 \\
1.113 \\
1.152 \\
1.125 \\
0.848 \\
0.848\end{array}$ & $\begin{array}{l}+2.3 \\
+4.0 \\
-0.5 \\
-4.8 \\
+0.4 \\
-1.2\end{array}$ & $\begin{array}{l}+19.0 \\
+4.5 \\
-3.1 \\
-8.6 \\
+12.5 \\
+10.7\end{array}$ \\
\hline & Rod & Average & & & & & 26.62 & 1.385 & 1.749 & 1.000 & 1.000 & 1.000 & & \\
\hline E6-C-f1 & 536 & Asymp & $\begin{array}{l}3 \\
3 \\
3 \\
3\end{array}$ & $\begin{array}{l}1 \\
1 \\
2 \\
6\end{array}$ & $\begin{array}{r}86.4 \\
86.4 \\
70.4 \\
6.4\end{array}$ & $\begin{array}{l}T-158 \\
N-17 \\
T-159 \\
T-160\end{array}$ & $\begin{array}{l}16.10 \\
16.44 \\
28.79 \\
20.44\end{array}$ & $\begin{array}{l}0.922 \\
0.922 \\
1.547 \\
1.077\end{array}$ & $\begin{array}{l}1.283 \\
1.283 \\
1.899 \\
1.474\end{array}$ & $\begin{array}{l}0.613 \\
0.626 \\
1.096 \\
0.778\end{array}$ & $\begin{array}{l}0.674 \\
0.674 \\
1.131 \\
0.787\end{array}$ & $\begin{array}{l}0.739 \\
j .739 \\
1.094 \\
0.849\end{array}$ & $\begin{array}{l}+10.0 \\
+7.7 \\
+3.2 \\
+1.2\end{array}$ & $\begin{array}{l}+20.6 \\
+18.0 \\
-0.2 \\
+9.1\end{array}$ \\
\hline & Rod & Average & & & & & 26.28 & 1.368 & 1.736 & 1.000 & 1.000 & 1.000 & & \\
\hline
\end{tabular}


TABLE 7-2 (cont)

COMPARISON OF GROSS GAMMA ACTIVITY WITH FUEL SAMPLE BURNUP IN EYC PHASE 3 FUEL RODS

\begin{tabular}{|c|c|c|c|c|c|c|c|c|c|c|c|c|c|c|}
\hline \multirow{3}{*}{$\begin{array}{l}\quad \text { Fuel } \\
\text { Rod } \\
\text { Core } \\
\text { Location }\end{array}$} & \multirow[b]{3}{*}{\begin{tabular}{|l} 
Fue1 \\
Rod \\
No.
\end{tabular}} & \multirow[b]{3}{*}{$\begin{array}{l}\text { Neutron } \\
\text { Spectrum }\end{array}$} & \multirow[b]{3}{*}{$\begin{array}{l}\text { EYC }^{(\mathrm{l})} \\
\text { Phase }\end{array}$} & \multirow[b]{3}{*}{$\begin{array}{c}\text { Axial } \\
\text { Sample } \\
\text { Zone }\end{array}$} & \multirow{3}{*}{$\begin{array}{l}\text { Nominal } \\
\text { Distance } \\
\text { From } \\
\text { Bottom } \\
\text { of Fuel } \\
\text { (inches) }\end{array}$} & \multirow[b]{3}{*}{$\begin{array}{l}\text { Sample }{ }^{(c} \\
\text { Code No. }\end{array}$} & \multirow{3}{*}{$=\mid \begin{array}{l}\text { Heavy } \\
\text { Element } \\
\text { Burnup } \\
\text { (GWD/MTU) }\end{array}$} & \multirow{3}{*}{$\begin{array}{c}\text { Gamma } \\
\text { Activity, } \\
\text { Ion } \\
\text { Chamber } \\
\text { amps }\left(x 10^{9}\right)\end{array}$} & \multirow{3}{*}{$\begin{array}{c}\text { Gamma } \\
\text { Activity, } \\
\text { Scin. } \\
\text { Crystal, } \\
\text { C/10 } \sec \left(\times 10^{-6}\right)\end{array}$} & \multicolumn{3}{|c|}{ Normalized to Rod Average } & \multirow{2}{*}{\multicolumn{2}{|c|}{ Percent Difference }} \\
\hline & & & & & & & & & & \multirow{2}{*}{\begin{tabular}{|l|} 
B \\
Burnup \\
\end{tabular}} & \multirow{2}{*}{\begin{tabular}{|c|}
$\left.\right|^{\mathrm{A}} \mathrm{I}$ \\
$\begin{array}{c}\text { Ton } \\
\text { Chamber } \\
\gamma \text {-Activity }\end{array}$
\end{tabular}} & \multirow{2}{*}{\begin{tabular}{|c|}
$\mathrm{A}_{\mathrm{Sc}}$ \\
Scin. \\
Crystal \\
$\gamma$-Activity
\end{tabular}} & & \\
\hline & & & & & & & & & & & & & $\frac{A_{I}-B}{B} \times 100 \%$ & $\frac{\mathrm{A}_{\mathrm{Sc}}{ }^{-\mathrm{B}}}{\mathrm{B}} \times 100 \%$ \\
\hline \multirow[t]{2}{*}{$\mathrm{E} 6-\mathrm{C}-\mathrm{f} 6$} & & Asymp & $\begin{array}{c}3 \\
3 \\
3(\text { EXT) } \\
3 \\
3 \\
3 \text { (EXT) } \\
3 \\
3 \\
3 \\
3\end{array}$ & $\begin{array}{l}1 \\
2 \\
2 \\
3 \\
3 \\
3 \\
4 \\
5 \\
5] \\
6\end{array}$ & $\begin{array}{r}86.4 \\
70.4 \\
69.7 \\
54.4 \\
54.4 \\
53.1 \\
38.4 \\
22.4 \\
22.4 \\
6.4\end{array}$ & $\begin{array}{l}\mathrm{T}-175 \\
\mathrm{~T}-176 \\
\mathrm{G}-125 \\
\mathrm{~T}-177 \\
\mathrm{~N}-26 \\
\mathrm{G}-126 \\
\mathrm{~T}-178 \\
\mathrm{~T}-179 \\
\mathrm{~N}-27 \\
\mathrm{~T}-180\end{array}$ & $\begin{array}{l}15.95 \\
27.84 \\
26.76 \\
30.90 \\
31.13 \\
29.15 \\
32.48 \\
31.29 \\
31.36 \\
20.19\end{array}$ & $\begin{array}{l}0.821 \\
1.567 \\
1.554 \\
1.649 \\
1.649 \\
1.664 \\
1.677 \\
1.567 \\
1.567 \\
1.115\end{array}$ & $\begin{array}{l}1.301 \\
1.948 \\
1.956 \\
1.987 \\
1.987 \\
1.966 \\
1.928 \\
1.865 \\
1.865 \\
1.409\end{array}$ & $\begin{array}{l}0.612 \\
1.067 \\
1.026 \\
1.185 \\
1.194 \\
1.118 \\
1.245 \\
1.200 \\
1.202 \\
0.774\end{array}$ & $\begin{array}{l}0.589 \\
1.124 \\
1.115 \\
1.183 \\
1.183 \\
1.194 \\
1.203 \\
1.124 \\
1.124 \\
0.800\end{array}$ & $\begin{array}{l}0.754 \\
1.129 \\
1.134 \\
1.152 \\
1.152 \\
1.140 \\
1.118 \\
1.081 \\
1.081 \\
0.817\end{array}$ & $\begin{array}{l}-3.8 \\
+5.3 \\
+8.7 \\
-0.2 \\
-0.9 \\
+6.8 \\
-3.4 \\
-6.3 \\
-6.5 \\
+3.4\end{array}$ & $\begin{array}{r}+23.2 \\
+5.8 \\
+10.5 \\
-2.8 \\
-3.5 \\
+2.0 \\
-10.2 \\
-9.9 \\
-10.1 \\
+5.6\end{array}$ \\
\hline & Rod & verage & & & & & 26.08 & 1.394 & 1.725 & 1.000 & 1.000 & 1.000 & & \\
\hline \multirow[t]{2}{*}{$\mathrm{E} 6-\mathrm{SE}-\mathrm{c} 2$} & 3198 & Inter & $\begin{array}{l}3 \\
3\end{array}$ & $\begin{array}{l}3 \\
5\end{array}$ & $\begin{array}{l}54.4 \\
22.4\end{array}$ & $\begin{array}{l}T-187 \\
T-188\end{array}$ & $\begin{array}{l}32.03 \\
31.41\end{array}$ & $\begin{array}{l}1.631 \\
1.637\end{array}$ & $\begin{array}{l}2.091 \\
1.917\end{array}$ & $\begin{array}{l}1.186 \\
1.163\end{array}$ & $\begin{array}{l}1.152 \\
1.156\end{array}$ & $\begin{array}{l}1.166 \\
1.069\end{array}$ & $\begin{array}{l}-2.9 \\
-0.6\end{array}$ & $\begin{array}{l}-1.7 \\
-8.1\end{array}$ \\
\hline & Rod & verage & & & & & 27.00 & 1.416 & 1.793 & 1.000 & 1.000 & 1.000 & & \\
\hline E6-SE-e4 & Rod & Iverage & & & & & 29.68 & 1.617 & 1.906 & 1.000 & 1.000 & 1.000 & & \\
\hline
\end{tabular}


TABLE 7-2 (cont)

COMPARISON OF GROSS GAMMA ACTIVITY WITH FUEL SAMPLE BURNUP IN EYC PHASE 3 FUEL RODS

\begin{tabular}{|c|c|c|c|c|c|c|c|c|c|c|c|c|c|c|}
\hline \multirow[b]{3}{*}{$\begin{array}{l}\text { Fuel } \\
\text { Rod } \\
\text { Core } \\
\text { Location }\end{array}$} & \multirow[b]{3}{*}{$\begin{array}{l}\text { Fuel } \\
\text { Rod } \\
\text { No. }\end{array}$} & \multirow[b]{3}{*}{$\begin{array}{l}\text { Neutron } \\
\text { Spectrum }\end{array}$} & \multirow[b]{3}{*}{$\begin{array}{c}\text { EYC } \\
\text { Phase }\end{array}$} & \multirow[b]{3}{*}{$\begin{array}{l}\text { Axial } \\
\text { Sample } \\
\text { Zone }\end{array}$} & \multirow{3}{*}{$\begin{array}{l}\text { Nominal } \\
\text { Distance } \\
\text { From } \\
\text { Bottom } \\
\text { of Fuel } \\
\text { (inches) }\end{array}$} & \multirow[b]{3}{*}{$\begin{array}{l}\text { Sample } \\
\text { Code No. }\end{array}$} & \multirow{3}{*}{$\begin{array}{l}\text { Heavy } \\
\text { Element } \\
\text { Burnup } \\
\text { (GWD/MTU) }\end{array}$} & \multirow{3}{*}{$\begin{array}{c}\text { Gamma } \\
\text { Activity, } \\
\text { Ion } \\
\text { Chamber } \\
\text { amps (x109) }\end{array}$} & \multirow{3}{*}{$\begin{array}{c}\text { Gamma } \\
\text { Activity, } \\
\text { Scin. } \\
\text { Crystal, } \\
\text { C/10 } \sec \left(x 10^{-6}\right)\end{array}$} & \multicolumn{3}{|c|}{ Normalized to Rod Average } & \multirow{2}{*}{\multicolumn{2}{|c|}{ Percent Difference }} \\
\hline & & & & & & & & & & \multirow{2}{*}{\begin{tabular}{|l|} 
B \\
Burnup \\
\end{tabular}} & \multirow{2}{*}{\begin{tabular}{|c|}
$I^{A}$ \\
Ion \\
Chlamber \\
$\gamma$-Activity
\end{tabular}} & \multirow{2}{*}{\begin{tabular}{|c|} 
Sc \\
Scin. \\
Crystal \\
$\gamma$-Activity
\end{tabular}} & & \\
\hline & & & & & & & & & & & & & $\frac{\mathrm{A}_{\mathrm{I}}-\mathrm{B}}{\mathrm{B}} \times 100 \%$ & $\frac{A S^{-B}}{B} \times 100 \%$ \\
\hline E6-SE-f5 & 3113 & Pert & $\begin{array}{l}3 \\
3 \\
3 \\
3 \\
3 \\
3 \\
3 \\
3 \\
3 \\
3 \\
3\end{array}$ & $\begin{array}{l}1 \\
3 \\
3 \\
3 \\
3 \\
3 \\
3 \\
5 \\
5] \\
6\end{array}$ & $\begin{array}{r}86.4 \\
54.4 \\
54.4 \\
54.4 \\
54.4 \\
54.4 \\
54.4 \\
22.4 \\
22.4 \\
6.4\end{array}$ & $\begin{array}{l}\mathrm{T}-181 \\
\mathrm{~T}-182 \\
\mathrm{~T}-213 \\
\mathrm{G}-110 \\
\mathrm{G}-124 \\
\mathrm{~N}-24 \\
\mathrm{~N}-28 \\
\mathrm{~T}-183 \\
\mathrm{~N}-25 \\
\mathrm{~T}-184\end{array}$ & $\begin{array}{l}18.22 \\
41.84 \\
41.63 \\
41.97 \\
41.24 \\
41.97 \\
41.82 \\
41.15 \\
41.30 \\
26.25 \\
----\end{array}$ & $\begin{array}{l}1.068 \\
2.356 \\
2.356 \\
2.356 \\
2.356 \\
2.356 \\
2.356 \\
2.236 \\
2.236 \\
1.514 \\
-\end{array}$ & $\begin{array}{l}1.562 \\
2.512 \\
2.512 \\
2.512 \\
2.512 \\
2.512 \\
2.512 \\
2.396 \\
2.396 \\
1.958 \\
---\end{array}$ & $\begin{array}{l}0.528 \\
1.213 \\
1.207 \\
1.216 \\
1.195 \\
1.216 \\
1.212 \\
1.193 \\
1.197 \\
0.761\end{array}$ & $\begin{array}{l}0.552 \\
1.218 \\
1.218 \\
1.218 \\
1.218 \\
1.218 \\
1.218 \\
1.156 \\
1.156 \\
0.783\end{array}$ & $\begin{array}{l}0.721 \\
1.160 \\
1.160 \\
1.160 \\
1.160 \\
1.160 \\
1.160 \\
1.106 \\
1.106 \\
0.904\end{array}$ & $\begin{array}{l}-1.1 \\
+0.4 \\
+0.9 \\
+0.2 \\
+1.9 \\
+0.2 \\
+0.5 \\
-3.1 \\
-3.4 \\
+2.9\end{array}$ & $\begin{array}{l}+36.6 \\
-4.4 \\
-3.9 \\
-4.6 \\
-2.9 \\
-4.6 \\
-4.3 \\
-7.3 \\
-7.6 \\
+18.8\end{array}$ \\
\hline & Rod A & verage & & & & & 34.50 & 1.934 & 2.166 & 1.000 & 1.000 & 1.000 & & \\
\hline E6-NW-al & 44 & Pert & \begin{tabular}{|ll} 
& 3 \\
& 3 \\
& 3 \\
& 3 \\
3 & $(\mathrm{EXT})$ \\
& 3 \\
& 3 \\
3 & $(\mathrm{EXT})$ \\
& 3 \\
& 3
\end{tabular} & $\begin{array}{l}1 \\
2 \\
3 \\
3] \\
3 \\
4 \\
4 \\
4 \\
5 \\
6\end{array}$ & $\begin{array}{r}86.4 \\
70.4 \\
54.4 \\
54.4 \\
52.4 \\
38.4 \\
38.4 \\
37.8 \\
22.4 \\
6.4\end{array}$ & $\begin{array}{l}\mathrm{T}-162 \\
\mathrm{~T}-163 \\
\mathrm{~T}-164 \\
\mathrm{~N}-21 \\
\mathrm{G}-127 \\
\mathrm{~T}-165 \\
\mathrm{~N}-22 \\
\mathrm{G}-128 \\
\mathrm{~T}-166 \\
\mathrm{~T}-167\end{array}$ & $\begin{array}{l}18.04 \\
38.08 \\
41.92 \\
42.57 \\
42.47 \\
43.18 \\
44.38 \\
42.55 \\
40.58 \\
24.56 \\
---\end{array}$ & $\begin{array}{l}1.090 \\
2.171 \\
2.235 \\
2.235 \\
2.208 \\
2.308 \\
2.308 \\
2.310 \\
2.298 \\
1.476 \\
-\end{array}$ & $\begin{array}{l}1.552 \\
2.392 \\
2.454 \\
2.454 \\
2.426 \\
2.420 \\
2.420 \\
2.426 \\
2.310 \\
1.770 \\
----\end{array}$ & $\begin{array}{l}0.524 \\
1.106 \\
1.218 \\
1.236 \\
1.234 \\
1.254 \\
1.289 \\
1.236 \\
1.179 \\
0.713 \\
----\end{array}$ & $\begin{array}{l}0.566 \\
1.128 \\
1.161 \\
1.161 \\
1.147 \\
1.199 \\
1.199 \\
1.200 \\
1.194 \\
0.767\end{array}$ & $\begin{array}{l}0.721 \\
1.111 \\
1.140 \\
1.140 \\
1.127 \\
1.124 \\
1.124 \\
1.127 \\
1.073 \\
0.822 \\
-----\end{array}$ & $\begin{array}{l}+8.0 \\
+2.0 \\
-4.7 \\
-6.1 \\
-7.0 \\
-4.4 \\
-7.0 \\
-2.9 \\
+1.3 \\
+7.6\end{array}$ & $\begin{array}{r}+37.6 \\
+0.4 \\
-6.4 \\
-7.8 \\
-8.7 \\
-10.4 \\
-12.8 \\
-8.8 \\
-9.0 \\
+15.3\end{array}$ \\
\hline & Rod A & verage & & & & & 34.43 & 1.925 & 2.153 & 1.000 & 1.000 & 1.000 & & \\
\hline
\end{tabular}

Notes: a. Refers to asymptotic neutron spectrum in central subassembly, the perturbed spectrum in corner rods adjacent to water slots, and the intermediate spectrum along the assembly diagonal between the assembly center and the corner fuel rods.

c. The Letters T, G, and $\mathrm{N}$ Indicate mass spectrometric analyses by TRACERLAB, General Electric-Vallecitos and New Brunswick, respectively. 
burnup distribution obtained from destructive analysis is about 9 percent on the average, ranging from as much as -12 percent in the central portions of the fuel rods to as much as +37 percent at the end portions of the fuel rods.

To estimate the degree to which the gross gamma activity distributions represent the burnup distribution in the XY plane, the rod average gamma activity and burnup of the fuel rods in the non-asymptotic neutron spectra (diagonal and corner positions) are taken relative to the corresponding rod averages of the fuel rods in the asymptotic neutron spectrum (center positions). The results are summarized in Table $7-3$ where it can ve seen that tine ion chamber gross gamma scans overpredict the corner-to-center burnup ratio by about 8 percent, while the scintillation crystal gross gamma scans underpredict the corner-to-center burnup ratio by about 5 percent.

The net discrepancy of about 13 percent between the results obtained with the two methods of gamma scanning in the XY plane is consistent with the 7 percent discrepancy between the two methods for results obtained in the axial plane (excluding the approximate 10-inch segments at the rod ends) in view of the fact that the neutron spectrum variations which exist along the corner-to corner diagonal of the assembly are considerably greater than the neutron spectrum variations which exist locally along the lengths of the individual fuel rods.

\subsection{MULTI-CHANNEL GAMMA SCANS}

To date, no measurements of axial power distributions have been made in a fuel assembly. which has undergone an average burnup of more than about $21,000 \mathrm{MWD} / \mathrm{MTU}$ in a large PWR. However, some measurements have been obtained for the fission product $2 r-95$ in the EYC Phase 3 fuel assembly which has experienced an average burnup of approximately 30,000 MWD/MTU. Since the $\mathrm{Zr}-95$ half-1ife of 65 days is short relative to the in-core residence time of the Phase 3 fuel assembly (about 3-1/4 years in Yankee Cores I, II, and IV), the distribution of $\mathrm{Zr}-95$ is indicative of the power distribution which existed in the recycled fuel assembly towards the end-of-life. 
TABLE 7-3

SUMMARY OF ROD AVERAGE GROSS GAMMA ACTIVITY AND BURNUP

\begin{tabular}{|c|c|c|c|c|c|c|c|c|c|c|}
\hline \multirow{3}{*}{$\begin{array}{l}\text { Fue1 } \\
\text { Rod } \\
\text { Core } \\
\text { Location }\end{array}$} & \multirow[b]{3}{*}{$\begin{array}{l}\text { Fue1 } \\
\text { Rod } \\
\text { No. }\end{array}$} & \multirow[b]{3}{*}{$\begin{array}{l}\text { Neutron } \\
\text { Spectrum }\end{array}$} & \multirow[b]{3}{*}{$\begin{array}{l}\text { Burnup } \\
\text { (GWD/MTU) }\end{array}$} & \multirow{3}{*}{$\begin{array}{c}\text { Gamma } \\
\text { Activity, } \\
\text { Ion } \\
\text { Chamber } \\
\text { amps }\left(x 10^{9}\right)\end{array}$} & \multirow{3}{*}{$\begin{array}{c}\text { Gamma } \\
\text { Activity, } \\
\text { Scin. } \\
\text { Crystal, } \\
\text { C/10 sec }(x 10-6)\end{array}$} & \multicolumn{3}{|c|}{ Normalized to Center Average } & \multirow{2}{*}{\multicolumn{2}{|c|}{ Percent Difference }} \\
\hline & & & & & & $\mathrm{B}$ & $\mathrm{I}_{\mathrm{I}}^{\mathrm{A}}$ & $\mathrm{Ac}_{\mathrm{Sc}}^{\mathrm{A}}$ & & \\
\hline & & & & & & Burnup & $\begin{array}{c}\text { Ion } \\
\text { Chamber } \\
\gamma \text {-Activity }\end{array}$ & $\begin{array}{c}\text { Scin. } \\
\text { Crystal } \\
\gamma \text {-Activity }\end{array}$ & $\frac{A_{I}-B}{B} \times 100 \%$ & $\frac{A_{S c}-B}{B} \times 100 \%$ \\
\hline E6-C-a1 & 369 & Asymp & 26.65 & 1.340 & 1.731 & 1.009 & 0.977 & 0.998 & -3.2 & -1.1 \\
\hline$E 6-C-a 6$ & 5352 & Asymp & 26.62 & 1.385 & 1.749 & 1.008 & 1.009 & 1.008 & +0.1 & 0.0 \\
\hline$E 6-C-f 1$ & 536 & Asymp & 26.28 & 1.368 & 1.736 & 0.995 & 0.997 & 1.000 & +0.2 & +0.5 \\
\hline E6-C-f6 & 354 & Asymp & 26.08 & 1.394 & 1.725 & 0.988 & 1.016 & 0.994 & +2.8 & +0.6 \\
\hline Center Av & erage & Asymp & 26.41 & 1.372 & 1.735 & 1.000 & 1.000 & 1.000 & & \\
\hline E6-SE-c2 & 3198 & Inter & 27.00 & 1.416 & 1.793 & 1.022 & 1.032 & 1.033 & +1.0 & +1.1 \\
\hline E6-SE-e4 & 3187 & Inter & 29.68 & 1.617 & 1.906 & 1.124 & 1.178 & 1.098 & +4.8 & -2.3 \\
\hline E6-SE-f5 & 3113 & Pert & 34.50 & 1.934 & 2.166 & 1.306 & 1.410 & 1.248 & +8.0 & -4.4 \\
\hline E6-NW-al & 44 & Pert & 34.43 & 1.925 & 2.153 & 1.304 & 1.403 & 1.241 & +7.6 & -4.8 \\
\hline
\end{tabular}


The Zr-95 activity in four Phase 3 fuel rods has been determined by both non-destructive and destructive analyses. The southeast corner and center pair of rods, E6-SE-f5 and E6-C-f6, were multi-channel gamma scanned at ten selected points along the length of each rod. The Zr-95 - Nb-95 peak height activity, determined by graphically stripping each multi-channel gamma scan, is shown in Figure 7-11. Although limits of uncertainty are not given for the data, it is clearly evident that a saddle-shaped axial power distribution existed in these fuel rods prior to reactor shutdown. These axial distributions of $\mathrm{Zr}-95$ - Nb-95 activity are to be compared with the $\mathrm{Zr}-95$ activity determined from radiochemical analysis of six pellet samples removed from each rod of the northwest pair of rods, E6-NW-al and E6-C-al. The results of the radiochemical analysis are shown in Figure 7-12, where the limits of uncertainty are 10 percent, relative. Again, it is evident that a saddleshaped axial power distribution existed in these rods; however, the magnitude of both the axial peaking and the assembly corner-to-center peaking are considerably greater than that observed from the multi-channel Zr-95 $\mathrm{Nb}-95$ data on Figure 7-11. From the gross gamma activity and burnup data presented in the previous section (Table 7-3), it can be concluded that the power was symmetrically distributed along the assembly corner-to-corner diagonal.

The two sets of Zr-95 axial distributions, each normalized to their respective rod averages, are displayed in Figure 7-13. Both the axial peaking factors and the assembly corner-to-center peaking factors are summarized in Table 7-4. Although the apparent discrepancy between the multi-channel $\mathrm{Zr}-95$ - Nb-95 data and the radiochemical $\mathrm{Zr}-95$ data has not been resolved, it is pointed out that, due to the high level of gamma activity in the fuel rods at the time of the multi-channel analysis, pulse pile-up may have occurred, and that the radiochemical data may be more representative of the actual $\mathrm{Zr}-95$ fission product distribution in the fuel.

It is stressed that the data shown herein are typical only of the conditions in the recycled Phase 3 fuel assembly, E6. Because of the relatively low initial reactivity and high burnup, this assembly is not representative of either the other Core IV assemblies, or of a typical recycled fuel assembly in a large PWR. 


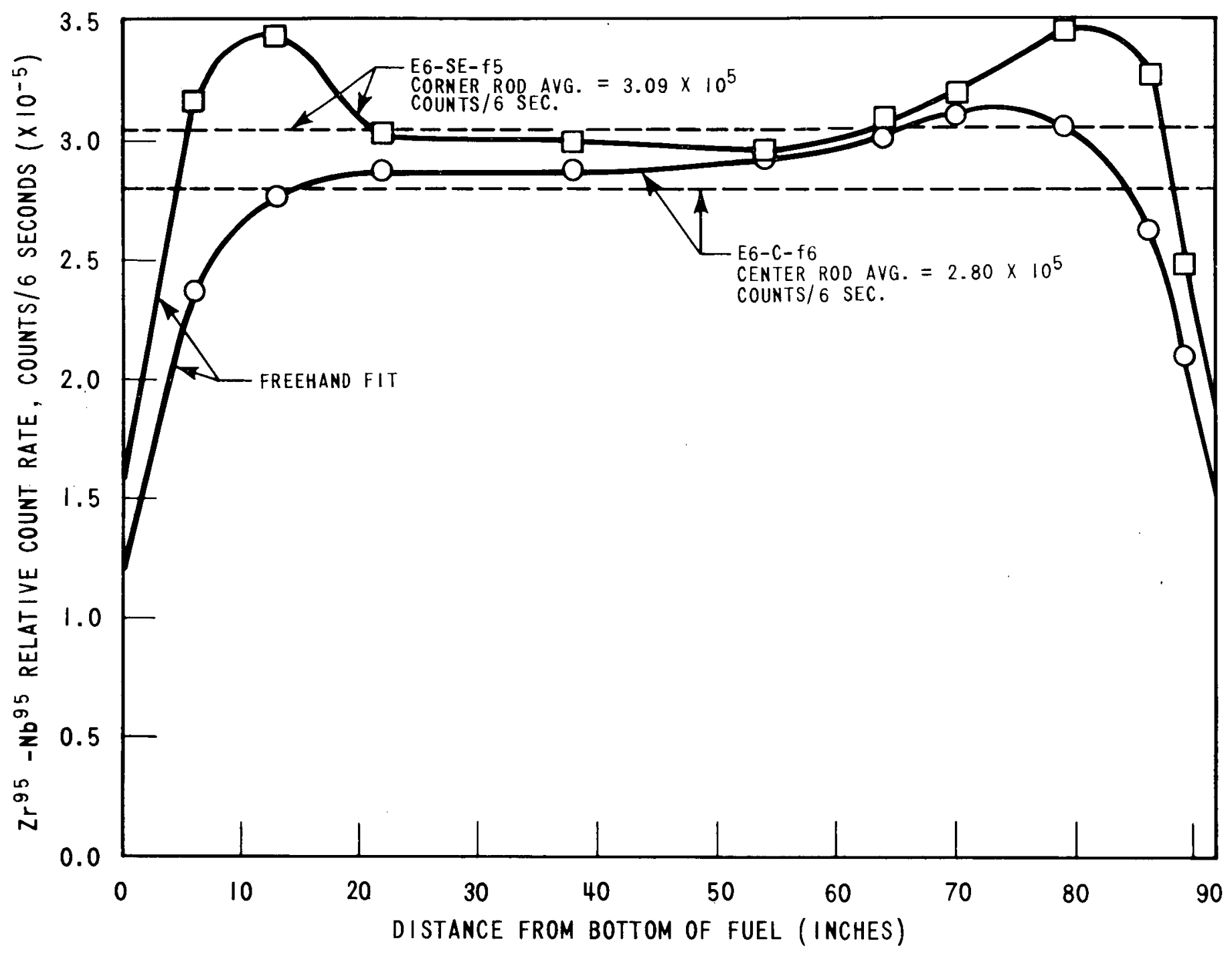

Figure 7-11. Axial Distribution of $\mathrm{Zr}-95-\mathrm{Nb}-95$ Activity in EYC Phase 3 Fuel Rods Determined from Multi-Channel Gamma Scans 


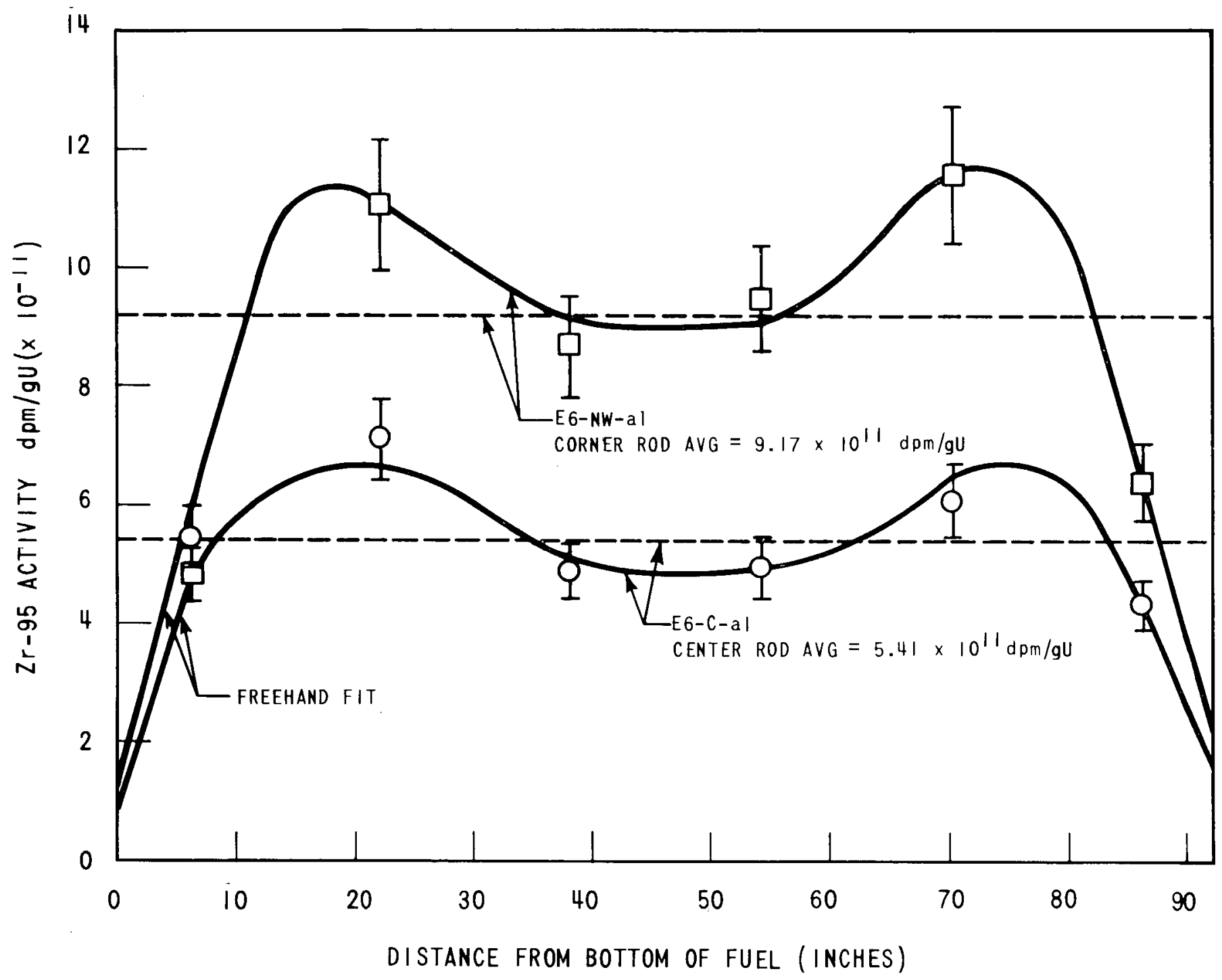

Figure 7-12. Axial Distribution of Zr-95 Activity, in EYC Phase 3 Fue.1 Rods Determined from Radiochemical Analysis 

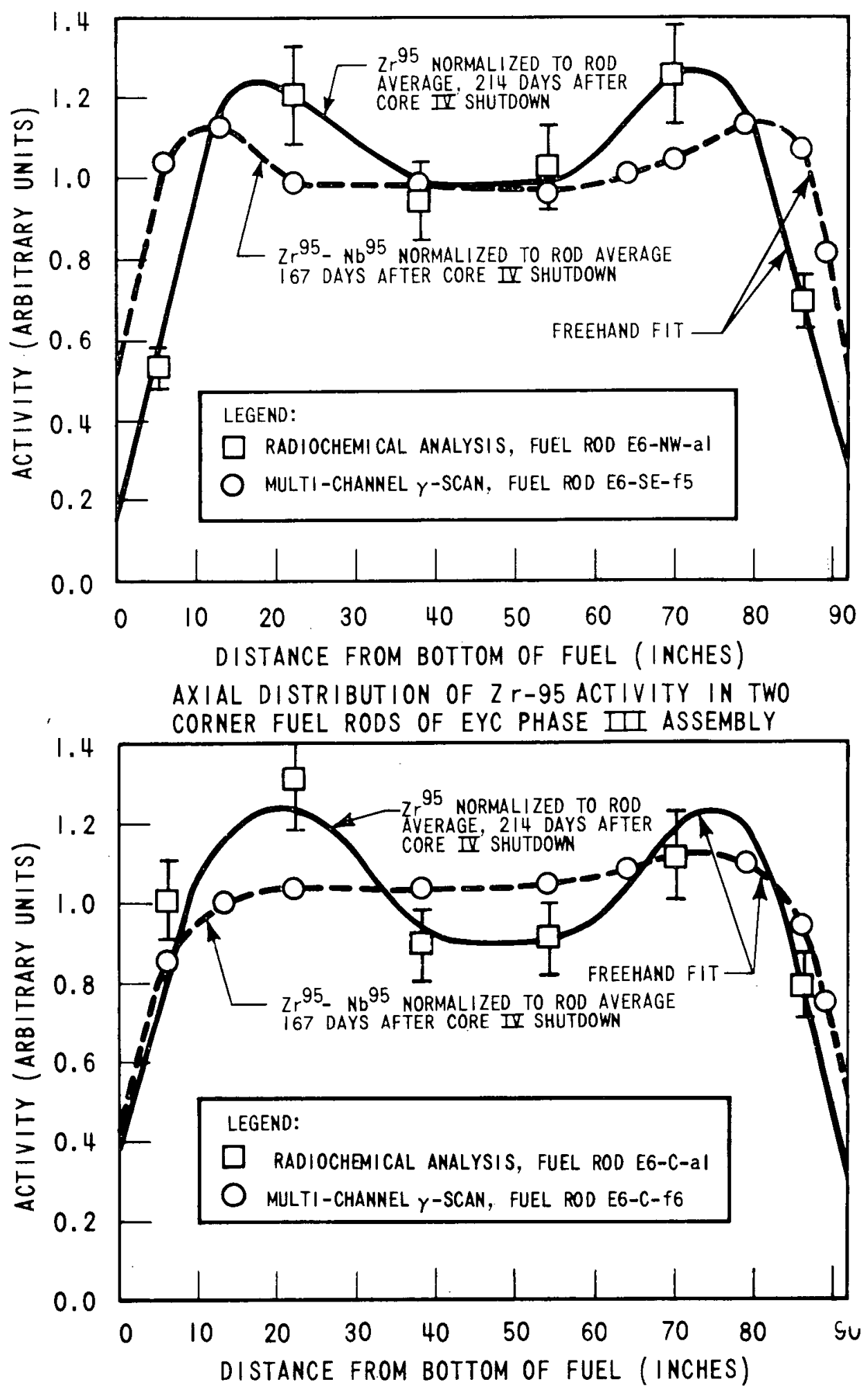

Figure 7-13. Axial Distribution of $\mathrm{Zr}-95$ in Several EYC Phase 3 Fuel Rodis Determined by Radiochemical and Gamma Spectrometric Analyses 
TABLE 7-4

SUMMARY OF GAMMA ACTIVITY PEAKING

FACTORS IN THE EYC PHASE 3 FUEL ASSEMBLY

\begin{tabular}{|c|c|c|c|c|c|c|}
\hline \multirow[b]{3}{*}{$\begin{array}{c}\text { Core } \\
\text { Location }\end{array}$} & \multirow{3}{*}{$\begin{array}{l}\text { Fuel } \\
\text { Rod } \\
\text { Number }\end{array}$} & \multicolumn{5}{|c|}{ Axial Maximum-to-Average in Fuel Rod } \\
\hline & & \multirow{2}{*}{$\begin{array}{c}\mathrm{Zr}-95-\mathrm{Nb}-95 \\
\text { Multi } \\
\text { Channel } \\
\gamma-\text { Scan }\end{array}$} & \multirow{2}{*}{$\begin{array}{l}\text { Zr-95 } \\
\text { Radio- } \\
\text { chemical } \\
\text { Analysis }\end{array}$} & \multicolumn{2}{|c|}{ Gross Gamma Activity } & \multirow[b]{2}{*}{ Burnup } \\
\hline & & & & $\begin{array}{c}\text { Scintillation } \\
\text { Crystal }\end{array}$ & $\begin{array}{c}\text { Ionization } \\
\text { Chamber }\end{array}$ & \\
\hline $\mathrm{E} 6-\mathrm{NW}-\mathrm{a} 1$ & 44 & \multirow{4}{*}{$\begin{array}{l}1.13 \\
1.13\end{array}$} & \multirow{4}{*}{$\begin{array}{l}1.27 \\
1.24\end{array}$} & \multirow{4}{*}{$\begin{array}{l}1.21 \\
1.27(a) \\
1.22 \\
1.24\end{array}$} & 1.22 & 1.28 \\
\hline $\mathrm{E} 6-\mathrm{C}-\mathrm{al}$ & 369 & & & & 1.23 & 1.24 \\
\hline E6-SE-f5 & 3113 & & & & 1.26 & 1.30 \\
\hline$E 6-C-f 6$ & 354 & & & & 1.22 & 1.25 \\
\hline \multicolumn{7}{|c|}{ Assembly Corner-to-Center Ratio } \\
\hline$\frac{\mathrm{E} 6-\mathrm{NW}-\mathrm{a} 1}{\mathrm{E} 6-\mathrm{C}-\mathrm{a} 1}$ & $\frac{\# 44}{\# 369}$ & \multirow[b]{2}{*}{1.10} & \multirow[t]{2}{*}{1.69} & 1.24 & 1.44 & 1.29 \\
\hline$\frac{\mathrm{E} 6-\mathrm{SE}-\mathrm{f} 5}{\mathrm{E} 6-\mathrm{C}-\mathrm{f} 6}$ & $\frac{\# 3113}{\# 354}$ & & & 1.26 & 1.39 & 1.32 \\
\hline
\end{tabular}

Note: (a) Second of two gross gamma scans of the same rod; the first scan gave a maximum-to-average value of 1.25 . 
SECTION 8

THEORY FOR CALCULATION OF ISOTOPES OF

ELEMENTS THORIUM THROUGH CURIUM

H. E. Krug, J. E. Olhoeft, and J. Alsina

The theoretical portion of the EYC Program Extension is the development of a method to calculate the production of the special isotopes U-232, U-233, $\mathrm{Pu}-236, \mathrm{~Np}-237, \mathrm{Pu}-238, \mathrm{Am}-241, \mathrm{Cm}-242, \mathrm{Am}-243$, and $\mathrm{Cm}-244$. Matrices and series are used in a general mathematical expression and programmed as a package of subroutines to calculate these isotopes as well as other isotopes in the chain. [3] This package, or link, which is general enough to be made part of any burnup code, has been incorporated, along with appropriate cross section data, into the LASER ${ }^{[21]}$ computer code. These subroutines are called HIC for Higher Isotope Calculation.

\subsection{CALCULATIONAL MODEL}

The HIC link calculates the vector $N(t+\Delta t)$ from the matrix exponential equation

$$
N(t+\Delta t)=[\operatorname{EXP}(A \Delta t)] N(t)
$$

where $A$ is a square matrix which may be singular.

When cast in the form of Equation (1), the nuclide transmutation ("burnup") equations can be very generally coupled. $[1,3,32,33]$ Many nuclide transmutation codes such as CINDER [34] make a number of approximations in order to employ certain computational schemes. These schemes do not permit general coupling. The matrix exponential method is general and exceptionally we11 suited for automated application; however, care must be taken in the computational scheme to reduce errors resulting from the finite size of the computer word. 
Equation (1) can be written

$$
\left(\begin{array}{c}
\mathrm{n}_{1} \\
\mathrm{n}_{2} \\
\vdots
\end{array}\right)_{\mathrm{t}+\Delta t}=\operatorname{ExP}\left(\begin{array}{cc}
\mathrm{a}_{11} & \mathrm{a}_{21} \ldots \\
\mathrm{a}_{12} & \mathrm{a}_{22} \ldots \\
\vdots & \vdots
\end{array}\right)_{t} \Delta t\left(\begin{array}{c}
\mathrm{n}_{1} \\
\mathrm{n}_{2} \\
\vdots
\end{array}\right)_{t}
$$

where $a_{i j}$ represents the source to isotope " $i$ " from the " $j$ th" isotope and $a_{i i}$ (the elements on the diagonal) represents the total loss of the " $i$ " isotope. Because the A matrix is square, the formulation allows a contribution to any isotope from any other isotope.

\subsubsection{Mathematical Description}

If, as shown in Figure 8-1 the concentration of a given isotope $\mathrm{N}_{i}$ is represented by a box and the possible nuclear reactions are indicated by arrows entering (production) or leaving (destruction) the box, the timedependent balance equation for isotope $N_{i}$ is fully described, and it can be written as:

$$
\begin{aligned}
& \dot{\mathrm{N}}_{i}=-\sum_{j=1}^{3}\left[\frac{\iint_{j} \sigma_{i}(E) \phi(E, r) d E d r}{V}+j_{i} \lambda_{i} N_{i}+\left[\frac{\int^{\sigma} \sigma_{h}(E) \phi(E, r) d E d r}{V}\right] N_{h}\right. \\
& +\left[\frac{\int{ }_{2}{ }^{\sigma}(E) \phi(E, r) d E d r}{V}\right] N_{k}+{ }_{1} \lambda q^{N} q+{ }_{2}{ }^{\lambda}{ }_{r}{ }_{r}+{ }_{3}^{\lambda} p^{N} N_{p}
\end{aligned}
$$

where

$\mathrm{N}_{i}$ is the concentration of the isotope of interest

$\mathrm{N}_{\mathrm{n}}, \mathrm{N}_{\mathrm{k}}, \mathrm{N}_{\mathrm{q}}, \mathrm{N}_{\mathrm{r}}, \mathrm{N}_{\mathrm{p}}$ are the concentrations of isotopes which are coupled to isotope $\mathrm{N}_{i}$ by the defined transmutation processes

Note: The expression $\frac{f f \sigma(E) \phi(E, r) d E d r}{V}$ represents the reaction rate per unit volume. $\mathrm{N}_{i}, \mathrm{~N}_{h}, \mathrm{~N}_{k}, \mathrm{~N}_{\mathrm{q}}, \mathrm{N}_{\mathrm{r}}$ and $\mathrm{N}_{\mathrm{p}}$ are assumed to have a flat distribution across the fuel pellet. 


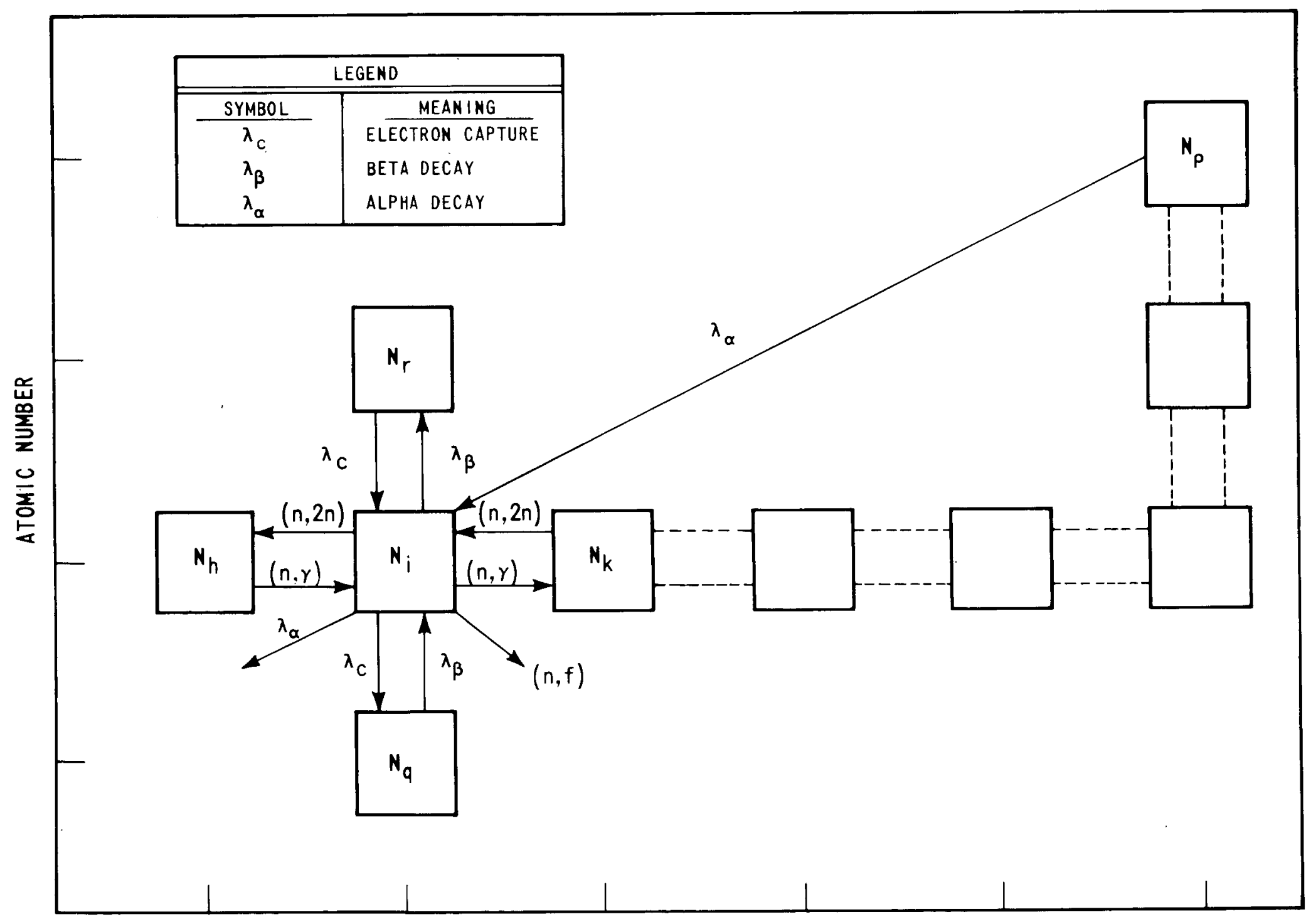

ATOMIC WEIGHT

Figure 8-1. Transmutation Processes Affecting Isotope $\mathrm{N}_{\mathrm{i}}$ 
$j^{\sigma}(E)$ is the energy dependent cross section of isotope $N_{i}$ for any of the following nuclear reactions:

$$
\begin{aligned}
& j=1, \quad(n, \gamma) \\
& j=2, \quad(n, 2 n) \\
& j=3, \quad(n, f i s s i o n)
\end{aligned}
$$

$j^{\lambda} i$ is the decay constant of isotope $N_{i}$ for any of the following modes of radioactive decay:

$$
\begin{aligned}
& j=1, \text { B decay } \\
& j=2, \text { electron capture } \\
& j=3, \text { a decay }
\end{aligned}
$$

$\mathrm{V}$ is the volume of the region under consideration $\phi(E, r)$ is the energy and space dependent neutron flux

Equation (3) can be written in the following general form

$$
\dot{N}_{i}=a_{i 1} N_{1}+a_{i 2} N_{2}+\ldots .+a_{i n} N_{n}=\sum_{k=1}^{n} a_{i k} N_{k}
$$

where $a_{i k}$ couples isotope $N_{i}$ with isotope $N_{k}$. When $k=i$, the coefficient $a_{i i}$ describes the destruction of isotope $N_{i}$. For instance, the term $a_{i i}$ in Equation (3) is

$$
a_{i i}=\sum_{j=1}^{3}\left[\frac{\iint_{j}^{\sigma_{i}}(E) \phi(E, r) d E d r}{V}+j_{i}^{\lambda}\right]
$$

Similarly, the coefficient coupling isotope $\mathrm{N}_{i}$ to isotope $\mathrm{N}_{\mathrm{q}}$ by $\beta$ decay is

$$
a_{i q}={ }_{1} \lambda q
$$


Expanding this process to encompass a chain of $\mathrm{n}$ interrelated isotopes of interest, it is possible to define $n$ equations in $n$ unknowns, as indicated below:

$$
\begin{aligned}
& \dot{\mathrm{N}}_{1}=a_{11} \mathrm{~N}_{1}+\mathrm{a}_{12} \mathrm{~N}_{2}+\mathrm{a}_{13 \mathrm{~N}_{3}}+\ldots \ldots \ldots+\mathrm{a}_{1 \mathrm{n} \mathrm{N}_{n}} \\
& \dot{\mathrm{N}}_{2}=\mathrm{a}_{21} \mathrm{~N}_{1}+\mathrm{a}_{22} \mathrm{~N}_{2}+\mathrm{a}_{23} \mathrm{~N}_{3}+\ldots \ldots \ldots+\mathrm{a}_{2 \mathrm{n}} \mathrm{N}_{n} \\
& \text {--- = - - - } \\
& \dot{\mathrm{N}}_{i}=a_{i 1} \mathrm{~N}_{1}+a_{i 2} \mathrm{~N}_{2}+a_{i 3} \mathrm{~N}_{3}+\ldots \ldots \ldots+a_{i n} \mathrm{~N}_{n} \\
& \dot{\mathrm{N}}_{\mathrm{n}}=\mathrm{a}_{\mathrm{n} 1} \mathrm{~N}_{\mathrm{n}}+\mathrm{a}_{\mathrm{n} 2} \mathrm{~N}_{2}+\mathrm{a}_{\mathrm{n} 3} \mathrm{~N}_{3}+\ldots \ldots \ldots+a_{n n} \mathrm{~N}_{n}
\end{aligned}
$$

Using matrix notation

$$
(\dot{N})=(A)(N)
$$

The solution of Equation (8) for a specific time step length is Equation (1).

\subsubsection{Numerical Solution}

The heart of the calculation is the evaluation of the matrix Taylor series:

$$
N=\left[I+\sum_{i=1}^{S C}(A t)^{i} / i !\right] N_{0}
$$

or

$$
N=\left[I+A+\sum_{i=2}^{S C}\left(\frac{A t}{i}\right)\left(\frac{(A t)^{i-1}}{(i-1) !}\right)\right] N_{0}
$$

where the series is summed to "solid convergence." Solid convergence (SC) occurs when the addition of the next term in a series to the partial sum of 
the series results in no change to the sum because of underflow during the addition.*

Direct evaluation of Equation (10) can be accelerated (for a given number of terms) by including the $N_{0}$ vector directly in the series as in Equation (11):

$$
\mathrm{N}=\mathrm{N}_{0}+\sum_{i=1}^{\mathrm{SC}}\left(\frac{\mathrm{At}}{i}\right)\left(\frac{(\mathrm{At})^{\mathrm{i}-1} \mathrm{~N}_{\mathrm{O}}}{(\mathrm{i}-1) !}\right)
$$

since all matrix into matrix multiplications are now replaced by matrix into vector multiplications. Unfortunately, experience shows that truncation error is still too large.

The basic problem is to reduce the norm ("magnitude") of the At matrix. In HIC, the sum of the absolute values of the magnitudes of the elements of At matrix is reduced to less than unity by a combination of time step subdivision and matrix scaling.

Time step subdivision is shown in Equation (12).

$$
\mathrm{N}=\left[\mathrm{e}^{A t / \tilde{n}}\right]^{\mathrm{n}} \mathrm{N}_{\mathrm{O}} \equiv\left[\mathrm{e}^{\tilde{\mathrm{A}}}\right]_{\mathrm{O}}^{\mathrm{n}} \mathrm{N}
$$

In the squaring procedure described next, the $\tilde{A}$ matrix is scaled by an integral power of 2 .

$$
e^{Z} \equiv e^{\tilde{A} / 2^{N}}
$$

Equation (11) is evaluated with the A matrix replaced by the $Z$ matrix. The resulting exponential matrix is successively squared as follows:

$$
e^{\tilde{\mathrm{A}} / 2^{\mathrm{N}}} * \mathrm{e}^{\tilde{\mathrm{A}} / 2^{\mathrm{N}}}=e^{\tilde{\mathrm{A}} / 2^{\mathrm{N}-1}}
$$

* This result depends upon the word size of the computer and whether single or double precision is used. 


$$
\begin{gathered}
\mathrm{e}^{\tilde{\mathrm{A}} / 2^{\mathrm{N}-1}} * \mathrm{e}^{\tilde{\mathrm{A}} / 2^{\mathrm{N}-1}}:=\mathrm{e}^{\tilde{\mathrm{A}} / 2^{\mathrm{N}-2}} \\
\tilde{\mathrm{A} / 2} * \mathrm{e}^{\tilde{\mathrm{A}} / 2}=e^{\tilde{A}}=e^{\mathrm{At} / \mathrm{n}}
\end{gathered}
$$

The $e^{A t}$ is calculated using Equation (12). Note that squaring requires matrix into matrix multiplication whereas time step sub-division does not.

\subsubsection{Computer Calculation}

The above formulation has been programmed in a set of double precision routines called PAC, CPAC, EXPM, COMPAR, TRANS, MATAD and MATMPY.

The fast and thermal cross sections for the special isotopes are read off a tape generated by program SAMAND which was written for this purpose.

The cross sections which are used in this program will be described later in Section 9.

For each time step the (A) matrix is constructed in PAC by combining the reaction rate components $\mathrm{R}(\mathrm{I}, \mathrm{J}) *$, obtained by CPAC from LASER, with the $\mathrm{D}(\mathrm{I}, \mathrm{J}) * *$ stored in CPAC.

In addition, PAC selects a reduced time interval and then scales the product of the (A) matrix and the reduced time interval by $2^{n}$, where $n$ is the smallest

* $R(I, J)$ represents the reaction rate of type $I$ with the isotope $J$ : I can be 1,2 or 3 .

$$
\begin{aligned}
& I=1 \text {, reaction rate for } n, \gamma \\
& I=2 \text {, reaction rate for } n, 2 n \\
& I=3 \text {, reaction rate for } n, f
\end{aligned}
$$

$* * \mathrm{D}(I, J)$ means the radioactive decay of the isotope $J$ by the $I$ mode of decay. I can be 1,2 , or 3 , representing $\beta$-decay, electron capture, and $\alpha$-decay, respectively.

$$
\begin{aligned}
& I=1, \text { for } \lambda_{\beta} \\
& I=2, \text { for } \lambda_{c} \\
& I=3, \text { for } \lambda_{\alpha}
\end{aligned}
$$


integer such that the sum of the squares of the absolute values of the scaled matrix is less than 1.0. The exponential of this scaled matrix is then unscaled and multiplied into the number density vector yielding the new number density vector. As a check on the computational accuracy, PAC also performs the inverse calculation to see if the initial number densities can be reconstructed from the new ones.

\subsubsection{Separation of the A Matrix}

A promising extension (not included in the scope of this program) of the present HIC calculational method which both saves computer time and provides more accurate results for very large time steps is to separate the A matrix into two matrices, ${ }^{[35]} \mathrm{D}$ and $\mathrm{R}$; i.e.

$$
e^{A t}=e^{(D+R) t}=e^{D t} e^{R t} e^{C_{2} t^{2}} e^{C_{3} t^{3}} \cdots
$$

where, for example,

$$
\mathrm{C}_{2}=(\mathrm{RD}-\mathrm{DR}) / 2
$$

With respect to nuclide transmutation calculations, all the decay constants can be placed in $D$ and all the reaction rate terms in $R$. With this specification three important things have been accomplished: 1) all the large elements in A appear in $D ; 2$ ) as a result of 1 ), products of $R$ and $D$ result in small elements making the $C_{i}$ terms small; and 3 ) since the D matrix is time step independent, $e^{D t}$ can be carefully precomputed using double precision and stored for a number of convenient time step lengths. The precomputation of $e^{D t}$ will save substantial computational time. Note that

$$
e^{(A+B)}=e^{A} e^{B} \text { only if } A B=B A
$$

With this procedure, considerable additional computational time can be saved for useful time step sizes because the relatively small norms of the Rt and $\mathrm{C}_{i} \mathrm{t}^{i}$ matrices means that scaling by a power of two (Equation 13) can be 
avoided; as a result 1) the matrix-into-vector computational scheme (Equation 11) can be employed and 2) as a result of 1), the "sparse" matrix routines ${ }^{[36]}$ can be effectively employed.

\subsubsection{Perturbation Calculations Using Experimental Data}

The results of the calculations reported herein have not been adjusted in any way; however, there are a number of ways in which experimental data can be used to provide "correction" or "bias" factors to modify the transmutation matrix. The following new method is suggested as an area for further investigation because of its high computational efficiency.

If Equation (8)

$$
\dot{\mathrm{N}}=\mathrm{AN}=\mathrm{AC}^{-1} \mathrm{CN}
$$

is multiplied on the left by $\mathrm{C}$ it becomes:

$$
C \frac{d N}{d t}=C^{-1}(C N)
$$

Defining $\mathrm{y}=\mathrm{CN}$ and $\hat{\mathrm{A}}=\mathrm{CAC}^{-1}$

Equation (18) becomes:

$$
\frac{\mathrm{dy}}{\mathrm{dt}}=\hat{\mathrm{A}} \mathrm{y} ; \mathrm{y}_{\mathrm{o}}=\mathrm{CN}_{\mathrm{o}}
$$

the solution of which is

$$
y=e^{\hat{A} t} y_{0}
$$

so that

$$
N=C^{-1} y=C^{-1} e^{\hat{A} t} y_{0}
$$


The case where $\mathrm{C}$ is a diagonal matrix is important since $\mathrm{C}^{-1}$ is then trivial to calculate. The application to a perturbation calculation is given below. Define a correction matrix, $C$, such that the calculated number density vector $\mathrm{N}$ is transformed into the experimental number density vector $\overline{\mathrm{N}}$, i.e.

$$
\bar{N}=C N
$$

We can then write

$$
e^{(I+E) A} \equiv e^{C^{-1}} C=C e^{A}
$$

where (I+E)A is the "corrected" A matrix.

The departure from zero of the elements of the E matrix indicates areas where more accurate data are required to bring into better agreement the calculated and measured isotopic concentrations.

\subsection{NUCLIDE TRANSMUTATION CHAIN DESCRIPTION}

The isotopes and nuclear reactions considered in the HIC calculational model are shown in the transmutation chains in Figure 8-2. The horizontal arrows pointing to the right and the left indicate the $(n, \gamma)$ and $(n, 2 n)$ reactions, respectively. The vertical arrows pointing up and down indicate $\beta$-decay and electron capture (EC), respectively; the arrows pointing diagonally downward and to the left indicate $\alpha$-decay. Due to the relatively short half-lives of some of the isotopes, some simplifications were made in the transmutation chain description to avoid computational errors; namely, captures in Th-232, $\mathrm{U}-238, \mathrm{Pu}-242$, and Am-243 result directly in the formation of $\mathrm{Pa}-233$, $\mathrm{Np}-239, \mathrm{Am}-243$, and $\mathrm{Cm}-244$, respectively, thus bypassing the isotopes $\mathrm{Th}-233$ $(22.1 \mathrm{~m}), \mathrm{U}-239(23.5 \mathrm{~m}), \mathrm{Pu}-243(4.98 \mathrm{~h})$, and Am-244 (25 m - $10 \mathrm{~h})$. The accuracy of the computations is not compromised by these simplifications. Some difficulties were encountered in representing the formation of the two isomers of Am-242 by neutron capture in $\mathrm{Am}-241$; also, the representation of the isomeric transition (IT) of Am-242A (152 y) to Am-242 (16 h) presented a 


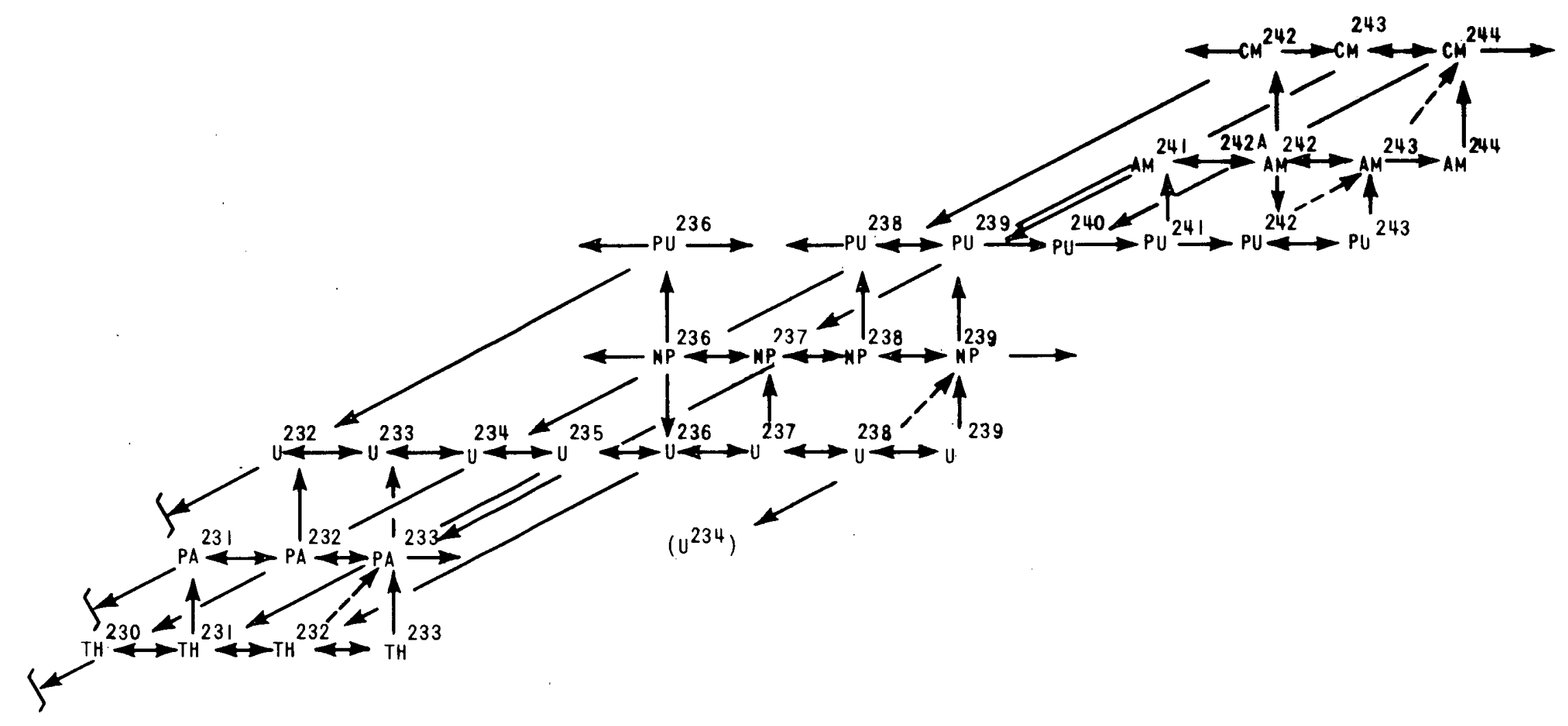

Figure 8-2. HIC Depletion Chains 
problem since the HIC model considers just three modes of decay - $\beta$, EC, and a. This portion of the chain is shown on Figure 8-3. These difficulties were overcome by taking advantage of the extreme degree of flexibility programmed into the HIC model. In the case of the Am-242 isomers, the $(n, \gamma)$ cross sections for Am-241 which lead to the formation of Am-242 (16 h) were programmed in the usual manner; however, the $(n, 2 n)$ cross sections for Am-241 were set equal to the $(n, \gamma)$ cross sections which lead to the formation of $\mathrm{Am}-242 \mathrm{~A}(152 \mathrm{y})$, and the model was programmed so that $(\mathrm{n}, 2 \mathrm{n})$ reactions in Am-241 result in the formation of Am-242A, instead of Am-240. In the case of the isomeric transition of Am-242A to Am-242, the EC decay constant of Am-242A (normally zero) was set equal to the IT decay constant, and the model was programmed so that EC decay of Am-242A leads to the formation of Am-242, instead of $\mathrm{Pu}-242$. 


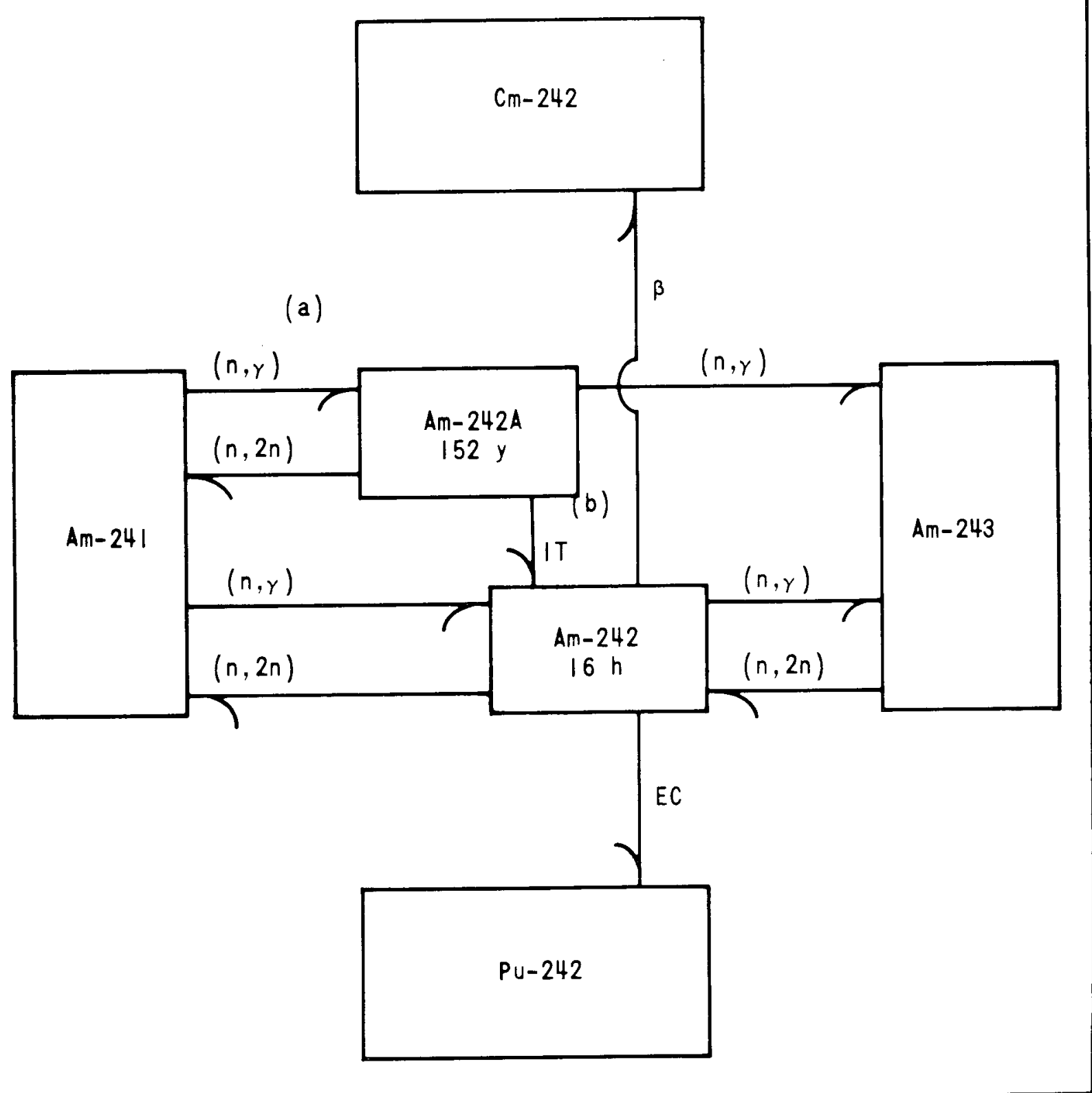

NOTE: (a) HIC SUBSTITUTES $(n, \gamma)$ CROSS SECTION VALUES FOR $(n, 2 n)$ CROSS SECTIONS, AMD IS PROGRAMMED SO THAT $(n, 2 n)$ REACTIONS IN Am-24I RESULT IN THE FORMATION OF Am-242A, INSTEAD OF Am- 240 .

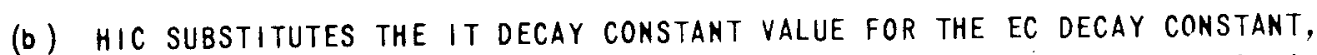
AND IS PROGRAMMED SO THAT THE EC DECAY MODE IN Am-242A RESULTS IN THE FORMATION OF AM-242, INSTEAD OF PU-242.

Figure 8-3. Transmutation Chain Description for Am- 242 


\section{SECTION 9}

\section{BASIC NUCLEAR DATA USED IN CALCULATIONS \\ N. Azziz, S. Semanderes, J. E. Olhoeft}

One of the main objectives of the EYC Program Extension is the calculation of the concentrations of the special isotopes U-232, U-233, Pu-236, Pu-238, $\mathrm{Np}-237, \mathrm{Am}-241, \mathrm{Am}-243, \mathrm{Cm}-242$, and $\mathrm{Cm}-244$ in the Yankee fuel. This section of the report is a documentation of the cross sections and decay constants used in the application of the calculational model described in section 8 . For completeness, the basic nuclear data used for the main chain $U$ and $\mathrm{Pu}$ isotopes are also, included.

\subsection{CROSS SECTION VALUES FOR EYC PROGRAM EXTENSION}

The group averaged cross sections, $\sigma_{\gamma}, \sigma_{f}, \sigma_{2 n}$, were generated for the isotopes listed in Table 9-1 and the energy structure listed in Tables 9-2 and 9-3. The main sources of information were $B N L-325^{[37]}, \operatorname{ENDF} / \mathrm{B}^{[38]}$, and BNWL-CC-325 ${ }^{[39]}$; cross sections that were not found reported in literature were calculated using appropriate nuclear models. [40] A summary of the sources of the multi-group cross sections used in the LASER-HIC program is given in Table 9-1, while the actual library values are tabulated in Appendix C.

A detailed account of the sources used for a specific energy range for each isotope is given in Table 9-4. Additional information for some of the special isotopes is given in the following paragraphs. 
TABLE 9-1

SUMMARY OF SOURCES OF CROSS SECTION DATA

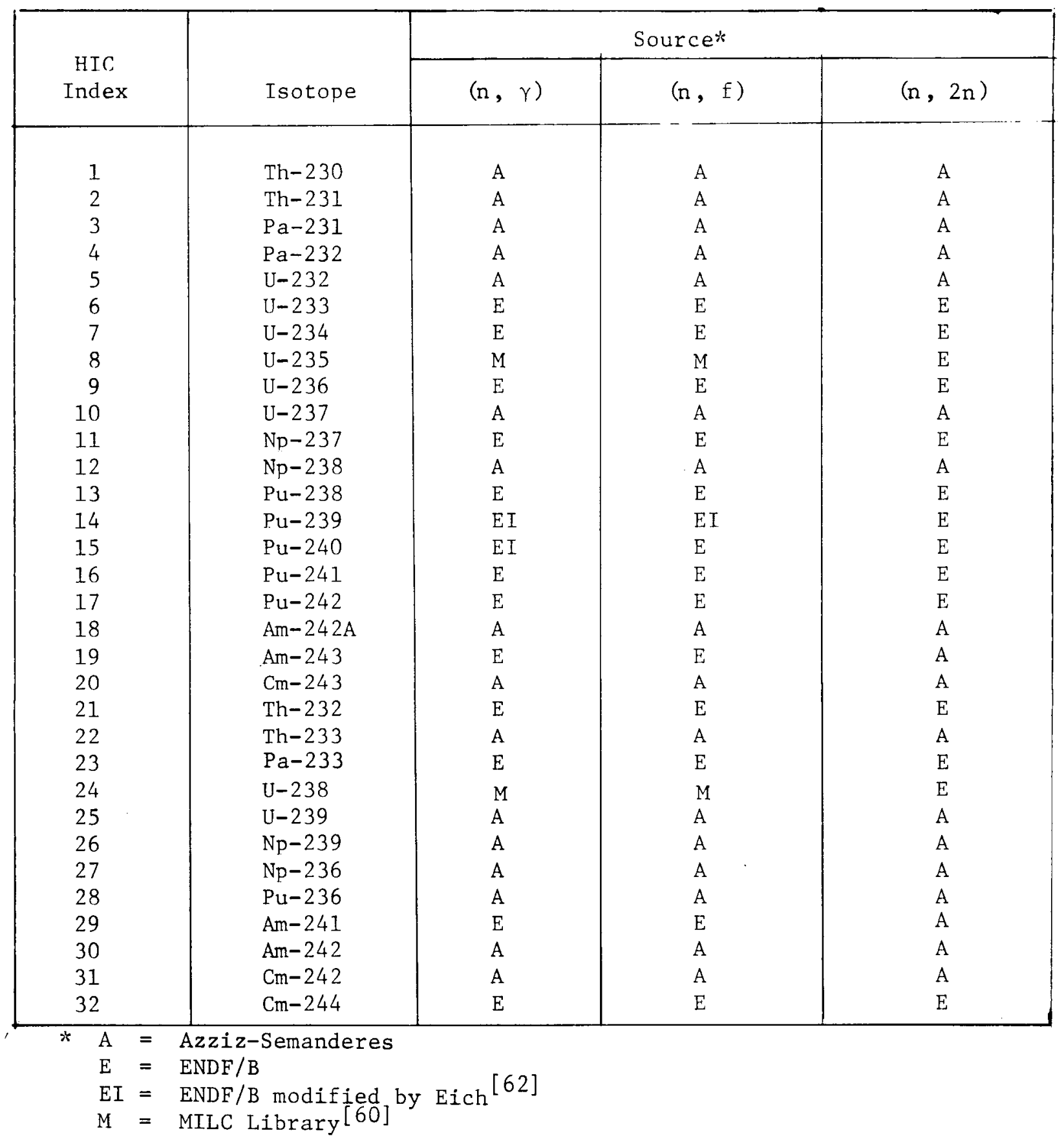


TABLE 9-2

ENERGY GROUP STRUCTURE FOR FAST RANGE

\begin{tabular}{|c|c|c|c|}
\hline Group & Energy Span (ev) & Group & Energy Span (ev) \\
\hline 1 & $1.0000 \times 10^{7}$ to $7.7900 \times 10^{6}$ & 26 & $5.5300 \times 10^{3}$ to $3.3500 \times 10^{3}$ \\
\hline 2 & $7.7900 \times 10^{6}$ to $6.0700 \times 10^{6}$ & 27 & $3.3500 \times 10^{3}$ to $2.0300 \times 10^{3}$ \\
\hline 3 & $6.0700 \times 10^{6}$ to $4.7200 \times 10^{6}$ & 28 & $2.0300 \times 10^{3}$ to $1.2300 \times 10^{3}$ \\
\hline 4 & $4.7200 \times 10^{6}$ to $3.6800 \times 10^{6}$ & 29 & $1.2300 \times 10^{3}$ to $7.5000 \times 10^{2}$ \\
\hline 5 & $3.6800 \times 10^{6}$ to $2.8600 \times 10^{6}$ & 30 & $7.5000 \times 10^{2}$ to $4.5400 \times 10^{2}$ \\
\hline 6 & $2.8600 \times 10^{6}$ to $2.2300 \times 10^{6}$ & 31 & $4.5400 \times 10^{2}$ to $2.7500 \times 10^{2}$ \\
\hline 7 & $2.2300 \times 10^{6}$ to $1.7400 \times 10^{6}$ & 32 & $2.7500 \times 10^{2}$ to $1.6700 \times 10^{2}$ \\
\hline 8 & $1.7400 \times 10^{6}$ to $1.3500 \times 10^{6}$ & 33 & $1.6700 \times 10^{2}$ to $1.3000 \times 10^{2}$ \\
\hline 9 & $1.3500 \times 10^{6}$ to $1.0500 \times 10^{6}$ & 34 & $1.3000 \times 10^{2}$ to $1.0100 \times 10^{2}$ \\
\hline 10 & $1.0500 \times 10^{6}$ to $8.2100 \times 10^{5}$ & 35 & $1.0100 \times 10^{2}$ to $7.8700 \times 10^{1}$ \\
\hline 11 & $8.2100 \times 10^{5}$ to $6.3900 \times 10^{5}$ & 36 & $7.8700 \times 10^{1}$ to $6.1300 \times 10^{1}$ \\
\hline 12 & $6.3900 \times 10^{5}$ to $4.9800 \times 10^{5}$ & 37 & $6.1300 \times 10^{1}$ to $4.7800 \times 10^{1}$ \\
\hline 13 & $4.9800 \times 10^{5}$ to $3.6700 \times 10^{5}$ & 38 & $4.7800 \times 10^{1}$ to $3.7200 \times 10^{1}$ \\
\hline 14 & $3.6700 \times 10^{5}$ to $3.0200 \times 10^{5}$ & 39 & $3.7200 \times 10^{1}$ to $2.9000 \times 10^{1}$ \\
\hline 15 & $3.0200 \times 10^{5}$ to $2.3500 \times 10^{5}$ & 40 & $2.9000 \times 10^{1}$ to $2.2600 \times 10^{1}$ \\
\hline 16 & $2.3500 \times 10^{5}$ to $1.8300 \times 10^{5}$ & 41 & $2.2600 \times 10^{1}$ to $1.7600 \times 10^{1}$ \\
\hline 17 & $1.8300 \times 10^{5}$ to $1.4300 \times 10^{5}$ & 42 & $1.7600 \times 10^{1}$ to $1.3700 \times 10^{1}$ \\
\hline 18 & $1.4300 \times 10^{5}$ to $1.1100 \times 10^{5}$ & 43 & $1.3700 \times 10^{1}$ to $1.0700 \times 10^{1}$ \\
\hline 19 & $1.1100 \times 10^{5}$ to $8.6500 \times 10^{4}$ & 44 & $1.0700 \times 10^{1}$ to 8.3200 \\
\hline
\end{tabular}


TABLE 9-2 (Cont)

ENERGY GROUP STRUCTURE FOR FAST RANGE

\begin{tabular}{|c|c|c|c|}
\hline Group & Energy Span (ev) & Group & Energy Span (ev) \\
\hline 20 & $8.6500 \times 10^{4}$ to $6.7400 \times 10^{4}$ & 45 & 8.3200 to 6.5000 \\
21 & $6.7400 \times 10^{4}$ to $4.0900 \times 10^{4}$ & 46 & 6.5000 to 5.1000 \\
22 & $4.0900 \times 10^{4}$ to $2.4800 \times 10^{4}$ & 47 & 5.1000 to 3.9700 \\
23 & $2.4800 \times 10^{4}$ to $1.5000 \times 10^{4}$ & 48 & 3.9700 to 3.0600 \\
24 & $1.5000 \times 10^{4}$ to $9.1200 \times 10^{3}$ & 49 & 3.0600 to 2.3800 \\
25 & $9.1200 \times 10^{3}$ to $5.5300 \times 10^{3}$ & 50 & 2.3800 to 1.8550 \\
\hline
\end{tabular}


TABLE $9-3$

ENERGY GROUP STRUCTURE FOR THERMAL RANGE

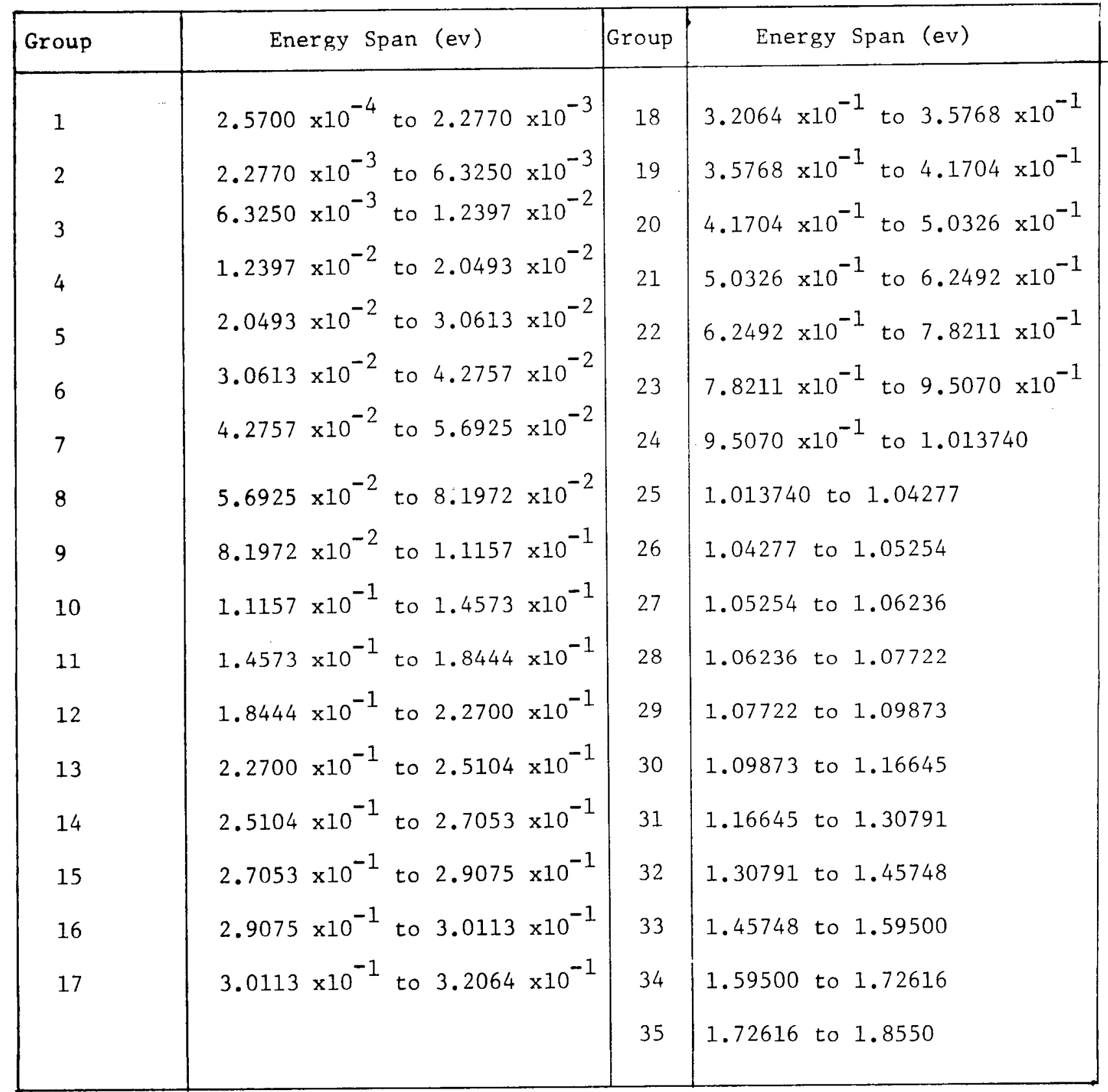


TABLE $9-4$

SOURCES OF INFORMATION ON THE CROSS SECTION

OF THE ISOTOPES EVALUATED IN THE EYC PROGRAM EXTENSION

\begin{tabular}{|c|c|c|c|}
\hline Isotope & Type Cross Section & Energy Range & Main Source \\
\hline \multirow{3}{*}{ Th-230 } & $(n, \gamma)$ & $\mathrm{E}=.0253 \mathrm{ev}$ & $\begin{array}{l}\text { BNL-325 } \\
\sigma_{\gamma}=23 \mathrm{~b}\end{array}$ \\
\hline & $(n, f)$ & $\begin{array}{l}E=2.5 \mathrm{Mev} \\
E=14.6 \mathrm{Mev}\end{array}$ & $\begin{array}{l}\text { BNL-325 } \\
\sigma_{f}=0.40 \mathrm{~b} \\
\sigma_{\mathrm{f}}=0.72 \mathrm{~b}\end{array}$ \\
\hline & $(n, 2 n)$ & Complete & Calculated \\
\hline Th -231 & $(n, 2 n)$ & Complete & Calculated \\
\hline \multirow{3}{*}{ Th-232 } & $(n, \gamma)$ & Complete & ENDF/B, 1038 \\
\hline & $(n, f)$ & Complete & ENDF/B, 1038 \\
\hline & $(n, 2 n)$ & Complete & ENDF / B , 1038 \\
\hline \multirow{3}{*}{ Th-233 } & $(n, \gamma)$ & $E=.0253 \mathrm{ev}$ & $\begin{array}{l}\text { BNL }-325 \\
\sigma_{Y}=1500 \mathrm{~b}\end{array}$ \\
\hline & $(n, f)$ & $E=.0253 \mathrm{ev}$ & $\begin{array}{l}\mathrm{BNL}=325 \\
\sigma_{\mathrm{f}}=14 \mathrm{~b}\end{array}$ \\
\hline & $(n, 2 n)$ & Complete & Calculated \\
\hline \multirow{3}{*}{$\mathrm{Pa}-231$} & $(n, y)$ & Complete & $\mathrm{BNWL}-\mathrm{CC}-325$ \\
\hline & $(n, f)$ & $\begin{array}{l}10^{-4}-10^{5} \mathrm{ev} \\
10^{5}-1.8 \times 10^{6} \mathrm{ev} \\
1.8 \times 10^{6}-10^{7} \mathrm{ev}\end{array}$ & $\begin{array}{l}\text { BNWL-CC-325 } \\
\text { BNL-325 } \\
\text { BNWL-CC-325 }\end{array}$ \\
\hline & $(n, 2 n)$ & Complete & Calculated \\
\hline $\mathrm{Pa}-232$ & $(n, \gamma)$ & $E=.0253 \mathrm{ev}$ & $\begin{array}{l}\text { BNWL-CC-325 } \\
\sigma_{Y}=760 \mathrm{~b}\end{array}$ \\
\hline
\end{tabular}


TABLE 9-4 (Cont)

SOURCES OF INFORMATION ON THE CROSS SECTION

OF THE ISOTOPES EVALUATED IN THE EYC PROGRAM EXTENSION

\begin{tabular}{|c|c|c|c|}
\hline Isotope & Type Cross Section & Energy Range & Main Source \\
\hline \multirow[t]{2}{*}{$\mathrm{Pa}-232$} & $(n, f)$ & $E=.0253 \mathrm{ev}$ & $\begin{array}{l}\mathrm{BNWL}-\mathrm{CC}-325 \\
\sigma_{f}=700 \mathrm{~b}\end{array}$ \\
\hline & $(n, 2 n)$ & Complete & Calculated \\
\hline \multirow{3}{*}{$\mathrm{Pa}-233$} & $(n, \gamma)$ & Complete & $\mathrm{ENDF} / \mathrm{B}, 1040$ \\
\hline & $(n, f)$ & Complete & ENDF / B, 1040 \\
\hline & $(n, 2 n)$ & Complete & ENDF/B, 1040 \\
\hline \multirow{4}{*}{$\mathrm{U}-232$} & $(n, \gamma)$ & Complete & BNWL-CC-325 \\
\hline & $(n, f)$ & $10^{-4}-4 \mathrm{ev}$ & BNWL-CC-325 \\
\hline & & $4-400 \mathrm{ev}$ & BNL-325 \\
\hline & $(n, 2 n)$ & Complete & Calculated \\
\hline \multirow{3}{*}{$U-233$} & $(n, \gamma)$ & Complete & ENDF/B, 1041 \\
\hline & $(n, f)$ & Complete & ENDF/B, 1041 \\
\hline & $(n, 2 n)$ & Complete & ENDF / B, 1041 \\
\hline \multirow{3}{*}{$\mathrm{U}-234$} & $(n, \gamma)$ & Complete & ENDF/B, 1043 \\
\hline & $(n, f)$ & Complete & $\mathrm{ENDF} / \mathrm{B}, 1043$ \\
\hline & $(n, 2 n)$ & Complete & $\mathrm{ENDF} / \mathrm{B}, 1043$ \\
\hline \multirow[t]{3}{*}{$\mathrm{U}-235$} & $(n, \gamma)$ & Complete & MILC Library \\
\hline & $(n, f)$ & Complete & MILC Library \\
\hline & $(n, 2 n)$ & Complete & ENDF/B, 1044 \\
\hline $\mathrm{U}-236$ & $(n, \gamma)$ & Complete & ENDF/B, 1046 \\
\hline
\end{tabular}


TABLE $9-4$ (Cont)

SOURCES OF INFORMATION ON THE CROSS SECTION

OF THE ISOTOPES EVALUATED IN THE EYC PROGRAM EXTENSION

\begin{tabular}{|c|c|c|c|}
\hline Isotope & Type Cross Section & Energy Range & Main Source \\
\hline$U-236$ & $\begin{array}{l}(n, f) \\
(n, 2 n)\end{array}$ & $\begin{array}{l}\text { Complete } \\
\text { Complete }\end{array}$ & $\begin{array}{l}\text { ENDF/B, } 1046 \\
\text { ENDF/B, } 1046\end{array}$ \\
\hline$U-237$ & $(n, 2 n)$ & Complete & Calculated \\
\hline$U-238$ & $\begin{array}{l}(n, \gamma) \\
(n, f) \\
(n, 2 n)\end{array}$ & $\begin{array}{l}\text { Complete } \\
\text { Complete } \\
\text { Complete }\end{array}$ & $\begin{array}{l}\text { MILC Library } \\
\text { MILC Library } \\
\text { ENDF/B, } 1047\end{array}$ \\
\hline$U-239$ & $\begin{array}{l}(n, \gamma) \\
(n, f) \\
(n, 2 n)\end{array}$ & $\begin{array}{l}\text { Complete } \\
\text { Complete } \\
\text { Complete }\end{array}$ & $\begin{array}{l}\text { BNWL-CC-325 } \\
\text { BNWL-CC-325 } \\
\text { Calculated }\end{array}$ \\
\hline$N p-237$ & $\begin{array}{l}(n, Y) \\
(n, f) \\
(n, 2 n)\end{array}$ & $\begin{array}{l}\text { Complete } \\
\text { Complete } \\
\text { Complete }\end{array}$ & $\begin{array}{l}\text { ENDF/B, } 1048 \\
\text { ENDF/B, } 1048 \\
\text { ENDF/B, } 1048\end{array}$ \\
\hline $\mathrm{Np}-238$ & $\begin{array}{l}(n, f) \\
(n, 2 n)\end{array}$ & $\begin{array}{l}\text { Complete } \\
\text { Complete }\end{array}$ & $\begin{array}{l}\text { BNWL-CC- } 325 \\
\text { Calculated }\end{array}$ \\
\hline $\mathrm{Np}-239$ & $\begin{array}{l}(n, \gamma) \\
(n, f) \\
(n, 2 n)\end{array}$ & $\begin{array}{l}\text { Complete } \\
\text { Complete } \\
\text { Complete }\end{array}$ & $\begin{array}{l}\text { BNWL-CC-325 } \\
\text { BNWL-CC-325 } \\
\text { Calculated }\end{array}$ \\
\hline $\mathrm{Pu}-236$ & $(n, f)$ & $E=.0253 \mathrm{ev}$ & $\begin{array}{l}\text { Chart of } \\
\text { Nuclides } \\
\text { AEC, Ninth } \\
\text { Ed., ō } 170 \mathrm{~b} \\
1966\end{array}$ \\
\hline
\end{tabular}


TABLE $9-4$ (Cont)

SOURCES OF INFORMATION ON THE CROSS SECTION

OF THE ISOTOPES EVALUATED IN THE EYC PROGRAM EXTENSION

\begin{tabular}{|c|c|c|c|}
\hline Isotope & Type Cross Section & Energy & Main Source \\
\hline Pu-236 & $(n, 2 n)$ & Complete & Calculated \\
\hline $\mathrm{Pu}-238$ & $\begin{array}{l}(n, \gamma) \\
(n, f) \\
(n, 2 n)\end{array}$ & $\begin{array}{l}\text { Complete } \\
\text { Complete } \\
\text { Complete }\end{array}$ & $\begin{array}{l}\text { ENDF/B, } 1050 \\
\text { ENDF/B, } 1050 \\
\text { ENDF/B, } 1050\end{array}$ \\
\hline $\mathrm{Pu}-239$ & $\begin{array}{l}(n, y) \\
(n, f) \\
(n, 2 n)\end{array}$ & $\begin{array}{l}\text { Complete } e^{* *} \\
\text { Complete } \\
\text { Complete }\end{array}$ & $\begin{array}{l}\text { ENDF/B, } 1051 \\
\text { ENDF/B, } 1051 \\
\text { ENDF/B, } 1051\end{array}$ \\
\hline $\mathrm{Pu}-240$ & $\begin{array}{l}(n, \gamma) \\
(n, f) \\
(n, 2 n)\end{array}$ & $\begin{array}{l}\text { Complete } \\
\text { Complete } \\
\text { Complete }\end{array}$ & $\begin{array}{l}\text { ENDF/B, } 1053 \\
\text { ENDF/B, } 1053 \\
\text { ENDF/B, } 1053\end{array}$ \\
\hline $\mathrm{Pu}-241$ & $\begin{array}{l}(n, y) \\
(n, f) \\
(n, 2 n)\end{array}$ & $\begin{array}{l}\text { Complete } \\
\text { Complete } \\
\text { Complete }\end{array}$ & $\begin{array}{l}\text { ENDF/B, } 1054 \\
\text { ENDF/B, } 1054 \\
\text { ENDF/B, } 1054\end{array}$ \\
\hline Pu -242 & $\begin{array}{l}(n, y) \\
(n, f) \\
(n, 2 n)\end{array}$ & $\begin{array}{l}\text { Complete } \\
\text { Complete } \\
\text { Complete }\end{array}$ & $\begin{array}{l}\text { ENDF/B, } 1055 \\
\text { ENDF/B, } 1055 \\
\text { ENDF/B, } 1055\end{array}$ \\
\hline $\mathrm{Pu}-243$ & $\begin{array}{l}(n, \gamma) \\
(n, 2 n)\end{array}$ & $\begin{array}{l}\mathrm{E}=.0253 \mathrm{ev} \\
\text { Complete }\end{array}$ & $\begin{array}{l}\text { BNWL-CC-325 } \\
\sigma_{\gamma}=170 \mathrm{~b} \\
\text { Calculated }\end{array}$ \\
\hline
\end{tabular}

** Modified by Eich

[62] 
TABLE 9-4 (Cont)

SOURCES OF INFORMATION ON THE CROSS SECTION

OF THE ISOTOPES EVALUATED IN THE EYC PROGRAM EXTENSION

\begin{tabular}{|c|c|c|c|}
\hline Isotope & Type Cross Section & Energy Range & Main Source \\
\hline$A m-241$ & $\begin{array}{l}(n, \gamma) \\
(n, f) \\
(n, 2 n)\end{array}$ & $\begin{array}{l}\text { Complete } \\
\text { Complete } \\
\text { Complete }\end{array}$ & $\begin{array}{l}\text { ENDF/B, } 1056 \\
\text { ENDF/B, } 1056 \\
\text { Calculated }\end{array}$ \\
\hline $\mathrm{Am}-242 \mathrm{~A}$ & $\begin{array}{l}(n, \gamma) \\
(n, f) \\
(n, 2 n)\end{array}$ & $\begin{array}{l}\text { Complete } \\
\text { Complete } \\
\text { Complete }\end{array}$ & $\begin{array}{l}\text { BNWL-CC-325 } \\
\text { BNWL-CC-325 } \\
\text { Calculated }\end{array}$ \\
\hline$A m-243$ & $\begin{array}{l}(n, \gamma) \\
(n, f) \\
(n, 2 n)\end{array}$ & $\begin{array}{l}\text { Complete } \\
\text { Complete } \\
\text { Complete }\end{array}$ & $\begin{array}{l}\text { ENDF/B, } 1057 \\
\text { ENDF/B, } 1057 \\
\text { Calculated }\end{array}$ \\
\hline$A m-244$ & $\begin{array}{l}(n, f) \\
(n, 2 n)\end{array}$ & $\begin{array}{l}\mathrm{E}=.0253 \mathrm{ev} \\
\text { Complete }\end{array}$ & $\begin{array}{l}\text { Chart of } \\
\text { nuclides AEC } \\
\text { Ninth Ed., } 1966, \\
\sigma_{f}=2300 \mathrm{~b} \\
\text { Calculated }\end{array}$ \\
\hline $\mathrm{Cm}-242$ & $\begin{array}{l}(n, \gamma) \\
(n, 2 n)\end{array}$ & $\begin{array}{l}E=.0253 \\
\text { Complete }\end{array}$ & $\begin{array}{l}\text { BNWL-CC-325 } \\
\sigma_{\gamma}=20 \mathrm{~b} \\
\text { Calculated }\end{array}$ \\
\hline $\mathrm{Cm}-243$ & $\begin{array}{l}(n, \gamma) \\
(n, f)\end{array}$ & $\begin{array}{l}\text { Complete } \\
\text { Complete }\end{array}$ & $\begin{array}{l}\text { BNWL-CC-325 } \\
\text { BNWL-CC-325 }\end{array}$ \\
\hline $\mathrm{Cm}-244$ & $\begin{array}{l}(n, \gamma) \\
(n, f) \\
(n, 2 n)\end{array}$ & $\begin{array}{l}\text { Complete } \\
\text { Complete } \\
\text { Complete }\end{array}$ & $\begin{array}{l}\text { ENDF/B, } 1058 \\
\text { ENDF/B, } 1058 \\
\text { ENDF/B, } 1058\end{array}$ \\
\hline
\end{tabular}




\subsubsection{Cross Sections for $(n, \gamma)$ Reaction}

It was found that the $(n, \gamma)$ cross sections of some of the isotopes were either not measured or were measured only at thermal energy. The isotopes Th-230, Th-233, Pa-232, Pu-243, and $\mathrm{Cm}-242$ have their cross sections reported only for $E=0253 \mathrm{ev}$. It is recommended that $\sigma_{\gamma} 1 / \mathrm{V}$ be taken for these 1sotopes. This approximation would be satisfactory for the thermal region, but in the resonance region the cross section would be much lower than expected. Above the resonance region this cross section would still be lower than the actual, but since $\sigma_{\gamma}$ is very small for these higher energies it would not affect calculations noticeably. The cross sections for these isotopes at $E=.0253 \mathrm{ev}$ given below:

$$
\begin{array}{ll}
\text { Th-230 } & \sigma_{Y}=23 \mathrm{~b} \\
\text { Th-233 } & \sigma_{Y}=1500 \mathrm{~b} \\
\mathrm{~Pa}-232 & \sigma_{\gamma}=760 \mathrm{~b} \\
\mathrm{Pu}-243 & \sigma_{Y}=170 \mathrm{~b} \\
\mathrm{Cm}-242 & \sigma_{\gamma}=20 \mathrm{~b}
\end{array}
$$

For the 1sotopes Th-231, U-237, Np-236, Np-238, Pu-236 and Am-244, no information is reported on $\sigma_{\gamma}$. In these cases it has been assumed that $\sigma_{\gamma}$ has a $1 / \mathrm{v}$ variation; at thermal energy, $E=.0253 \mathrm{ev}$, the $\sigma_{\gamma}$ for these isotopes was taken as follows:

$$
\begin{aligned}
& \text { Th-231 } \sigma_{\gamma}=\text { Th-233 } \sigma_{\gamma} \\
& \text { U-237 } \sigma_{\gamma}=\mathrm{U}-235 \sigma_{\gamma} \\
& \text { Np-236 } \sigma_{\gamma}=40 \text { barns } \\
& \text { Np-238 } \sigma_{\gamma}=40 \text { barns } \\
& \text { Pu-236 } \sigma_{\gamma}=\text { Pu-238 } \sigma_{\gamma} \\
& \text { Am-244 } \sigma_{\gamma}=\text { Am-242 } \sigma_{\gamma}
\end{aligned}
$$




\subsubsection{Cross Section for $(n, f)$ Reaction}

For the isotopes Th-231, U-237, Np-236, Pu-243, and $\mathrm{Cm}-242$, the (n,fission) cross sections were not given in any source. A calculation or estimation of $\sigma_{f}$ based on nuclear models was not intended. Hence, $\sigma_{f}=0$ has been taken for these isotopes until some information does become available. For the isotopes Th-233 and $\mathrm{Pa}-232, \sigma_{f}$ has a $1 / \mathrm{v}$ variation, and its values at $\mathrm{E}=.0253 \mathrm{ev}$ are

$$
\begin{array}{ll}
\mathrm{Th}-233 & \sigma_{f}=14 \mathrm{~b} \\
\mathrm{~Pa}-232 & \sigma_{\mathrm{f}}=700 \mathrm{~b}
\end{array}
$$

For the isotopes Th-230, $\mathrm{Pu}-236, \mathrm{Am}-244, \sigma_{\mathrm{f}}$ was given in BNL-325, Volume III at one or two energies. These values are given below but again no variation of $\sigma_{f}$ with energy can be recommended.

$$
\begin{aligned}
& \text { Th-230 } \sigma_{f}(2.5 \mathrm{Mev})=0.40 \mathrm{~b} \\
& \text { Th-230 } \sigma_{f}(14.6 \mathrm{Mev})=0.72 \mathrm{~b} \\
& \text { Pu-236 } \sigma_{f}(.0253 \mathrm{ev})=170 \mathrm{~b} \\
& \text { Am-244 } \sigma_{f}(.0253 \mathrm{ev})=2300 \mathrm{~b}
\end{aligned}
$$

\subsubsection{Cross Sections for $(n, 2 n)$ Reaction}

Table 9-4 shows that there are only a few isotopes whose $(n, 2 n)$ cross sections have been measured. Hence, the $(n, 2 n)$ cross sections of most isotopes were predicted. The statistical model of nuclear reactions (a coming report will describe in detail the model as well as some of its predictions ${ }^{[40]}$ was used to calculate the $(n, 2 n)$ cross sections. Because of the threshold reaction of $(n, 2 n)$, cross sections are given for only the upper three groups of the fast spectrum. The cross section below this energy range is zero. 


\subsubsection{Cross Sections for Isomers of Am-242}

The production and transitions of the isomeric states of Am-242 is included since there is an important effect on the daughter products. The reported transition schemes of these isomers have undergone marked change in the last several years, but the ones used in this study are taken from the recent 6 th Edition of the Table of Isotopes [43]. According to this reference, the ground state of Am-242 decays with a 16.0 hour half-life,with 84 percent of the transitions specified as beta-decay to $\mathrm{Cm}-242$ and 16 percent being electron capture to $\mathrm{Pu}-242$. It is noted there is no alpha decay or isomeric transition. The isomeric state, Am-242M, designated as Am-242A in the library, decays with a 152 year half-life.

Its disintegration scheme is reported as 99.5 percent isomeric transition to Am-242 ( 16.0 hour state) and 0.5 percent alpha decay to Np-238. The alpha decay mode was neglected in this study.

According to BNL-325, 2nd Edition [37], the 740 barn thermal ( $n, \gamma)$ cross section of Am-241 is subdivided so that 620 barns (or 83.8 percent) produces the Am-242 (16 hour state) and 120 barns (or 16.2 percent) produces Am-242M (152 year state). This same proportioning is assumed for the full neutron energy spectrum of the reactor. This appears to be at least approximately consistent with the 900 barns resonance integral measurements of Am-241 (n, $\gamma$ ) Am-242 (16 hour) ${ }^{[37]}$ and the total epithermal $(n, \gamma)$ cross section of Am-241 on the ENDF/B Tape 115 .

The thermal fission cross section of Am-242 (16 hour) appears to be the only reported value. The fission and $(n, \gamma)$ cross sections of Am-242 (16 hour) for the full reactor spectrum were determined by the ratio of $\sigma_{\mathrm{f}}$ for Am-242 (16 hour) to $\sigma_{f} \mathrm{Am}-242 \mathrm{M}$ (152 year), i.e., ref. the ratio 3000 barns $/ 6400$ barns, taken from the Chart of Nuclides ${ }^{[42]}$.

\subsubsection{Definition of Group Structure and Group Cross Section}

The energy range of the neutrons was split into two ranges--fast and thermal. The energy group structure in Table 9-2 is for the fast range and that in Table 9-3 is for the thermal range. This group structure is consistent with that used in the LASER code. 
Group cross sections were obtained ${ }^{*}$ for reported data using the following definition:

$$
\bar{\sigma}_{j}=\frac{\int_{j+1}^{E_{j}} \sigma(E) \phi(E) d E}{\int_{j}^{E_{j+1}} \phi(E) d E}
$$

where

$$
\begin{aligned}
& \bar{\sigma}_{j}=\text { average cross section for group } j \\
& \sigma(E)=\text { microscopic cross section in group } j \text { as a function of energy } \\
& E_{j+1}=\text { lower energy limit of } j \\
& E_{j}=\text { upper energy limit of group } j . \\
& \phi(E) \quad 1.0 \text { for isotopes labeled A in Table } 9-1 . \\
& \quad 1 / E \text { for isotopes labeled } E \text { in Table } 9-1 .
\end{aligned}
$$

* Library tapes edited from cross section files maintained by C. Beard and D. Kusner of Mathematics and Applied Programming Section, Westinghouse Nuclear Energy Systems, using the ETOM-1 code. [46] 


\subsection{DECAY CONSTANT VALUES FOR EYC PROGRAM EXTENSION}

The values of decay constants used in this study are summarized in Table 9-5. These data, and their specific sources, can be found in References 42, 43, and 44. The half-1ife for Pu-241 is from Reference 45. 
TABLE 9-5

VALUES OF DECAY CONSTANTS USED IN EYC PROGRAM EXTENSION

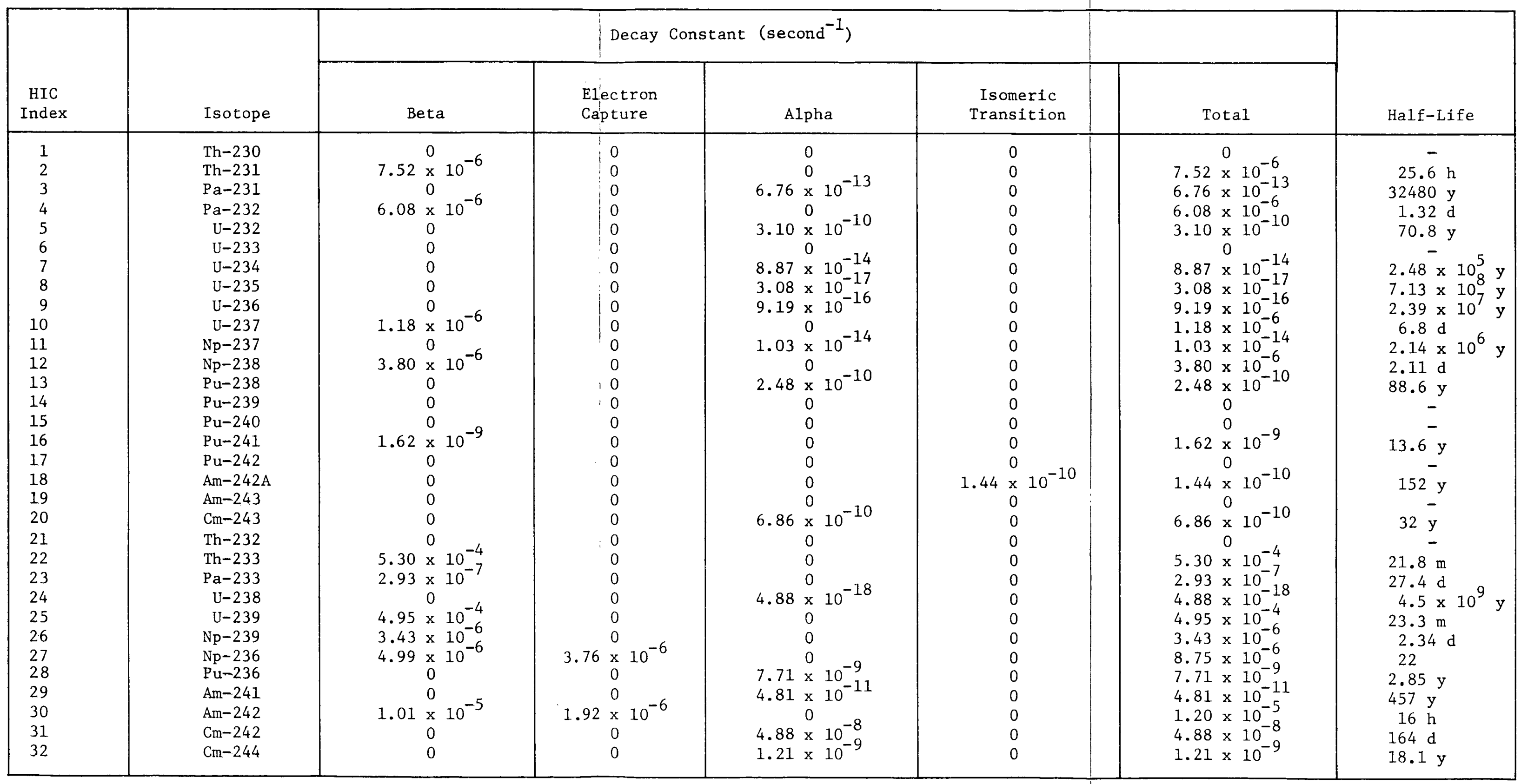


SECTION 10

DESCRIPTION OF UNIT CELL CALCULATIONS

PERFORMED IN THE EYC PROGRAM

Three basic computer codes were used for the unit cell calculations performed in the EYC Program; these are LEOPARD, ${ }^{[22]}$ LASER, and LASER-HIC*.

The LEOPARD calculations, which consider the main chain uranium and plutonium 1sotopes (U-234, $-235,-236,-238$, and $\mathrm{Pu}-239,-240,-241,-242$ ), but do not include the special isotopes (U-232, U-233, Pu-236, $\mathrm{Pu}-238, \mathrm{~Np}-237, \mathrm{Am}-241$, Am-243, Cm-242, and $\mathrm{Cm}-244)$ investigated in the EYC Program Extension, were performed in Phases 1, 2, and 3 of the program.

The LASER calculations also consider only the main chain uranium and plutonium isotopes (but not U-234), and also were performed in Phases 1, 2, and 3 .

The LASER-HIC calculations, which consider thirty isotopes from Th-23I up through $\mathrm{Cm}-244$, were performed in the Program Extension. The general objectives of the use of these codes in the EYC Program are outlined below.

The LEOPARD code was used to:

1. Provide initial number densities and microscopic and macroscopic group constants for input to one- and two-dimensional few-group diffusion-theory depletion codes; a WAPD version of the CANDLE[47] code was used to obtain an estimate of the effects of the Yankee Core IV environment on the neutron spectrum in the recycled Phase 3 fuel assembly, ${ }^{[48]}$ and the TURBO* [49] and ZIP ${ }^{[50]}$ codes

\footnotetext{
*The LASER computer program was developed in the EYC Program and is documented in References 4 and 21; the LASER-HIC computer program was developed in the EYC Program Extension and is documented in Sections 8 and 9 of this report. **A modified TURBO[51] code, known as "turbo-star" (TURBO*).
} 
were used to calculate the spatial distribution and inventory of uranium and plutonium isotopes in the Yankee Core I fue ${ }^{[11]}$.

2. Provide fuel nuclear parameters for input to the REBUF data reducing code; these include burnup-dependent values of $\alpha^{49}, \alpha^{41}$, $\delta^{28}, \mathrm{MeV} /$ fission, and $\mathrm{U}-236$ and $\mathrm{Pu}-242$ burnout correction factors.

3. Provide survey calculations for uranium and plutonium isotopic production to evaluate the relative consistency of measured data.

The LASER code was used to:

1. Analyze the burnup dependence of the moderator temperature coefficient and excess reactivity in Yankee Core I. [52]

2. Evaluate the dissimilar treatment of the thermal energy range in the LASER and LEOPARD. calculational models. [52]

3. Provide calculated values of uranium and plutonium isotopic distribution within the fuel pellet for comparison with measured data obtained for radial microsamples; calculated and measured results for the Phase 1 and Phase 2 radial microsamples are evaluated in Reference 4; calculated and measured results for the Phase 3 radial microsamples are evaluated in Reference 53, and are not included in this report.

The LASER-HIC code was used primarily to calculate the production and depletion of the special isotopes and to provide data for comparison with isotopic measurements on pellet-size samples; these comparisons form the basis for the evaluation of the calculational model (LASER-HIC) and basic nuclear data (cross sections and decay constants) used in this study. Although the LASER portion of the LASER-HIC calculations results in the radial distributions of the main chain uranium and plutonium isotopes within the pellet, as well as the integrated-average for the pellet, no comparisons have been made between these calculated radial distributions (resulting from the use of the cross section library documented in this report) and the Phase 1, 2, and 3 measurements on radial microsamples. The LASER-HIC calculations were also used to provide fuel nuclear parameters for input to the REBUF data reducing code; these include $\alpha^{49}, \alpha^{41}, \delta^{28}$, and $U-236$ and $\mathrm{Pu}-242$ burnout correction factors. 
The degree of validity which can be attached to any detailed comparisons between unit cell calculations and measurements of isotopic concentrations (i.e., evaluation of calculational models and basic nuclear data) is strongly dependent on the following three items:

1. Accurate representation of irradiation history

2. Representation of neutron spectrum

3. Initial isotopic composition

These items are discussed more fully in the following paragraphs.

\subsection{REPRESENTATION OF IRRADIATION HISTORY IN UNIT CELL CALCULATIONS}

In evaluating the calculated and measured main chain plutonium production, it is important to consider that, although each sample in a given phase was exposed to the same irradiation time, each sample was subjected to a different power density (i.e., fuel temperature) and moderator temperature during irradiation. The plutonium production is dependent upon the U-238 resonance absorption, which in turn is dependent on fuel temperature and neutron spectrum.

In the case of the special isotopes, it is even more important to accurately represent the irradiation history due to the relatively short half-lives of some of the isotopes. For the Phase 2 samples, particular attention must be paid to the four-month shutdown between Core I and Core II operation; for the Phase 3 samples, the one-year cooling time between Core II and Core IV operation* must absolutely be accounted for in the unit cell calculations if meaningful comparisons are to be made with measurements for the special isotopes in the spent fuel samples.

Since most of the Program Extension samples (upon which measurements for the special isotopes were made) were obtained from symmetrically located fuel assemblies -- core position $\mathrm{E} 5$ in Phase 1, core position $\mathrm{F} 5$ in Phase 2, and core position E6 in Phase 3 -- the LASER-HIC unit cell calculations, in terms

*The recycled Phase 3 assembly was stored in the Yankee fuel storage pit during Core III operation. 
of fuel temperature, moderator temperature, power density, boron concentration, etc., were set up similar to the LEOPARD unit cell zone calculations described in Reference 54. For purpose of reference, this information is summarized in Table 10-1. In-core flux-wire and thermocouple data, reduced with the YADAR [55] code, were used to determine the average moderator temperature and power level for each of the four axial sample zones listed in Table 10-1. Values of $\mathrm{T}_{\text {eff }}$ were computed with Sha's correlation. [56]

The present version of the LASER-HIC computer code does not allow input data (i.e., time step length, power density, fuel temperature, etc.) to be varied as the calculation proceeds from time step to time step in a single run (i.e., a single submission to the CDC-6600 computer). To make these changes, the calculation is terminated, a restart data package is punched out on cards, the required changes are made in the restart package, and the calculation is then restarted (i.e., resubmitted to the CDC-6600). Successive restarts must be interfaced with a short time step which is itself a restart. This procedure is clarified in Table 10-2, which is a summary of the time steps followed in the final LASER-HIC unit cell zone calculations.

Note that each of the four zone calculations required 10 restarts and 34 time steps including the initial Xe time step and the Sm time step. For these final calculations, this is a total of 40 restarts and 136 time steps.

The average running time on the CDC-6600 computer for the current version of the LASER-HIC program is 81 seconds CP time and 29 seconds PP time, per time step.

10.2 YANKEE CORE IV ENVIRONMENTAL EFFECT ON NEUTRON SPECTRLM Initial evaluations [48, 53] of plutonium data for Phase 3 samples were performed by plotting a11 the Phase 3 data on the same plot with Phase 1 and Phase 2 data (but not including samples from the intermediate and perturbed neutron spectra). The plutonium data were correlated with burnup according to phase, rather than according to axial sample zone. The result was two correlations, one representing plutonium production versus burnup for the Phase 1 and Phase 2 fuel, and a second representing plutonium production 
TABLE 10-1

SUMMARY OF LEOPARD UNIT CELL CALCULATIONS FOR EYC AXIAL ZONES 1, 3, 5, AND 6

\begin{tabular}{|c|c|c|c|c|c|c|c|c|c|c|c|c|c|c|c|c|}
\hline \multirow[b]{2}{*}{$\begin{array}{c}\text { Yankee } \\
\text { Core }\end{array}$} & \multirow[b]{2}{*}{$\begin{array}{c}\text { EYC } \\
\text { Phase } \\
\end{array}$} & \multirow[b]{2}{*}{$\begin{array}{l}\text { Assembly }^{(a)} \\
\text { Location }\end{array}$} & \multirow{2}{*}{$\begin{array}{l}\begin{array}{l}\text { LEOPARD } \\
\text { Run } \\
\text { Number }\end{array} \\
\end{array}$} & \multirow[b]{2}{*}{$\begin{array}{l}\text { Axial } \\
\text { Zone } \\
\end{array}$} & \multirow[b]{2}{*}{$\begin{array}{c}\text { Power (d) } \\
\text { (MWT) }\end{array}$} & \multirow{2}{*}{$\begin{array}{c}\text { Specific } \\
\text { Power } \\
\text { (kw/kg) }\end{array}$} & \multicolumn{4}{|c|}{ Temperatures $\quad\left({ }^{\circ} \mathrm{F}\right)$} & \multirow[b]{2}{*}{ e) $w / U$} & \multirow[b]{2}{*}{$\mathrm{p}^{28}$} & \multicolumn{2}{|c|}{ L-Factors } & \multirow{2}{*}{\begin{tabular}{|c|} 
Burnup at \\
Beginning \\
of \\
Leopard \\
Run \\
(GWD/MTU) \\
\end{tabular}} & \multirow[b]{2}{*}{$\phi_{1} / \phi_{2}{ }^{(f)}$} \\
\hline & & & & & & & Moderator $^{(\mathrm{d})}$ & Cladding & \begin{tabular}{|l} 
Pellet \\
Average
\end{tabular} & $\begin{array}{l}\text { Resonance } \\
\text { Effective }\end{array}$ & & & $\mathrm{U}-238$ & Pu-240 & & \\
\hline I & 1 & E5 & I & $\begin{array}{l}1 \\
3 \\
5 \\
6\end{array}$ & $\begin{array}{l}295 \\
624 \\
636 \\
402\end{array}$ & $\begin{array}{l}14.0920 \\
29.8540 \\
30.4430 \\
19.2270\end{array}$ & $\begin{array}{l}527 \\
514 \\
496 \\
489\end{array}$ & $\begin{array}{l}544 \\
549 \\
532 \\
512\end{array}$ & $\begin{array}{r}928 \\
1219 \\
1203 \\
998\end{array}$ & $\begin{array}{r}900 \\
1149 \\
1134 \\
958\end{array}$ & $\begin{array}{l}2.089 \\
2.124 \\
2.165 \\
2.178\end{array}$ & $\left|\begin{array}{l}0.7080 \\
0.7074 \\
0.7115 \\
0.7156\end{array}\right|$ & $\left|\begin{array}{l}0.8602 \\
0.8752 \\
0.8712 \\
0.8582\end{array}\right|$ & $\mid \begin{array}{l}0.8346 \\
0.8416 \\
0.8376 \\
0.8296\end{array}$ & $\begin{array}{l}0 \\
0 \\
0 \\
0\end{array}$ & $\begin{array}{l}8.99 \\
8.98 \\
8.84 \\
8.74\end{array}$ \\
\hline II & 2 & F5 & II & $\begin{array}{l}1 \\
3 \\
5 \\
6\end{array}$ & $\begin{array}{l}293 \\
693 \\
646 \\
423\end{array}$ & $\begin{array}{l}14.0250 \\
33.1280 \\
30.8800 \\
20.2140\end{array}$ & $\begin{array}{l}529 \\
514 \\
496 \\
488\end{array}$ & $\begin{array}{l}546 \\
553 \\
533 \\
512\end{array}$ & $\begin{array}{r}928 \\
1268 \\
1213 \\
1014\end{array}$ & $\begin{array}{r}900 \\
1188 \\
1141 \\
973\end{array}$ & $\begin{array}{l}2.099 \\
2.156 \\
2.198 \\
2.202\end{array}$ & $\left|\begin{array}{l}0.7082 \\
0.7084 \\
0.7130 \\
0.7165\end{array}\right|$ & $\left|\begin{array}{l}0.8612 \\
0.8790 \\
0.8730 \\
0.8598\end{array}\right|$ & $\mid \begin{array}{l}0.8350 \\
0.8430 \\
0.8379 \\
0.8300\end{array}$ & $\begin{array}{r}6.363 \\
13.480 \\
13.746 \\
8.682\end{array}$ & $\begin{array}{l}10.78 \\
11.90 \\
11.60 \\
10.69\end{array}$ \\
\hline IV & 3 & E6 & III & $\begin{array}{l}1 \\
3 \\
5 \\
6\end{array}$ & $\begin{array}{l}382 \\
509 \\
519 \\
371\end{array}$ & $\begin{array}{l}18.2970 \\
24.2910 \\
24.8310 \\
17.7600\end{array}$ & $\begin{array}{l}528 \\
513 \\
501 \\
496\end{array}$ & $\begin{array}{l}550 \\
542 \\
530 \\
517\end{array}$ & $\begin{array}{r}1017 \\
1119 \\
1118 \\
973\end{array}$ & $\begin{array}{r}980 \\
1066 \\
1064 \\
938\end{array}$ & $\begin{array}{l}2.113 \\
2.183 \\
2.210 \\
2.200\end{array}$ & $\left|\begin{array}{l}0.7078 \\
0.7118 \\
0.7143 \\
0.7162\end{array}\right|$ & $\left|\begin{array}{l}0.8671 \\
0.8718 \\
0.8696 \\
0.8592\end{array}\right|$ & $\begin{array}{l}0.8379 \\
0.8382 \\
0.8358 \\
0.8300\end{array}$ & $\begin{array}{l}11.034 \\
24.514 \\
24.032 \\
15.415\end{array}$ & $\begin{array}{l}12.16 \\
13.54 \\
13.17 \\
12.16\end{array}$ \\
\hline
\end{tabular}

Notes: (a) Assemblies E5, F5, and E6 are symmetrically located with respect to both core geometry and control rod withdrawal programs.

(b) The duration of LEOPARD Runs 1,2 , and 3 were $10,837,7,994$, and 8,088 hours, respectively. No boron was present in Runs 1 and 2; however, in Run 3, boron was included, varying from $845 \mathrm{ppm}$ at 0 hours to $0 \mathrm{ppm}$ at 5,000 hours. All unit cell calculations controlled with material buckling.

(c) Axial sample zones $1,3,5$ and 6 are located 86.4, 54.4, 22.4, and 6.4 inches above the bottom of the fuel, respectively

(d) Estimated from in-core flux wire and thermocouple measurements. The power given here is the product of the average normalized pointwise power distribution and the core average power.

(e) Obtalned with Sha's correlation - statistical weighting factor for non-uniform fuel temperature not included.

(f) Neutron flux, E> $0.625 \mathrm{eV}$ relative to neutron $\mathrm{f} 1 \mathrm{ux}, \mathrm{E}<0.625 \mathrm{ev}$. 
TABLE $10-2$

TIME STEP DESCRIPTION IN LASER-HIC ZONE CALCULATIONS

\begin{tabular}{|c|c|c|c|c|}
\hline $\begin{array}{l}\text { Time } \\
\text { Step }\end{array}$ & $\begin{array}{l}\Delta \mathrm{T} \\
\text { (Hours) }\end{array}$ & $\begin{array}{l}\text { Accum. } \\
\text { Hours } \\
\text { at End } \\
\text { of Step }\end{array}$ & $\begin{array}{l}\text { Boron } \\
\text { Conc. } \\
\text { (ppm) }\end{array}$ & Condition \\
\hline $\mathrm{Xe}$ & 100.0 & 100.0 & 0 & Core I BOL/At Power \\
\hline $\mathrm{Sm}$ & 900.0 & 1000.0 & 0 & \\
\hline 1 & 983.6 & 1983.6 & 0 & \\
\hline 2 & 983.6 & 2967.2 & 0 & \\
\hline 3 & 983.6 & 3950.8 & 0 & \\
\hline 4 & 983.6 & 4934.4 & 0 & \\
\hline 5 & 983.6 & 5918.0 & 0 & \\
\hline 6 & 983.6 & 6901.6 & 0 & \\
\hline 7 & 983.6 & 7885.2 & 0 & \\
\hline 8 & 983.6 & 8868.8 & 0 & \\
\hline 9 & 983.6 & 9852.4 & 0 & \\
\hline 10 & 983.6 & 10836.0 & 0 & \\
\hline 11 & 1.0 & 10837.0 & 0 & Core I EOL/Restart 1/Zero Power \\
\hline 12 & 3095.0 & 13932.0 & 0 & Core I-II SD/Restart 2/Zero Power \\
\hline 13 & 1.0 & 13933.0 & 0 & Core II BOL/Restart 3/At Power \\
\hline 14 & 999.125 & 14932.125 & 0 & Core II/Restart 4/At Power \\
\hline 15 & 999.125 & 15931.25 & 0 & \\
\hline 16 & 999.125 & 16930.375 & 0 & \\
\hline 17 & 999.125 & 17929.5 & 0 & \\
\hline 18 & 999.125 & 18928.625 & 0 & \\
\hline 19 & 999.125 & 19927.75 & 0 & \\
\hline 20 & 999.125 & 20926.875 & 0 & \\
\hline 21 & 999.125 & 21926.0 & 0 & \\
\hline 22 & 1.0 & 21927.0 & 0 & Core II EOL/Restart $5 /$ Zero Power \\
\hline 23 & 8879.0 & 30806.0 & 0 & Core II-IV Cooling/Restart 6/Zero Power \\
\hline 24 & 1.0 & 30807.0 & 835 & Core IV BOL/Restart 7/At Power \\
\hline 25 & 1011.0 & 31818.0 & 707 & Core IV/Restart 8/At Power \\
\hline 26 & 1011.0 & 32829.0 & 707 & \\
\hline 27 & 1011.0 & 33840.0 & 337 & Core IV/Restart 9/At Power \\
\hline 28 & 1011.0 & 34851.0 & 337 & \\
\hline 29 & 1011.0 & 35862.0 & 0 & Core IV/Restart 10/At Power \\
\hline 30 & 1011.0 & 36873.0 & 0 & \\
\hline 31 & 1011.0 & 37884.0 & 0 & \\
\hline 32 & 1011.0 & 38895.0 & 0 & \\
\hline
\end{tabular}


versus burnup for the Phase 3 fuel. Each correlation included samples from different core positions; as a consequence, Zone 1 samples, which were subjected to relatively low fuel temperatures and relatively high moderator temperatures, Zones 3 and 5 samples, which were subjected to relatively high fuel temperatures and about average moderator temperatures, and Zone 6 samples, which were subjected to relatively low fuel temperatures and relatively low moderator temperatures, were all included in the same correlation.

The initial conclusion drawn from these preliminary evaluations was that the plutonium production in the recycled Phase 3 fuel was some 8 to 10 percent lower than the plutonium production in the Phase 1 and Phase 2 fuel, and at first, all of this offset was attributed to a Core IV environmental effect on the neutron spectrum in the recycled Phase 3 fuel assembly. During Core IV operation, the recycled Phase 3 fuel assembly (highly depleted 3.4 w/o fuel) was surrounded by assemblies containing moderately depleted 3.4 w/o and $4.1 \mathrm{w} / \mathrm{o}$ fuel. (The fuel cycling performed through the first four Yankee cores is shown on Figure 2-2.) Subsequent evaluations revealed that approximately three-quarters of the offset in plutonium production was due to the manner in which the initial comparisons were made (i.e., including in the same correlation data obtained from samples which were exposed to different temperatures), and approximately one-quarter of the offset was due to the Core IV environmental effect on the neutron spectrum in the Phase 3 fuel (i.e., softening of the spectrum). In other words, about three-quarters of the offset is apparent, and about one-quarter is real.

The significance of the offset in plutonium production in the Phases 1 and 2 fuel and the plutonium production in the Phase 3 fuel is obvious; instead of a single asymptotic neutron spectrum which could be represented by a single unit cell calculation, there are in fact many "asymptotic" neutron spectra, corresponding to the different zone positions and different phases (i.e, irradiation histories).

To make valid comparisons between theory and experiment, the different asymptotic neutron spectra corresponding to different zone positions were accounted for to some extent by performing several zone calculations as described in Subsection 10.1 . 
There is no way that a unit cell calculation (zero-dimensional) can be made to include the Core IV enviornmental effect on the neutron spectrum in the recycled Phase 3 fuel. However, the degree of the effect on plutonium production can, and was, evaluated by one-dimensional calculations as described below.

One-dimensional calculations were performed with a WAPD version of the CANDLE Code, [47] to obtain an estimate of the effects of the Yankee Core IV environment on the neutron spectrum in the recycled Phase 3 assembly. Two sets of calculations were performed. The first considered a batch-loaded core with 76 assemblies of $3.4 \mathrm{w} / 0$ fuel. In the calculation, the fuel was allowed to deplete from 0 to 25,060 MWD/MTU burnup, which is the sum of the core average burnups of Yankee Core I (8470 MWD/MTU), Core II (7870 MWD/MTU), and Core IV (8720 MWD/MTU). The second set of calculations also started with 76 assemblies of $3.4 \mathrm{w} / \mathrm{o}$ fuel, but after the first $8470 \mathrm{MWD} / \mathrm{MTU}$ of burnup, followed the actual fuel cycling shown on Figure 2-2 as closely as one-dimensional cylindrical geometry will permit. The LEOPARD ${ }^{[22]}$ code was used to generate fast and thermal microscopic cross section libraries at burnups of 2000, 16,340, and 25,000 MWD/MTU. These libraries were used as a function of burnup in both sets (batchloaded and cycled) of calculations.

The fast-to-thermal flux ratio* at the center of the Phase 3 assembly, as obtained with the one-dimensional calculations described above, is shown on Figure 10-1. The fast-to-thermal flux ratio corresponding to the Phase 1 and Phase 2 conditions are nearly identical in the batch-loaded and cycled cases; however, the Phase 3 results, indicated at the extreme right on Figure 10-1, clearly demonstrate that a softening of the neutron spectrum has occurred in the recycled assembly due to the Core IV environment. The results of the decrease in the fast-to-thermal flux ratio in the recycled assembly are shown in Figure 10-2, on which the calculated Pu-239 production is plotted. Notice that the set of calculations which approximate the fuel cycling result in a relatively lower plutonium production in the Phase 3 assembly.

\footnotetext{
*Ratio of the flux in the energy range $0.625 \mathrm{eV}<\mathrm{E}<10 \mathrm{MeV}$ to the flux in the energy range $0<E<0.625 \mathrm{eV}$.
} 


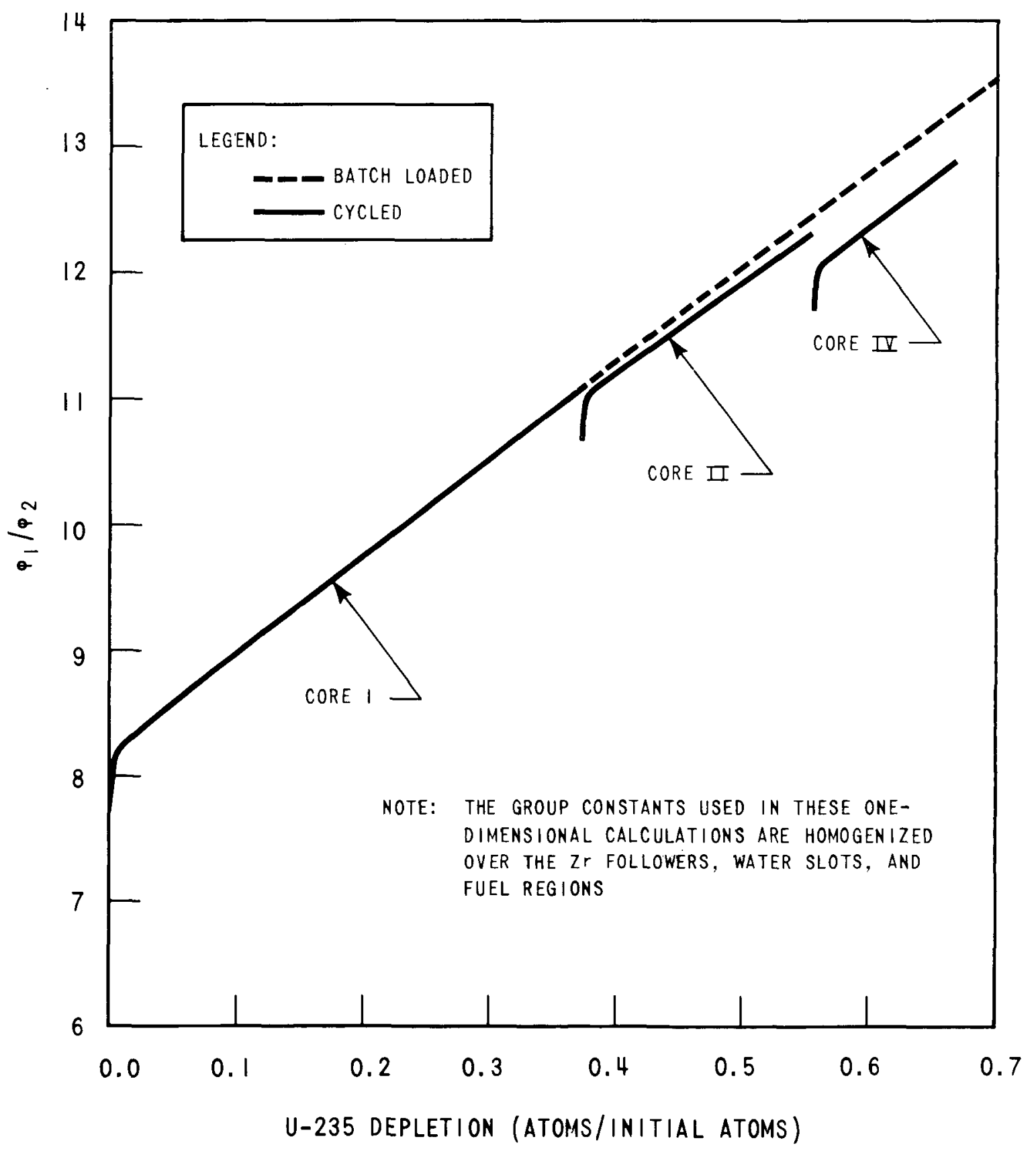

Figure 10-1. Calculated Fast-to-Thermal Flux Ratio versus U-235 Depletion in the Yankee

Recycled Fuel Assembly 


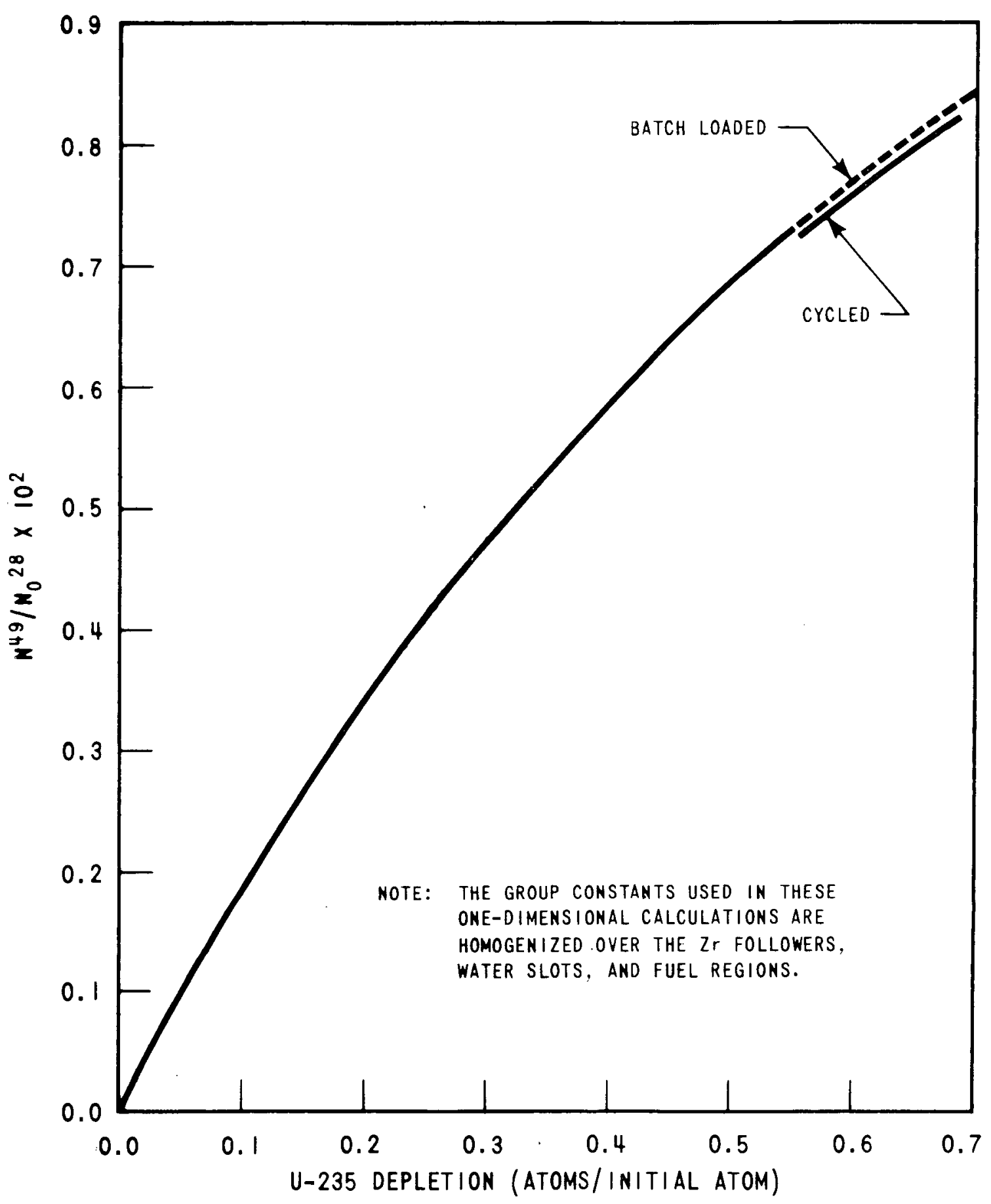

Figure 10-2. Calculated Pu-239 Atom Density versus U-235 Depletion in Yankee Cores I, II, and IV 
Based on these one-dimensional calculations, it is concluded that the Core IV environmental effect on the neutron spectrum results in about $1-1 / 2$ percent decrease in plutonium production in the Phase 3 fuel. Thus, any conclusions made regarding the validity of the LASER-HIC calculational model and the accuracy of the basic nuclear data used in the calculations, and based on the comparisons between theory and experiment in Section 11, must be tempered by the fact that the Phase 3 samples were exposed to a somewhat different neutron spectrum (i.e., softer) than that calculated by the LASER-HIC unit cell computer program. As will be shown in Section 11, the final LASER-HIC zone calculations tend to overpredict the plutonium production in the Phase 3 fuel. 


\subsection{INITIAL ISOTOPIC COMPOSITION OF YANKEE CORE I FUEL}

Accurate knowledge of the initial isotopic composition of the pre-irradiated fuel is absolutely necessary if a meaningful comparison is to be made between theory and experiment. Values used in this program are based on measurements made on unirradiated fuel believed to be representative of Yankee Core I fuel in the case of the initial concentrations of the main chain uranium isotopes. The Yankee Core I fuel pellets were obtained from two vendors: Mallinckrodt and the Westinghouse Atomic Fuel Department (WAFD). The initial composition of the fuel supplied by the two vendors is given on page 15 in Reference 13, and is repeated in Table 10-3.

TABLE $10-3$

CHEMICAL COMPOSITION OF UO ${ }_{2}$ PELLETS IN YANKEE CORE I

\begin{tabular}{|c|c|c|c|}
\hline & Mnalysis & Walinckrodt & Weighted Average \\
\hline U-235/U (weight \%) & 3.391 & 3.406 & 3.399 \\
Total U (weight \%) & 88.07 & 88.05 & 88.06 \\
O/U (atomic ratio) & 1.996 & 1.98 & 1.988 \\
& & & \\
\hline
\end{tabular}

a. The weighted average is based on an equal mixture of the respective loadings of the two vendors' pellets.

In addition, shipping records indicate an average enrichment of $3.4053 \pm 0.0040$ w/o of U-235 in uranium, where the two-standard deviations of the mean was calculated from the analysis of 72 tanks of $\mathrm{UF}_{6}$.

Analyses for uranium initial isotopic composition also were made on samples of some pellets and on samples of $\mathrm{U}_{3} \mathrm{O}_{8}$, believed to be representative of Yankee fuel. Results of these analyses, along with the U-235 data from 
Table 10-3, are summarized in Table 10-4. The values for U-234, U-235, U-236, and $U-238$ are consistent with values obtained by least squares fitting the results from mass spectrometric analysis on many irradiated fuel samples, and extrapolating back to zero burnup.

No plutonium was detectable in the unirradiated samples.

Measurements for initial concentrations of the special isotopes U-232 and $\mathrm{U}-233$, and their precursors $\mathrm{Pa}-231$ and $\mathrm{Th}-232$, were made on the $\mathrm{U}_{3} \mathrm{O}_{8}$ samples by the Technical Services Laboratories of Westinghouse Advanced Reactors Division (WARD).

Chemical spectrographic measurements for thorium impurities in the unirradiated sample are summarized in Table 10-5.

Alpha spectrographic analysis for $\mathrm{Pa}-231$ impurity in the unirradiated sample on January 24, 1969 gave an upper limit of $3.5 \times 10^{-9}$ atoms of $\mathrm{Pa}-231$ relative to U-235 atoms.* A calcualation of the $\mathrm{Pa}-231 / \mathrm{U}-235$ atom ratio, assuming 622 days elapsed time from $\mathrm{UF}_{6}$ delivery in 1959 to the Yankee Core I beginningof-life (at power January 16,1961), results in a value of $1.6 \times 10^{-9}$. However, further calculations to decay correct the $1.6 \times 10^{-9}$ value to the time of the alpha spectrographic analysis, January 24, 1969, gives a value of $9.4 \times 10^{-9}$ for the $\mathrm{Pa}-231 / \mathrm{U}-235$ atom ratio. The inconsistency between the calculated value (based on $\alpha$-decay of $\mathrm{U}-235$ and $\mathrm{Pa}-231$, and $\beta$-decay of $\mathrm{Th}-231$ ) and the measured upper limit, both referenced to January 24, 1969, is not surprising in view of the difficulty of sample preparation and measurement. The ca1culated value of $1.6 \times 10^{-9}$ for the initial $\mathrm{Pa-231/U-235}$ ratio is used in this study.

Results of alpha spectrographic measurements for U-232/U-235 atom ratio in the unirradiated $\mathrm{U}_{3} \mathrm{O}_{8}$ sample on October 16, 1968 are given in Table 10-6.

*Analysis run in duplicate using a $100 \mathrm{mg} \mathrm{U}_{3} \mathrm{O}_{8}$ sample. 
TABLE $10-4$

MEASURED URANIUM ISOTOPIC COMPOSITION OF YANKEE CORE I PRE-IRRADIATED FUEL

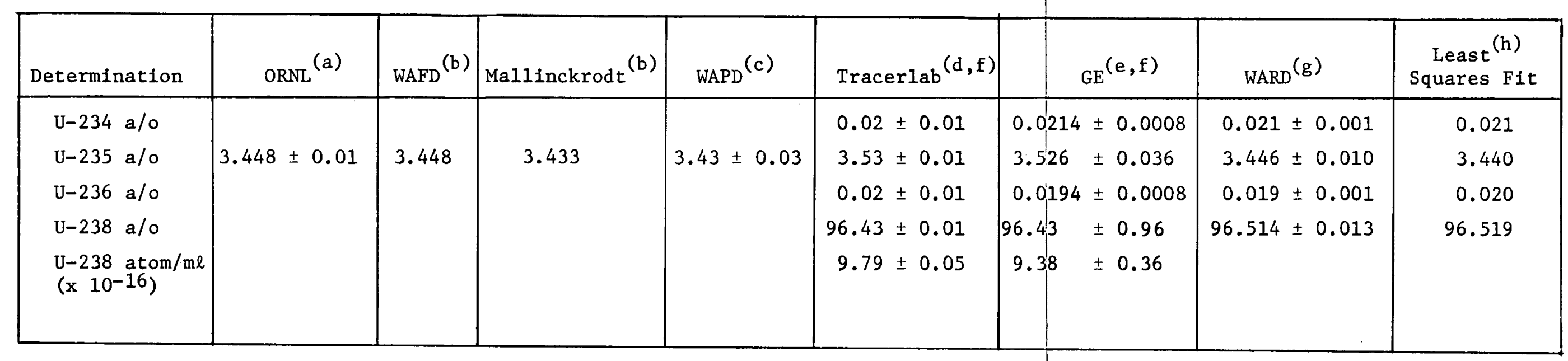

a. Average ORNL gas analysis of unirradiated material, Reference 15.

b. Chemical analysis by vendor, Reference 13 .

c. Optical emission spectrographic analysis on a sample of the uranium dioxide

fuel employed in fabrication of Yankee Core I, Reference 15.

d. Mass spectrometric analysis on a cross check sample of uranium dioxide, analyzed May 1963.

e. Mass spectrometric analysis on a cross check sample of uranium dioxide, analyzed July 1963.

f. It is strongly suspected that the unirradiated fuel pellet (or posstbly each of the sample solutions made from it) was contaminated prior to analysis.

g. Mass spectrometric analysis on sample of $\mathrm{U}_{3} \mathrm{O}_{8}$, analyzed November 1968.

h. Estimated from least squares fit of $\mathrm{N}^{1} / \mathrm{N}_{\mathrm{O}} 28$ versus $\mathrm{Ln}\left(\mathrm{N}_{\mathrm{o}} 25 / \mathrm{N} 25\right)$ as inferred from EYC Phase 1 and Phase 2 mass spectrometric analysis of irradiated fuel samples. 
TABLE $10-5$

MEASURED CONCENTRATION OF THORIUM IMPURITY

IN YANKEE CORE I UNIRRADIATED FUEL

\begin{tabular}{|c|c|c|c|}
\hline $\operatorname{Run}(a)$ & Analysis & \multicolumn{2}{|c|}{$\begin{array}{l}\text { Thorium Concentration } \\
\text { (ppm, relative to } \mathrm{UO}_{2} \text { ) }\end{array}$} \\
\hline \multirow[t]{6}{*}{1} & 1 & & 0.31 \\
\hline & 2 & & 0.31 \\
\hline & 3 & & 0.27 \\
\hline & 4 & & 0.35 \\
\hline & 5 & & 0.31 \\
\hline & 6 & & 0.35 \\
\hline \multirow[t]{3}{*}{2} & 7 & & 0.40 \\
\hline & 8 & & 0.40 \\
\hline & & Weighted average & 0.35 \\
\hline \multicolumn{4}{|c|}{$\mathrm{N}^{02} / \mathrm{N}^{28} \approx\left(0.35 \times 10^{-6} \times 270\right) /(0.96519 \times 232)=0.42 \times 10^{-6}$} \\
\hline
\end{tabular}

a. Run 1 analysis on 3-gram samples of $\mathrm{U}_{3} \mathrm{O}_{8}$.

Run 2 analysis on 6-gram samples of $\mathrm{U}_{3} \mathrm{O}_{8}$.

b. Relative uncertainty is \pm 30 percent. Analyses

performed during April 1966. 
TABLE $\quad 10-6$

MEASURED U-232 CONCENTRATION IN

YANKEE CORE I UNIRRADIATED FUEL

\begin{tabular}{|l|l|}
\hline \multirow{2}{*}{ Analysis } & $\begin{array}{c}\text { U-232/U-235 } \\
\text { Atom Ratio }( \pm 2 \sigma)\end{array}$ \\
\hline 1 & $0.9 \pm 0.6 \times 10^{-9}$ \\
2 & $1.4 \pm 1.1 \times 10^{-9}$ \\
3 & $0.6 \pm 0.6 \times 10^{-9}$ \\
\hline
\end{tabular}

Weighted Average $\mathrm{N} 22 / \mathrm{N} 25=0.989 \times 10^{-9}$ on October 16, 1968. $\mathrm{N}^{22} / \mathrm{N}^{25}=1.06 \times 10^{-9}$, at Yankee Core I BOL (January 16, 1961). $\mathrm{N}^{22} / \mathrm{N}^{28}=3.8 \times 10^{-11}$, at Yankee Core I BOL.

TABLE $10-7$

SUMMARY OF YANKEE CORE I FUEL BEGINNING-OF-LIFE ISOTOPIC COMPOSITION

\begin{tabular}{|c|l|c|c|c|}
\hline Isotope $i$ & $\begin{array}{l}\text { Weight } \\
\text { Percent }\end{array}$ & $\begin{array}{c}\text { Atom } \\
\text { Percent }\end{array}$ & $\mathrm{N}^{\mathrm{i}} / \mathrm{N}^{28}$ & $\mathrm{~N}^{\mathrm{i}} / \mathrm{N}^{25}$ \\
\hline Th-232 & $4.0 \times 10^{-5}$ & $4.0 \times 10^{-5}$ & $4.2 \times 10^{-7}$ & $1.2 \times 10^{-5}$ \\
$\mathrm{~Pa}-231$ & $5.5 \times 10^{-9}$ & $5.7 \times 10^{-9}$ & $5.9 \times 10^{-11}$ & $1.6 \times 10^{-9}$ \\
$\mathrm{U}-232$ & $3.6 \times 10^{-9}$ & $3.7 \times 10^{-9}$ & $3.8 \times 10^{-11}$ & $1.1 \times 10^{-9}$ \\
$\mathrm{U}-233$ & $9.0 \times 10^{-5}$ & $9.2 \times 10^{-5}$ & $9.5 \times 10^{-7}$ & $2.7 \times 10^{-5}$ \\
$\mathrm{U}-234$ & 0.021 & 0.021 & 0.00022 & 0.0061 \\
$\mathrm{U}-235$ & 3.40 & 3.44 & 0.0356 & 1.0 \\
$\mathrm{U}-236$ & 0.020 & 0.020 & 0.00021 & 0.0058 \\
$\mathrm{U}-238$ & 96.560 & 96.519 & 1.0 & 28.048 \\
Total & 100.00 & 100.00 & 1.03603 & 29.060 \\
\hline
\end{tabular}


Two analyses on November 13, 1968 for the U-233 abundance in the unirradiated

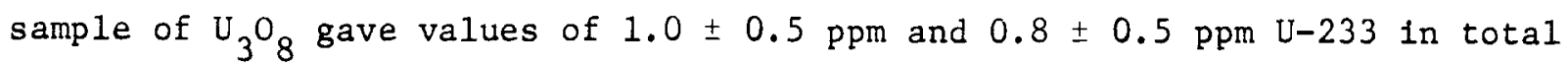
uranium. An average value of $0.9 \mathrm{ppm} \mathrm{U-233}$ in uranium is used in this program. The initial ratio of $\mathrm{U}-233$ to $\mathrm{U}-238$ is:

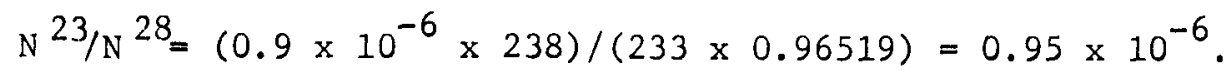

The beginning-of-life isotopic abundances, used to initialize LASER-HIC unit cell calculations are summarized in Table 10-7. 


\subsection{CROSS SECTIONS USED IN LASER-HIC CALCULATIONS}

In addition to the four LASER-HIC zone calculations described in Subsection 10.1, a flat-out calculation (i.e., fourteen at-power time steps with no shutdowns and no restarts) was performed. The calculated isotopic concentrations $\left(\mathrm{N}^{i} / \mathrm{N}_{0}^{28}\right)$ are shown in Figure $10-3$ as a function of total accumulated fissions $\left(\mathrm{F}^{\mathrm{i}} / \mathrm{N}_{\mathrm{O}}^{28}\right)$. (This calculation corresponds to the Zone 3 axial sample zone, and is one run of fourteen time steps, 2000 hours per time step, and no boron.) The purpose of this figure is to provide a quick reference for estimating the abundance of a particular isotope, relative to the others. The values of $\mathrm{F}^{\mathrm{i}} / \mathrm{N}_{\mathrm{o}}^{28}$ can be related to burnup on Figure $6-6$.

The $2200 \mathrm{mps}$ cross sections $(E=0.0253 \mathrm{eV})$ used in the LASER-HIC calculations are listed in Table 10-8 for reference. The source of these values is given in Section 9.

The fast and thermal cross sections for the reactor spectrum at 0 and 30,800 MWD/MTU are 1isted in Tables $10-9$ and 10-10, respectively, for the few-group energy structure with the thermal energy breakpoint at $1.855 \mathrm{eV}$; corresponding cross section data for the few-group energy structure with the thermal energy breakpoint at $0.625 \mathrm{eV}$ are listed in Tables $10-11$ and 10-12. These cross section values are edited in the LASER-HIC calculation. It is pointed out that the LASER calculational model uses a thermal energy breakpoint of $1.855 \mathrm{eV}$ to better represent the Pu-240 1-eV resonance, while the LEOPARD calculational model uses the historical MUFT thermal cutoff value of $0.625 \mathrm{eV}$. The calculated data on Figure 10-3 and Tables 10-9 through 10-12 can be used to estimate the reactivity effects of the special isotopes. An example is presented in the following paragraphs.

\subsection{ESTIMATE OF REACTIVITY EFFECT DUE TO BUILDUP OF SPECIAL ISOTOPES}

Many unit cell codes and diffusion-theory depletion codes do not ordinarily include the calculation of the special isotopes (e.g., Np-237, Pu-238, Am-241). Although the objectives of this program do not include the evaluation of the reactivity effects due to the buildup of the special isotopes, it is of interest to determine which of the special isotopes contribute significantly 


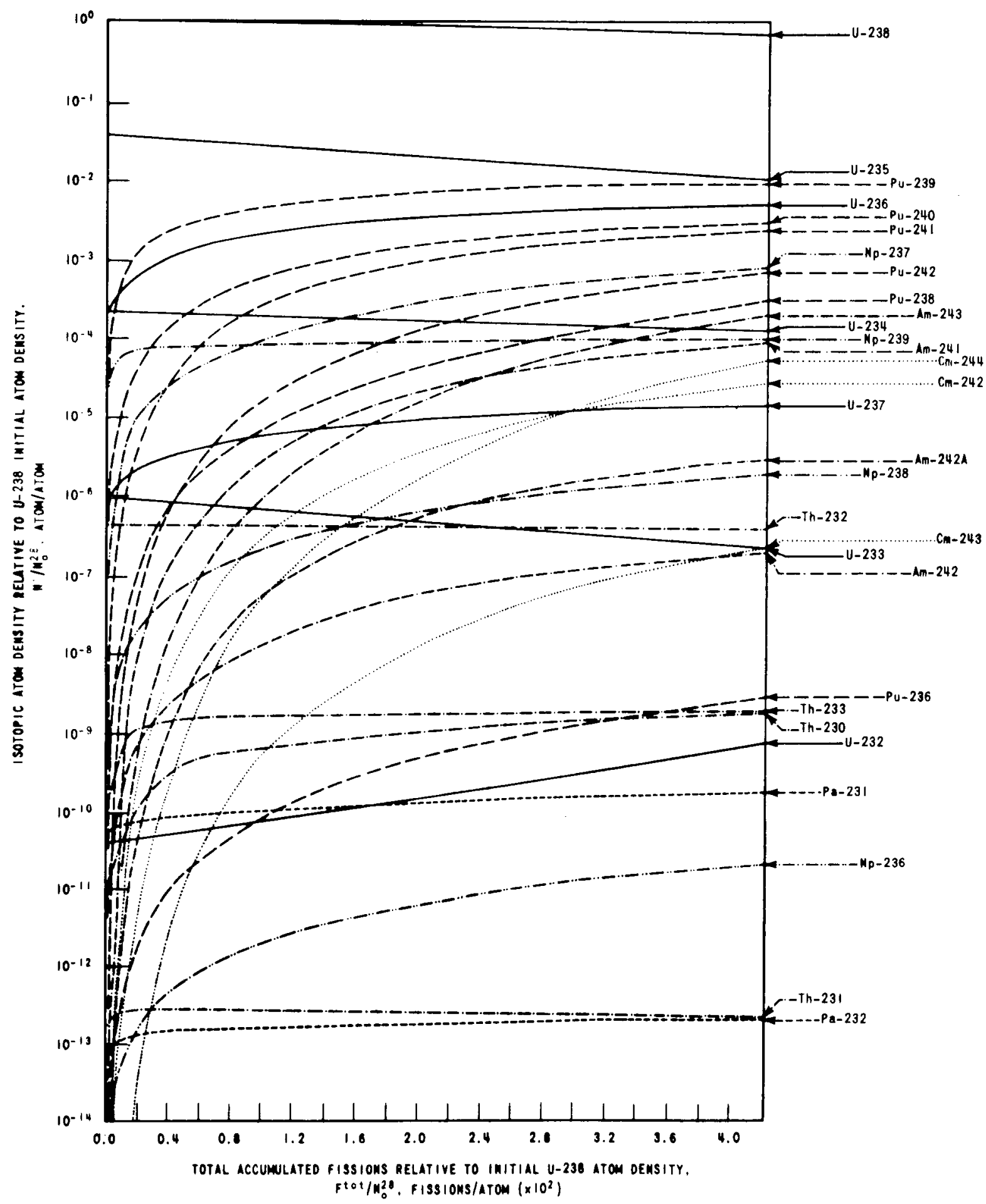

Figure 10-3. Calculated Concentrations of the Isotopes of the Elements Thorium Through Curium versus Accumulated Fissions in Yankee Core I Fuel 
TABLE $10-8$

THERMAL MICROSCOPIC CROSS SECTIONS USED IN LASER-HIC CALCULATIONS (2200 mps) (a)

\begin{tabular}{|c|c|c|c|c|}
\hline \multirow[b]{2}{*}{ Isotope } & \multirow{2}{*}{$\begin{array}{l}\text { HIC } \\
\text { Index }\end{array}$} & \multicolumn{3}{|c|}{ Microscopic Cross Section (barns) } \\
\hline & & $\sigma_{\gamma}$ & $\sigma_{\mathrm{f}}$ & $\sigma_{\mathrm{a}}$ \\
\hline Th -230 & 1 & 23. & 0 & 23. \\
\hline Th-231 & 2 & 1500 . & 0 & 1500. \\
\hline Th-232 & 21 & 7.31 & 0 & 7.31 \\
\hline Th-233 & 22 & 1500. & 14. & 1514 \\
\hline $\mathrm{Pa}-231$ & 3 & 200. & 0.010 & 200 . \\
\hline $\mathrm{Pa}-232$ & 4 & 760. & 700 & 1460. \\
\hline $\mathrm{Pa}-233$ & 23 & 40.2 & 0 & 40.2 \\
\hline $\mathrm{U}-232$ & 5 & 78.0 & 80.0 & 158. \\
\hline$U-233$ & 6 & 47.4 & 525. & 572. \\
\hline $\mathrm{U}-234$ & 7 & 76.2 & 0 & 76.2 \\
\hline $\mathrm{U}-235$ & 8 & 99. & 580. & 679. \\
\hline $\mathrm{U}-236$ & 9 & 6.10 & 0 & 6.10 \\
\hline $\mathrm{U}-237$ & 10 & 99.8 & 0 & 99.8 \\
\hline $\mathrm{U}-238$ & 24 & 2.73 & 0 & 2.73 \\
\hline $\mathrm{U}-239$ & 25 & 22. & 14. & 36. \\
\hline$N p-236$ & 27 & 40. & 0 & 40. \\
\hline $\mathrm{Np}-237$ & 11 & 169. & 0.0190 & 169. \\
\hline $\mathrm{Np}-238$ & 12 & 40 & 1735 . & 1775 . \\
\hline$N p-239$ & 26 & 30.6 & 2.0 & 32.6 \\
\hline$P u-236$ & 28 & 500. & 170. & 670. \\
\hline $\mathrm{Pu}-238$ & 13 & 546. & 16.3 & 562. \\
\hline$P u-239$ & 14 & 286 . & 742 & 1028 \\
\hline $\mathrm{Pu}-240$ & 15 & 276. & 0.0523 & 276 \\
\hline $\mathrm{Pu}-241$ & 16 & 361. & 1011. & 1372 . \\
\hline$P u-242$ & 17 & 30.0 & 0 & 30.0 \\
\hline
\end{tabular}

a. LASER-HIC thermal energy group 5, $E=0.0253 \mathrm{eV}$. 
TABLE 10-8 (Cont)

THERMAL MICROSCOPIC CROSS SECTIONS USED IN LASER-HIC CALCULATIONS (2200 mps) (a)

\begin{tabular}{|l|c|c|c|c|}
\hline \multirow{2}{*}{ Isotope } & \multirow{2}{*}{$\begin{array}{l}\text { HIC } \\
\text { Index }\end{array}$} & \multicolumn{2}{|c|}{ Microscopic Cross Section (barns) } \\
\cline { 2 - 5 } & & $\sigma_{\gamma}$ & $\sigma_{f}$ & $\sigma_{a}$ \\
\hline Am-241 & 29 & 487. & 3.27 & 490. \\
Am-242 & 30 & 750. & 3000. & 3750. \\
Am-242A & 18 & 1600. & 6400. & 8000. \\
Am-243 & 19 & 179. & 0 & 179. \\
& 31 & 20.0 & 0 & 20.0 \\
Cm-242 & 20 & 154. & 154. & 308. \\
Cm-243 & 32 & 25.2 & 1.67 & 26.9 \\
& & & & \\
\hline
\end{tabular}


TABLE 10-9

FEW-GROUP MICROSCOPIC CROSS SECTIONS FOR YANKEE REACTOR SPECTRUM AT ZERO BURNUP (THERMAL BREAKPOINT AT $1.855 \mathrm{eV}$ )

\begin{tabular}{|c|c|c|c|c|c|c|c|c|}
\hline \multirow[b]{2}{*}{ Isotope } & \multirow{2}{*}{$\begin{array}{l}\text { HIC } \\
\text { Index }\end{array}$} & \multirow{2}{*}{$\begin{array}{l}\text { Atom } \\
\text { Density } \\
\text { in Fuel } \\
(a / b-c m)\end{array}$} & \multicolumn{3}{|c|}{$\begin{array}{c}\text { Fast Cross Section, barns } \\
(\mathrm{E}>1.855 \mathrm{eV})\end{array}$} & \multicolumn{3}{|c|}{$\begin{array}{c}\text { Thermal Cross Section, barns } \\
(\mathrm{E}<1.855 \mathrm{eV})\end{array}$} \\
\hline & & & $\sigma_{Y}$ & $\sigma_{2 n}$ & $\sigma_{f}$ & $\sigma_{\gamma}$ & $\sigma_{2 \mathrm{n}}$ & $\sigma_{f}$ \\
\hline Th-230 & 1 & 0 & 0.165 & 0.00304 & 0 & 9.86 & 0 & 0 \\
\hline Th-231 & 2 & 0 & 10.8 & 0.00472 & 0 & 643. & 0 & 0 \\
\hline Th-232 & 21 & $9.0628(E-09)$ & 3.15 & 0.00440 & 0.0237 & 3.04 & 0 & 0 \\
\hline Th-233 & 22 & (b) & 10.8 & 0.0102 & 0.100 & 643. & 0 & 5.99 \\
\hline $\mathrm{Pa}-231$ & 3 & $1.2731(E-12)$ & 4.49 & 0.00454 & 0.410 & 255. & 0 & 0.0127 \\
\hline $\mathrm{Pa}-232$ & 4 & 0 & 5.46 & 0.00612 & 5.01 & 326. & 0 & 300 \\
\hline $\mathrm{Pa}-233$ & 23 & 0 & 28.4 & 0.00423 & 0.103 & 102 & 0 & 0 \\
\hline $\mathrm{U}-232$ & 5 & $8.1996(\mathrm{E}-13)$ & 4.60 & 0.00335 & 12.1 & 33.4 & 0 & 34.3 \\
\hline $\mathrm{U}-233$ & 6 & $2.0499(E-08)$ & 3.01 & 0.00272 & 15.3 & 27.1 & 0 & 257. \\
\hline $\mathrm{U}-234$ & 7 & 4.6948(E-06) & 15.5 & 0.000427 & 0.485 & 28.1 & 0 & 0 \\
\hline $\mathrm{U}-235$ & 8 & $7.6931(\mathrm{E}-04)$ & 4.00 & 0.00321 & 7.43 & 38.2 & 0 & 218. \\
\hline $\mathrm{U}-236$ & 9 & $4.4703(E-06)$ & 8.42 & 0.00256 & 0.204 & 2.54 & 0 & 0 \\
\hline$U-237$ & 10 & 0 & 0.716 & 0.000658 & 0 & 42.8 & 0 & 0 \\
\hline $\mathrm{U}-238$ & 24 & $2.1578(E-02)$ & 0.842 & 0.00485 & 0.104 & 1.16 & 0 & 0 \\
\hline $\mathrm{U}-239$ & 25 & (b) & 0.474 & 0.00104 & 0.218 & $9: 46$ & 0 & 6.00 \\
\hline $\mathrm{Np}-236$ & 27 & 0 & 0.287 & 0.00106 & 0 & 17.2 & 0 & 0 \\
\hline$N p-237$ & 11 & 0 & 12.9 & 0.000559 & 0.587 & 120. & 0 & 0.00596 \\
\hline$N p-238$ & 12 & 0 & 0.287 & 0.000238 & 13.8 & 17.2 & 0 & 752 \\
\hline $\mathrm{Np}-239$ & 26 & 0 & 0.236 & 0.000238 & 0.720 & 13.2 & 0 & 0.858 \\
\hline $\mathrm{Pu}-236$ & 28 & 0 & 3.59 & 0.00323 & 1.22 & 214. & 0 & 72.9 \\
\hline $\mathrm{Pu}-238$ & 13 & 0 & 4.42 & 0.000158 & 1.82 & 161. & 0 & 4.76 \\
\hline Pu-239 & 14 & 0 & 5.56 & 0.000681 & 10.7 & 388. & 0 & 644. \\
\hline $\mathrm{Pu}-240$ & 15 & 0 & 6.06 & 0.00140 & 0.577 & 1576 . & 0 & 0.299 \\
\hline $\mathrm{Pu}-241$ & 16 & 0 & 3.83 & 0.00619 & 17.9 & 231. & 0 & 601. \\
\hline $\mathrm{Pu}-242$ & 17 & 0 & 31.5 & 0.00190 & 0.486 & 14.5 & 0 & 0 \\
\hline$A m-241$ & 29 & 0 & $16.2^{(\mathrm{c})}$ & $3.14^{(\mathrm{d})}$ & 0.813 & $588 .^{(c)}$ & $114 .^{(d)}$ & 4.38 \\
\hline $\mathrm{Am}-242$ & 30 & 0 & 5.39 & 0.00380 & 21.6 & 322. & 0 & 1287. \\
\hline$A m-242 A$ & 18 & 0 & 11.5 & 0.00810 & 46.0 & 686. & 0 & 2745 . \\
\hline Am-243 & 19 & 0 & 8.96 & 0.00118 & 0.373 & 213. & 0 & 0 \\
\hline $\mathrm{Cm}-242$ & 31 & 0 & 0.143 & 0.00113 & 0 & 8.56 & 0 & 0 \\
\hline $\mathrm{Cm}-243$ & 20 & 0 & 1.89 & 0 & 1.89 & 73.6 & 0 & 73.6 \\
\hline $\mathrm{Cm}-244$ & 32 & 0 & 13.8 & 0.000434 & 1.16 & 10.5 & 0 & 0.665 \\
\hline
\end{tabular}

Notes: (a) Unit cell averaged number densities can be obtained by multiplying these values by the fuel volume fraction.

(b) By-passed in LASER-HIC calculational model of transmutation chains.

(c) Value for $(\mathrm{h}, \gamma)$ reaction which leads to production of Am-242 (16h).

Value for $(n, \gamma)$ reaction which leads to production of Am-242A (152y). HIC is programed so that ( $n, 2 n$ ) reactions in Am-241 result in the formation of Am-242A, instead of Am-240. 
TABLE $10-10$

FEW-GROUP MICROSCOPIC CROSS SECTIONS FOR YANKEE REACTOR SPECTRUM AT 30,800 MWD/MTU (THERMAL/BREAKPOINT AT 1.855 eV)

\begin{tabular}{|c|c|c|c|c|c|c|c|c|}
\hline \multirow[b]{2}{*}{ Isotope } & \multirow{2}{*}{$\begin{array}{l}\text { HIC } \\
\text { Index }\end{array}$} & \multirow{2}{*}{$\begin{array}{l}\text { Atom }(a) \\
\text { Density } \\
\text { in Fue1 } \\
(a / b-c m)\end{array}$} & \multicolumn{3}{|c|}{$\begin{array}{l}\text { Fast Cross Section, barns } \\
\quad(E>1.855 \mathrm{eV})\end{array}$} & \multicolumn{3}{|c|}{$\begin{array}{l}\text { Thermal Cross Section, barns } \\
(E<1.855 \mathrm{eV})\end{array}$} \\
\hline & & & $\sigma_{\gamma}$ & $\sigma_{2 \mathrm{n}}$ & $\sigma_{f}$ & $\sigma_{\gamma}$ & $\sigma_{2 \mathrm{n}}$ & $\sigma_{\mathrm{f}}$ \\
\hline Th-230 & 1 & 2.9142 (E-11) & 0.157 & 0.00309 & 0 & 9.46 & 0 & 0 \\
\hline Th-231 & 2 & $4.6701(\mathrm{E}-15)$ & 10.2 & 0.00479 & 0 & 617 & 0 & 0 \\
\hline Th-232 & 21 & $8.2523(\mathrm{E}-09)$ & 3.07 & 0.00448 & 0.0240 & 2.89 & 0 & 0 \\
\hline Th-233 & 22 & (b) & 10.2 & 0.0103 & 0.0954 & 617. & 0 & 5.74 \\
\hline $\mathrm{Pa}-231$ & 3 & $2.8728(\mathrm{E}-12)$ & 4.25 & 0.00462 & 0.416 & 234. & 0 & 0.0117 \\
\hline $\mathrm{Pa}-232$ & 4 & $4.1580(\mathrm{E}-15)$ & 5.19 & 0.00622 & 4.76 & 312. & 0 & 288. \\
\hline $\mathrm{Pa}-233$ & 23 & $3.1482(E-11)$ & 27.4 & 0.00430 & 0.104 & 143. & 0 & 0 \\
\hline$U-232$ & 5 & $8.1487(\mathrm{E}-12)$ & 4.25 & 0.00340 & 11.6 & 32.1 & 0 & 32.9 \\
\hline $\mathrm{U}-233$ & 6 & $5.9166(\mathrm{E}-09)$ & 2.85 & 0.00276 & 14.6 & 28.4 & 0 & 260 \\
\hline $\mathrm{U}-234$ & 7 & $2.9105(\mathrm{E}-06)$ & 14.2 & 0.000434 & 0.492 & 26.6 & 0 & 0 \\
\hline $\mathrm{U}-235$ & 8 & $2.7340(\mathrm{E}-04)$ & 4.11 & 0.00326 & 7.50 & 35.8 & 0 & 208. \\
\hline U-236 & 9 & $9.3798(\mathrm{E}-05)$ & 7.82 & 0.00260 & 0.208 & 2.47 & 0 & 0 \\
\hline $\mathrm{U}-237$ & 10 & $2.5054(\mathrm{E}-07)$ & 0.683 & 0.000669 & 0 & 41.0 & 0 & 0 \\
\hline U-238 & 24 & $2.0956(\mathrm{E}-02)$ & 0.834 & 0.00493 & 0.106 & 1.12 & 0 & 0 \\
\hline U-239 & 25 & (b) & 0.465 & 0.00106 & 0.214 & 9.07 & 0 & 5.76 \\
\hline $\mathrm{Np}-236$ & 27 & $2.8225(\mathrm{E}-13)$ & 0.273 & 0.00108 & 0 & 16.4 & 0 & 0 \\
\hline $\mathrm{Np}-237$ & 11 & $1.2633(\mathrm{E}-05)$ & 12.4 & 0.000568 & 0.594 & 129. & 0 & 0.00610 \\
\hline $\mathrm{Np}-238$ & 12 & $2.7337(E-08)$ & 0.273 & 0.000242 & 13.2 & 16.4 & 0 & 721. \\
\hline Np-239 & 26 & $1.9745(\mathrm{E}-06)$ & 0.225 & 0.000242 & 0.728 & 12.6 & 0 & 0.822 \\
\hline $\mathrm{Pu}-236$ & 28 & $3.2803(\mathrm{E}-11)$ & 3.41 & 0.00328 & 1.16 & 205. & 0 & 69.9 \\
\hline $\mathrm{Pu}-238$ & 13 & $3.4532(\mathrm{E}-06)$ & 4.26 & 0.000161 & 1.82 & 151. & 0 & 4.48 \\
\hline $\mathrm{Pu}-239$ & 14 & $1.8402(\mathrm{E}-04)$ & 4.96 & 0.000692 & 10.0 & 329. & 0 & 559. \\
\hline $\mathrm{Pu}-240$ & 15 & $5.0676(\mathrm{E}-05)$ & 5.37 & 0.00142 & 0.583 & 999. & 0 & 0.189 \\
\hline $\mathrm{Pu}-241$ & 16 & $3.8497(E-05)$ & 3.67 & 0.00629 & 17.1 & 205 & 0 & 538. \\
\hline $\mathrm{Pu}-242$ & 17 & $9.0025(\mathrm{E}-06)$ & 26.9 & 0.00193 & 0.493 & 14.5 & 0 & 0 \\
\hline $\mathrm{Am}-241$ & 29 & $1.2803(\mathrm{E}-06)$ & $15.4^{(c)}$ & $2.98^{(\mathrm{d})}$ & 0.813 & 582. (c) & 113 (d) $^{(d)}$ & 4.19 \\
\hline$A m-242$ & 30 & $2.4941(\mathrm{E}-09)$ & 5.12 & 0.00386 & 20.5 & 308. & 0 & 1233. \\
\hline $\mathrm{Am}-242 \mathrm{~A}$ & 18 & $4.0182(\mathrm{E}-08)$ & 10.9 & 0.00823 & 43.7 & 658. & 0 & 2631. \\
\hline $\mathrm{Am}-243$ & 19 & $1.9398 \quad(\mathrm{E}-06)$ & 8.58 & 0.00120 & 0.378 & 286. & 0 & 0 \\
\hline $\mathrm{Cm}-242$ & 31 & $3.3213(\mathrm{E}-07)$ & 0.136 & 0.00115 & 0 & 8.20 & 0 & 0 \\
\hline $\mathrm{Cm}-243$ & 20 & 2.1511 (E-09) & 1.81 & 0 & 1.81 & 70.8 & 0 & 70.8 \\
\hline $\mathrm{Cm}-244$ & 32 & $4.2275(\mathrm{E}-07)$ & 13.1 & 0.000441 & 1.15 & 10.0 & 0 & 0.631 \\
\hline
\end{tabular}

Notes: (a) Unit cell averaged number densities can be obtained by multiplying these values by the fuel volume firaction. volume fractions for fuel, cladding, and moderator are $0.38193,0.12825$, and 0.48981 , respectively.

(b) By-passed in LASER-HIC calculational model of transmutation chains.

(c) Value for $(n, \gamma)$ reaction which leads to production of Am-242 (16h).

(d) Value for $(n, y)$ reaction which leads to production of Am-242A (152y). HIC is programmed so that $(n, 2 n)$ reactions in $A m-241$ result in the formation of Am-242A, instead of $A m-240$. 
TABLE $10-11$

FEW-GROUP MICROSCOPIC CROSS SECTIONS FOR YANKEE REACTOR SPECTRUM AT ZERO BURNUP (THERMAL| BREAKPOINT AT $0.625 \mathrm{eV}$ )

\begin{tabular}{|c|c|c|c|c|c|c|c|c|}
\hline \multirow[b]{2}{*}{ Isotope } & \multirow{2}{*}{$\begin{array}{l}\text { HIC } \\
\text { Index }\end{array}$} & \multirow{2}{*}{$\begin{array}{l}\text { Atom }(a) \\
\text { Density } \\
\text { in Fuel } \\
(a / b-c m)\end{array}$} & \multicolumn{3}{|c|}{$\begin{array}{l}\text { Fast Cross Section, barns } \\
\quad(E>0.625 \mathrm{eV})\end{array}$} & \multicolumn{3}{|c|}{$\begin{array}{l}\text { Therma1 Cross Section, barns } \\
(\mathrm{E}<0.625 \mathrm{eV})\end{array}$} \\
\hline & & & $\sigma_{\gamma}$ & $\sigma_{2 n}$ & $\sigma_{f}$ & $\sigma_{\gamma}$ & $\sigma_{2 n}$ & $\sigma_{f}$ \\
\hline $\begin{array}{l}\text { Th-230 } \\
\text { Th-231 }\end{array}$ & $\frac{1}{2}$ & 0 & 0.265 & 0.00296 & 0 & 11.5 & 0 & 0 \\
\hline $\begin{array}{l}\text { Th-231 } \\
\text { Th-232 }\end{array}$ & 2 & $\begin{array}{c}0 \\
90628 \times 10^{-9}\end{array}$ & 17.3 & 0.00458 & 0 & 748 & 0 & 0 \\
\hline $\begin{array}{l}\text { Th-232 } \\
\text { Th-233 }\end{array}$ & $\begin{array}{l}21 \\
22\end{array}$ & $\begin{array}{l}9.0628 \times 10^{-9} \\
\text { (b) }\end{array}$ & $\begin{array}{l}3.09 \\
17.3\end{array}$ & 0.00428 & $\begin{array}{l}0.0230 \\
0.161\end{array}$ & $\begin{array}{r}3.58 \\
748\end{array}$ & 0 & $\begin{array}{c}0 \\
6.97\end{array}$ \\
\hline $\mathrm{Pa}-231$ & & $1.2731 \times 10^{-12}$ & & 0 & 0.00 & & & \\
\hline $\mathrm{Pa}-232$ & 4 & 0 & $\begin{array}{l}5.24 \\
8.76\end{array}$ & $\begin{array}{l}0.00441 \\
0.00594\end{array}$ & $\begin{array}{l}0.399 \\
8.05\end{array}$ & $\begin{array}{l}312 . \\
379 .\end{array}$ & $\begin{array}{l}0 \\
0\end{array}$ & $\begin{array}{l}0.0156 \\
349 .\end{array}$ \\
\hline $\mathrm{Pa}-233$ & 23 & 0 & 39.9 & 0.00411 & 0.0999 & 19.3 & 0 & 0 \\
\hline U-232 & 5 & $8.1996 \times 10_{-8}^{-13}$ & 4.82 & 0.00325 & 12.1 & 38.9 & 0 & 39.9 \\
\hline U-233. & 6 & $2.0499 \times 10^{-6}$ & 3.86 & 0.00264 & 21.5 & 25.7 & 0 & 264. \\
\hline U-234 & 7 & $4.6948 \times 10^{-0}$ & 15.2 & 0.000414 & 0.471 & 34.0 & 0 & 0 \\
\hline U-235 & 8 & $7.6931 \times 10^{-4} 6$ & 4.06 & 0.00312 & 8.49 & 46.4 & 0 & 262 . \\
\hline U-236 & 9 & $4.4703 \times 10^{-6}$ & 8.20 & 0.00248 & 0.199 & 2.97 & 0 & 0 \\
\hline $\begin{array}{l}\mathrm{U}-237 \\
\mathrm{U}-238\end{array}$ & 10 & $\begin{array}{cc}0 & -2\end{array}$ & 1.15 & 0.000639 & 0 & 49.7 & 0 & 0 \\
\hline U-238 & 24 & $2.1578 \times 10^{-2}$ & 0.828 & 0.00471 & 0.101 & 1.35 & 0 & 0 \\
\hline U-239 & 25 & (b) & 0.561 & 0.00101 & 0.276 & 11.0 & 0 & 6.98 \\
\hline $\mathrm{Np}-236$ & 27 & 0 & 0.461 & 0.00103 & 0 & 19.9 & 0 & 0 \\
\hline $\mathrm{Np}-237$ & 11 & 0 & 15.2 & 0.000543 & 0.570 & 127 & 0 & 0.00684 \\
\hline $\mathrm{Np}-238$ & 12 & 0 & 0.461 & 0.000231 & 21.5 & 19.9 & 0 & 873. \\
\hline Np -239 & 26 & 0 & 0.369 & 0.000231 & 0.708 & 15.3 & 0 & 0.997 \\
\hline $\mathrm{Pu}-236$ & 28 & 0 & 5.76 & 0.00314 & 1.96 & 249. & 0 & 84.8 \\
\hline Pu-238 & 13 & 0 & 4.50 & 0.000154 & 1.78 & 200. & 0 & 5.92 \\
\hline Pu-239 & 14 & 0 & 5.49 & 0.000661 & 11.0 & 487. & 0 & 804. \\
\hline $\begin{array}{l}\mathrm{Pu}-240 \\
\mathrm{P}_{1-2}-241\end{array}$ & 15 & 0 & 209. & 0.00136 & 0.599 & 182. & 0 & 0.0342 \\
\hline $\begin{array}{l}\text { Pu-241 } \\
\text { Pu-242 }\end{array}$ & $\begin{array}{l}16 \\
17\end{array}$ & $\begin{array}{l}0 \\
0\end{array}$ & $\begin{array}{l}3.62 \\
30.9\end{array}$ & $\begin{array}{l}0.00601 \\
0.00184\end{array}$ & $\begin{array}{l}17.3 \\
0.472\end{array}$ & $\begin{array}{l}291 . \\
15.7\end{array}$ & $\begin{array}{l}0 \\
0\end{array}$ & $\begin{array}{l}756 . \\
0\end{array}$ \\
\hline $\mathrm{Am}-241$ & 29 & 0 & $26.1^{(c)}$ & $5.05^{(d)}$ & 0.867 & 647. (c) & $125 .^{(d)}$ & \\
\hline Am-242 & 30 & 0 & 8.64 & 0.00369 & 34.6 & 374. & 0 & 1496.01 \\
\hline $\mathrm{Am}-242 \mathrm{~A}$ & 18 & 0 & 18.4 & 0.00786 & 73.8 & 798. & 0 & 3191. \\
\hline $\mathrm{Am}-243$ & 19 & 0 & 33.5 & 0.00114 & 0.362 & 48.0 & 0 & 0 \\
\hline $\mathrm{Cm}-242$ & 31 & 0 & 0.230 & 0.00110 & 0 & 9.95 & 0 & 0 \\
\hline $\mathrm{Cm}-243$ & 20 & 0 & 2.77 & 0 & 2.77 & 84.2 & 0 & 84.2 \\
\hline $\mathrm{Cm}-244$ & 32 & 0 & 13.5 & 0.000421 & 1.13 & 12.4 & 0 & 0.801 \\
\hline
\end{tabular}

Notes: (a) Unit cell averaged number densities can be obtained by multiplying these values by the fuel volume fraction. Volume fractions for the fuel, cladding, and moderator are $0.38193,0.12825$, and 0.48981 , respectively.

(b) By-passed in LASER-HIC calculational model of transmutation chains.

(c) Value for $(n, \gamma)$ reaction which leads to production of Am-242 (16 h).

(d) Value for $(n, \gamma)$ reaction which leads to production of Am-242A (152y). HIC is programmed so that (, $2 n$ ) reactions in Am-241 result in the formation of Am-242A, instead of Am-240. 
TABLE $10-12$

FEW-GROUP MICROSCOPIC CROSS SECTIONS FOR YANKEE REACTOR SPECTRUM AT 30,800 MWD/MTU (THERMAL BREAKPOINT AT 0.625 eV)

\begin{tabular}{|c|c|c|c|c|c|c|c|c|}
\hline \multirow[b]{2}{*}{ Isotope } & \multirow{2}{*}{$\begin{array}{c}\text { HIC } \\
\text { Index }\end{array}$} & \multirow{2}{*}{$\begin{array}{l}\text { Atom (a) } \\
\text { Density } \\
\text { in Fuel } \\
(a / b-c m)\end{array}$} & \multicolumn{3}{|c|}{$\begin{array}{c}\text { Fast Cross Section, barns } \\
(\mathrm{E}>0.625 \mathrm{eV})\end{array}$} & \multicolumn{3}{|c|}{$\begin{array}{l}\text { Thermal Cross Section } \\
(\mathrm{E}<0.625 \mathrm{eV})\end{array}$} \\
\hline & & & $\sigma_{\gamma}$ & $\sigma_{2 n}$ & $\sigma_{f}$ & $\sigma_{\gamma}$ & $\sigma_{2 n}$ & $\sigma_{\mathrm{f}}$ \\
\hline Th-230 & 1 & $2.9142(\mathrm{E}-11)$ & 0.233 & 0.00302 & 0 & 11.5 & 0 & 0 \\
\hline Th-231 & 2 & $4.6701(\mathrm{E}-15)$ & 15.2 & 0.00469 & 0 & 747. & 0 & 0 \\
\hline Th-232 & 21 & $8.2523(\mathrm{E}-09)$ & 3.02 & 0.00438 & 0.0235 & 3.58 & 0 & 0 \\
\hline Th-233 & 22 & (b) & 15.2 & 0.0101 & 0.141 & 747 & 0 & 6.96 \\
\hline $\mathrm{Pa}-231$ & 3 & $2.8728(\mathrm{E}-12)$ & 4.79 & 0.00451 & 0.407 & 304. & 0 & 0.0152 \\
\hline $\mathrm{Pa}-232$ & 4 & $4.1580(\mathrm{E}-15)$ & 7.70 & 0.00608 & 7.08 & 379. & 0 & 349. \\
\hline $\mathrm{Pa}-233$ & 23 & $3.1482(E-11)$ & 38.0 & 0.00420 & 0.102 & 19.8 & 0 & 0 \\
\hline $\mathrm{U}-232$ & 5 & $8.1487(E-12)$ & 4.42 & 0.00333 & 11.6 & 38.9 & 0 & 39.8 \\
\hline $\mathrm{U}-233$ & 6 & $5.9166(\mathrm{E}-09)$ & 3.61 & 0.00270 & 19.8 & 25.5 & 0 & 264. \\
\hline $0-234$ & 7 & $2.9105(\mathrm{E}-06)$ & 14.0 & 0.000424 & 0.481 & 33.9 & 0 & 0 \\
\hline $\mathrm{U}-235$ & 8 & $2.7340(E-04)$ & 4.16 & 0.00319 & 8.36 & 45.8 & 0 & 262. \\
\hline$U-236$ & 9 & $9.3798(\mathrm{E}-05)$ & 7.67 & 0.00254 & 0.203 & 2.99 & 0 & 0 \\
\hline$U-237$ & 10 & $2.5054(\mathrm{E}-07)$ & 1.01 & 0.000654 & 0 & 49.7 & 0 & 0 \\
\hline $\mathrm{U}-238$ & 24 & $2.0956(\mathrm{E}-02)$ & 0.825 & 0.00482 & 0.103 & 1.36 & 0 & 0 \\
\hline U-239 & 25 & (b) & 0.531 & 0.00103 & 0.258 & 11.0 & 0 & 6.97 \\
\hline $\mathrm{Np}-236$ & 27 & $2.8225(E-13)$ & 0.405 & 0.00105 & 0 & 19.9 & 0 & 0 \\
\hline $\mathrm{Np}-237$ & 11 & $1.2633(\mathrm{E}-05)$ & 14.4 & 0.000556 & 0.581 & 138. & 0 & 0.00719 \\
\hline$N p-238$ & 12 & $2.7337(\mathrm{E}-08)$ & 0.405 & 0.000236 & 19.1 & 19.9 & 0 & 872 . \\
\hline$N p-239$ & 26 & $1.9745(\mathrm{E}-06)$ & 0.326 & 0.000236 & 0.719 & 15.3 & 0 & 0.996 \\
\hline $\mathrm{Pu}-236$ & 28 & $3.2803(\mathrm{E}-11)$ & 5.06 & 0.00321 & 1.72 & 249. & 0 & 84.7 \\
\hline Pu-238 & 13 & $3.4532(\mathrm{E}-06)$ & 4.32 & 0.000157 & 1.78 & 201. & 0 & 5.93 \\
\hline Pu-239 & 14 & $1.8402(\mathrm{E}-04)$ & 5.22 & 0.000677 & 10.7 & 435. & 0 & 736. \\
\hline Pu-240 & 15 & $5.0676(\mathrm{E}-05)$ & 80.4 & 0.00139 & 0.584 & 186. & 0 & 0.0349 \\
\hline Pu-241 & 16 & $3.8497(\mathrm{E}-05)$ & 3.70 & 0.00615 & 17.2 & 273. & 0 & 715. \\
\hline Pu-242 & 17 & $9.0025(\mathrm{E}-06)$ & 26.6 & 0.00189 & 0.482 & 15.8 & 0 & 0 \\
\hline Am-241 & 29 & $1.2803(\mathrm{E}-06)$ & $23.6^{(c)}$ & $4.56^{(d)}$ & 0.859 & 649. (c) & 126 (d) $^{(d)}$ & 4.62 \\
\hline$A m-242$ & 30 & $2.4941(\mathrm{E}-09)$ & 7.60 & 0.00377 & 30.4 & 374. & 0 & 1494. \\
\hline$A m-242 A$ & 18 & $4.0182(E-08)$ & 16.2 & 0.00805 & 64.9 & 797. & 0 & 3188. \\
\hline $\mathrm{Am}-243$ & 19 & $1.9398(\mathrm{E}-06)$ & 30.2 & 0.00117 & 0.370 & 49.1 & 0 & 0 \\
\hline $\mathrm{Cm}-242$ & 31 & $3.3213(E-07)$ & 0.202 & 0.00113 & 0 & 9.94 & 0 & 0 \\
\hline $\mathrm{Cm}-243$ & 20 & $2.1511(\mathrm{E}-09)$ & 2.48 & 0 & 2.48 & 84.1 & 0 & 84.1 \\
\hline $\mathrm{Cm}-244$ & 32 & $4.2275(E-07)$ & 12.9 & 0.000431 & 1.13 & 12.4 & 0 & 0.800 \\
\hline
\end{tabular}

NOTES: (a) Unit cell averaged number densities can be obtained by multiplying these values by the fuel volume fraction. Volume fractions for the fuel, cladding, and moderator are $0.38193,0.12825$, and 0.48981 , respectively.

(b) By-passed in LASER-HIC calculational model of transmutation chains.

(c) Value for $(n, \gamma)$ reaction which leads to production of Am-242 (16 h).

(d) Value for $(n, \gamma)$ reaction which leads to production of Am-242A (152y). HIC is programmed so that ( $n$, 2n) reactions in Am-241 result in formation 
to the reactivity, and to provide a rough estimate of the magnitude of the contribution.

Data obtained from the LASER-HIC calculation for Yankee axial sample Zone 3, described in Subsection 10.4, is used in an example. Reference values of macroscopic few-group constants, edited in the LASER nortion of the LASER-

HIC calculation at a burnup of $30,800 \mathrm{MWD} / \mathrm{MTU}\left(\mathrm{F}^{\mathrm{TOT}} / \mathrm{N}_{\mathrm{O}}^{28}=0.0336\right)$, were used to calculate a reference value of k-infinity, whicn is a measure of the reactivity potential of the fuel:

$$
\mathrm{k}_{\infty}^{\mathrm{ref}}=\frac{v \sum_{f_{1}}^{r e f}}{\sum_{a_{1}}^{r e f}+\sum_{r_{1}}^{r e f}}+\frac{v \sum_{f_{2}}^{r e f} \sum_{r_{1}}^{r e f}}{\left(\sum_{a_{1}}^{r e f}+\sum_{r_{1}}^{r e f}\right) \sum_{a_{2}}^{r e f}}
$$

where $\sum^{\text {ref }}$ includes the main chain uranium and plutonium isotopes and the moderator and cladding materials, and subscripts $a, f$, and $r$ denote absorption, fission, and removal respectively, and the numerals 1 and 2 denote the fast $(E>1.855 \mathrm{eV}$ ) and thermal ( $\mathrm{E}<1.855 \mathrm{eV}$ ) energy groups, respectively. Then, considering each special isotope one at a time, a new value of $k$-infinity was calculated with:

$$
k_{\infty}^{i}=\frac{v^{i} \sum_{f_{1}}^{i}}{\sum_{a_{1}}^{i}+\sum_{r_{1}}^{i}}+\frac{v \sum_{f_{2}}^{i} \sum_{r_{1}}^{i}}{\left(\sum_{a_{1}}^{i}+\sum_{r_{1}}^{i}\right) \sum_{a_{2}}^{i}}
$$

where

$$
\Sigma^{i}=\sum^{r e f}+\Delta \sum^{i}
$$

and

$$
\Delta \sum^{i}=\sigma^{i} N^{i} \text {, for the special isotope } i
$$


Values of $v^{i}$ were obtained from the general references given in subsection 9.1; values of $2.61,2.75,2.96$, and 3.23 neutrons per fission were used for $\mathrm{Np}-237, \mathrm{Pu}-238, \mathrm{Am}-241$, and $\mathrm{Cm}-244$, respectively. Some assumptions made, regarding the special isotopes, for the purpose of illustrating the example are:
1) $\sigma_{a}^{i}=\sigma_{\gamma}^{i}+\sigma_{f}^{i}$
2) $\sigma_{r}^{i}=0$
3) $v_{1}^{i}=v_{2}^{i}$, and

4) the values of $v$ for any isotopes of the same element are equal.

The calculated data on Figure 10-3 and Table 10-10 were utilized in the above expressions.

The reactivity effect due to the presence of each special isotope was estimated with:

$$
(\Delta p)^{i}=\frac{k_{\infty}^{i}-k_{\infty}^{\text {ref }}}{k_{\infty}^{\text {ref }} k_{\infty}^{i}}
$$

The results are summarized in Table 10-13.

It is noted that the total effect on reactivity due to the buildup of the isotopes listed in Table $10-13$ is on the order of a negative one percent $\Delta p$, with Np-237, Am-241, Am-243, and perhaps Pu-238 contributing most significantly to the total. It is pointed out that for the Phase 3 fuel assembly, irradiated in Yankee Cores I, II, and IV (but not in Core III), the reactivity defect would be somewhat greater due to the Pu-241 B-decay to Am-241 during the 4-month cooling time between Core I and Core II operation, and the 1-year cooling time during Core III operation. 
TABLE $10-13$

ESTIMATED EFFECT ON REACTIVITY DUE TO BUILDUP OF

SPECIAL ISOTOPES IN YANKEE CORE I FUEL

\begin{tabular}{|c|c|c|c|c|}
\hline Isotope & $\begin{array}{l}\text { LASER-HIC } \\
\text { Index }\end{array}$ & Half-Life & $\begin{array}{l}\text { Atom } \\
\text { Density } \\
\text { in Unit } \\
\text { Cell } \\
(\mathrm{a} / \mathrm{b}-\mathrm{cm})\end{array}$ & Percent $\Delta \rho^{(a)}$ \\
\hline$N p-237$ & 11 & $2.14 \times 10^{6} \mathrm{y}$ & $4.82 \times 10^{-6}$ & -0.536 \\
\hline$N p-238$ & 12 & $2.11 \mathrm{~d}$ & $1.04 \times 10^{-8}$ & +0.006 \\
\hline$N p-239$ & 26 & $2.34 \mathrm{~d}$ & $7.54 \times 10^{-7}$ & -0.0006 \\
\hline $\mathrm{Pu}-238$ & 13 & $88.6 \mathrm{y}$ & $1.32 \times 10^{-6}$ & -0.097 \\
\hline$A m-241$ & 29 & $457 \mathrm{y}$ & $4.89 \times 10^{-7}$ & -0.201 \\
\hline$A m-242$ & 30 & $16 \mathrm{~h}$ & $9.52 \times 10^{-10}$ & +0.0009 \\
\hline$A m-242 A$ & 18 & $152 \mathrm{y}$ & $1.53 \times 10^{-8}$ & +0.031 \\
\hline$A m-243$ & 19 & -- & $7.41 \times 10^{-7}$ & -0.129 \\
\hline $\mathrm{Cm}-242$ & 31 & $164 \mathrm{~d}$ & $1.27 \times 10^{-7}$ & -0.0006 \\
\hline $\mathrm{Cm}-243$ & 20 & $32 y$ & $8.22 \times 10^{-10}$ & +0.00003 \\
\hline $\mathrm{Cm}-244$ & 32 & $18.1 \mathrm{y}$ & $1.62 \times 10^{-7}$ & -0.0075 \\
\hline Total & & & & -0.934 \\
\hline
\end{tabular}

a. Calculated at a burnup of $30,800 \mathrm{MWD} / \mathrm{MTU}$. 
In Zr-clad cores (as opposed to SS-clad cores), which have a softer (i.e., more thermal) neutron spectrum, it is expected that the reactivity defect due to $\mathrm{Np}-237$ would increase, while the reactivity defect due to $\mathrm{Am}-241$ and Am-243 would decrease. In cores which utilize mixed oxide fuel (i.e., $\mathrm{UO}_{2}-\mathrm{PuO}_{2}$ instead of $\mathrm{UO}_{2}$ ), it is expected that the reactivity defect due to Np-237 would decrease, while the reactivity defect due to the americium and curium isotopes would increase.*

\footnotetext{
*Use of natural $\mathrm{U}$ in $\mathrm{UO}_{2}-\mathrm{PuO}_{2}$ reduces the sources for $\mathrm{Np}-237$ production; the relatively large initial concentration of $\mathrm{Pu}$ increases the source for $\mathrm{Am}$ and $\mathrm{Cm}$ production.
} 


\subsection{COMPARISON OF HIC AND LASER CALCULATIONS OF MAIN CHAIN URANIUM AND PLUTONIUM ISOTOPIC CONCENTRATIONS}

Before going into the theory-experiment comparisons in the following section of this report, a brief discussion of the differences in the isotopic concentrations calculated in HIC and in LASER is in order.

The main chain uranium and plutonium isotopes (but not U-234) are calculated in the usual maner ${ }^{[4]}$ in the LASER portion of LASER-HIC, concurrently with the HIC matrix exponential calculation of thirty isotopes of the elements thorium through curium, including the main chain uranium and plutonium isotopes. It is pointed out that the concurrent HIC and LASER calculations do not result in exactly the same values of isotopic buildup and depletion; the differences are burnup dependent, with the HIC calculation giving about $1 / 2$ percent more U-235 and about 1 percent more $\mathrm{Pu}$ at high burnup. Table $10-14$ is a summary of the comparison between the two calculations. For this comparison, all values of isotopic atom densities are taken directly from the LASER-HIC printout. No adjustments (i.e., increases) have been made to the HIC values of $\mathrm{Pu}-239$ concentrations to account for holdup in $\mathrm{Np}-239 . *$

The major part of the differences is attributed to the more exact treatment of the transmutation chains in HIC; for example, LASER considers just one source of $\mathrm{Pu}-239$-- the $(\mathrm{n}, \mathrm{\gamma})$ reaction in U-238 leading directly to Pu-239, while HIC considers four sources of Pu-239 (albeit three are relatively minor) -- the $(n, \gamma)$ reaction in $U-238, * *$ the $(n, \gamma)$ reaction in $P u-238$, the $(n, 2 n$ ) reaction in $\mathrm{Pu}-240$, and the $\alpha$-decay of $\mathrm{Cm}-243$. It is also noted that HIC and LASER use different values of half-1ife for Pu-24I $\beta$-decay; HIC uses 13.6 years while LASER uses 13.0 years.

\footnotetext{
*After a burnup of 32,700 MWD/MTU, essentially all the Np-239 (2.34 d) would $\beta$-decay to $\mathrm{Pu}-239$ after a relatively short cooling time; the result would be a 0.8 percent increase in $\mathrm{Pu}-239$.

$* *$ The $(n, \gamma)$ reaction directly to $\mathrm{Np}-239$ (by-passing $\mathrm{U}-239$ ), and $\beta$-decay of $\mathrm{Np}-239$.
} 
TABLE 10-14

COMPARISON OF CONCURRENT HIC AND LASER CALCULATIONS FOR MAIN CHAIN

U AND PU ISOTOPIC CONCENTRATIONS IN YANKEE AXIAL SAMPLE ZONE 3

\begin{tabular}{|c|c|c|c|c|c|c|c|c|c|c|}
\hline \multirow{3}{*}{ Isotope } & \multirow{3}{*}{$\begin{array}{c}\text { HIC } \\
\text { Index } \\
\end{array}$} & \multicolumn{3}{|c|}{$\begin{array}{l}\text { At End of Core I } \\
\text { (13479 } \mathrm{MWD} / \mathrm{MTU})\end{array}$} & \multicolumn{3}{|c|}{$\begin{array}{l}\text { At End of Core II (b) } \\
(24513 \mathrm{MWD} / \mathrm{MTU})\end{array}$} & \multicolumn{3}{|c|}{$\begin{array}{l}\text { At End of Core IV } \\
(32701 \mathrm{MWD} / \mathrm{MTU})\end{array}$} \\
\hline & & \multicolumn{2}{|c|}{$\begin{array}{l}\text { Atom Density } \\
\text { in Fuel }(\mathrm{a} / \mathrm{b}-\mathrm{cm})\end{array}$} & $\begin{array}{l}\text { Percent } \\
\text { Difference }\end{array}$ & \multicolumn{2}{|c|}{$\begin{array}{l}\text { Atom Density } \\
\text { in Fue1 }(\mathrm{a} / \mathrm{b}-\mathrm{cm})\end{array}$} & $\begin{array}{l}\text { Percent } \\
\text { Difference }\end{array}$ & \multicolumn{2}{|c|}{$\begin{array}{l}\text { Atom Density } \\
\text { in Fue1 }(\mathrm{a} / \mathrm{b}-\mathrm{cm})\end{array}$} & $\begin{array}{l}\text { Percent } \\
\text { Difference }\end{array}$ \\
\hline & & & & & & & & & & \\
\hline U-235 & 8 & $4.8768(E-04)$ & $4.8702(E-04)$ & +0.14 & $3.4136(\mathrm{E}-04)$ & $3.4042(\mathrm{E}-04)$ & +0.28 & $2.6208(E-04)$ & $2.6103(\mathrm{E}-04)$ & +0.40 \\
\hline$U-236$ & 9 & $5.8732(E-05)$ & $5.8697(E-05)$ & +0.06 & 8. $399.1(\mathrm{E}-05)$ & $8.3896(E-05)$ & +0.11 & $9.5486(\mathrm{E}-05)$ & $9.5339(\mathrm{E}-05)$ & +0.15 \\
\hline $\mathrm{U}-238$ & 24 & $2.1321(E-02)$ & $2.1322(\mathrm{E}-02)$ & -0.005 & $2.1096(\mathrm{E}-02)$ & $2.1099(E-02)$ & -0.014 & $2.0922(\mathrm{E}-02)$ & $2.0926(\mathrm{E}-02)$ & -0.019 \\
\hline $\mathrm{Pu}-239^{(\mathrm{e})}$ & 14 & $1.2539(\mathrm{E}-04)$ & $1.2534(\mathrm{E}-04)$ & +0.04 & $1.6948(E-04)$ & $1.6848(E-04)$ & +0.6 & $1.8924(E-04)$ & $1.8712(E-04)$ & +1.1 \\
\hline $\mathrm{Pu}-240$ & 15 & $2.1283(E-05)$ & $2.1422(E-05)$ & -0.6 & $4.0958(E-05)$ & $4.0673(E-05)$ & +0.7 & $5.3646(E-05)$ & $5.2760(E-05)$ & +1.7 \\
\hline $\mathrm{Pu}-241$ & 16 & $1.1800(\mathrm{E}-05)$ & $1.2084(E-05)$ & -2.4 & $2.8981(E-05)$ & $2.9393(E-05)$ & -1.4 & $3.9371(E-05)$ & $3.9705(E-05)$ & -0.8 \\
\hline Pu-242 & 17 & $1.1503(\mathrm{E}-06)$ & $1.1090(\mathrm{E}-06)$ & +3.7 & $5.2363(\mathrm{E}-06)$ & $5.0765(\mathrm{E}-06)$ & +3.1 & $9.6589(E-06)$ & $9.1989(E-06)$ & +5.0 \\
\hline
\end{tabular}

a. 10837 hours at power.

b. 10837 hours at power in Core I, 3096 hours Core I - Core II shutdown, and 7994 hours at power in Core II

c. 10837 hours at power in Core I, 3096 hours Core I - Core II shutdown, 7994 hours at power in Core II, 8800 hours Core II - Core IV cooling, and 8088 hours at power in Core IV.

d. Percent difference $=($ HIC - LASER $) /($ LASER $) \times 100 \%$.

e. As calculated; the HIC values do not include holdup in Np-239. 
SECTION 11

COMPARISON BETWEEN CALCULATED AND MEASURED

ISOTOPIC BUILDUP AND DEPLETION IN YANKEE CORE I FUEL

The results obtained from the LASER-HIC zone calculations described in Section 10 have been compared with the results obtained from mass spectrometric and alpha spectrometric analyses on the Phase 1, 2, and 3 spent fuel samples. For all isotopes, the concentrations calculated with the matrix exponential method in the HIC portion of LASER-HIC are used in the comparisons. The evaluations are made only for those isotopes which were measured; these include the main chain uranium and plutonium isotopes -- U-234, U-235, U-236, U-238, Pu-239, Pu-240, Pu-241, and Pu-242, and the special isotopes U-232, U-233, Np-237, Pu-236, Pu-238, Am-241, Am-243, Cm-242, and $\mathrm{Cm}-244$. To place the caluclated and measured data on the same basis, all measured and calculated data are referenced to core end-of-life (i.e., Phase 1 data are referenced to Core I EOL, Phase 2 data are referenced to Core II EOL, and Phase 3 data are referenced to Core IV EOL) by appropriately adjusting the measured and calculated data to account for radioactive decay during the time intervals between core end-of-life and time of analysis. In the case of the measured data, these adjustments, or decay corrections, include:

$$
\begin{array}{ll}
\text { U-232 } & \mathrm{U}-232 \alpha \text {-decay and Pu-236 } \alpha \text {-decay } \\
\text { Pu-236 } & \text { Pu-236 } \alpha \text {-decay } \\
\text { Pu-238 } & \text { Pu-238 } \alpha \text {-decay and Cm-242 } \alpha \text {-decay } \\
\text { Pu-241 } & \text { Pu-241 } \beta \text {-decay } \\
\text { Am-241 } & \text { Am-241 } \alpha \text {-decay and Pu-241 } \beta \text {-decay } \\
\text { Cm-242 } & \text { Cm-242 } \alpha \text {-decay } \\
\text { Cm-244 } & \mathrm{Cm}-244 \alpha \text {-decay }
\end{array}
$$

The method used to decay-correct the measured data was outlined previously in Subsection 5.2. In the case of the calculated data, these adjustments, or decay corrections, include: 


$$
\begin{array}{lll}
\mathrm{Np}-237 & \mathrm{U}-237 & \beta \text {-decay } \\
\mathrm{Pu}-236 & \mathrm{~Np}-236 & \beta \text {-decay } \\
\mathrm{Pu}-238 & \mathrm{~Np}-238 & \beta \text {-decay } \\
\mathrm{Pu}-239 & \mathrm{~Np}-239 & \beta \text {-decay } \\
\mathrm{Cm}-242 & \mathrm{Am}-242 & \beta \text {-decay }
\end{array}
$$

In view of the relatively short half-lives involved, the method used to decaycorrect the calculated data was simply to add together appropriate values of calculated concentrations. For example, the calculated value of $\mathrm{Np}-237$ used in the comparisons was the sum of the values of $\mathrm{Np}-237$ and U-237 printed out in the HIC calculation at each time step; similarly, the calculated value of $\mathrm{Pu}-236$ used in the comparisons was the sum of the $\mathrm{Pu}-236$ value and 0.57 times the Np-236 value printed out in the HIC calculation. The magnitudes of these corrections to the calculated isotopic concentrations can be estimated from the data in Figure 10-3.

In the case of the main chain uranium and plutonium isotopes, the agreement between theory and experiment is excellent. In the case of the special isotopes, the agreement between theory and experiment is generally good, with a few exceptions.

The comparisons are made by plotting the calculated and measured parameters (e.g., a/o or $\mathrm{N}^{i} / \mathrm{N}_{0}^{28}$ ) as functions of total accumulated fissions (i.e., $\left.\mathrm{F}^{\mathrm{TOT}} / \mathrm{N}_{\mathrm{O}}^{28}\right) . *$ For those isotopes whose concentrations are not significantly dependent upon the axial zone, only the Zone 3 calculation is shown; for those isotopes whose concentrations are significantly dependent upon axial zone (i.e., irradiation history), the results of more than one zone calculation are shown. The comparisons are discussed in more detail in the following paragraphs.

*U-235 fractional depletion and fuel burnup can be related to total accumulated fissions on Figures $6-5$ and 6-6, respectively; it is noted that, to a first approximation, the value of fuel burnup in MWD/MTU is about $10^{6}$ times the value of $\mathrm{FTOT}_{\mathrm{N}} 28$ (e.g., a value of $\mathrm{F}_{\mathrm{O} O T} / \mathrm{N}_{\mathrm{O}} 28$ of 0.03 corresponds to a burnup value of about 30,000 MWD/MTU). 


\subsection{MAIN CHAIN U AND PU ISOTOPIC ABUNDANCES AND PU/U MASS RATIO}

The calculated and measured uranium isotopic abundances are shown as a function of total accumulated fissions on Figure 11-1. The agreement between theory and experiment is excellent, except for U-234 abundance in the high burnup Phase 3 fuel where the calculation underpredicts U-234 depletion by about 3 to 8 percent, relative. (Throughout Phase 1, 2, and 3 of the EYC Program, it was observed that the scatter in the U-234 data was somewhat greater than that which would be expected from the quoted uncertainties on the measurements.) The calculated and measured plutonium isotopic abundances are shown as a function of total accumulated fissions in Figure 11-2. The overall agreement between theory and experiment is quite good, and in the case of Pu-239 and $\mathrm{Pu}-240$, is excellent; at a value of $\mathrm{F}^{\mathrm{TOT}} / \mathrm{N}_{0}^{28}$ of about 0.01 , the calculation tends to underpredict the Pu-241 and Pu-242 abundance somewhat, even though the calculated curves pass through many of the data points. The results for the Phase 1, 2, and 3 samples are represented by different symbols. It can be observed that any influence that the Core IV environment may have exerted on the neutron spectrum in the Phase 3 fuel assembly, discussed in Subsection 10.2, apparently has little, if any, effect on the plutonium isotopic abundance. The calculated and measured $\mathrm{Pu} / \mathrm{U}$ mass ratio is compared in Figure 11-3. Neither the calculation nor the measurements include $\mathrm{Pu}-238$, which is treated separately in a later section of this report. The discontinuities in the calculated curves, at a value of $\mathrm{F}^{\mathrm{TOT}} / \mathrm{N}_{0} 28$ of about 0.025 , reflect the Pu-241 $\beta$-decay during the 1-year cooling time for the Phase 3 assembly during Core III operation.

The agreement between theory and experiment is good for the Phase 1 and Phase 2 data; however the calculated and measured values for $\mathrm{Pu} / \mathrm{U}$ mass ratio in the Phase 3 fuel are different by about 6 percent for the Zone 1 samples and about 4 percent difference for the Zones 3, 5, and 6 samples. (For the Phase 3 fuel samples, the zone 1 data are plotted at a value of $\mathrm{F}^{\mathrm{TOT}} / \mathrm{N}_{0}^{28}$ of about 0.018 , the Zone 6 data are plotted at a value of $\mathrm{F}^{\mathrm{TOT}} / \mathrm{N}_{\mathrm{O}}^{28}$ of about 0.022 , and the zones $2,3,4$, and 5 data are plotted at values of $\mathrm{F}^{\mathrm{TOT}} / \mathrm{N}_{\mathrm{o}}{ }^{28}$ ranging from 0.030 to 0.035 ; the Zone 1 calculated curve falls very nearly on the Zone 3 calculated curve and is not shown in Figure 11-3.) It is 


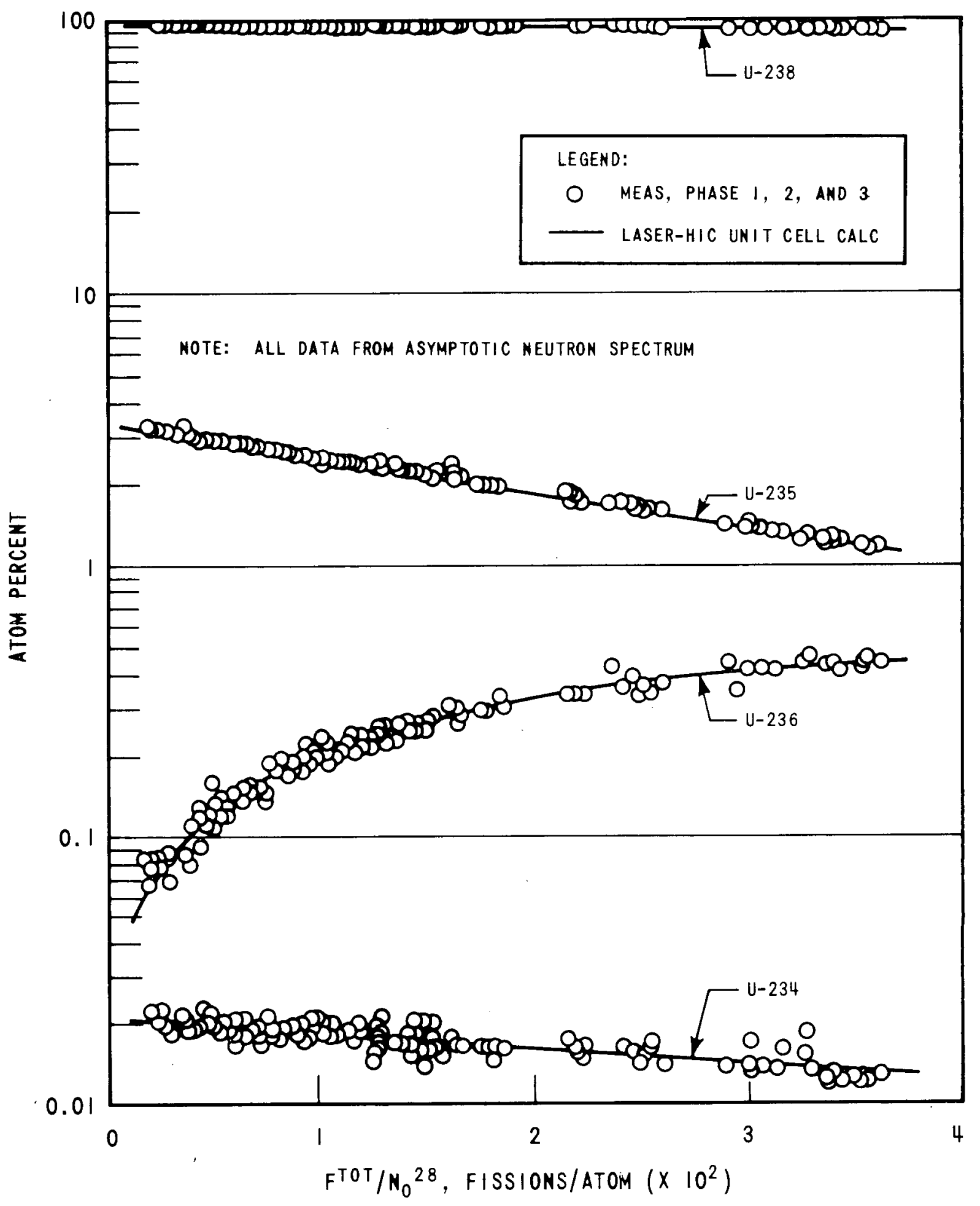

Figure 11-1. Uranium Isotopic Abundance versus Total Fissions 


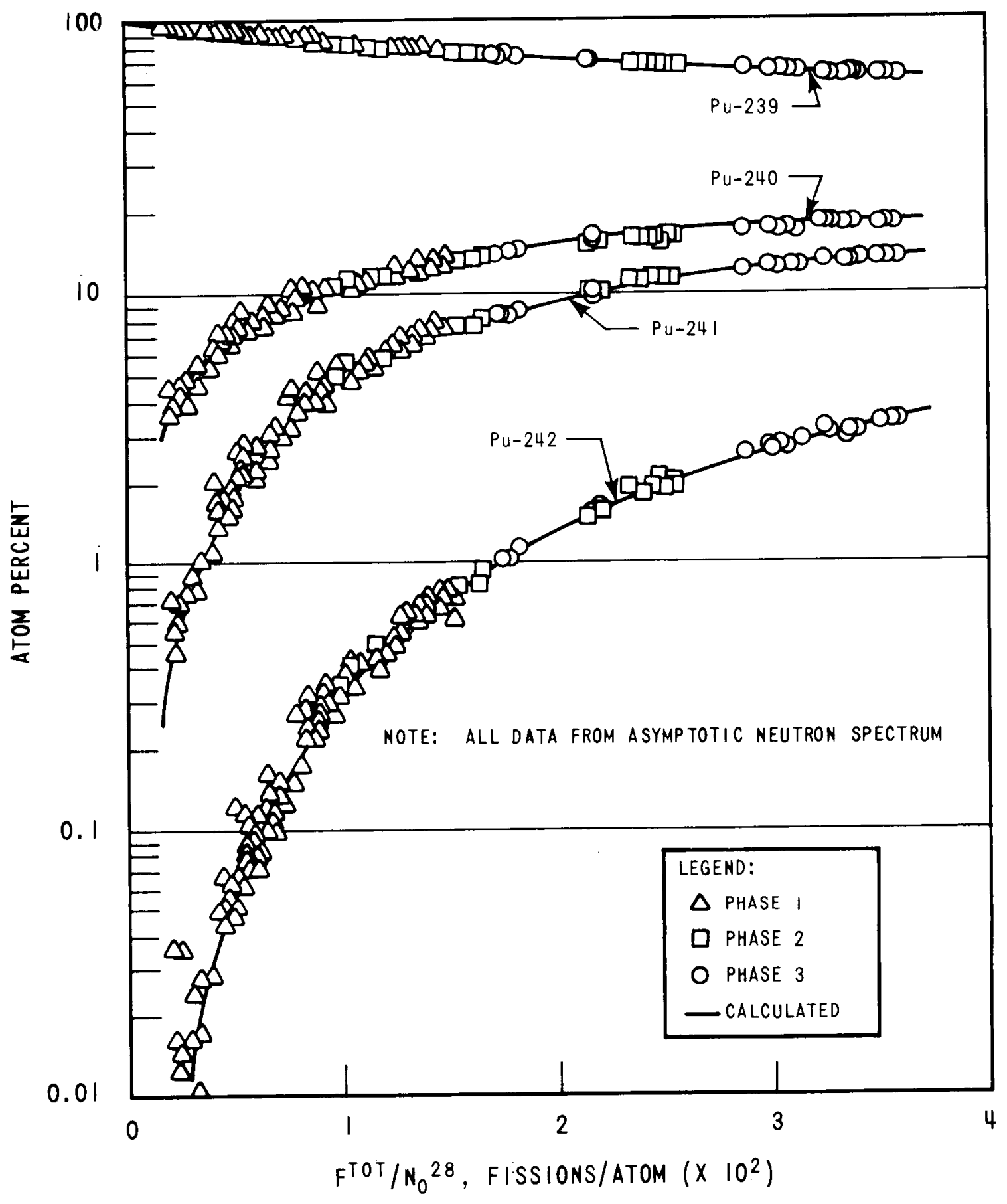

Figure 11-2. Plutonium Isotopic Abundance versus Total Fissions 


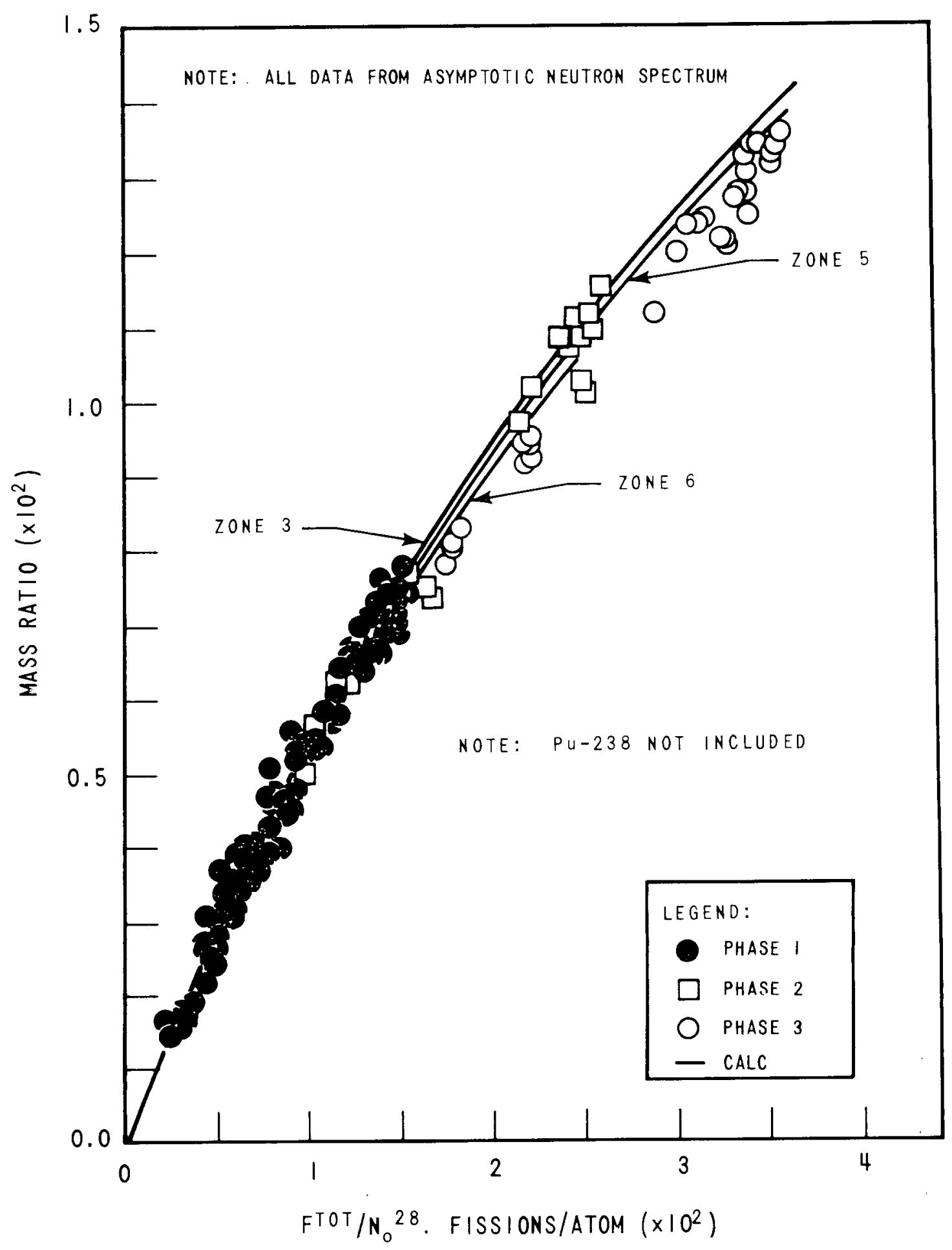

Figure 11-3. Calculated and Measured Pu/U Mass Ratio versus Total Fissions 
believed that part of these discrepancies are due to the Core IV environmenta1 effect on the neutron spectrum in the Phase 3 fuel assembly (which cannot be accounted for in a unit cell calculation). Based on the results of a one-dimensional diffusion-theory depletion calcualtion (see Figure 10-2), the unit cell calculations were expected to overpredict the plutonium production in the Phase 3 fuel by about $11 / 2$ to 2 percent. Part of the discrepancies are due also to the use of only approximately known fuel temperatures in the unit cell calculations corresponding to Phase 3 . As was discussed in Subsection 10.1, results of in-core flux-wire and thermocouple measurements were used to estimate the average power density and moderator temperature to which each of the axial sample zones was exposed during each of the three irradiation phases; it is pointed out, however, that Yankee core location $\mathrm{E} 6$, in which the Phase 3 fuel assembly was irradiated, does not contain a flux-wire thimble, and as a consequence some approximations were necessary to determine the power distribution in this assembly during Core IV operation.

Due to scatter in the data, and the uncertainties in irradiation history (i.e., fuel temperature and moderator temperature), the discrepancy between calculated and measured $\mathrm{Pu} / \mathrm{U}$ mass ratio cannot be resolved to anything less than about 2 percent.

\subsection{MAIN CHAIN URANIUM AND PLUTONIUM ISOTOPIC CONCENTRATION}

Calculated and measured uranium isotopic concentrations (i.e., $\mathrm{N}^{i} / \mathrm{N}_{0}^{28}$ ) are shown as a function of total accumulated fissions (i.e., $\mathrm{F}^{\mathrm{TOT}} / \mathrm{N}_{0}{ }^{28}$ ) in Figures 11-4 through 11-7.

Despite the great deal of scatter in the U-234 data as shown in Figure 11-4, it can be concluded that the calculated and measured U-234 concentrations are different by 3 to 8 percent. Some of the discrepancy may be due to the fact that the Yankee Core I fuel was supplied by two vendors, and as a consequence, fuel samples obtained from different spent fuel assemblies may have contained different initial concentrations of U-234 prior to irradiation. The initial composition data presented in Subsection 10.3 (and the data in Figures 11-1 and 11-4) indicates that the initial abundance of U-234 was greater by a 


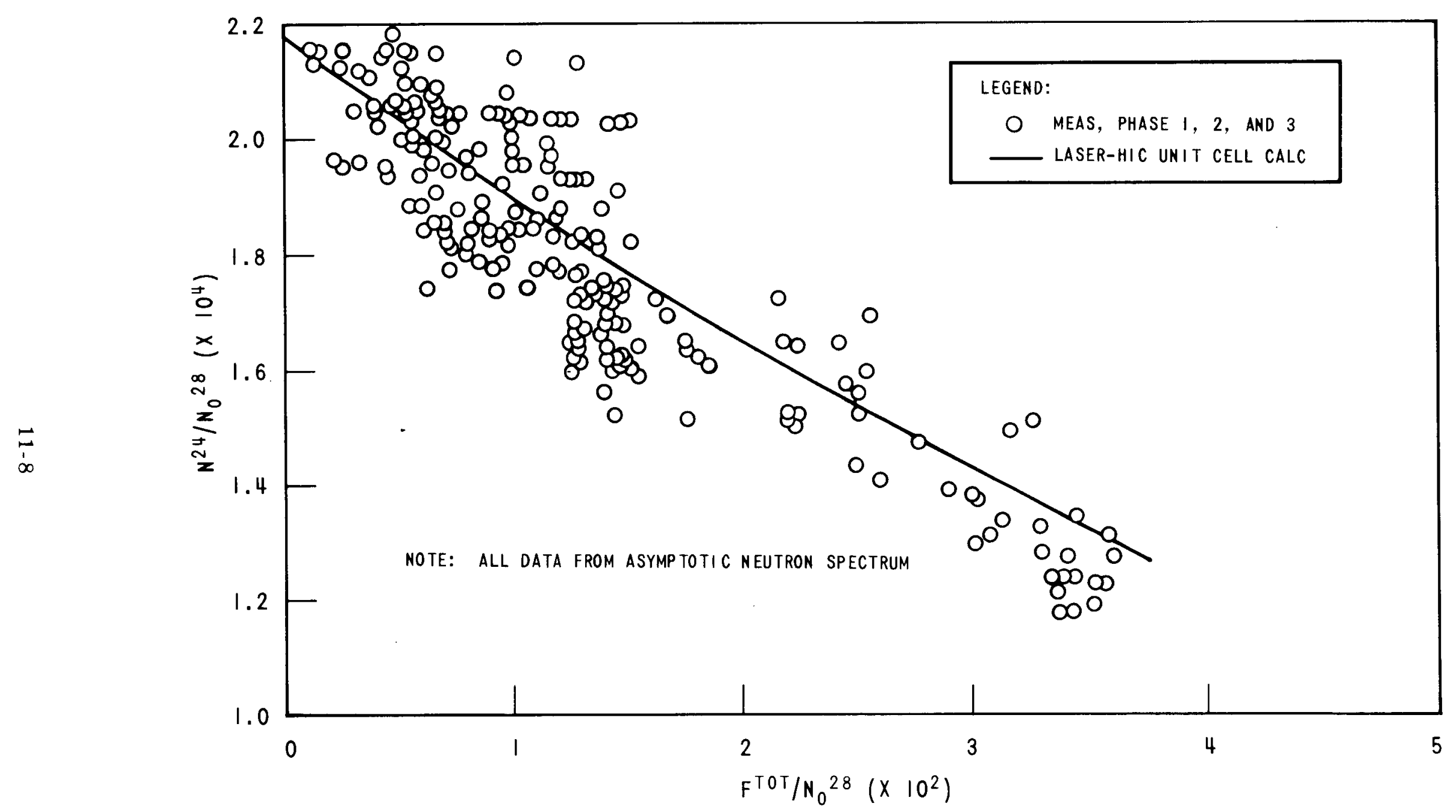

Figure 11-4. U-234 Concentrations versus Accumulated Fissions 


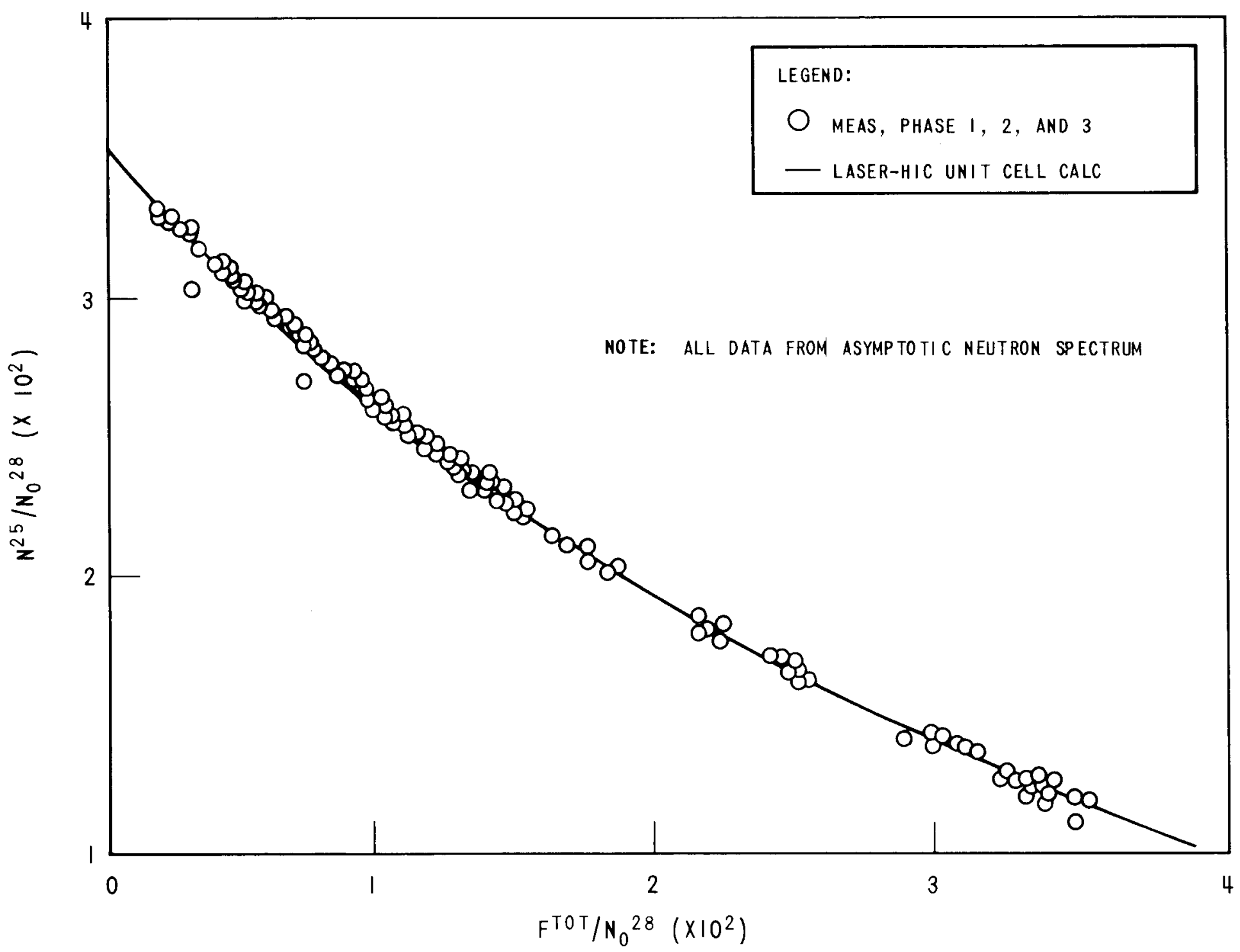

Figure 11-5. U-235 Concentrations versus Accumulated Fissions 


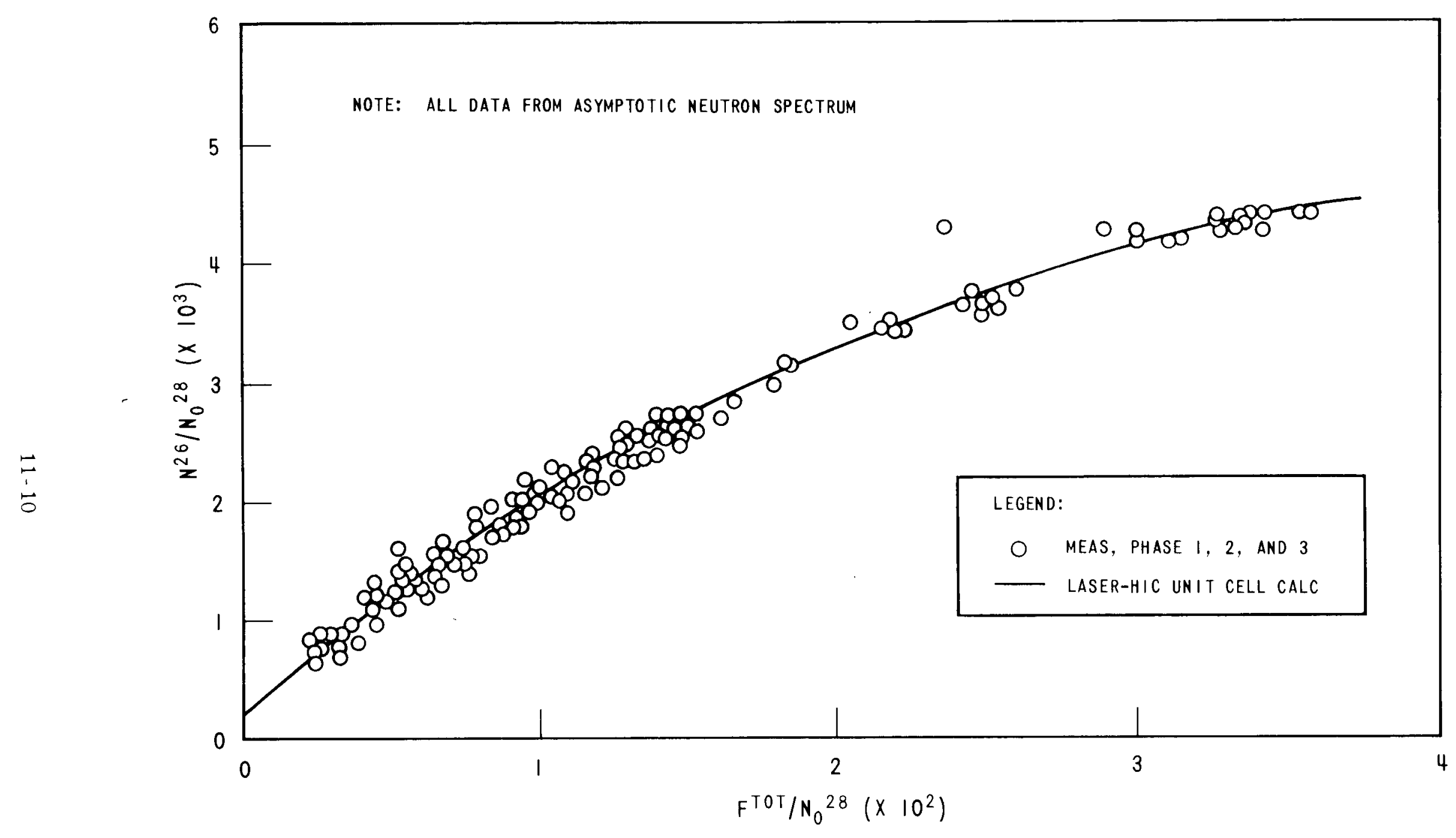

Figure 11-6. U-236 Concentrations versus Accumulated Fissions 


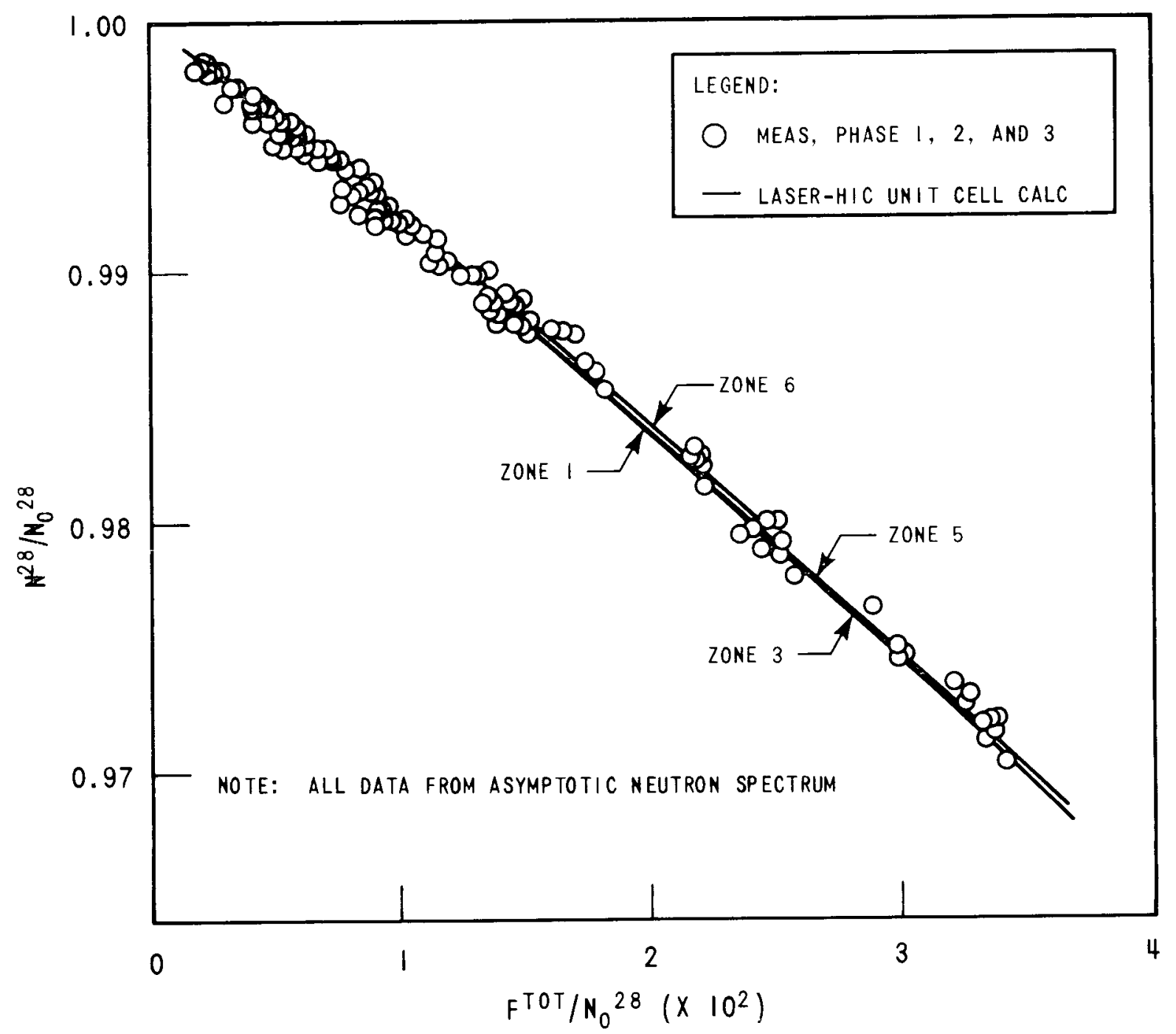

Figure 11-7. U-238 Concentrations versus Accumulated Fissions 
factor of 3.7 (i.e., 0.021 a/o versus 0.0057 a/o) than that which occurs naturally. The ratio of U-235 initial enrichment relative to the naturally occurring abundance of $U-235$ is 4.8 (i.e., 3.44 a/o versus 0.72 a/o).

Due to the large scatter in the U-234 measured data, taken together with the uncertainty in the U-234 initial concentration in every spent fuel sample, the discrepancy between calculated and measured U-234 concentration cannot be resolved to anything less than about 8 percent.

The U-235 data shown in Figure 11-5 indicate very good agreement between theory and experiment. This conclusion should be tempered by the fact that the independent variable (i.e., $\mathrm{F}^{\mathrm{TOT}} / \mathrm{N}_{0}{ }^{28}$ ) with which the $\mathrm{U}-235$ concentration (i.e., $\mathrm{N}^{25} / \mathrm{N}_{\mathrm{o}}^{28}$ ) is correlated, is not truly independent. As was described in Subsection 6.1, the total accumulated fissions, determined with the Heavy Element (HE) method, is the sum of the accumulated fissions in U-235, U-238, $\mathrm{Pu}-239$, and $\mathrm{Pu}-241$. Since the determination of accumulated fissions in U-235 is based, in large part, on the difference between the initial and final concentrations of U-235 (see Subsection 5.1), a kind of normalizing, or selfcompensating, effect takes place in the correlation. That is, if for any reason, the measured (or calculated) U-235 concentration is too high, the difference between initial and final concentrations will be too low, and the resulting total accumulated fissions will be too low. The net effect, in the case of the correlation shown in Figure 11-5, would be to move the measured (or calculated) value of $\mathrm{N}^{25} / \mathrm{N}_{\mathrm{o}}{ }^{28}$ up and to the left, thus remaining nearly always (to the extent to which accumulated U-235 fissions contribute to total accumulated fissions, which is burnup dependent) on the measured (or calculated) correlation.

However, the total accumulated fissions as determined with the HE method used in this study was shown to be in statistical agreement with the total accumulated fissions determined from measurements of the fission product Nd-148 (see Subsection 6.3). For this reason, the comparison made between calculated and measured U-235 concentrations in Figure $11-5$ is considered to be valid.

The calculated and measured U-236 data are shown in Figure 11-6; the agreement between theory and experiment is considered excellent. It is pointed out that U-236 is not a naturally occurring isotope; consequently, its presence 
in the fuel prior to irradiation is an indication that all or part of the fuel which was supplied for Yankee Core I may have been previously irradiated and reprocessed in connection with the operation of some other reactor. Since the U-236 concentration in the Yankee spent fuel samples is much larger (depending on the burnup) than the initial concentration of U-236 prior to irradiation, any reasonable uncertainty in the value of initial U-236 abundance used in the calculations or data reduction performed in this study has little significance.

The calculated and measured U-238 data are shown in Figure 11-7. The agreement between theory and experiment is quite good, except for the high burnup Phase 3 samples where the calculation overpredicts the U-238 depletion. This is consistent with $\mathrm{Pu} / \mathrm{U}$ data shown previously, in Figure 11-3, where the calculation overpredicted plutonium production in the high burnup Phase 3 fuel. As was discussed earlier, this discrepancy is attributed in part to the Core IV environmental effect on the neutron spectrum in the recycled Phase 3 fuel assembly, and in part to a less than accurate knowledge of the irradiation history of the Phase 3 axial sample zones.

Using the data reduction methods incorporated in the REBUF code, values of the U-235 effective capture-to-fission ratio $\left(\alpha^{25}\right) *$ were determined from the $\mathrm{U}-235$ and U-236 isotopic measurements. Calculated and measured values of effective $\alpha^{25}$ are shown on Figure 11-8. The calculated values of effective $\alpha^{25}$ were edited in the LASER portion of the LASER-HIC zone calculations. The agreement between theory and experiment is excellent.

Calculated and measured plutonium isotopic concentrations (i.e., $\mathrm{N}^{\mathrm{i}} / \mathrm{N}_{0}{ }^{28}$ ) are shown as a function of total accumulated fissions (i.e., $\mathrm{F}^{\mathrm{TOT}} / \mathrm{N}_{0}{ }^{28}$ ) in Figures 11-9 through 11-12. The difference in the degree of scatter in the measured data in Figure 11-9 and on the other three figures is only apparent; the Pu-239 data span one decade and are plotted on a linear scale; the $\mathrm{Pu}-240$, $\mathrm{Pu}-241$, and Pu-242 data span more than one decade and are plotted on logarithmic scales. The discontinuities in the calculated curves of Pu-241 buildup, in

*Effective alpha-25 is the ratio of accumulated captures in U-235 relative to accumulated fissions in U-235. 


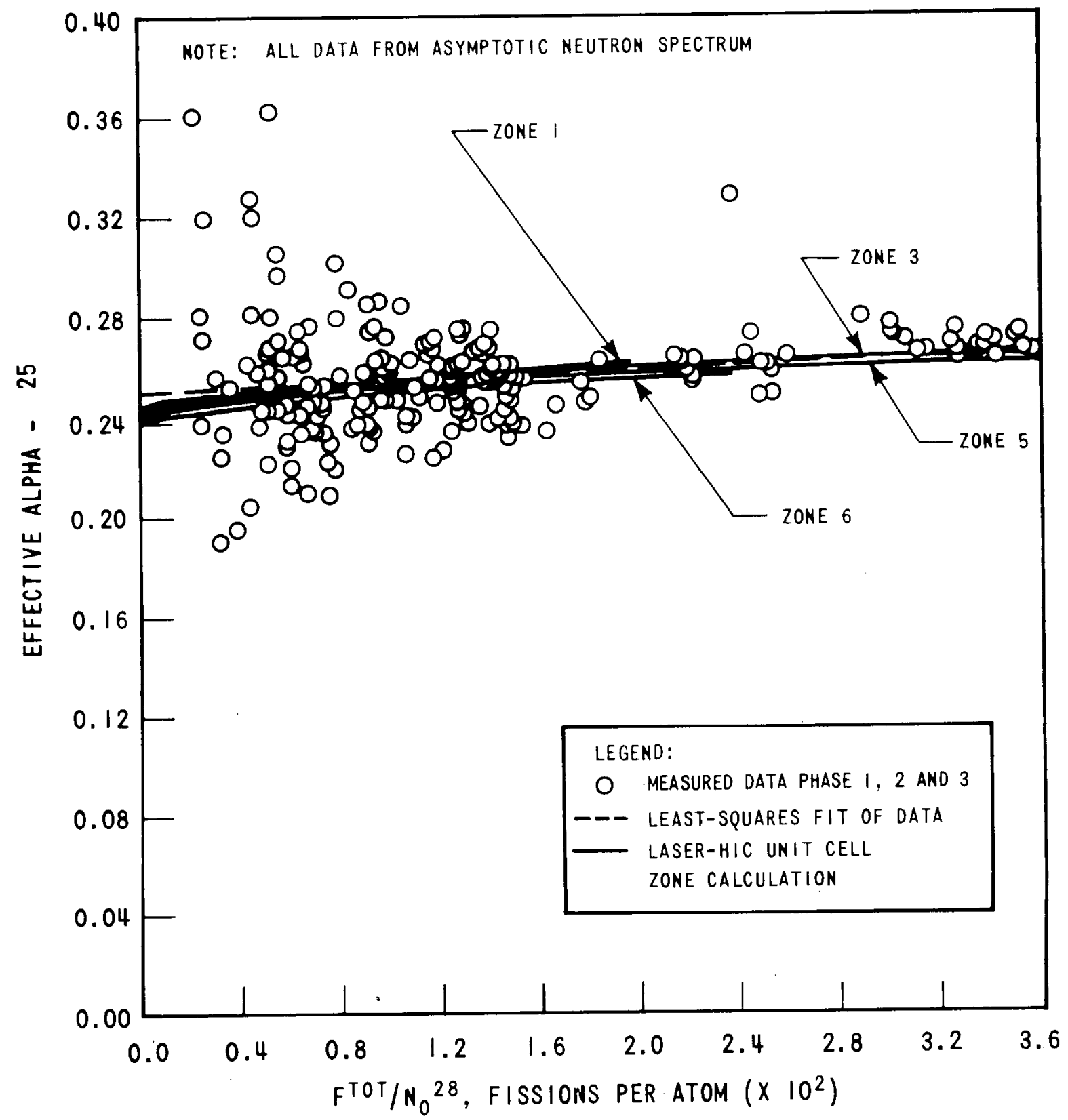

Figure 11-8. Effective Capture-to-Fission Ratio in U-235 versus Total Accumulated Fissions 


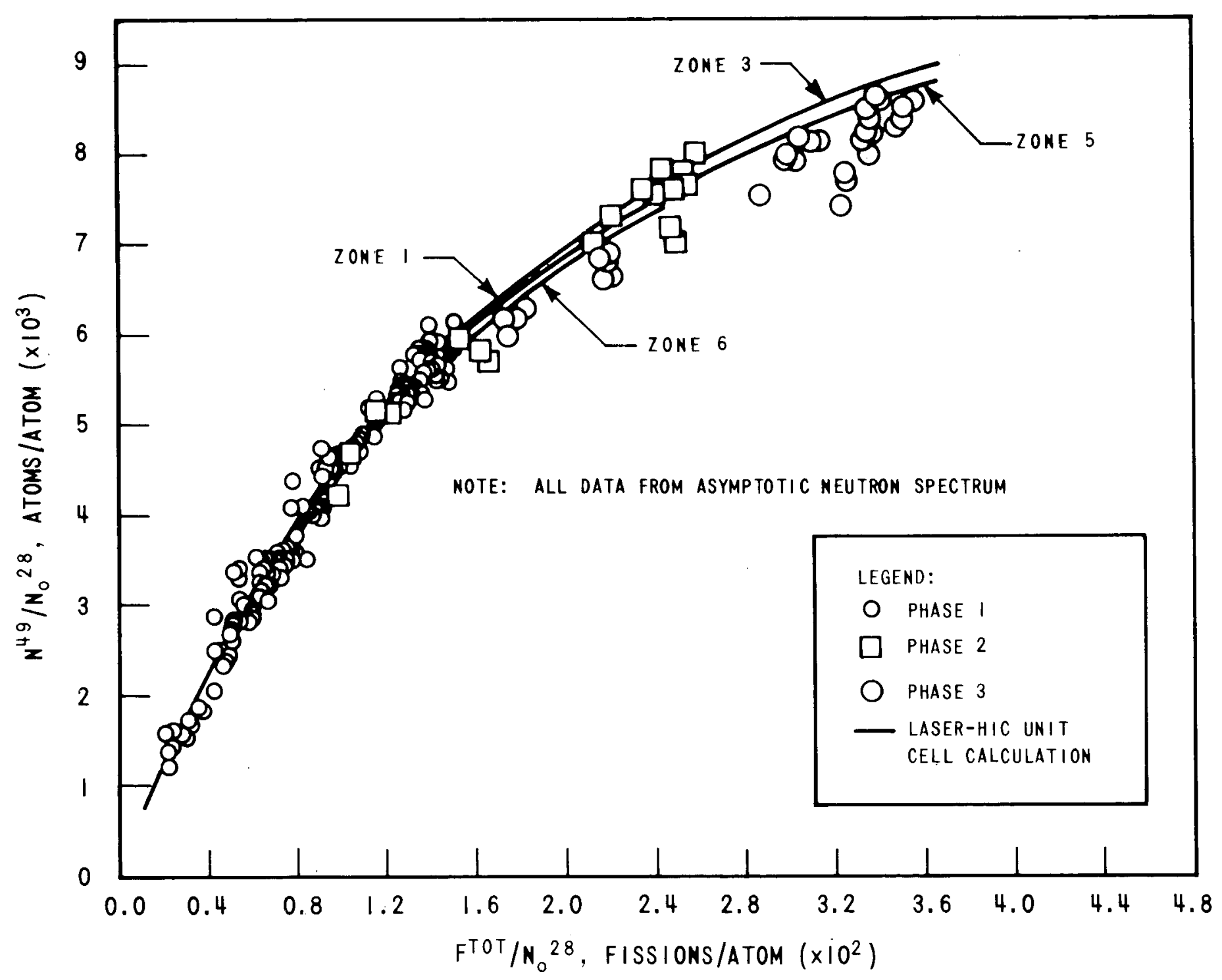

Figure 11-9. Pu-239 Concentrations versus Accumulated Fissions 


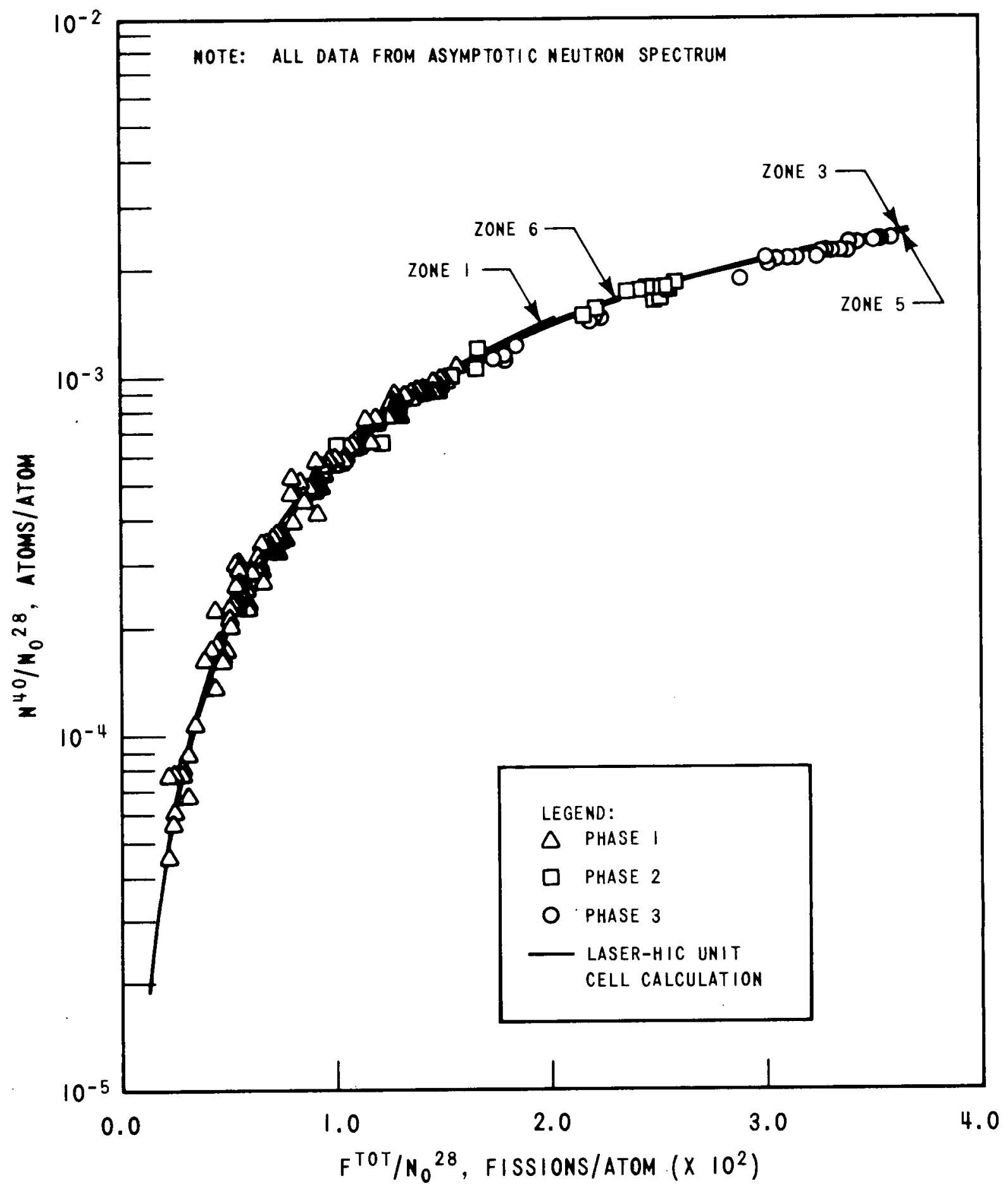

Figure 11-10. Pu-240 Concentrations versus Accumulated Fissions 


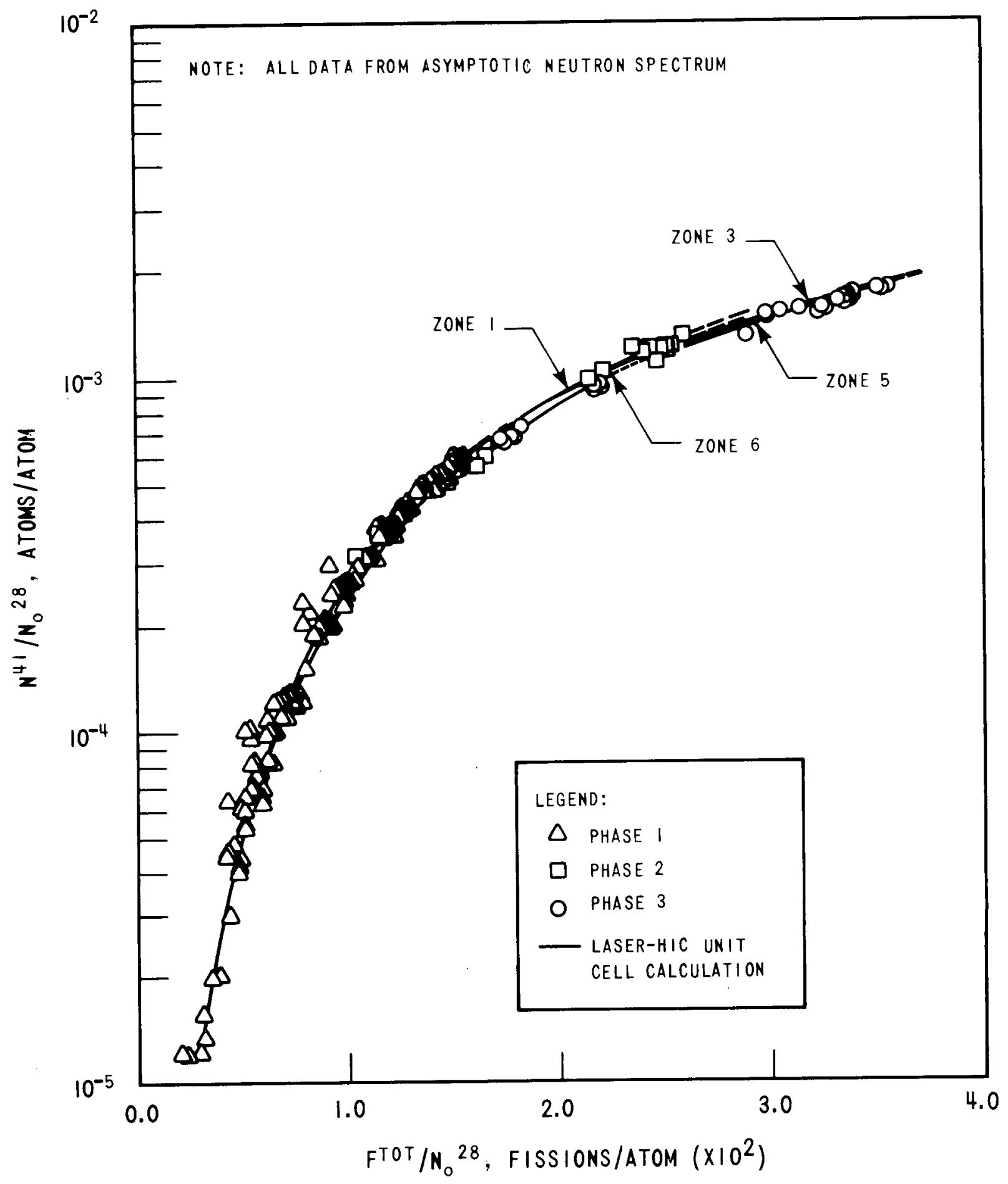

Figure 11-11. Pu-241 Concentrations versus Accumulated Fissions 


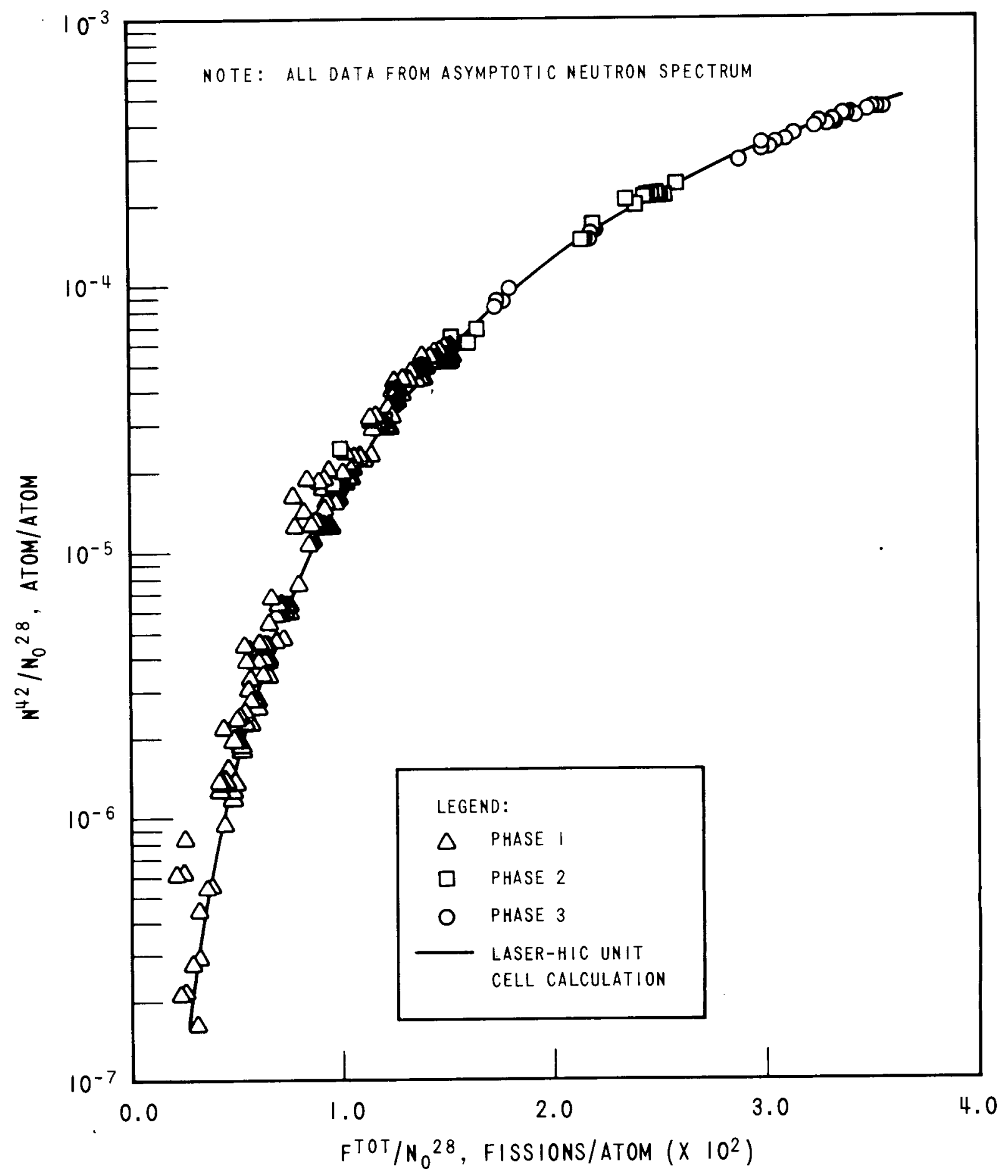

Figure 11-12... Pu-242 Concentrations versus Accumulated Fissions 
Figure 11-11, reflect the $\beta$-decay during cooling intervals between irradiation phases.

The overall agreement between theory and experiment is excellent for the Phase 1 and Phase 2 data; however, in the case of the Phase 3 data, the calculation overpredicts the plutonium isotopic concentrations by about 4 percent. As was discussed previously, part of this discrepancy between the calculated and measured Phase 3 data is due to the Core IV environmental effect (unaccounted for in the unit cell zone calculations) and part of the discrepancy is due to uncertainty in the irradiation history (only approximately known fuel temperatures and moderator temperatures used in the unit cell zone calculations).

There exist many definitions of the conversion ratio (see, for example, Reference 20$) \%$. One of these, the number of fissile atoms discharged (i.e., fissile atoms in place at a given burnup) relative to the number of fissile atoms loaded, is shown on Figure 11-13. Again, the agreement between theory and experiment is excellent for the Phase 1 and Phase 2 data, and a 4 percent bias shows up in the Phase 3 data.

Another kind of conversion ratio, which is a measure of fuel performance, is the net number of grams of fissile material destroyed per megawat day of energy generated (NGD/MWD). In Figure 11-14, the calculated values of NGD/MWD are compared with measured values determined with the use of the REBUF data reducing code. The agreement between theory and experiment reflects the overall accuracy in the calculation of U-235 depletion and Pu-239 and Pu-241 buildup.

\footnotetext{
*For mixed oxide fuel $\left(\mathrm{UO}_{2}-\mathrm{PuO}_{2}\right)$, the conversion ratios corresponding to most definitions are difficult to apply to the results of mass spectrometric isotopic measurements on spent fuel samples, and in most cases, result in negative values.
} 


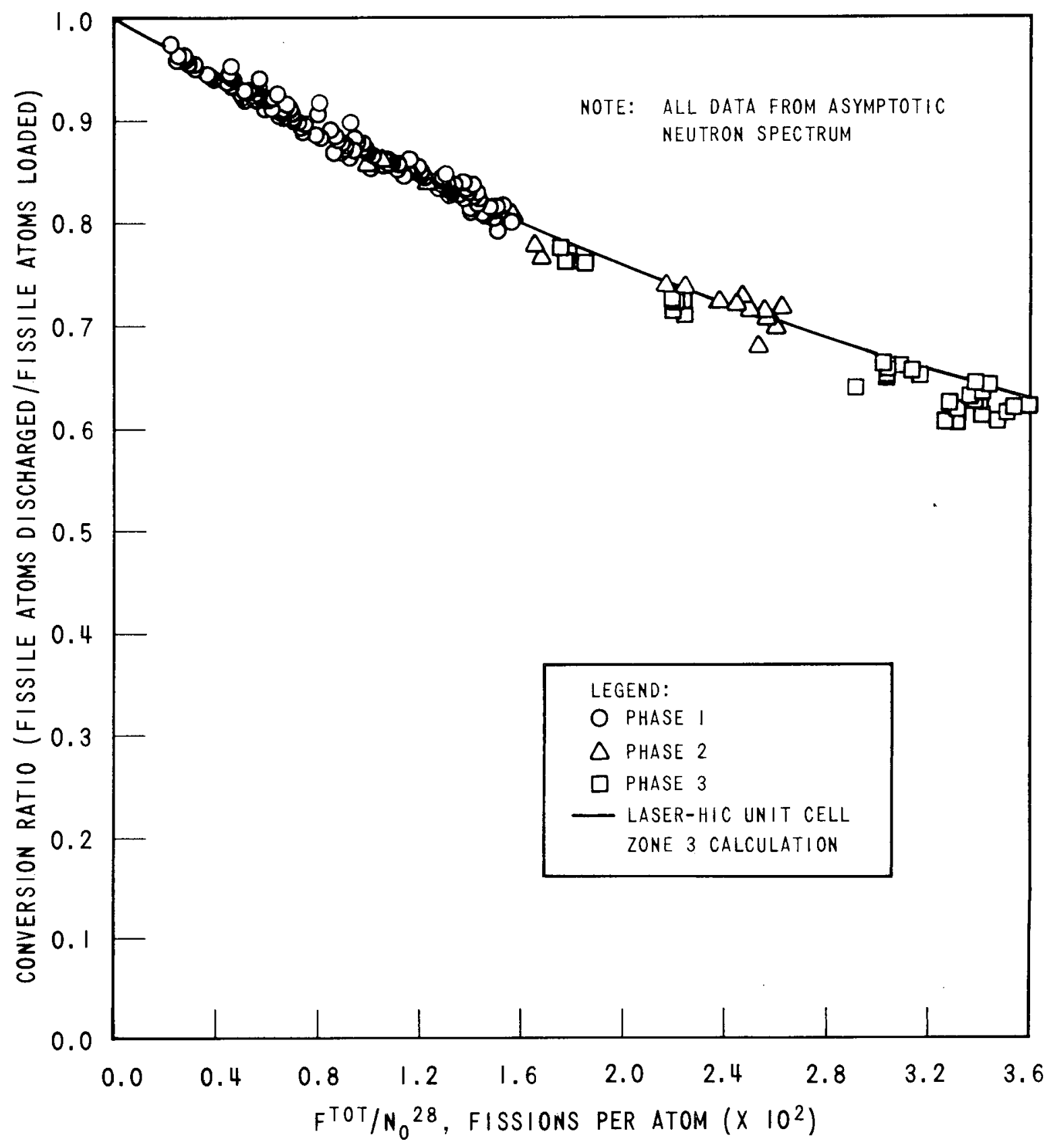

Figure 11-13. Fissile Atoms Discharged/Fissile Atoms Loaded versus Total Accumulated Fissions 


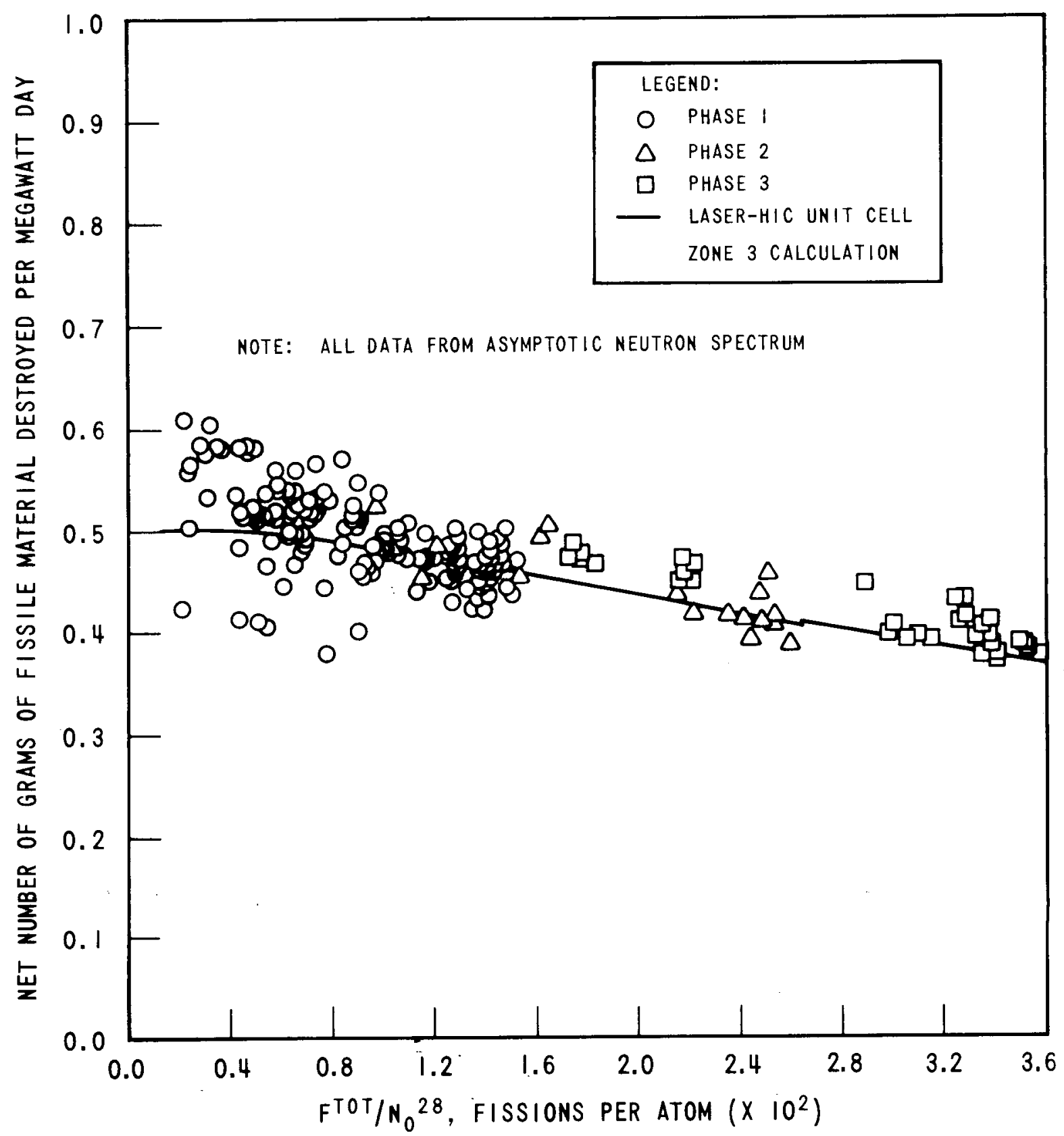

Figure 11-14. - NGD/MWD versus Total Accumulated Fissions 


\subsection{CONCENTRATION OF SPECIAL ISOTOPES}

The comparisons between theory and experiment for each special isotope is given more or less in an order corresponding to the location of the isotope in the transmutation chains in accordance with its proximity to the main chain. The results of the measurements for the special isotopes are documented in Appendix $\mathrm{D}$, where values are given both at time of analysis and referenced to core end-of-life.

The calculated and measured concentrations of Np-237 are shown in Figure 11-15. At low values of burnup, the results of theory and experiment are in fair agreement; however, at high values of burnup, the calculation overpredicts Np-237 production by approximately 8 percent, even though the calculated curve intercepts the error flags on a few of the measured data points. A significant portion of the $\mathrm{Np}-237$ production is due to the $\mathrm{n}, 2 \mathrm{n}$ reaction in $\mathrm{U}-238$. Since the $\mathrm{n}, 2 \mathrm{n}$ cross section has a threshold at very high energies, the manner in which the basic cross section data are weighted significantly affects the resultant group average (i.e., the first two groups in the fast energy range) value of U-238 n,2n'cross section used in the calculation, and significantly affects the calculated value of $\mathrm{Np}-237$ concentration. The group average $n, 2 n$ cross sections. used in the LASER-HIC final calculations performed in this study were $1 / \mathrm{E}$ weighted. Initial test run and debug calculations used values of $n, 2 n$ cross section for U-238 which were flatweighted, resulting in calculated $\mathrm{Np}-237$. concentrations even higher than that shown in Figure 11-15. Other preliminary calculations used values of $\mathrm{n}, 2 \mathrm{n}$ cross sections for $\mathrm{U}-238$ which were weighted by the fission spectrum; the resulting $\mathrm{Np}-237$ concentrations in these calculations were roughly 4 percent lower than the calculated values shown in Figure 11-15, cutting the discrepancy between theory and experiment in half.

At this point, one would conclude that fission spectrum weighting the $n, 2 n$ cross sections gives more accurate results in the unit cell calculations. In the case of $\mathrm{Np}-237$ production, this appears to be true; however, the improvement in the accuracy of the Np-237 calculation is made at the expense of the accuracy of the $\mathrm{Pu}-236$ and Pu-238 calculations, each of which 


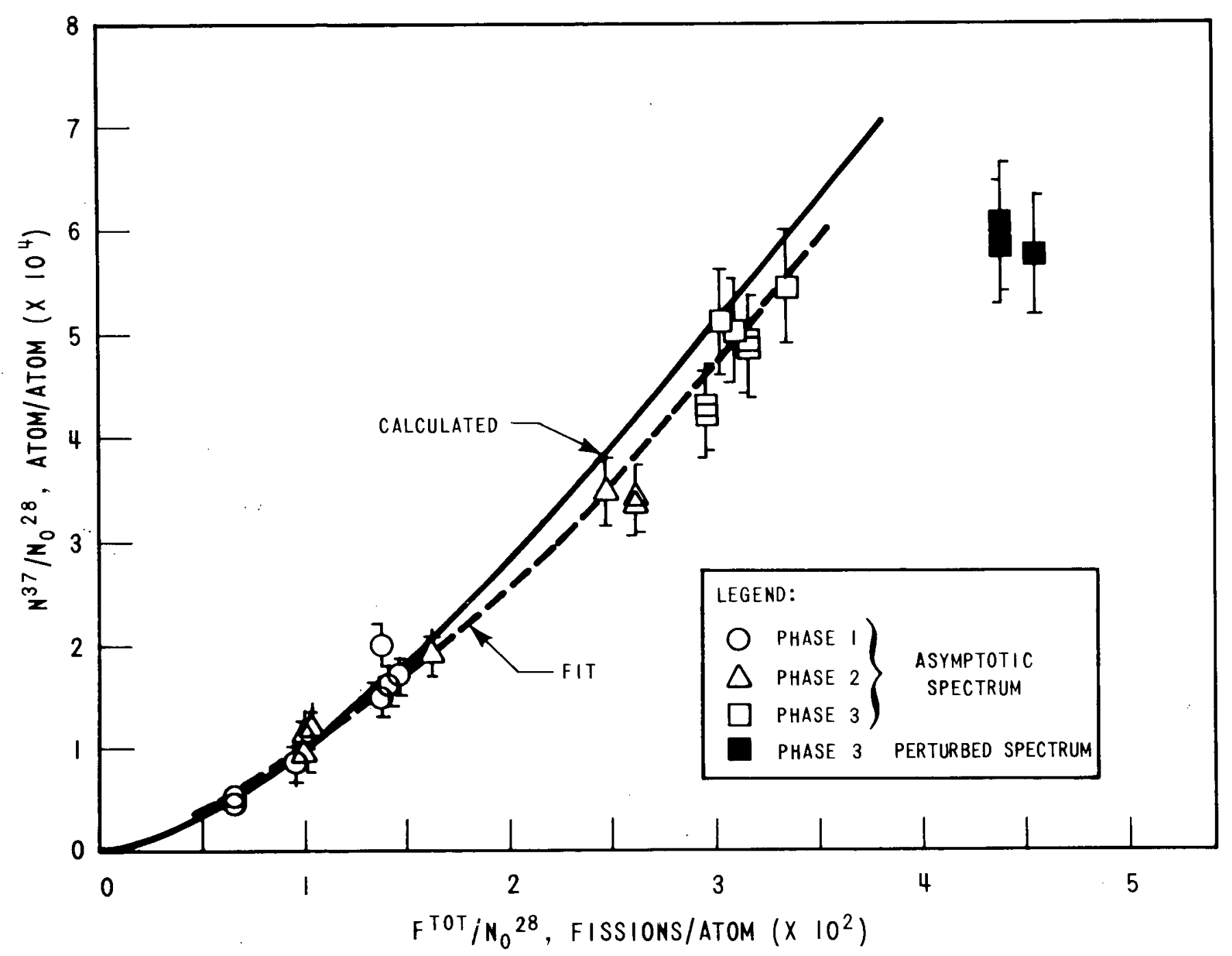

Figure 11-15. Calculated and Measured Np-237 as a Function of Accumulated Fissions 
is dependent on the Np-237 calculation. As will be shown later, the calculations using $1 / \mathrm{E}$ weighted $\mathrm{n}, 2 \mathrm{n}$ cross sections for $\mathrm{U}-238$ tend to underpredict the $\mathrm{Pu}-236$ and $\mathrm{Pu}-238$ concentrations; using fission spectrum weighted U-238 $\mathrm{n}, 2 \mathrm{n}$ cross sections would result in even lower calculated values of $\mathrm{Pu}-236$ and $\mathrm{Pu}-238$. Furthermore, if it can be argued that fission spectrum weighting should be used in the case of the U-238 n, $2 n$ cross section, then for the sake of consistency, fission spectrum weighting should be used in the case of the $\mathrm{Np}-237 \mathrm{n}, 2 \mathrm{n}$ cross section. However, in the test calculations mentioned above, the use of fission spectrum weighted $n, 2 n$ cross sections for Np-237 resulted in a 25 percent decrease in the calculated Pu-236 concentration, and a corresponding decrease in the calculated U-232 concentration, which, if anything, is already too low even when $1 / E$ weighted $n, 2 n$ cross sections are used.

Thus, from the consideration of best overall agreement between theory and experiment, it was decided to use $1 / E$ weighting for the final LASER-HIC zone calculations. There is no doubt that cancellation of errors is taking place to some degree; however, further resolution of discrepancies between theory and experiment would require more measurements and more calculations, both of which are beyond the scope of this program.

Considering 1) the scatter in the measured Np-237 data, 2) the relatively large measurement uncertainties, 3) the uncertainties associated in weighting the $n, 2 n$ cross sections, 4) the uncertainties in the Phase 3 sample irradiation history, and 5) the uncertainty of the effect of the Core IV environment on Np-237 production, an 8 percent discrepancy between theory and experiment is not too unreasonable.

The calculated and measured Pu-238 concentrations are shown in Figure 11-16. The calculated Pu-238 production is somewhat dependent on axial zone and irradiation history. For the sake of clarity, some simplifications were made in presenting the calculated data in Figure 11-16; for example, the Zone 6 calculation is not shown, and the Zones 3 and 5 calculations are represented by a single curve. Without showing the complexities of the final zone calculations, it can be concluded that the calculations underpredict Pu-238 concentration by about 5 percent in the Phase 1 and Phase 3 samples, 


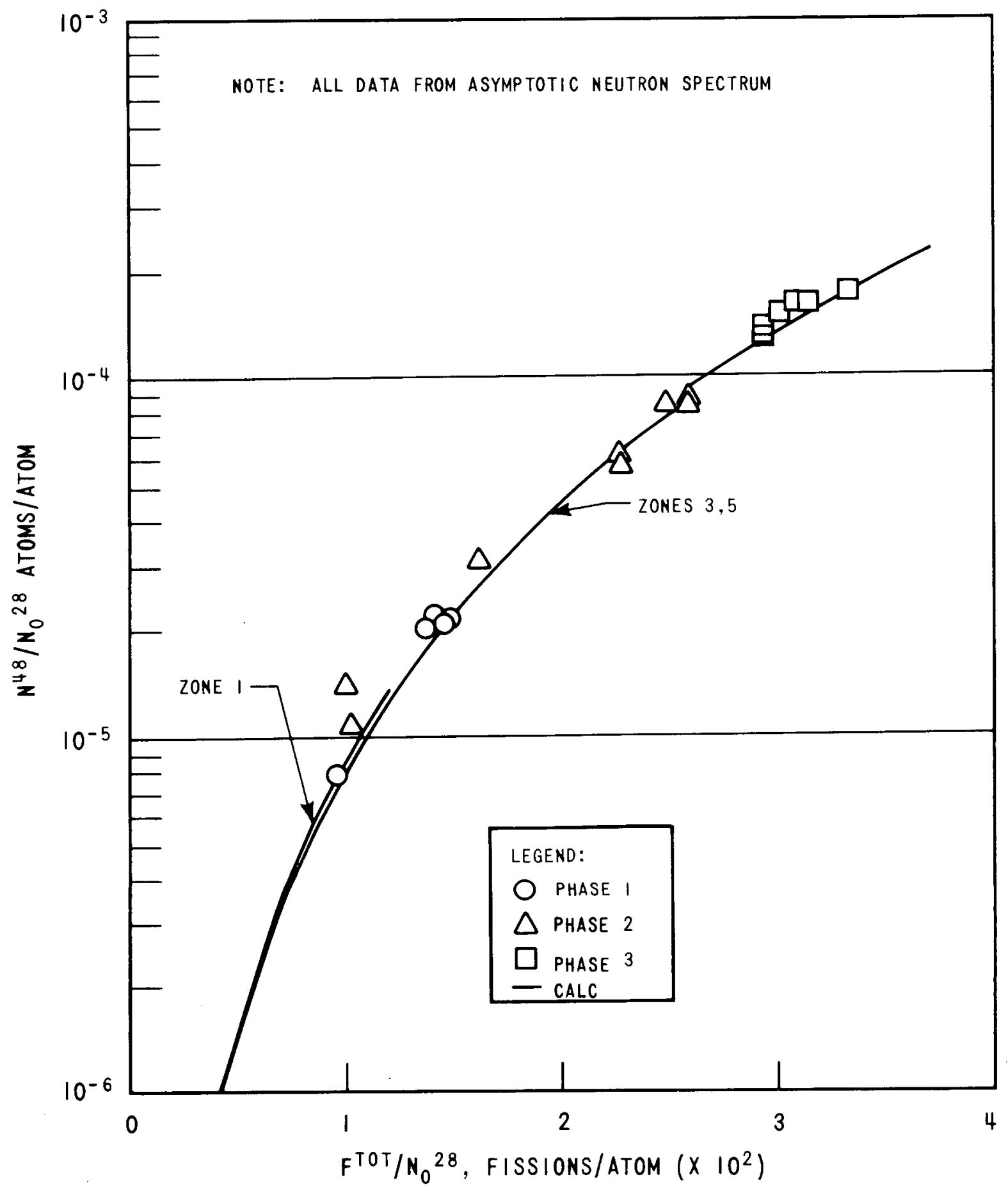

Figure 11-16. Calculated and Measured $\mathrm{Pu}-238$ Concentration as a Function of Accumulated Fissions 
and in the low burnup Phase 2 samples; however, the agreement between theory and experiment is quite good in the case of the high burnup Phase 2 samples. The discontinuity in the calculated curve of $\mathrm{Pu}-238$ production, at a value of $\mathrm{F}^{\mathrm{TOT}} / \mathrm{N}_{\mathrm{O}}{ }^{28}$ of about 0.026 , is due to the a-decay of $\mathrm{Cm}-242$ during the cooling time interval between Core II and Core IV operation.

The calculated and measured Pu-236 concentrations are shown in Figure 11-17. As was the case with the $\mathrm{Pu}-238$ data, the calculated Pu-236 concentrations are dependent on irradiation history (even more so due to the relatively short half-life of $\mathrm{Pu}-236,2.85$ years), and some simplifications were made in the presentation of the calculated $\mathrm{Pu}-236$ data in Figure 11-17. Although the calculation is somewhat low in the case of the low burnup samples, the calculated curve intercepts most of the error flags on the measured data points; in view of the scatter in the data for the low burnup samples and the relatively large uncertainties in the measurements, the agreement between theory and experiment is considered good. It is pointed out that, in the case of the Phase 1 samples, the elapsed time from Core I EOL to time of analysis for $\mathrm{Pu}-236$ was about five years, or about two $\mathrm{Pu}-236$ halflives.

Again, mention is made of the fact that the $n, 2 n$ cross sections used for Np-237 and U-238 were $1 / E$ weighted; the use of fission spectrum weighted $\mathrm{n}, 2 \mathrm{n}$ cross sections would decrease the calculated $\mathrm{Pu}-236$ concentration by roughly 25 percent.

The calculated and measured concentrations of U-232 are compared in Figure 11-18, where the data is presented in the form of the U-232/U-235 atom ratio. The discontinuities in the calculated curves indicate $\mathrm{Pu}-236 \alpha$-decay to U-232 during the time intervals between Core I EOL and Core II BOL, and between Core II EOL and Core IV BOL. The relative uncertainties on the U-232 measured data range from 60 to 100 percent on the cold samples (indicated by the solid symbols at zero burnup in Figure 11-18), and from 14 to 200 percent on the irradiated samples (including the statistical addition of the uncertainty in the Pu-236 measurements, a consequence of the necessity of referring the $\mathrm{U}-232$ data to core end-of-life by accounting for $\mathrm{Pu}-236$ a-decay during the time interval from core end-of-life to time of the analyses). 


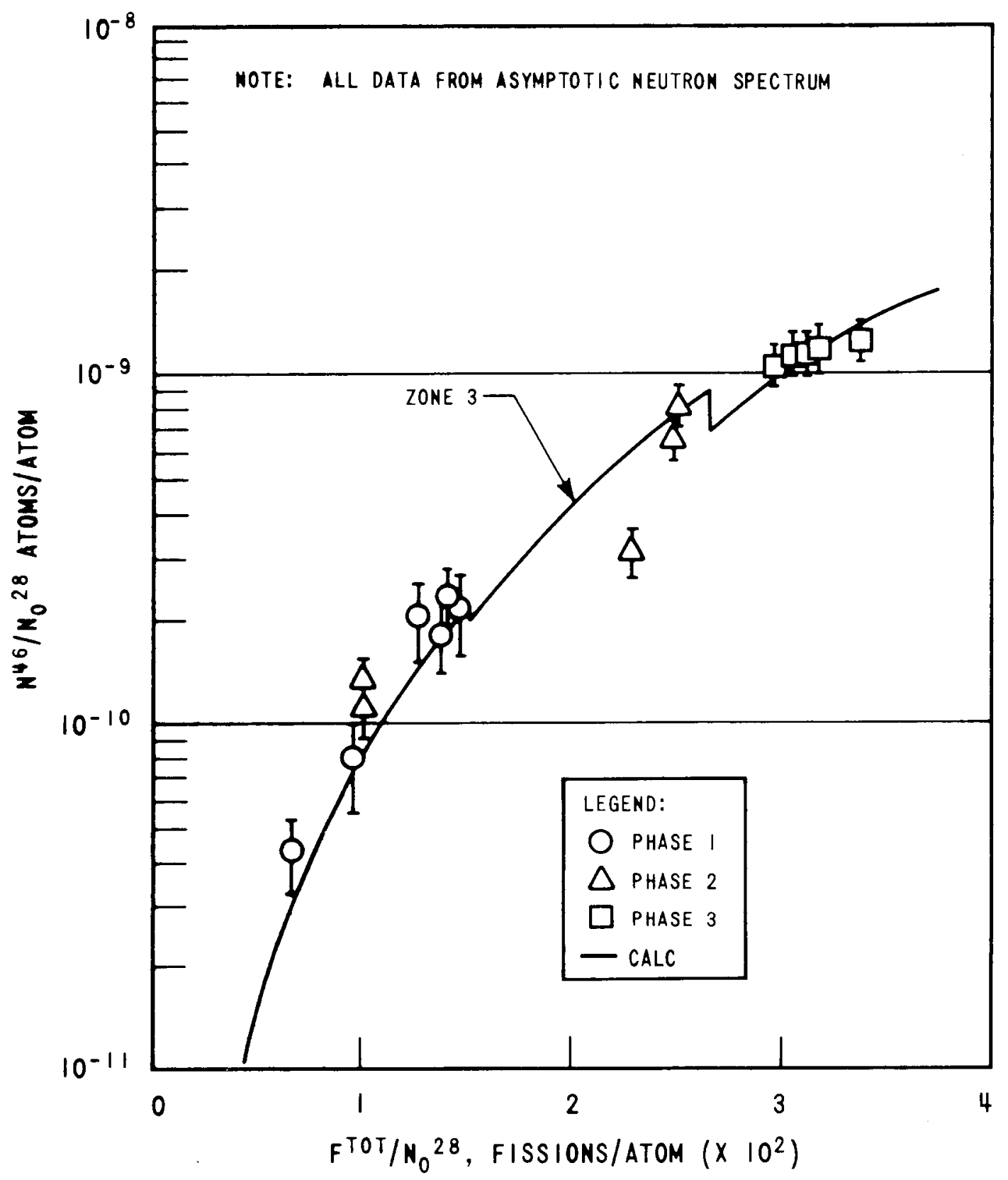

Figure 11-17. Calculated and Measured Pu-236 Concentration as a Function of Accumulated Fissions 


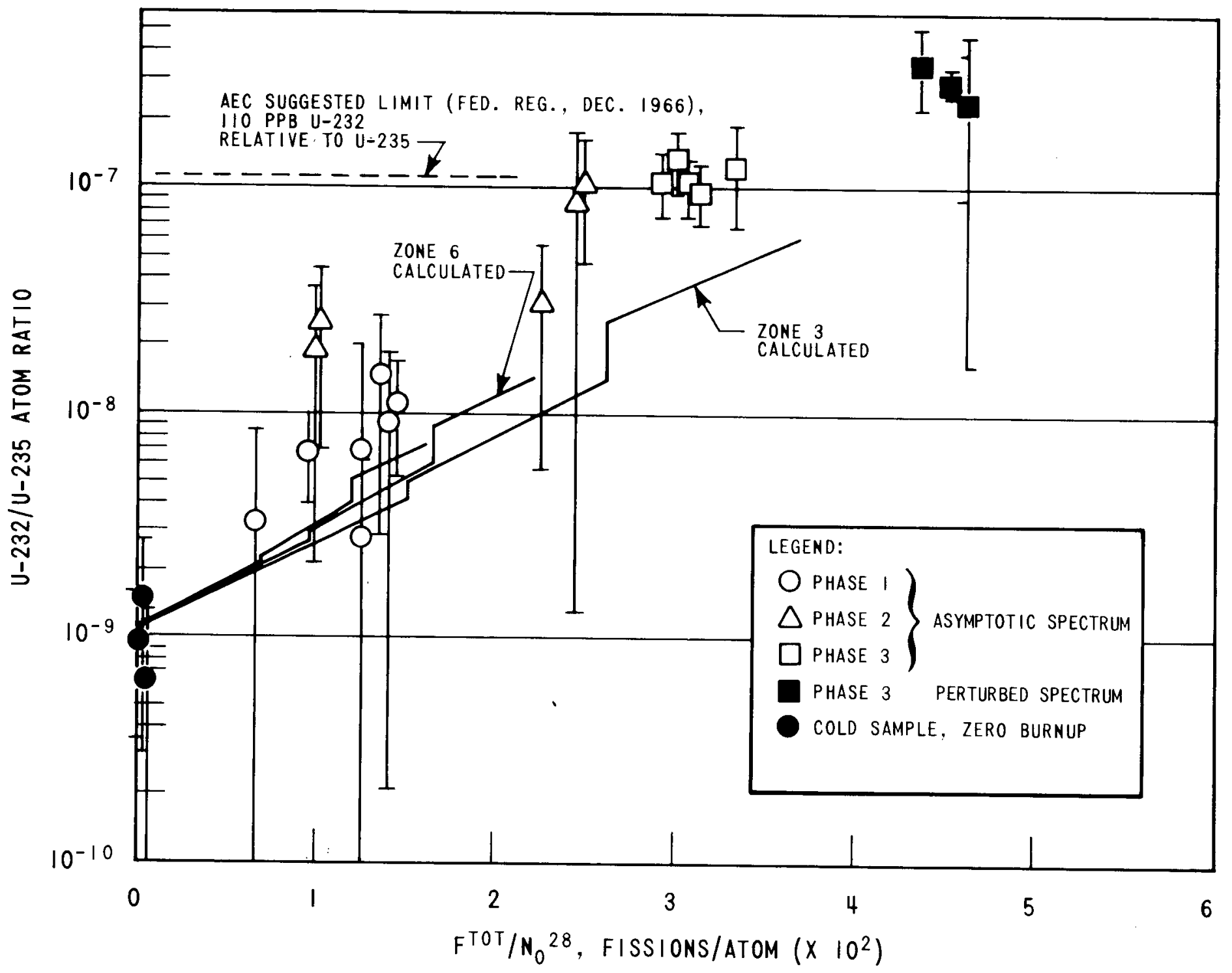

Figure 11-18. Calculated and Measured U-232 Concentrations versus Total Fissions 
The calculated U-232 concentration is low by a factor of two when the calculation is initialized at the average value of U-232 concentration determined from analyses on three solutions of an unirradiated sample of $\mathrm{U}_{3} \mathrm{O}_{8}$.

An anomaly is observed in the U-232 measured data in Figure 11-18. The Phase 1 and Phase 3 measured U-232 data appear to be more or less consistent; however, the Phase 2 measurements appear to be rather high. Although there is no conclusive evidence to explain the anomaly in the U-232 measured data, it is believed that, since the Yankee Core I fuel was provided by two different vendors, the fuel samples obtained in the different irradiation phases may have contained different initial concentrations of the U-232 contaminant, prior to irradiation.

In Figure 11-18, it is observed that the U-232 concentration in the high burnup samples (about 30,000 MWD/MTU) exceeds the AEC suggested limit of $0.11 \mathrm{ppm} \mathrm{U}-232$ relative to U-235. It is pointed out that the high burnup samples are taken from high burnup fuel assemblies, purposely recycled for extended burnup. Also, the Zone 3 calculation, at a burnup of about $25,000 \mathrm{MWD} / \mathrm{MTU}$, indicates a doubling of the U-232 concentration due to Pu-236 a-decay during the 1-year cooling time between Core II EOL and Core IV BOL*.

Some preliminary test run and debug calculations were performed in which the initial U-232 concentration was set equal to zero; other preliminary calculations were performed in which the U-232 concentration was initialized at a value corresponding to about four times the initial value of U-232/U-235 ratio shown for the final calculations in Figure 11-18. Also, some early program modification calculations were performed before it was thought to include in the scope of the study the analyses for initial Pa-231 in the cold fuel. Although no specific controlled series of burnup calculations

* It should be kept in mind that the U-232 concentrations shown on Figure 11-18 are referenced to core EOL; the U-232 concentration in the fueI at some subsequent time is a function of cooling time between discharge from the reactor and separation of plutonium from uranium at the reprocessor. 
were performed to evaluate the sensitivity of the U-232 concentration to the initial isotopic composition of the fuel prior to irradiation, the results of the many preliminary calculations mentioned above were examined and the following conclusions were reached:

1. If the value of U-232 initial concentration is assumed to be zero, instead of the initial value shown in Figure 11-18, the resulting calculated value of U-232 would be lower by roughly a factor of six at a value of $\mathrm{F}^{\mathrm{TOT}} / \mathrm{N}_{0}^{28}$ of 0.015 , and lower by about 25 percent at a value of $\mathrm{F}^{\mathrm{TOT}} / \mathrm{N}_{\mathrm{o}}^{28}$ of 0.030 .

2. If the initial U-232 concentration is a factor of three greater than that shown on Figure 11-18 (which corresponds roughly to the upper limit on the highest of the three measurements for U-232 in the cold fuel sample), the resulting calculated value of U-232 would be about 50 percent more at a value of $\mathrm{F}^{\mathrm{TOT}} / \mathrm{N}_{\mathrm{o}}{ }^{28}$ of 0.015 , and only somewhat more than the calculated values shown at relatively high burnup on Figure 11-18.

3. The sensitivity of net U-232 production (not in-place concentration) to initial $\mathrm{Pa}-231$ concentration is burnup dependent and ranges from approximately 0.2 atoms $\mathrm{U}-232$ per initial atom $\mathrm{Pa}-231$, at a burnup of about $7000 \mathrm{MWD} / \mathrm{MTU}$, to approximately 0.4 atoms U-232 per initial atom $\mathrm{Pa}-231$ at a burnup of about 20,000 MWD/MTU. It is estimated that as much as 25 percent relative error can be introduced into the calculation of U-232 production if the initial concentration of $\mathrm{Pa}-231$ is neglected.

Based on the above considerations, it is concluded that the calculational model and basic nuclear data used in this study can predict the $\mathrm{U}-232$ concentration in depleted fuel to within a factor of two, provided that the initial isotopic composition of the fuel, prior to irradiation, is reasonably well known.

Measurement for the U-233 concentration in the Yankee fuel was not included in the scope of the EYC Program Extension. However, some measurements for U-233 were performed in order to provide additional information on the 
composition of the pre-irradiated fuel*; at the same time an analysis for U-233 was carried out on the Yankee control sample 0994-3. The results of the analysis on the cold sample are given in Subsection 10.3. The result of a single measurement on the irradiated sample is $0.6 \pm 0.5 \mathrm{ppm} \mathrm{U}-233$, relative to uranium.

The calculated and measured concentration of U-233 are compared in Figure 11-19, where the U-233 data are presented as ppm relative to U-235. Included on the same figure, for reference, are some of the U-232 data shown previously in Figure 11-18. With regard to the Yankee Core I fuel, the data in Figure 11-19 indicate:

1. The U-233 is burning out, and the agreement between theory and experiment is good, even though just three measurements are available;

2. The U-233 concentration is about 100 times greater than the $\mathrm{U}-232$ concentration; and

3. The U-233 concentration is about 10 times less than the AEC

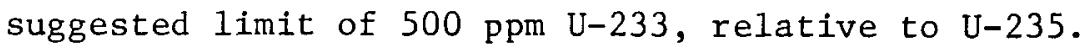

* Preliminary calculations resulted in calculated concentrations of U-232 which, relative to the measurements for $\mathrm{U}-232$, were low by factors of 20 , 10,6 , and 2 , depending on the stage of the LASER-HIC computer program deve1opment and modification at which these preliminary calculations were performed. For example, calculations in which the initial concentrations of U-232, U-233, and $\mathrm{Pa}-231$ were assumed to be zero, and in which the $\mathrm{Np}-236$ beta decay/ electron capture decay constant ratio was considerably different than that used in the final calculations, resulted in a calculated value of U-232 concentration some 20 times smaller than the measured values. At the time, it was thought that this large discrepancy in the U-232 calculation might possibly be due to neglect of $n, 2 n$ reaction in any $U-233$ that might have been initially present in the fuel, prior to irradiation; to investigate this possibility, the U-233 measurements were performed. 
$10^{3}$

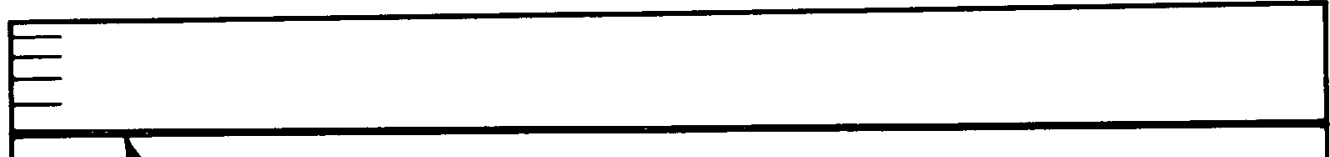

10

$10^{2}$

500 PPM

LIMIT
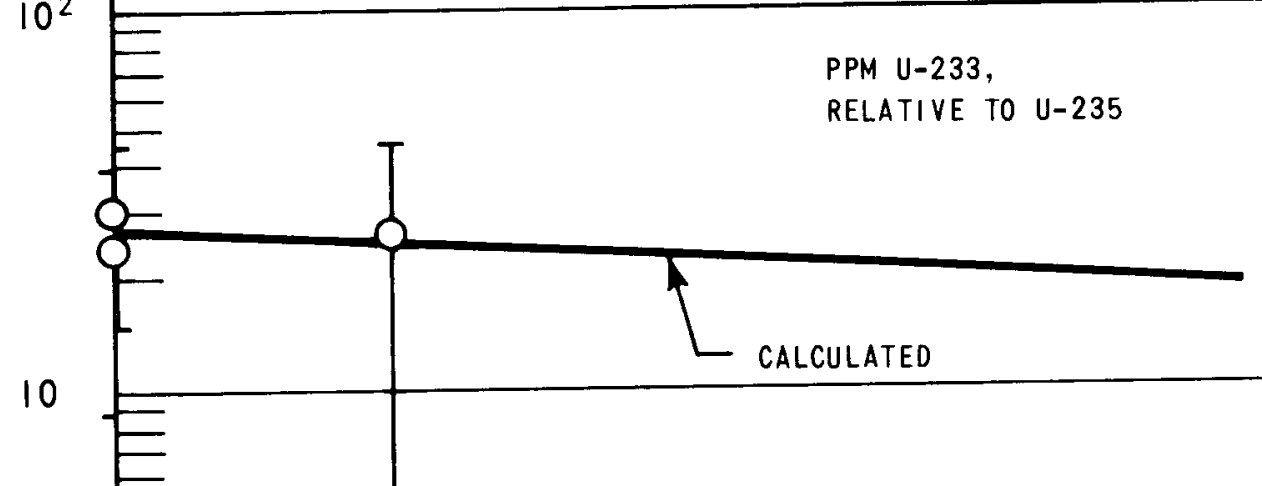

1.0

PPM U-232

RELATIVE TO U-235

0.1

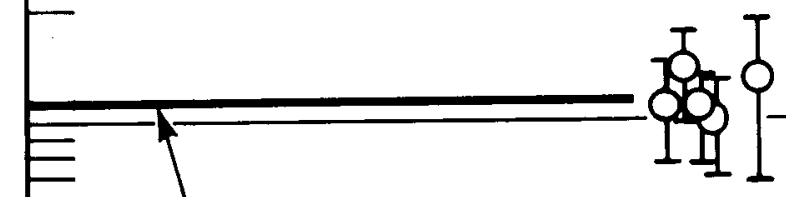

0.01

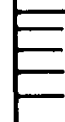

0.11 PPM

LIMIT

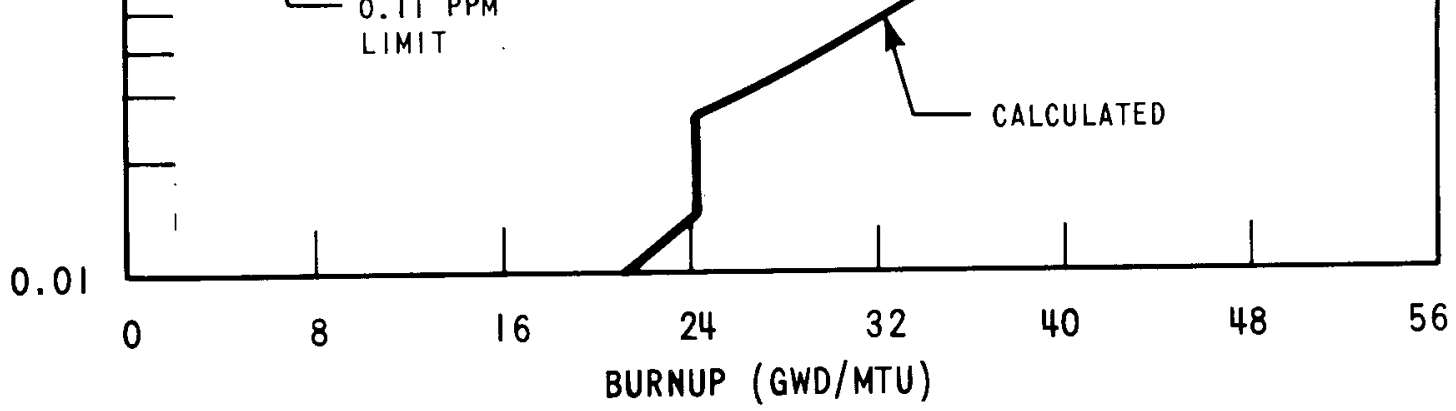

Figure 11-19. Calculated and Measured U-233 Concentrations versus Burnup in Yankee Core I Fuel 
The calculated and measured concentrations of Am-241 are compared in Figure 11-20, where the results of the measurements are referenced to core end-of1ife. The most immediate, and somewhat disconcerting, observations made with regard to the measured data are:

1. The results of some of the measurements, referenced to Core I EOL, have negative values (the data are purposely plotted on a linear scale in order to show the negative values as an example of the difficulties encountered in the interpretation of data when measurements are made some 5 to 6 years after the end of the irradiation period);

2. The scatter in the measured data is considerable; and

3. The error flags on the data are relatively large.

The apparently poor quality of the measured Am-241 data, as shown on Figure 11-20, is due to the necessity of referring the results of the measurements to core end-of-life in order to place the calculated and measured data on the same basis. The explanation for the negative values is that the magnitude of the correction for Pu-241 $\beta$-decay is larger than the measured value of Am-241 to which the decay correction is being applied.

In general, the scatter in the Am-241 data referenced to core EOL, and the large error flags, as shown in Figure 11-20, are due to the corrections for out-of-core Pu-241 B-decay; in effect, the difference between two values of nearly equal magnitudes, each having an associated value of uncertainty, results in a relatively small value with a relatively large uncertainty.

Some features of the calculated curves of Am-241 concentration shown in Figure 11-20 are:

1. During the 4-month cooling time between Core I EOL and Core II BOL, the concentration of Am-241 doubles due to Pu-241 B-decay; during the 1-year cooling time between Core II EOL and Core IV BOL, the concentration of Am-241 trebles due to Pu-241 $\beta$-decay, and

2. During the irradiation period corresponding to Core IV operation (Phase 3) the initial peak Am-241 burns out for a while, and then begins to increase on a new buildup curve. 


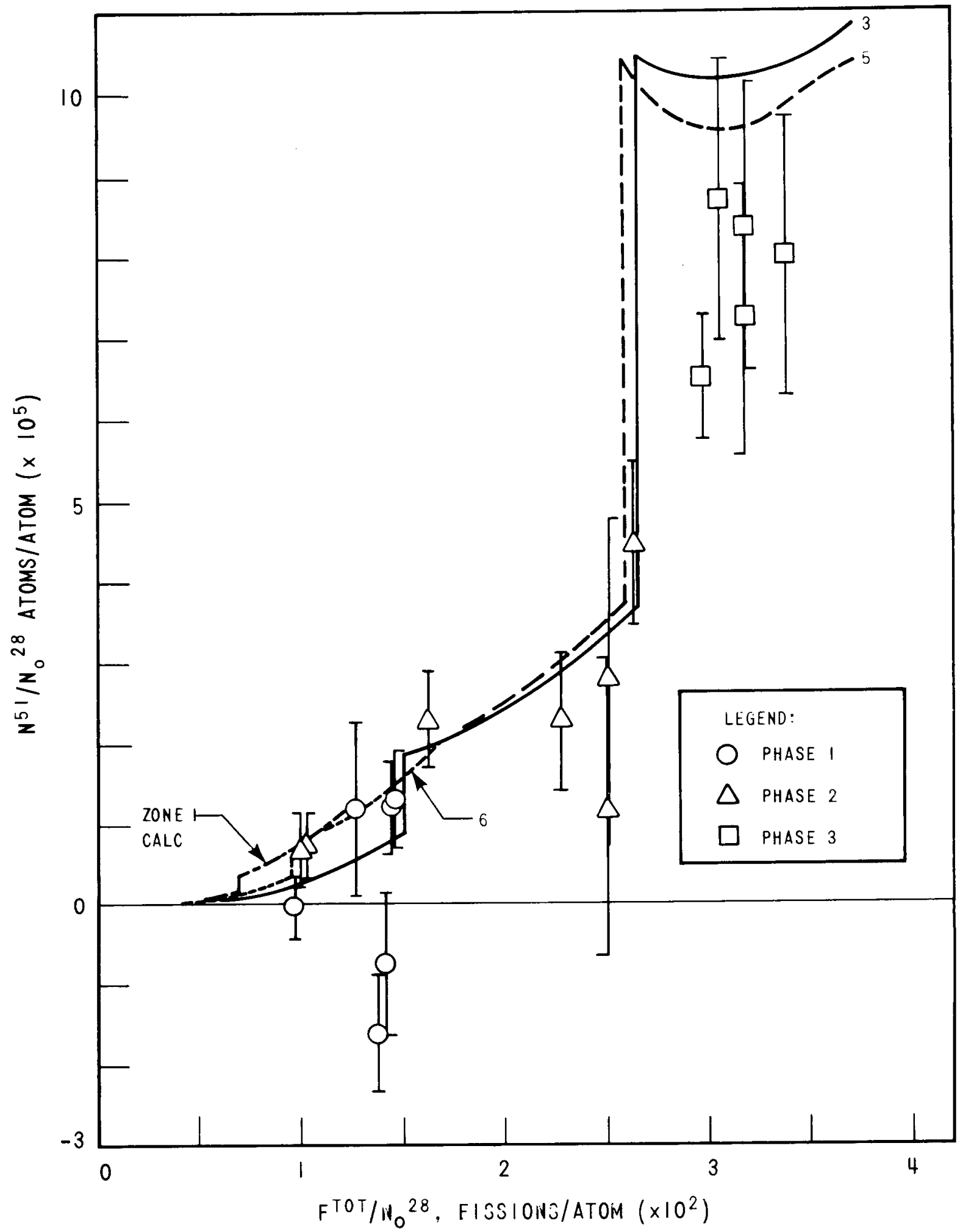

Figure 11-20. Calculated and Measured Am-241 Concentration as a Function of Accumulated Fissions 
The Phase 1 calculations for Am-241 are difficult to evaluate due to the poor resolution of the results of the Phase 1 measurements. The results of the Phase 2 measurements appear to be adequately resolved such that a fair evaluation of the calculations can be made; considering the seven Phase 2 data points (including two in Zone 1 and one in Zone 6), the calculated curves intercept all the error flags but one, with data points falling both above and below the calculated curves. The Phase 3 calculations for Am-24I concentration overpredict the measured data by about 20 percent; however, some part of this discrepancy is probably due to the Core IV environmental effect on the neutron spectrum in the Phase 3 fuel assembly (for example, a decrease in plutonium production, which in turn would cause a decrease in americium production), which cannot be accounted for in the unit cell calculations. Based most1y on the Am-241 data for Phase 2, it is concluded that theory and experiment agree to within about 10 percent.

Only five samples, a11 from Phase 3, were analyzed for Am-243; the results of four of the measurements (two from the asymptotic neutron spectrum, and two from the perturbed neutron spectrum) are shown plotted in Figure 11-21, along with the calculated curve. The results obtained from measurements on a fifth sample (from the perturbed neutron spectrum) seem to be inconsistent, giving a value of $\mathrm{N}^{53} / \mathrm{N}{ }^{28}$ of $21.6 \times 10^{-5}( \pm 17 \%)$ at a value of $\mathrm{F}^{\mathrm{TOT}} / \mathrm{N}_{\mathrm{O}}{ }^{28}$ of 0.044 . Referring to Figure $11-21$, it is concluded that the calculation overpredicts the Am-243 concentration; however, the possibility exists that the high value of $\mathrm{N}^{53} / \mathrm{N}_{0}^{28}$ for the fifth sample is valid, and it is the results for the other four samples, shown on the figure, which are inconsistent. This interpretation of the measured data would place the calculation in a more favorable light, if it is kept in mind that the calculation represents the asymptotic neutron spectrum and that the results for the fifth sample represents the perturbed neutron spectrum.

To pursue this argument further, the results of some analyses on Yankee fuel performed by the Pacific Northwest Laboratory, as part of the Plutonium Utilization Program conducted by Battelle Memorial Institute, and reported in Reference 57 were compared with the calculated curve for Am-243 concentration shown on Figure 11-21. This comparison (not shown in this report) indicates good agreement between theory and experiment. 


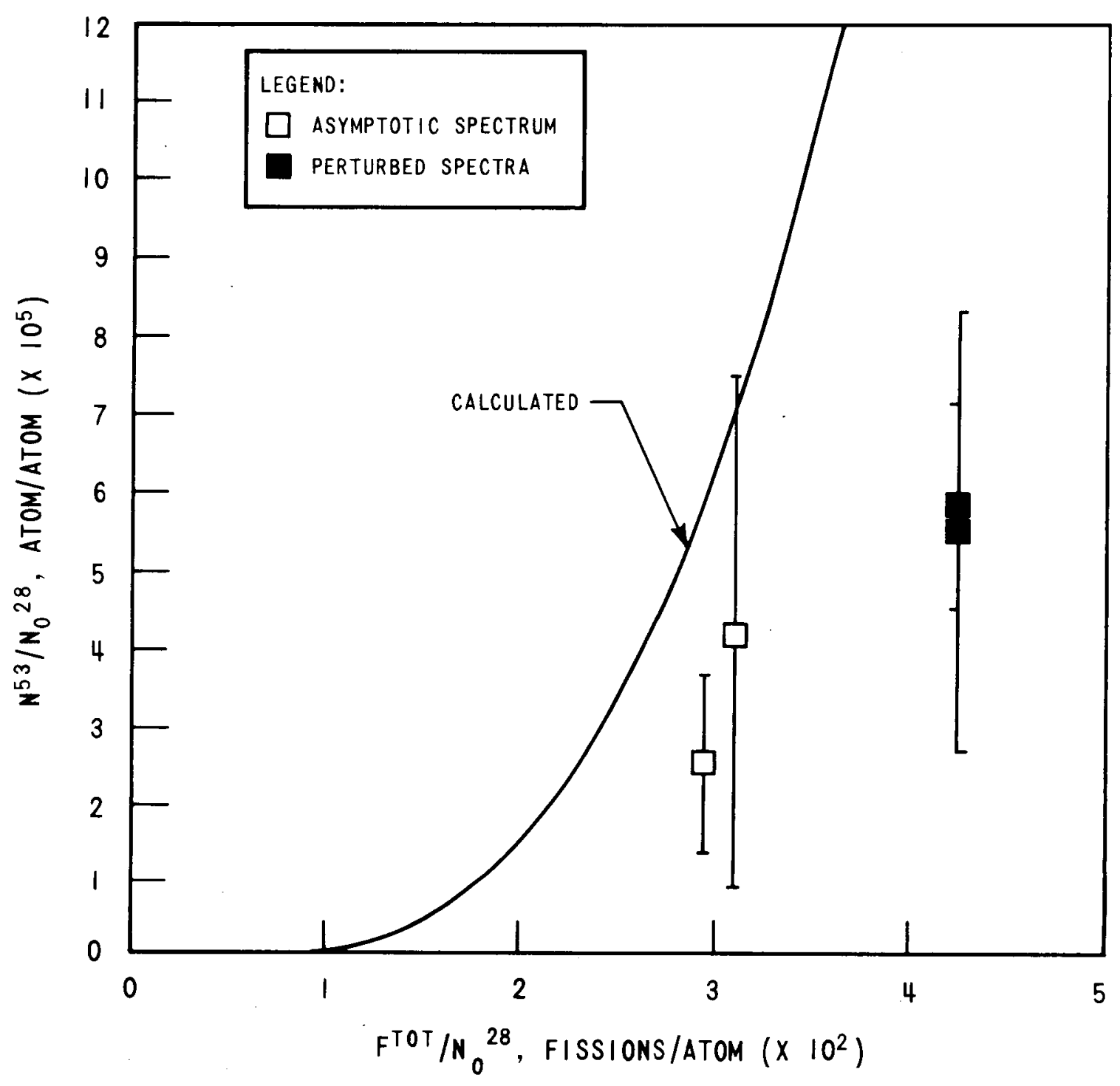

Figure 11-21. Calculated and Measured Am-243 Concentrations as a Function of Accumulated Fissions 
The calculated and measured $\mathrm{Cm}-242$ concentrations are compared in Figure 11-22. Discontinuties in the calculated curves indicate $\mathrm{Cm}-242$ a-decay during the cooling time intervals between Core I EOL and Core II BOL, and between Core II EOL and Core IV BOL. The calculation grossly underpredicts the $\mathrm{Cm}-242$ concentration in the Phase 1 samples; however, it is pointed out that the Phase 1 samples were analyzed for the special isotopes some 5 to 6 years after Core I end-of-life. To account for Cm-242 (164d) $\alpha$-decay from Core I EOL to time of analysis, correction factors ranging from 1700 to 2400 were applied to the measured data. Any uncertainty in the $\mathrm{Cm}-242$ half-1ife would significantly affect the experimentally determined value of $\mathrm{Cm}-242$ concentration, when referenced to Core I EOL. In addition, at the time of the analysis for $\mathrm{Cm}-242$ in the Phase 1 samples, the concentration of $\mathrm{Cm}-242$ was relatively low, making the analysis difficult. For these reasons, the validity of the comparison between the calculated and measured Phase 1 data is questionable. In the case of the Phase 2 and Phase 3 data, the agreement between theory and experiment is quite good; however, it is again pointed out that, due to uncertainties in irradiation history and the Core IV environmental effect, some cancellation of errors may be taking place. The measured $\mathrm{Cm}-242$ data in Figure $11-22$ are consistent with the results of measurements reported in Reference 57.

The calculated and measured $\mathrm{Cm}-244$ concentrations are compared in Figure 11-23. There is a consistent bias between the calculated and measured results, with the calculation underpredicting the $\mathrm{Cm}-244$ concentration by approximately 30 percent. The measured $\mathrm{Cm}-244$ data on Figure 11-23 are consistent with the results of measurements reported in Reference 57. It is suspected that the $\mathrm{Cm}-244 \mathrm{n}, \gamma$ cross sections used in the calculations performed in this study are too large.

In concluding the discussion of the comparisons between theory and experiment given in this section, the LASER-HIC final zone calculations must be qualified in the case of the higher isotopes Am-241, Am-243, Cm-242, and Cm-244. The final zone calculations, described in Subsection 10.1, were performed with the model and cross section library documented in Sections 8 and 9 , with one exception. In the case of the isomers of Am-242, the LASER-HIC 


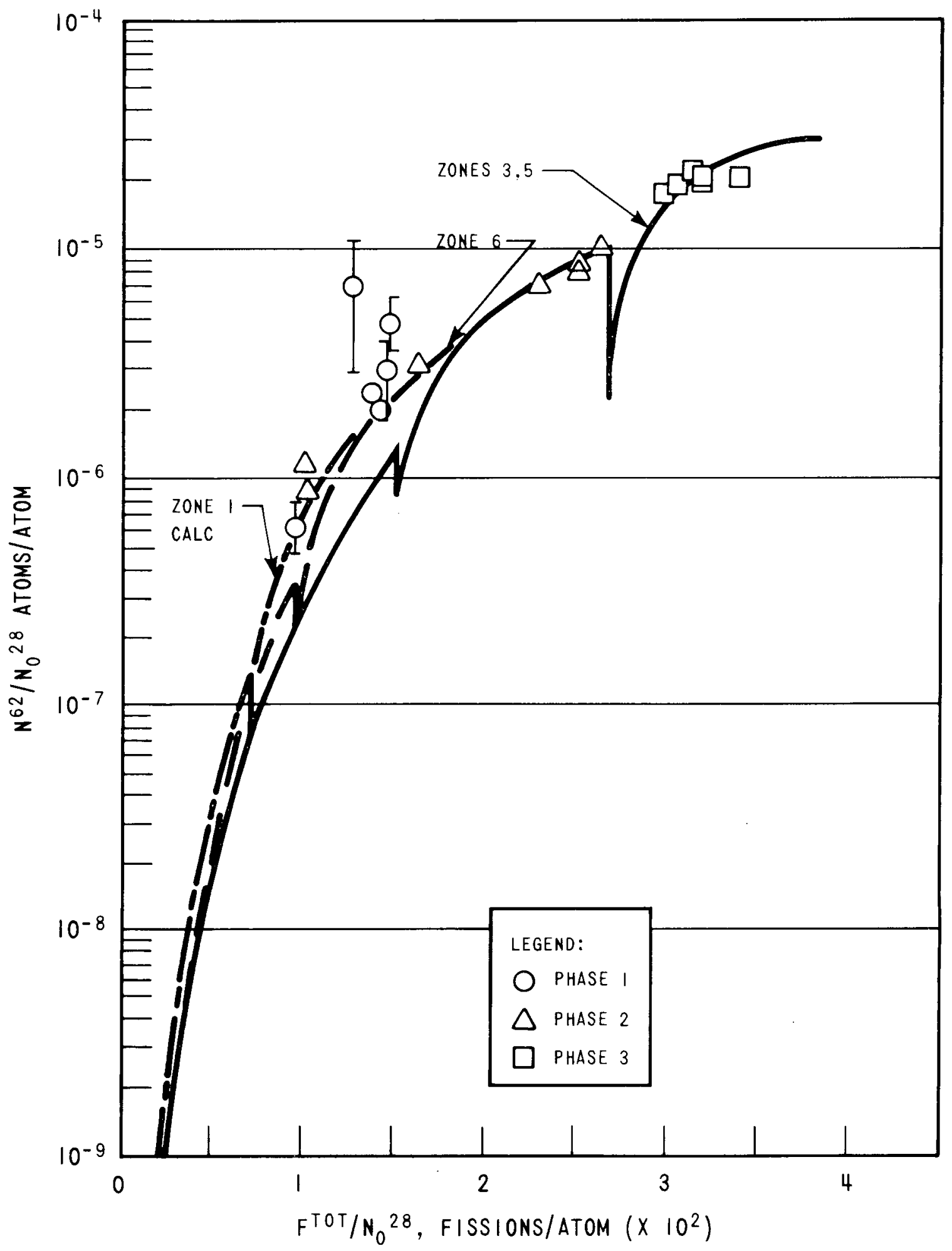

Figure 11-22. Calculated and Measured Cm-242 Concentration as a Function of Accumulated Fissions 


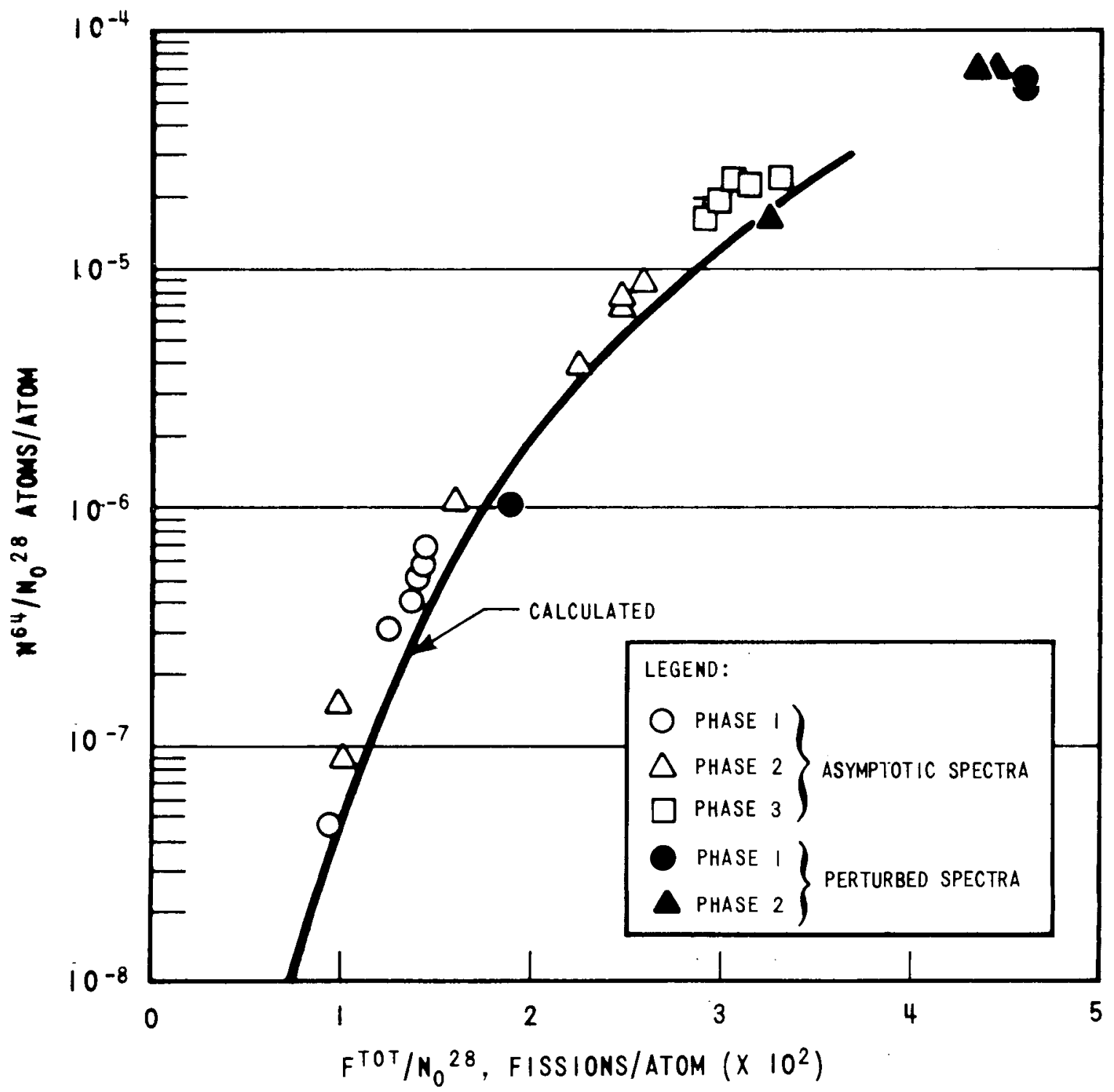

Figure 11-23. Calculated and Measured $\mathrm{Cm}-244$ Concentration as a Function of Accumulated Fissions 
final zone calculations utilized the transmutation chain description shown in Reference 58, rather than the description as shown on Figure 8-3; as a consequence, the cross sections used for Am-241 and Am-242 were somewhat different than those documented in Section 9 and Appendix C. It is believed that the chain description and cross section values given in Sections 8 and 9 and Appendix $C$ are more accurate than those used in the LASER-HIC final zone calculations as described above.

To evaluate the effect on the calculated concentrations of Am-241, Am-243, $\mathrm{Cm}-242$, and $\mathrm{Cm}-244$ due to these differences in the representation of the Am-242 isomers, the flat-out Zone 3 calculation (i.e., one run at power with no shutdowns and no restarts), described in Section 10, was performed with the LASER-HIC model and cross sections represented exactly as described in Sections 8 and 9 and Appendix $C$. Based on comparisons of results obtained from the flat-out Zone 3 calculation using the corrected version of the LASER-HIC program with results obtained from the final Zone 3 calculations, it appears that a coincidental and fortunate cancellation of errors occured to minimize the effects on the calculated isotopic concentrations ( in the case of the Phase 1 data); the effects of the change in the transmutation chain description (i.e., Am-242 and Am-242A reversed) were compensated by the effects of the corresponding changes in the cross section values for Am-241 and Am-242. It is estimated that the effect of the discrepancy in the representation of the Am-242 isomers with respect to the calculated concentrations of Am-241, Am-243, and $\mathrm{Cm}-242$ amounts to just a few percent; in the case of $\mathrm{Cm}-244$, it is estimated that the corrected LASER-HIC model would result in calculated values approximately 5 percent higher than the final zone calculations represented by the curve on Figure 11-23. 
SECTION 12

SUMMARY AND CONCLUSIONS

The EYC Phase 3 fuel assembly, irradiated in Yankee Cores I, II, and IV to a peak pellet burnup of 46,000 MWD/MTU, was destructively analyzed for the main chain uranium and plutonium isotopic composition. An extension to the EYC Program included, in addition to analyses for main chain uranium and plutonium isotopic composition, analyses for special isotopes of the elements thorium through curium in selected spent fuel samples from Phases 1 , 2, and 3; the Program Extension also included the development and evaluation of a theoretical model for the calculation of the special isotopes. Both EYC Phase 3 and the Program Extension included the evaluation of several methods of burnup determination; these included gross gamma scans for relative burnup distributions, and the Heavy Element (HE), Cs-137, and Nd-148 methods for absolute burnup determination.

The eight fuel rods removed from the Phase 3 fuel assembly were gross gamma scanned with two different detectors -- a miniature ionization chamber and a sodium iodide scintillation crystal -- and the resulting normalized gamma activity distributions were compared with each other, and with the normalized burnup distribution determined from destructive analysis of the fuel. It was found that the two gamma scan detectors did not give identical results; in the vertical plane, the scintillation crystal scans indicate a somewhat flatter gamma activity distribution than that measured with the miniature ionization chamber, with the scintillation crystal giving an axial peak-toaverage ratio about 7 percent less than the ion chamber; in the XY plane of the assembly, the assembly corner-to-center peaking in the two gamma activity distributions was different by about 12 percent, with the scintillation crystal giving consistently lower values. 
Comparisons of the normalized gamma activity distributions with the normalized burnup distribution in the axial plane indicated that the burnup distribution was skewed somewhat towards the bottom of the fuel assembly, while the gamma activity distributions were more nearly symmetrical about the mid-plane. In the axial plane, the average difference between the normalized ion chamber scans and the normalized burnup was 4 percent, ranging from as much as -7 percent in the central portions of the fuel rods to as much as +10 percent at the end portions of the rods; the average difference between the normalized scintillation crystal scans and the normalized burnup was about 9 percent, ranging from as much as -12 percent in the central portions of the fuel rods to as much as +37 percent at the end portions of the rods. In the XY plane of the assembly, it was observed that the ion chamber scans overpredicted the corner-to-center burnup ratio by about 7 percent, while the scintillation crystal scans underpredicted the corner-to-center burnup ratio by about 5 percent.

The discrepancy between the gamma activity distributions obtained with the ion chamber and the scintillation crystal is attributed to differences in the response of the two detectors to the spatially varying composition of gamma-emitters in the fuel (and cladding), which in turn is due to the burnup distribution and varying neutron spectra in both the axial and radial planes. The differences between the gross gamma activity distributions and the burnup distribution, assuming a linear correlation between gamma activity and burnup, are primarily functions of the time interval between core end-of-life and the gamma activity measurements; after relatively short cooling times, the gross gamma activity distribution is indicative of some combination of the end-of-life power distribution and average burnup, while after relatively long cooling times, the gross gamma activity distribution is more representative of the actual burnup distribution.

One of the objectives of the EYC Program Extension was the evaluation of the use of the fission product $\mathrm{Nd}-148$ as an accurate burnup indicator; however, as it turned out, the Nd-148 burnup data were used instead to evaluate the $\mathrm{HE}$ and Cs-137 burnup results. Throughout Phases 1, 2, and 3 of the EYC Program, a consistent burnup-dependent bias was observed between the results 
obtained with the $\mathrm{HE}$ and $\mathrm{Cs}-137$ methods, with the Cs-137 data indicating up to about 7 percent more burnup in high-burnup samples.

Two sets of fission yield constants were used in the determination of the burnup from the results of Nd-148 measurements. Comparisons of the HE burnup with the $\mathrm{Nd}-148$ burnup showed that the $\mathrm{HE}$ and $\mathrm{Nd}-148$ data were in statistical agreeement, regardless of which set of Nd-148 yields was employed; use of one set of yields resulted in agreement at low burnups and an average +2 percent difference at high burnups; use of the second set of $\mathrm{Nd}-148$ yields resulted in agreement at high burnups and an agerage -2 percent difference at low burnups. Relative to the Nd-148 burnup values, the $\mathrm{Cs}-137$ burnup values were high by as much as 3 or 5 percent on the average, depending upon which set of fission yield constants were used in the determination of the Nd-148 burnup.

The HIC computer program was developed for the solution of the isotopic transmutation equations based on the matrix exponential method, and was incorporated into the LASER code. Utilizing ENDF/B cross section data for those isotopes for which such data are available, and calculated cross section data for those isotopes for which no cross section data were available, LASER-HIC unit cell calculations were performed to determine the buildup and depletion of thirty isotopes of the actinide series of elements; these included, in addition to the main chain uranium and plutonium isotopes, the special isotopes whose concentrations were measured in the spent fuel samples -- U-232, U-233, Np-237, Pu-236, Pu-238, Am-241, Am-243, $\mathrm{Cm}-242$, and Cm-244.

Comparisons between calculated and measured isotopic concentrations showed good agreement between theory and experiment in general, thus verifying the adequacy of the calculational model and the relative accuracy of the basic nuclear data used in the calculations. In the particular conclusions given below, allowances have been made for the effects of uncertainties in the initial isotopic composition of the pre-irradiated fuel, the effects of uncertainties in specific sample irradiation history, and the Core IV environmental effect on the neutron spectrum in the Phase 3 fuel assembly. 
The agreement between the calculated and measured main chain uranium isotopic abundances is excellent, except for U-234 abundance in high-burnup samples where the calculation overpredicts the U-234 abundance by about 3 to 8 percent, relative. The overall agreement between calculated and measured main chain plutonium isotopic abundances is quite good, and in the case of Pu-239 and $\mathrm{Pu}-240$ is excellent; in the case of $\mathrm{Pu}-241$ and $\mathrm{Pu}-242$, the calculation is in good agreement with measurements in high-burnup samples, but tends to underpredict the abundance in low-burnup samples, even though the calculated curves of $\mathrm{Pu}-241$ and $\mathrm{Pu}-242$ pass through many of the measured data points. The calculated and measured main chain uranium and plutonium isotopic concentrations agree to within about 2 to 5 percent which is approximately the degree of uncertainty in the measurements; due to scatter in the data, it appears that resolution of the discrepancy between theory and experiment below this level is not possible.

In the case of the special isotopes, the agreement between theory and experiment varies from isotope to isotope, and appears to be related to the location of a particular isotope in the transmutation chains with respect to its proximity to the main chain. Due to the uncertainties associated with weighting the $n, 2 n$ cross sections used in the calculation, it is believed that the agreement between theory and experiment is due, in some part, to fortuitous cancellation of error.

With the methods used in this study, the calculations predicted the Np-237, $\mathrm{Pu}-238$, and Pu-236 concentrations to within approximately 8, 5, and 15 percent, respectively. The U-232 concentration was calculated low by roughly a factor of two, relative to the measurements.

The results of measurements for Am-241 were difficult to interpret due to the necessity of accounting for Pu-241 $\beta$-decay during the cooling time interval between core end-of-life and the analyses; however, based on some measured data which appeared to be fairly well resolved, it is estimated that the calculations predict the Am-241 concentration to roughly 10 percent. 
In the case of Am-243, the results from the few measurements made in this program are highly suspect; however, comparison of the calculated concentrations with results of measurements reported by others [57] indicates good agreement between theory and experiment.

Comparisons between calculated and measured $\mathrm{Cm}-242$ concentrations in the Phase 1 samples indicated a gross discrepancy between theory and experiment; however, the validity of the comparisons is questionable due to the magnitude of correction factors (on the order of 2000) applied to the measurements to account for $\mathrm{Cm}-242$-decay during the 5- to 6-year cooling time interval between Core I EOL and the time of the Cm-242 analysis. In the case of the Phase 2 and Phase 3 samples, however, the calculated and measured $\mathrm{Cm}-242$ concentrations agreed to about 5 percent, which is approximately the uncertainty in the measurements.

Comparisons between calculated and measured $\mathrm{Cm}-244$ concentrations showed a gross bias between theory and experiment, with the calculations underpredicting the $\mathrm{Cm}-244$ concentration by approximately 25 percent; it is believed that the discrepancy is due largely to the use of $\mathrm{Cm}-244 \mathrm{n}, \gamma$ cross sections which are too large.

A description of the calculational model and the cross section library used in this study is fully documented, along with the results of all the isotopic measurements. 
SECTION 13

\section{LIST OF REFERENCES}

1. O'Boyle, M. J., et al., "Yankee Core Evaluation Program Quarterly Progress Report for the Period Ending June 30, 1967," WCAP-6085 (October 1967).

2. O'Boyle, M. J., et. al., "Yankee Core Evaluation Program Semi-Annual Progress Report for the Period Ending March 31, 1967," WCAP-6083 (August 1967).

3. Duane, B. H., "Transmutation Logic for Sn Transport Analysis," Proceedings of the Conference on the Application of Computing Methods to Reactor Problems, May 17-19, 1965, ANL-7050 (August 1965), pp. $291-298$.

4. Poncelet, C. G., "Burnup Physics of Heterogeneous Reactor Lattices," WCAP-6069 (January 1965).

5. Nodvik, R. J., "Evaluation of Mass Spectrometric and Radiochemical Analyses of Yankee Core I Spent Fuel, "WCAP-6068 (March 1966).

6. Paxson, E. and Smalley, W. R., "Engineering and Metallurgical Evaluation of the Yankee Core I Spent Fue1, "WCAP-6084 (June 1967).

7. Nodvik, R. J., "Evaluation of Gamma Activity Distributions in Yankee Core I and Core II, "WCAP-6067 (June 1965).

8. Picone, L. F. and Taylor, G. R., "Chemical Evaluation of Yankee Core I Fuel Cladding Corrosion Products,"WCAP-6072 (August 1966).

9. Jedruch, J. and Nodvik, R. J., "Experimentally Determined Burnup and Spent Fuel Composition of Yankee Core I, "WCAP-6071 (July 1965).

10. Nodvik, R. J., Guthrie, C. E., and Corbett, J. A., "Evaluation of Fue1 Accountability Methods Applied to Yankee Fuel," WCAP-3896-1 (June 1968).

11. Lacey, P. G. and Radcliffe, R. E., "Diffusion-Theory Depletion Analysis of the Yankee Core," WCAP-6077 (1966).

12. Yankee Atomic Electric Company, "Technical Information and Final Hazards Summary Report," AEC Docket No. 50-29, Part B, License Application, Vol. I. 
REFERENCES (cont)

13. Graves, H. W. Jr., et a1., "The Nuclear Design of the Yankee Core,: YAEC-136 (February 1961).

14. Poncelet, C. G., "Analysis of the Reactivity Characteristics of Yankee Core I," WCAP-6050 (January 1963).

15. Chajson, L., et al., "Yankee Core Evaluation Program Quarterly Progress Report for the Period Ending June 30, 1963," WCAP-6055 (July 1963).

16. James, B., Payne, D. D., and Walchli, H. E., "Experience in the Shipment of Yankee Fuel Assemblies for Post-Irradiation Examination," WCAP-6062 (February 1965).

17. Rider, B. F., et al., "Accurate Nuclear Fuel Burnup Analysis, Eighteenth Quarterly Progress Report, March-May, 1966" GEAP-5174.

18. Chajson, L., et al., "Yankee Core Evaluation Program Semi-Annual Progress Report for the Period Ending March 31, 1964." WCAP-6061 (April 1964).

19. Rider, B. F. et al., "The Determination of Uranium Burnup in MWD/Ton," GEAP-3373 (March 1960).

20. Jedruch, J. and Nodvik, R. J., "Determination of Core Burnup, Isotopic Inventory, and Conversion Ratio from Selective Sampling of Spent Reactor Fuel," Nuclear Applications, 3, 507-518 (August 1967).

21. Poncelet, C. G., "LASER - A Depletion Program for Lattice Calculations Based on Muft and Thermos," WCAP-6073 (April 1966).

22. Barry, R. F., "LEOPARD - A Spectrum Dependent Non-Spatial Depletion Code for the IMB-7094." WCAP-3269-26 (September 1963).

23. Englnad, T. R., "Time-Dependent Fission-Product Thermal and Resonance Absorption Cross Sections," WAPD-TM-333 (November 1962).

24. Rider, B. F., Ruiz, C. P., Peterson, J. P., Jr., and Smith, F. R., "A Survey and Evaluation of Thermal Fission Yields for U-235, Pu-239, U-233, and Pu-241," GEAP-5356 (September 1967).

25. Private communication (May 1969) - Corbett, J. A., Analytical Service Laboratories, Westinghouse Advanced Reactors Division, Waltz Mill, Pa.

26. Ruiz, C. P., Peterson, J. P., Jr., and Rider, B. F., "Therma1-Neutron Cross-Section and Resonance Capture Integral of Neodymium-148," Trans., Am. Nuc1. Soc., 7, 270 (November 1964). 
REFERENCES (cont)

27. Matsen, R. P., Busselman, G. J., Holeman, R. H., and Liikala, R. C., "An Analysis of Uranium Fuel Irradiated in Yankee Reactor," Trans., Am. Nucl. Soc., 12, 31 (June 1969).

28. Kern, R. and Bonaca, M., "Comparisons Between Simple Calculations and Measurements of Yankee Rowe Fuel Inventories," Trans., Am. Nuc1. Soc., 12, 32 (June 1969).

29. Nodvik, R. J., Guthrie, C. E., and Corbett, J. A., "Evaluation of Fuel Accountability Methods Applied to Yankee Fuel, "Trans., Am. Nucl. Soc., 12, 92 (June 1969).

30. Lisman, F. L. et a1., "Burnup Determination of Nuclear Fuels Project Report for the Quarter April 1 to June 301968 and Final Report." IN-1277 (March 1969).

31. Krug, H. E., Nodvik, R. J., Corbett, J. A., and Azziz, N., "Matrix Exponential Calculations and Comparison with Measurements of Isotopic Concentrations in Yankee Core I," Trans., Am. Nucl. Soc., 12, 52 (June 1969).

32. Private communication (1967) - Poncelet, C. G., Reactor Development, Westinghouse Nuclear Energy Systems.

33. Ball, S. J. and Adams, R. K., "MATEXP, A General Purpose Digital Computer Program for Solving Ordinary Differential Equations by the Matrix Exponential Method, "ORNL-TM-1933 (August 30, 1967).

34. England, T. R., "CINDER - A One-Point Depletion and Fission Product Program," WAPD-TM-334 (August 1962).

35. Bellman, R., "Perturbation Techniques in Mathematics, Physics, and Engineering," Holt, Rinehart and Winston, Inc. (1964).

36. Private communication (1968) - Kasdorf, J. R., Digital Computation Services, Reactor Development, Westinghouse Nuclear Energy Systems.

37. Stehn, J. R., et al., "Neutron Cross Sections Volume III, $Z=88$ to 98 ," BNL-325, Second Edition, Supplement No. 2 (February 1965).

38. Pearlstein, S., and Honeck, H., ENDF/B, BNL-50066, Tapes 103, 114, 115, 1967-68.

39. Stewart, K. B., "BNW Master Library," BNWL-CC-325 (September 1965). 


\section{REFERENCES (cont)}

40. Azziz, N., and Connelley, J. W., "Interpreting Fast Reaction Cross Sections by Statistical Methods," WCAP-7280 (1969).

41. Eich, W. J., and Nodvik, R. J., "Applications of Modified ENDF/B Pu Cross Sections to Mixed-Oxide Critical Experiments and Isotopic Buildup," Trans., Am. Nucl. Soc., 12, 228 (June 1969).

42. Goldman, D. T., and Roesser, J. R. (prepared by), "Chart of the Nuclides," Ninth Edition-Revised to July 1966, Knolls Atomic Power Laboratories, Operated by the General Electric Company under direction of Naval Reactors, U. S. Atomic Energy Commission.

43. Lederer, C. M., Hollander, J. M., and Perlman, I., "Table of Isotopes," Sixth Edition, John Wiley \& Sons, Inc., New York, N. Y. (1967).

44. Weast, R. C. (editor), "Handbook of Chemistry and Physics," Fortyninth Edition, 1968-1969, The Chemical Rubber, Co., Cleveland, Ohio.

45. Stepan, I. E., and Nisle, R. G., "Measurement of Plutonium-241 Half-Life by a Reactivity Method," Trans., Am. Nuc1. Soc., 9, 451 (OctoberNovember 1966).

46. Dannels, R. A., and Kusner, D. E., "ETOM-1 -A FORTRAN IV Program to Process Data from the ENDF/B File to the MUFT Format," WCAP-3688-1 (May 1968).

47. Marlowe, O. J., and Ombrellaro, P. A., "CANDLE, A One-Dimensional Few Group Depletion Code for the IBM-704," WAPD-TM-53 (1957).

48. O'Boyle, M. J. (compiled and edited by), "Yankee Core Evaluation Program Quarterly Progress Report for the Period Ending March 31, 1966," WCAP-6080 (June 1966).

49. Hendley, S. M., and Mangan, R. A., "TURBO - A Two-Dimensional FewGroup Depletion Code for the IBM-7090," WCAP-6059 (March 1964).

50. Pfiefer, C. F., and Urbanus, F. R., "ZIP-2, A One-Dimensional, Few-Group Depletion Program for the Philco-2000 Computer," WAPD-TM-228 (November 1961).

51. Callaghan, J. B., et al., "TURBo - A Two Dimensional Few Group Depletion Code for the IBM-704," WAPD-TM-95 (1957).

52. Poncelet, C. G., "Effects of Fuel Burnup on Reactivity and Reactivity Coefficients in Yankee Core I," WCAP-6076 (October 1965). 


\section{REFERENCES (cont)}

53. O'Boyle, M. J. (compiled and edited by), "Yankee Core Evaluation Program Quarterly Progress Report for the Period Ending June 30, 1966," WCAP-6081 (August 1966).

54. O'Boyle, M. J. (compiled and edited by), "Yankee Core Evaluation Program Quarterly Progress Report for the Period Ending September 30, 1966," WCAP-6082 (Apri1 1967).

55. McGaugh, J. D. and Chastain, R. H., "Power Density and Burnup Distributions in Yankee Core I," WCAP-6051 (January 1963).

56. Sha, W. T., "Effective Resonance Temperature Correlation of $\mathrm{UO}_{2}$," Nucl. App1., 1, (December 1965), pp. 538-545.

57. Matsen, R. P., "An Analysis of Yankee-Rowe Burnup Data," BNWL-1122 (July 1969).

58. Radiological Health Handbook, Revised Edition September 1960, compiled and edited by Division of Radiological Health, U. S. Department of Health, Education, and Welfare.

59. Meek, M. E. and Rider, B. F., "Summary of Fission Product Yields for U-235, U-238, Pu-239, and Pu-241 At Therma1, Fission Spectrum and 14 MeV Neutron Energies," APED-5398 (March 1, 1968).

60. MILC Library, available from PHILCO Corporation, Willow Grove, Pa. (July 1, 1961).

61. Smalley, W. R., "Saxton Plutonium Project Quarterly Progress Letter for the Period Ending September 30, 1969," WCAP-3385-21 (November 1969).

62. Eich, W. J., "A Status Report for the Current Evaluation of Modified ENDF/B Representations for the Isotopes of Plutonium: 239, 240, 241, and 242", WCAP-7365 (October 1969). 
APPENDIX A

SUMMARY OF RESULTS FROM MASS SPECTROMETRIC AND RADIOCHEMICAL ANALYSES

The results of mass spectrometric and radiochemical analyses are shown in Table A-1. The plutonium abundance data (atom percent) and the Cs-137 and Sr-90 activities are referenced to core end-of-life; the data for Phase 1 samples are referenced to Core I EOL, the data for Phase 2 samples are referenced to Core II EOL, and the data for Phase 3 samples are referenced to Core IV EOL. The relative uncertainties on the $U$ and $\mathrm{Pu}$ abundance data are of the order of a few tenths of a percent, ranging from about $\pm 0.2 \%$ to about $\pm 0.9 \%$. The relative uncertainties on the Cs-137 and Sr-90 data are about $\pm 6 \%$ and $\pm 8 \%$, respectively.

Also shown in Table A-1 are the results of alpha spectrometric analysis for Pu-236 and Pu-238 by two laboratories. The Pu-236 and Pu-238 data are given in terms of atomic ratios (relative to either U-238 or $\mathrm{Pu}-239$ ) at the time of analysis. Note that no corrections have been made for $\mathrm{Pu}-236, \mathrm{Pu}-238$, and Cm-242 out-of-core alpha decay.

The $\mathrm{Pu} / \mathrm{U}$ mass ratio data obtained from mass spectrometric analyses and $\mathrm{X}$-ray fluorescence analyses are summarized in Table A-2. The relative uncertainties on the mass spectrometric data are of the order of \pm 2 to $\pm 3 \%$, while the relative uncertainties on the $x$-ray fluorescence data are of the order of $\pm 4 \%$ to $\pm 5 \%$.

The measured data in Tables $\mathrm{A}-1$ and $\mathrm{A}-2$, including quoted uncertainties, were initially reported in References $1,2,48,53$, and 54. 


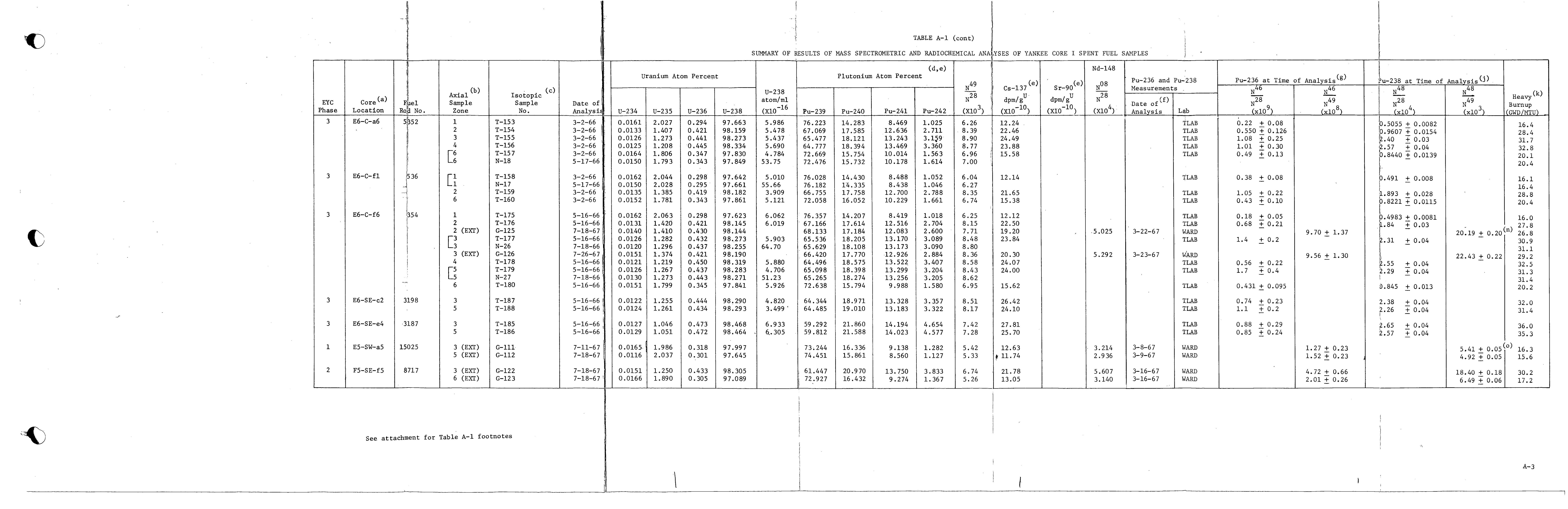


NOTES.

(a) In the fuel rod designation, the letter $\mathrm{C}$ indicates asymptotic neutron spectrum; the letters SE, SW, and NW indicate non-asymptatic neutron spectra.

(b) Entries in brackets Indicate Interlaboratory crosscheck analyses; EXT in parentheses Indicates EYC Program Extension samples.

(c) The letters $T, G$, and $N$ indicate mass spectrometric analysis for main chain U and Pu isotopes by TRACERLAB, GE-Vallecitos, and New Brunswick, respectively. All analyses for $\mathrm{Cs}-137$ and $\mathrm{Sr}-90$ by Westinghouse Advanced Reactors Division (WARD).

(d) Pu-236 and Pu-238 not included.

(e) Referenced to core end-of-life (EOL); Phase 1, 2, and 3 data referenced to Core I, II, and IV EOL, respectively.

(f) Date of analysis for Pu-236 and Pu-238 by Westinghouse Advanced Reactors Division (WARD); dates for TRACERLAB analyses taken to be those indicated for mass spectrometric analysis for main chain $\mathrm{U}$ and $\mathrm{Pu}$ isotopes.

(g) Not corrected for $\alpha$-decay from core EOL to time of analysis.

(h) Analyzed in fuel accountability evaluation program -- Reference 10.

(1) Analyzed in SAXTON Plutonium Project -- Reference 61.

(j) Not corrected for Pu-238 and $\mathrm{Cm}-242 \alpha$-decay from core EOL to time of analysis.

(k) Units on burnup are $10^{9}$ watt-days per $10^{6}$ grams initial uranium loading.

(1) Re-analysis for $\mathrm{N}^{48} / \mathrm{N}^{49}$ gave $9.61( \pm 0.20) \times 10^{-3}$ atoms/atom.

(m) Re-analysis for $\mathrm{N}^{48} / \mathrm{N}^{49}$ gave $13.5( \pm 0.4) \times 10^{-3}$ atoms/atom.

(n) Re-analysis for $\mathrm{N}^{48} / \mathrm{N}^{49}$ gave $20.6( \pm 0.4) \times 10^{-3}$ atoms/atom.

(o) Re-analysis for $\mathrm{N}^{48} / \mathrm{N}^{49}$ gave $5.46( \pm 0.15) \times 10^{-3}$ atoms/atom.

(p) $\mathrm{N}^{48} / \mathrm{N}^{28}$ analyzed by TRACERLAB on $5 / 1 / 67$.

(q) $\mathrm{N}^{48} / \mathrm{N}^{28}$ analyzed by TRACERLAB on $6 / 20 / 67$. 
TABLE A-2

COMPARISON OF EYC PU/U/MASS RÁTIO FROM MASS SPECTROMETRIC AND X-RAY FLUORESCENCE ANALYSES

\begin{tabular}{|c|c|c|c|c|c|c|c|c|c|c|c|c|c|}
\hline \multirow[b]{2}{*}{$\begin{array}{l}\text { Core } \\
\text { Location }\end{array}$} & \multirow[b]{2}{*}{$\begin{array}{l}\text { Fuel } \\
\text { Rod No. }\end{array}$} & \multirow{2}{*}{$\begin{array}{l}\text { Axial } \\
\text { Sample } \\
\text { Zone }\end{array}$} & \multirow[b]{2}{*}{$\begin{array}{l}\text { Sample } \\
\text { Code No. }\end{array}$} & \multicolumn{2}{|c|}{$\begin{array}{c}\text { (a) } \\
\text { Mass Ratio } \\
\left(\times 10^{2}\right)\end{array}$} & \multirow[b]{2}{*}{$\begin{array}{c}\text { Deviation } \\
\frac{\text { X-ray-Iso }}{I \text { so }} \times 100 \%\end{array}$} & \multirow[b]{2}{*}{$\begin{array}{c}\text { Core } \\
\text { Location }\end{array}$} & \multirow[b]{2}{*}{$\begin{array}{l}\text { Fue I } \\
\text { Rod No. }\end{array}$} & \multirow{2}{*}{$\begin{array}{l}\text { Axial } \\
\text { Sample } \\
\text { Zone }\end{array}$} & \multirow[b]{2}{*}{$\begin{array}{l}\text { Sample } \\
\text { Code No. }\end{array}$} & \multicolumn{2}{|c|}{$\begin{array}{c}\text { (a) } \\
\text { Mass Ratio }\left(\times 10^{2}\right)\end{array}$} & \multirow[b]{2}{*}{$\begin{array}{c}\text { Deviation } \\
\frac{\text { X-ray-Iso }}{\text { Iso }} \times 100 \%\end{array}$} \\
\hline & & & & $\begin{array}{l}\text { Isotopic } \\
\mathrm{Pu} / \mathrm{U}\end{array}$ & $\begin{array}{l}\mathrm{X}-\mathrm{ray} \\
\mathrm{Pu} / \mathrm{U}\end{array}$ & & & & & & $\begin{array}{l}\text { Isotopic } \\
\text { Pu/U }\end{array}$ & $\begin{array}{r}\mathrm{X}-\mathrm{ray} \\
\mathrm{Pu} / \mathrm{U}\end{array}$ & \\
\hline F4-C-f1 & 8977 & $\begin{array}{l}1 \\
4 \\
5\end{array}$ & $\begin{array}{l}G-113 \\
G-114 \\
G-115\end{array}$ & $\begin{array}{l}0.393 \\
0.744 \\
0.701\end{array}$ & $\begin{array}{l}0.347 \\
0.740 \\
0.728\end{array}$ & $\begin{array}{l}-11.7 \\
-0.5 \\
+3.8\end{array}$ & $\mathrm{E} 6-\mathrm{C}-\mathrm{f} 6$ & 354 & $\begin{array}{l}1 \\
2 \\
2\end{array}$ & $\begin{array}{l}T-175 \\
T-176 \\
G-125\end{array}$ & $\begin{array}{l}0.809 \\
1.216 \\
1.132\end{array}$ & $\begin{array}{l}0.695 \\
1.273 \\
1.157\end{array}$ & $\begin{array}{l}-14.1 \\
+\quad 4.7 \\
+\quad 2.2\end{array}$ \\
\hline $\mathrm{F} 4-\mathrm{C}-\mathrm{f} 6$ & 19579 & $\begin{array}{l}3 \\
4\end{array}$ & $\begin{array}{l}G-116 \\
G-117\end{array}$ & $\begin{array}{l}0.698 \\
0.636\end{array}$ & $\begin{array}{l}0.703 \\
0.678\end{array}$ & $\begin{array}{l}+0.7 \\
+6.6\end{array}$ & & & $\begin{array}{l}3 \\
3\end{array}$ & $\begin{array}{l}T-177 \\
\text { NB-26 }\end{array}$ & $\begin{array}{l}1.301 \\
1.349\end{array}$ & 1.272 & $\begin{array}{l}-2.2 \\
-5.7\end{array}$ \\
\hline E5-C-a6 & 7992 & $\begin{array}{l}3 \\
5 \\
6\end{array}$ & $\begin{array}{l}\text { G-101 } \\
\text { G-102 } \\
\text { G-103 }\end{array}$ & $\begin{array}{l}0.708 \\
0.662 \\
0.475\end{array}$ & $\begin{array}{l}0.750 \\
0.664 \\
0.467\end{array}$ & $\begin{array}{l}+5.9 \\
+0.3 \\
-1.7\end{array}$ & & & $\begin{array}{l}3 \\
4 \\
5 \\
5\end{array}$ & $\begin{array}{l}G-126 \\
T-178 \\
T-179 \\
N B-27\end{array}$ & $\begin{array}{l}1.261 \\
1.339 \\
1.303 \\
1.328\end{array}$ & $\begin{array}{l}\mathbf{1} .265 \\
1.245 \\
1.331\end{array}$ & $\begin{array}{l}+0.3 \\
-7.0 \\
+2.1 \\
+0.2\end{array}$ \\
\hline F5-C-a1 & 553 & $\begin{array}{l}1 \\
3 \\
3\end{array}$ & $\begin{array}{l}\text { G-104 } \\
\text { G-105 } \\
T-211\end{array}$ & $\begin{array}{l}0.500 \\
1.092 \\
1.080\end{array}$ & $\begin{array}{l}0.452= \\
0.922\end{array}$ & $\begin{array}{l}-9.6 \\
-15.6 \\
-14.6\end{array}$ & E6-SE-c2 & 3198 & $\begin{array}{l}6 \\
3 \\
5\end{array}$ & $\begin{array}{l}T-180 \\
T-187 \\
T-188\end{array}$ & $\begin{array}{l}0.950 \\
1.332 \\
1.274\end{array}$ & $\begin{array}{l}0.957 \\
0.978 \\
1.341\end{array}$ & $\begin{array}{l}+0.7 \\
-26.6 \\
+5.2\end{array}$ \\
\hline F5-C-f6 & 541 & $\begin{array}{l}1 \\
2 \\
4 \\
5 \\
6\end{array}$ & $\begin{array}{l}G-106 \\
G-118 \\
G-119 \\
G-120 \\
G-121\end{array}$ & $\begin{array}{l}0.559 \\
0.973 \\
1.020 \\
1.032 \\
0.739\end{array}$ & $\begin{array}{l}0.544 \\
0.823 \\
0.984 \\
1.001 \\
0.727\end{array}$ & $\begin{array}{l}-2.7 \\
-15.4 \\
-3.5 \\
-3.0 \\
-1.6\end{array}$ & $\begin{array}{l}\text { E6-SE-e4 } \\
\text { E5-SW-a5 }\end{array}$ & $\begin{array}{r}3187 \\
15025\end{array}$ & $\begin{array}{l}3 \\
5 \\
3 \\
5\end{array}$ & $\begin{array}{l}T \\
T-185 \\
T-186 \\
G-111 \\
G-112\end{array}$ & $\begin{array}{l}1.268 \\
1.230 \\
0.730 \\
0.705\end{array}$ & $\begin{array}{l}1.241 \\
1.051 \\
0.750 \\
0.705\end{array}$ & $\begin{array}{r}-2.1 \\
-14.6 \\
+\quad 2.7 \\
0.0\end{array}$ \\
\hline E6-C-a1 & 369 & $\begin{array}{l}1 \\
2 \\
2 \\
3 \\
3 \\
3 \\
3 \\
3 \\
4 \\
4 \\
5 \\
5 \\
6\end{array}$ & $\begin{array}{l}\mathrm{T}-168 \\
\mathrm{~T}-169 \\
\mathrm{G}-107 \\
\mathrm{~T}-170 \\
\mathrm{NB}-19 \\
\mathrm{G}-108 \\
\mathrm{~T}-212 \\
\mathrm{~T}-319 \\
\mathrm{~T}-171 \\
\mathrm{NB}-20 \\
\mathrm{~T}-172 \\
\mathrm{G}-109 \\
\mathrm{~T}-173\end{array}$ & $\begin{array}{l}0.836 \\
1.215 \\
1.218 \\
1.291 \\
1.367 \\
1.184 \\
1.224 \\
1.190 \\
1.349 \\
1.383 \\
1.270 \\
1.235 \\
0.920\end{array}$ & $\begin{array}{l}1.380 \\
1.300 \\
0.756\end{array}$ & $\begin{array}{l}-9.9 \\
-0.7 \\
-4.3 \\
-9.6\end{array}$ & $\begin{array}{l}\text { F5-SE-f5 } \\
\text { E6-NW-a1 }\end{array}$ & $\begin{array}{r}8717 \\
44\end{array}$ & $\begin{array}{l}3 \\
6 \\
1 \\
2 \\
3 \\
3 \\
3 \\
4 \\
4 \\
4 \\
5 \\
6\end{array}$ & $\begin{array}{l}\mathrm{G}-122 \\
\mathrm{G}-123 \\
\mathrm{~T}-162 \\
\mathrm{~T}-163 \\
\mathrm{~T}-164 \\
\mathrm{NB}-21 \\
\mathrm{G}-127 \\
\mathrm{~T}-165 \\
\mathrm{~N}-22 \\
\mathrm{G}-128 \\
\mathrm{~T}-166 \\
\mathrm{~T}-167\end{array}$ & $\begin{array}{l}1.097 \\
0.713 \\
0.857 \\
1.196 \\
1.248 \\
1.287 \\
1.195 \\
1.255 \\
1.305 \\
1.187 \\
1.205 \\
0.922\end{array}$ & $\begin{array}{l}0.858 \\
0.719 \\
0.818 \\
0.842 \\
1.014 \\
0.825 \\
0.809 \\
0.681 \\
1.185 \\
0.903\end{array}$ & $\begin{array}{l}-21.8 \\
+0.8 \\
-4.6 \\
-29.6 \\
-18.8 \\
-21.2 \\
-31.0 \\
-35.5 \\
-38.0 \\
-42.6 \\
-1.6 \\
-2.1\end{array}$ \\
\hline E6-C-a6 & 5352 & $\begin{array}{l}1 \\
2 \\
3 \\
4 \\
6 \\
6\end{array}$ & $\begin{array}{l}T-153 \\
T-154 \\
T-155 \\
T-156 \\
T-157 \\
\text { NB-18 }\end{array}$ & $\begin{array}{l}0.812 \\
1.256 \\
1.368 \\
1.364 \\
0.951 \\
0.960\end{array}$ & $\begin{array}{l}0.796 \\
1.196 \\
1.320 \\
1.411 \\
1.002\end{array}$ & $\begin{array}{l}-2.0 \\
-4.8 \\
-3.5 \\
+3.4 \\
+5.4 \\
+4.4\end{array}$ & E6-SE-f5 & 3113 & $\begin{array}{l}1 \\
3 \\
3 \\
3 \\
3 \\
3 \\
3\end{array}$ & $\begin{array}{l}T-181 \\
T-182 \\
T-213 \\
T-218 \\
G-110 \\
G-124 \\
\text { NB-24 }\end{array}$ & $\begin{array}{l}0.953 \\
1.258 \\
1.256 \\
1.263 \\
1.238 \\
1.184 \\
1.259\end{array}$ & $\begin{array}{l}0.706 \\
1.184 \\
1.151\end{array}$ & $\begin{array}{l}-25.9 \\
-5.9 \\
-8.6 \\
-5.0 \\
-8.6\end{array}$ \\
\hline E6-C-f1 & 536 & $\begin{array}{l}1 \\
1 \\
2 \\
6\end{array}$ & $\begin{array}{l}T-158 \\
\text { NB-17 } \\
T-159 \\
T-160\end{array}$ & $\begin{array}{l}0.786 \\
0.814 \\
1.256 \\
0.930\end{array}$ & $\begin{array}{l}0.812 \\
1.579 \\
0.951\end{array}$ & $\begin{array}{l}+3.3 \\
-0.2 \\
+25.7 \\
+2.2\end{array}$ & & & $\begin{array}{l}3 \\
5 \\
5 \\
6\end{array}$ & $\begin{array}{l}\mathrm{NB}-28 \\
\mathrm{~T}-183 \\
\mathrm{NB}-25 \\
\mathrm{~T}-184\end{array}$ & $\begin{array}{l}1.259 \\
1.233 \\
1.239 \\
0.971\end{array}$ & $\begin{array}{l}1.195 \\
0.836\end{array}$ & $\begin{array}{l}0.0 \\
-3.1 \\
-3.6 \\
-13.9\end{array}$ \\
\hline
\end{tabular}




\section{APPENDIX B}

SUMMARY OF BURNUP, ATOM DENSITIES, AND FUEL CHARACTERISTICS

DETERMINED FROM MASS SPECTROMETRIC, ALPHA SPECTROGRAPHIC, AND RADIOCHEMICAL ANALYSES

Results obtained from analysis of EYC Phase 3 and Program Extension samples are summarized in Table $B-1$. Note that Table $B-1$ includes all the results for Pu-236 and Pu-238 measurements only on Program Extension samples. A complete listing of a11 the $\mathrm{Pu}-236$ and $\mathrm{Pu}-238$ data from Phase 3 and Program Extension analyses is given in Table $\mathrm{A}-1$. 


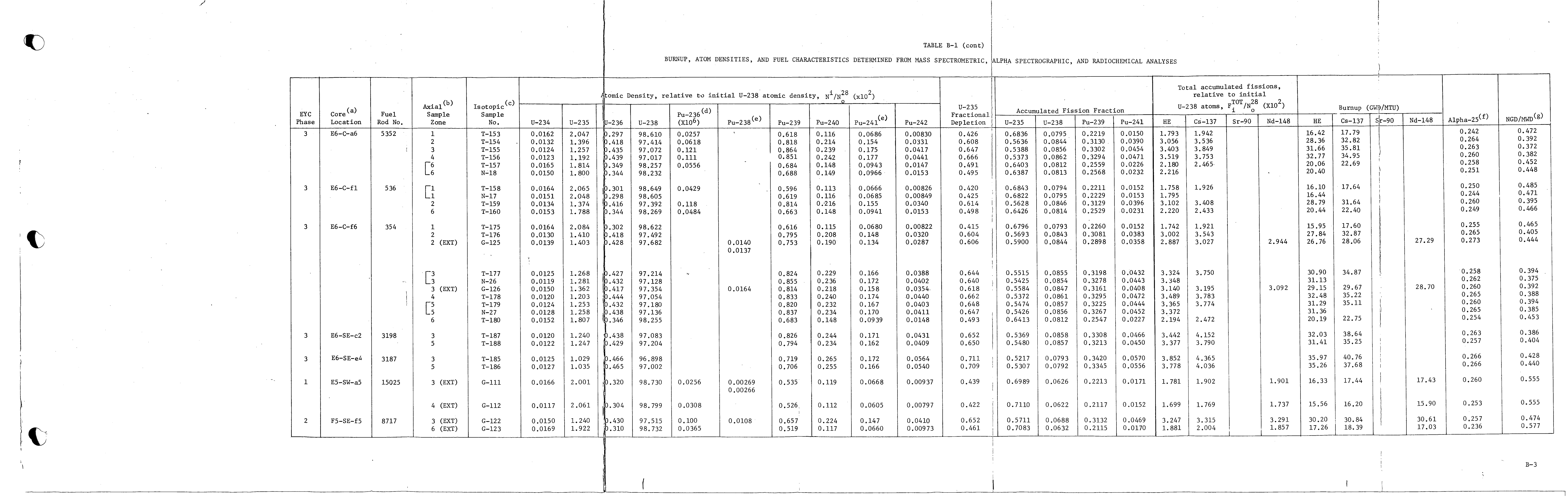




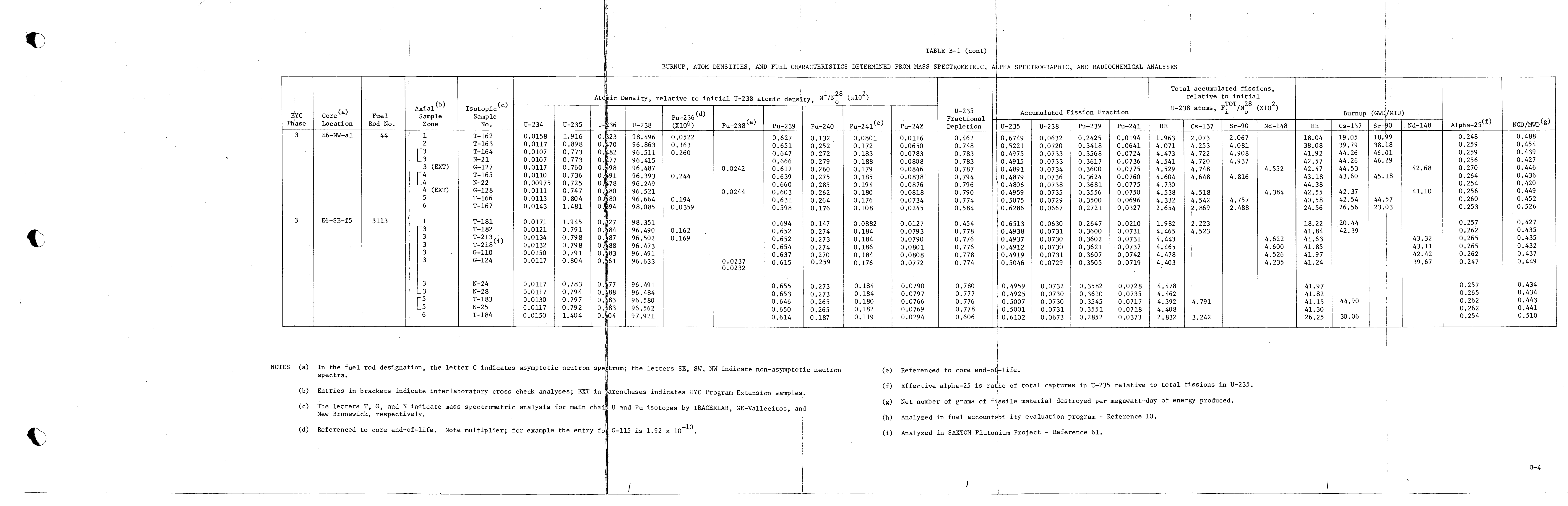




\section{APPENDIX C}

\section{CROSS SECTION LIBRARY FOR LASER-HIC}

The isotopes and corresponding identification numbers used in the LASER-HIC program are listed in Table $\mathrm{C}-1$. The LASER thermal energy mesh description is given in Table $\mathrm{C}-2$.

The library values used in the LASER-HIC calculations performed in the EYC Program Extension are documented in Table $\mathrm{C}-3$. Note that the $(n, y)$ and $(n, f)$ cross sections for the main chain uranium and plutonium isotopes (U-235, U-236, U-238, Pu-239, Pu-240, Pu-241, and Pu-242) normally considered in LASER are not included in Table $\mathrm{C}-3$. 
TABLE $\mathrm{C}-1$

HIC ISOTOPIC IDENTIFICATION NUMBERS

\begin{tabular}{|c|c|c|c|}
\hline Isotope & $\begin{array}{l}\text { HIC } \\
\text { ID }\end{array}$ & Isotope & $\begin{array}{l}\text { HIC } \\
\text { ID }\end{array}$ \\
\hline $\begin{array}{l}\text { Th-230 } \\
\text { Th-231 } \\
\text { Th-232 } \\
\text { Th-233 } \\
\text { Pa-231 } \\
\mathrm{Pa}-232 \\
\mathrm{~Pa}-233 \\
\mathrm{U}-232 \\
\mathrm{U}-233 \\
\mathrm{U}-234 \\
\mathrm{U}-235 \\
\mathrm{U}-236 \\
\mathrm{U}-237 \\
\mathrm{U}-238 \\
\mathrm{U}-239 \\
\mathrm{~Np}-236 \\
\mathrm{~Np}-237 \\
\mathrm{~Np}-238 \\
\mathrm{~Np}-239\end{array}$ & $\begin{array}{r}1 \\
2 \\
21 \\
22 \\
\\
3 \\
4 \\
23 \\
\\
5 \\
6 \\
7 \\
8 \\
9 \\
10 \\
24 \\
25 \\
\\
27 \\
11 \\
12 \\
26\end{array}$ & $\begin{array}{l}\mathrm{Pu}-236 \\
\mathrm{Pu}-238 \\
\mathrm{Pu}-239 \\
\mathrm{Pu}-240 \\
\mathrm{Pu}-241 \\
\mathrm{Pu}-242 \\
\mathrm{Am}-241 \\
\mathrm{Am}-242 \\
\mathrm{Am}-242 \mathrm{~A} \\
\mathrm{Am}-243 \\
\mathrm{Cm}-242 \\
\mathrm{Cm}-243 \\
\mathrm{Cm}-244\end{array}$ & $\begin{array}{l}28 \\
13 \\
14 \\
15 \\
16 \\
17 \\
\\
29 \\
30 \\
18 \\
19 \\
\\
31 \\
20 \\
32\end{array}$ \\
\hline
\end{tabular}


TABLE $\quad \mathrm{C}-2$

LASER THERMAL ENERGY MESH

\begin{tabular}{|c|c|c|c|c|}
\hline$i$ & $\operatorname{sped}_{i}^{a}$ & $\begin{array}{l}\text { Mesh Width } \\
\qquad \Delta v_{i}\end{array}$ & $\begin{array}{l}\text { Energy } \\
\mathrm{E}_{i} \quad(\mathrm{eV})\end{array}$ & $E_{\text {int }}(\mathrm{eV})$ \\
\hline 1 & .2 & .2 & .001012 & .002277 \\
\hline 2 & .4 & .2 & .004048 & .006325 \\
\hline 3 & .6 & .2 & .009108 & .012397 \\
\hline 4 & .8 & .2 & .016192 & .020493 \\
\hline 5 & 1.0 & .2 & .0253 & .030613 \\
\hline 6 & 1.2 & .2 & .036432 & .042757 \\
\hline 7 & 1.4 & .2 & .049588 & .056925 \\
\hline 8 & 1.65 & .3 & .068879 & .081972 \\
\hline 9 & 1.95 & .3 & .096203 & .11157 \\
\hline 10 & 2.25 & .3 & .12808 & .14573 \\
\hline 11 & 2.55 & .3 & .16451 & .18444 \\
\hline 12 & 2.85 & .3 & .20550 & .22770 \\
\hline 13 & 3.075 & .15 & .23923 & .25104 \\
\hline 14 & 3.21 & .12 & .26069 & .27053 \\
\hline 15 & 3.33 & .12 & .28055 & .29075 \\
\hline 16 & 3.42 & .06 & .29592 & .30113 \\
\hline 17 & 3.505 & .11 & .31081 & .32064 \\
\hline 18 & 3.66 & .2 & .33891 & .35768 \\
\hline 19 & 3.91 & .3 & .38679 & .41704 \\
\hline 20 & 4.26 & .4 & .45913 & .50326 \\
\hline 21 & 4.715 & .51 & .56245 & .62493 \\
\hline 22 & 5.265 & .59 & .70132 & . 78211 \\
\hline 23 & 5.845 & .57 & .86435 & . 95070 \\
\hline 24 & 6.23 & .2 & .98197 & 1.01374 \\
\hline 25 & 6.375 & .09 & 1.02821 & 1.04277 \\
\hline 26 & 6.435 & .03 & 1.04765 & 1.05254 \\
\hline 27 & 6.465 & .03 & 1.05744 & 1.06236 \\
\hline 28 & 6.495 & .03 & 1.06728 & 1.07222 \\
\hline 29 & 6.55 & .08 & 1.08543 & 1.09873 \\
\hline 30 & 6.69 & .2 & 1.13233 & 1.16645 \\
\hline 31 & 6.99 & .4 & 1.23616 & 1.30791 \\
\hline 32 & 7.39 & .4 & 1.38169 & 1.45748 \\
\hline 33 & 7.765 & .35 & 1.52547 & 1.59500 \\
\hline 34 & 8.10 & .32 & 1.65993 & 1.72616 \\
\hline 35 & 8.41135 & .3027 & 1.79000 & 1.85500 \\
\hline
\end{tabular}

a 1 unit $=2200 \mathrm{~m} / \mathrm{sec}$ 
TABLE C-3

LIBRARY OF CROSS SECTIONS FOR LASER-HIC CALCULATIONS LASE GROHP STRUCTUHF
THEOAAL RAHGF

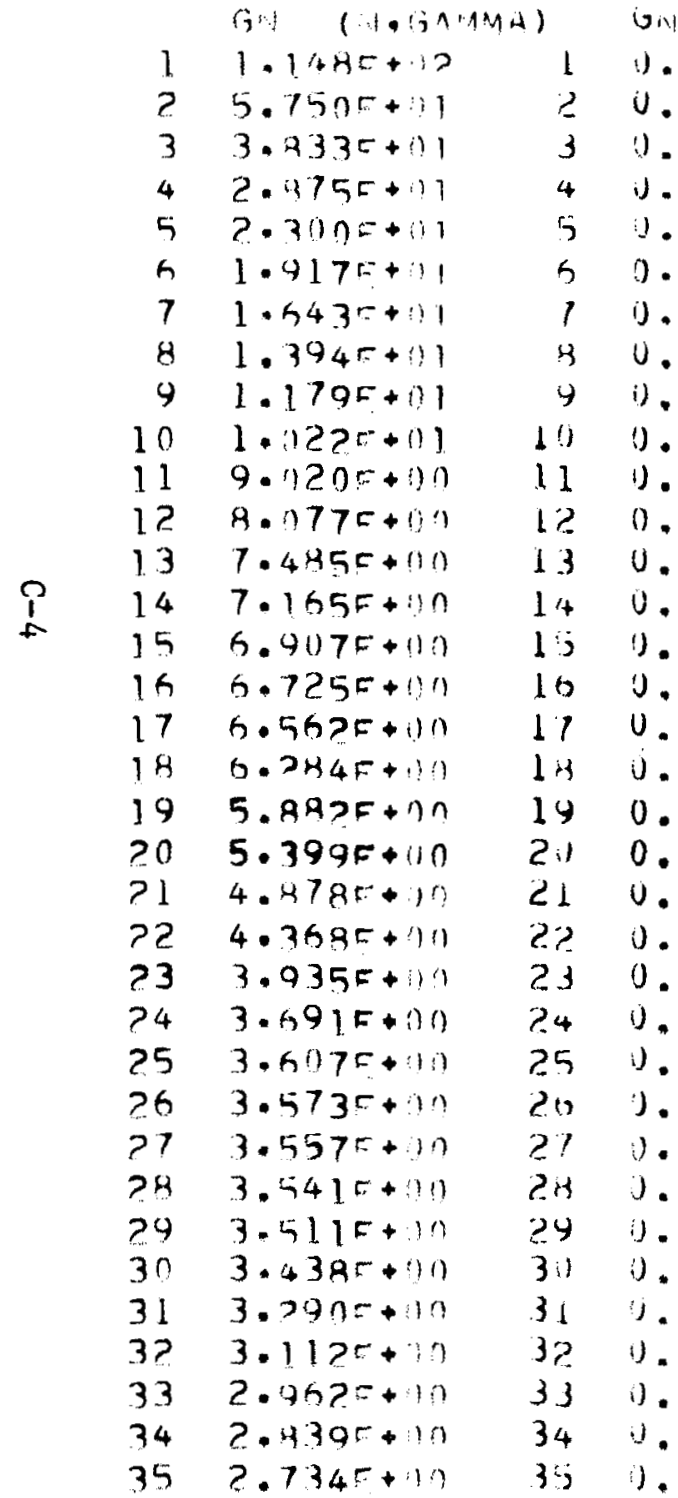

AVERAGF (VAMMAA) INO $(v, F I S S$.

FAST RANOE

\begin{tabular}{|c|c|c|}
\hline \multicolumn{2}{|c|}{$(N,(; A): A \Delta)$} & $(N, G)$ \\
\hline 1.?.?9F- -3 & 35 & $4.341 E-01$ \\
\hline $1.39 ? F-13$ & 37 & $963 E-01$ \\
\hline $1.57\{F-13$ & 34 & $E=0$ \\
\hline $1.79 Q F-03$ & 39 & $E-0$ \\
\hline $.027 F-03$ & $4 n$ & $? 1$ \\
\hline $.298 F-13$ & 41 & $E=0$ \\
\hline $.6 \cap 2 F-113$ & $4 ?$ & \\
\hline $7.444 F-03$ & 4.3 & +0 \\
\hline $3.346 F=0.3$ & 44 & \\
\hline $3.79 \cap E-13$ & 45 & $6 E+0$ \\
\hline $4 . ? 90 F-\cap 3$ & 46 & \\
\hline $4.861 F=03$ & 47 & $721 E+0$ \\
\hline $5.579 F-03$ & $4 A$ & $E+00$ \\
\hline $4.333 F-03$ & 49 & $2.222 F+00$ \\
\hline $7.074 F-13$ & $5 n$ & $2.519 E+0$ \\
\hline
\end{tabular}

$8.014 F-03$

9.079F-0.3

1. $0 ?(9 F-\cap ?$

1. IGGF-ח?

1.3?1F-11?

$1.544 F=n ?$

?. $034 F-n ?$

?. $514 F-0 ?$

3. $357 F-11 ?$

4. $317 F-\cap ?$

$5.533 F-0 ?$

7. $108 F-11 ?$

Q. $131 F-n ?$

$1.171 F-01$

$1.503 \mathrm{~F}-01$

$1.931 \mathrm{~F}=111$

$2.4 n \cap E-01$

3. $) \cap A F-01$

3. $411 F-\cap 1$

3. $467 E-n 1$
(LASFR HIC ID 1)

IIV BARNS

(N.FISS.)

\begin{tabular}{|c|c|c|c|}
\hline (A) & in & $(N, F[S S)$. & $(N, F I$ \\
\hline l & $n$ & 35 & 0. \\
\hline 2 & $\eta$ & 37 & 0. \\
\hline 3 & 0 & 39 & 0. \\
\hline 4 & $n$. & 39 & 0 \\
\hline 5 & $n$ & $4 !$ & $n$. \\
\hline 6 & 0) & 41 & 0 . \\
\hline 7 & $\eta$ & 4? & $n$ \\
\hline$H$ & 0 & 43 & 0. \\
\hline 9 & 0 & 44 & 0. \\
\hline $\ln$ & $n$. & 45 & 0 \\
\hline 11 & 0 & 46 & 0 . \\
\hline 12 & 0 & 47 & 0 \\
\hline 13 & 0 & 42 & 0. \\
\hline 14 & 0 & 47 & 0. \\
\hline 15 & $n$ & $5 n$ & 0 \\
\hline 16 & n. & & \\
\hline 17 & 0 . & & $(v, ?, v)$ \\
\hline 10 & 0 . & 1 & 1. $1 \angle 5 F+00$ \\
\hline 19 & ?. & 2 & $? .11005-01$ \\
\hline 20 & !. & 3 & $n$ \\
\hline
\end{tabular}


TABLE C-3 (Continued)

LIBRARY OF CROSS SECTIONS FOR LASER-HIC CALCULATIONS

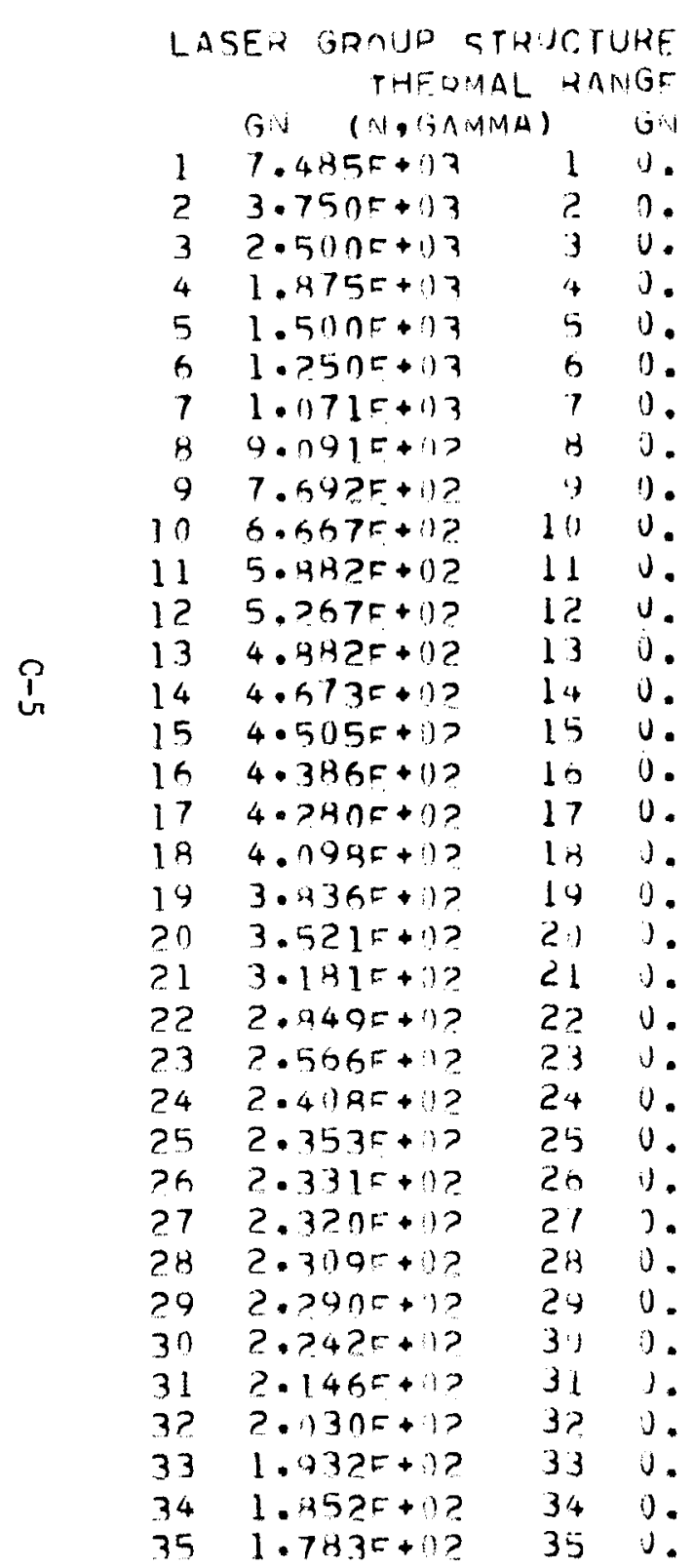

\begin{tabular}{|c|c|}
\hline$(N, F I \subseteq S)$, & (No SAMAC \\
\hline 1 & Q. $\cap 1 \supset F-0 ?$ \\
\hline$?$ & $9.1191 F-0 ?$ \\
\hline 3 & $1.10 \geq 95-n 1$ \\
\hline 4 & 1. IASE-O1 \\
\hline 5 & $1.3225-01$ \\
\hline 6 & $1.498 F-n 1$ \\
\hline 7 & $1.6975-111$ \\
\hline 3 & $1.423 E-01$ \\
\hline 9 & $? .182 F-01$ \\
\hline 10 & $2.471 \mathrm{~F}-01$ \\
\hline 11 & $2.798 F-01$ \\
\hline 1? & $3,171 E-11$ \\
\hline 13 & $3.638 F-n 1$ \\
\hline 14 & $4.130 F-01$ \\
\hline 15 & $4.6(4 F-1) !$ \\
\hline 16 & $5.229 \mathrm{~F}-01$ \\
\hline 17 & $5.9 ? \mid F-111$ \\
\hline 14 & $5.708 F \rightarrow 01$ \\
\hline 19 & $7.6075-01$ \\
\hline 2:) & $8.618 F-01$ \\
\hline 21 & $1 . n 33 F+n 0$ \\
\hline 22 & $1.3275+100$ \\
\hline 23 & $1.704 \mathrm{~F}+00$ \\
\hline 24 & $2,1895+00$ \\
\hline 25 & $2.8 \cap 9 F+00$ \\
\hline 26 & $3.5085+00$ \\
\hline$? 7$ & $4.536 E+100$ \\
\hline 24 & $5.955 F+00$ \\
\hline 24 & $7.640 F+00$ \\
\hline $3 n$ & $9.900 E+00$ \\
\hline 31 & $1.259 F+01$ \\
\hline 32 & $1.6175+111$ \\
\hline 33 & $1.9525+01$ \\
\hline 34 & $2.224 E+01$ \\
\hline 35 & $2.522 F+111$ \\
\hline
\end{tabular}

SS.) CROSS
FAST RAVGE

AST RANGE
(NN GAMMA)

$36 \quad 2.857 E+01$

$3 . ? 3 / E+01$

3. $R$ ATE +01

$4,15 S E+01$

$4.700 F+01$

$5.332 E+01$

$6.143 E+01$

5. $944 E+n 1$

$7.752 E+01$

$9.781 F+01$

$9.925 E+01$

$1.123 E+02$

$1.275 E+02$

$1.450 E+02$

$1.443 F+02$

SECTIUU FOR THZ31

MA)

GN

(N,FTSE.

(LASER HIC ID 2)

Iiv BARNS

GN (N,FISS.)

\begin{tabular}{|c|c|c|}
\hline 1 & 0. & 36 \\
\hline 2 & n. & 37 \\
\hline 3 & $n$. & 38 \\
\hline 4 & n. & 39 \\
\hline 5 & 0. & $4 !$ \\
\hline 6 & ). & 41 \\
\hline 7 & 0 . & 42 \\
\hline 8 & $n$. & 43 \\
\hline 9 & 0. & 44 \\
\hline 10 & 0. & 45 \\
\hline 11 & 0 . & 46 \\
\hline 12 & 0. & 47 \\
\hline 13 & 0. & 48 \\
\hline 14 & 0. & 49 \\
\hline 15 & 0. & 50 \\
\hline
\end{tabular}

(iv, ZN)

17

170.

$19 \pi$

201.

?1 0

$\begin{array}{ll}22 & 19 \\ 23 & 0\end{array}$

240

250.

250

$>70$

280

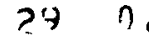

30 ?

310.

320.

$33 n$

340 
TABLE C-3 (Continued)

LIBRARY OF CROSS SECTIONS FOR LASER-HIC CALCULATIONS

LASER GRIJP STRUCTUIFE AVERAGE (N,AMMAA) ANI) (N,FISS.) CHISS SECTIUN FOR PAP3) (LASER HIC ID 3 ) THERAAL HANGF FAST PANGE

\begin{tabular}{|c|c|c|c|c|c|c|}
\hline$(n, g)(j \wedge)$ & & $(N, F[$ & & $\operatorname{lng}, G \triangle A$ & & GN \\
\hline $9.9 B D F+0 ?$ & 1 & $+.991 E-1) 2$ & 1 & $n$. & 36 & $2.912 E+00$ \\
\hline $5.000=+10 ?$ & ? & $2.501 E-02$ & ? & $n$. & 37 & $3.420 E+00$ \\
\hline $3 \cdot 333=+12$ & 3 & $1.667 E-112$ & 3 & $n$ & 38 & $4.018 E+00$ \\
\hline $2.500 F+07$ & 4 & 1.2SOE -02 & 4 & $n$. & 39 & $.7 \geq 2 E+00$ \\
\hline $2.0(1) 05+i) 2$ & 5 & $00 F-02$ & 5 & $F-03$ & 40 & 0 \\
\hline $1.667 F+02$ & 6 & d. $335 E-03$ & h & $7.041 \mathrm{E}-0 ?$ & 41 & $.517 F+00$ \\
\hline $1.429 c+1 ? ?$ & 7 & $7.144 E-1) 3$ & 7 & $? .290 F-0 ?$ & 4? & $7.659 E+00$ \\
\hline $1.212 F+1 ?$ & 8 & 6. $062 E-03$ & a & $\supset .6 Z R E-0 ?$ & 4.3 & $2.153 E+01$ \\
\hline $1 \cdot 040 F+1) ?$ & 9 & $3 \cdot 199 E-03$ & 9 & $2.977 F-02$ & 44 & $.456 E+02$ \\
\hline $1.042 F+0 ?$ & 1is & $5.212 E-113$ & 10 & $3.359 E-n ?$ & 45 & $48 C E+01$ \\
\hline $1 \cdot 080=+1) 7$ & 11 & $5.400 E-1) 3$ & 11 & $3.89 .3 F-02$ & 46 & $3 E+01$ \\
\hline $1 \cdot 2225+12$ & 12 & $6.108 E-03$ & $1 ?$ & $4.319 E-\cap 2$ & 47 & 02 \\
\hline - 420 $5+11 ?$ & 1.3 & $1.728 E-102$ & 13 & 4.6QOE-0? & 49 & $E+01$ \\
\hline $2.4005+0 ?$ & 14 & $1.200 E-02$ & 14 & $4.965 F=n ?$ & 49 & $1 E+01$ \\
\hline $3 \cdot \cap 32 E+1 ?$ & 15 & $1.516 E-02$ & 15 & $5.150 F-02$ & $5 ?$ & $.545 F+01$ \\
\hline
\end{tabular}

4. $014 F+1 ? \quad 16 \quad 2.007 E-02$ is $5.317 E-0 ?$

9.3ANE+11? $17 \quad 4.190 F-02 \quad 17 \quad 5.446 E-0 ?$

$3.768 F+13$ lB $1.944 E-111$ 14 $5.547 F-0 ?$

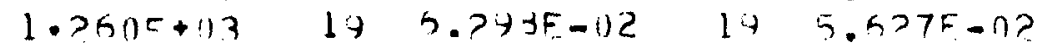

$2.634 \pi+12$ 20 $1.3175-112$ 21) $5.5 A A E-n ?$

$1.485 F+1 ? 21 \quad 1.427 F-113 \quad 215.752 E=0 ?$

$3.365 F+1122 \quad 1.683 E-03$ 22 5.919F-n?

$1.561 F+1) 1$

$1 \cdot 6175+11$

$1.7175+! 1$

237 , $218 \mathrm{~F}-04$

2.4 $9.09 \times E-104$

$25 \quad 0.5905-114$

$1 \cdot 7425+11$

$1.2870+?$

2h $\quad 8.977 F-114$

2. $137 E+11$

$3.7895+11$

$9.448 E-114$

$2 \cdot 747=+1 ?$

$28 \quad 1.070 \mathrm{O}-03$

$29 \quad 1.896 E-13$

$301.374 E-1) 2$

$2.4535+01$

$31 \quad 1.432 E-1) 3$

$7.1875+10$

32 3.604E-04

$4.426 x+11 n$

$332.223 E-1) 4$

$5.0075+100$

$34 \quad 2.514 F-114$

$7.645 \mathrm{~F}=n$ ?

$24 \quad 1.05 \mathrm{GF}-01$

$25 \quad 1.457 F-01$

2h ?.014F-n1

2? ?.743F-01

2. 3. $846 F-01$

$29 \quad 5.305 F-01$

3) $7.317 F-01$

$31 \quad 1.012 E+00$

32. $1.397 F+00$

$33 \quad 1.792 F+00$

$34 ? .107 F+10$

$35 \quad 1.545 F+11$

$7.7325-114$

$2.47 \mathrm{RE}+00$

GN (IN,FISS,

$1.349 E+00$

1. $345 E+00$

$1.344 F+0 n$

$1.343 F+00$

$1.34 ? E+00$

1. $341 E+00$

$1.334 F+00$

$1.197 F+n 0$

1. $003 E+00$

9. $D 53 E-n 1$

6.98BE-01

4.910E-O1

1. $409 E-01$

9. $060 E-02$

8. $127 E-0$ ?

$5.064 E-02$

4. $>91 E-0$ ?

2. $942 E-02$

?. $034 E-0$ ?

1. $454 E-17$ ?

$1.312 E-02$

$9.926 E-03$

$1.984 F-03$

5. $808 F-03$

6. $\cap 97 E-03$

$5.454 E-03$

$5.402 F-03$

$5 .>43 F-03$

$5.147 E-03$

$5 . \cap 89 E-03$

$5.053 E-n 3$

$5.031 F-03$

5.ก?lF-n3

5. O1GE-03

5. D I E -03
IN BARNS

GN (N,FISS.)

3h 5.009E-03

$37 \quad 5.007 E-03$

38 5.1UUSE-03

$39 \quad 5.003 E-03$

$40 \quad 5.002 E-03$

$415.0015-03$

4? $5.0015-03$

$435.176=-03$

$447.2 y 3 E-03$

$45 \quad 4.330 E-03$

$44 \quad 3.552 E-03$

$47 \quad 1.0585-02$

$48 \quad 1.853 F-03$

49 6.168F-04

$507.735 E-04$

$(N, 2 N)$

1.30 $05+00$

$3.700=-01$

0 . 
TABLE C-3 (Continued)

LIBRARY OF CROSS SECTIONS FOR LASER-HIC CALCULATIONS

LASER GROIJP GTRUCTUHE AVERAIE (N, GAMMA) ANI) (N.FISS,) CHOSS SECTIUN FOH PAZ3? THEQAAL KANGF

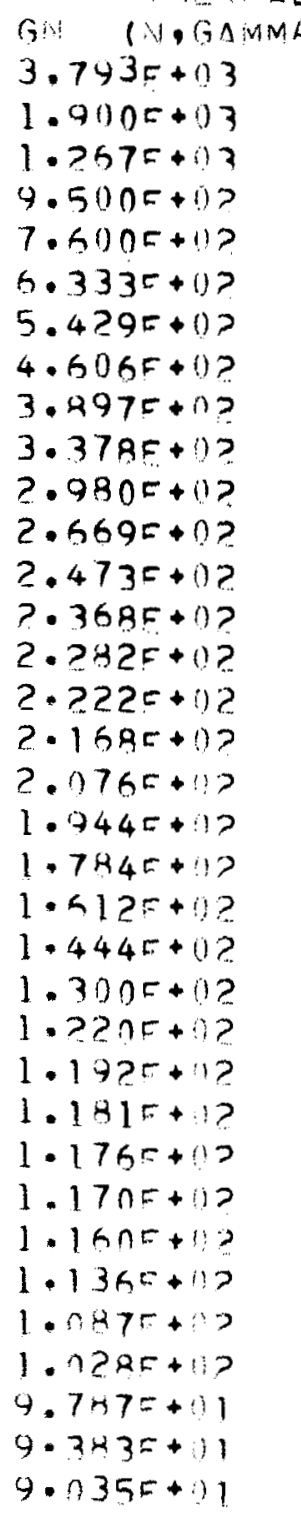

\section{GNi $(, N, F I S S O)$}

\begin{abstract}
$3.49 C E+1) 3$
\end{abstract}
$1.749 F+113$

$1.166 E+103$

$8.747 \mathrm{~F}+112$

$6.997 E+112$

$5.931 E+02$

$4.998 E+102$

$4.241 E+02$

$3.588 \mathrm{E}+112$

$3.110 E+112$

$2.744 E+102$

$2.457 E+112$

2. $277 E+12$

$2.180 E+102$

$2.101 E+02$

$2.046 E+12$

1.995E+02

$1.912 E+02$

1. $740 F+02$

$1.543 E+112$

$1.404 E+112$

1. $329 E+112$

$1.197 E+1) 2$

$1.123 E+02$

1. $948 E+112$

1. 1$) \rightarrow 7 E+12$

1. $082 E+12$

1.077E+02

1. $0685+02$

$1.0465+12$

$1.001 E+02$

$\forall .463 E+01$

$9.011 E+01$

$0.639 F+1$

b. $31 \rightarrow E+01$
FAST RAIVGE

$$
\text { GN (N,GAMMA) GN (N,GAMMA) }
$$

$$
4.061 E-0 ?
$$

$4.601 F-0 ?$

$5.215 F-0 ?$

$5.910 E-0 ?$

$4.698 F=n$ ?

$7.592 F-02$

8. $5965-0$ ?

$9.745 F-n 2$

1. $106 E-01$

$1.252 E-01$

$1.418 F=01$

$1.606 E-01$

$1.843 E-n 1$

2. $093 \mathrm{~F}-01$

?. $337 F-n$ I

2. $549 E-n 1$

3. OOOF-OI

$3.399 F-01$

$3.854 \mathrm{~F}=n 1$

4. $366 F-01$

$5.235 F-01$

$4.721 F-01$

$4.636 \mathrm{~F}-01$

$1.109 F+00$

$1.423 F+n 0$

2h $1.428 F+n 0$

$27 ? .349 F+00$

2. $3.017 E+00$

?a $3.871 F+00$

$30 \quad 4.955 E+0 n$

$31 \quad 4.391 E+00$

32 $\quad .194 E+0 n$

$33 \quad .939 F+n$

$34 \quad 1.127 E+111$

$35 \quad 1.278 F+01$

$\begin{array}{ll}36 & 1.448 E+01 \\ 37 & 1.540 E+01 \\ 38 & 1.358 E+01 \\ 39 & 2.105 E+01 \\ 4 n & 2.385 E+01 \\ 41 & 2.702 E+01 \\ 47 & 3.062 E+01 \\ 43 & 3.467 E+01 \\ 44 & 3.928 E+01 \\ 45 & 4.449 E+01 \\ 46 & 5.029 E+01 \\ 47 & 5.688 E+01 \\ 44 & 6.461 E+01 \\ 49 & 7.344 E+01 \\ 50 & 8.373 E+01\end{array}$

. $373 F+01$

(LASER HIC ID 4)

A) GN (NOFISS.) 10. 20 . 30 . 40. 50 . 0 . 70 . 0 . 90 .

$\begin{array}{ll}10 & 9.129 E-02 \\ 11 & 1.305 E-01\end{array}$

$12 \quad 1.479 \mathrm{~F}-01$

$13 \quad 1.597 F-01$

$14 \quad 1.927 E-01$

$152.152 E-01$

$16 ? .439 \mathrm{~F}-01$

$17 \quad 2.762 \mathrm{~F}-01$

$183.129 F-0$

$193.549 F-01$

$20 \quad 40020 F-0$

$214.920 F-01$

22 $6.199 \mathrm{AE}-0$

$237.951 \mathrm{~F}-01$

$24 \quad 1 . n 2 l F+00$

$>5 \quad 1.310 E+00$

2h $1.683 \mathrm{~F}+100$

$27 \quad 2.163 F+00$

$28 \quad 2.77 \mathrm{AF}+00$

$29 \quad 3.564 E+00$

30 $4.571 E+00$

31

32

33

34

35
IN BARNS

3h $1.333 E+01$

$37 \quad 1.510 E+01$

$38 \quad 1.711 F+01$

$39 \quad 1.936 E+01$

$40 \quad 2.195 E+01$

$412.487 E+01$

$42 ? .919 F+01$

$433.1935+01$

$443.616 E+01$

$454.096 E+01$

$46 \quad 4.630 E+01$

$47 \quad 5.237 E+01$

$48 \quad 5.949 E+01$

$496.762 F+01$

$507.6635+01$

$(N, 2 \times 1)$

$11.000 F+00$

? $6.300=-01$

$34.0005=02$ 
TABLE C-3 (Continued)

LIBRARY OF CROSS SECTIONS FOR LASER-HIC CALCULATIONS

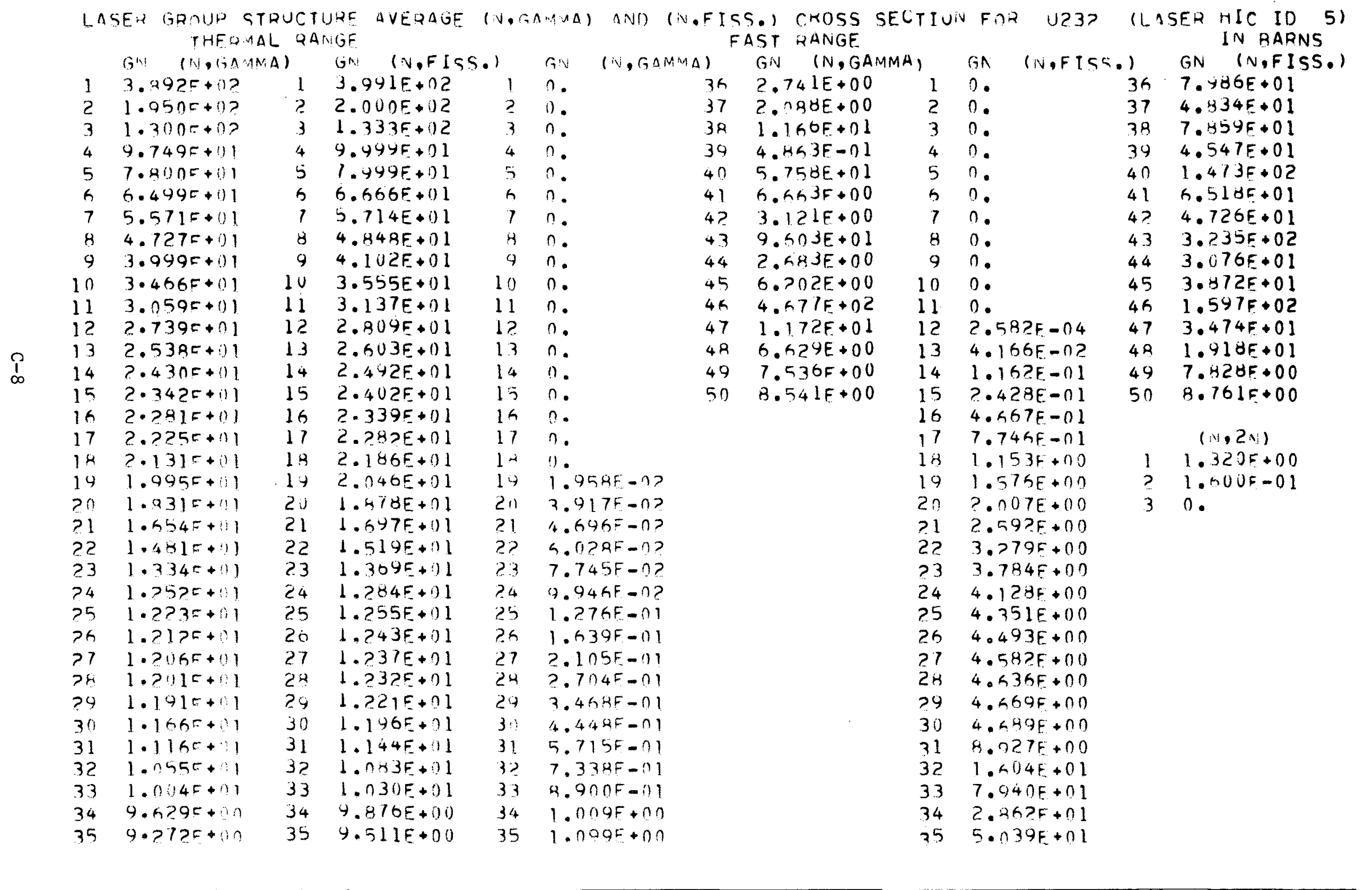


TABLE C-3 (Continued)

LIBRARY OF CROSS SECTIONS FOR LASER-HIC CALCULATIONS

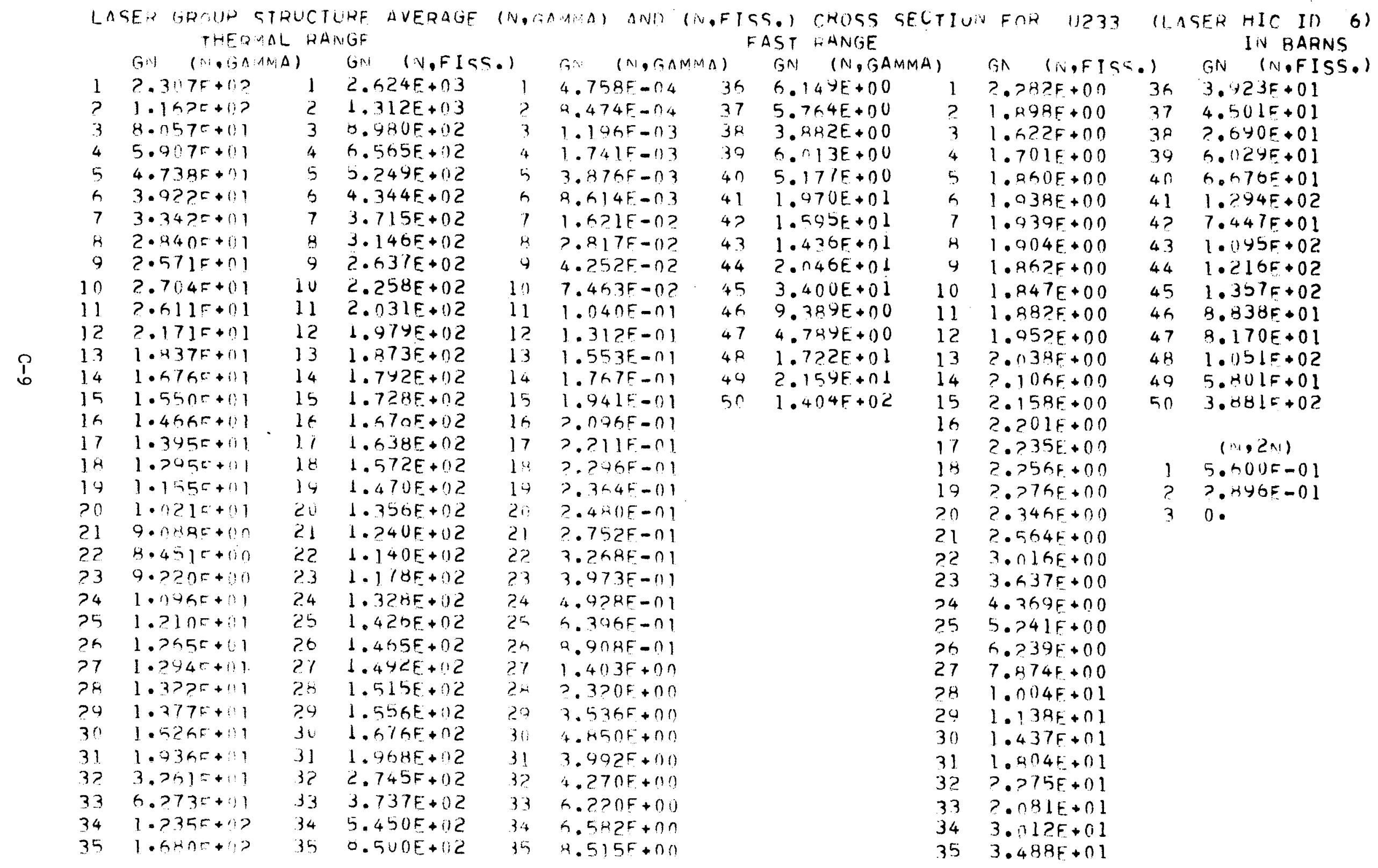


TABLE C-3 (Continued)

LIBRARY OF CROSS SECTIONS FOR LASER-HIC CALCULATIONS

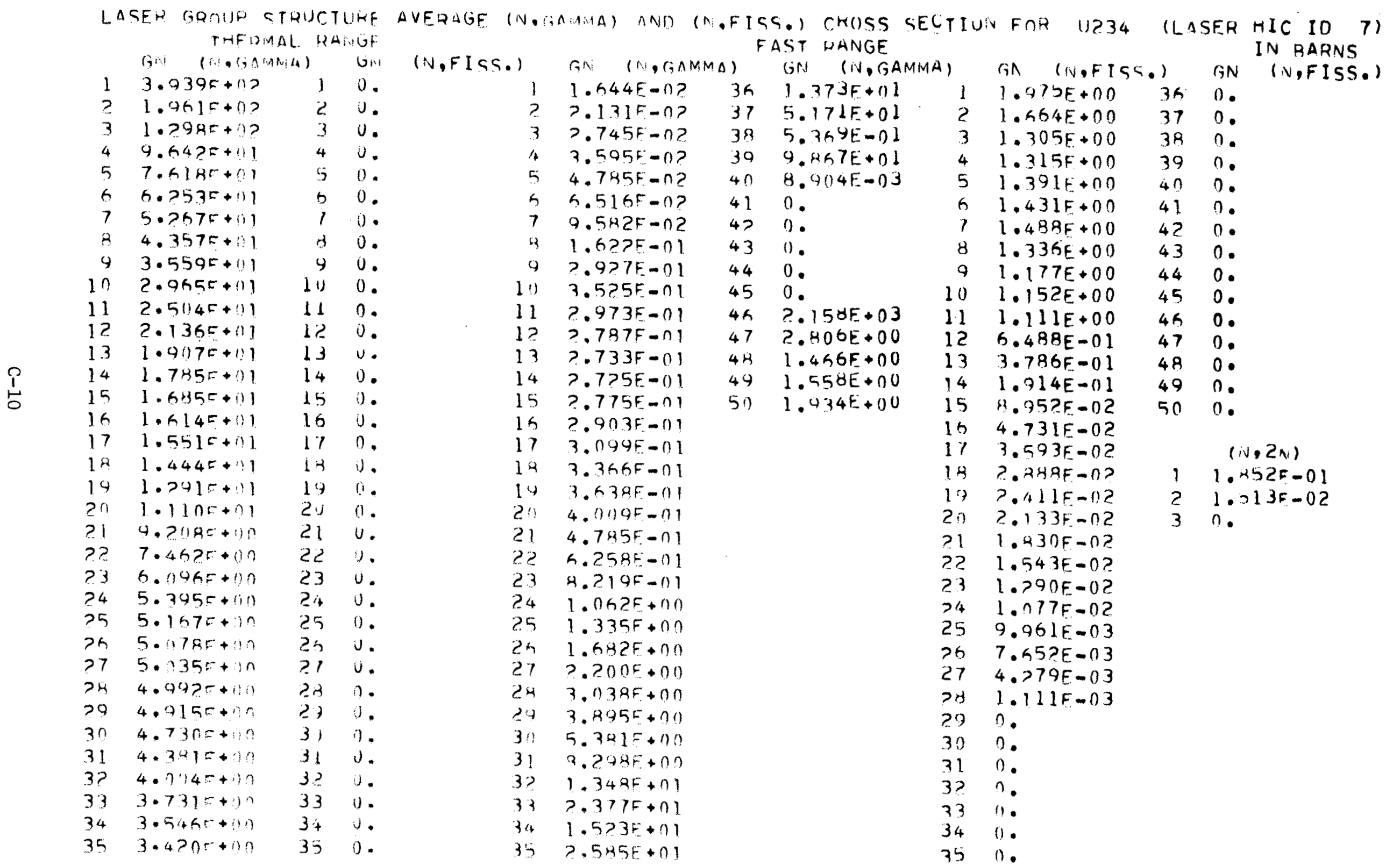


TABLE C-3 (Continued)

LIBRARY OF CROSS SECTIONS FOR LASER-HIC CALCULATIONS

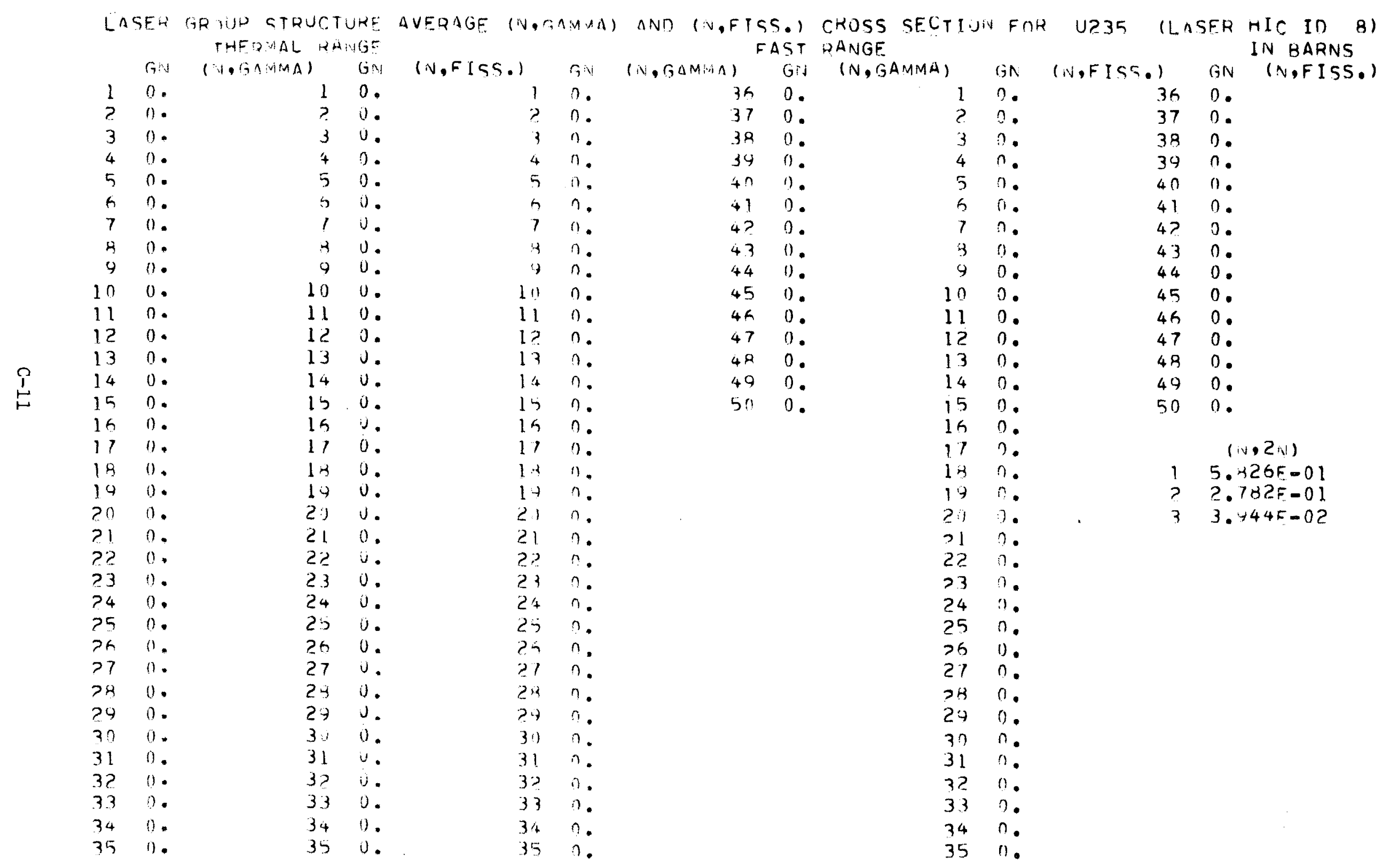


TABLE C-3 (Continued)

LIBRARY OF CROSS SECTIONS FOR LASER-HIC CALCULATIONS

\begin{tabular}{|c|c|c|c|c|c|c|c|c|c|c|c|c|}
\hline L. & ASEL & $\begin{array}{c}\text { IIR IJP GTRUC } \\
\text { THEDMAL H }\end{array}$ & $\begin{array}{l}\text { URE } \\
\text { VGE }\end{array}$ & AVERAGE (N, & $(M M A)$ & AND IN,FIS & $\begin{array}{l}5.1 \\
A S T\end{array}$ & $\begin{array}{l}\text { CKOSS SECTIUIV } \\
\text { RANGE }\end{array}$ & $F \cap R$ & บ236 & ILn & $\begin{array}{l}\text { FER HIC ID 9) } \\
\text { IN BARNS }\end{array}$ \\
\hline & Giv & $(N,(; \Delta M M \Delta)$ & GN & $(N, F I S S)$. & Gid & $(N, G \triangle M M A)$ & GN & $(N, G A M M A)$ & $5 n$ & $1 N, F I S 5$. & ) & $(N, F I S S)$. \\
\hline 1 & 0. & 1 & u. & 1 & $n$ & $3 h$ & 0. & 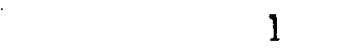 & 0. & & 36 & 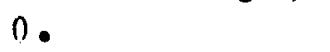 \\
\hline 2 & 0 & 2 & u. & $?$ & 0 . & 37 & 0. & 2 & 0. & & 37 & 0. \\
\hline 3 & 0 & 3 & 0 & 3 & $n$. & 38 & 0 & 3 & $n$ & & 38 & 0 . \\
\hline 4 & $0 \cdot$ & 4 & 0 & 4 & $n$. & 39 & 0. & 4 & 0 & & 39 & 0 . \\
\hline 5 & 0 . & 5 & 0 & 5 & $n$. & 40 & 0. & 5 & $n$ & & 40 & 0 . \\
\hline 6 & 0 - & 6 & 0 & G & $n$. & 41 & 0. & 6 & 0 & & 41 & 0. \\
\hline 7 & 0 - & 7 & j. & 7 & $n$. & 4? & 0. & 7 & 0. & & 42 & 0. \\
\hline 8 & 0 - & 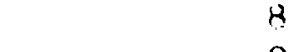 & $0 \cdot$ & 3 & $n$. & 43 & 0 & 8 & 0 & & 43 & $0 \cdot$ \\
\hline 9 & 0 . & 9 & 0 & 9 & $n$. & 44 & 0 . & 9 & 0 & & 44 & 0 . \\
\hline 10 & 0. & 10 & U. & 10 & $n$. & 45 & 0. & 10 & 0. & & 45 & 0. \\
\hline 11 & 0. & 11 & 0. & 11 & 0. & 46 & 0. & 11 & 0. & & $4 h$ & 0 . \\
\hline 12 & 0 & 12 & 0. & 12 & 0. & 47 & 0. & 12 & 0. & & 47 & 0 . \\
\hline 13 & 0. & 13 & l. & 13 & $n$. & 44 & 0. & 13 & 0. & & 49 & 0. \\
\hline 14 & 0. & 14 & u. & 14 & 0. & 49 & 0. & 14 & 0 & & 49 & 0 . \\
\hline 15 & 0. & 15 & 0. & 15 & 0 . & 50 & 0. & 15 & $n$. & & 50 & 0. \\
\hline 16 & 0. & 16 & j. & 16 & $n$. & & & 16 & 0. & & & \\
\hline 17 & 0 & 11 & u. & 17 & $n$. & & & 17 & $1)$ & & & $(1,2 n)$ \\
\hline 18 & 0 . & 18 & 0. & $1^{\mu}$ & $n$. & & & 18 & 0. & & 1 & $9.374 E-01$ \\
\hline 19 & 1) & 19 & (i. & 14 & $n$. & & & 19 & 0. & & $?$ & $1.756 \mathrm{~F}-01$ \\
\hline 20 & 0 . & 2 & 0. & 20 & $n$. & & & 20 & 0. & & 3 & 0 \\
\hline 21 & $0 \cdot$ & 21 & 0. & $? 1$ & $?$ & & & 21 & 0. & & & \\
\hline $2 ?$ & 0. & $2 ?$ & 0 . & 2? & $n$. & & & 2? & 0. & & & \\
\hline$? 3$ & $n \cdot$ & 23 & 0 & 2.3 & $n$. & & & 23 & 0 . & & & \\
\hline$? 4$ & 0. & 2.4 & a. & 24 & $n$. & & & 24 & 0. & & & \\
\hline 25 & 0. & 25 & 0. & 25 & $n$. & & & 25 & $n$ & & & \\
\hline 26 & 0 . & 26 & 0. & 25 & $n$. & & & 26 & $n$ & & & \\
\hline 27 & 0 & $? 1$ & 0 . & 27 & $n$. & & & 27 & $n$. & & & \\
\hline ? 8 & $0 *$ & 24 & i). & 29 & o. & & & 28 & $n$. & & & \\
\hline$? 9$ & $0 \cdot$ & 29 & 0 . & 24 & $n$. & & & 29 & $n$ & & & \\
\hline 30 & 0. & 30 & 0 . & 30 & $n$. & & & 30 & 0. & & & \\
\hline 31 & 1). & 31 & $n$, & 31 & 1). & & & 31 & $n$ & & & \\
\hline 32 & • & 32 & 9. & 32 & $n$. & & & 32 & 0. & & & \\
\hline 3.3 & 0 & 33 & 0. & 33 & n. & & & 33 & 0. & & & \\
\hline 34 & 0. & 34 & 11. & 34 & $n$ & & & 34 & 0 & & & \\
\hline $3^{h}$ & $0 \cdot$ & 35 & ". & 35 & 0. & & & 35 & 0. & & & \\
\hline
\end{tabular}


TABLE C-3 (Continued)

LIBRARY OF CROSS SECTIONS FOR LASER-HIC CALCULATIONS

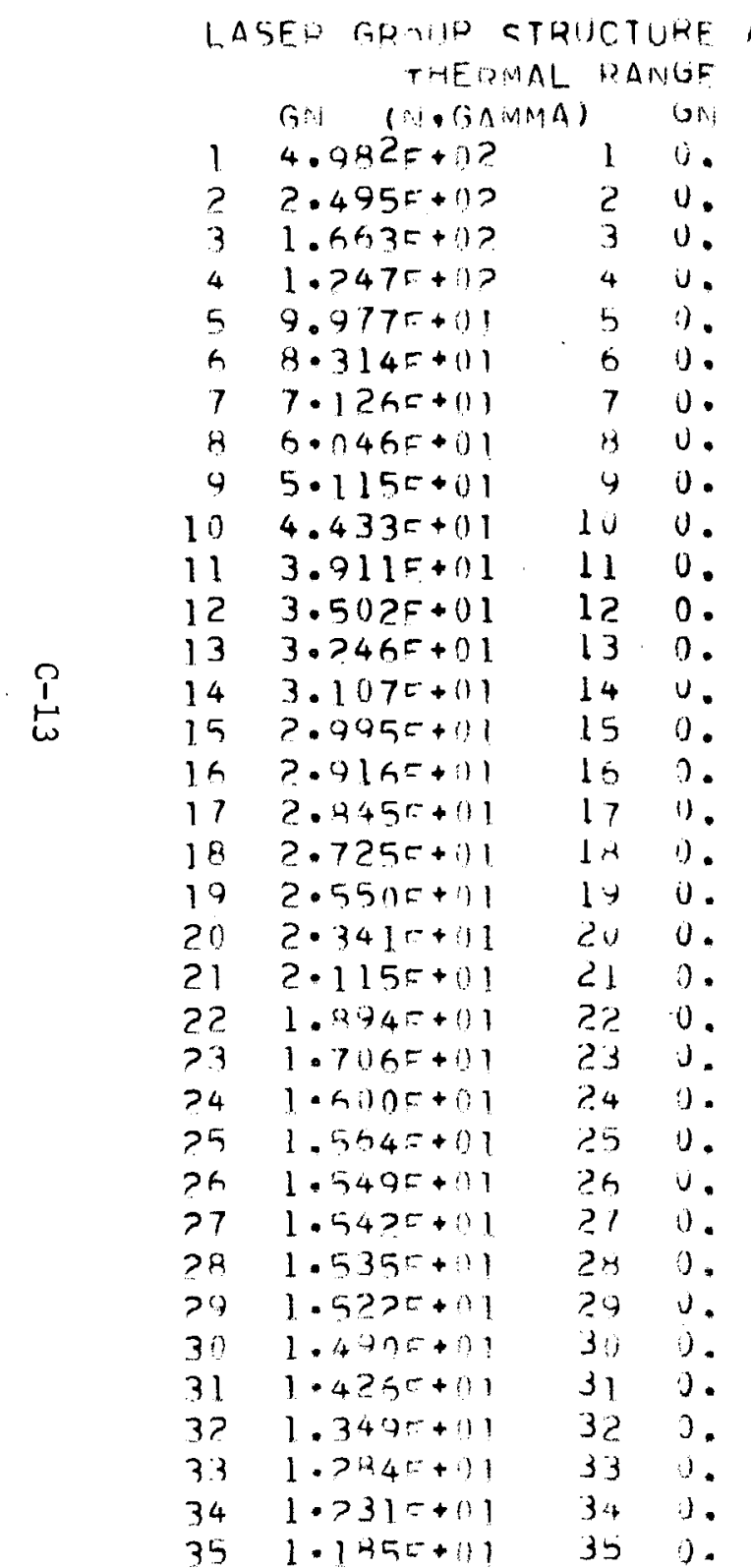

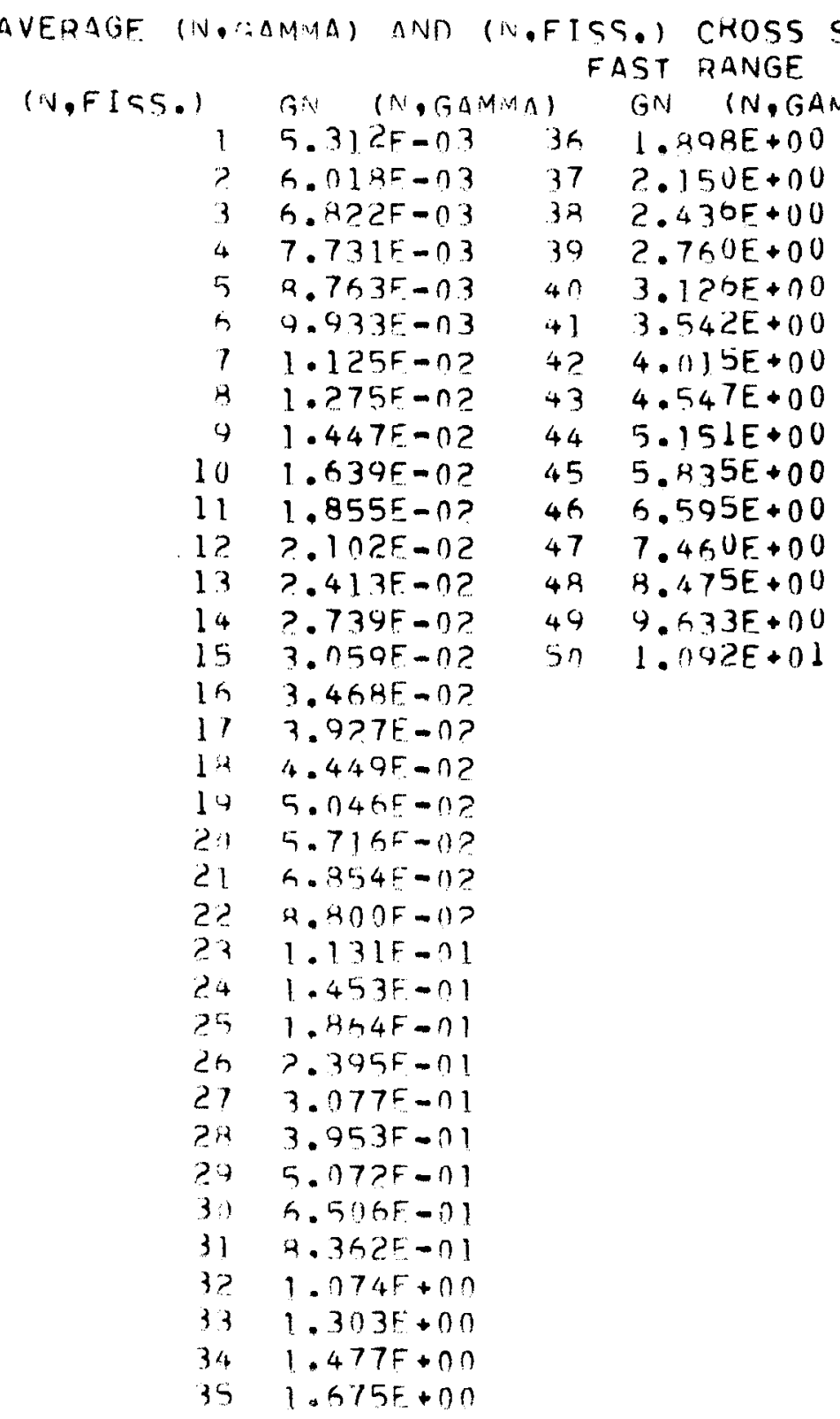

ECTIUN FOR $G N$ $\begin{array}{ll}1 & 6 \\ 2 & 0 \\ 3 & 0\end{array}$ 20 40. $5 n$ 50 . 70 . 711 90 100

110
110 130. 140.
U237 (LASER HIC ID 10) (N,FISS.) IN BARNS

GN (N.FISS.)

$\begin{array}{ll}1 & \text { GN } \\ 36 & 0 . \\ 37 & 0 . \\ 38 & 0 \\ 39 & 0 \\ 40 & 0 \\ 41 & 0 \\ 42 & 0 \\ 43 & 0 \\ 44 & 0 . \\ 45 & 0 . \\ 46 & 0 . \\ 47 & 0 . \\ 48 & 0 \\ 49 & 0 \\ 50 & 0 .\end{array}$

\section{(in, 5N)}

1 ?. $1000-01$

? $5 \cdot$ i $1005-0$ ?

$3 n$. 
TABLE C-3 (Continued)

LIBRARY OF CROSS SECTIONS FOR LASER-HIC CALCULATIONS

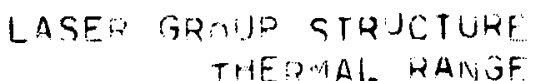

$$
\text { GN (U, GNMAA) }
$$

\section{Q. $452 t+0 ?$}

4.?25F+1?

2. $917=+0 ?$

$2 \cdot 1125+0 ?$

$1 \cdot 690=+0 ?$

$1 \cdot 4095+11 ?$

$1 \cdot 208 r+0 ?$

$1.0 ? 5 \mathrm{~s}+1) ?$

$8.6755+11$

B. $) 44 t+01$

$7 \cdot 34.3=+(1)$

6.920. $5+11$

$6 \cdot 4 ! 3 F+11$

$6 \cdot 9425+111$

$6.973=+11$

$7 \cdot 15 R F+1) 1$

$7.415 F+01$

H. $>38 E+()$

1. 1 MAE +112

$6.9275+11 ?$

$1.5135+112$

$4.5605+i)$

$3.4575+11$

$3.3725+11$

$3 \cdot 4415+01$

$3 \cdot 494 F+? !$

$3.5775+11$

$3.5665+!$

$3.653 F+01$

4.006F+0!

6.99nद+ 11

2. $140 x+11 ?$

4. $3111+11 ?$

$4.942=+1$

$3.1935+11$

\begin{abstract}
isn (N,FISS.)
\end{abstract}
$9.53 \cap E-02$

$4.764 E-02$

$3.174 F-1) 2$

$2.37 \rightarrow F-02$

1. $903 E-1) 2$

$1.585 F-112$

$1.35 \partial F-12$

$1.152 F-112$

$9.747 E-03$

3. $441 F-04$

$3.602 E=04$

$4.022 F=04$

$4.608 E-1) 4$

5. $148 E-114$

5.31 LE- . 4

6. $481 F=04$

7. 2त5E-114

$9.5 U R E-114$

1. $914 E=113$

1. $455 E-1) 2$

2. $418 F-13$

$4.477 E-114$

$3.195 F-114$

$4.232 F-114$

$5.175 E-i 14$

$5.7 ? 7 E-04$

6. $050 F-14$

6. 41 ? $E-14$

1.19BE-14

1. กट7F-13

3. $939 F-13$

1.2U0F- 2

7. $838 \mathrm{~F}-13$

7. $333 F-14$

$2.865 E-14$
(N. IAMMA) AND

$3.518 F+01$

4. $030 \mathrm{~F}+01$

$4.6|1 E+n|$
FAST. QANGF

$\begin{array}{ll}41 & 6 N \\ 36 & 5.770 E+01 \\ 37 & 6.017 E+01 \\ 38 & 6.967 E+01 \\ 34 & 5.597 E+01 \\ 40 & 1.316 E+02 \\ 41 & 1.721 E+01 \\ 42 & 4.525 E+01 \\ 43 & 2.737 E+02 \\ 44 & 6.580 E+01 \\ 45 & 3.043 E+01 \\ 46 & 1.339 E+02 \\ 47 & 3.195 E+01 \\ 48 & 1.431 E+02 \\ 49 & 1.444 E+01 \\ 50 & 4.202 E+01\end{array}$

A)$$
14
$$

FOR INP?37

GN (N,FISC.)

2. $344 E+00$

$1.973 E+00$

$1.578 F+00$

$1.535 E+00$

$1.711 \mathrm{~F}+00$

$1.739 F+00$

$1.714 F+n O$

$1.648 F+00$

1. $566 E+00$

$1.417 E+00$

1. $\cap 48 E+00$

6.? $13 E-01$

2. $187 E-01$

3. $120 E-02$

5. $356 E-0 ?$

4. $018 E-0$ ?

3. $14 \cap E-\cap 2$

$2.54 \mathrm{IF}=02$

2. $997 F-\cap 2$

1. $759 E-02$

$1.439 F-02$

1.227F-0?

1. $370 \mathrm{~F}-0$ ?

2. $006 F=0$ ?

2. $R 91 E-02$

$3.86 ? F-02$

$5.142 F-n 2$

6. $946 F-n ?$

$7.410 \mathrm{~F}-02$

$9.829 F-02$

$1.310 F-01$

1. $735 E-01$

2. $129 E-01$

2. $440 F=01$

2. $794 F-01$
(I ASER HIC ID 11 )

IN BARNS

GN (N,FISS.)

$36 \quad 3.194 E-01$

$373.6495-01$

$38 \quad 4.165 F-01$

$396.116 E-02$

$40 \quad 0$.

41 0 .

4? 0

430.

440.

450 .

460.

470.

480 .

490 .

500 .

$(N, 2 N)$

$12.229 F-01$

? $2.584 \mathrm{~F}-02$

30 . 
TABLE C-3 (Continued)

LIBRARY OF CROSS SECTIONS FOR LASER-HIC CALCULATIONS

LASF" GROUP GTRUCTURE AVERIUE (N, AAAHA) ANI (H.FISS.) CHOSS SECTTUN FOR NPZ3R (LASFR HIC IO IZ) THFONAL KANGF

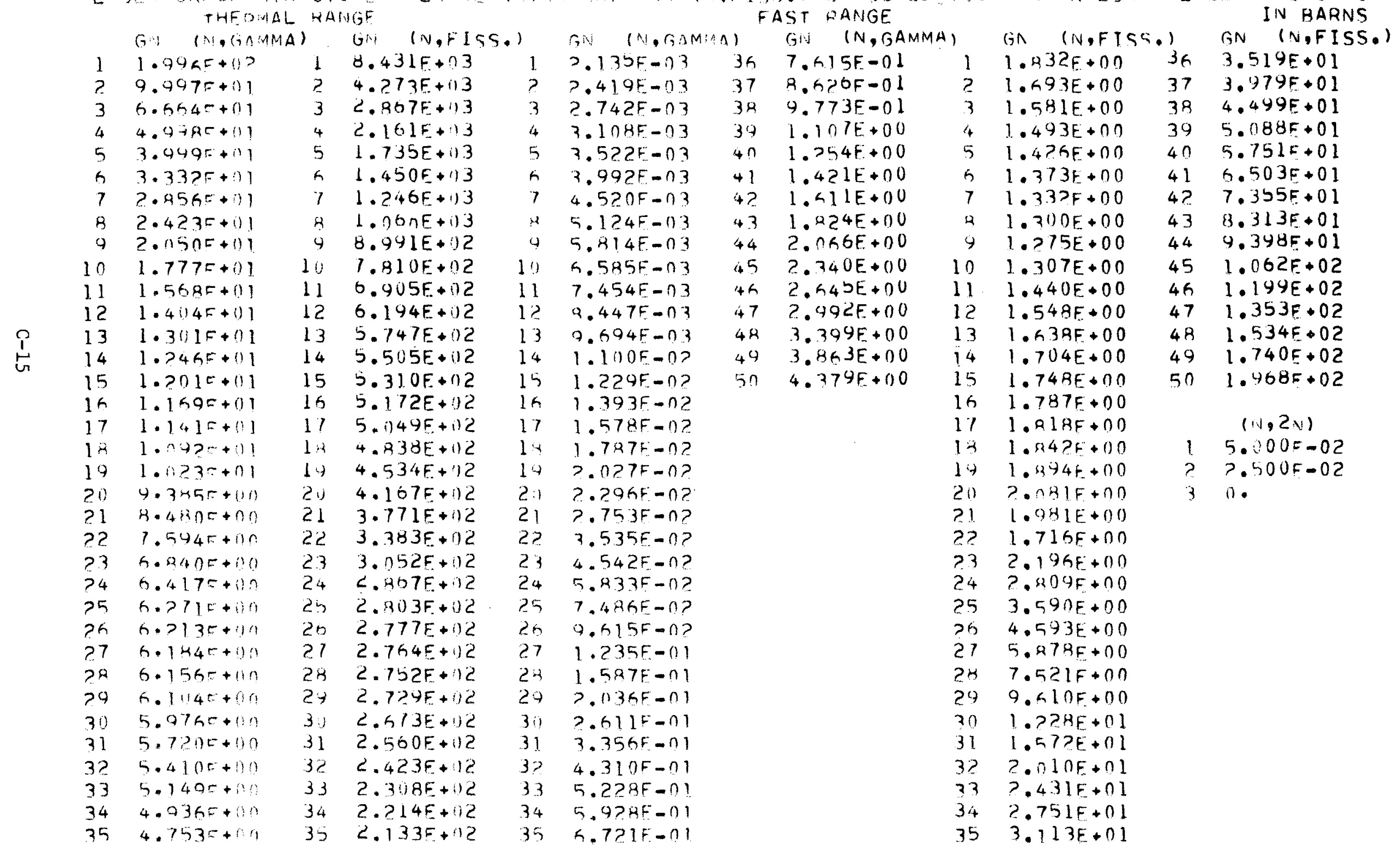


TABLE C-3 (Continued)

LIBRARY OF CROSS SECTIONS FOR LASER-HIC CALCULATIONS

LASEH GRTUP CTRUCTURE
THF MAL QANGF

\begin{tabular}{|c|c|c|c|c|c|}
\hline & $1: 1$ & & $(N, F)$ & & GN \\
\hline 1 & 3. ARGE + O? & $i$ & $9.151 E+01$ & 1 & $5.553 F-0.3$ \\
\hline$?$ & $1.50 R E+07$ & 2 & $4.500 F+01$ & ? & $7.6 \geq 9 E-n 3$ \\
\hline 3 & $9.9273=+19 ?$ & 3 & $2.420 F+111$ & 3 & $0.295 E-03$ \\
\hline 4 & $7.1715+12$ & 4 & $2.0 \$ 6 E+11$ & 4 & $1.195 F_{-}-n 2$ \\
\hline 5 & $5.4630+12$ & 5 & $1.62 \Delta E_{-}+01$ & 5 & $1.547 F-02$ \\
\hline 6 & $4.3245+17 ?$ & 6 & $1.288 E+111$ & $h$ & $? .047 F-0 ?$ \\
\hline 7 & $3.5: 115+11 ?$ & 1 & $1.04 \supset E+01$ & 7 & ?.42RF-n? \\
\hline h & $2.7375+112$ & 4 & $8.136 F+00$ & 2 & ?. ก5BF-0? \\
\hline 9 & $2.072 \%+112$ & $\dot{9}$ & $6.1+9 E+00$ & 9 & $3.990 F-0 ?$ \\
\hline 10 & $1.5625+112$ & 10 & $4.623 E+00$ & 10 & $4.130 F-n 2$ \\
\hline 1 & $1.2145+0 ?$ & 11 & $3.582 E+100$ & 11 & $4.678 F-n ?$ \\
\hline 12 & $4.6395+01$ & 12 & $2.838 E+100$ & 1? & $6.544 \mathrm{~F}-0 ?$ \\
\hline 13 & 7.90GE+II & 13 & $2.347 E+110$ & 13 & R. $6 \cap 5 F-0 ?$ \\
\hline 14 & $7 \cdot 1965+11$ & 14 & $2.108 E+00$ & 14 & $1.05 G F-01$ \\
\hline 15 & $6.5765+01.1$ & 15 & $1.924 E+1) 0$ & 15 & $1.240 F-01$ \\
\hline 16 & $6 \cdot 160 F+01$ & 16 & $1.800 E+00$ & 16 & $1.425 F-01$ \\
\hline 17 & $5.7575+11$ & 17 & $1.678 E+100$ & 17 & $1.598 F-01$ \\
\hline 18 & $5 \cdot 070 r+111$ & In & $1.474 E+00$ & 15 & $1.816 F=01$ \\
\hline 19 & $4 \cdot \mid \not \partial D+01$ & 14 & $1.210 E+1) U$ & 10 & $? .094 F-01$ \\
\hline 20 & $6=+11$ & 20 & $9.358 F-01$ & 20 & $2.474 \mathrm{~F}-01$ \\
\hline 21 & $2.4225+11 !$ & 21 & $6.906 F-01$ & 21 & $3.314 F-01$ \\
\hline ?? & $1.7060+1 ! 1$ & 22 & $4.794 \mathrm{E}-111$ & $2 ?$ & $4.353 F-n 1$ \\
\hline 23 & $1 \cdot 209+1$ & 23 & $3.348 E-111$ & 23 & $5.404 E-01$ \\
\hline 24 & $9.2155 t+10$ & 24 & $2.688 F-01$ & 24 & $5.793 F-01$ \\
\hline$? 5$ & $4.35>0-11$ & 25 & $2.2+5 E-1,2$ & 25 & $4.835 F-11$ \\
\hline 26 & $4 \cdot>43>0-11]$ & 26 & $2.268 F-112$ & $2 h$ & $1.302 F+00$ \\
\hline 27 & $4, ? Y 25-11$ & 27 & $2.270 E-12$ & ?.7 & $1.966 F+00$ \\
\hline 28 & $4.315 \pi-111$ & $2 \mathrm{H}$ & $2.273 F-112$ & 24 & $3.009 F+00$ \\
\hline ?9 & $4.347 t-1$ & 29 & $2.278 E-12$ & 29 & $4.624 F+n$ \\
\hline 30 & $4.4300-11$ & 3 & $2.294 F-112$ & $3:$ & $7.090 F+00$ \\
\hline 31 & $4 \cdot \sin 5=-1$ & 31 & $2,3+8 E-12$ & 31 & $1.044 F+11$ \\
\hline 32 & $5.16 .70-1$ & $3 ?$ & $2.414 F-112$ & $3 ?$ & D. $307 F+01$ \\
\hline 33 & $5.240=-1$ & 33 & $2.670 E-112$ & 33 & $5.004 E+00$ \\
\hline 34 & $6.726=-1: 1$ & 34 & $2.94[F-12$ & 34 & $4.757 F+01$ \\
\hline 35 & $7.438=-1$ & 35 & $.323 F_{-112}$ & 35 & $2.736 F+0$ \\
\hline
\end{tabular}

\section{FAST DANGE}

$\begin{array}{ll}\text { 1) } & \text { GN } \quad(N, G A M M A) \\ 36 & 6.530 E+00 \\ 37 & 6.345 E+00 \\ 36 & 1.441 E-03 \\ 39 & 0 . \\ 4 n & 0 . \\ 41 & 1.473 E+02 \\ +2 & 5.736 E-02 \\ 43 & 1.969 E-02 \\ 44 & 2.415 E+01 \\ 45 & 2.929 E-02 \\ 46 & 1.779 E-02 \\ 47 & 0 . \\ 48 & 0 . \\ 49 & 1.465 E+02 \\ 5 n & 2.571 E-01\end{array}$

$5 \cap 2.5 ? 1 E-01$
FOH PIJZ3R

(

GN (N,FISS,

?. $525 E+00$

$2.635 E+00$

?. $637 F+00$

$2.596 \bar{E}+00$

?. $475 E+00$

$2.334 E+00$

2. $184 E+00$

$2.155 E+00$

?. $076 E+100$

2. $034 E+00$

$1.965 E+00$

$1.735 E+00$

1. $345 E+00$

1. $017 E+00$

7. $998 E-01$

$6.461 E-01$

$5.569 F-01$

$5.197 F-01$

$5.040 F-01$

5. $200 F-01$

$5.7696-01$

$6.758 E-01$

8. $1995-01$

$1.093 F+00$

$1.496 E+00$

$1.714 F+00$

$1.984 E+00$

$2.305 E+00$

$2.673 F+00$

3. $179 E+10$

$3.403 E+00$

6. $2233 \mathrm{~F}+00$

?. $850 \mathrm{~F}+00$

$7.722 E+00$

$3.481 E+00$
(LASER HIC ID 13) IN BARNS

GN (N,FISS.)

36 $1.451 F+00$

$37 \quad 5.385 F-01$

$32 \quad 7.328 E-04$

$39 \quad 5.485 E-04$

$40 ? .0695-04$

$41 \quad 6.342 F+00$

4? $? .484 E-03$

$43 \quad 1.355 E-04$

$446.295 F+00$

$45 \quad 4.714 E-03$

$46 \quad 3.944 E-03$

$47 \quad 3.297 E-03$

480 .

$49 \quad 4.427 F+00$

$50 \quad 1.3215=02$

$$
(1,2 N)
$$

$7.7>4 F-02$

$2.1715-0.3$

0 . 
TABLE C-3 (Continued)

LIBRARY OF CROSS SECTIONS FOR LASER-HIC CALCULATIONS

\begin{tabular}{|c|c|c|c|c|c|c|c|c|c|c|c|}
\hline L & $-A S E$ & $\begin{array}{c}\text { WROID CTRUC } \\
\text { THFOMAL } \\
\text { (OAMMA) }\end{array}$ & & AVERAGE IN, & A:M:A) & ANI $(\because, F T$ & $\begin{array}{l}5.1 \\
45 T\end{array}$ & CROSS SECTIU & & 011230 & $\begin{array}{l}\text { SER HIC ID 14) } \\
\text { IN RARNS }\end{array}$ \\
\hline r. & G:1 & $(\sin (\sin 4)$ & $6 \mathrm{M}$ & & 54 & $(A, \beta \triangle M M A)$ & $G N$ & $(N, G A M M A)$ & GN & $(N, F[S \rightarrow)$, & GN $\quad(N, F I S S)$, \\
\hline 1 & 11. & $\perp$ & J. & 1 & $n$. & 36 & 0 . & 1. & 0. & 36 & 0 . \\
\hline 2 & ). & $?$ & u. & $?$ & 0. & 37 & ). & $?$ & 0. & 37 & 0 \\
\hline 3 & 0. & 3 & 0. & 3 & $n$. & 30 & 0. & 3 & $n$ & 39 & 0. \\
\hline 4 & 0 . & 4 & 1. & 4 & 0 . & 39 & 0. & 4 & $n$. & 39 & 0. \\
\hline 5 & i) & 5 & 0. & 5 & $n$. & 40 & 1) & 5 & $\pi$ & 40 & 0. \\
\hline h & $? \cdot$ & h & U. & 5 & a. & 41 & 0 & 6 & $n$. & 41 & (1). \\
\hline 7 & 0 . & 7 & 0 . & 7 & 0 & $4 ?$ & 0 & 7 & 0 & 42 & 0 . \\
\hline a & $1 \cdot$ & q & i). & A & $n$ & 43 & 0 & $B$ & 0 . & 4.3 & 0 . \\
\hline 9 & 0 . & 9 & i) & 4 & $n$. & 44 & 0 & 9 & 0 . & 44 & 0 . \\
\hline 10 & 0 . & 10 & 0 . & 10 & 0. & 45 & 0 . & 10 & $n$. & 45 & 0 . \\
\hline 11 & 0 . & 11 & 0 . & 11 & 0. & 45 & 0 & 11 & 0 & 46 & 0 \\
\hline 12 & ). & 12 & 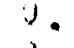 & $1 ?$ & $n$. & 47 & 0 . & 12 & $n$. & 47 & 0 . \\
\hline 13 & 0 . & 13 & 0. & 13 & $n$. & 49 & 0 . & 13 & $n$. & 48 & $n$ \\
\hline 14 & 11. & 14 & 0 & 14 & 0 . & 49 & 0. & 14 & 0 . & 49 & 0 . \\
\hline 15 & 0 . & 15 & j. & 15 & 1. & 50 & 0. & 15 & ?. & 50 & 0. \\
\hline 16 & $n$. & Ih & 0 . & lis & 0 & & & 16 & 0 & & \\
\hline 17 & i). & 17 & $J$. & 17 & $n$. & & & 17 & $n$ & & $(N, 2 N)$ \\
\hline 18 & i. & 14 & i). & 14 & $n$. & & & 18 & 0. & 1 & $1.405 F-01$ \\
\hline 19 & 1) & 17 & 0 . & 19 & 0 & & & 19 & $n$. & 2 & $6.149 E-02$ \\
\hline$? 0$ & $1 \cdot$ & 20 & J. & 211 & ). & & & $?$ & $n$ & 3 & $1.3305-03$ \\
\hline 21 & $0 \cdot$ & 21 & ). & 21 & 0 & & & 21 & 9 & & \\
\hline$? ?$ & $n$. & $2 ?$ & $j$. & $2 ?$ & $n$. & & & $3 ?$ & $n$. & & \\
\hline 23 & ? & 23 & $\lambda$ & 23 & $n$. & & & 2.3 & 0 . & & \\
\hline 24 & 0 - & 24 & j). & $? 4$ & ?. & & & $? 4$ & 0 & & \\
\hline$? 5$ & 1) & 25 & v. & 25 & 7 . & & & 25 & 0 . & & \\
\hline ?h & ). & 24 & . & 26 & 7. & & & 36 & $n$ & & \\
\hline$? 7$ & $0 \cdot$ & 27 & 0 & $? 7$ & $n$. & & & 27 & 0 & & \\
\hline 24 & 0 & 20 & U. & 30 & $\therefore$ & & & $\begin{array}{l}38 \\
29\end{array}$ & i. & & \\
\hline 30 & 0. & 3 & 0 . & 30 & 0 & & & $\begin{array}{l}27 \\
30\end{array}$ & $n$ & & \\
\hline 31 & 0 . & 31 & $u$ & $3 !$ & 0 & & & 31 & $n$. & & \\
\hline 32 & 0. & 32 & 0 & $3 ?$ & 0 & & & 32 & $n$. & & \\
\hline 33 & 0. & 33 & i). & 33 & $\eta$ & & & 33 & 0 & & \\
\hline 34 & a. & 34 & l. & 34 & 11. & & & 34 & $n$. & & \\
\hline 35 & ). & 35 & 1). & 35 & $n$ & & & 35 & 0 & & \\
\hline
\end{tabular}


TABLE C-3 (Continued)

LIBRARY OF CROSS SECTIONS FOR LASER-HIC CALCULATIONS

\begin{tabular}{|c|c|c|c|c|c|c|c|c|c|c|c|c|}
\hline $\mathrm{L}$ & $A C F R$ & $\begin{array}{l}\text { (AROUP STRUC } \\
\text { THEOMAL }\end{array}$ & $\begin{array}{l}J R F \\
\text { IGF }\end{array}$ & AVERAGE IN, & $\triangle(1: A A)$ & ANI $(I, F I C$ & .15 & $\begin{array}{l}\text { CROSS SECTTUN } \\
\text { PANGE }\end{array}$ & $F \cap$ & PUج4n & ILA & $\begin{array}{l}\text { SER HIC ID I5) } \\
\text { IN BARNS }\end{array}$ \\
\hline & 6:4 & $(\because, O A A \operatorname{MA} A)$ & GN & $(N, F I \subset S)$, & 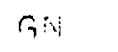 & $(N,(B \cap M N \cap)$ & $G N$ & $(N, G A M M A)$ & GA & $(N, F[S S)$. & 1 & GN $\quad(N, F I S S)$. \\
\hline 1 & 0 & 1 & ). & 1 & $n$. & 36 & 0 & J & 0. & & 36 & 0. \\
\hline 2 & 0. & 2 & i). & 2 & $n$ & 37 & 0. & 2 & 0. & & 37 & 0. \\
\hline 3 & $0 \cdot$ & 3 & 0. & 3 & $n$ & 38 & 0 & 3 & $n$ & & 38 & $n$ \\
\hline 4 & 0 . & 4 & 0 , & 4 & $n$ & 30 & $n$ & 4 & 0. & & 39 & 0. \\
\hline 5 & $n \cdot$ & 5 & 0. & 5 & $n$ & $4 i$ & !). & 5 & $n$ & & $4 n$ & 0. \\
\hline h & $0 \cdot$ & 6 & $\therefore$ & G & 7. & 41 & ). & 6 & $n$. & & 41 & 0 . \\
\hline 7 & 0 - & 7 & i). & 7 & n. & +7 & $n$ & 7 & $n$ & & 4? & 0. \\
\hline R & 0 & 4 & i). & 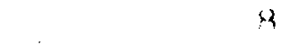 & $n$. & 43 & ). & $y$ & !) & & 43 & 0. \\
\hline 9 & $9 \cdot$ & 9 & 0. & $\dot{y}$ & 0. & 44 & 0 . & 9 & $n$. & & 44 & 0. \\
\hline 10 & 0 . & 10 & u. & 10 & 0 & 45 & 0 & 10 & 0 . & & 45 & 0. \\
\hline 11 & 0 & 11 & 0 & 11 & $n$. & $4 h$ & 0 & 1.1 & 0 . & & 46 & 0 \\
\hline 12 & 1). & 12 & 0. & $1 ?$ & 0. & 47 & 0 . & 12 & 0 . & & 47 & 0 . \\
\hline 1.3 & $0 \cdot$ & 13 & 0. & 1.3 & 0. & 49 & 0 & 13 & 0. & & 48 & 0 \\
\hline 14 & 0 & 14 & 0. & 14 & $n$. & 49 & 9 & 14 & $n$. & & 49 & 0. \\
\hline 15 & $0 \cdot$ & 15 & 0. & 15 & $n$. & 50 & 1). & 15 & $n$. & & 50 & 0. \\
\hline $1 \mathrm{t}$ & 0. & 16 & . & 16 & $n$ & & & 16 & 0. & & & \\
\hline 17 & $0 \cdot$ & 17 & 1). & 17 & 0 & & & 17 & $n$. & & & $(N, 2 N)$ \\
\hline 1.8 & 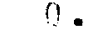 & La & i). & 18 & ") & & & $1^{\alpha}$ & $n$. & & 1 & 4. $\cup \cup S F-01$ \\
\hline 19 & 0 & 19 & ). & 19 & i. & & & 19 & $n$. & & 2 & $x .+21 F=02$ \\
\hline 20 & 0 & 20 & ). & 211 & $?$ & & & 20 & $n$ & & 3 & 0. \\
\hline 21 & 0 . & 21. & 1). & ?.1 & ?. & & & $? 1$ & 0 . & & & \\
\hline 22 & $0 \cdot$ & $2 c$ & 0 . & 27 & $n$. & & & 22 & 0 & & & \\
\hline 23 & 0. & 23 & u. & 23 & 11. & & & $? 3$ & 0. & & & \\
\hline 24 & 0 & 24 & i). & 24 & $n$ & & & 24 & $n$ & & & \\
\hline 25 & 0 & 25 & 0. & 25 & a. & & & $? 5$ & $n$. & & & \\
\hline $2 h$ & $n \cdot$ & 26 & U. & $2 \mathrm{~h}$ & $n$ & & & 26 & 0 & & & \\
\hline 27 & 0 . & 27 & i. & 27 & $n$. & & & 27 & 0. & & & \\
\hline$? 8$ & 0 & $? 4$ & U. & 24 & . & & & $? 8$ & 0 & & & \\
\hline$? 9$ & $n \cdot$ & 29 & 1). & 29 & i. & & & 29 & 0. & & & \\
\hline 30 & 0 & 30 & 0. & 30 & 0. & & & 30 & 0 . & & & \\
\hline 31 & . & 31 & i), & 31 & ). & & & 31 & $n$. & & & \\
\hline $3 ?$ & 0 & 32 & 0. & 3? & $n$. & & & 32 & $n$ & & & \\
\hline 33 & 0 & 3.3 & i). & 3.3 & 0 & & & 33 & ). & & & \\
\hline 34 & $a$ & 34 & U. & 34 & $n$ & & & 34 & 0. & & & \\
\hline 35 & $n \cdot$ & 35 & 0. & 35 & $n$. & & & 35 & 0. & & & \\
\hline
\end{tabular}


TABLE C-3 (Continued)

LIBRARY OF CROSS SECTIONS FOR LASER-HIC CALCULATIONS

LASER GRIIO GTHUCTURE AVERAGF (N, IAMMA) ANO (N.FISS.) CROSS SECTIUIV FOH PU24I (LASER HIC ID 16)

\begin{tabular}{|c|c|c|c|c|c|c|c|c|c|c|c|}
\hline & $G \times$ & $(O,(B A N A)$ & $G^{n-1}$ & $(N, F \mid s s)$. & GN & $(N, G \wedge M P A)$ & $\begin{array}{l}\text { AST } \\
\text { GN }\end{array}$ & $\begin{array}{l}\text { HANGE } \\
\text { (N, GAMMA) }\end{array}$ & GN & & IN RARNS \\
\hline 1 & 0. & 1 & 0. & 1 & $n$. & 36 & 0 & 1 & 0 & $(I N A F I S S \cdot)_{3 G}$ & $\begin{array}{ll}G N & (N, F I S S,) \\
0 . & \end{array}$ \\
\hline 2 & 0 . & 2 & 0. & $?$ & $n$. & 37 & 0. & $?$ & 0. & 37 & 0 \\
\hline 3 & 0 . & 3 & 0. & 3 & $n$. & 38 & 0. & 3 & 0. & 38 & 0. \\
\hline 4 & 0 . & 4 & u. & 4 & $\eta$ & 39 & 0 & 4 & $n$ & 39 & 0. \\
\hline 5 & 1). & 5 & v. & 5 & $n$. & 40 & 0 & 5 & 0 & $4 n$ & 0. \\
\hline h & 0 & 6 & 0 . & h & $n$. & 41 & 0. & 6 & 0. & 41 & 0. \\
\hline 7 & 0 & 7 & u. & 7 & $n$. & $4 ?$ & 0. & 7 & $n$ & $4 ?$ & 0. \\
\hline$B$ & 0 & H & 0. & M & $n$ & 43 & 0 & a & 0. & 43 & 0. \\
\hline 9 & 0. & 9 & 0. & 9 & 0. & 44 & 0 & 9 & $n$ & 44 & 0. \\
\hline 10 & 0 . & 10 & 0. & 10 & 0. & 45 & 0. & 10 & 0. & 45 & 0. \\
\hline 11 & 0. & 11 & 0. & 11 & 0. & $4 h$ & 0. & 11 & $n$ & $4 h$ & 0. \\
\hline 12 & 0. & 12 & 0. & 12 & 0. & 47 & 0 & 12 & 0. & 47 & 0 \\
\hline 13 & 0 . & 13 & 0. & 13 & 0. & $4 R$ & 0 & 13 & $n$. & $4 R$ & $n$ \\
\hline 14 & 0 . & 14 & 0. & 14 & $n$. & 49 & 0. & 14 & 0. & 49 & 0. \\
\hline 15 & $0 \cdot$ & 15 & 0. & 15 & $n$ & 50 & 0. & 15 & 0 & 50 & 0. \\
\hline 15 & $9 \cdot$ & 16 & u. & 16 & $n$. & & & 16 & 0. & & \\
\hline 17 & 0 . & 17 & 0. & 17 & ). & & & 17 & 0 & & $(N, 2 N)$ \\
\hline 18 & 0 & $1 \%$ & u. & $\ln$ & $n$ & & & $1^{H}$ & $n$ & 1 & $7.1645-01$ \\
\hline 19 & is. & 19 & 0 & 14 & $n$ & & & 19 & 0 & $?$ & $6.700 F=01$ \\
\hline 20 & 0 & 20 & U. & 21 & $n$. & & & 20 & $n$ & 3 & $0.146 F=02$ \\
\hline 21 & $0 \cdot$ & 21 & 0 & $? ?$ & $n$ & & & $>1$ & 0 & & \\
\hline $2 ?$ & 0 . & 22 & 0 & 27 & 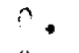 & & & 22 & $n$ & & \\
\hline $\begin{array}{l}73 \\
74\end{array}$ & 0. & 23 & ). & 23 & 0. & & & 23 & 0. & & \\
\hline$? 5$ & 0 & $\begin{array}{l}24 \\
25\end{array}$ & 0. & 24 & 0. & & & 24 & 0. & & \\
\hline 36 & 0 & $\begin{array}{l}25 \\
26\end{array}$ & u. & 25 & 1) & & & 75 & 0. & & \\
\hline 27 & 0 . & 27 & 0. & 27 & 0 & & & $\begin{array}{l}26 \\
27\end{array}$ & $n$ & & \\
\hline 28 & 0. & 20 & 0. & $2 \times$ & 0. & & & $\begin{array}{l}27 \\
28\end{array}$ & $n \cdot$ & & \\
\hline 74 & 0 & 29 & u. & 29 & 0. & & & $\begin{array}{l}28 \\
39\end{array}$ & $n$. & & \\
\hline 30 & 11. & 30 & 0 & 30 & 0 & & & $31)$ & 0. & & \\
\hline 31 & 0 & 31 & ". & 31 & $n$ & & & 31 & 0. & & \\
\hline 32 & 0. & 32 & 0 & 32 & $n$ & & & $3 ?$ & 0 & & \\
\hline 33 & 0 & 33 & 0 & 33 & $n$. & & & 33 & 0. & & \\
\hline 34 & 0 & $3 / 4$ & v. & 34 & $n$. & & & 34 & $n$ & & \\
\hline 35 & $n$. & 35 & 0. & 35 & 0. & & & 35 & 0. & & \\
\hline
\end{tabular}


TABLE C-3 (Continued)

LIBRARY OF CROSS SECTIONS FOR LASER-HIC CALCULATIONS

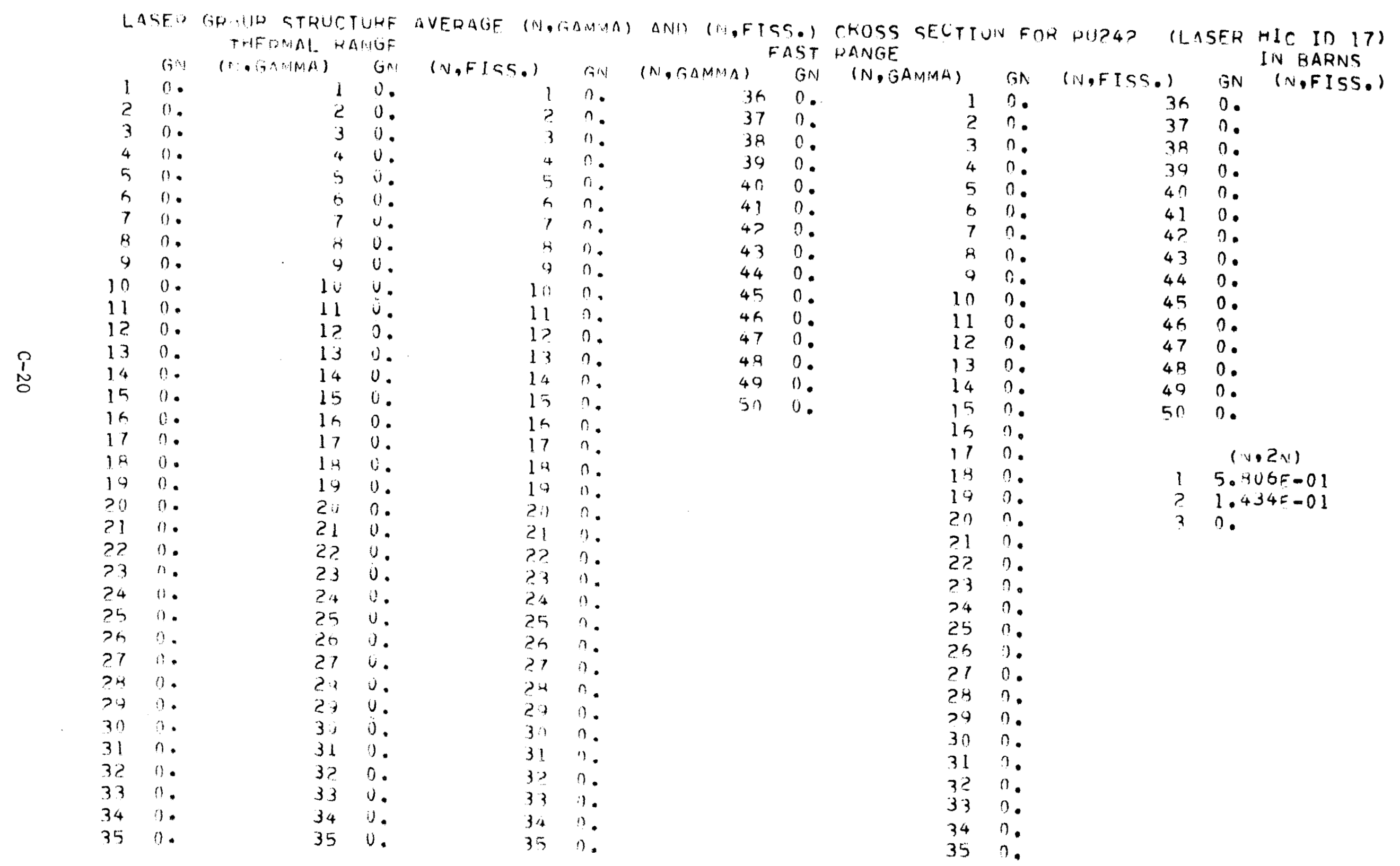


TABLE C-3 (Continued)

LIBRARY OF CROSS SECTIONS FOR LASER-HIC CALCULATIONS

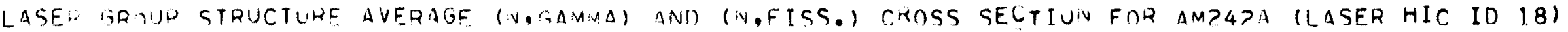
THFQMAL RANGF

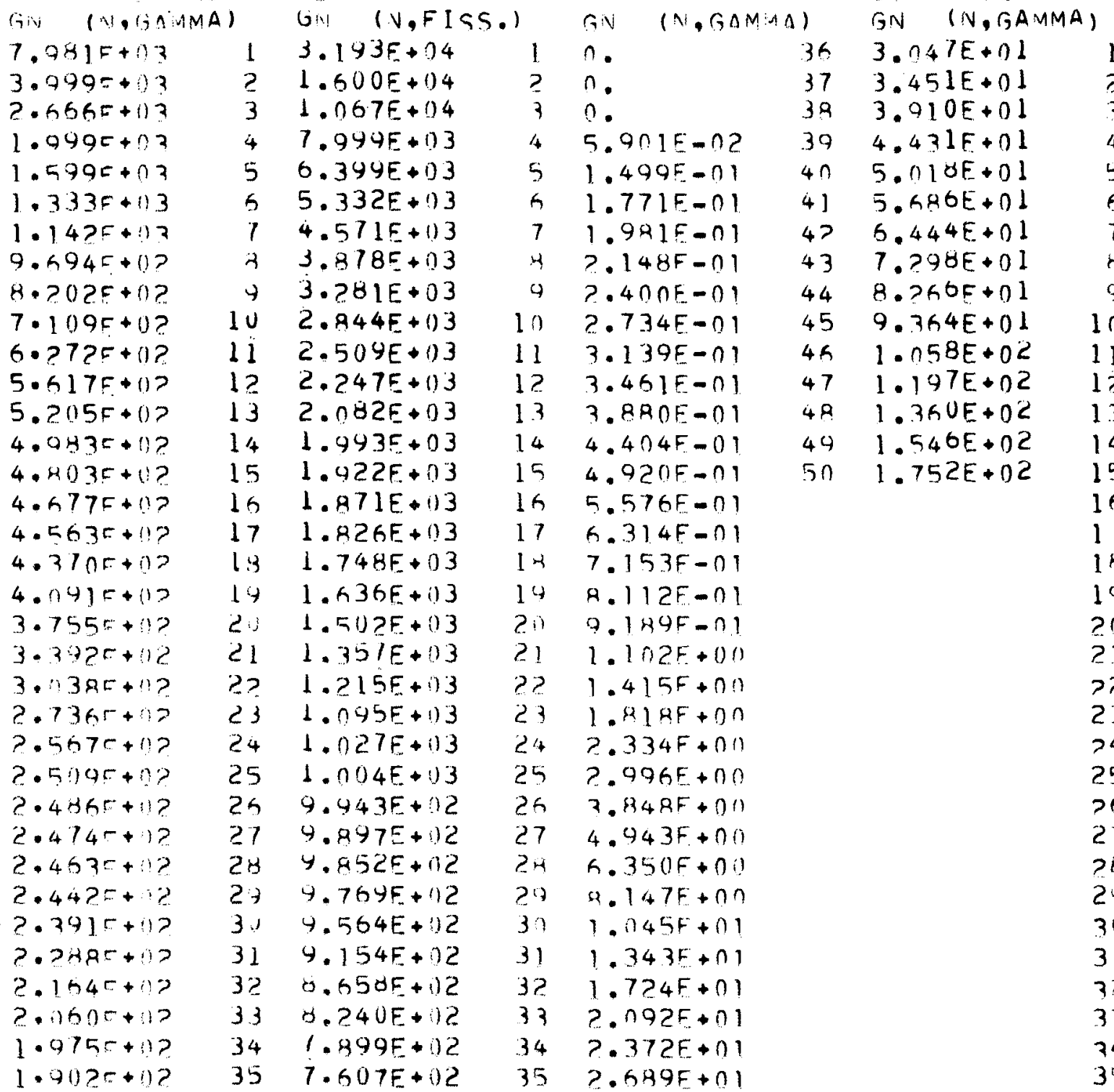

GN $(N, F[5, \cdot)$

$3.476 E-0]$

$4.066 E-01$

4. $530 F-01$

$5.129 F-01$

$6.751 E-01$

7. $\cap 19 F-01$

7. $.71 \mathrm{~F}-01$

P. $50 ? F=01$

$9.450 F-01$

$1.017 E+00$

1.? $46 E+00$

$1.382 \mathrm{E}+00$

$1.55 ? E+00$

1. $761 E+00$

$1.968 E+00$

2. $230 \mathrm{~F}+00$

?. $525 E+00$

2. $861 \mathrm{~F}+00$

3. $>44 E+00$

3. $675 F+00$

$4.406 E+00$

$5.658 E+00$

7. ? $7 \mathrm{OF}+00$

$9.337 E+00$

1.19RE+01

$1.539 \mathrm{~F}+0 \mathrm{I}$

$1.977 \mathrm{~F}+01$

2. $540 F+01$

3. $259 \mathrm{~F}+01$

4. $180 \mathrm{~F}+01$

$5.372 E+01$

$6.998 E+01$

$4.367 E+01$

$9.488 E+01$

$1 . \cap 76 F+02$

IN BARNS

GN (N,FISS.)

$36 \quad 1.219 E+02$

$37 \quad 1.381 E+02$

$38 \quad 1.564 E+02$

$39 \quad 1.772 E+02$

$40 \quad 2.007 F+02$

$412.274 F+02$

$422.578 E+02$

$43 ? .919 E+02$

$443.307 E+02$

$453.746 F+02$

$46 \quad 4.234 E+02$

$47 \quad 4.788 F+02$

$48 \quad 5.440 E+02$

$49 \quad 6.183 F+02$

$507.008 F+02$

$(N, 2 N)$

$11.500=+00$

? $9.000 \mathrm{~F}-01$

30. 
TABLE C-3 (Continued)

LIBRARY OF CROSS SECTIONS FOR LASER-HIC CALCULATIONS

LASFR GPNIP STRJCTUHE AVERAGF (N.AAMAA) IND (N.FTSS.) CKOSS SECTIUN FOR AMZ43 THFOAAL RANGF

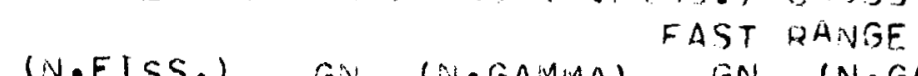

3
1
1
1
1
1
1
1
1
1
1
2
2
2
2
2
2
2
2
3
3
3
3
3
3
3
3

(LASER HIC ID 19) IIV RARNS 
TABLE C-3 (Continued)

LIBRARY OF CROSS SECTIONS FOR LASER-HIC CALCULATIONS

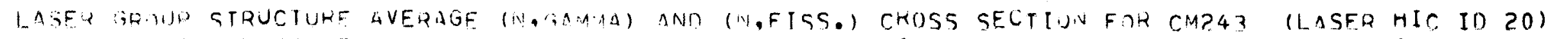
THEDAL RANGF FAST RANGE

\section{G.) (MAMMA) \\ GV $\quad(V, F I 5 S$. \\ GN $($ N. $($; M MAA $)$ \\ GN $(N, G A M M A)$}

$5.91330+12$

$3.3465+1 ?$

$2.3750+i ?$

1. $46 ?=+1 ?$

$1 \cdot 5+1=+12$

1. $321 F+1 ?$

$1.159 r+112$

$1 \cdot 0119 r+02$

8. $7555+11$

$7.7565+01$

$6.075 F+i 1$

$6.353 r+1) i$

$5.9575+01$

$5.740 \%+11$

$5 \cdot 564 E+01$

$5 \cdot 4+n \pi+? !$

5. 3?RE+?

$5 \cdot 1,36=+11$

4. $.257=+11$

$4.516 r+11$

$4.144 \div+11$

3. $7745+i 1$

$3.455 x+1$

$3.2735+11$

3. $21 n r+11$

$3 \cdot 184 t+11$

3.1720+i1

3. $1611 r+11$

3. $1375+1$

3. लि15+11

?. $469 r+11$

2. 3? $3+01$

?.716F+11

2. $620=+1$

2. $538 F+1$
5. $993 E+112$

3. $346 E+112$

c. $375 F+12$

$1.962 E+12$

$1.541 E+12$

$1.321 E+112$

$1.159 E+02$

$1.009 F+112$

b. $755 E+01$

1.756E+ 1

$6.975 E+11$

6. $353 F+1) 1$

$5.957 E+1) 1$

$5.740 E+1) 1$

$5.564 E+11$

$5.44 \cup E+01$

๖. $328 F_{n}+111$

๖. $136 F+111$

$4.857 E+11$

4.516F+11

$4.144 E+11$

$3.774 E+111$

$3.455 E+11$

$3.273 E+01$

3. $210 E+01$

$3.184 F+01$

$3.172 F+01$

3. $160 E+01$

$3.137 E+111$

3. 0 ⿰ा $F+1$

2. $964 F+01$

c.4.325+01

$2.716 E+111$

$2.62 \cap F+1$

2. $538 E+11$
$3.705 F=0 ?$

4. IIRF-O?

$4.579 F=02$

5. $191 \mathrm{~F}=0 ?$

$5.561 F-0 ?$

4. $2945-n$ ?

$6.993 F=0 ?$

$7.777 F-0 ?$

a. $655 \mathrm{~F}=n$ ?

Q.617F-0?

$111.068 E-01$

$121.14 R F-01$

$131.335 E-01$

$14 \quad 1.486 F=01$

$15 \quad 1.632 F-01$

is $1.415 \mathrm{~F}-n$

$17>.016 \mathrm{~F}-11$

$142 . ?+1 F-01$

$172.493 \mathrm{~F}-01$

2i) $? .771 F-01$

$213.23 \cap F-n 1$

22 $3.992 F-01$

$234.936 F=01$

टA $5.102 E-01$

$257.537 E-01$

DA $9.31 R F-01$

$27 \quad 1.152 F+00$

$241.425 F+0 n$

20 $1.759 F+n n$

$317 ? .172 F+n n$

$312.697 F+00$

3) ?.321F+0

$33 \quad 3.912 \mathrm{~F}+0 \mathrm{O}$

$3 / 4.351 F+00$

$354.440 F+00$
35 $5.379 E+10$

$375.979 E+100$

$32 \quad 6.640 E+100$

$397.388 E+00$

4 ? $3 . ?] 0 E+00$

$419.120 E+00$

$+? \quad 1.015 E+1) 1$

$431.127 E+01$

$44 \quad 1.253 E+01$

$45 \quad 1.393 E+01$

$46 \quad 1.545 E+01$

$47 \quad 1.715 E+01$

$49 \quad 1.910 E+01$

49 2.129E+01

$5 n \quad 2.367 E+01$
I: HARNS

GN (N,FISS.) GN (N,FISS.) $3.70 \supset E-0 ? \quad 36 \quad 5.37 Y F+00$ $4.11 A E-n>\quad 37 \quad 5.479 E+00$ 4.579E-n? $38 \quad 6.546 E+00$

$5.091 E-n ? \quad 39 \quad 7.388 E+00$

$5.451 F-02 \quad 40 \quad 4.210 E+00$

6.?94E-0? $41 \quad 9.126 E+00$

$6.393 E-12 \quad 42 \quad 1.015 E+01$

$7.777 F=0 ?$

9. $455 E-0 ?$

$9.517 E-O ?$

1. $068 E-01$

]. $188 \mathrm{E}-01$

$1.335 E-01$

$1.486 E-01$

$1.632 E-01$

1. $815 E-01$

2. $1 G E-01$

?. $241 F-01$

$2.493 E-01$

?.771F-OI

3. $>3 \cap F-11$

3. $792 F-01$

$4.936 F-01$

6. $10 ? E-01$

$7.537 E-01$

$9.31 R F-01$

$1.152 E+00$

$1.425 \mathrm{~F}+100$

$1.759 F+n n$

$? .172 \mathrm{~F}+00$

?. $687 \mathrm{E}+00$

$3.321 E+00$

3. $.12 E+00$

$4.351 E+00$

$4.840 E+00$

$431 \cdot 127 E+01$

$441 \cdot 253 E+01$

$451.393 F+01$

$46 \quad 1.545 E+01$

$47 \quad 1.715 E+01$

$48 \quad 1.910 E+01$

$49 ? .129 E+01$

$50 ? \cdot 367 E+01$

$(v, 2 y)$

0

? 0 .

30 . 
TABLE C-3 (Continued)

LIBRARY OF CROSS SECTIONS FOR LASER-HIC CALCULATIONS

LASEH GPOUP STPUCTUHE
THFOMAL HAIGF GN (M, (INMMA) GN

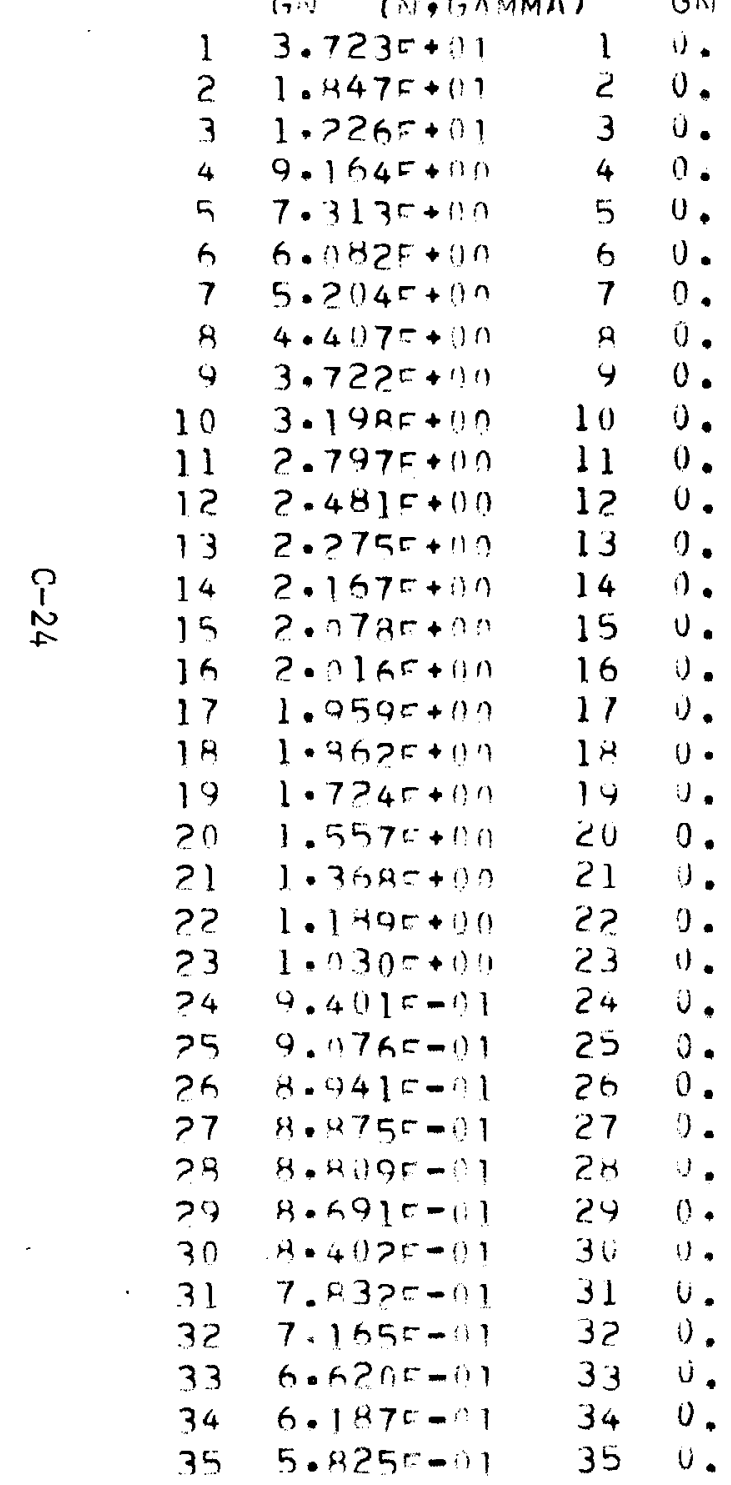

AVEQAUE (N,CIAMMA) AND (N,FISS.) CKOSS SECTIUN FOK THZ3? FAST RANGE

(IV.FISS.) GMi (N,GAMMA) (KN (N,GAMMA)

$\begin{array}{lll}9.243 F-03 & 34 & 5.540 E+01 \\ 1.237 F-02 & 37 & 1.621 E+01 \\ 1.701 F-02 & 39 & 3.267 E-02 \\ 7.345 F-0 ? & 34 & 1.745 E-02 \\ 7.275 F-0 ? & 40 & 9.420 E+01 \\ 4.644 E-0 ? & 41 & 6.475 E+01 \\ 6.635 F-0 ? & 47 & 5.265 F-02 \\ 9.201 F-0 ? & 43 & 8.483 E-02 \\ 1.248 F-01 & 44 & 1.681 E-01 \\ 1.579 F-01 & 45 & 1.462 E-01 \\ 1.749 F-01 & 46 & 1.850 E-01 \\ 1.797 F-01 & 47 & 2.425 E-01 \\ 1.791 F-01 & 49 & 3.149 F-01 \\ 1.794 F-01 & 49 & 4.027 F-01 \\ 1.783 F-01 & 50 & 5.078 E-01\end{array}$

$1.783 F-01$

1. $8 R 5 E-n 1$

?. $057 F-n ?$

?. $297 F-n !$

ว. $\$ 41 F-n 1$

3. $135 E-\cap 1$

4. $1 \cap 1 F_{-}-11$

$5.410 F-01$

$4.713 F-01$

Q. $150 \mathrm{~F}=01$

1. $000 E+00$

$1.320 F+00$

$1.70 \mathrm{AF}+00$

2. $359 F+00$

$3.349 F+0 n$

$4.370 F+00$

$7.099 F+00$

$1.45 \cap E+01$

1. $298 F-n !$

?. $699 F+n 1$

1. $56.3 F-n ?$
(L $\triangle S E R$ HIC ID 21 ) IN BARNS

SN $(N, F T S S$.

GN

(N.FISS.)

$\begin{array}{llll}1 & 3.104 E-01 & 36 & 0 . \\ 2 & 2.751 E-01 & 37 & 0 . \\ 3 & 1.414 E-01 & 38 & 0 . \\ 4 & 1.431 F-01 & 39 & 0 . \\ 5 & 1.333 F-01 & 40 & 0 . \\ 6 & 1.151 E-01 & 41 & 0 . \\ 7 & 1.012 E-01 & 42 & 0 . \\ 4 & 9.142 E-02 & 43 & 0 . \\ 1 & 5.058 F-03 & 44 & 0 . \\ 10 & 0 . & 45 & 0 . \\ 11 & 0 . & 46 & 0 . \\ 12 & 0 . & 47 & 0 . \\ 13 & 0 . & 49 & 0 . \\ 14 & 0 . & 49 & 0 . \\ 15 & 0 . & 50 & 0 . \\ 16 & 0 . & & \end{array}$

$(i v, 2 N)$

$1.617 F+00$

? $2.474 F-01$

30 . 
TABLE C-3 (Continued)

LIBRARY OF CROSS SECTIONS FOR LASER-HIC CALCULATIONS

LASF GHGUP STRUCTUHE AVERAGE (N. TAMMA) DND (N.FISS.) CKOSS SELTTUIN FOR THZZZ THFDAAL RANGE FAST PANGE

\begin{tabular}{|c|c|c|c|c|c|c|c|c|c|}
\hline & $\cos \cdot \cos$ & & $(N, F I s$ & . $)$ & $(N, C ; A M$ & & (N,GAN & & $(N, F I$ \\
\hline 1 & $7 \cdot 485 F+13$ & 1 & $6.977 F_{-}+111$ & 1 & $9.015 F-n ?$ & 36 & $2.85 \%=01$ & 1 & $7.437 F=n 4$ \\
\hline 2 & $3.7505+113$ & $?$ & $3.494 E+191$ & $?$ & $9.0 R \perp F-\cap P$ & 37 & $3 .>37 E+01$ & 2 & A. $426 E-04$ \\
\hline 3 & $2.500 F+03$ & 3 & $2.329 E+101$ & 3 & $1.029 F-11$ & 38 & 3. $K G / F+n I$ & 3 & $4.550 E-04$ \\
\hline 4 & 1. $475 \sigma+02$ & 4 & $1.747 F+01$ & 4 & $1.166 F=01$ & 39 & $4.155 F+101$ & 4 & 1. $082 E-13$ \\
\hline 5 & $1.5005+113$ & 5 & $1.397 E+11$ & 5 & $1.3 ? 2 F=11$ & 40 & $4.7016 F+01$ & 5 & $1.227 F-03$ \\
\hline 6 & $1.750 F+03$ & 6 & $1.164 E+01$ & h & $1.498 \mathrm{~F}-n !$ & 41 & $5.332 F+01$ & 6 & $1.391 F-03$ \\
\hline 7 & $1 \cdot 071 F+0 ?$ & 7 & $9.979 E+00$ & 7 & $1.697 F-01$ & 47 & $6.043 F+n 1$ & 7 & $1.575 F-03$ \\
\hline 8 & $9 \cdot 0.915+17$ & 9 & $8.465 E+00$ & $y$ & $1.923 F-01$ & 4.3 & $6.944 E+01$ & 9 & 1. $785 F-03$ \\
\hline 9 & $7 \cdot 6925+0 ?$ & $\varphi$ & $7 \cdot 163 E+010$ & 9 & $? .192 F=01$ & 44 & $7.752 E+01$ & $\vartheta$ & $2.026 F-0.3$ \\
\hline 10 & $6.6675+i 7$ & 10 & $D . ? 08 E+100$ & 10 & $? .471 E-01$ & 45 & $8.791 E+01$ & 10 & ?. $294 E-03$ \\
\hline 11 & $5 \cdot x 425+112$ & 11 & $5.477 F+00$ & 11 & $2.798 E-01$ & 46 & $9.925 F+01$ & 11 & $2.597 E-0.3$ \\
\hline 12 & $5.2670+12$ & 12 & $4.904 E+00$ & 12 & $3.171 F-01$ & 47 & $1.123 F+02$ & 12 & $2.943 F=n 3$ \\
\hline 13 & $4.3 \beta>r+11 ?$ & 13 & $4.545 F+00$ & 13 & $3.638 E=01$ & $4 R$ & $1 . ? 75 F+02$ & 13 & $3.378 F-03$ \\
\hline 14 & $4.6735+0 ?$ & 14 & $4.351 E+00$ & 14 & $4.13 \cap F-n 1$ & 49 & $1.450 E+02$ & 14 & $3.935 E-03$ \\
\hline 15 & $4.51050+1 ?$ & 15 & $4.194 F+00$ & 15 & $4.614 F-n 1$ & $5 n$ & $1.643 F+02$ & 15 & 4.? $83 E=03$ \\
\hline 16 & $4 \cdot 3 \& 6 F+11 ?$ & 16 & $4 \cdot 083 E+100$ & 15 & $5.229 F-01$ & & & 16 & $4.955 E-03$ \\
\hline 17 & 4. $2 A n E+12$ & 17 & $3.944 E+10$ & 17 & $5.4>1 F=n 1$ & & & 17 & $5.498 E-03$ \\
\hline 39 & $4 \cdot 1420+2$ & 18 & $3.915 E+01)$ & $1 \times$ & $4.7085-01$ & & & 18 & $5 .>P 9 F-03$ \\
\hline 19 & $3 \cdot 036 F+112$ & 14 & $3.572 F+00$ & 19 & $7.607 E-01$ & & & 14 & $7 . n 64 F-03$ \\
\hline 20 & $3.5215+112$ & $2 v$ & $3.278 E+110$ & 211 & $9.618 F-01$ & & & 20 & $\mathrm{H} \cdot 003 \mathrm{~F}-03$ \\
\hline 21 & $3 \cdot 1 \times 15+1 ! 2$ & 21 & $2.9625+110$ & 21 & $1.033 F+n \pi$ & & & 21 & $9.576 F-03$ \\
\hline$? ?$ & $? \cdot 2400+? ?$ & 22 & $2.652 F+110$ & $2 ?$ & $1.327 F+00$ & & & 22 & $1.232 E-02$ \\
\hline 23 & $? .5665+i 12$ & 23 & $2.384 E+00$ & $2 ?$ & $1.7045+00$ & & & 23 & $1.583 E-0 ?$ \\
\hline$? 4$ & $? .408=+17 ?$ & 24 & $2.241 E+110$ & 24 & $7.189 E+00$ & & & 24 & $2 \cdot \cap 34 F-02$ \\
\hline 25 & $2.3535+1 ! ?$ & 25 & $2.1405+10$ & 25 & $? \cdot R \cap 9 E+00$ & & & 25 & $? .610 E-0 ?$ \\
\hline 26 & $2 \cdot 331=+2$ & 26 & $2.17 \cup E+110$ & 26 & $3.608 F+00$ & & & 26 & $3.353 E-02$ \\
\hline 27 & $2.32 n c+02$ & 27 & $2.160 E+100$ & 27 & $4.536 F+00$ & & & 27 & $4.308 t-02$ \\
\hline 26 & $2 \cdot 3190+?$ & $2 R$ & $\angle .150 E+00$ & 24 & $5.955 F+100$ & & & 28 & $5.535 E-02$ \\
\hline 29 & $2.24 n=+12$ & 29 & $2.132 E+110$ & 29 & $7.640 F+00$ & & & 24 & $7.10>F-02$ \\
\hline 30 & $2.2425+1 ?$ & 30 & $2.08 / E+1) 0$ & $3 ! 1$ & 9.4005 .00 & & & 30 & $9.110 F-02$ \\
\hline 31 & $2.146 \pi+10$ & 31 & $1.997 E+100$ & 31 & $1.259 F+n 1$ & & & 31 & $1.171 F-01$ \\
\hline 32 & $2.1130+2$ & 32 & $1.989 E+100$ & 32 & $1.617 F+111$ & & & 32 & $1.504 F-01$ \\
\hline 33 & $1 \cdot 932 r+?$ & 33 & $1.7 Y B E+110$ & 33 & $1.962 F+01$ & & & 33 & $1.47 .4 E-01$ \\
\hline 34 & $1.9525+?$ & 34 & $1.724 F+100$ & 34 & $2.224 F+01$ & & & 34 & $2.069 F-01$ \\
\hline 35 & $1.7335+0 ?$ & 35 & $1.650 F+00$ & 35 & $2.5225+01$ & & & 35 & $2.345 E-01$ \\
\hline
\end{tabular}

(LASER HIC ID 22) IN RARNS

GN $(N, F I S S$.

GN (N,F I
$2.657 F-0)$

$373.010 F-01$

$3 A \quad 3.411 E-01$

$39 \quad 3.465 F-01$

$404.3785-01$

$414.469 \mathrm{~F}-01$

$425.622 F-01$

$43 \quad 6.367 F=01$

$447.212 F-01$

$45 \quad 8.171 E=01$

$46 \quad 9.235 E-01$

$47 \quad 1.045 F+00$

$48 \quad 1.187 E+00$

$491.349 E+00$

$5 \cap \quad 1.529 E+00$

$\left(v, 2 n^{\prime}\right)$

$1 \cdot 125 F+00$

B. $750 \mathrm{~F}-0]$

?. $3006-01$ 
TABLE $\mathrm{C}-3$ (Continued)

LIBRARY OF CROSS SECTIONS FOR LASER-HIC CALCULATIONS

LASER GRMIP STRUCTULE AVERAGE (N,GAMMA) AND (V,FTSS.) CROSS SELTTUIN FOR PAZ33 (LASER HIC ID 23) PWFOAL HANGF

SN (N.FISS.)

IN BARNS

(N,FISS.)

\section{GN} 1. $! 21 E+00 \quad 36 \quad 0$. $9.614 E-01 \quad 370$. $5.770 F-01 \quad 38 \quad 0$. 
TABLE C-3 (Continued)

LIBRARY OF CROSS SECTIONS FOR LASER-HIC CALCULATIONS

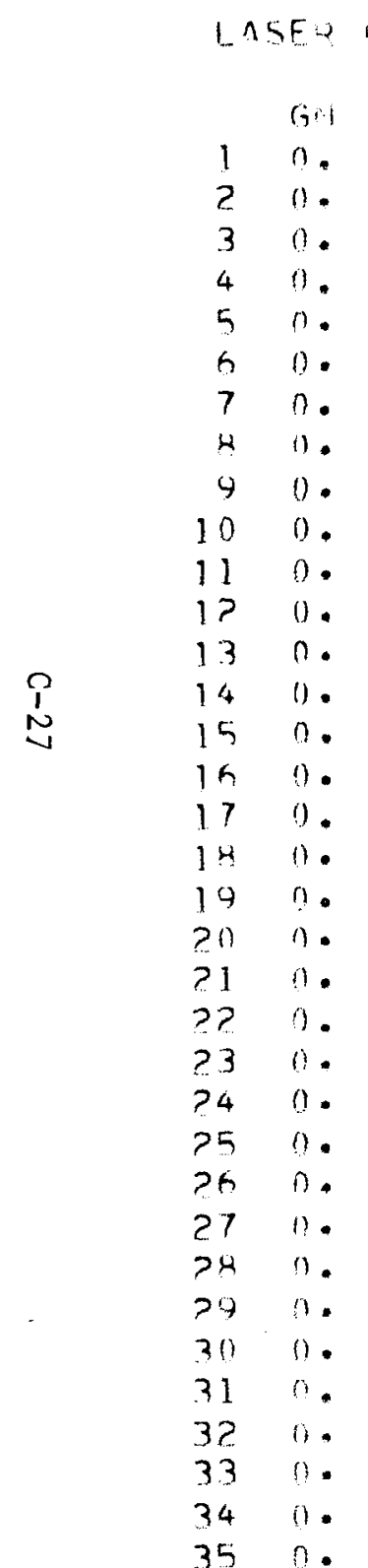

POU STRUCTURF
THFOAAL HANGE

(a, GA:AMA

\begin{tabular}{|c|c|c|c|c|}
\hline$|A|$ & GN & $(N, F I \subseteq S)$, & $\sin$ & (in, Gavas) \\
\hline$l$ & 0 & 1 & 0. & 36 \\
\hline ? & U. & $\lambda$ & 0 . & 37 \\
\hline 3 & u. & 3 & ก. & 30 \\
\hline 4 & 0 . & 4 & $n$. & 34 \\
\hline 5 & 0 . & 5 & $n$. & $4 \pi$ \\
\hline$G$ & u. & h & $n$. & 41 \\
\hline 7 & 0 . & 7 & $n$. & 47 \\
\hline 3 & U. & 9 & 0 . & 43 \\
\hline 9 & 0. & $\theta$ & 0. & 44 \\
\hline 10 & 0. & 111 & 0 . & 45 \\
\hline 11 & 0. & 11 & $n$ & $4 \mathrm{~K}$ \\
\hline 12 & 0. & 12 & $n$. & 47 \\
\hline 13 & 0. & 13 & $n$. & 48 \\
\hline 14 & 0. & 14 & 0 & 47 \\
\hline 15 & 0. & 15 & $n$ & 50 \\
\hline
\end{tabular}

FAST RANGE
(N, GAMMA)
11238

(LASER HIC ID 24) IN BARNS

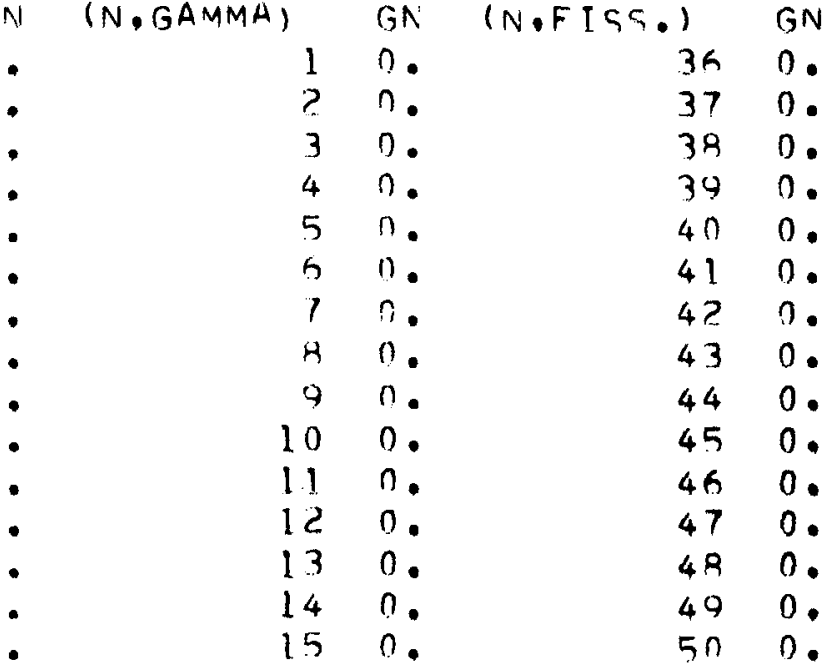

is $n$.

l) n.

$1+0$

190.

2i) $n$.

2) 0 .

2? $n$.

$23 n$.

240

250

2h 0.

270

$\begin{array}{ll}24 & 0 \\ 20 & 10\end{array}$

30 .

$31 n$.

3. 0 .

330.

340.

350
(N.FISS.)

.

0 .

.

0 .

0.

.

0.

0.

.

\section{(N, $2 \mathrm{~N})$}

$1.2635+00$

? $4.345 F-01$

30 . 
TABLE C-3 (Continued)

LIBRARY OF CROSS SECTIONS FOR LASER-HIC CALCULATIONS

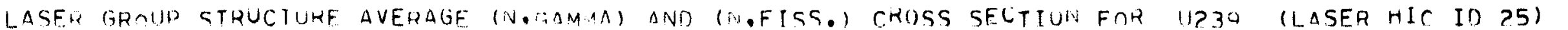
THFUMAL HANGF

\section{$6.986 E+01$}

$3.500 F+01$

$5.5 ! 8=+1 ! 1$

$3.673 r+111$

$2.7555+11]$

2. $2 ! 14 \pi+i !$

$1.937 \div+19$

$1.5750+1$

$1 \cdot 3365+! !$

$1 \cdot 1315+!$

9. $800=+0 !$

$8.6475+1)$

$7 \cdot 7435+0 n$

$7 \cdot 177=+1)$

$6.4705+11 !$

6. 42? $=+11$

6. $4495+11 ?$

$6.2925+1) !$

$6 \cdot 125=+01$

$5 \cdot+405+11$

$5 \cdot 1770+1$

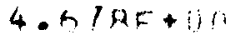

4. $14 Q F+1)$

$3 \cdot 774=-0 ?$

$3.5415+111$

$3 \cdot 460=+00$

$3 \cdot 4245+2 n$

$3 \cdot 4120+10$

3. 39R +11

3. 36Rr+i

3. $297 r+11$

$3 \cdot 1560+1 n$

$2 \cdot 925 m+n$

?. $241=+11$

?. $723:+11$

$2 \cdot 4 ? 3 r+9 ?$

$2.333 F+i) 1$

$1.750 \mathrm{E}+01$

1. $400 E+01$

$1.166 E+01$

$9.948 E+01$

$8.483 F+00$

$7.17 \mathrm{BE}+110$

$6.221 E+00$

$5.489 E+100$

$4.915 F+00$

$4.555 F+00$

$4.361 F+00$

$4.203 F+00$

4. . $93 E+00$

3. $994 F+00$

3. $924 E+00$

3. $580 E_{-}+0$

3. $286 F+10$

C. $96 Y F+100$

$2.559 E+10$

$2.3 y 5 E+00$

$2.2475+00$

$2.196 E+010$

$2.175 E+01$

2. $165 E+100$

2. $155 E+10$

C. $137 E+1)$

¿. $19 ? F+11$

C. $002 F+00$

1. $>3 \rightarrow 4 F+100$

$1.20 J E+10$

1. $720 E+00$

$1.604 F+11)$

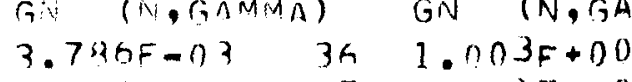

?. 36, $6 F-0 ?$

4. $120 F-0 ?$

$5.496 F-0 ?$

6. $549 F-\cap ?$

7.377F-n?

Q. ISGF-ח?

Q. $975 F-0 ?$

$9.714 \mathrm{~F}-02$

1. $\cap 2 R F=01$

1. $n 72 F-n$ ?

1. $2945-\cap 1$

?. $005 F-01$

2. $534 F-n 1$

?. $890 F-0$ ?

3. $2\lfloor 1 . E-0 !$

$3.4505-01$

$3.6545-n 1$

3. $\$ 7 R F=11$

4. $363 F-n !$

4.9? IF-O)

$5.441 F-01$

5. 75PF-01

$5.96,3 F-n 1$

6. $544=-n 1$

7. $236 F-n 1$

$7.524 F-n 1$

7. $96 \cap 5-01$

A. OGGF-ก1

4. $653 F-n !$

Q.) $59 F-01$

9. PQRF -01

$9.419 F-\cap 1$

$9.474 F-01$

$0.631 F-n !$
$37 \quad \operatorname{na} 3 \pm+00$

$32 \quad 1.052 \mathrm{~F}+00$

$391.069 E+00$

$4 n \quad 1.042 F+00$

$41 \quad 1.192 F+00$

4? $1.100 E+00$

$43 \quad 1.10 E+00$

$441.146 E+10$

$45 \quad 1.292 E+00$

$4 \mathrm{~h} \quad 1.460 E+00$

$47 \quad 1.651 E+00$

$4 A \quad 1.876 E+00$

$49 \quad 2.132 F+00$

$50 \quad 2.41 .6 F+00$
GN (N,FISS.)

1. $759 E-01$

$2.500 F-01$

?. $500 F-01$

2.50OF-01

?. $493 F-01$

?. $485 F-01$

?. $454 F-? !$

?.373F-?

?. $ว 1 E-01$

?.ว?9F-01

2. $131 E-01$

?. $005 F-01$

1. $487 E-01$

1. $\cap 99 E-01$

Q. $373 E-0 ?$

$6 . \cap 16 E-n 2$

4. $195 F-n$ ?

2. $769 F-n ?$

1. $831 F=0 ?$

1. $005 E-02$

?. $108 F-0$ ?

2. $297 \mathrm{E}-0 ?$

?. $+12 E=02$

$2.51 B E-n ?$

$3.986 E-0 ?$

$5.589 F-0 ?$

6.56IF-ก?

7. $150 F-0 ?$

8. $434 F-0$ ?

1. $462 F-01$

1. $.97 F-01$

?. $144 F-01$

?. ?73F-01

?. $33 ? F-n 1$

?. $989 F-n 1$
IN BARNS

GN (N,FISS.)

$36 \quad 3.420 F-01$

$37 \quad 4.723 F-01$

$38 \quad 5.350 F-01$

$395.839 F-01$

4? 6.? $18=-01$

4) $5.515=-01$

4? $5.746 E-01$

$436.926 F-01$

$447.263 F-01$

$45 \quad 8.194 F-01$

$469.261 F-01$

$47 \quad 1.047 F+00$

$48 \quad 1.190 F+00$

$49 \quad 1.353 F+00$

$501.5335+00$

$(N, 2 \times)$

$1.500 F-01$

? $7.500 \mathrm{~F}-02$

$3 ? .500=-0$ ? 
TABLE C-3 (Continued)

LIBRARY OF. CROSS SECTIONS FOR LASER-HIC CALCULATIONS

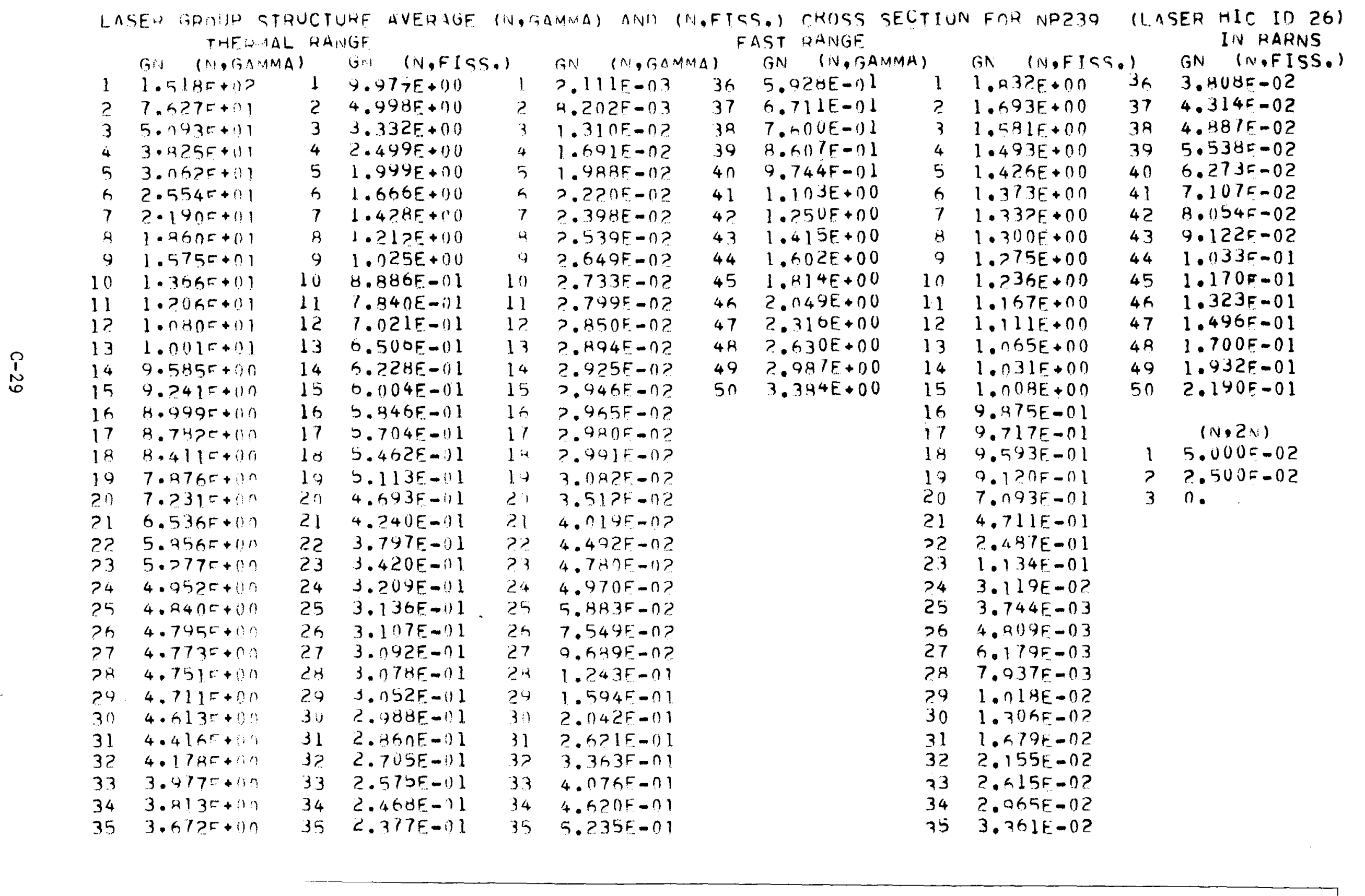


TABLE C-3 (Continued)

LIBRARY OF CROSS SECTIONS FOR LASER-HIC CALCULATIONS

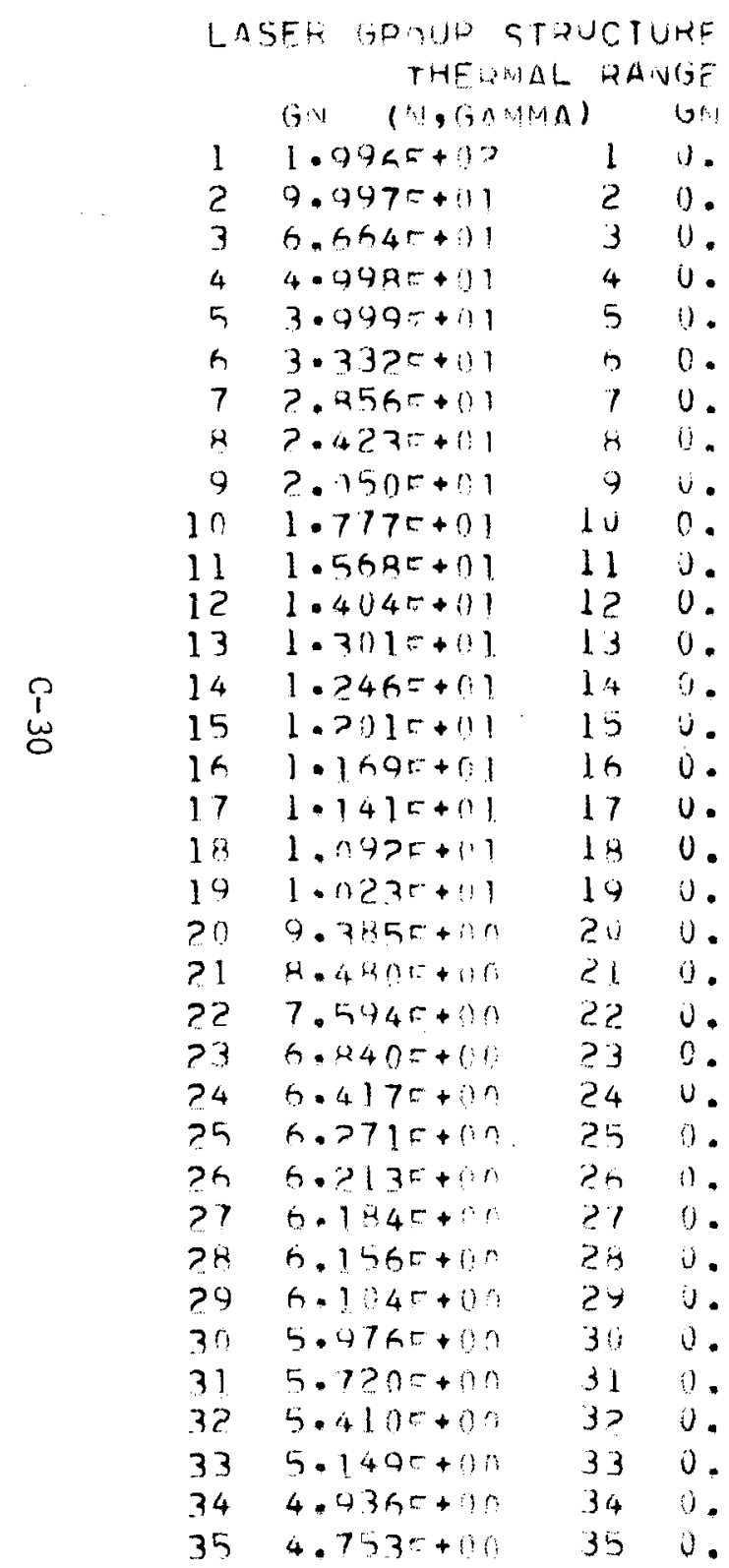

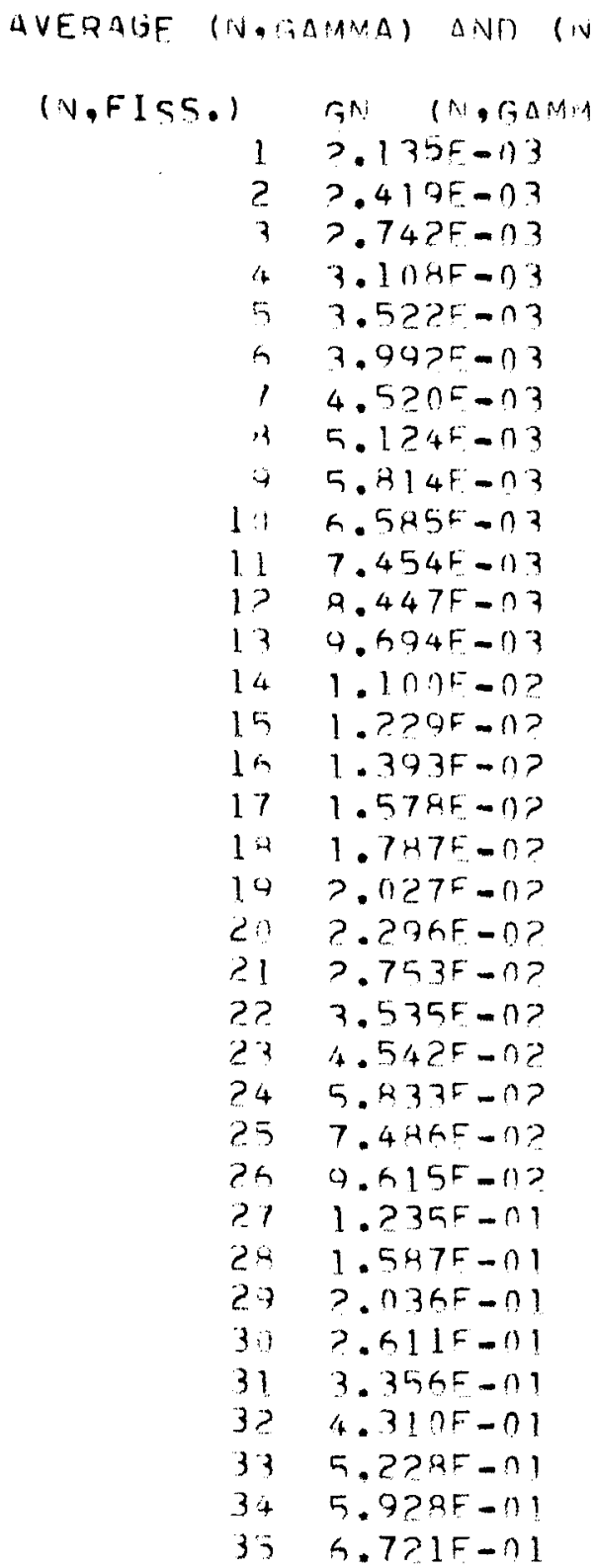

ISS.) CROSS SECTIUN FOH WPZ36 FAST TANGE (JN) (N,GAMMA)

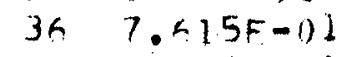

$37 \quad 0.626 \mathrm{~F}-01$

$38 \quad 9.773 E-01$

$39 \quad 1.107 F+0 U$

$40 \quad 1.254 F+00$

$41 \quad 1.421 E+00$

$42 \quad 1.511 E+00$

$47 \quad 1.424 E+00$

$44 ? .166 \mathrm{~F}+10 \mathrm{O}$

$452.340 F+00$

$46 ? .645 E+10$

$472.992 F+00$

$48 \quad 3.399 F+00$

$493.963 E+00$

$5 n \quad 4.279 E+00$

A) GN (IV,FISS.

(LASER HIC ID ?7)

IN HARNS

$\begin{array}{llll}1 & 0 . & 36 & 0 . \\ 2 & 0 . & 37 & 0 . \\ 3 & 0 . & 39 & 0 . \\ 4 & 0 & 39 & 0 . \\ 5 & 0 . & 40 & 0 . \\ 6 & 0 . & 41 & 0 . \\ 7 & 0 . & 42 & 0 . \\ 9 & 0 . & 43 & 0 . \\ 9 & 0 . & 44 & 0 . \\ 10 & 0 . & 45 & 0 . \\ 11 & 0 . & 45 & 0 . \\ 12 & 0 . & 47 & 0 . \\ 13 & 0 . & 49 & 0 . \\ 14 & 0 . & 49 & 0 . \\ 15 & 0 . & 50 & 0 .\end{array}$

$(1, \cdot 2 \cdots)$

5. $1100=-0$

? $2.500 F-0 ?$

30 . 
TABLE C-3 (Continued)

LIBRARY OF CROSS SECTIONS FOR LASER-HIC CALCULATIONS

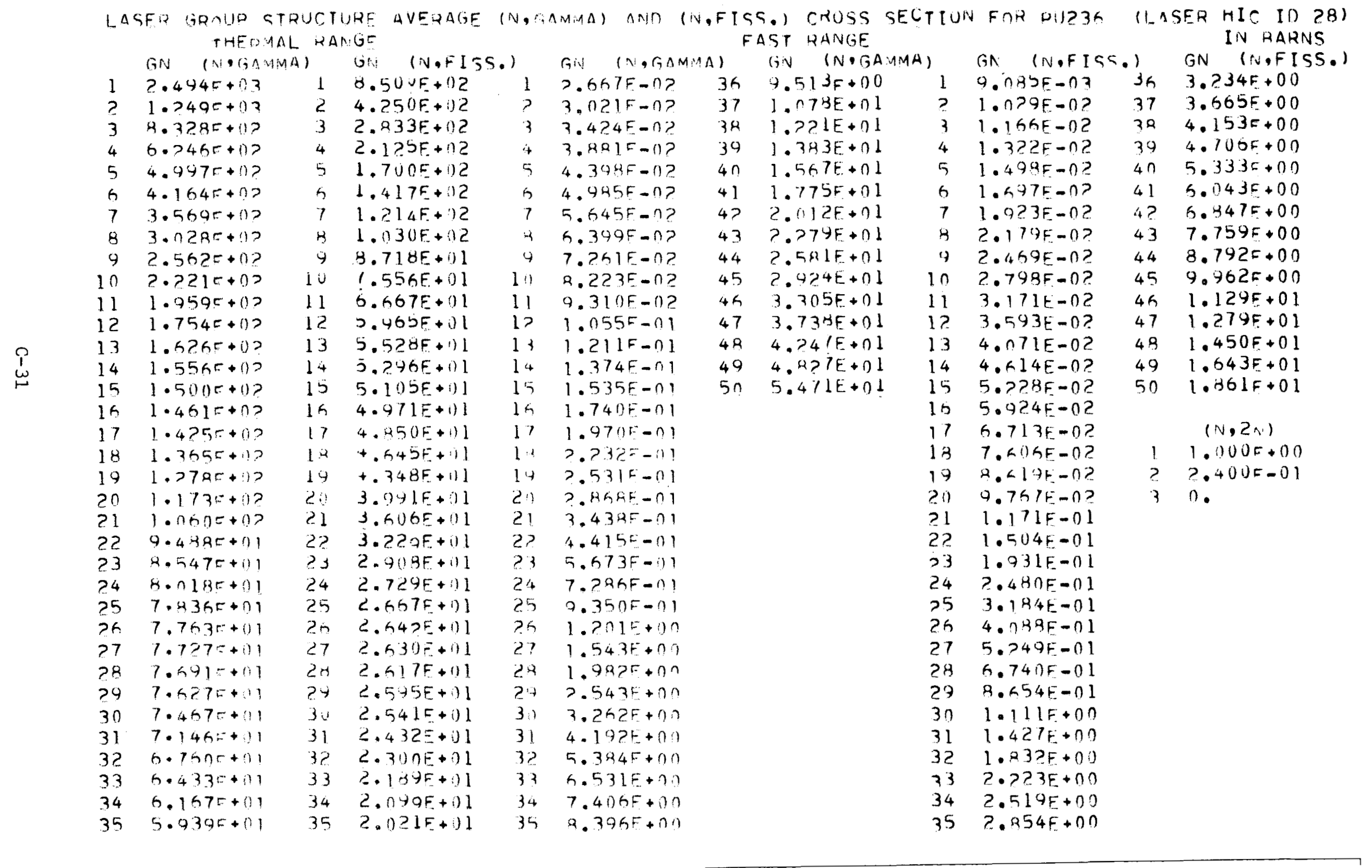


TABLE C-3 (Continued)

LIBRARY OF CROSS SECTIONS FOR LASER-HIC CALCULATIONS

LASER GROUP GTRUCTURE AVERAGE (N,GAIAMA) ANI) (V.FIGS,) CHOSS SECTIUIV FOR AMZ4) (LASFR HIC ID ?9) TIAEDIMAL. RANGF

\begin{tabular}{|c|c|c|c|c|}
\hline \multicolumn{2}{|c|}{$(\because, G \wedge M M A)$} & \multicolumn{2}{|c|}{$(N, F I S S)}$, & (w, Gn \\
\hline $2.6500+1)^{2}$ & 1 & $1.7 \supset 0 E+11$ & 1 & $1.041 F-03$ \\
\hline $1 . ? 9 n=+03$ & 2 & $0.74 ? E+1) 0$ & $?$ & $1.609 E-03$ \\
\hline R. $36 n=+0 ?$ & 3 & $5.826 F+100$ & 7 & $? .40 \cap E-03$ \\
\hline $6.195 F+10 ?$ & 4 & $4.236 F+110$ & 4 & $3.587 F-03$ \\
\hline $4 \cdot 47 ? 0+1 ?$ & 5 & $3.2745+70$ & 3 & 5.3 ARF -03 \\
\hline $3.494 F+0 ?$ & 6 & $2.5415+100$ & a & $4.043 F=03$ \\
\hline $3.4015+1 ?$ & $!$ & $2.258 E+00$ & 7 & 1. $207 F-0 ?$ \\
\hline $7.4755+10 ?$ & H & $1.932 F+00$ & $x$ & $1.911 F-0)>$ \\
\hline $2.4200+112$ & $\dot{y}$ & $1.750 E_{-}+00$ & 4 & $2.721 F-112$ \\
\hline $2 \cdot ? 465+0 ?$ & 10 & $1.844 F+00$ & 11 & 4. กQ9F-ก? \\
\hline $2.3625+12 ?$ & 11 & $2.120 E+100$ & 11 & $4.149 F-n ?$ \\
\hline$? .9175+02$ & 12 & $2.8+8 E+00$ & 12 & $4.9625-02$ \\
\hline $5 \cdot 1560+1)$ & 13 & $4.944 E+00$ & 13 & $1.153 F-n 1$ \\
\hline $9.344 \pi+1 ?$ & 14 & $1.445 E+00$ & 14 & $1.4935-n !$ \\
\hline $1.998=+12$ & 15 & $1.7345+01$ & 15 & $1.934=-n 1$ \\
\hline $4 \cdot 015=+13$ & 16 & $3.3 \times>E+11$ & la & ?. $505 r-111$ \\
\hline $5.1045+113$ & 17 & $+.2745+111$ & 11 & $3 . ? 45 F-111$ \\
\hline $1 \cdot 6 / 50+112$ & 18 & $1.318 F+01$ & 14 & $4.2 \cap 4 F-01$ \\
\hline $4 \cdot 467-112$ & 19 & $3.373 E+00$ & 17 & $5.284 F-01$ \\
\hline $2.9510+112$ & 215 & 1. . $20 D E+110$ & $2 ! 1$ & $4 .>5>5-n 1$ \\
\hline $2.5730+03$ & 21 & $1.3235+01$ & 21 & $8.170 F-n !$ \\
\hline 1.74 $1 \%+0 ?$ & 22 & $1.017 E+00$ & 22 & $1.151 F+100$ \\
\hline $1.077 F+11 ?$ & 23 & $6.925 F-1) 1$ & 23 & $1.6145+00$ \\
\hline $1 \cdot 127=+12$ & 24 & $8,1(18 F-1) 1$ & 24 & $? .250 \mathrm{f}+00$ \\
\hline $1.351=+97$ & 25 & $9.512 E-01$ & 25 & $3.117 t+011$ \\
\hline $1.4605+1 ?$ & 26 & $1.042 F+100$ & 24 & $4.2395+00$ \\
\hline $1 \cdot 527=+1 ?$ & 27 & $1.048 F_{-}+00$ & 2.7 & $5.4545+00$ \\
\hline $1.606=+0 ?$ & 20 & $1.162 E+1) 0$ & $2 \cdot 3$ & $7.933 F+00$ \\
\hline $1.7978+1 ?$ & 24 & $1.311 E+00$ & 27 & $1.0575+01$ \\
\hline $2 \cdot 4310+1 ;$ & 30 & $2.002 E+00$ & 30 & $1.426 F+11$ \\
\hline $2 \cdot 414 F+33$ & 31 & $1.943 E+111$ & 31 & $1.493 F+1 !$ \\
\hline $3.2975+n$ & 32 & $2.5 ל 1 E+100$ & 32 & $2.4045+11$ \\
\hline $1.154 F+n ?$ & 33 & $7.175 F-111$ & 33 & 3. IGOF+ II \\
\hline $7.998 \%+111$ & 34 & $4.706 E-01$ & 34 & $3.503 F+101$ \\
\hline $9 \cdot 467=+11 ?$ & 35 & $4.336 F-01$ & 35 & $4.01175+01$ \\
\hline
\end{tabular}

FAST RANGE

GN (N, SAMMMA)

4. $57 B E+01$

5. $226 E+11$

$5.941 E+01$

$0.795 F+01$

$7.741 F+01$

$41 \quad 8.413 F+01$

47 $1.310 E+02$

$43 \quad 4.39 / F+01$

$449.473 F+01$

45 ?. $43^{\prime} \mathrm{EE}+01$

$4 \mathrm{~h} 2.92 \mathrm{DE}+12$

$47 ? .054 E+02$

$48 \quad 2.994 E+01$

$49 \quad 2.34 D E+02$

$50 \quad 3.483 F+n 2$

IN RAKNS

GN (N,FISS.)

GN (N.FISS.)

?. $64 \mathrm{HF}+0 \mathrm{O}$

2. $32 \mathrm{HF}+00$

1. Q? $7 E+00$

$1.773 E+00$

$1.751) \mathrm{F}+00$

$1.750 \mathrm{~F}+100$

$1.7 !) 7 F+00$

$1.532 F+00$

$1.419 F+00$

$9.541 E-n 1$

3. $759 F-01$

$9.87 ? E-\cap 2$

?.12กE-0?

$5.30 D F-03$

$3.354 F-03$

$6.753 E-03$

4. $n$ B5F-0?

$1.530 E-0 ?$

$1.455 E-0 ?$

?. $95 \cap F-13$

$4.711 E-n ?$

$2.165 E-01$

5. $459 F-01$

Q. $949 F-n 1$

$5.11 \cap F-01$

$5.703 F-01$

1. $>19 E+00$

$1.365 F+00$

$1.465 E+00$

$1.461++00$

1. $620 \mathrm{~F}+00$

$7.369 \mathrm{~F}-\mathrm{n} 1$

$7.48 P F-11$

7. $>84 F-01$

$4.273 E-n 1$
3G $1.273 E+0 n$

$37 \quad 4.442 F-01$

$38 \quad 6.416 E-01$

$397.318 F-01$

$40 \quad 1.033 F+00$

$413.351 F-01$

$429.5015=01$

$439.402 E-02$

$44 \quad 8.715 E-01$

$451.203 F-01$

$46 \quad 2.941 F+00$

$47 ? .691 F-01$

$48 \quad 1.765 F-01$

49 S.081F-01

$50 \quad 1.150 F+00$

(iv, 2N)

?. $993=-04$

$3.115 E-04$

4.64 bF -04 
TABLE C-3 (Continued)

LIBRARY OF CROSS SECTIONS FOR LASER-HIC CALCULATIONS

\begin{tabular}{|c|c|c|c|c|c|c|c|c|c|c|c|}
\hline LASER & \multicolumn{2}{|c|}{$\begin{array}{l}\text { GROUP STRUCTURF } \\
\text { THEOMAL RANGF }\end{array}$} & $\begin{array}{l}\text { IRF AVERAGF } \\
\text { IGF }\end{array}$ & \multicolumn{2}{|c|}{ (IN, $\triangle A M M A)$ ANN } & $\begin{array}{r}(N, F I S C,) \\
F A S T\end{array}$ & $\begin{array}{l}\text { CKOSS } \\
\text { RANGE }\end{array}$ & SECTI UN & FOR $\triangle M 24 ?$ & \multicolumn{2}{|c|}{ (LASER HIC ID 30$)$} \\
\hline & 10,19000 & & IN,FIS & .1 & $\ln , G \cap N$ & & GN $\quad(N, G A$ & MMA) & $(N, F T S$ & - $)$ & GN (N,FISS.) \\
\hline 1 & $3.741=+17$ & 1 & $1.497 E+0.4$ & 1 & $n$. & 36 & $1.4 ? 8 E+01$ & 1 & 1. $629 E-01$ & 36 & $5.713 \mathrm{~F}+01$ \\
\hline 2 & $1.874 F+03$ & 2 & $7.499 E+113$ & 2 & $n$. & .37 & $1.618 E+01$ & 2 & $1.906 F-01$ & 37 & $6.411 F+01$ \\
\hline 3 & $1 . ? 50 F+0 ?$ & 3 & $4.99 Y E+03$ & 3 & 0 & $3 R$ & $1.933 F+01$ & 3 & $2 \cdot 123 \mathrm{~F}-01$ & 38 & $7.332 F+01$ \\
\hline 4 & $9.372 F+0 ?$ & 4 & $3.749 E+03$ & 4 & $2.766 F-03$ & 39 & $2.07 ? E+01$ & 4 & $2.357 E-01$ & 39 & $8.308 F+01$ \\
\hline 5 & $7.4975+112$ & 5 & $3.000 E+03$ & 5 & $7.025 F-02$ & 40 & $2.352 E+01$ & 5 & $2.236 E-01$ & 40 & $9.410 f+01$ \\
\hline 6 & $6 .>49 F+117$ & 6 & $2.500 E+133$ & 6 & $9.300 \mathrm{~F}=02$ & 41 & $? . A G D E+n I$ & 6 & $3.318 E-01$ & 41 & $1.0665+02$ \\
\hline 7 & $5.355 F+02$ & 7 & $2.142 E+03$ & 7 & $9.2845-n ?$ & 4? & $3.1120 E+01$ & 7 & $3.690 E-01$ & $4 ?$ & $1.208 E+02$ \\
\hline 8 & $4 \cdot 544=+0 ?$ & 8 & $1.918 E+113$ & 8 & $1.007 F-01$ & 4.3 & $3.421 E+01$ & 8 & $3.385 E-01$ & 43 & $1.3685+02$ \\
\hline 9 & $3.845 F+07$ & 9 & $1.538 E+113$ & 4 & $1.125 \mathrm{~F}-11$ & 44 & $3.875 E+01$ & 9 & $4.430 E-01$ & 44 & $1.550 E+02$ \\
\hline 10 & $3 \cdot 3325+02$ & 10 & $1.333 E+13$ & 10 & $1.282 E-01$ & 45 & 4. $389 E+01$ & 10 & $5.046 E-01$ & 45 & $1.756 F+02$ \\
\hline 11 & $2.940 F+0 ?$ & 11 & $1.176 F+03$ & 11 & $1.471 E-01$ & $4 h$ & $4.961 E+01$ & 11 & 5. $.942 E-01$ & $4 \pi$ & $1.985 E+02$ \\
\hline 12 & $2.433 F+0 ?$ & 12 & $1.053 E+103$ & 12 & $1.6 ? 2 F=01$ & 47 & $5.6]+E+01$ & 12 & $6.476 E-01$ & 47 & $2.2455+02$ \\
\hline 13 & $? .440 F+0 ?$ & 13 & $9.761 \mathrm{~F}+0 ?$ & 13 & $1.819 F-01$ & 48 & $6.374 E+01$ & 13 & $7.274 F-01$ & 48 & $2.550 E+02$ \\
\hline 14 & $7.3365+112$ & 14 & $9.344 E+132$ & 14 & $? .0645-01$ & 49 & $7 . ?+5 F_{-}+01$ & 14 & $A .>57 E-01$ & 49 & $2.898 F+02$ \\
\hline 15 & $? \cdot 251=+0 ?$ & 15 & $9.00 / F_{-}+02$ & 15 & $2.30 G F-01$ & $5 n$ & $9 . ? 11 \mathrm{~F}+01$ & 15 & $9 . ? 23 F-01$ & 50 & $3.285 E+02$ \\
\hline 16 & $2 \cdot 1925+17$ & 10 & $8.77 \cap E+102$ & If & $? .614 E=01$ & & & 16 & $1.045 E+00$ & & \\
\hline 17 & $2 \cdot 139 \pi+12$ & 17 & $8.557 F+112$ & 17 & $2.959 F-n 1$ & & & 17 & $1.184 E+00$ & & $(N, 2)$, \\
\hline 18 & $? \cdot 1044 E+11 ?$ & 18 & $3.195 E+12$ & 12 & $3.353 \mathrm{~F}-11$ & & & 18 & $1.341 E+00$ & 1 & $6 F-01$ \\
\hline 19 & $1.9175+0 ?$ & 19 & $1.671 F+12$ & 19 & $3.41125-01$ & & & 19 & $1.5 ? .1 E+00$ & $?$ & 4.?195-01 \\
\hline$? 0$ & $1 \cdot 76 \cap 5+0 ?$ & 20 & $7.041 E+12$ & 21 & $4.3 n 7 F-n 1$ & & & 20 & $1.723 F+0 n$ & 3 & $0 \bullet$ \\
\hline ?1 & $1 \cdot 590 \pi+1) ?$ & 21 & $6.361 E+112$ & $? 1$ & $5.154 F-01$ & & & $? 1$ & 2. OAGE + OD & & \\
\hline ?? & $1 \cdot 4 ? 45+0 ?$ & 22 & $5.697 \mathrm{E}+02$ & 22 & $6.631 F-01$ & & & 2? & $2.65 ? F+00$ & & \\
\hline $\begin{array}{l}23 \\
24\end{array}$ & $1.2435+12$ & 23 & $\left.3.13 \mid F_{-}+1\right) ?$ & 23 & $2.5>0 F-01$ & & & 23 & $3.41) 8 F+00$ & & \\
\hline$?$ & $\begin{array}{l}1.2135+0 ? \\
1.176 F+n ?\end{array}$ & $\begin{array}{l}24 \\
25\end{array}$ & $\begin{array}{l}\text { 4. . } 14 E+02 \\
405 E+0 ?\end{array}$ & $\begin{array}{l}? 4 \\
25\end{array}$ & $1.07+5+00$ & & & 24 & $4.377 E+00$ & & \\
\hline ?h. & $1.1555+0 ?$ & 26 & $4.661 E+1) 2$ & $2 h$ & $1.804 F+00$ & & & 26 & 7.214E+00 & & \\
\hline 27 & $1.1600+10$ & $2 !$ & $4.639 E+12$ & 21 & $2.317 E+011$ & & & 27 & 9.> $6 A E+01$ & & \\
\hline 28 & $1 \cdot 1545+1 ?$ & 24 & $4.618 E_{-}+12$ & ?Q & $2.977 F+n n$ & & & 28 & $1 \cdot 1^{9} 1 E+01$ & & \\
\hline 29 & $1 \cdot 1+5 F+?$ & 29 & $4.57 .7 E+02$ & 29 & $3.4195+00$ & & & 29 & $1.527 F+01$ & & \\
\hline 30 & $1 \cdot 1 \geq 15+1)>$ & 30 & $4.403 E+02$ & 30 & $4.999 .9 F+00$ & & & 30 & $1.959 E+01$ & & \\
\hline 31 & $1 . n 730+10$ & 31 & $4.291 E+12$ & 31 & $5.275 F+100$ & & & 31 & $2.517 F+01$ & & \\
\hline 32 & $1 \cdot 0155+02$ & 32 & $4.05 Y F+02$ & 32 & $2.17 \times 4 F+00$ & & & 32 & $3.333 F+01$ & & \\
\hline 33 & $9.6555+1$ & 33 & $3.963 E+112$ & 33 & $9.805 E+00$ & & & 33 & $3.92 \supset F+01$ & & \\
\hline 34 & $9.2565+11$ & 34 & $3.703 F+02$ & 34 & $1.112 F+01$ & & & 34 & $4.448 E+01$ & & \\
\hline 35 & $8.9135+01$ & 35 & $3.565 E+02$ & 35 & $1.261 F+01$ & & & 35 & $5 \cdot 042 E+01$ & & \\
\hline
\end{tabular}


TABLE C-3 (Continued)

LIBRARY OF CROSS SECTIONS FOR LASER-HIC CALCULATIONS

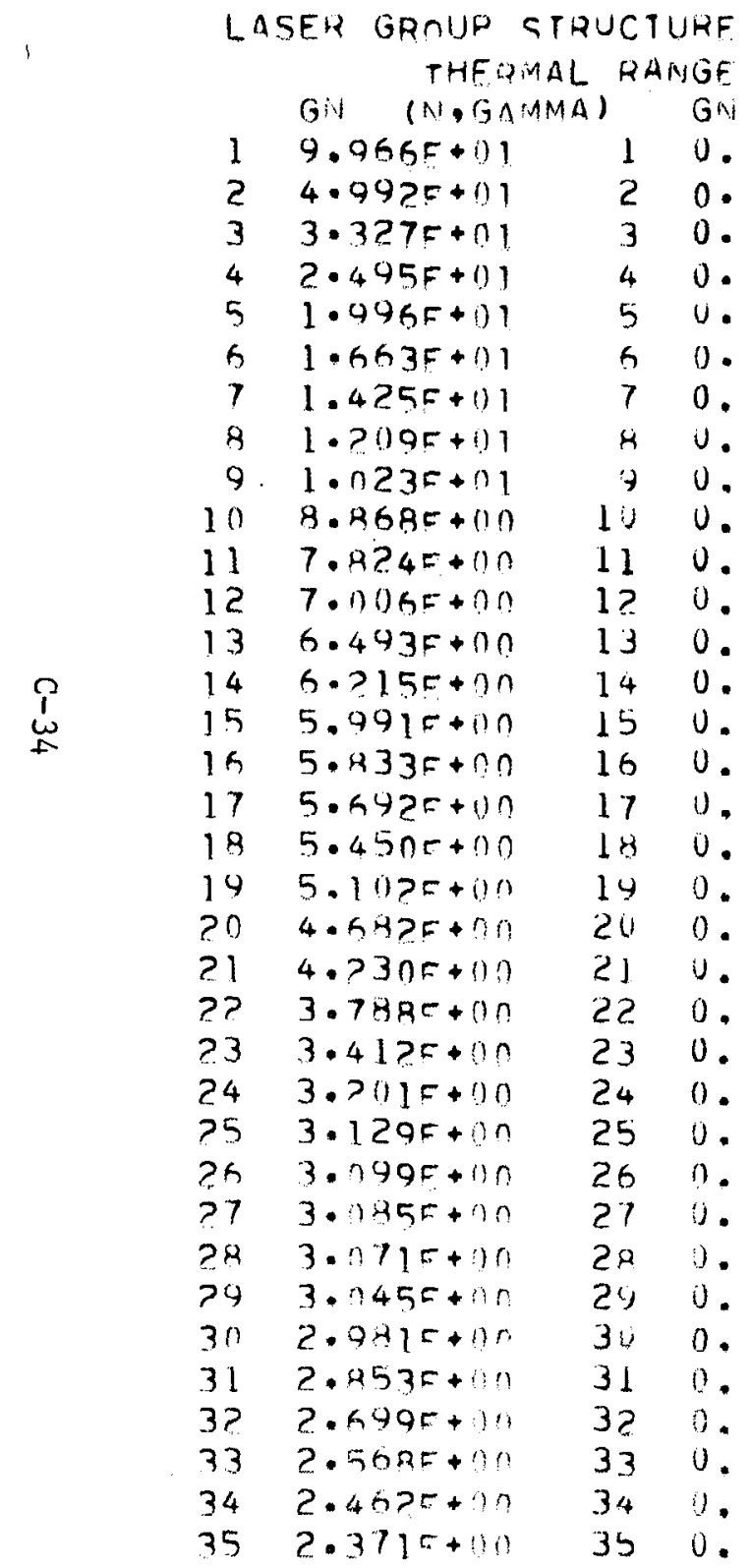

AVERAGE (N, GAMMA) ANI) (N,FIGS.) CKUSS SECTIUN FOR CMZ4?

(N,FISS.) GN (N,GAMMA) GAST RANGE

$1.062 F-03 \quad 36 \quad 3.790 E-01$

$1.204 F-03$

$1.364 F-0.3$

1. $546 F-n 3$

$1.753 F-03$

$1.987 F-03$

ว.?50E-03

2. $550 E-03$

2. $894 F-0.3$

3. $277 E-0.3$

$3.710 \mathrm{E}-03$

4. $205 E-n 3$

4. $R>5 F-13$

5. 4 ? HF -03

4. $119 \mathrm{~F}-03$

$6.036 \mathrm{~F}=\mathrm{n} ?$

7. $454 F-03$

R. $899 F-113$

1. OOQF -0 ?

1. $143 F=0 ?$

$1.371 \mathrm{~F}-n$ ?

$1.760 F-0 ?$

?. $2625=02$

?. $905 F-0 ?$

$3.7 ? 9 F=0 ?$

$4.790 F-02$

6. $154 \mathrm{~F}-n$ ?

$7.907 \mathrm{~F}-0$ ?

1. $114 \mathrm{~F}-01$

$1.301 \mathrm{~F}-01$

$1.6735-01$

?.148F-n1

?. $506 F-01$

2. $955 E-01$

$3.350 F-01$
(LASER HIC ID 31 )

IN RARNS

$\begin{array}{llllll}36 & 3.790 E-01 & 1 & 0 . & 36 & 0 . \\ 37 & 4.30 \cup E-01 & 2 & 0 . & 37 & 0 . \\ 38 & 4.072 E-01 & 3 & 0 . & 38 & 0 . \\ 39 & 5.521 E-01 & 4 & 0 . & 39 & 0 . \\ 40 & 6.254 E-01 & 5 & 0 . & 40 & 0 . \\ 41 & 7.045 E-01 & 6 & 0 . & 41 & 0 . \\ 42 & 8.030 E-01 & 7 & 0 . & 42 & 0 . \\ 43 & 9.095 F-01 & 9 & 0 . & 43 & 0 . \\ 44 & 1.03 \cup E+00 & 9 & 0 . & 44 & 0 . \\ 45 & 1.157 E+00 & 10 & 0 . & 45 & 0 . \\ 46 & 1.719 E+00 & 11 & 0 . & 46 & 0 . \\ 47 & 1.492 E+00 & 12 & 0 . & 47 & 0 . \\ 49 & 1.695 E+00 & 13 & 0 . & 48 & 0 . \\ 49 & 1.927 F+00 & 14 & 0 . & 49 & 0 . \\ 5 n & 2.144 E+00 & 15 & 0 . & 50 & 0 .\end{array}$

$(N, 2 N)$

$1.400 F-01$

? $1.5005-01$

30 . 
TABLE C-3 (Continued)

LIBRARY OF CROSS SECTIONS FOR LASER-HIC CALCULATIONS

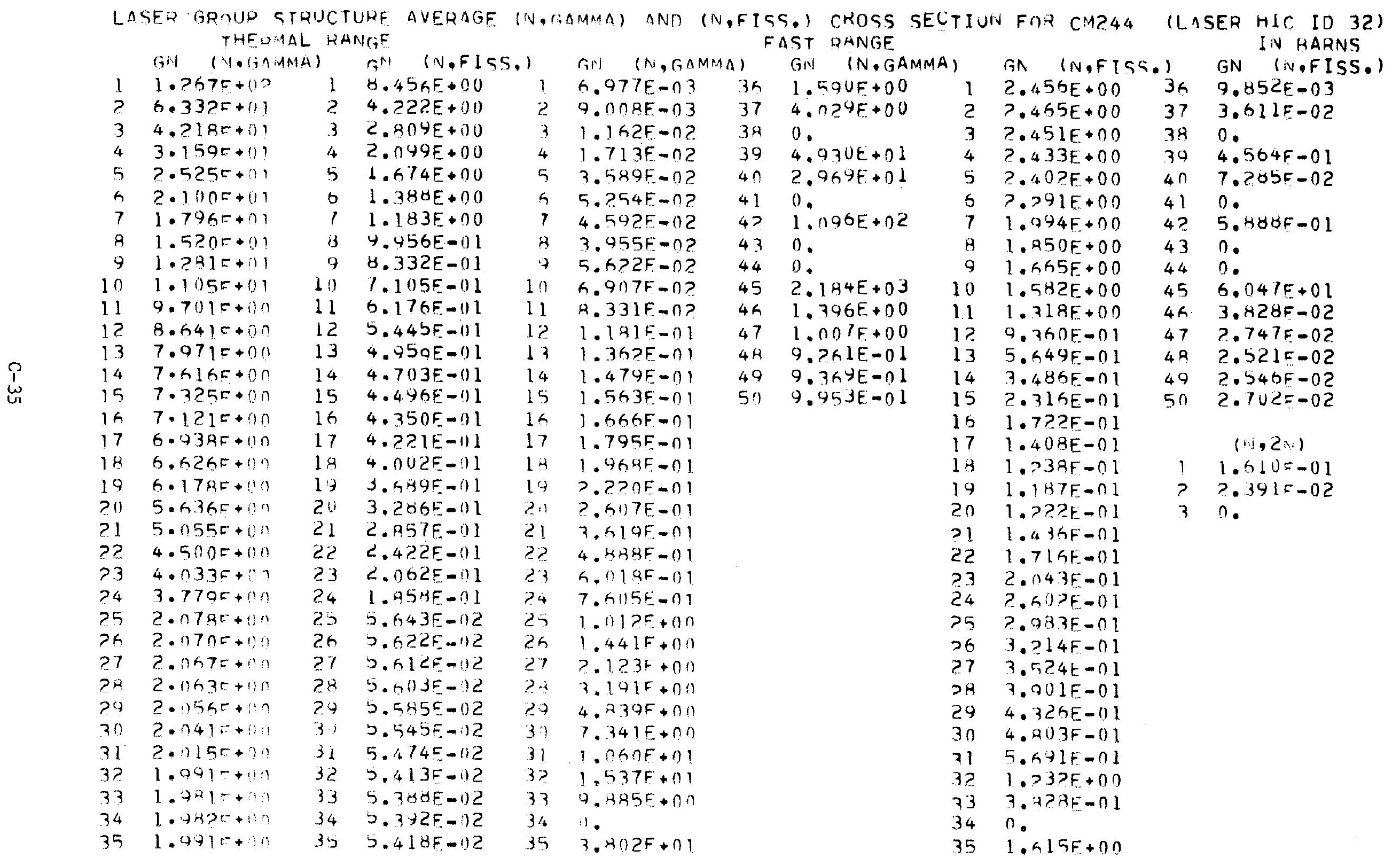


APPENDIX D

SUMMARY OF MEASUREMENTS FOR SPECIAL ISOTOPES

The results of measurements for the special isotopes are summarized in Tables D-1 through D-5. Both the time-of-analysis and the corresponding end-of-life values are shown; the data for Phase 1 samples are referenced to Core I EOL on May 18, 1962, the data for Phase 2 samples are referenced to Core II EOL on September 2, 1963, and the data for Phase 3 samples are referenced to Core IV EOL on August 9, 1965. The equations given in Subsection 5.2 were used to make the decay corrections from time of analysis to core end-of-1ife. (The value of elapsed time listed in the tables is based on the reported date of analysis, which in some cases, may differ from the actual date of analysis by as much as several days.) The uncertainty on the measurements is estimated at 2 standard deviations, precision.

The tables are self-explanatory, however, a few comments are in order.

The results of measurements for Am-241 are summarized in Table D-3; also shown are the corresponding Pu-241 data used to decay-correct the Am-241 data to core end-of-life. Unfortunately, for a few samples, the correction for Pu-241 B-decay is larger than the measured value of Am-241 at time of analysis, thus resulting in a negative value of Am-241 when referenced to core end-of-life.

The results of measurements for Am-243 are summarized in Table D-4. Notice that the value for sample 44-4 is somewhat high relative to the others; a check of these data with results of measurements by others [57] indicates that the high value for sample 44-4 may be reasonable, and the relatively low values may be inconsistent.

The results of twelve sets of TRACERLAB analyses for Am-241, Cm-242, and $\mathrm{Cm}-244$ were reported in terms of $\mathrm{dpm} / \mathrm{ml}$; these are indicated with footnote $\mathrm{k}$ 
in Tables D-3 and D-5. In order to relate these data to the Pu-239 concentrations, the TRACERLAB $\mathrm{dpm} / \mathrm{ml}$ data were normalized using the $\mathrm{Cm}-244 \mathrm{dpm} / \mathrm{ml}$ data and the Cm-244/Pu-239 atom ratio measured for these samples by WARD. 
TABLE D-1

SUMMARY OF MEASUREMENTS FOR U-232

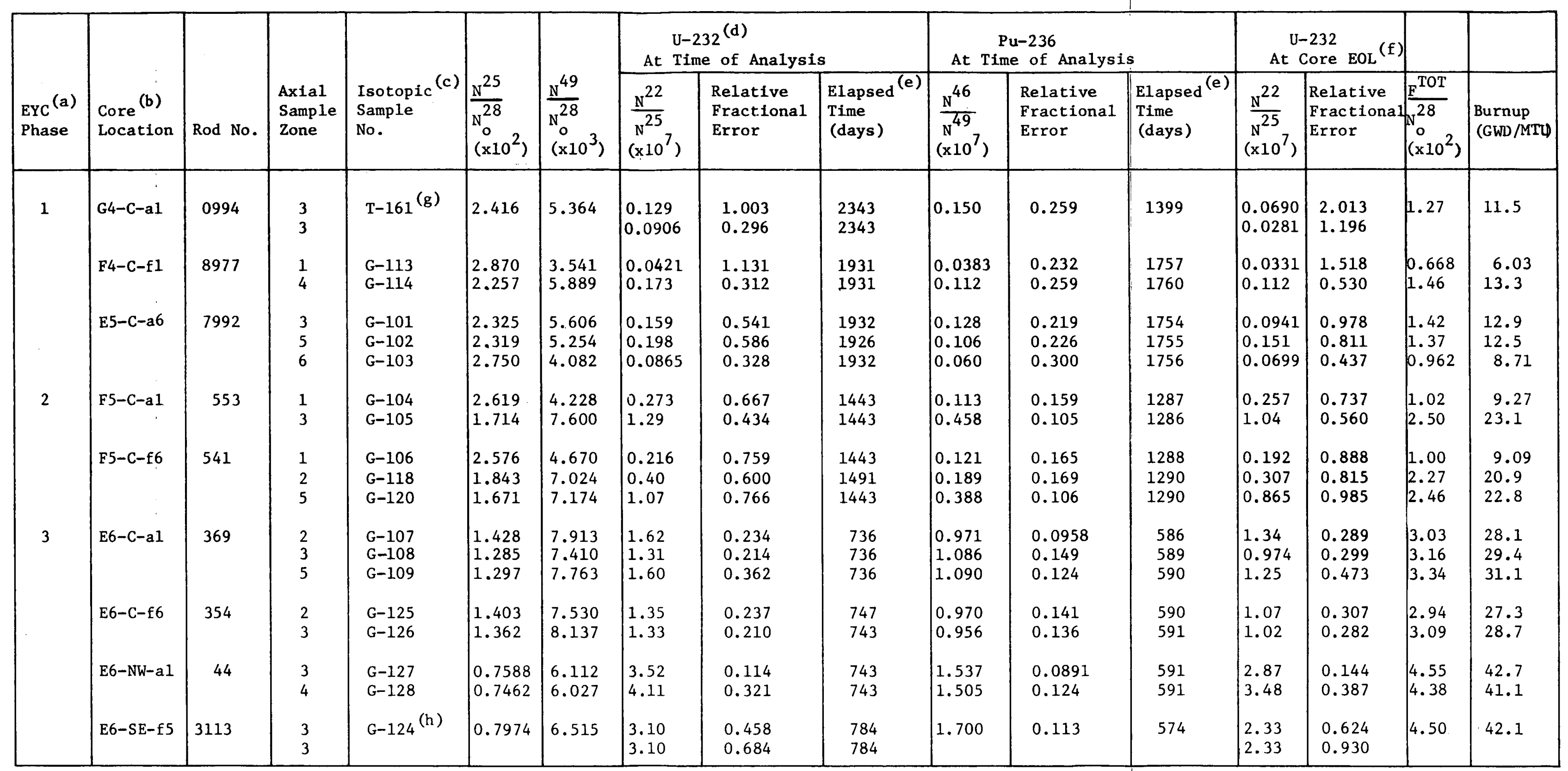

Notes: (a) Phase 1 samples were irradiated in Yankee Core I; Phase 2 samples were irradiated in Cores I and II; Phase 3 samples were irradiated in Cores I, II, and IV.

(b) Subassembly designation $C$ indicates asymptotic neutron spectrum; subassembly designations NW and SE indicate non-asymptotic neutron spectra.

(c) The letters $\mathrm{T}$ and $\mathrm{G}$ indicate mass spectrometric analysis for main chain $U$ and Pu isotopes by TRACERLAB and General Electric-Vallecitos,

respectively.

(d) Analysis for U-232 by WARD; relative fractic

(f) Phase 1, 2, and 3 data referenced to Core I, Core II, and Core IV end-of-life, respectively. Decay constants for U-232 and Pu-236 = $0.0000268 \mathrm{~d}^{-1}$ and $0.000666 \mathrm{~d}^{-1}$, respective1y.

(g) EYC Phase 1 control sample.
(h) EYC Phase 3 control sample. 


\begin{tabular}{|c|c|c|c|c|c|c|c|c|c|c|}
\hline $\begin{array}{l}\text { EYC } \\
\text { Phase }\end{array}$ & $\begin{array}{l}\text { Core }{ }^{(b)} \\
\text { Location }\end{array}$ & Rod No. & $\begin{array}{l}\text { Axial } \\
\text { Sample } \\
\text { Zone }\end{array}$ & $\begin{array}{l}\text { Isotopic (c) } \\
\text { Sample } \\
\text { No. }\end{array}$ & $\begin{array}{l}\text { U-238 } \\
\text { Atom } \\
\text { Percent }\end{array}$ & $\frac{\mathrm{N}^{28}}{\mathrm{~N}_{\mathrm{o}}^{28}}$ & $\begin{array}{l}\mathrm{Np}-237^{(d)} \\
(\mathrm{ppm})\end{array}$ & $\begin{array}{l}\frac{\mathbb{N}^{37}}{128} \\
N_{0}^{28} \\
\left(\times 10^{-4}\right)\end{array}$ & $\begin{array}{l}\frac{\mathrm{F}^{\mathrm{TOT}}}{\mathrm{N}_{\mathrm{O}}^{28}} \\
\left(\mathrm{x} 10^{2}\right) \\
\end{array}$ & $\begin{array}{l}\text { Burnup } \\
\text { (GWD/MTU) }\end{array}$ \\
\hline \multirow[t]{2}{*}{1} & F4-C-f1 & 8977 & $\begin{array}{l}1 \\
1(e) \\
4\end{array}$ & $\begin{array}{l}\text { G-113 } \\
\text { G-114 }\end{array}$ & $\begin{array}{l}97.037 \\
97.487\end{array}$ & $\begin{array}{l}0.99463 \\
0.98809\end{array}$ & $\begin{array}{r}46 \\
52 \\
169\end{array}$ & $\begin{array}{l}0.47 \\
0.54 \\
1.72\end{array}$ & $\begin{array}{l}0.668 \\
1.46\end{array}$ & $\begin{array}{l}6.03 \\
13.3\end{array}$ \\
\hline & E5-C-a6 & 7992 & $\begin{array}{l}3 \\
5 \\
5(e) \\
6\end{array}$ & $\begin{array}{l}G-101 \\
G-102 \\
G-103\end{array}$ & $\begin{array}{l}97.430 \\
97.439 \\
97.099\end{array}$ & $\begin{array}{l}0.98868 \\
0.98939 \\
0.99316\end{array}$ & $\begin{array}{r}159 \\
197 \\
146 \\
90\end{array}$ & $\begin{array}{l}1.62 \\
2.01 \\
1.49 \\
0.92\end{array}$ & $\begin{array}{l}1.42 \\
1.37 \\
0.962\end{array}$ & $\begin{array}{l}12.9 \\
12.5 \\
8.71\end{array}$ \\
\hline \multirow[t]{2}{*}{2} & $\mathrm{~F} 5-\mathrm{C}-\mathrm{a} 1$ & 553 & 1 & G-104 & 97.206 & 0.99256 & 114 & 1.17 & 1.02 & 9.27 \\
\hline & F5-C-f6 & 541 & $\begin{array}{l}1 \\
1(e) \\
4 \\
4(e) \\
5 \\
6\end{array}$ & $\begin{array}{l}G-106 \\
G-119 \\
G-120 \\
G-121\end{array}$ & $\begin{array}{l}97.229 \\
97.988 \\
97.962 \\
97.612\end{array}$ & $\begin{array}{l}0.99158 \\
0.98028 \\
0.98019 \\
0.98763\end{array}$ & $\begin{array}{r}92 \\
112 \\
341 \\
339 \\
348 \\
188\end{array}$ & $\begin{array}{l}0.94 \\
0.15 \\
1.15 \\
3.43 \\
3.41 \\
3.50 \\
1.91\end{array}$ & $\begin{array}{l}1.00 \\
2.62 \\
2.46 \\
1.62\end{array}$ & $\begin{array}{l}9.09 \\
24.2 \\
\\
22.8 \\
14.8\end{array}$ \\
\hline \multirow[t]{3}{*}{3} & E6-C-a1 & 369 & $\begin{array}{l}2 \\
3 \\
3(e) \\
5\end{array}$ & $\begin{array}{l}\text { G-107 } \\
\text { G-108 } \\
\text { G-109 }\end{array}$ & $\begin{array}{l}98.120 \\
98.253 \\
98.233\end{array}$ & $\begin{array}{l}0.97472 \\
0.97392 \\
0.97304\end{array}$ & $\begin{array}{l}516 \\
493 \\
497 \\
552\end{array}$ & $\begin{array}{l}5.15 \\
4.91 \\
4.95 \\
5.49\end{array}$ & $\begin{array}{l}3.03 \\
3.16 \\
\\
3.34\end{array}$ & $\begin{array}{l}28.1 \\
29.4 \\
\\
31.1\end{array}$ \\
\hline & E6-C-f6 & 354 & $\begin{array}{l}2 \\
2(\mathrm{e}) \\
3\end{array}$ & $\begin{array}{l}G-125 \\
G-126\end{array}$ & $\begin{array}{l}98.144 \\
98.190\end{array}$ & $\begin{array}{l}0.97682 \\
0.97354\end{array}$ & $\begin{array}{l}433 \\
425 \\
507\end{array}$ & $\begin{array}{l}4.33 \\
4.25 \\
5.05\end{array}$ & $\begin{array}{l}2.94 \\
3.09\end{array}$ & $\begin{array}{l}27.3 \\
28.7\end{array}$ \\
\hline & E6-NW-a1 & 44 & $\begin{array}{l}3 \\
4 \\
4(e)\end{array}$ & $\begin{array}{l}G-127 \\
G-128\end{array}$ & $\begin{array}{l}98.702 \\
98.734\end{array}$ & $\begin{array}{l}0.96487 \\
0.96521\end{array}$ & $\begin{array}{l}591 \\
619 \\
603\end{array}$ & $\begin{array}{l}5.80 \\
6.07 \\
5.92\end{array}$ & $\begin{array}{l}4.55 \\
4.38\end{array}$ & $\begin{array}{l}42.7 \\
41.1\end{array}$ \\
\hline
\end{tabular}

Notes: (a) Phase 1 samples were irradiated in Yankee Core I; Phase 2 samples were irradiated in Cores I and II; Phase 3 samples were irradiated in Cores I, II, IV. (b) Subassembly designation $C$ indicates asymptotic neutron spectrum; subassembly designation NW indicates non-asymptotic neutron spectrum.

(c) The letter $G$ indicates mass spectrometric analysis for main chain $U$ and Pu isotopes by General Electric-Vallecitos.

(d) Analysis for $\mathrm{Np}-237$ by WARD; uncertainties are $\pm 10 \%$, relative.

(e) Duplicate analysis. 


\begin{tabular}{|c|c|c|c|c|c|c|c|c|c|c|c|c|c|c|c|c|}
\hline \multirow[b]{2}{*}{$\begin{array}{l}\text { EYC } \\
\text { Phase }\end{array}$} & \multirow[b]{2}{*}{$\begin{array}{l}\text { Core }{ }^{(b)} \\
\text { Location }\end{array}$} & \multirow[b]{2}{*}{ Rod No. } & \multirow[b]{2}{*}{$\begin{array}{c}\text { Axial } \\
\text { Sample } \\
\text { Zone }\end{array}$} & \multirow[b]{2}{*}{$\begin{array}{c}\text { Isotopic }(c) \\
\text { Sample } \\
\text { No. }\end{array}$} & \multirow[b]{2}{*}{$\begin{array}{l}\frac{\mathrm{N}^{49}}{\mathrm{~N}_{0}^{28}} \\
\left(\times 10^{3}\right)\end{array}$} & \multicolumn{3}{|c|}{$\begin{array}{c}\text { Pu-241 } \\
\text { At Time of Analysis }\end{array}$} & \multicolumn{3}{|c|}{$\begin{array}{c}\text { Am-241 } \\
\text { At Time of Analysis }\end{array}$} & & \multicolumn{2}{|c|}{$\begin{array}{l}\text { Am-241 } \\
\text { At Core EOL }\end{array}$} & \multirow[b]{2}{*}{$\mid \begin{array}{l}\mathrm{F}^{\mathrm{TOT}} \\
\mathrm{N}_{0}^{28} \\
\left(\mathrm{x} 10^{2}\right)\end{array}$} & \multirow[b]{2}{*}{$\begin{array}{l}\text { Burnup } \\
\text { (GWD/MTU) }\end{array}$} \\
\hline & & & & & & $\frac{n^{41}}{N^{49}}$ & $\begin{array}{l}\text { Relative } \\
\text { Fractional } \\
\text { Error }\end{array}$ & $\begin{array}{c}\text { Elapsed } \\
\text { Time } \\
\text { (days) }\end{array}$ & Lab & 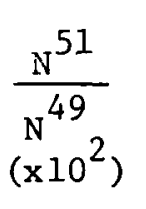 & $\begin{array}{l}\text { Relative } \\
\text { Fractional } \\
\text { Error }\end{array}$ & $\begin{array}{c}\text { Elapsed } \\
\text { Time } \\
\text { (days) }\end{array}$ & $\mid \begin{array}{l}\frac{N^{51}}{N_{0}^{28}} \\
\left(x 10^{5}\right)\end{array}$ & $\begin{array}{l}\text { Relative } \\
\text { Fractional } \\
\text { Error }\end{array}$ & & \\
\hline \multirow[t]{3}{*}{1} & G4-C-al & 0994 & 3 & $T-161^{(f)}$ & 5.364 & 0.06623 & 0.00168 & 1384 & WARD (h) & 2.47 & 0.0810 & 2373 & 1.18 & 0.918 & 1.27 & 11.5 \\
\hline & $\mathrm{F} 4-\mathrm{C}-\mathrm{f} 1$ & 8977 & $\begin{array}{l}4 \\
4 \\
5 \\
5\end{array}$ & $\begin{array}{l}G-114 \\
G-115\end{array}$ & $\begin{array}{l}5.888 \\
5.556\end{array}$ & $\begin{array}{l}0.0740 \\
0.0731\end{array}$ & $\begin{array}{c}0.0136 \\
\vdots \\
0.0105\end{array}$ & $\begin{array}{l}1880 \\
1880\end{array}$ & $\begin{array}{l}\operatorname{TLAB}(k) \\
\operatorname{WARD}(\mathrm{h}) \\
\operatorname{TLAB}(k) \\
\operatorname{WARD}(\mathrm{h})\end{array}$ & $\begin{array}{l}3.63 \\
2.46 \\
2.50 \\
2.43\end{array}$ & $\begin{array}{l}0.0551 \\
0.0406 \\
0.0800 \\
0.0412\end{array}$ & $\begin{array}{l}1910 \\
1910 \\
1910 \\
1910\end{array}$ & $\begin{array}{l}8.24 \\
1.31 \\
1.60 \\
1.21\end{array}$ & $\begin{array}{l}0.146 \\
0.473 \\
0.702 \\
0.477\end{array}$ & $\begin{array}{l}1.46 \\
1.44\end{array}$ & $\begin{array}{l}13.3 \\
13.2\end{array}$ \\
\hline & E5-C-a6 & 7992 & $\begin{array}{l}3 \\
5 \\
6\end{array}$ & $\begin{array}{l}G-101 \\
G-102 \\
G-103\end{array}$ & $\begin{array}{l}5.605 \\
5.253 \\
4.082\end{array}$ & $\begin{array}{l}0.07448 \\
0.07255 \\
0.04200\end{array}$ & $\begin{array}{l}0.00447 \\
0.00447 \\
0.00447\end{array}$ & $\begin{array}{l}1789 \\
1789 \\
1789\end{array}$ & $\begin{array}{l}\operatorname{TLAB}(j) \\
\operatorname{TLAB}(j) \\
\operatorname{TLAB}(j)\end{array}$ & $\begin{array}{l}2.01 \\
1.78 \\
1.20\end{array}$ & $\begin{array}{l}0.0796 \\
0.0786 \\
0.0833\end{array}$ & $\begin{array}{l}1828 \\
1828 \\
1828\end{array}$ & \begin{tabular}{|l|}
-0.751 \\
-1.62 \\
-0.0356
\end{tabular} & $\begin{array}{c}1.209 \\
0.456 \\
11.60\end{array}$ & $\begin{array}{l}1.42 \\
1.37 \\
0.962\end{array}$ & $\begin{array}{c}12.9 \\
12.5 \\
8.71\end{array}$ \\
\hline \multirow[t]{2}{*}{2} & F5-C-a1 & 553 & $\begin{array}{l}1 \\
3 \\
3\end{array}$ & $\begin{array}{l}G-104 \\
G-105\end{array}$ & $\begin{array}{l}4.228 \\
7.600\end{array}$ & $\begin{array}{l}0.05077 \\
0.1348\end{array}$ & $\begin{array}{l}0.00447 \\
0.00447\end{array}$ & $\begin{array}{l}1317 \\
1317\end{array}$ & $\begin{array}{l}\operatorname{TLAB}(j) \\
\operatorname{TLAB}(1) \\
\operatorname{WARD}(\mathrm{h})\end{array}$ & $\begin{array}{l}1.22 \\
3.15 \\
2.93\end{array}$ & $\begin{array}{l}0.0820 \\
0.0825 \\
0.0819\end{array}$ & $\begin{array}{l}13.56 \\
1356 \\
1353\end{array}$ & $\begin{array}{l}0.731 \\
2.80 \\
1.16\end{array}$ & $\begin{array}{l}0.584 \\
0.710 \\
1.592\end{array}$ & $\begin{array}{l}1.02 \\
2.50\end{array}$ & $\begin{array}{l}9.27 \\
23.1\end{array}$ \\
\hline & F5-C-f6 & 541 & $\begin{array}{l}1 \\
2 \\
2 \\
4 \\
4 \\
6 \\
6\end{array}$ & $\begin{array}{l}\text { G-106 } \\
\text { G-118 } \\
\text { G-119 } \\
\text { G-121 }\end{array}$ & $\begin{array}{l}4.670 \\
7.024 \\
7.018 \\
5.708\end{array}$ & $\begin{array}{l}0.05673 \\
0.117 \\
0.138 \\
0.0870\end{array}$ & $\begin{array}{c}0.00447 \\
0.00956 \\
0.00844 \\
\vdots \\
0.0119 \\
1\end{array}$ & $\begin{array}{l}1317 \\
1393 \\
1415 \\
1393\end{array}$ & $\begin{array}{l}\operatorname{TLAB}(j) \\
\operatorname{TLAB}(k) \\
\operatorname{WARD}(\mathrm{h}) \\
\operatorname{TLAB}(k) \\
\operatorname{WARD}(\mathrm{h}) \\
\operatorname{TLAB}(k) \\
\operatorname{WARD}(\mathrm{h})\end{array}$ & $\begin{array}{l}1.32 \\
2.76 \\
2.87 \\
3.47 \\
3.65 \\
2.31 \\
2.30\end{array}$ & $\begin{array}{l}0.0758 \\
0.0580 \\
0.0418 \\
0.0576 \\
0.0384 \\
0.0693 \\
0.0435\end{array}$ & $\begin{array}{l}11356 \\
1421 \\
1421 \\
1424 \\
1424 \\
1425 \\
1425\end{array}$ & $\begin{array}{l}0.696 \\
1.50 \\
2.28 \\
3.19 \\
4.46 \\
2.36 \\
2.30\end{array}$ & $\begin{array}{l}0.678 \\
0.762 \\
0.378 \\
0.447 \\
0.225 \\
0.392 \\
0.256\end{array}$ & $\begin{array}{l}1.00 \\
2.27 \\
2.62 \\
1.62\end{array}$ & $\begin{array}{l}9.09 \\
20.9 \\
24.2 \\
14.8\end{array}$ \\
\hline 3 & E6-C-a1 & 369 & $\begin{array}{l}2 \\
3 \\
3 \\
5\end{array}$ & $\begin{array}{l}\text { G-107 } \\
\text { G-108 } \\
\text { G-109 }\end{array}$ & $\begin{array}{l}7.914 \\
7.411 \\
7.764\end{array}$ & $\begin{array}{l}0.1742 \\
0.1894 \\
0.1883\end{array}$ & $\begin{array}{l}0.00447 \\
0.00447 \\
0.00447\end{array}$ & $\begin{array}{l}610 \\
610 \\
610\end{array}$ & $\begin{array}{l}\operatorname{TLAB}(j) \\
\operatorname{TLAB}(i) \\
\operatorname{WARD}(\mathrm{h}) \\
\operatorname{TLAB}(j)\end{array}$ & $\begin{array}{l}2.74 \\
2.76 \\
2.90 \\
2.80\end{array}$ & $\begin{array}{l}0.0803 \\
0.0797 \\
0.0828 \\
0.0786\end{array}$ & $\begin{array}{l}649 \\
649 \\
647 \\
649\end{array}$ & $\begin{array}{l}8.70 \\
7.26 \\
8.37 \\
8.00\end{array}$ & $\begin{array}{l}0.200 \\
0.224 \\
0.213 \\
0.214\end{array}$ & $\begin{array}{l}3.03 \\
3.16 \\
3.34\end{array}$ & $\begin{array}{l}28.1 \\
29.4 \\
\\
31.1\end{array}$ \\
\hline 3 & E6-C-f6 & 354 & $\begin{array}{l}2 \\
2 \\
3 \\
3\end{array}$ & $\begin{array}{l}G-125 \\
G-126\end{array}$ & $\begin{array}{l}7.531 \\
8.138\end{array}$ & $\begin{array}{l}0.1606 \\
0.1761\end{array}$ & $\begin{array}{l}0.00933 \\
0.00870\end{array}$ & $\begin{array}{l}708 \\
686\end{array}$ & $\begin{array}{l}\text { TLAB }(k) \\
\text { WARD }(h) \\
\operatorname{TLAB}(k) \\
\text { WARD }(h)\end{array}$ & $\begin{array}{l}2.45 \\
2.54 \\
2.77 \\
3.43\end{array}$ & $\begin{array}{l}0.0653 \\
0.0394 \\
0.0578 \\
0.0408\end{array}$ & $\begin{array}{r}714 \\
714 \\
714 \\
714 \\
714\end{array}$ & $\begin{array}{c}5.84 \\
6.51 \\
7.63 \\
13.0\end{array}$ & $\begin{array}{l}0.208 \\
0.118 \\
0.172 \\
0.0881\end{array}$ & $\begin{array}{l}2.94 \\
3.09\end{array}$ & $\begin{array}{l}27.3 \\
28.7\end{array}$ \\
\hline 1 & E5-SW-a5 & 15025 & $\begin{array}{l}3 \\
3\end{array}$ & G-111 & 5.351 & 0.0960 & 0.0114 & 1880 & $\begin{array}{l}\operatorname{TLAB}(k) \\
\operatorname{WARD}(\mathrm{h})\end{array}$ & $\begin{array}{l}3.31 \\
2.95\end{array}$ & $\begin{array}{l}0.0604 \\
0.0407\end{array}$ & $\begin{array}{l}1897 \\
1897\end{array}$ & $\begin{array}{l}2.27 \\
0.326\end{array}$ & $\begin{array}{l}0.481 \\
2.052\end{array}$ & 1.90 & 17.4 \\
\hline
\end{tabular}


TABLE D-3 (cont)

SUMMARY OF MEASUREMENTS FOR Am-241

\begin{tabular}{|c|c|c|c|c|c|c|c|c|c|c|c|c|c|c|c|c|}
\hline \multirow[b]{2}{*}{$\begin{array}{l}\text { RYC (a) } \\
\text { Phase }\end{array}$} & \multirow[b]{2}{*}{$\begin{array}{l}\text { Core } \mathrm{e}^{(\mathrm{b})} \\
\text { Location }\end{array}$} & \multirow[b]{2}{*}{ Rod No. } & \multirow[b]{2}{*}{$\begin{array}{l}\text { Axial } \\
\text { Sample } \\
\text { Zone }\end{array}$} & \multirow[b]{2}{*}{$\begin{array}{c}\text { Isotopic } \\
\text { Sampie } \\
\text { No. }\end{array}$} & \multirow[b]{2}{*}{$\begin{array}{l}\frac{\mathrm{N}^{49}}{\mathrm{~N}_{\mathrm{O}}^{28}} \\
\left(\mathrm{x} 10^{3}\right)\end{array}$} & \multicolumn{3}{|c|}{$\begin{array}{l}\text { Pu-241 } \\
\text { At Time of Analysis }\end{array}$} & \multicolumn{3}{|c|}{$\begin{array}{c}\text { Am-241 } \\
\text { At Time of Analysis }\end{array}$} & & \multicolumn{2}{|c|}{$\begin{array}{l}\mathrm{Am}-241 \\
\text { At }\end{array}$} & \multirow[b]{2}{*}{$\begin{array}{l}\frac{\mathrm{F}^{\mathrm{TOT}}}{\mathrm{N}_{\mathrm{O}}^{28}} \\
\left(\mathrm{x} 10^{2}\right)\end{array}$} & \multirow[b]{2}{*}{$\begin{array}{l}\text { Burnup } \\
\text { (GWD/MTU) }\end{array}$} \\
\hline & & & & & & $\frac{N^{41}}{N^{49}}$ & $\begin{array}{l}\text { Relative } \\
\text { Fractional } \\
\text { Error }\end{array}$ & $\begin{array}{c}\text { Elapsed } \\
\text { Time } \\
\text { (days) }\end{array}$ & Lab & $\begin{array}{l}\frac{N^{51}}{N^{49}} \\
\left(\times 10^{2}\right)\end{array}$ & $\begin{array}{l}\text { Relative } \\
\text { Fractional } \\
\text { Error }\end{array}$ & $\begin{array}{c}\text { Elapsed } \\
\text { T1me } \\
\text { (days) }\end{array}$ & $\left|\begin{array}{l}\frac{\mathrm{N}^{51}}{\mathrm{~N}^{28}} \\
\mathrm{o} \\
\left(\mathrm{x} 10^{5}\right)\end{array}\right|$ & $\begin{array}{l}\text { Relative } \\
\text { Fractional } \\
\text { Error }\end{array}$ & & \\
\hline 2 & F5-SE-f 5 & 8717 & $\begin{array}{l}3 \\
3\end{array}$ & G-122 & 6.571 & 0.1836 & 0.00892 & 1415 & $\begin{array}{l}\operatorname{TLAB}(k) \\
\operatorname{WARD}(h)\end{array}$ & $\begin{array}{l}4.37 \\
5.23\end{array}$ & $\begin{array}{l}0.0595 \\
0.0382\end{array}$ & $\begin{array}{l}1425 \\
1425\end{array}$ & $\begin{array}{l}2.31 \\
8.02\end{array}$ & $\begin{array}{l}0.750 \\
0.167\end{array}$ & 3.29 & 30.6 \\
\hline 3 & E6-NW-a1 & $?^{44}$ & $\begin{array}{l}3 \\
3 \\
4 \\
4\end{array}$ & $\begin{array}{l}\text { G-127 } \\
\text { G-128 }\end{array}$ & $\begin{array}{l}6.112 \\
6.027\end{array}$ & $\begin{array}{l}0.2644 \\
0.2709\end{array}$ & $\begin{array}{l}0.00993 \\
0.00856\end{array}$ & $\begin{array}{l}708 \\
708\end{array}$ & $\begin{array}{l}\operatorname{TLAB}(k) \\
\operatorname{WARD}(h) \\
\operatorname{TLAB}(k) \\
\operatorname{WARD}(h)\end{array}$ & $\begin{array}{l}3.77 \\
4.26 \\
3.84 \\
5.34\end{array}$ & $\begin{array}{l}0.0584 \\
0.0422 \\
0.0573 \\
0.0374\end{array}$ & $\begin{array}{l}712 \\
712 \\
712 \\
712\end{array}$ & \begin{tabular}{|c|}
6.23 \\
9.23 \\
6.15 \\
15.2
\end{tabular} & $\begin{array}{l}0.219 \\
0.120 \\
0.218 \\
0.0802\end{array}$ & $\begin{array}{l}4.55 \\
4.38\end{array}$ & $\begin{array}{l}42.7 \\
41.1\end{array}$ \\
\hline & E6-SE-f5 & $\begin{array}{l}3113 \\
\vdots\end{array}$ & $\begin{array}{l}3 \\
3 \\
3 \\
3\end{array}$ & $G-124^{(g)}$ & 6.141 & 0.2550 & 0.00870 & 708 & $\begin{array}{l}\text { TLAB (i) } \\
\text { WARD (h) } \\
\text { TLAB }(k) \\
\text { WARD (h) }\end{array}$ & $\begin{array}{l}3.39 \\
3.10 \\
3.47 \\
3.91\end{array}$ & $\begin{array}{l}0.0826 \\
0.0774 \\
0.0576 \\
0.0409\end{array}$ & $\begin{array}{l}649 \\
647 \\
718 \\
718\end{array}$ & $\begin{array}{l}5.90 \\
4.15 \\
6.39 \\
7.61\end{array}$ & $\begin{array}{l}0.294 \\
0.358 \\
0.194 \\
0.131\end{array}$ & 4.50 & 42.1 \\
\hline
\end{tabular}

Notes: (a) Phase 1 samples were irradiated in Yankee Core I; Phase 2 samples were irradiated in Cores I and II; Phase 3 samples were irradiated in

(b) Subassembly designation $\mathrm{C}$ indicates asymptotic neutron spectrum; subassembly designations NW, SE, and SW indicate non-asymptotic neutron

(c) The letters $T$ and $G$ indicate mass spectrometric analysis for main chain $U$ and Pu isotopes by TRACERLAB and General Electric-Vallecitos, respectively.

(d) From core end-of-life to time of analysis. $0.00000415 \mathrm{~d}^{-1}$ and $0.000140 \mathrm{~d}^{-1}$, respectively.

(f) EYC Phase 1 control sample.

(g) EYC Phase 3 control sample.

(i) Measurements by TRACERLAB; Am by alpha spectrometry and Pu by mass spectrometry.

(j) From TRACERLAB alpha spectrometry for Am as $\mathrm{dpm} / \mathrm{m} 1$, and use of gross alpha spectrometry on sample measured at WARD.

(k) From TRACERLAB measurements for Am-241 and Cm-244 dpm/ml; TRACERLAB data converted using Cm-244/Pu-239 atom ratio measured by wARD. 
TABLE D-4

SUMMARY OF MEASUREMENTS FOR Am-243

\begin{tabular}{|c|c|c|c|c|c|c|c|c|c|c|}
\hline $\begin{array}{l}\text { EYC (a) } \\
\text { Phase }\end{array}$ & $\begin{array}{l}\text { Core }{ }^{(b)} \\
\text { Location }\end{array}$ & Rod No. & $\begin{array}{l}\text { Axial } \\
\text { Sample } \\
\text { Zone }\end{array}$ & $\begin{array}{l}\text { Isotopic (c) } \\
\text { Sample } \\
\text { No. }\end{array}$ & $\begin{array}{l}\frac{\mathrm{N}^{49}}{\mathrm{~N}_{\mathrm{O}}^{28}} \\
\left(\mathrm{x} 10^{2}\right)\end{array}$ & 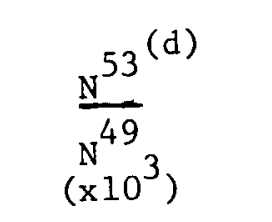 & $\frac{\mathrm{N}^{53}}{\mathrm{~N}_{0}^{28}}$ & & $\begin{array}{l}\frac{\mathrm{F}^{\mathrm{TOT}}}{\mathrm{N}_{\mathrm{O}}^{28}} \\
\left(\mathrm{x} 10^{2}\right)\end{array}$ & $\begin{array}{l}\text { Burnup } \\
\text { (GWD/MTU) }\end{array}$ \\
\hline \multirow[t]{5}{*}{3} & \multirow[t]{2}{*}{$E 6-C-f 6$} & \multirow[t]{2}{*}{354} & 2 & $G-125$ & 0.7530 & $3.34 \pm 1.52$ & 2.52 & \pm 1.15 & 2.94 & 27.3 \\
\hline & & & 3 & $G-126$ & 0.8137 & $5.14 \pm 4.06$ & 4.18 & \pm 3.30 & 3.09 & 28.7 \\
\hline & E6-NW-a1 & 44 & 4 & G-128 & 0.6027 & $35.8 \pm 6.0$ & 21.6 & \pm 3.62 & 4.38 & 41.1 \\
\hline & \multirow[t]{2}{*}{ E6-SE-f5 } & \multirow[t]{2}{*}{3113 . } & 3 & $G-124$ & 0.6141 & $9.46 \pm 2.14$ & 5.81 & \pm 1.31 & 4.50 & 42.1 \\
\hline & & & 3 & & & $8.94 \pm 4.56$ & 5.49 & \pm 2.80 & & \\
\hline
\end{tabular}

Notes: (a) Phase 3 samples were irradiated in Yankee Cores I, II, and IV.

(b) Subassembly designation $C$ indicates asymptotic neutron spectrum; subassembly designation NW and SE indicates non-asymptotic neutron spectra.

(c) The letter $G$ indicates mass spectrometric analvsis for main chain $U$ and $P u$ isotopes by General Electric-Vallecitos.

(d) From alpha spectrometric analysis by WARD; uncertainties are $2 \sigma$. 


\begin{tabular}{|c|c|c|c|c|c|c|c|c|c|c|c|c|c|c|c|c|c|}
\hline \multirow[b]{2}{*}{$\begin{array}{l}\text { EYC } \\
\text { Phase }\end{array}$} & \multirow[b]{2}{*}{$\begin{array}{l}\text { Core }^{(b)} \\
\text { Location }\end{array}$} & \multirow[b]{2}{*}{ Rod No. } & \multirow[b]{2}{*}{$\begin{array}{l}\text { Axial } \\
\text { Sample } \\
\text { Zone }\end{array}$} & \multirow[b]{2}{*}{$\begin{array}{l}\text { Isotopic } \\
\text { Sample } \\
\text { No. }\end{array}$} & \multirow[b]{2}{*}{$\begin{array}{l}\frac{\mathrm{N}^{49}}{\mathrm{~N}_{\mathrm{o}}^{28}} \\
\left(\mathrm{x} 10^{3}\right)\end{array}$} & \multicolumn{6}{|c|}{$\begin{array}{l}\mathrm{Cm}-242 \text { and } \mathrm{Cm}-244 \\
\text { At Time of Analysis }\end{array}$} & \multicolumn{4}{|c|}{$\begin{array}{l}\mathrm{Cm}-242 \text { and } \mathrm{Cm}-244 \\
\text { at Core EOL }(\mathrm{e})\end{array}$} & \multirow[b]{2}{*}{$\begin{array}{l}\frac{\mathrm{F}^{\mathrm{TOT}}}{\mathrm{N}_{\mathrm{O}}^{28}} \\
\left(\mathrm{x} 10^{2}\right)\end{array}$} & \multirow[b]{2}{*}{$\begin{array}{l}\text { Burnup } \\
\text { (GWD/MTU) }\end{array}$} \\
\hline & & & & & & Lab & 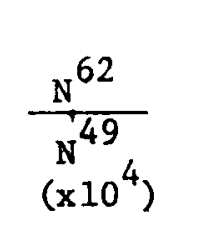 & $\begin{array}{l}\text { Relative } \\
\text { Fractional } \\
\text { Error on } \\
\mathrm{N}^{62} / \mathrm{N}^{49}\end{array}$ & 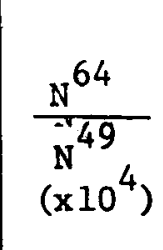 & $\begin{array}{l}\text { Relative } \\
\text { Fractional } \\
\text { Error on } \\
\mathrm{N}^{64} / \mathrm{N}^{49}\end{array}$ & $\begin{array}{l}\text { Elapsed } \\
\text { Time } \\
\text { (days) }\end{array}$ & $\frac{N^{62}}{N^{49}}$ & 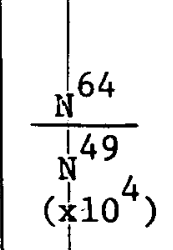 & $\begin{array}{l}\frac{\mathrm{N}^{62}}{\mathrm{~N}_{0}^{28}} \\
\left(\mathrm{x} 10^{5}\right)\end{array}$ & $\begin{array}{l}\frac{N^{64}}{N_{0}^{28}} \\
\left(x 10^{5}\right)\end{array}$ & & \\
\hline \multirow[t]{3}{*}{1} & G4-C-a 1 & 0994 & 3 & $T-161^{(f)}$ & 5.364 & WARD (h) & 0.000585 & 0.588 & 0.464 & 0.0948 & 2373 & 12.9 & $0 ! 595$ & 0.694 & 0.0319 & 1.27 & 11.5 \\
\hline & F4-C-f1 & 8977 & $\begin{array}{l}4 \\
4 \\
5 \\
5\end{array}$ & $\begin{array}{l}G-114 \\
G-115\end{array}$ & $\begin{array}{l}5.888 \\
5.556\end{array}$ & $\begin{array}{l}\operatorname{TLAB}(k) \\
\operatorname{WARD}(\mathrm{h}) \\
\operatorname{TLAB}(k) \\
\operatorname{WARD}(\mathrm{h})\end{array}$ & $\begin{array}{l}0.00164 \\
0.00254 \\
0.00159 \\
0.00168\end{array}$ & $\begin{array}{l}0.0732 \\
0.236 \\
0.0755 \\
0.357\end{array}$ & $\begin{array}{l}0.96 \\
0.96 \\
0.84 \\
0.84\end{array}$ & $\begin{array}{l}0.0625 \\
0.0833 \\
0.0714 \\
0.119\end{array}$ & $\begin{array}{l}1910 \\
1910 \\
1910 \\
1910\end{array}$ & $\begin{array}{l}5.15 \\
7.98 \\
5.00 \\
5.28\end{array}$ & $1 \prod_{0}^{2}$ & $\begin{array}{l}0.303 \\
0.470 \\
0.278 \\
0.293\end{array}$ & $\begin{array}{l}0.069 \\
0.057\end{array}$ & $\begin{array}{l}1.46 \\
1.44\end{array}$ & $\begin{array}{l}13.3 \\
13.2\end{array}$ \\
\hline & E5-C-a6 & 7992 & $\begin{array}{l}3 \\
5 \\
6\end{array}$ & $\begin{array}{l}G-101 \\
G-102 \\
G-103\end{array}$ & $\begin{array}{l}5.605 \\
5.253 \\
4.082\end{array}$ & $\begin{array}{l}\operatorname{TLAB}(j) \\
\operatorname{TLAB}(j) \\
\operatorname{TLAB}(j)\end{array}$ & $\begin{array}{l}0.00157 \\
0.00204 \\
0.000669\end{array}$ & $\begin{array}{l}0.0637 \\
0.0588 \\
0.138\end{array}$ & $\begin{array}{l}0.765 \\
0.643 \\
0.094\end{array}$ & $\begin{array}{l}0.0810 \\
0.0809 \\
0.128\end{array}$ & $\begin{array}{l}1828 \\
1828 \\
1828\end{array}$ & $\begin{array}{l}3.49 \\
4.54 \\
1.49\end{array}$ & $\begin{array}{l}0,927 \\
0 ! 779 \\
0: 11\end{array}$ & $\begin{array}{l}0.196 \\
0.238 \\
0.0607\end{array}$ & $\begin{array}{l}0.0519 \\
0.0409 \\
0.0046\end{array}$ & $\begin{array}{l}1.42 \\
1.37 \\
0.962\end{array}$ & $\begin{array}{c}12.9 \\
12.5 \\
8.71\end{array}$ \\
\hline \multirow[t]{2}{*}{$2 \cdot$} & F5-C-a 1 & 553 & $\begin{array}{l}1 \\
3 \\
3\end{array}$ & $\begin{array}{l}\text { G-104 } \\
\text { G-105 }\end{array}$ & $\begin{array}{l}4.228 \\
7.600\end{array}$ & $\begin{array}{l}\operatorname{TLAB}(j) \\
\operatorname{TLAB}(1) \\
\operatorname{WARD}(h)\end{array}$ & $\begin{array}{l}0.00696 \\
0.0368 \\
0.0379\end{array}$ & $\begin{array}{l}0.0603 \\
0.0598 \\
0.0580\end{array}$ & $\begin{array}{l}0.179 \\
8.61 \\
8.21\end{array}$ & $\begin{array}{l}0.0447 \\
0.0372 \\
0.0390\end{array}$ & $\begin{array}{l}1353 \\
1353 \\
1353\end{array}$ & $\begin{array}{l}2.09 \\
11.0 \\
11.4\end{array}$ & $\begin{array}{l}0.206 \\
9.93 \\
9.46\end{array}$ & $\begin{array}{l}0.0883 \\
0.839 \\
0.864\end{array}$ & $\begin{array}{l}0.00872 \\
0.754 \\
0.719\end{array}$ & $\begin{array}{l}1.02 \\
2.50\end{array}$ & $\begin{array}{c}9.27 \\
23.1\end{array}$ \\
\hline & F5-C-f6 & 541 & $\begin{array}{l}1 \\
2 \\
2 \\
4 \\
4 \\
6 \\
6\end{array}$ & $\begin{array}{l}\text { G-106 } \\
\text { G-118 } \\
\text { G-119 } \\
\text { G-121 }\end{array}$ & $\begin{array}{l}4.670 \\
7.024 \\
7.018 \\
5.708\end{array}$ & $\begin{array}{l}\operatorname{TLAB}(j) \\
\operatorname{TLAB}(\mathrm{k}) \\
\operatorname{WARD}(\mathrm{h}) \\
\operatorname{TLAB}(\mathrm{k}) \\
\text { WARD (h) } \\
\operatorname{TLAB}(\mathrm{k}) \\
\operatorname{WARD}(\mathrm{h})\end{array}$ & $\begin{array}{l}0.00817 \\
0.0253 \\
0.0251 \\
0.0344 \\
0.0356 \\
0.0153 \\
0.0136\end{array}$ & $\begin{array}{l}0.0588 \\
0.0553 \\
0.0398 \\
0.0523 \\
0.0337 \\
0.0654 \\
0.0441\end{array}$ & \begin{tabular}{|l}
0.274 \\
4.68 \\
4.68 \\
10.6 \\
10.6 \\
1.56 \\
1.56
\end{tabular} & $\begin{array}{l}0.0365 \\
0.0598 \\
0.0385 \\
0.0566 \\
0.0377 \\
0.0513 \\
0.0256\end{array}$ & $\begin{array}{l}1356 \\
1421 \\
1421 \\
1424 \\
1424 \\
1425 \\
1425\end{array}$ & $\begin{array}{l}2.48 \\
10.1 \\
10.0 \\
13.9 \\
14.4 \\
6.22 \\
5.53\end{array}$ & $\begin{array}{c:c}0 & 316 \\
5 & 43 \\
12 & 31 \\
1 & 81\end{array}$ & $\begin{array}{l}0.116 \\
0.710 \\
0.705 \\
0.977 \\
1.01 \\
0.355 \\
0.316\end{array}$ & $\begin{array}{l}0.0148 \\
0.382 \\
0.864 \\
0.103\end{array}$ & $\begin{array}{l}1.00 \\
2.27 \\
2.62 \\
1.62\end{array}$ & $\begin{array}{l}9.09 \\
20.9 \\
24.2 \\
14.8\end{array}$ \\
\hline 3 & E6-C-al & 369 & $\begin{array}{l}2 \\
3 \\
3 \\
5\end{array}$ & $\begin{array}{r}G-107 \\
G-108 \\
G-109\end{array}$ & $\begin{array}{l}7.914 \\
7.411 \\
7.764\end{array}$ & $\begin{array}{l}\operatorname{TLAB}(j) \\
\operatorname{TLAB}(i) \\
\operatorname{WARD}(h) \\
\operatorname{TLAB}(j)\end{array}$ & $\begin{array}{l}1.54 \\
1.76 \\
1.74 \\
1.72\end{array}$ & $\begin{array}{l}0.0390 \\
0.0341 \\
0.0345 \\
0.0349\end{array}$ & $\begin{array}{l}23.0 \\
29.1 \\
29.9 \\
30.7\end{array}$ & $\begin{array}{l}0.0435 \\
0.0412 \\
0.0401 \\
0.0391\end{array}$ & $\begin{array}{l}649 \\
649 \\
647 \\
649\end{array}$ & $\begin{array}{l}23.8 \\
27.2 \\
26.6 \\
26.5\end{array}$ & $\begin{array}{l}24: 6 \\
31 ! 2 \\
32 ! 0 \\
32 ! 9\end{array}$ & $\begin{array}{l}1.88 \\
2.01 \\
1.97 \\
2.06\end{array}$ & $\begin{array}{l}1.95 \\
2.31 \\
2.37 \\
2.55\end{array}$ & $\begin{array}{l}3.03 \\
3.16 \\
\\
3.34\end{array}$ & $\begin{array}{l}28.1 \\
29.4 \\
31.1\end{array}$ \\
\hline 3 & E6-C-f6 & 354 & $\begin{array}{l}2 \\
2 \\
3 \\
3\end{array}$ & $\begin{array}{l}G-125 \\
G-126\end{array}$ & $\begin{array}{l}7.531 \\
8.138\end{array}$ & $\begin{array}{l}\operatorname{TLAB}(k) \\
\operatorname{WARD}(h) \\
\operatorname{TLAB}(k) \\
\operatorname{WARD}(h)\end{array}$ & $\begin{array}{l}1.18 \\
1.16 \\
1.32 \\
1.28\end{array}$ & $\begin{array}{l}0.0508 \\
0.0345 \\
0.0606 \\
0.0312\end{array}$ & $\begin{array}{l}20.5 \\
20.5 \\
27.2 \\
27.2\end{array}$ & $\begin{array}{l}0.0585 \\
0.0390 \\
0.0588 \\
0.0368\end{array}$ & $\begin{array}{l}714 \\
714 \\
714 \\
714\end{array}$ & $\begin{array}{l}23.9 \\
23.5 \\
26.8 \\
26.0\end{array}$ & 22.1 & $\begin{array}{l}1.80 \\
1.77 \\
2.18 \\
2.11\end{array}$ & $\begin{array}{l}1.66 \\
2.39\end{array}$ & $\begin{array}{l}2.94 \\
3.09\end{array}$ & $\begin{array}{l}27.3 \\
28.7\end{array}$ \\
\hline
\end{tabular}


TABLE D-5 (cont)

SUMMARY OF MEASUREMENTS FOR Cm-242 AND Cm-244

\begin{tabular}{|c|c|c|c|c|c|c|c|c|c|c|c|c|c|c|c|c|c|}
\hline \multirow[b]{2}{*}{$\begin{array}{l}\text { EYC } \\
\text { Phase }\end{array}$} & \multirow[b]{2}{*}{$\begin{array}{l}\text { Core }{ }^{(b)} \\
\text { Location }\end{array}$} & \multirow[b]{2}{*}{ Rod No. } & \multirow[b]{2}{*}{$\begin{array}{l}\text { Axial } \\
\text { Sample } \\
\text { Zone }\end{array}$} & \multirow[b]{2}{*}{$\begin{array}{l}\text { Isotopic } \\
\text { Sample } \\
\text { No. }\end{array}$} & \multirow[b]{2}{*}{$\begin{array}{l}\frac{N^{49}}{N_{0}^{28}} \\
\left(\times 10^{3}\right)\end{array}$} & \multicolumn{6}{|c|}{$\begin{array}{l}\mathrm{Cm}-242 \text { and } \mathrm{Cm}-244 \\
\text { At Time of Analysis }\end{array}$} & \multicolumn{4}{|c|}{$\begin{array}{l}\mathrm{Cm}-242 \text { and } \mathrm{Cm}-244 \\
\text { at Core EOL }\end{array}$} & \multirow[b]{2}{*}{$\begin{array}{l}\frac{F^{\text {TOT }}}{N_{0}^{28}} \\
\left(x 10^{2}\right)\end{array}$} & \multirow[b]{2}{*}{$\begin{array}{l}\text { Burnup } \\
\text { (GWD/MTU) }\end{array}$} \\
\hline & & & & & & Lab & $\frac{\frac{N^{62}}{N^{49}}}{\left(\times 10^{4}\right)}$ & $\begin{array}{l}\text { Relative } \\
\text { Fractional } \\
\text { Error on } \\
\mathrm{N}^{62} / \mathrm{N}^{49}\end{array}$ & $\begin{array}{l}\frac{N^{64}}{N^{49}} \\
\left(\times 10^{4}\right)\end{array}$ & $\begin{array}{l}\text { Relative } \\
\text { Fractional } \\
\text { Error on } \\
\mathrm{N}^{64} / \mathrm{N}^{49}\end{array}$ & $\begin{array}{c}\text { Elapsed } \\
\text { Time } \\
\text { (days) }\end{array}$ & 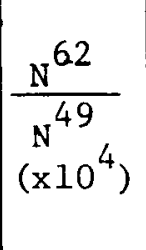 & $\frac{N^{64}}{N^{49}}\left(\begin{array}{l}\left.x^{4}\right) \\
\left(10^{4}\right)\end{array}\right.$ & $\begin{array}{l}\frac{\mathrm{N}^{62}}{\mathrm{~N}_{0}^{28}} \\
\left(\mathrm{x} 10^{5}\right)\end{array}$ & $\begin{array}{l}\frac{N^{64}}{N_{0}^{28}} \\
\left(x 10^{5}\right)\end{array}$ & & \\
\hline 1 & E5-SW-a5 & 15025 & $\begin{array}{l}3 \\
3\end{array}$ & G-111 & 5.350 & $\begin{array}{l}\text { TLAB (k) } \\
\operatorname{WARD}(\mathrm{h})\end{array}$ & \begin{tabular}{l|l}
0.00187 \\
0.00204
\end{tabular} & $\begin{array}{l}0.0642 \\
0.235\end{array}$ & $\begin{array}{l}1.56 \\
1.56\end{array}$ & $\begin{array}{l}0.0641 \\
0.0385\end{array}$ & $\begin{array}{l}1897 \\
1897\end{array}$ & $\begin{array}{l}5.56 \\
6.07\end{array}$ & 1.90 & $\begin{array}{l}0.298 \\
0.325\end{array}$ & 0.102 & 1.90 & 17.4 \\
\hline 2 & F5-SE-f5 & 8717 & $\begin{array}{l}3 \\
3\end{array}$ & $G-122$ & 6.572 & $\begin{array}{l}\text { TLAB (k) } \\
\operatorname{WARD}(\mathrm{h})\end{array}$ & \begin{tabular}{|l|}
0.0586 \\
0.0581
\end{tabular} & $\begin{array}{l}0.0512 \\
0.0344\end{array}$ & $\begin{array}{l}21.1 \\
21.1\end{array}$ & $\begin{array}{l}0.0569 \\
0.0379\end{array}$ & $\begin{array}{l}1425 \\
1425\end{array}$ & $\begin{array}{l}23.8 \\
23.6\end{array}$ & 24.5 & $\begin{array}{l}1.56 \\
1.55\end{array}$ & 1.61 & 3.29 & 30.6 \\
\hline 3 & E6-NW-a 1 & 44 & $\begin{array}{l}3 \\
3 \\
4 \\
4\end{array}$ & $\begin{array}{l}G-127 \\
G-128\end{array}$ & $\begin{array}{l}6.120 \\
6.035\end{array}$ & $\begin{array}{l}\text { TLAB (k) } \\
\text { WARD (h) } \\
\text { TLAB (k) } \\
\text { WARD (h) }\end{array}$ & \begin{tabular}{|l|}
2.78 \\
2.61 \\
2.78 \\
2.72
\end{tabular} & $\begin{array}{l}0.0576 \\
0.0383 \\
0.0576 \\
0.0368\end{array}$ & $\begin{array}{l}104 . \\
104 . \\
105 . \\
105 .\end{array}$ & $\begin{array}{l}0.0577 \\
0.0577 \\
0.0571 \\
0.0571\end{array}$ & $\begin{array}{l}712 \\
712 \\
712 \\
712\end{array}$ & $\begin{array}{l}55.9 \\
52.5 \\
55.9 \\
54.7\end{array}$ & $\begin{array}{l}112 . \\
113 .\end{array}$ & $\begin{array}{l}3.42 \\
3.21 \\
3.38 \\
3.30\end{array}$ & $\begin{array}{l}6.86 \\
6.83\end{array}$ & $\begin{array}{l}4.55 \\
4.38\end{array}$ & $\begin{array}{l}42.7 \\
41.1\end{array}$ \\
\hline & E6-SE-f5 & 3113 & $\begin{array}{l}3 \\
3 \\
3 \\
3\end{array}$ & $G-124^{(g)}$ & 6.148 & $\begin{array}{l}\text { TLAB (i) } \\
\text { WARD (h) } \\
\text { TLAB (k) } \\
\text { WARD (h) }\end{array}$ & \begin{tabular}{|l|}
3.19 \\
3.25 \\
2.46 \\
2.39
\end{tabular} & $\begin{array}{l}0.0376 \\
0.0431 \\
0.0569 \\
0.0418\end{array}$ & $\begin{array}{l}93.2 \\
90.6 \\
88.9 \\
88.9\end{array}$ & $\begin{array}{l}0.0386 \\
0.0397 \\
0.0540 \\
0.0405\end{array}$ & $\begin{array}{l}649 \\
647 \\
718 \\
718\end{array}$ & $\begin{array}{l}48.8 \\
49.7 \\
37.6 \\
49.3\end{array}$ & $\begin{array}{l}99.8 \\
97.8 \\
95.8\end{array}$ & $\begin{array}{l}3.00 \\
3.06 \\
2.31 \\
3.03\end{array}$ & $\begin{array}{l}6.13 \\
5.96 \\
5.89\end{array}$ & 4.50 & 42.1 \\
\hline
\end{tabular}

Notes: (a) Phase I samples were irradiated in Yankee Core I; Phase 2 samples were irradiated in Cores I and II; Phase 3 samples were irradiated in Cores I, II, and IV.

(b) Subassembly designation $C$ indicates asymptotic neutron spectrum; subassembly designations NW, SE, and SW indicate non-asymptotic

(c) The letters $T$ and $G$ indicate mass spectrometric analysis for main chain $U$ and Pu isotopes by TRACERLAB and General Electric-Vallecitos,

(d) From core end-of-life to time of analysis; the results of cross check analysis for $\mathrm{dpm} / \mathrm{m} 1$ by TRACERLAB are referenced to WARD time of analysis

(e) Phase 1,2 , and 3 data are referenced to Core I, Core II, and Core IV end-of-1ife, respectively. Decay constants for Cm-242 are $0.004216 \mathrm{~d}^{-1}$ and $0.000105 \mathrm{~d}^{-1}$, respectively.

(f) EYC Phase 1 control sample.

(g) EYC Phase 3 control sample.

(h) From alpha spectrometric measurements by WARD.

(i) Measurements by TRACERLAB; Cm by alpha spectrometry and Pu by mass spectrometry.

metry on sample measured at WARD.

(k) From 